\title{
Análise de um modelo de regressão com erros nas variáveis multivariado com intercepto nulo
}

\author{
Cibele Maria Russo
}

Orientadora: $\operatorname{Prof}^{a}$. Dr ${ }^{a}$. Reiko Aoki

Dissertação apresentada ao Instituto de Ciências Matemáticas e de Computação (ICMC-USP), como parte dos requisitos para obtenção do título de Mestre em Ciências de Computação e Matemática Computacional.

USP - São Carlos

Maio / 2006 
Análise de um modelo de regressão com erros nas variáveis multivariado com intercepto nulo 
Análise de um modelo de regressão com erros nas variáveis multivariado com intercepto nulo 



\section{Agradecimentos}

Agradeço a Deus, por não me abandonar um minuto sequer nesta jornada, mesmo em momentos em que duvidei de Sua existência.

A meus pais Luiz e Izabel, e meus irmãos Luiz Roberto, Renato e Bruna, pelo apoio e companheirismo fora do comum, e por não deixarem nunca de acreditar que eu conseguiria.

À minha orientadora Reiko pela dedicação e paciência em nossas reuniões, pela sensatez com que me guiou neste trabalho, pelo dinamismo em suas aulas, pela amizade e pelos conselhos, que foram essenciais para minhas decisões acadêmicas e profissionais.

A pessoas especiais que fizeram parte da minha vida durante esses anos: ao Vladimir e sua família, que estiveram sempre presentes incondicionalmente, aos meus amigos de graduação e pós-graduação, em especial à Márcia, Renato, Amanda, Daniel, Gecirlei e Fabrizio. Aos meus amigos de sempre Cristiane, Ângela, Ana, Rodrigo, Thiago, Cassio e Creu. A todos os amigos que fiz durante minha estada em São Carlos, em especial àqueles que me fizeram ser uma pessoa melhor.

Aos meus professores, em especial à Míriam, Sandra, Hildebrando, Ladeira, Dorival e Mário, por me darem exemplos extraordinários de como se deve ser professor.

Aos funcionários do ICMC, por tornarem o trabalho menos desgastante. Em especial aos seguranças dos laboratórios pela companhia e amizade.

Ao CNPQ pelo financiamento concedido para o desenvolvimento deste trabalho.

Finalmente, à Universidade de São Paulo, por me permitir uma boa formação. 
Análise de um modelo de regressão com erros nas variáveis multivariado com intercepto nulo 


\section{Resumo}

Para analisar características de interesse a respeito de um conjunto de dados reais da área de Odontologia apresentado em Hadgu \& Koch (1999), ajustaremos um modelo de regressão linear multivariado com erros nas variáveis com intercepto nulo. Este conjunto de dados é caracterizado por medições de placa bacteriana em três grupos de voluntários, antes e após utilizar dois líquidos de bochecho experimentais e um líquido de bochecho controle, com medições (sujeitas a erros de medição) no início do estudo, após três e seis meses de utilização dos líquidos. Neste caso, uma possível estrutura de dependência entre as medições feitas em um mesmo indivíduo deve ser incorporada ao modelo e, além disto, temos duas variáveis resposta para cada indivíduo. Após a apresentação do modelo estatístico, iremos obter estimativas de máxima verossimilhança dos parâmetros utilizando o algoritmo iterativo EM e testaremos as hipóteses de interesse utilizando testes assintóticos de Wald, razão de verossimilhanças e score. Como neste caso não existe um teste ótimo, faremos um estudo de simulação para verificar o comportamento das três estatísticas de teste em relação a diferentes tamanhos amostrais e diferentes valores de parâmetros. Finalmente, faremos um estudo de diagnóstico buscando identificar possíveis pontos influentes no modelo, considerando o enfoque de influência local proposto por Cook (1986) e a medida de curvatura normal conformal desenvolvida por Poon \& Poon (1999). 


\section{Abstract}

To analyze some characteristics of interest in a real odontological data set presented in Hadgu \& Koch (1999), we propose the use of a multivariate null intercept errors-in-variables regression model. This data set is composed by measurements of dental plaque index (with measurement errors), which were measured in volunteers who were randomized to two experimental mouth rinses (A and $\mathrm{B}$ ) or a control mouth rinse. The measurements were taken in each individual, before and after the use of the respective mouth rinses, in the beginning of the study, after three months from the baseline and after six months from the baseline. In this case, a possible structure of dependency between the measurements taken within the same individual must be incorporated in the model. After presenting the statistical model, we obtain the maximum likelihood estimates of the parameters using the numerical algorithm EM, and we test the hypotheses of interest considering asymptotic tests (Wald, likelihood ratio and score). Also, a simulation study to verify the behavior of these three test statistics is presented, considering different sample sizes and different values for the parameters. Finally, we make a diagnostic study to identify possible influential observations in the model, considering the local influence approach proposed by Cook (1986) and the conformal normal curvature proposed by Poon \& Poon (1999). 


\section{Sumário}

$\begin{array}{lr}\text { Agradecimentos } & 3\end{array}$

$\begin{array}{lr}\text { Resumo } & 5\end{array}$

$\begin{array}{ll}\text { Abstract } & 7\end{array}$

1 Introdução $\quad 19$

2 O modelo estatístico $\quad 23$

2.1 O conjunto de dados . . . . . . . . . . . . . . . . . . . . . . . 23

2.1 .1 Descrição dos dados . . . . . . . . . . . . . . . . . . . 23

2.1 .2 Objetivo do estudo . . . . . . . . . . . . . . . . . . . 24

2.2 Modelo de regressão com erros nas variáveis . . . . . . . . . . . . . . . 25

2.3 Função escore . . . . . . . . . . . . . . . . . . . . . . . . . . . . . . . . . . 28

3 Estimação dos parâmetros $\quad 31$

3.1 Algoritmo EM . . . . . . . . . . . . . . . . . . . . . . . . . 31

3.2 Desenvolvimento do algoritmo EM . . . . . . . . . . . . . . . . . 32

4 Distribuição assintótica dos estimadores de máxima verossimilhança $\quad 37$

5 Testes de hipóteses assintóticos $\quad 45$

5.1 Estimativas de máxima verossimilhança sob $H_{0} \ldots \ldots \ldots$. . . . . . . . . 48

5.2 Resultados e conclusões sobre as hipóteses de interesse . . . . . . . . . . . . 53

$\begin{array}{lll}6 & \text { Estudos de simulação } & 57\end{array}$

6.1 Simulação do tamanho dos testes assintóticos . . . . . . . . . . . . . . 58

6.1.1 Conclusões das simulações do tamanho dos testes assintóticos . . . . . . 58

6.1 .2 Gráficos de quantis . . . . . . . . . . . . . . . . . . . . 59

6.2 Simulação do poder dos testes assintóticos . . . . . . . . . . . . . . . . 67 
6.2.1 Conclusões das simulações do poder dos testes assintóticos . . . . . . . 68

7 Diagnóstico de influência local $\quad 71$

7.1 Influência local de Cook (1986) . . . . . . . . . . . . . . . 73

7.1.1 Ponderação de casos . . . . . . . . . . . . . . . . . . . 75

7.1 .2 Perturbação na variável explanatória . . . . . . . . . . . . 76

7.1.3 Perturbação nas variáveis resposta . . . . . . . . . . . . . 76

7.1.4 Perturbação na variância dos erros de medição no início do estudo . . . . 77

7.1.5 Perturbação nas variâncias dos erros após o uso dos líquidos . . . . . . . 78

7.1.6 Aplicação Numérica . . . . . . . . . . . . . . . . . . . . . 79

7.2 Exclusão de casos . . . . . . . . . . . . . . . . . . . . . 88

7.3 Novos enfoques para influência local . . . . . . . . . . . . . . . . . 91

7.3.1 Curvatura normal conformal . . . . . . . . . . . . . . . 93

7.4 Conclusões dos estudos de diagnóstico de influência . . . . . . . . . . . . 108

$\begin{array}{ll}\text { Conclusões } & 111\end{array}$

$\begin{array}{ll}\text { Referências Bibliográficas } & 118\end{array}$

$\begin{array}{ll}\text { A Conjunto de Dados } & 119\end{array}$

B Derivadas vetoriais e propriedades $\quad 123$

B.1 Notação . . . . . . . . . . . . . . . . . . . . . . . 123

B.2 Definições . . . . . . . . . . . . . . . . . . . . . . 124

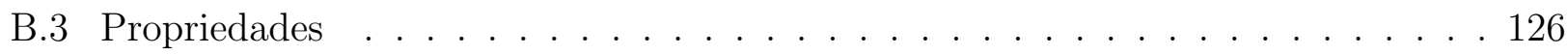

$\begin{array}{ll}\text { C Cálculo da função escore } & 137\end{array}$

$\begin{array}{ll}\text { D Matriz de informação observada } & 141\end{array}$

$\begin{array}{ll}\text { E Resultados das simulações do tamanho do teste } & 145\end{array}$

F Resultados das simulações do poder do teste $\quad 173$ 


\section{Lista de Figuras}

2.1 Índice médio de placa dentária no início do estudo e após três e seis meses . . . 25

6.1 Gráficos de quantis para $\sigma^{2}=0.01$ e $\sigma_{x}^{2}=0.01$ para o teste $H_{0}: \beta_{11}=\beta_{13}$ versus $H_{0}: \beta_{11} \neq \beta_{13}$ com $\mu=2.5 \ldots \ldots \ldots \ldots$. . . . . . . . . . . . . . .

6.2 Gráficos de quantis para $\sigma^{2}=0.01$ e $\sigma_{x}^{2}=0.1$ para o teste $H_{0}: \beta_{11}=\beta_{13}$ versus $H_{0}: \beta_{11} \neq \beta_{13} \operatorname{com} \mu=2.5 \ldots \ldots \ldots \ldots \ldots$. . . . . . . . . . . . . . .

6.3 Gráficos de quantis para $\sigma^{2}=0.05$ e $\sigma_{x}^{2}=0.1$ para o teste $H_{0}: \beta_{11}=\beta_{13}$ versus

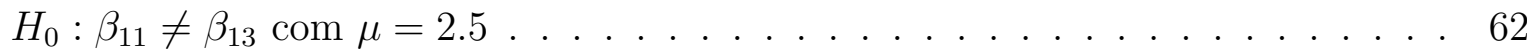

6.4 Gráficos de quantis para $\sigma^{2}=0.1$ e $\sigma_{x}^{2}=0.1$ para o teste $H_{0}: \beta_{11}=\beta_{13}$ versus $H_{0}: \beta_{11} \neq \beta_{13}$ com $\mu=2.5 \ldots \ldots \ldots \ldots \ldots$

6.5 Gráficos de quantis para $\sigma^{2}=0.01$ e $\sigma_{x}^{2}=0.5$ para o teste $H_{0}: \beta_{11}=\beta_{13}$ versus $H_{0}: \beta_{11} \neq \beta_{13} \operatorname{com} \mu=2.5 \ldots \ldots \ldots \ldots \ldots \ldots$

6.6 Gráficos de quantis para $\sigma^{2}=0.05$ e $\sigma_{x}^{2}=0.5$ para o teste $H_{0}: \beta_{11}=\beta_{13}$ versus $H_{0}: \beta_{11} \neq \beta_{13} \operatorname{com} \mu=2.5$

6.7 Gráficos de quantis para $\sigma^{2}=0.1$ e $\sigma_{x}^{2}=0.5$ para o teste $H_{0}: \beta_{11}=\beta_{13}$ versus $H_{0}: \beta_{11} \neq \beta_{13}$ com $\mu=2.5$

6.8 Gráficos de quantis para $\sigma^{2}=0.5$ e $\sigma_{x}^{2}=0.5$ para o teste $H_{0}: \beta_{11}=\beta_{13}$ versus $H_{0}: \beta_{11} \neq \beta_{13}$ com $\mu=2.5 \ldots \ldots \ldots \ldots \ldots$

7.1 Gráfico de $d_{\max }$ para o esquema de perturbação de ponderação de casos . . . 80

7.2 Gráfico de $L D(\omega)$ para o esquema de perturbação de ponderação de casos . . . . 81

7.3 Mudanças relativas nas estimativas dos parâmetros no esquema de perturbação de ponderação de casos . . . . . . . . . . . . . . . . . . . . . . . . . . . 81

7.4 Gráfico de $d_{\max }$ para o esquema de perturbação nas variáveis explanatórias . 82

7.5 Mudanças relativas nas estimativas dos parâmetros no esquema de perturbação nas variáveis explanatórias . . . . . . . . . . . . . . . . . . 83

7.6 Gráfico de $d_{\max }$ para o esquema de perturbação nas variáveis resposta $\ldots$. . . . 84

7.7 Gráfico de $L D(\omega)$ para o esquema de perturbação nas variáveis resposta . . . . . 84 
7.8 Mudanças relativas nas estimativas dos parâmetros no esquema de perturbação nas variáveis resposta . . . . . . . . . . . . . . . . . 85

7.9 Mudanças relativas nas estimativas dos parâmetros de variância dos erros após o uso dos líquidos no esquema de perturbação nas variáveis resposta . . . . . . . 85

7.10 Gráfico de $d_{\max }$ para o esquema de perturbação na variância dos erros de medição no início do estudo . . . . . . . . . . . . . . . . . . 86

7.11 Gráfico de $L D(\omega)$ para o esquema de perturbação na variância dos erros de medição no início do estudo . . . . . . . . . . . . . . . . . 87

7.12 Mudanças relativas nas estimativas dos parâmetros no esquema de perturbação na variância dos erros de medição no início do estudo . . . . . . . . . . . . . 87

7.13 Gráfico de $d_{\max }$ para o esquema de perturbação na variância dos erros após o uso dos líquidos . . . . . . . . . . . . . . . . . . . . 88

7.14 Gráfico de $L D(\omega)$ para o esquema de perturbação na variância dos erros após o uso dos líquidos . . . . . . . . . . . . . . . . . . . . . . . . . 89

7.15 Mudanças relativas nas estimativas dos parâmetros no esquema de perturbação na variância dos erros após o uso dos líquidos

7.16 Mudanças relativas nas estimativas dos parâmetros de variância dos erros após o uso dos líquidos no esquema de perturbação na variância dos erros após o uso

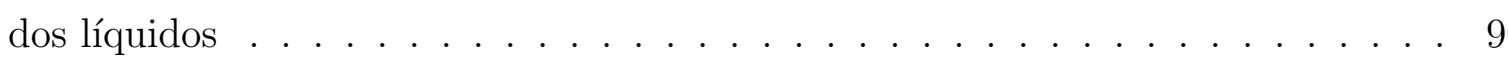

7.17 (a) Autovalores normalizados em módulo, $\mu_{i}$, com valores de $q$ e (b) influência devida à contribuição agregada de todos os autovetores para o esquema de ponderação de casos. . . . . . . . . . . . . . . . . . . . . . . . 97

7.18 Contribuição agregada dos autovetores correspondentes aos dois maiores autovalores $(q=4)$, provenientes da perturbação de ponderação de casos. . . . . . . 97

7.19 Autovetores e autovalores normalizados, $E_{1}$, com $\mu_{1}=0.559$ (a), $E_{2}$, com $\mu_{2}=$ 0.429 (b), $E_{3}$, com $\mu_{3}=0.329$ (c), $E_{4}$, com $\mu_{4}=0.313$ (d) provenientes da perturbação de ponderação de casos.

7.20 (a) Autovalores normalizados em módulo, $\mu_{i}$, com valores de $q$ e (b) influência devida à contribuição agregada de todos os autovetores para o esquema de perturbação na variável explanatória. . . . . . . . . . . . . . . . . 100

7.21 Gráfico de $L D(\omega)$ para o esquema de perturbação nas variáveis explanatórias 100

7.22 Contribuição agregada dos autovetores correspondentes aos três maiores autovalores $(q=1)$, provenientes da perturbação nas variáveis explanatórias. . . . . 101 
7.23 Autovetores e autovalores normalizados, $E_{1}$, com $\mu_{1}=0.845$ (a), $E_{2}$, com $\mu_{2}=0.401$ (b), $E_{3}$, com $\mu_{3}=0.317$ (c) provenientes da perturbação na variável explanatória. . . . . . . . . . . . . . . . . . 102

7.24 (a) Autovalores normalizados em módulo, $\mu_{i}$, com valores de $q$ e (b) influência devida à contribuição agregada de todos os autovetores para o esquema de perturbação nas variáveis resposta. . . . . . . . . . . . . . . . . 103

7.25 Contribuição agregada dos autovetores correspondentes aos três maiores autovalores $(q=4)$, provenientes da perturbação nas variáveis resposta. . . . . . . 103

7.26 Autovetores e autovalores normalizados, $E_{1}$, com $\mu_{1}=0.511$ (a), $E_{2}$, com $\mu_{2}=$ 0.467 (b), $E_{3}$, com $\mu_{3}=0.361$ (c) provenientes da perturbação na variável resposta.104

7.27 (a) Autovalores normalizados em módulo, $\mu_{i}$, com valores de $q$ e (b) influência devida à contribuição agregada de todos os autovetores para o esquema de perturbação na variância dos erros de medição no início do estudo. . . . . . . . . . . 105

7.28 Autovetor e autovalor normalizado, $E_{1}$, com $\mu_{1}=0.999$ provenientes da perturbação na variância dos erros de medição no início do estudo. . . . . . . . . . . 106

7.29 (a) Autovalores normalizados em módulo $\mu_{i}$ com valores de $q$ e (b) influência devida à contribuição agregada de todos os autovetores para o esquema de perturbação na variância dos erros após o uso dos líquidos. . . . . . . . . . . . . . . 107

7.30 Contribuição agregada dos autovetores correspondentes aos três maiores autovalores $(q=3)$, provenientes da perturbação nas variâncias dos erros de medição após o uso dos líquidos. . . . . . . . . . . . . . . . . . . . . . 107

7.31 Autovetores e autovalores normalizados, $E_{1}$, com $\mu_{1}=0.707$ (a), $E_{2}$, com $\mu_{2}=$ 0.363 (b), $E_{3}$, com $\mu_{3}=0.295$ (c) provenientes da perturbação na variável resposta.108 


\section{Lista de Tabelas}

2.1 Características dos voluntários no início do estudo . . . . . . . . . . . . . . . 24

3.1 Estimativas iniciais obtidas pelo método dos momentos (EMM) e estimativas de máxima verossimilhança obtidas pelo algoritmo EM (EMV) . . . . . . . . 36

4.1 Estimativas de máxima verossimilhança dos parâmetros (com desvios padrão) 44

5.1 Estimativas de máxima verossimilhança irrestritas e restritas às hipóteses $H_{0}$. . 53

5.2 Valores obtidos pelas estatísticas Wald, razão de verossimilhanças e score e pvalor para testes entre líquidos. . . . . . . . . . . . . . . 53

5.3 Valores obtidos pelas estatísticas Wald, razão de verossimilhanças e score e pvalor para testes intra-líquidos. . . . . . . . . . . . . . . . . 54

7.1 Estimativas de máxima verossimilhança considerando a exclusão de casos para as observações detectadas utilizando técnicas de influência local . . . . . . . 91

7.2 Medidas de influência utilizando a curvatura normal conformal para o esquema de ponderação de casos. . . . . . . . . . . . . . . . . . . . . . 99

7.3 Medidas de influência utilizando a curvatura normal conformal para o esquema de perturbação na variável explanatória. . . . . . . . . . . . . . . . . . 99

7.4 Medidas de influência utilizando a curvatura normal conformal para o esquema de perturbação nas variáveis resposta. . . . . . . . . . . . . . 105

7.5 Medidas de influência utilizando a curvatura normal conformal para o esquema de perturbação na variância dos erros de medição no início do estudo . . . . . . . 105

7.6 Medidas de influência utilizando a curvatura normal conformal para o esquema de perturbação na variância dos erros após o uso dos líquidos. . . . . . . . . . 106

E.1 Tamanhos empíricos dos testes Wald, razão de verossimilhança e score para o teste $H_{0}: \beta_{11}=\beta_{12}$, com $\mu=1$. . . . . . . . . . . . . 146

E.2 Tamanhos empíricos dos testes Wald, razão de verossimilhança e score para o

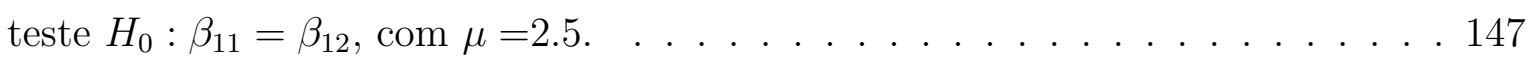


E.3 Tamanhos empíricos dos testes Wald, razão de verossimilhança e score para o teste $H_{0}: \beta_{11}=\beta_{12}$, com $\mu=5$.

E.4 Tamanhos empíricos dos testes Wald, razão de verossimilhança e score para o teste $H_{0}: \beta_{11}=\beta_{13}$, com $\mu=1$.

E.5 Tamanhos empíricos dos testes Wald, razão de verossimilhança e score para o

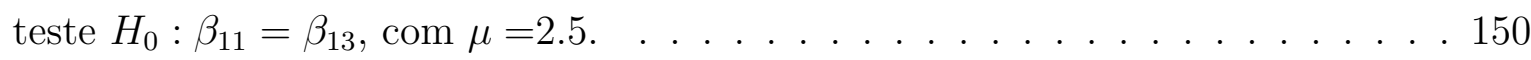

E.6 Tamanhos empíricos dos testes Wald, razão de verossimilhança e score para o

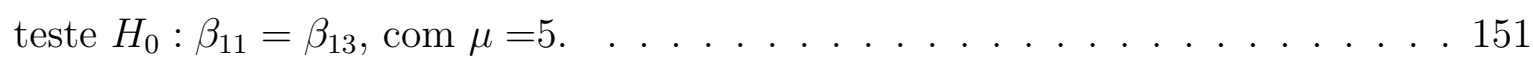

E.7 Tamanhos empíricos dos testes Wald, razão de verossimilhança e score para o teste $H_{0}: \beta_{12}=\beta_{13}$, com $\mu=1$.

E.8 Tamanhos empíricos dos testes Wald, razão de verossimilhança e score para o teste $H_{0}: \beta_{12}=\beta_{13}$, com $\mu=2.5$.

E.9 Tamanhos empíricos dos testes Wald, razão de verossimilhança e score para o teste $H_{0}: \beta_{12}=\beta_{13}$, com $\mu=5$.

E.10 Tamanhos empíricos dos testes Wald, razão de verossimilhança e score para o teste $H_{0}: \beta_{21}=\beta_{22}$, com $\mu=1$.

E.11 Tamanhos empíricos dos testes Wald, razão de verossimilhança e score para o

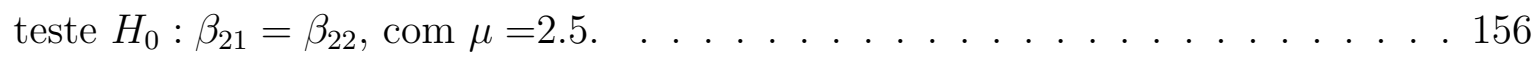

E.12 Tamanhos empíricos dos testes Wald, razão de verossimilhança e score para o

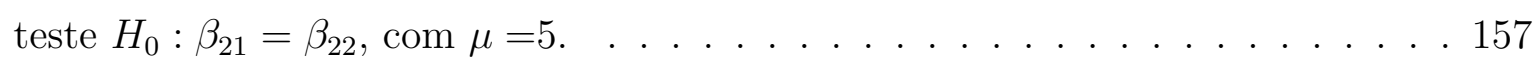

E.13 Tamanhos empíricos dos testes Wald, razão de verossimilhança e score para o teste $H_{0}: \beta_{21}=\beta_{23}, \operatorname{com} \mu=1$.

E.14 Tamanhos empíricos dos testes Wald, razão de verossimilhança e score para o teste $H_{0}: \beta_{21}=\beta_{23}$, com $\mu=2.5$.

E.15 Tamanhos empíricos dos testes Wald, razão de verossimilhança e score para o teste $H_{0}: \beta_{21}=\beta_{23}$, com $\mu=5$.

E.16 Tamanhos empíricos dos testes Wald, razão de verossimilhança e score para o teste $H_{0}: \beta_{22}=\beta_{23}$, com $\mu=1$.

E.17 Tamanhos empíricos dos testes Wald, razão de verossimilhança e score para o teste $H_{0}: \beta_{22}=\beta_{23}$, com $\mu=2.5$.

E.18 Tamanhos empíricos dos testes Wald, razão de verossimilhança e score para o teste $H_{0}: \beta_{22}=\beta_{23}$, com $\mu=5$.

E.19 Tamanhos empíricos dos testes Wald, razão de verossimilhança e score para o teste $H_{0}: \beta_{11}=\beta_{21}$, com $\mu=1$. 
E.20 Tamanhos empíricos dos testes Wald, razão de verossimilhança e score para o teste $H_{0}: \beta_{11}=\beta_{21}$, com $\mu=2.5$.

E.21 Tamanhos empíricos dos testes Wald, razão de verossimilhança e score para o teste $H_{0}: \beta_{11}=\beta_{21}$, com $\mu=5$.

E.22 Tamanhos empíricos dos testes Wald, razão de verossimilhança e score para o teste $H_{0}: \beta_{12}=\beta_{22}$, com $\mu=1$.

E.23 Tamanhos empíricos dos testes Wald, razão de verossimilhança e score para o teste $H_{0}: \beta_{12}=\beta_{22}$, com $\mu=2.5$.

E.24 Tamanhos empíricos dos testes Wald, razão de verossimilhança e score para o teste $H_{0}: \beta_{12}=\beta_{22}$, com $\mu=5$.

E.25 Tamanhos empíricos dos testes Wald, razão de verossimilhança e score para o teste $H_{0}: \beta_{13}=\beta_{23}$, com $\mu=1$.

E.26 Tamanhos empíricos dos testes Wald, razão de verossimilhança e score para o teste $H_{0}: \beta_{13}=\beta_{23}$, com $\mu=2.5$.

E.27 Tamanhos empíricos dos testes Wald, razão de verossimilhança e score para o teste $H_{0}: \beta_{13}=\beta_{23}$, com $\mu=5$.

F.1 Poder simulado dos testes para a hipótese $H_{0}: \beta_{11}=\beta_{12}$, com $\mu=1$. . . . . 174

F.2 Poder simulado dos testes para a hipótese $H_{0}: \beta_{11}=\beta_{12}$, com $\mu=2.5$. . . . 175

F.3 Poder simulado dos testes para a hipótese $H_{0}: \beta_{11}=\beta_{12}$, com $\mu=5$. . . . . 176

F.4 Poder simulado dos testes para a hipótese $H_{0}: \beta_{11}=\beta_{13}$, com $\mu=1$. . . . . 177

F.5 Poder simulado dos testes para a hipótese $H_{0}: \beta_{11}=\beta_{13}$, com $\mu=2.5$. . . . 178

F.6 Poder simulado dos testes para a hipótese $H_{0}: \beta_{11}=\beta_{13}$, com $\mu=5$. . . . . . 179

F.7 Poder simulado dos testes para a hipótese $H_{0}: \beta_{12}=\beta_{13}$, com $\mu=1$. . . . . . 180

F.8 Poder simulado dos testes para a hipótese $H_{0}: \beta_{12}=\beta_{13}$, com $\mu=2.5$. . . . . 181

F.9 Poder simulado dos testes para a hipótese $H_{0}: \beta_{12}=\beta_{13}$, com $\mu=5$. . . . . . 182

F.10 Poder simulado dos testes para a hipótese $H_{0}: \beta_{21}=\beta_{22}$, com $\mu=1$. . . . . 183

F.11 Poder simulado dos testes para a hipótese $H_{0}: \beta_{21}=\beta_{22}$, com $\mu=2.5$. . . . . 184

F.12 Poder simulado dos testes para a hipótese $H_{0}: \beta_{21}=\beta_{22}$, com $\mu=5$. . . . . 185

F.13 Poder simulado dos testes para a hipótese $H_{0}: \beta_{21}=\beta_{23}$, com $\mu=1$. . . . . 186

F.14 Poder simulado dos testes para a hipótese $H_{0}: \beta_{21}=\beta_{23}$, com $\mu=2.5$. . . . . 187

F.15 Poder simulado dos testes para a hipótese $H_{0}: \beta_{21}=\beta_{23}$, com $\mu=5$. . . . . 188

F.16 Poder simulado dos testes para a hipótese $H_{0}: \beta_{22}=\beta_{23}$, com $\mu=1$. . . . . . 189

F.17 Poder simulado dos testes para a hipótese $H_{0}: \beta_{22}=\beta_{23}$, com $\mu=2.5$. . . . 190

F.18 Poder simulado dos testes para a hipótese $H_{0}: \beta_{22}=\beta_{23}$, com $\mu=5$. . . . . 191 
F.19 Poder simulado dos testes para a hipótese $H_{0}: \beta_{11}=\beta_{21}$, com $\mu=1$. . . . . . 192

F.20 Poder simulado dos testes para a hipótese $H_{0}: \beta_{11}=\beta_{21}$, com $\mu=2.5$. . . . . 193

F.21 Poder simulado dos testes para a hipótese $H_{0}: \beta_{11}=\beta_{21}$, com $\mu=5$. . . . . . 194

F.22 Poder simulado dos testes para a hipótese $H_{0}: \beta_{12}=\beta_{22}$, com $\mu=1$. . . . . 195

F.23 Poder simulado dos testes para a hipótese $H_{0}: \beta_{12}=\beta_{22}$, com $\mu=2.5$. . . . . 196

F.24 Poder simulado dos testes para a hipótese $H_{0}: \beta_{12}=\beta_{22}$, com $\mu=5 \ldots$. . . . 197

F.25 Poder simulado dos testes para a hipótese $H_{0}: \beta_{13}=\beta_{23}$, com $\mu=1$. . . . . 198

F.26 Poder simulado dos testes para a hipótese $H_{0}: \beta_{13}=\beta_{23}$, com $\mu=2.5$. . . . . 199

F.27 Poder simulado dos testes para a hipótese $H_{0}: \beta_{13}=\beta_{23}$, com $\mu=5$. . . . . . 200 
18 Análise de um modelo de regressão com erros nas variáveis multivariado com intercepto nulo 


\section{Capítulo 1}

\section{Introdução}

O desenvolvimento deste trabalho foi motivado por um conjunto de dados clínicos dentários apresentado em Hadgu \& Koch (1999). Neste experimento, 109 voluntários de ambos os sexos com índice de placa dentária pré-existente foram aleatorizados entre dois líquidos de bochecho experimentais (A e B) e um líquido de bochecho controle. O interesse primordial deste estudo foi a verificação da eficiência de dois líquidos de bochecho experimentais A e B, em relação ao líquido de bochecho controle na prevenção do desenvolvimento do índice de placa dentária. Estes índices foram coletados no início do estudo e após decorridos três e seis meses do início do estudo. Como a obtenção do índice de placa dentária está sujeita a erros de medição, uma alternativa na análise deste conjunto de dados seria a utilização de modelos de regressão com erros nas variáveis. Neste caso, teríamos um modelo multivariado, com uma possível estrutura de dependência entre os índices de placa dentária obtidos, decorridos três e seis meses do início do estudo.

O início do estudo de modelos de regressão onde a variável explanatória está sujeita a erros de medição data do século XIX. Sprent (1990) descreve o seu desenvolvimento histórico e um estudo detalhado pode ser encontrado em Kendall \& Stuart (1961), Moran (1971) e Fuller (1987). Estes modelos são denominados modelos com erros de medição ou modelos com erros nas variáveis. Um modelo de regressão linear simples com erros nas variáveis pode ser definido da seguinte forma.

Considere um conjunto finito de pares $\left(\xi_{1}, \eta_{1}\right), \ldots,\left(\xi_{n}, \eta_{n}\right)$ satisfazendo a seguinte relação linear:

$$
\eta_{i}=\alpha+\beta \xi_{i}
$$

$i=1, \ldots, n$, onde $\left(\xi_{i}, \eta_{i}\right)$ não podem ser observados diretamente, mas com erros através dos 
pares $\left(x_{i}, y_{i}\right)$, ou seja,

$$
\begin{aligned}
& x_{i}=\xi_{i}+u_{i}, \\
& y_{i}=\eta_{i}+\varepsilon_{i},
\end{aligned}
$$

$i=1, \ldots, n$. Os erros $u_{i}$ e $\varepsilon_{i}$ são variáveis aleatórias com médias iguais a 0 e variâncias finitas $\sigma^{2} \mathrm{e}$ $\sigma_{\varepsilon}^{2}$, respectivamente, e independentemente distribuídos para diferentes valores de $i, i=1, \ldots, n$. Nestas condições, podemos ter pelo menos três modelos diferentes: o primeiro, onde os $\xi_{i}$ são constantes desconhecidas; o segundo, quando os $\xi_{i}$ são variáveis aleatórias independentes com a mesma distribuição e o terceiro, onde os $\xi_{i}$ são variáveis aleatórias independentes com médias diferentes e variâncias em comum. No primeiro (segundo) caso em que os $\xi_{i}$ são fixos (aleatórios) o modelo especificado pela equação (1.1) é denominado modelo funcional (estrutural), como considerado em Kendall \& Stuart (1973) e Moran (1971). Esta distinção entre as denominações funcional e estrutural foi claramente exposta pela primeira vez por Kendall $(1951,1952)$. O terceiro modelo é denominado modelo ultraestrutural e foi proposto por Dolby (1976). Este modelo é uma generalização dos modelos funcional e estrutural, sendo que ao considerarmos que as médias do verdadeiro valor da covariável $\left(\xi_{i}\right)$ são iguais, este se reduz ao modelo estrutural e ao assumirmos que a variância do verdadeiro valor da covariável é nula, este se reduz ao modelo funcional. Neste trabalho, consideramos o modelo estrutural.

É bem conhecido que nestas condições a distribuição conjunta de $\left(x_{i}, y_{i}\right)$ não é identificável (Kendall e Stuart, 1973) e portanto não existem estimadores consistentes para $\alpha$ e $\beta$. O único parâmetro identificável é $\mu\left(E\left(\xi_{i}\right)\right)$. A fim de contornar este problema de identificabilidade, procedimentos mais comumente utilizados consideram: (i)- a razão das variâncias dos erros $\left(\lambda=\frac{\sigma_{e}^{2}}{\sigma^{2}}\right)$ conhecida; (ii)- uma das variâncias dos erros $\left(\sigma_{e}^{2}\right.$ ou $\left.\sigma^{2}\right)$ conhecida; (iii)- ambas as variâncias dos erros $\left(\sigma_{e}^{2}\right.$ e $\left.\sigma^{2}\right)$ conhecidas; (iv)- o coeficiente de atenuação $\left(k_{x}=\sigma^{2} /\left(\sigma^{2}+\sigma_{x}^{2}\right)\right)$ conhecido; (v)- $\alpha$ conhecido e $\mu \neq 0$.

Qualquer uma das suposições acima reduz o número de parâmetros da distribuição conjunta de $\left(x_{i}, y_{i}\right)$, tornando o modelo identificável. Suposições a respeito de $\beta$ não são feitas, pois este é o parâmetro de interesse primordial. A suposição (i) é a mais utilizada e a que possui o maior número de resultados teóricos publicados (Cheng \& Van Ness 1999). O caso (iv), onde o intercepto é suposto conhecido pode ser encontrado em Chan \& Mak (1979), Patefield (1985) e Aoki et al. (2001, 2002). Em Cheng \& Van Ness (1999) são consideradas áreas de aplicações dos modelos estruturais com cada uma das suposições citadas acima.

Na prática existem várias situações onde a variável explanatória não pode ser observada diretamente, mas com erros, como quando estas observações são obtidas através de análises laboratoriais resultando em observações que são estimativas da covariável e não no verdadeiro 
valor da covariável. Em Aoki et al. (2001) foi feito um estudo sobre modelos de regressão com erros nas variáveis com intercepto nulo e com possíveis estruturas de dependência entre as populações. A motivação para tal estudo, veio de um conjunto de dados citado em Singer \& Andrade (1997) e que resultou de um estudo conduzido na Faculdade de Odontologia da USP, correspondente a medidas de índices de placa dentária antes e após a escovação com a utilização de uma escova experimental e de uma escova convencional. Observe que neste caso, se o índice de placa dentária antes da escovação for nulo, depois da escovação também o será. Desta forma teríamos um modelo com intercepto nulo. Um outro fato foi a utilização das mesmas crianças para a coleta dos índices de placa dentária com os dois tipos de escovas, ou seja, é necessário um modelo que leve em consideração possíveis dependências existentes entre as medidas tomadas no mesmo indivíduo. Motivado pelo conjunto de dados apresentado em Hadgu \& Koch (1999), estendemos o modelo definido em Aoki et al. (2001) para um modelo com erros nas variáveis multivariado, onde neste caso temos independência entre os grupos (diferentes indivíduos foram utilizados, para cada um dos três tipos de líquidos para bochechar) e dependência nas medições de um mesmo indivíduo (foram coletados índices de placa dentária no início do estudo e decorridos três e seis meses do início do estudo). Desta forma, um possível modelo é dado por

$$
\begin{aligned}
\mathbf{x}_{i} & =\xi_{i}+\delta_{i}, \\
\mathbf{y}_{i} & =\mathbf{X}_{i} \boldsymbol{\beta}_{i}+\varepsilon_{i}, \quad i=1, \ldots, p,
\end{aligned}
$$

onde $\mathbf{x}_{i}=\left(x_{i_{1}}, \ldots, x_{i_{n_{i}}}\right)^{\prime}$ e $\mathbf{y}_{i}=\left(\mathbf{y}_{1 i}^{\prime}, \mathbf{y}_{2 i}^{\prime}\right)^{\prime}=\left(y_{1 i_{1}}, \ldots, y_{1 i_{n_{i}}}, y_{2 i_{1}}, \ldots, y_{2 i_{n_{i}}}\right)^{\prime}$, representam os vetores observados, com $\left(x_{i_{j}}, y_{1 i_{j}}, y_{2 i_{j}}\right)^{\prime}$ as medições realizadas no $j$-ésimo indivíduo que testou o $i$-ésimo líquido de bochecho, sendo $x_{i_{j}}$ o valor observado do índice de placa dentária no início do estudo, $y_{1 i_{j}}$ o valor observado do índice de placa dentária após três meses e $y_{2 i_{j}}$ o valor observado do índice de placa dentária após seis meses. $\xi_{i_{j}}$ representa o verdadeiro valor do índice de placa dentária (não-observável) no início do estudo para o $j$-ésimo indivíduo que testou o $i$-ésimo líquido, e $\mathbf{X}_{i}=\left(\begin{array}{cc}\xi_{i} & 0 \\ 0 & \xi_{i}\end{array}\right)$. No exemplo dos dados odontológicos, teríamos $p=3$ líquidos de bochecho. No Capítulo 2 iremos descrever o modelo com mais detalhes.

Considerando-se o modelo (1.2), apresentaremos o algoritmo iterativo EM para a obtenção das estimativas de máxima verossimilhança dos parâmetros, assim como estatísticas de testes assintóticos de Wald, score e razão de verossimilhanças para testar a eficiência dos líquidos de bochecho A e B em relação ao líquido controle. Além disso, será apresentado um estudo de simulação com o intuito de comparar as três estatísticas de teste quanto ao nível de significância e ao poder do teste. Em seguida, faremos um estudo de diagnóstico de influência local (Cook , 
22 Análise de um modelo de regressão com erros nas variáveis multivariado com intercepto nulo

1986) considerando-se vários tipos de perturbações no modelo e nos dados. 


\section{Capítulo 2}

\section{O modelo estatístico}

Neste capítulo descreveremos o conjunto de dados odontológicos que serviu de motivação ao nosso trabalho e apresentaremos um modelo de regressão multivariado com erros nas variáveis com intercepto nulo para estes dados. Em seguida obteremos a função de verossimilhança e apresentaremos a função escore.

\subsection{O conjunto de dados}

Descreveremos nesta seção o conjunto de dados da área de Odontologia apresentado em Hadgu \& Koch (1999), cujo objetivo de estudo foi avaliar a eficácia de dois líquidos de bochecho experimentais em relação a um líquido controle na prevenção do desenvolvimento de placa dentária. Descreveremos aqui, de uma forma geral, as características desse conjunto de dados e a forma com que os dados foram coletados no decorrer do estudo.

\subsubsection{Descrição dos dados}

Visando estudar a eficácia de dois líquidos de bochecho experimentais A e B em relação a um líquido controle, o experimento apresentado em Hadgu \& Koch (1999) contou com 109 voluntários, que foram aleatorizados em três grupos para o uso dos respectivos líquidos durante seis meses. O grupo de voluntários passou inicialmente por testes em que se excluíram pessoas com grave patologia oral, como também pessoas em tratamento com medicamentos antibióticos, antibacterianos e anti-inflamatórios. Além disso, foram selecionados para o estudo apenas pessoas consideradas sadias, com dentes naturais e índice mínimo de placa dentária 2.0. O grupo selecionado contou com adultos de ambos os sexos, com idade média de aproximadamente 28 anos.

Os voluntários de cada grupo foram orientados a utilizar $20 \mathrm{ml}$ do respectivo produto por 30 segundos pela manhã e à tarde, todos os dias, ao longo dos seis meses, e tiveram acom- 
panhamento de um supervisor do estudo. Durante o uso dos produtos, os voluntários foram instruídos a manter seus hábitos normais de higiene e alimentação, porém foram solicitados a abster-se de outros produtos comerciais de higiene bucal e informar aos supervisores o uso de medicamentos antibióticos ou anti-inflamatórios.

Os dados foram coletados no início do estudo e decorridos três e seis meses do início do estudo. Ao final do estudo havia-se perdido dados de quatro voluntários, sendo três do grupo que testou o líquido controle e um do grupo que testou o líquido A. O conjunto de dados ficou portanto com 36 voluntários no grupo controle, 33 no grupo A e 36 no grupo B. O conjunto de dados encontra-se no Apêndice A.

Algumas informações adicionais foram obtidas dos voluntários, como status de fumante e idade, mas concluiu-se em Hadgu \& Koch (1999) que estas informações tinham pouca ou nenhuma relação com a quantidade de placa dentária existente. Os grupos eram razoavelmente homogêneos, apesar de haver mais mulheres no grupo A que nos demais. A Tabela 2.1 descreve resumidamente os três grupos.

Tabela 2.1: Características dos voluntários no início do estudo

\begin{tabular}{|rccc|}
\hline & $\begin{array}{c}\text { Controle } \\
(\mathrm{n}=36)\end{array}$ & $\begin{array}{c}\mathbf{A} \\
(\mathrm{n}=33)\end{array}$ & $\begin{array}{c}\mathbf{B} \\
(\mathrm{n}=36)\end{array}$ \\
\hline \hline Sexo & & & \\
Feminino & 13 & 23 & 19 \\
Masculino & 23 & 10 & 17 \\
\hline Status de fumante & & & \\
Não fumante & 31 & 27 & 31 \\
Fumante & 5 & 6 & 5 \\
\hline Idade & & & \\
Mediana & 27 & 26 & 25 \\
Média & 27.47 & 29.21 & 27.42 \\
(Min, Max) & $(23,38)$ & $(22,48)$ & $(23,47)$ \\
\hline \hline Índice de Placa & & & \\
Mediana & 2.5 & 2.51 & 2.43 \\
Média & 2.56 & 2.57 & 2.48 \\
(Min, Max) & $(2.07,3.3)$ & $(2.04,3.57)$ & $(2.02,3.13)$ \\
\hline
\end{tabular}

\subsubsection{Objetivo do estudo}

O principal objetivo do experimento apresentado em Hadgu \& Koch (1999) era avaliar a eficiência dos três líquidos de bochecho após três e seis meses do início do estudo. Ou seja, 


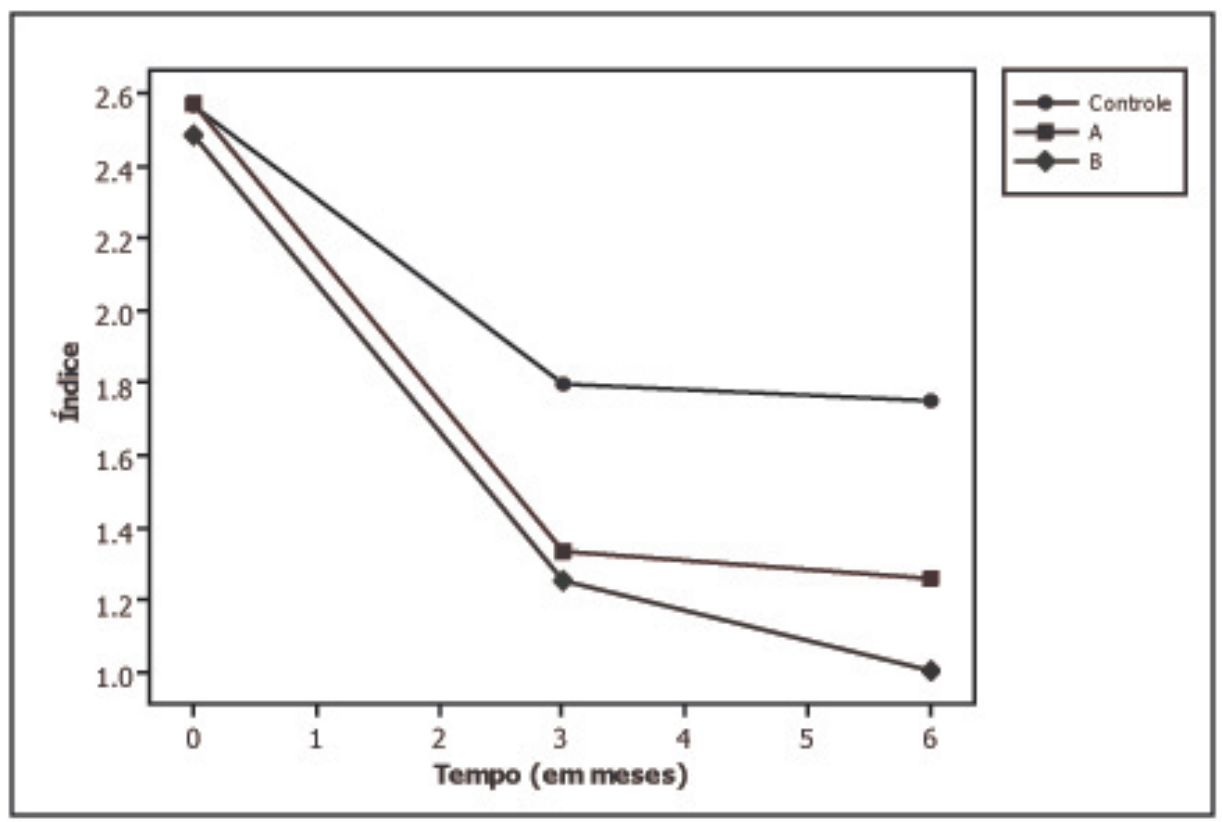

Figura 2.1: Índice médio de placa dentária no início do estudo e após três e seis meses

desejava-se saber se os dois líquidos experimentais apresentavam maior diminuição de placa dentária do que o líquido controle, se um dos líquidos experimentais era mais eficiente do que o outro, e se havia diferenças consideráveis em cada grupo entre a diminuição de placa no decorrer dos três meses e seis meses, isto é, se os líquidos de bochecho continuavam a ter efeito a longo prazo ou se a quantidade de placa se estabilizava depois dos três primeiros meses. Pôde-se perceber, como mostra o gráfico da Figura 2.1, que o uso dos líquidos, em média, surtiu efeito, tanto nos líquidos experimentais quanto no líquido controle. No entanto, aparentemente apenas o líquido experimental B continua a diminuir significativamente o índice de placa dentária após o primeiro período de uso.

Com um enfoque diferente de Hadgu \& Koch (1999), em que foi empregado um modelo linear generalizado sem considerar erros de medição, o nosso objetivo é analisar o mesmo conjunto de dados utilizando um modelo com erros nas variáveis para comparar a eficácia dos três líquidos. Na Seção 2.2, discutiremos a utilização do modelo com erros nas variáveis para este conjunto de dados.

\subsection{Modelo de regressão com erros nas variáveis}

Para os dados de Hadgu \& Koch (1999), descritos na Seção 2.1, um modelo alternativo seria o modelo de regressão com erros nas variáveis, devido ao fato das medições de placa dentária 
não serem exatas. A estrutura de dependência entre as variáveis decorre do fato de se tomar as medidas em um mesmo indivíduo (o modelo estrutural incorpora essa dependência), ou seja, o comportamento dos líquidos pode ser diferente para cada indivíduo, e além disto, é esperado que a quantidade de placa dentária após três e seis meses seja menor em indivíduos que já possuíam menor quantidade de placa, ou seja, a quantidade de placa em cada indivíduo depende da quantidade inicial que este possuía. Da mesma forma, se um indivíduo não possuía placa dentária inicialmente, é natural que não possua após utilizar os líquidos de bochecho, o que justifica o intercepto nulo.

Como mostra o Apêndice $\mathrm{A}$, temos três medições para cada indivíduo. Por exemplo, as medições do primeiro indivíduo que testou o líquido controle (Líquido 1) são dadas por $(2.46,1.73,1.58)$, ou seja, estas são respectivamente as medições no início do estudo, após três e seis meses de uso do líquido controle. De uma forma geral, a primeira medição do $j$-ésimo indivíduo que testou o $i$-ésimo líquido será denotada por $x_{i_{j}}$ (variável explanatória) e as duas medições seguintes serão denotadas pelo par $\left(y_{1 i_{j}}, y_{2 i_{j}}\right)$ (variáveis resposta), onde $i=1,2,3$ indicam respectivamente o grupo que testou o líquido controle, o grupo que testou o líquido A e o grupo que testou o líquido B. Desta forma, temos que $x_{1_{1}}=2.46, y_{11_{1}}=1.73$ e $y_{21_{1}}=1.58$.

Assim, um modelo estrutural adequado poderia ser da forma

$$
\left\{\begin{array}{l}
\mathbf{x}_{i}=\boldsymbol{\xi}_{i}+\boldsymbol{\delta}_{i}, \\
\mathbf{y}_{i}=\mathbf{X}_{i} \boldsymbol{\beta}_{i}+\mathbf{e}_{i}, \quad i=1, \ldots, p,
\end{array}\right.
$$

onde, para os $n_{i}$ indivíduos que testaram o líquido $i(i=1, \ldots, p)$ :

- o vetor observado $\mathbf{x}_{i}=\left(x_{i_{1}}, \ldots, x_{i_{n_{i}}}\right)^{\prime}$ representa o índice de placa dentária no início do estudo,

- o vetor observado $\mathbf{y}_{i}=\left(\mathbf{y}_{1 i}^{\prime}, \mathbf{y}_{2 i}^{\prime}\right)^{\prime}$, com $\mathbf{y}_{1 i}=\left(y_{1 i_{1}}, \ldots, y_{1 i_{n_{i}}}\right)^{\prime}$ e $\mathbf{y}_{2 i}=\left(y_{2 i_{1}}, \ldots, y_{2 i_{n_{i}}}\right)^{\prime}$, representa, respectivamente, os índices de placa dentária após três e seis meses do início do estudo,

- o vetor $\boldsymbol{\xi}_{i}=\left(\xi_{i_{1}}, \ldots, \xi_{i_{n_{i}}}\right)^{\prime}$ representa os verdadeiros valores do índice de placa dentária no início do estudo,

- $\mathbf{X}_{i}=\left(\begin{array}{cc}\xi_{i} & 0 \\ 0 & \xi_{i}\end{array}\right)$

- $\boldsymbol{\delta}_{i}=\left(\delta_{i_{1}}, \ldots, \delta_{i_{n_{i}}}\right)^{\prime}, \mathbf{e}_{i}=\left(\mathbf{e}_{1 i}^{\prime}, \mathbf{e}_{2 i}^{\prime}\right)^{\prime}, \operatorname{com} \mathbf{e}_{1 i}=\left(e_{1 i_{1}}, \ldots, e_{1 i_{n_{i}}}\right)^{\prime}$ e $\mathbf{e}_{2 i}=\left(e_{2 i_{1}}, \ldots, e_{2 i_{n_{i}}}\right)^{\prime}$ representam os erros de medição no início do estudo e decorridos três e seis meses do início do estudo, respectivamente. 
- $\boldsymbol{\beta}_{i}=\left(\beta_{1 i}, \beta_{2 i}\right)^{\prime}$ representa a proporção de placa dentária restante após o uso do líquido de bochecho $i$, decorridos três e seis meses respectivamente.

No caso de Hadgu \& Koch (1999), temos 3 líquidos de bochecho e então $p=3$. Neste caso, $n_{1}=36, n_{2}=33$ e $n_{3}=36$.

Assumimos como suposições que $\delta_{i_{j}} \sim$ ind. $N\left(0, \sigma^{2}\right), e_{1 i_{j}} \sim$ ind. $N\left(0, \sigma_{e_{1 i}}^{2}\right), e_{2 i_{j}} \sim$ ind. $N\left(0, \sigma_{e_{2 i}}^{2}\right)$ e $\xi_{i_{j}} \sim$ ind. $N\left(\mu, \sigma_{x}^{2}\right), i=1, \ldots, p ; j=1, \ldots, n_{i}$. Como os voluntários foram aleatorizados em 3 grupos, assume-se que no início os grupos são razoavelmente homogêneos e assim consideramos a mesma variância dos erros nos três grupos $\left(\sigma^{2}\right)$ no início do estudo. No entanto, como não conhecemos o comportamento dos três líquidos ao longo do estudo, as variâncias podem não ser as mesmas ao longo dos três e seis meses, ou seja, consideramos as variâncias dos erros diferentes para cada grupo após o início do estudo, isto é, $\boldsymbol{\sigma}_{e_{i}}^{2}=\left(\sigma_{e_{1 i}}^{2}, \sigma_{e_{2 i}}^{2}\right)^{\prime}$ para o grupo que testou o líquido $i$. Pode ser mostrado facilmente que as correlações entre as medidas, tomadas em um mesmo indivíduo, $x_{i_{j}}, y_{1 i_{j}}$ e $y_{2 i_{j}}$ são dadas por

$$
\begin{aligned}
& \rho\left(x_{i_{j}}, y_{1 i_{j}}\right)=\frac{\beta_{1 i} \sigma_{x}^{2}}{\sqrt{\left(\sigma_{x}^{2}+\sigma^{2}\right)\left(\beta_{1 i}^{2} \sigma_{x}^{2}+\sigma_{e_{1 i}}^{2}\right)}}, \\
& \rho\left(x_{i_{j}}, y_{2 i_{j}}\right)=\frac{\beta_{2 i} \sigma_{x}^{2}}{\sqrt{\left(\sigma_{x}^{2}+\sigma^{2}\right)\left(\beta_{2 i}^{2} \sigma_{x}^{2}+\sigma_{e_{2 i}}^{2}\right)}}, \mathrm{e} \\
& \rho\left(y_{1 i_{j}}, y_{2 i_{j}}\right)=\frac{\beta_{1 i} \beta_{2 i} \sigma_{x}^{2}}{\sqrt{\left(\beta_{1 i}^{2} \sigma_{x}^{2}+\sigma_{e_{1 i}}^{2}\right)\left(\beta_{2 i}^{2} \sigma_{x}^{2}+\sigma_{e_{2 i}}^{2}\right)}} .
\end{aligned}
$$

Seja $\mathbf{z}_{i_{j}}$ o vetor de observações do $j$-ésimo indivíduo que utilizou o $i$-ésimo líquido de bochecho. Temos que

$$
\mathbf{z}_{i_{j}}=\left(\begin{array}{c}
x_{i_{j}} \\
y_{1 i_{j}} \\
y_{2 i_{j}}
\end{array}\right) \sim N_{3}\left(\left(\begin{array}{c}
\mu \\
\beta_{1 i} \mu \\
\beta_{2 i} \mu
\end{array}\right),\left(\begin{array}{ccc}
\sigma_{x}^{2}+\sigma^{2} & \beta_{1 i} \sigma_{x}^{2} & \beta_{2 i} \sigma_{x}^{2} \\
\beta_{1 i} \sigma_{x}^{2} & \beta_{1 i}^{2} \sigma_{x}^{2}+\sigma_{e_{1 i}}^{2} & \beta_{1 i} \beta_{2 i} \sigma_{x}^{2} \\
\beta_{2 i} \sigma_{x}^{2} & \beta_{1 i} \beta_{2 i} \sigma_{x}^{2} & \beta_{2 i}^{2} \sigma_{x}^{2}+\sigma_{e_{2 i}}^{2}
\end{array}\right)\right) .
$$

Definindo

$$
\mathbf{m}_{i}=\left(\begin{array}{c}
\mu \\
\beta_{1 i} \mu \\
\beta_{2 i} \mu
\end{array}\right) \text { e } \mathbf{V}_{i}=\left(\begin{array}{ccc}
\sigma_{x}^{2}+\sigma^{2} & \beta_{1 i} \sigma_{x}^{2} & \beta_{2 i} \sigma_{x}^{2} \\
\beta_{1 i} \sigma_{x}^{2} & \beta_{1 i}^{2} \sigma_{x}^{2}+\sigma_{e_{1 i}}^{2} & \beta_{1 i} \beta_{2 i} \sigma_{x}^{2} \\
\beta_{2 i} \sigma_{x}^{2} & \beta_{1 i} \beta_{2 i} \sigma_{x}^{2} & \beta_{2 i}^{2} \sigma_{x}^{2}+\sigma_{e_{2 i}}^{2}
\end{array}\right)
$$

temos que a função densidade de probabilidade para a $j$-ésima observação do grupo que testou o $i$-ésimo líquido de bochecho é dada por

$$
f_{i_{j}}\left(\mathbf{z}_{i_{j}}, \boldsymbol{\theta}\right)=(2 \pi)^{-\frac{3}{2}}\left|\mathbf{V}_{i}\right|^{-\frac{1}{2}} \exp \left\{-\frac{1}{2}\left(\mathbf{z}_{i_{j}}-\mathbf{m}_{i}\right)^{\prime} \mathbf{V}_{i}^{-1}\left(\mathbf{z}_{i_{j}}-\mathbf{m}_{i}\right)\right\}
$$

com $\boldsymbol{\theta}=\left(\boldsymbol{\beta}_{1}^{\prime}, \ldots, \boldsymbol{\beta}_{p}^{\prime}, \mu, \sigma^{2}, \sigma_{x}^{2}, \boldsymbol{\sigma}_{e_{1}}^{2^{\prime}}, \ldots, \boldsymbol{\sigma}_{e_{p}}^{2^{\prime}}\right)^{\prime}$. A função densidade de probabilidade conjunta para os indivíduos que testaram o líquido $i$, para $i=1, \ldots, p$ é dada então por

$$
f_{i}\left(\mathbf{z}_{i}, \boldsymbol{\theta}\right)=(2 \pi)^{-\frac{3 n_{i}}{2}}\left|\mathbf{V}_{i}\right|^{-\frac{n_{i}}{2}} \exp \left\{-\frac{1}{2} \sum_{j=1}^{n_{i}}\left(\mathbf{z}_{i_{j}}-\mathbf{m}_{i}\right)^{\prime} \mathbf{V}_{i}^{-1}\left(\mathbf{z}_{i_{j}}-\mathbf{m}_{i}\right)\right\}
$$


onde $\mathbf{z}_{i}=\left(z_{i_{1}}, \ldots, z_{i_{n_{i}}}\right)^{\prime}$, e a função densidade de probabilidade conjunta para todas as observações é dada por

$$
f(\mathbf{z}, \boldsymbol{\theta})=(2 \pi)^{-\frac{3 N}{2}} \prod_{i=1}^{p}\left|\mathbf{V}_{i}\right|^{-\frac{n_{i}}{2}} \exp \left\{-\frac{1}{2} \sum_{i=1}^{p} \sum_{j=1}^{n_{i}}\left(\mathbf{z}_{i_{j}}-\mathbf{m}_{i}\right)^{\prime} \mathbf{V}_{i}^{-1}\left(\mathbf{z}_{i_{j}}-\mathbf{m}_{i}\right)\right\},
$$

onde $\mathbf{z}=\left(\mathbf{z}_{1}^{\prime}, \ldots, \mathbf{z}_{p}^{\prime}\right)^{\prime}$ e $N=\sum_{i=1}^{p} n_{i}$. Seja $L(\mathbf{z}, \boldsymbol{\theta})=\log f(\mathbf{z}, \boldsymbol{\theta})$. Temos então a função $\log$ verossimilhança do modelo,

$$
L(\mathbf{z}, \boldsymbol{\theta})=-\frac{3 N}{2} \log (2 \pi)-\frac{1}{2} \sum_{i=1}^{p} n_{i} \log \left|\mathbf{V}_{i}\right|-\frac{1}{2} \sum_{i=1}^{p} \sum_{j=1}^{n_{i}}\left(\mathbf{z}_{i_{j}}-\mathbf{m}_{i}\right)^{\prime} \mathbf{V}_{i}^{-1}\left(\mathbf{z}_{i_{j}}-\mathbf{m}_{i}\right),
$$

que pode ser expressa como

$$
\begin{aligned}
& L(\mathbf{z}, \boldsymbol{\theta})=-\frac{3 N}{2} \log (2 \pi)-\frac{1}{2} \sum_{i=1}^{p} n_{i} \log \nu_{i} \\
& -\frac{1}{2} \sum_{i=1}^{p}\left(\frac{\sigma_{e_{1 i}}^{2} \sigma_{e_{2 i}}^{2}+\sigma_{x}^{2}\left(\beta_{1 i}^{2} \sigma_{e_{2 i}}^{2}+\beta_{2 i} \sigma_{e_{1 i}}^{2}\right)}{\nu_{i}}\right) \sum_{j=1}^{n_{i}}\left(x_{i_{j}}-\mu\right)^{2} \\
& +\sum_{i=1}^{p} \beta_{1 i}\left(\frac{\sigma_{x}^{2} \sigma_{e_{2 i}}^{2}}{\nu_{i}}\right) \sum_{j=1}^{n_{i}}\left(x_{i_{j}}-\mu\right)\left(y_{1 i_{j}}-\beta_{1 i} \mu\right)+\sum_{i=1}^{p} \beta_{2 i}\left(\frac{\sigma_{x}^{2} \sigma_{e_{1 i}}^{2}}{\nu_{i}}\right) \sum_{j=1}^{n_{i}}\left(x_{i_{j}}-\mu\right)\left(y_{2 i_{j}}-\beta_{2 i} \mu\right) \\
& -\frac{1}{2} \sum_{i=1}^{p} \frac{1}{\nu_{i}}\left(\left(\sigma^{2}+\sigma_{x}^{2}\right) \sigma_{e_{2 i}}^{2}+\sigma_{x}^{2} \sigma^{2} \beta_{2 i}^{2}\right) \sum_{j=1}^{n_{i}}\left(y_{1 i_{j}}-\beta_{1 i} \mu\right)^{2} \\
& -\frac{1}{2} \sum_{i=1}^{p} \frac{1}{\nu_{i}}\left(\left(\sigma^{2}+\sigma_{x}^{2}\right) \sigma_{e_{1 i}}^{2}+\sigma_{x}^{2} \sigma^{2} \beta_{1 i}^{2}\right) \sum_{j=1}^{n_{i}}\left(y_{2 i_{j}}-\beta_{2 i} \mu\right)^{2} \\
& +\sum_{i=1}^{p} \frac{1}{\nu_{i}}\left(\beta_{1 i} \beta_{2 i} \sigma_{x}^{2} \sigma^{2}\right) \sum_{j=1}^{n_{i}}\left(y_{1 i_{j}}-\beta_{1 i} \mu\right)\left(y_{2 i_{j}}-\beta_{2 i} \mu\right)
\end{aligned}
$$

onde $\nu_{i}=\left(\sigma_{x}^{2}+\sigma^{2}\right)\left(\sigma_{e_{1 i}}^{2} \sigma_{e_{2 i}}^{2}\right)+\sigma_{x}^{2} \sigma^{2}\left(\beta_{1 i}^{2} \sigma_{e_{2 i}}^{2}+\beta_{2 i}^{2} \sigma_{e_{1 i}}^{2}\right)$.

\subsection{Função escore}

Apresentaremos nesta seção as formas das derivadas de primeira ordem de $L(\mathbf{z}, \boldsymbol{\theta})$ em relação a $\boldsymbol{\theta}$, ou seja, a função escore, que será utilizada na obtenção das estatísticas de teste. O cálculo destas derivadas se encontra no Apêndice C. 
Para facilitar os cálculos, definimos $\mathbf{a}_{i}$ e $\mathbf{A}_{i}$ tal que $\mathbf{m}_{i}=\mu \mathbf{a}_{i}$ e $\mathbf{V}_{i}=\mathbf{A}_{i}+\sigma_{x}^{2} \mathbf{a}_{i} \mathbf{a}_{i}^{\prime}$, ou seja,

$$
\mathbf{a}_{i}=\left(1, \boldsymbol{\beta}_{i}^{\prime}\right)^{\prime}=\left(1, \beta_{1 i}, \beta_{2 i}\right)^{\prime} \quad \text { e } \quad \mathbf{A}_{i}=\mathbf{D}\left(\sigma^{2}, \boldsymbol{\sigma}_{e_{i}}^{2}\right)^{\prime}=\mathbf{D}\left(\sigma^{2}, \sigma_{e_{1 i}}^{2}, \sigma_{e_{2 i}}^{2}\right)^{\prime},
$$

onde $\mathbf{D}(\mathbf{v})$ representa uma matriz quadrada com os elementos do vetor $\mathbf{v}$ na diagonal principal e todos os outros elementos nulos, isto é, representa uma matriz diagonal.

Na função $\log$-verossimilhança $L(\mathbf{z}, \boldsymbol{\theta})$ (equação 2.4), precisamos da matriz inversa $\mathbf{V}_{i}^{-1}$. Para este cálculo, utilizamos o Teorema 8.3.3, p. 189 de Graybill (1983) e as formas definidas em (2.6), e assim escrevemos $\mathbf{V}_{i}^{-1}$ na forma

$$
\begin{gathered}
\mathbf{V}_{i}^{-1}=\mathbf{A}_{i}^{-1}-\sigma_{x}^{2} b_{i}^{-1} \mathbf{B}_{i} \text { e }\left|\mathbf{V}_{i}\right|=b_{i}\left|\mathbf{A}_{i}\right|, \quad \text { onde } \\
b_{i}=1+\sigma_{x}^{2} \mathbf{a}_{i}^{\prime} \mathbf{A}_{i}^{-1} \mathbf{a}_{i}=1+\sigma_{x}^{2}\left[\sigma^{-2}+\boldsymbol{\beta}_{i}^{\prime} \mathbf{D}^{-1}\left(\boldsymbol{\sigma}_{e_{i}}^{2}\right) \boldsymbol{\beta}_{i}\right] \quad \text { e } \quad \mathbf{B}_{i}=\mathbf{A}_{i}^{-1} \mathbf{a}_{i} \mathbf{a}_{i}^{\prime} \mathbf{A}_{i}^{-1} .
\end{gathered}
$$

Os componentes do vetor de derivadas de primeira ordem de $L(\mathbf{z}, \boldsymbol{\theta})$ em relação a $\boldsymbol{\theta}=$ $\left(\boldsymbol{\beta}_{1}^{\prime}, \ldots, \boldsymbol{\beta}_{p}^{\prime}, \mu, \sigma^{2}, \sigma_{x}^{2}, \boldsymbol{\sigma}_{e_{1}}^{2^{\prime}}, \ldots, \boldsymbol{\sigma}_{e_{p}}^{2^{\prime}}\right)^{\prime}$ são dados por

1. $\frac{\partial L(\mathbf{z}, \boldsymbol{\theta})}{\partial \boldsymbol{\beta}_{i}}=\sum_{j=1}^{n_{i}}\left\{-\sigma_{x}^{2} b_{i}^{-1}\left\{1+\sigma_{x}^{2} b_{i}^{-1}\left[\left(\mathbf{z}_{i_{j}}-\mathbf{m}_{i}\right)^{\prime} \mathbf{B}_{i}\left(\mathbf{z}_{i_{j}}-\mathbf{m}_{i}\right)\right]+\mu\left[\mathbf{a}_{i}^{\prime} \mathbf{A}_{i}^{-1}\left(\mathbf{z}_{i_{j}}-\mathbf{m}_{i}\right)\right]\right\}\right.$

$$
\left.\mathbf{D}^{-1}\left(\boldsymbol{\sigma}_{\mathbf{e}_{i}}^{2}\right) \boldsymbol{\beta}_{i}+\left\{\mu+\sigma_{x}^{2} b_{i}^{-1}\left[\mathbf{a}_{i}^{\prime} \mathbf{A}_{i}^{-1}\left(\mathbf{z}_{i_{j}}-\mathbf{m}_{i}\right)\right]\right\} \mathbf{D}^{-1}\left(\boldsymbol{\sigma}_{\mathbf{e}_{i}}^{2}\right)\left(\mathbf{y}_{i_{j}}-\boldsymbol{\beta}_{i} \mu\right)\right\}, i=1, \ldots, p
$$

2. $\frac{\partial L(\mathbf{z}, \boldsymbol{\theta})}{\partial \mu}=\sum_{i=1}^{p} \sum_{j=1}^{n_{i}} b_{i}^{-1}\left[\mathbf{a}_{i}^{\prime} \mathbf{A}_{i}^{-1}\left(\mathbf{z}_{i_{j}}-\mathbf{m}_{i}\right)\right]$,

3. $\frac{\partial L(\mathbf{z}, \boldsymbol{\theta})}{\partial \sigma^{2}}=-\frac{1}{2} \sum_{i=1}^{p} \sum_{j=1}^{n_{i}}\left\{\sigma^{-2}\left[1-\sigma_{x}^{2} \sigma^{-2} b_{i}^{-1}-\sigma^{-2}\left(x_{i_{j}}-\mu\right)^{2}\right]\right.$

$$
\left.+\sigma_{x}^{2} \sigma^{-4} b_{i}^{-1}\left\{2\left(x_{i_{j}}-\mu\right)\left[\mathbf{a}_{i}^{\prime} \mathbf{A}_{i}^{-1}\left(\mathbf{z}_{i_{j}}-\mathbf{m}_{i}\right)\right]-\sigma_{x}^{2} b_{i}^{-1}\left[\left(\mathbf{z}_{i_{j}}-\mathbf{m}_{i}\right)^{\prime} \mathbf{B}_{i}\left(\mathbf{z}_{i_{j}}-\mathbf{m}_{i}\right)\right]\right\}\right\},
$$

4. $\frac{\partial L(\mathbf{z}, \boldsymbol{\theta})}{\partial \sigma_{x}^{2}}=-\frac{1}{2} \sum_{i=1}^{p} \sum_{j=1}^{n_{i}}\left\{\sigma_{x}^{-2}\left(1-b_{i}^{-1}\right)-b_{i}^{-2}\left[\left(\mathbf{z}_{i_{j}}-\mathbf{m}_{i}\right)^{\prime} \mathbf{B}_{i}\left(\mathbf{z}_{i_{j}}-\mathbf{m}_{i}\right)\right]\right\}$,

5. $\frac{\partial L(\mathbf{z}, \boldsymbol{\theta})}{\partial \boldsymbol{\sigma}_{\mathbf{e}_{i}}^{2}}=\sum_{j=1}^{n_{i}}\left\{\frac{1}{2} \mathbf{D}\left(\mathbf{y}_{i_{j}}-\boldsymbol{\beta}_{i} \mu\right) \mathbf{D}^{-2}\left(\boldsymbol{\sigma}_{\mathbf{e}_{i}}^{2}\right)\left(\mathbf{y}_{i_{j}}-\boldsymbol{\beta}_{i} \mu\right)+\right.$

$$
\begin{aligned}
& +\frac{1}{2} \sigma_{x}^{2} b_{i}^{-1}\left\{\sigma_{x}^{2} b_{i}^{-1}\left[\left(\mathbf{z}_{i_{j}}-\mathbf{m}_{i}\right)^{\prime} \mathbf{B}_{i}\left(\mathbf{z}_{i_{j}}-\mathbf{m}_{i}\right)\right]+1\right\} \mathbf{D}\left(\boldsymbol{\beta}_{i}\right) \mathbf{D}^{-2}\left(\boldsymbol{\sigma}_{\mathbf{e}_{i}}^{2}\right) \boldsymbol{\beta}_{i} \\
& \left.-\sigma_{x}^{2} b_{i}^{-1}\left[\mathbf{a}_{i}^{\prime} \mathbf{A}_{i}^{-1}\left(\mathbf{z}_{i_{j}}-\mathbf{m}_{i}\right)\right] \mathbf{D}\left(\boldsymbol{\beta}_{i}\right) \mathbf{D}^{-2}\left(\boldsymbol{\sigma}_{\mathbf{e}_{i}}^{2}\right)\left(\mathbf{y}_{i_{j}}-\boldsymbol{\beta}_{i} \mu\right)-\frac{1}{2} \mathbf{D}^{-1}\left(\boldsymbol{\sigma}_{\mathbf{e}_{i}}^{2}\right) \mathbf{1}_{2}\right\}, i=1, \ldots, p,
\end{aligned}
$$


onde $\boldsymbol{a}_{i}, \mathbf{A}_{i}, b_{i}$ e $\mathbf{B}_{i}$ são dados em (2.6) e (2.7).

Poderíamos obter as estimativas de máxima verossimilhança para os parâmetros com a solução do sistema de equações simultâneas

$$
\begin{gathered}
\frac{\partial L(\mathbf{z}, \boldsymbol{\theta})}{\partial \boldsymbol{\theta}}=\mathbf{0 , \text { ou seja }} \\
\frac{\partial L(\mathbf{z}, \boldsymbol{\theta})}{\partial \boldsymbol{\theta}}=\left(\begin{array}{c}
\frac{\partial L(\mathbf{z}, \boldsymbol{\theta})}{\partial \boldsymbol{\beta}_{1}} \\
\vdots \\
\frac{\partial L(\mathbf{z}, \boldsymbol{\theta})}{\partial \boldsymbol{\beta}_{p}} \\
\frac{\partial L(\mathbf{z}, \boldsymbol{\theta})}{\partial \mu} \\
\frac{\partial L(\mathbf{z}, \boldsymbol{\theta})}{\partial \sigma^{2}} \\
\frac{\partial L(\mathbf{z}, \boldsymbol{\theta})}{\partial \sigma_{x}^{2}} \\
\frac{\partial L(\mathbf{z}, \boldsymbol{\theta})}{\partial \boldsymbol{\sigma}_{e_{1}}^{2}} \\
\vdots \\
\frac{\partial L(\mathbf{z}, \boldsymbol{\theta})}{\partial \boldsymbol{\sigma}_{e_{p}}^{2}}
\end{array}\right)=\mathbf{0 .}
\end{gathered}
$$

Entretanto, as expressões das derivadas primeiras de $L(\mathbf{z}, \boldsymbol{\theta})$ em relação aos parâmetros são complexas e não-lineares, o que nos leva a adotar um procedimento iterativo para obter as estimativas de máxima verossimilhança para estes parâmetros. Apresentaremos na próxima seção um método iterativo para a obtenção das estimativas de máxima verossimilhança para os parâmetros do modelo. 


\section{Capítulo 3}

\section{Estimação dos parâmetros}

Utilizaremos neste capítulo um algoritmo iterativo para obter as estimativas dos parâmetros numericamente, já que não é simples obter os estimadores explicitamente, dada a complexidade das expressões das derivadas apresentadas na seção anterior. Descreveremos a seguir o algoritmo EM, que é muito utilizado como uma alternativa na obtenção de estimativas de máxima verossimilhança numericamente.

\subsection{Algoritmo EM}

O algoritmo EM (Expectation-Maximization algorithm) tem sido um método amplamente utilizado para se obter estimativas de máxima verossimilhança numericamente. Primeiramente proposto em Dempster et al. (1977), juntamente com exemplos e aplicações, este algoritmo é utilizado principalmente quando os dados do modelo podem ser tratados como dados incompletos. Por exemplo, num estudo de uma doença epidemiológica, onde muitos doentes podem não resistir até o fim do estudo. Apesar de ter sido apresentado formalmente em 1977, o algoritmo EM já teria sido utilizado anteriormente de maneira intuitiva por diversos autores para se obter estimativas de máxima verossimilhança em modelos com dados incompletos, como citam Dempster et al. (1977) e McLachlan \& Krishnan (1997). O algoritmo EM pode ser utilizado também quando não é tão imediato de se observar que há dados incompletos, por exemplo em modelos com efeitos aleatórios ou variáveis latentes.

Uma grande vantagem do algoritmo EM sobre outros algoritmos iterativos é a simplicidade de sua estrutura, que consiste de dois passos, o passo E (expectation-step ou simplesmente E-step) e o passo M (maximization-step ou M-step). O primeiro passo consiste na obtenção da esperança condicional da log-verossimilhança dos dados completos dadas as observações e o passo M maximiza, em relação aos parâmetros, a esperança condicional obtida no passo E. A simplicidade deste último passo ocorre quando a verossimilhança dos dados "completos" tem 
uma forma muito mais simples do que a dos dados "incompletos". Definidas as expressões dos dois passos, e utilizando um valor inicial (um ponto de partida para o processo iterativo), o algoritmo EM consiste da repetição dos passos E e M utilizando os valores calculados no passo anterior, até que se obtenha convergência. Apesar de ser um processo relativamente simples, a velocidade de convergência do algoritmo EM pode depender da condição inicial, por isto muitas vezes utiliza-se uma estimativa para os parâmetros obtida utilizando-se um método alternativo de estimação, como por exemplo o método dos momentos.

No modelo de regressão com erros nas variáveis, uma alternativa simplificadora é considerar que o verdadeiro valor dos dados (variável latente) é uma informação perdida, e tomar como dados completos as observações mais a variável latente. A função de verossimilhança do modelo completo, neste caso, é muito mais simples do que a função de verossimilhança original, como veremos na Seção 3.2, onde desenvolveremos as expressões dos passos E e M e aplicaremos o algoritmo aos dados do nosso problema.

\subsection{Desenvolvimento do algoritmo EM}

Como foi visto na seção anterior, para o modelo de regressão com erros nas variáveis uma alternativa simplificadora é considerar o verdadeiro valor dos dados (variável latente) como dado perdido, isto é, tomar como vetor de dados completos $\mathbf{w}_{i_{j}}=\left(\xi_{i_{j}}, x_{i_{j}}, y_{1 i_{j}}, y_{2 i_{j}}\right)^{\prime}=\left(\xi_{i_{j}}, \mathbf{z}_{i_{j}}\right)^{\prime}$ e trabalhar com o algoritmo estimando os valores de $E\left[\xi_{i_{j}} / \mathbf{z}, \boldsymbol{\theta}\right]$ e $E\left[\xi_{i_{j}}^{2} / \mathbf{z}, \boldsymbol{\theta}\right]$ (passo E) e maximizando a esperança condicional da log-verossimilhança dos dados completos dadas as observações (passo $\mathrm{M})$.

Para o modelo com dados completos temos que

$$
\mathbf{w}_{i_{j}} \sim N_{4}\left(\left(\begin{array}{c}
\mu \\
\mu \\
\beta_{1 i} \mu \\
\beta_{2 i} \mu
\end{array}\right),\left(\begin{array}{cccc}
\sigma_{x}^{2} & \sigma_{x}^{2} & \beta_{1 i} \sigma_{x}^{2} & \beta_{2 i} \sigma_{x}^{2} \\
\sigma_{x}^{2} & \sigma_{x}^{2}+\sigma^{2} & \beta_{1 i} \sigma_{x}^{2} & \beta_{2 i} \sigma_{x}^{2} \\
\beta_{1 i} \sigma_{x}^{2} & \beta_{1 i} \sigma_{x}^{2} & \beta_{1 i}^{2} \sigma_{x}^{2}+\sigma_{e_{1 i}}^{2} & \beta_{1 i} \beta_{2 i} \sigma_{x}^{2} \\
\beta_{2 i} \sigma_{x}^{2} & \beta_{2 i} \sigma_{x}^{2} & \beta_{1 i} \beta_{2 i} \sigma_{x}^{2} & \beta_{2 i}^{2} \sigma_{x}^{2}+\sigma_{e_{2 i}}^{2}
\end{array}\right)\right)
$$

Seja $\Sigma_{i}$ a matriz de covariâncias do modelo completo. $\mathrm{O}$ determinante de $\Sigma_{i}$ e sua inversa $\Sigma_{i}^{-1}$ são dados por 


$$
\left|\Sigma_{i}\right|=\sigma^{2} \sigma_{x}^{2} \sigma_{e_{1 i}}^{2} \sigma_{e_{2 i}}^{2} \quad \text { e } \quad \Sigma_{i}^{-1}=\left(\begin{array}{ccccc}
\frac{1}{\sigma^{2}}+\frac{1}{\sigma_{x}^{2}}+\frac{\beta_{1 i}^{2}}{\sigma_{e_{1 i}}^{2}}+\frac{\beta_{2 i}^{2}}{\sigma_{e_{2 i}}^{2}} & -\frac{1}{\sigma^{2}} & -\frac{\beta_{1 i}}{\sigma_{e_{1 i}}^{2}} & -\frac{\beta_{2 i}}{\sigma_{e_{2 i}}^{2}} \\
-\frac{1}{\sigma^{2}} & \frac{1}{\sigma^{2}} & 0 & 0 \\
-\frac{\beta_{1 i}}{\sigma_{e_{1 i}}^{2}} & 0 & \frac{1}{\sigma_{e_{1 i}}^{2}} & 0 \\
-\frac{\beta_{2 i}}{\sigma_{e_{2 i}}^{2}} & 0 & 0 & \frac{1}{\sigma_{e_{2 i}}^{2}}
\end{array}\right) .
$$

Neste caso, a função log-verossimilhança é dada por

$$
\begin{aligned}
& L(\mathbf{w}, \boldsymbol{\theta})=-2 N \log (2 \pi)-\frac{N}{2} \log \sigma^{2}-\frac{N}{2} \log \sigma_{x}^{2}-\frac{1}{2} \sum_{i=1}^{p} n_{i} \log \sigma_{e_{1 i}}^{2}-\frac{1}{2} \sum_{i=1}^{p} n_{i} \log \sigma_{e_{2 i}}^{2} \\
& -\frac{1}{2 \sigma_{x}^{2}} \sum_{i=1}^{p} \sum_{j=1}^{n_{i}}\left(\xi_{i_{j}}-\mu\right)^{2}-\frac{1}{2 \sigma^{2}} \sum_{i=1}^{p} \sum_{j=1}^{n_{i}}\left(x_{i_{j}}-\xi_{i_{j}}\right)^{2}-\frac{1}{2} \sum_{i=1}^{p} \frac{1}{\sigma_{e_{1 i}}^{2}} \sum_{j=1}^{n_{i}}\left(y_{1 i_{j}}-\beta_{1 i} \xi_{i_{j}}\right)^{2} \\
& -\frac{1}{2} \sum_{i=1}^{p} \frac{1}{\sigma_{e_{2 i}}^{2}} \sum_{j=1}^{n_{i}}\left(y_{2 i_{j}}-\beta_{2 i} \xi_{i_{j}}\right)^{2},
\end{aligned}
$$

que é claramente mais simples do que (2.5).

Calculando o valor esperado condicional de $L(\mathbf{w}, \boldsymbol{\theta})$ dadas as observações, obtemos

$$
\begin{aligned}
& E[L(\mathbf{w}, \boldsymbol{\theta}) / \mathbf{z}, \boldsymbol{\theta}]=-2 N \log (2 \pi)-\frac{N}{2} \log \left(\sigma^{2}\right)-\frac{N}{2} \log \left(\sigma_{x}^{2}\right)-\frac{1}{2} \sum_{i=1}^{p} n_{i} \log \sigma_{e_{1 i}}^{2} \\
& -\frac{1}{2} \sum_{i=1}^{p} n_{i} \log \sigma_{e_{2 i}}^{2}-\frac{1}{2 \sigma_{x}^{2}} \sum_{i=1}^{p} \sum_{j=1}^{n_{i}}\left(E\left[\xi_{i_{j}}^{2} / \mathbf{z}, \boldsymbol{\theta}\right]-2 \mu E\left[\xi_{i_{j}} / \mathbf{z}, \boldsymbol{\theta}\right]+\mu^{2}\right) \\
& -\frac{1}{2 \sigma^{2}} \sum_{i=1}^{p} \sum_{j=1}^{n_{i}}\left(x_{i_{j}}^{2}-2 x_{i_{j}} E\left[\xi_{i_{j}} / \mathbf{z}, \boldsymbol{\theta}\right]+E\left[\xi_{i_{j}}^{2} / \mathbf{z}, \boldsymbol{\theta}\right]\right) \\
& -\frac{1}{2} \sum_{i=1}^{p} \frac{1}{\sigma_{e_{1 i}}^{2}} \sum_{j=1}^{n_{i}}\left(y_{1 i_{j}}^{2}-2 y_{1 i_{j}} \beta_{1 i} E\left[\xi_{i_{j}} / \mathbf{z}, \boldsymbol{\theta}\right]+\beta_{1 i}^{2} E\left[\xi_{i_{j}}^{2} / \mathbf{z}, \boldsymbol{\theta}\right]\right) \\
& -\frac{1}{2} \sum_{i=1}^{p} \frac{1}{\sigma_{e_{2 i}}^{2}} \sum_{j=1}^{n_{i}}\left(y_{2 i_{j}}^{2}-2 y_{2 i_{j}} \beta_{2 i} E\left[\xi_{i_{j}} / \mathbf{z}, \boldsymbol{\theta}\right]+\beta_{2 i}^{2} E\left[\xi_{i_{j}}^{2} / \mathbf{z}, \boldsymbol{\theta}\right]\right) .
\end{aligned}
$$

Para calcular $E\left[\xi_{i_{j}} / \mathbf{z}, \boldsymbol{\theta}\right]$ e $E\left[\xi_{i_{j}}^{2} / \mathbf{z}, \boldsymbol{\theta}\right]$ adotamos a seguinte partição no vetor observado para o modelo completo

$$
\mathbf{w}_{i_{j}}=\left(\begin{array}{c}
\xi_{i_{j}} \\
\mathbf{z}_{i_{j}}
\end{array}\right) \text {, com vetor de médias }\left(\begin{array}{c}
\mu \\
\mathbf{m}_{i}
\end{array}\right) \text { e matriz de covariâncias }\left(\begin{array}{cc}
\sigma_{x}^{2} & \Sigma_{i_{12}} \\
\Sigma_{i_{21}} & \mathbf{V}_{i}
\end{array}\right) \text {, }
$$


onde $\Sigma_{i_{12}}=\left(\sigma_{x}^{2}, \beta_{1 i} \sigma_{x}^{2}, \beta_{2 i} \sigma_{x}^{2}\right), \Sigma_{i_{21}}=\Sigma_{i_{12}}^{\prime}$, e além disto $\mathbf{m}_{i}$ e $\mathbf{V}_{i}$ são respectivamente o vetor de médias e a matriz de covariâncias do modelo incompleto, dados em (2.1).

Utilizando o Teorema 1.2.11 (b), p. 12 de Muirhead (1982) temos que

$$
\xi_{i_{j}} / \mathbf{z}_{i_{j}} \sim N\left(\mu+\Sigma_{i_{12}} \mathbf{V}_{i}^{-1}\left(\mathbf{z}_{i_{j}}-\mathbf{m}_{i}\right), \sigma_{x}^{2}-\Sigma_{i_{12}} \mathbf{V}_{i}^{-1} \Sigma_{i_{21}}\right)
$$

O processo adotado pelo algoritmo EM consiste em estimar $\boldsymbol{\theta}$ maximizando (3.1) em relação aos parâmetros, onde os valores de $E\left[\xi_{i_{j}} / \mathbf{z}, \boldsymbol{\theta}\right]$ e $E\left[\xi_{i_{j}}^{2} / \mathbf{z}, \boldsymbol{\theta}\right]$ são substituídos pelas suas estimativas utilizando $\widehat{\boldsymbol{\theta}}$ da iteração anterior. Em seguida, estima novamente $E\left[\xi_{i_{j}} / \mathbf{z}, \boldsymbol{\theta}\right]$ e $E\left[\xi_{i_{j}}^{2} / \mathbf{z}, \boldsymbol{\theta}\right]$ utilizando as estimativas atualizadas dos parâmetros e assim sucessivamente até que se obtenha convergência. Executando os cálculos de $E\left[\xi_{i_{j}} / \mathbf{z}, \boldsymbol{\theta}\right]$ e $E\left[\xi_{i_{j}}^{2} / \mathbf{z}, \boldsymbol{\theta}\right]$ e calculando as derivadas de (3.1) com relação aos parâmetros obtemos os passos do algoritmo

\section{Passo E (esperança)}

$$
\begin{gathered}
\xi_{i_{j}}^{(k+1)}=E\left[\xi_{i_{j}} / \mathbf{z}, \boldsymbol{\theta}\right]=\frac{\left(\frac{\mu^{(k)}}{\sigma_{x}^{2(k)}}+\frac{x_{i_{j}}}{\sigma^{2(k)}}+\frac{y_{1 i_{j}} \beta_{1 i}^{(k)}}{\sigma_{e_{1 i}}^{2(k)}}+\frac{y_{i_{j}} \beta_{2 i}^{(k)}}{\sigma_{e_{2 i}}^{2(k)}}\right)}{\left(\frac{1}{\sigma^{2(k)}}+\frac{1}{\sigma_{x}^{2(k)}}+\frac{\left(\beta_{1 i}^{(k)}\right)^{2}}{\sigma_{e_{1 i}}^{2(k)}}+\frac{\left(\beta_{2 i}^{(k)}\right)^{2}}{\sigma_{e_{2 i}}^{2(k)}}\right)} \\
\left(\xi_{i_{j}}^{2}\right)^{(k+1)}=E\left[\xi_{i_{j}}^{2} / \mathbf{z}, \boldsymbol{\theta}\right]=\left(\xi_{i_{j}}^{(k+1)}\right)^{2}+\frac{1}{\left(\frac{1}{\sigma^{2(k)}}+\frac{1}{\sigma_{x}^{2(k)}}+\frac{\left(\beta_{1 i}^{(k)}\right)^{2}}{\sigma_{e_{1 i}}^{2(k)}}+\frac{\left(\beta_{2 i}^{(k)}\right)^{2}}{\sigma_{e_{2 i}}^{2(k)}}\right)}
\end{gathered}
$$

\section{Passo M (maximização)}

$$
\begin{aligned}
\mu^{(k+2)} & =\frac{1}{N} \sum_{i=1}^{p} \sum_{j=1}^{n_{i}} \xi_{i_{j}}^{(k+1)} \\
\sigma^{2^{(k+2)}}= & \frac{1}{N} \sum_{i=1}^{p} \sum_{j=1}^{n_{i}}\left[x_{i_{j}}^{2}-2 x_{i_{j}} \xi_{i_{j}}^{(k+1)}+\left(\xi_{i_{j}}^{2}\right)^{(k+1)}\right], \\
\sigma_{x}^{2^{(k+2)}}= & \frac{1}{N} \sum_{i=1}^{p} \sum_{j=1}^{n_{i}}\left[\left(\xi_{i_{j}}^{2}\right)^{(k+1)}-2 \mu^{(k+2)} \xi_{i_{j}}^{(k+1)}+\left(\mu^{(k+2)}\right)^{2}\right] \\
\beta_{1 i}^{(k+2)}= & \frac{\sum_{j=1}^{n_{i}} y_{1 i_{j}} \xi_{i_{j}}^{(k+1)}}{\sum_{i}\left(\xi_{i_{j}}^{2}\right)^{(k+1)}}, \quad \beta_{2 i}^{(k+2)}=\frac{\sum_{j=1}^{n_{i}} y_{2 i_{j}} \xi_{i_{j}}^{(k+1)}}{\sum_{j=1}^{n_{i}}\left(\xi_{i_{j}}^{2}\right)^{(k+1)}}
\end{aligned}
$$




$$
\begin{aligned}
& \sigma_{e_{1 i}}^{{ }^{2}(k+2)}=\frac{1}{n_{i}} \sum_{i=1}^{p} \sum_{j=1}^{n_{i}}\left[y_{1 i_{j}}^{2}-2 \beta_{1 i}^{(k+2)} y_{1 i_{j}} \xi_{i_{j}}^{(k+1)}+\beta_{1 i}^{2}\left(\xi_{i_{j}}^{2}\right)^{(k+1)}\right], \\
& \sigma_{e_{2 i}}^{2^{(k+2)}}=\frac{1}{n_{i}} \sum_{i=1}^{p} \sum_{j=1}^{n_{i}}\left[y_{2 i_{j}}^{2}-2 \beta_{2 i}^{(k+2)} y_{2 i_{j}} \xi_{i_{j}}^{(k+1)}+\beta_{2 i}^{2}\left(\xi_{i_{j}}^{2}\right)^{(k+1)}\right],
\end{aligned}
$$

onde o índice superior $(k)$ indica a $k$-ésima iteração, $n_{i}$ é o número de voluntários que testaram o líquido de bochecho $i, i=1, \ldots, p$ e $N=\sum_{i=1}^{p} n_{i}$. Observe que a maximização da esperança condicional da log-verossimilhança dos dados completos em relação a $\boldsymbol{\theta}$ é bastante simples e tem forma explícita. Desta forma, a obtenção das estimativas de máxima verossimilhança com a utilização do algoritmo EM, neste caso, é bastante simples e não-custoso computacionalmente.

Para se utilizar o algoritmo EM é necessário ter uma aproximação inicial dos parâmetros, já que no primeiro passo $E\left[\xi_{i_{j}} / \mathbf{z}, \boldsymbol{\theta}\right]$ e $E\left[\xi_{i_{j}}^{2} / \mathbf{z}, \boldsymbol{\theta}\right]$ dependem deles. Para esta aproximação inicial podemos utilizar por exemplo o método dos momentos, que estima os momentos populacionais pelos momentos amostrais. Um dos inconvenientes de se utilizar o método dos momentos, no entanto, é que um parâmetro pode ter mais de um estimador. Os estimadores que utilizamos, obtidos pelo método dos momentos, foram os seguintes:

$$
\begin{aligned}
& \widehat{\mu}=\frac{1}{N} \sum_{i=1}^{3} \sum_{j=1}^{n_{i}} x_{i_{j}}, \\
& \widehat{\beta}_{11}=\frac{1}{\widehat{\mu} n_{1}} \sum_{j=1}^{n_{1}} y_{11 j}, \quad \widehat{\beta}_{12}=\frac{1}{\widehat{\mu} n_{2}} \sum_{j=1}^{n_{2}} y_{12 j}, \quad \widehat{\beta}_{13}=\frac{1}{\widehat{\mu} n_{3}} \sum_{j=1}^{n_{3}} y_{13 j}, \\
& \widehat{\beta}_{21}=\frac{1}{\widehat{\mu} n_{1}} \sum_{j=1}^{n_{1}} y_{21 j}, \quad \widehat{\beta}_{22}=\frac{1}{\widehat{\mu} n_{2}} \sum_{j=1}^{n_{2}} y_{22 j}, \\
& \widehat{\sigma_{x}^{2}}=\left\{\frac{1}{\beta_{1 i} n_{i}} \sum_{j=1}^{n_{i}} x_{i_{j}} y_{1 i_{j}}-\mu^{2}, \frac{1}{\beta_{2 i} n_{i}} \sum_{j=1}^{n_{i}} x_{i_{j}} y_{2 i_{j}}-\mu^{2}, \frac{1}{\beta_{1 i} \beta_{2 i} n_{i}} \sum_{j=1}^{n_{i}} y_{1 i_{j}} y_{2 i_{j}}-\mu^{2}\right\},
\end{aligned}
$$

onde $i=1,2,3$. Neste caso, foi considerado como valor de $\widehat{\sigma_{x}^{2}}$ a função acima com um dos três valores para o índice $i$. 


$$
\begin{array}{ll}
\widehat{\sigma^{2}}=\frac{1}{3} \sum_{i=1}^{3} \frac{1}{n_{i}} \sum_{j=1}^{n_{i}} x_{i_{j}}^{2}-\widehat{\sigma_{x}^{2}}-\widehat{\mu}^{2}, & \\
\widehat{\sigma}_{e_{11}}^{2}=\frac{1}{n_{1}} \sum_{j=1}^{n_{1}} y_{11 j}^{2}-\widehat{\beta}_{11}^{2} \widehat{\sigma_{x}^{2}}-\widehat{\beta}_{11}^{2} \widehat{\mu}^{2}, \quad \widehat{\sigma}_{e_{12}}^{2}=\frac{1}{n_{2}} \sum_{j=1}^{n_{2}} y_{12 j}^{2}-\widehat{\beta}_{12}^{2} \widehat{\sigma_{x}^{2}}-\widehat{\beta}_{12}^{2} \widehat{\mu}^{2}, \\
\widehat{\sigma}_{e_{13}}^{2}=\frac{1}{n_{3}} \sum_{j=1}^{n_{3}} y_{13 j}^{2}-\widehat{\beta}_{13}^{2} \widehat{\sigma_{x}^{2}}-\widehat{\beta}_{13}^{2} \widehat{\mu}^{2}, \quad \widehat{\sigma}_{e_{21}}^{2}=\frac{1}{n_{1}} \sum_{j=1}^{n_{1}} y_{21 j}^{2}-\widehat{\beta}_{21}^{2} \widehat{\sigma_{x}^{2}}-\widehat{\beta}_{21}^{2} \widehat{\mu}^{2}, \\
\widehat{\sigma}_{e_{22}}^{2}=\frac{1}{n_{2}} \sum_{j=1}^{n_{2}} y_{22 j}^{2}-\widehat{\beta}_{22}^{2} \widehat{\sigma_{x}^{2}}-\widehat{\beta}_{22}^{2} \widehat{\mu}^{2}, \quad \widehat{\sigma}_{e_{23}}^{2}=\frac{1}{n_{3}} \sum_{j=1}^{n_{3}} y_{23 j}^{2}-\widehat{\beta}_{23}^{2} \widehat{\sigma_{x}^{2}}-\widehat{\beta}_{23}^{2} \widehat{\mu}^{2} .
\end{array}
$$

sendo que os estimadores de $\mu, \beta_{1 i}$ e $\beta_{2 i}, i=1, \ldots, 3$ foram obtidos estimando primeiros momentos populacionais pelos respectivos momentos amostrais e os estimadores de $\sigma_{x}^{2}, \sigma^{2}, \sigma_{e_{1 i}}^{2}$ e $\sigma_{e_{2 i}}^{2}, i=1, \ldots, 3$ foram obtidos estimando segundos momentos populacionais pelos respectivos momentos amostrais.

A Tabela 3.1 mostra as estimativas iniciais obtidas pelo método dos momentos e as estimativas de máxima verossimilhança obtidas pelo algoritmo EM considerando os dados odontológicos apresentados no Apêndice A.

Tabela 3.1: Estimativas iniciais obtidas pelo método dos momentos (EMM) e estimativas de máxima verossimilhança obtidas pelo algoritmo EM (EMV)

\begin{tabular}{cccccccccc}
\hline Parâmetro & $\beta_{11}$ & $\beta_{21}$ & $\beta_{12}$ & $\beta_{22}$ & $\beta_{13}$ & $\beta_{23}$ & $\mu$ & $\sigma^{2}$ \\
\hline EMM & 0.695 & 0.686 & 0.526 & 0.497 & 0.495 & 0.407 & 2.535 & 0.060 \\
EMV & 0.703 & 0.687 & 0.525 & 0.502 & 0.508 & 0.414 & 2.534 & 0.010 \\
\hline \multicolumn{10}{c}{${ }_{10}$} \\
\hline Parâmetro & $\sigma_{x}^{2}$ & $\sigma_{e_{11}}^{2}$ & $\sigma_{e_{21}}^{2}$ & $\sigma_{e_{12}}^{2}$ & $\sigma_{e_{22}}^{2}$ & $\sigma_{e_{13}}^{2}$ & $\sigma_{e_{23}}^{2}$ \\
\hline EMM & 0.050 & 0.459 & 0.320 & 0.486 & 0.524 & 0.282 & 0.189 \\
EMV & 0.103 & 0.312 & 0.234 & 0.430 & 0.431 & 0.255 & 0.192 \\
\hline
\end{tabular}

No próximo capítulo obteremos a distribuição assintótica dos estimadores, que utilizaremos no desenvolvimento dos testes assintóticos. 


\section{Capítulo 4}

\section{Distribuição assintótica dos estimadores de máxima verossimilhança}

Como no modelo proposto não temos as expressões exatas dos estimadores de máxima verossimilhança dos parâmetros, iremos utilizar os testes assintóticos de Wald, da razão de verossimilhanças e score para verificar a eficiência dos líquidos em questão. Para isto, é necessário obter a distribuição assintótica dos estimadores de máxima verossimilhança.

No nosso modelo, o vetor de parâmetros é dado por

$$
\boldsymbol{\theta}_{(4 p+3) \times 1}=\left(\boldsymbol{\beta}_{1}^{\prime}, \ldots, \boldsymbol{\beta}_{p}^{\prime}, \mu, \sigma^{2}, \sigma_{x}^{2}, \boldsymbol{\sigma}_{e_{1}}^{2^{\prime}}, \ldots, \boldsymbol{\sigma}_{e_{p}}^{2^{\prime}}\right)^{\prime}
$$

onde $\boldsymbol{\beta}_{i}=\left(\beta_{1 i}, \beta_{2 i}\right)^{\prime}$, e $\boldsymbol{\sigma}_{e_{i}}^{2}=\left(\sigma_{e_{1 i}}^{2}, \sigma_{e_{2 i}}^{2}\right)^{\prime}, i=1, \ldots, p$. A matriz de informação de Fisher esperada é dada por

$$
I(\boldsymbol{\theta})=-E\left[\frac{\partial^{2} L(\mathbf{z}, \boldsymbol{\theta})}{\partial \boldsymbol{\theta} \partial \boldsymbol{\theta}^{\prime}}\right]
$$

No nosso modelo, as observações não são identicamente distribuídas para os grupos que testaram os líquidos de bochecho. Existem parâmetros específicos de cada grupo, por exemplo, $\beta_{1 i}$ é parâmetro somente do grupo que testou o líquido $i$, para cada $i=1, \ldots, p$, assim como também existem parâmetros comuns a todos os grupos $\left(\mu, \sigma_{x}^{2}\right.$ e $\left.\sigma^{2}\right)$. Bradley \& Gart (1962) classifica tais grupos como populações associadas (populações com distribuições diferentes, porém que podem ter alguns parâmetros em comum). Sob condições de regularidade, Bradley \& Gart (1962) estudam a distribuição assintótica dos estimadores de máxima verossimilhança de $\boldsymbol{\theta}$ quando os dados têm estas características, mais precisamente:

Teorema 4.1 Seja $(\Omega, \mathcal{F}, P)$ um espaço de probabilidade e $\mathbf{z}_{i}$ um vetor aleatório com função densidade de probabilidade $f_{i}=f_{i}\left(\mathbf{z}_{i}, \boldsymbol{\theta}\right)$, onde $\mathbf{z}_{i}$ assume valores em uma região $R_{i}$ independente de $\boldsymbol{\theta}=\left(\theta_{1}, \ldots, \theta_{k}\right) \in \Theta$ para todo $i=1, \ldots, p$ e $\mathbf{z}_{i}$ independente de $\mathbf{z}_{j}$, para $i, j=1, \ldots, p, i \neq j$. 
Não é necessário que cada $f_{i}$ dependa de todos os parâmetros $\theta_{1}, \ldots, \theta_{k}$. Sejam $\mathbf{z}_{i_{1}}, \ldots, \mathbf{z}_{i_{n_{i}}}$ $n_{i}$ observações independentes de $\mathbf{z}_{i}, i=1, \ldots, p$, com função densidade de probabilidade $f_{i_{j}}=$ $f_{i_{j}}\left(\mathbf{z}_{i_{j}}, \boldsymbol{\theta}\right)$ para $i=1, \ldots, p$ e $j=1, \ldots, n_{i}$. Temos então a função densidade de probabilidade conjunta

$$
f(\mathbf{z}, \boldsymbol{\theta})=\prod_{i=1}^{p} \prod_{j=1}^{n_{i}} f_{i_{j}}\left(\mathbf{z}_{i_{j}}, \boldsymbol{\theta}\right)
$$

e as equações normais

$$
\frac{\partial \log f(\mathbf{z}, \boldsymbol{\theta})}{\partial \boldsymbol{\theta}_{r}}=0, r=1, \ldots, k .
$$

Supondo válidas as condições de regularidade

1. Para quase todo $\boldsymbol{z}_{i} \in R_{i}$ e para todo $\boldsymbol{\theta} \in \Theta$,

$$
\frac{\partial \log f_{i}}{\partial \theta_{r}}, \frac{\partial^{2} \log f_{i}}{\partial \theta_{r} \partial \theta_{s}} \quad e \frac{\partial^{3} \log f_{i}}{\partial \theta_{r} \partial \theta_{s} \partial \theta_{t}}
$$

existem para $r, s, t=1, \ldots, k ; i=1, \ldots, p$

2. Para toda densidade $f_{i}$, para quase todo $\boldsymbol{z}_{i} \in R_{i}$ e para todo $\boldsymbol{\theta} \in \Theta$

$$
\left|\frac{\partial f_{i}}{\partial \theta_{r}}\right|<F_{i r}\left(\boldsymbol{z}_{i}\right),\left|\frac{\partial^{2} f_{i}}{\partial \theta_{r} \partial \theta_{s}}\right|<F_{\text {irs }}\left(\boldsymbol{z}_{i}\right) \text { e }\left|\frac{\partial^{3} f_{i}}{\partial \theta_{r} \partial \theta_{s} \partial \theta_{t}}\right|<H_{\text {irst }}\left(\boldsymbol{z}_{i}\right),
$$

onde $F_{i r}\left(\boldsymbol{z}_{i}\right), F_{\text {irs }}\left(\boldsymbol{z}_{i}\right)$ são integráveis em $R_{i}$ e

$$
\int_{R_{i}} H_{i r s t}\left(\boldsymbol{z}_{i}\right) f_{i} d \boldsymbol{z}_{i}<M_{i}
$$

$(i=1, \ldots, p ; r, s, t=1, \ldots, k)$, onde $M_{i}$ são constantes positivas e finitas. Analogamente, para toda função de probabilidade $f_{i}$,

$$
\sum_{\boldsymbol{z}_{i} \in R_{i}} \frac{\partial f_{i}}{\partial \theta_{r}} \text { e } \sum_{\boldsymbol{z}_{i} \in R_{i}} \frac{\partial^{2} f_{i}}{\partial \theta_{r} \partial \theta_{s}}
$$

convergem uniformemente para todo $\boldsymbol{\theta} \in \Theta$ e

$$
\left|\frac{\partial^{3} f_{i}}{\partial \theta_{r} \partial \theta_{s} \partial \theta_{t}}\right|<H_{i r s t}\left(\boldsymbol{z}_{i}\right)
$$

onde

$$
\sum_{\boldsymbol{z}_{i} \in R_{i}} H_{\text {irst }}\left(\boldsymbol{z}_{i}\right) f_{i}<M_{i}
$$

para todo $\boldsymbol{\theta} \in \Theta(r, s, t=1, \ldots, k ; i=1, \ldots, p)$ e com $M_{i}$ constantes positivas e finitas. Estas condições permitirão intercambiar a ordem da diferenciação e integração ou somatória. 
3. Para todo $\boldsymbol{\theta} \in \Theta$, a matriz $J=\left[J_{r s}(\boldsymbol{\theta})\right]$ com

$$
J_{r s}(\boldsymbol{\theta})=\sum_{i=1}^{p} m_{i} \int_{R_{i}} \frac{\partial \log f_{i}}{\partial \theta_{r}} \frac{\partial \log f_{i}}{\partial \theta_{s}} f_{i} d \boldsymbol{z}_{i}
$$

é positiva definida com determinante finito (para o caso discreto basta substituir integração por somatória), com $m_{i}=\frac{n_{i}}{N}$ e $N=\sum_{i=1}^{p} n_{i}$.

Então, se $\widehat{\boldsymbol{\theta}}$ é o estimador de máxima verossimilhança de $\boldsymbol{\theta}_{0}$ (verdadeiro valor de $\boldsymbol{\theta}$ ), temos que $\sqrt{N}\left(\widehat{\boldsymbol{\theta}}-\boldsymbol{\theta}_{0}\right)$ tem distribuição assintótica (quando $N \rightarrow \infty$ ) normal multivariada com vetor de médias 0 e matriz de covariâncias $J_{0}^{-1}$, onde

$$
J_{0}=\left.\sum_{i=1}^{p} m_{i} E\left(\frac{\partial \log f_{i}}{\partial \theta_{r}} \frac{\partial \log f_{i}}{\partial \theta_{s}}\right)\right|_{\boldsymbol{\theta}=\boldsymbol{\theta}_{0}}=-\left.\sum_{i=1}^{p} m_{i} E\left(\frac{\partial^{2} \log f_{i}}{\partial \theta_{r} \partial \theta_{s}}\right)\right|_{\boldsymbol{\theta}=\boldsymbol{\theta}_{0}}
$$

Prova em Bradley \& Gart (1962).

Ou seja,

$$
\begin{gathered}
\sqrt{N}\left(\widehat{\boldsymbol{\theta}}-\boldsymbol{\theta}_{0}\right) \rightarrow \mathcal{N}_{k}\left(\mathbf{0}, J_{0}^{-1}\left(\boldsymbol{\theta}_{0}\right)\right) \text { quando } N \rightarrow \infty, \text { com } \\
J(\boldsymbol{\theta})=\sum_{i=1}^{p} \frac{n_{i}}{N} I_{i}(\boldsymbol{\theta})=-\sum_{i=1}^{p} \frac{n_{i}}{N} E\left[\frac{\partial^{2} \log f_{i}}{\partial \boldsymbol{\theta} \partial \boldsymbol{\theta}^{\prime}}\right],
\end{gathered}
$$

e $k$ é a dimensão de $\boldsymbol{\theta}$, (no nosso caso, $k=4 p+3$ ), $N=\sum_{i=1}^{p} n_{i}$ e $\widehat{\boldsymbol{\theta}}$ é o estimador de máxima verossimilhança de $\boldsymbol{\theta}$, e $I_{i}(\boldsymbol{\theta})$ é a matriz de informação de Fisher para cada grupo que testou os líquidos de bochecho, com $f_{i}$ dada em (2.2). Utilizando Dolby (1976), para cada $i=1, \ldots, p$, $\operatorname{com} L\left(\mathbf{z}_{i}, \boldsymbol{\theta}\right)=\log f_{i}$, temos que

$$
I_{i}(\boldsymbol{\theta})=-E\left[\frac{\partial^{2} L\left(\mathbf{z}_{i}, \boldsymbol{\theta}\right)}{\partial \theta_{r} \partial \theta_{s}}\right]=\left\{\frac{1}{2} \operatorname{tr}\left(\mathbf{V}_{i}^{-1} \mathbf{V}_{i_{\theta_{r}}} \mathbf{V}_{i}^{-1} \mathbf{V}_{i_{\theta_{s}}}\right)+\mathbf{d}_{i_{\theta_{r}}} \mathbf{V}_{i}^{-1} \mathbf{d}_{i_{\theta_{s}}}\right\},
$$

onde $\boldsymbol{V}_{i_{\theta_{r}}}$ e $\mathbf{d}_{i_{\theta_{r}}}$ são respectivamente a matriz e o vetor de derivadas elemento a elemento (em relação ao parâmetro $\left.\theta_{r}\right)$ da matriz de covariâncias $\left(\mathbf{V}_{i}\right)$ e da média de desvios $\left(\mathbf{d}_{i}\right), i=1, \ldots, p$, ou seja, no nosso caso,

$$
\mathbf{V}_{i}=\left(\begin{array}{ccc}
\sigma_{x}^{2}+\sigma^{2} & \beta_{1 i} \sigma_{x}^{2} & \beta_{2 i} \sigma_{x}^{2} \\
\beta_{1 i} \sigma_{x}^{2} & \beta_{1 i}^{2} \sigma_{x}^{2}+\sigma_{e_{1 i}}^{2} & \beta_{1 i} \beta_{2 i} \sigma_{x}^{2} \\
\beta_{2 i} \sigma_{x}^{2} & \beta_{1 i} \beta_{2 i} \sigma_{x}^{2} & \beta_{2 i}^{2} \sigma_{x}^{2}+\sigma_{e_{2 i}}^{2}
\end{array}\right) \text { e } \mathbf{d}_{i}=\left(\begin{array}{c}
\frac{1}{n_{i}} \sum_{j=1}^{n_{i}} x_{i_{j}}-\mu \\
\frac{1}{n_{i}} \sum_{j=1}^{n_{i}} y_{1 i_{j}}-\beta_{1 i} \mu \\
\frac{1}{n_{i}} \sum_{j=1}^{n_{i}} y_{2 i_{j}}-\beta_{2 i} \mu
\end{array}\right)
$$


Assim, por exemplo

$$
V_{i_{\sigma_{x}^{2}}}=\left(\begin{array}{ccc}
1 & \beta_{1 i} & \beta_{2 i} \\
\beta_{1 i} & \beta_{1 i}^{2} & \beta_{1 i} \beta_{2 i} \\
\beta_{2 i} & \beta_{1 i} \beta_{2 i} & \beta_{2 i}^{2}
\end{array}\right), \quad \text { e } \quad \mathbf{d}_{i_{\mu}}=\left(\begin{array}{c}
-1 \\
-\beta_{1 i} \\
-\beta_{2 i}
\end{array}\right), \quad i=1, \ldots, p .
$$

Utilizando a matriz $J(\boldsymbol{\theta})$ definida em (4.2), obteremos as estatísticas do teste, definidas na próxima seção, que utilizaremos para testar hipóteses de interesse do conjunto de dados de Hadgu \& Koch (1999), e tirar conclusões a respeito da eficiência dos líquidos de bochecho durante o estudo.

Apresentaremos aqui os elementos da matriz de informação do nosso modelo. A matriz de informação esperada, para cada grupo $i$, é dada por

$$
I_{i}(\theta)=-E\left[\frac{\partial^{2} L\left(\mathbf{z}_{i}, \theta\right)}{\partial \boldsymbol{\theta} \partial \boldsymbol{\theta}^{\prime}}\right]
$$

onde $\boldsymbol{\theta}_{(4 p+3) \times 1}=\left(\boldsymbol{\beta}_{1}^{\prime}, \ldots, \boldsymbol{\beta}_{p}^{\prime}, \mu, \sigma^{2}, \sigma_{x}^{2}, \boldsymbol{\sigma}_{\mathbf{e}_{1}}^{2^{\prime}}, \ldots, \boldsymbol{\sigma}_{\mathbf{e}_{p}}^{2^{\prime}}\right)^{\prime}$, com $p$ representando o número de líquidos de bochecho.

Definindo $\boldsymbol{\beta}^{\prime}=\left(\boldsymbol{\beta}_{1}^{\prime}, \ldots, \boldsymbol{\beta}_{p}^{\prime}\right)$ e $\boldsymbol{\sigma}_{\mathbf{e}}^{2^{\prime}}=\left(\boldsymbol{\sigma}_{\mathbf{e}_{1}}^{2^{\prime}}, \ldots, \boldsymbol{\sigma}_{\mathbf{e}_{p}}^{2^{\prime}}\right)$, temos que

$$
\begin{aligned}
& J(\boldsymbol{\theta})=-\sum_{i=1}^{p} \frac{n_{i}}{N} E\left(\begin{array}{ccccc}
\frac{\partial^{2} L\left(\mathbf{z}_{i}, \boldsymbol{\theta}\right)}{\partial \boldsymbol{\beta} \partial \boldsymbol{\beta}^{\prime}} & \frac{\partial^{2} L\left(\mathbf{z}_{i}, \boldsymbol{\theta}\right)}{\partial \boldsymbol{\beta} \partial \mu} & \frac{\partial^{2} L\left(\mathbf{z}_{i}, \boldsymbol{\theta}\right)}{\partial \boldsymbol{\beta} \partial \sigma^{2}} & \frac{\partial^{2} L\left(\mathbf{z}_{i}, \boldsymbol{\theta}\right)}{\partial \boldsymbol{\beta} \partial \sigma_{x}^{2}} & \frac{\partial^{2} L\left(\mathbf{z}_{i}, \boldsymbol{\theta}\right)}{\partial \boldsymbol{\beta} \partial \boldsymbol{\sigma}_{\mathbf{e}}^{2^{\prime}}} \\
\frac{\partial^{2} L\left(\mathbf{z}_{i}, \boldsymbol{\theta}\right)}{\partial \mu \partial \boldsymbol{\beta}^{\prime}} & \frac{\partial^{2} L\left(\mathbf{z}_{i}, \boldsymbol{\theta}\right)}{\partial \mu \partial \mu} & \frac{\partial^{2} L\left(\mathbf{z}_{i}, \boldsymbol{\theta}\right)}{\partial \mu \partial \sigma^{2}} & \frac{\partial^{2} L\left(\mathbf{z}_{i}, \boldsymbol{\theta}\right)}{\partial \mu \partial \sigma_{x}^{2}} & \frac{\partial^{2} L\left(\mathbf{z}_{i}, \boldsymbol{\theta}\right)}{\partial \mu \partial \boldsymbol{\sigma}_{\mathbf{e}}^{2^{\prime}}} \\
\frac{\partial^{2} L\left(\mathbf{z}_{i}, \boldsymbol{\theta}\right)}{\partial \sigma^{2} \partial \boldsymbol{\beta}^{\prime}} & \frac{\partial^{2} L\left(\mathbf{z}_{i}, \boldsymbol{\theta}\right)}{\partial \sigma^{2} \partial \mu} & \frac{\partial^{2} L\left(\mathbf{z}_{i}, \boldsymbol{\theta}\right)}{\partial \sigma^{2} \partial \sigma^{2}} & \frac{\partial^{2} L\left(\mathbf{z}_{i}, \boldsymbol{\theta}\right)}{\partial \sigma^{2} \partial \sigma_{x}^{2}} & \frac{\partial^{2} L\left(\mathbf{z}_{i}, \boldsymbol{\theta}\right)}{\partial \sigma^{2} \partial \boldsymbol{\sigma}_{\mathbf{e}}^{2^{\prime}}} \\
\frac{\partial^{2} L\left(\mathbf{z}_{i}, \boldsymbol{\theta}\right)}{\partial \sigma_{x}^{2} \partial \boldsymbol{\beta}^{\prime}} & \frac{\partial^{2} L\left(\mathbf{z}_{i}, \boldsymbol{\theta}\right)}{\partial \sigma_{x}^{2} \partial \mu} & \frac{\partial^{2} L\left(\mathbf{z}_{i}, \boldsymbol{\theta}\right)}{\partial \sigma_{x}^{2} \partial \sigma^{2}} & \frac{\partial^{2} L\left(\mathbf{z}_{i}, \boldsymbol{\theta}\right)}{\partial \sigma_{x}^{2} \partial \sigma_{x}^{2}} & \frac{\partial^{2} L\left(\mathbf{z}_{i}, \boldsymbol{\theta}\right)}{\partial \sigma_{x}^{2} \partial \boldsymbol{\sigma}_{\mathbf{e}}^{2^{\prime}}} \\
\frac{\partial^{2} L\left(\mathbf{z}_{i}, \boldsymbol{\theta}\right)}{\partial \boldsymbol{\sigma}_{\mathbf{e}}^{2} \partial \boldsymbol{\beta}^{\prime}} & \frac{\partial^{2} L\left(\mathbf{z}_{i}, \boldsymbol{\theta}\right)}{\partial \boldsymbol{\sigma}_{\mathbf{e}}^{2} \partial \mu} & \frac{\partial^{2} L\left(\mathbf{z}_{i}, \boldsymbol{\theta}\right)}{\partial \boldsymbol{\sigma}_{\mathbf{e}}^{2} \partial \sigma^{2}} & \frac{\partial^{2} L\left(\mathbf{z}_{i}, \boldsymbol{\theta}\right)}{\partial \boldsymbol{\sigma}_{\mathbf{e}}^{2} \partial \sigma_{x}^{2}} & \frac{\partial^{2} L\left(\mathbf{z}_{i}, \boldsymbol{\theta}\right)}{\partial \boldsymbol{\sigma}_{\mathbf{e}}^{2} \partial \boldsymbol{\sigma}_{\mathbf{e}}^{2^{\prime}}}
\end{array}\right)=
\end{aligned}
$$

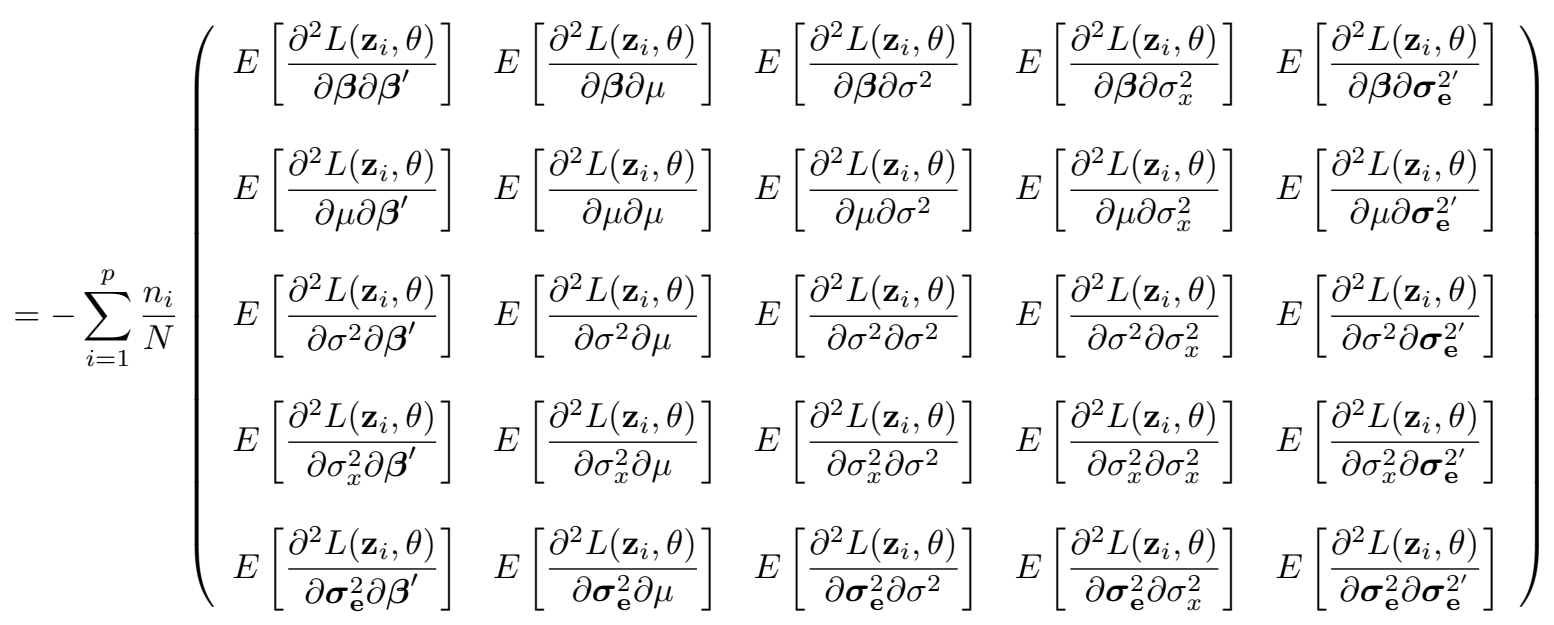


onde

$$
\frac{\partial^{2} L\left(\mathbf{z}_{i}, \boldsymbol{\theta}\right)}{\partial \boldsymbol{\beta} \partial \boldsymbol{\theta}_{l}^{\prime}}=\left(\begin{array}{c}
\frac{\partial^{2} L\left(\mathbf{z}_{i}, \boldsymbol{\theta}\right)}{\partial \boldsymbol{\beta}_{1} \partial \boldsymbol{\theta}_{l}^{\prime}} \\
\vdots \\
\frac{\partial^{2} L\left(\mathbf{z}_{i}, \boldsymbol{\theta}\right)}{\partial \boldsymbol{\beta}_{p} \partial \boldsymbol{\theta}_{l}^{\prime}}
\end{array}\right)_{2 p \times r} \quad \text { e } \frac{\partial^{2} L\left(\mathbf{z}_{i}, \boldsymbol{\theta}\right)}{\partial \boldsymbol{\sigma}_{\mathbf{e}}^{2} \partial \boldsymbol{\theta}_{l}^{\prime}}=\left(\begin{array}{c}
\frac{\partial^{2} L\left(\mathbf{z}_{i}, \boldsymbol{\theta}\right)}{\partial \boldsymbol{\sigma}_{\mathbf{e}_{1}}^{2} \partial \boldsymbol{\theta}_{l}^{\prime}} \\
\vdots \\
\frac{\partial^{2} L\left(\mathbf{z}_{i}, \boldsymbol{\theta}\right)}{\partial \boldsymbol{\sigma}_{\mathbf{e}_{p}}^{2} \partial \boldsymbol{\theta}_{l}^{\prime}}
\end{array}\right)_{2 p \times r}, l=1, \ldots, 2 p+3
$$

$\operatorname{com} \boldsymbol{\beta}_{j}=\left(\beta_{1 j}, \beta_{2 j}\right)^{\prime}$ e $\boldsymbol{\sigma}_{\mathbf{e}_{j}}^{2}=\left(\sigma_{\mathbf{e}_{1 j}}^{2}, \sigma_{\mathbf{e}_{2 j}}^{2}\right)^{\prime}, i, j=1, \ldots, p$.

Sejam $\mathbf{a}_{i}, \mathbf{A}_{i}, b_{i}$ e $\mathbf{B}_{i}$ como em (2.6) e (2.7) e seja

$$
J_{\boldsymbol{\theta}_{j}, \boldsymbol{\theta}_{l}^{\prime}}=-\sum_{i=1}^{p} \frac{n_{i}}{N} E\left[\frac{\partial^{2} L\left(\mathbf{z}_{i}, \theta\right)}{\partial \boldsymbol{\theta}_{j} \partial \boldsymbol{\theta}_{l}^{\prime}}\right], j, l=1, \ldots, 2 p+3 .
$$

Após manipulações algébricas e utilizando (4.2) e (4.3), e observando que

$$
J_{\boldsymbol{\theta}_{j}, \boldsymbol{\theta}_{l}^{\prime}}=\left(J_{\boldsymbol{\theta}_{l}, \boldsymbol{\theta}_{j}^{\prime}}\right)^{\prime}, \text { para } j \neq l,
$$

obtivemos as expressões de cada elemento $J_{\boldsymbol{\theta}_{j}, \boldsymbol{\theta}_{l}^{\prime}}$ de $J(\boldsymbol{\theta})$, onde $\boldsymbol{\theta}=\left(\boldsymbol{\beta}_{1}^{\prime}, \ldots, \boldsymbol{\beta}_{p}^{\prime}, \mu, \sigma^{2}, \sigma_{x}^{2}, \boldsymbol{\sigma}_{e_{1}}^{2^{\prime}}, \ldots, \boldsymbol{\sigma}_{e_{p}}^{2^{\prime}}\right)^{\prime}=\left(\boldsymbol{\theta}_{1}, \ldots, \boldsymbol{\theta}_{2 p+3}\right)^{\prime}$.

As expressões são dadas por:

$$
\begin{aligned}
& J_{\boldsymbol{\beta}_{\boldsymbol{i}}, \boldsymbol{\beta}_{\boldsymbol{i}}{ }^{\prime}}=\frac{n_{i}}{N}\left\{\left[2\left(\sigma_{x}^{2}\right)^{2}\left(b_{i}\right)^{-2}+\sigma_{x}^{2} b_{i}^{-1} \mu^{2}\right] \mathbf{D}^{-1}\left(\boldsymbol{\sigma}_{\mathbf{e}_{i}}^{2}\right) \boldsymbol{\beta}_{\boldsymbol{i}} \boldsymbol{\beta}_{\boldsymbol{i}}{ }^{\prime} \mathbf{D}^{-1}\left(\boldsymbol{\sigma}_{\mathbf{e}_{i}}^{2}\right)+\right. \\
& \left.+\left(\sigma_{x}^{2}-\sigma_{x}^{2} b_{i}^{-1}+\mu^{2}\right) \mathbf{D}^{-1}\left(\boldsymbol{\sigma}_{\mathbf{e}_{i}}^{2}\right)-\left(\sigma_{x}^{2}+2 \mu^{2}\right)\left[b_{i} \sigma_{x}^{2} \mathbf{D}\left(\boldsymbol{\beta}_{\boldsymbol{i}}\right) \mathbf{D}^{-1}\left(\boldsymbol{\sigma}_{\mathbf{e}_{i}}^{2}\right) \mathbf{1 1}^{\prime} \mathbf{D}^{-1}\left(\boldsymbol{\sigma}_{\mathbf{e}_{i}}^{2}\right) \mathbf{D}\left(\boldsymbol{\beta}_{\boldsymbol{i}}\right)\right]\right\}, \\
& i=1, \ldots, p \\
& J_{\boldsymbol{\beta}_{\boldsymbol{i}}, \boldsymbol{\beta}_{\boldsymbol{j}}{ }^{\prime}=0, \quad i \neq j ; i, j=1, \ldots, p} \\
& J_{\boldsymbol{\beta}_{\boldsymbol{i}}, \mu}=\frac{n_{i}}{N} b_{i}^{-1} \mu \mathbf{D}^{-1}\left(\boldsymbol{\sigma}_{\mathbf{e}_{i}}^{2}\right) \boldsymbol{\beta}_{\boldsymbol{i}}, i=1, \ldots, p \\
& J_{\boldsymbol{\beta}_{\boldsymbol{i}}, \sigma^{2}}=-\frac{n_{i}}{N}\left(\sigma_{x}^{2}\right)^{2} b_{i}^{-2}\left(\sigma^{2}\right)^{-2} \mathbf{D}^{-1}\left(\boldsymbol{\sigma}_{\mathbf{e}_{i}}^{2}\right) \boldsymbol{\beta}_{\boldsymbol{i}}, i=1, \ldots, p \\
& J_{\boldsymbol{\beta}_{\boldsymbol{i}}, \sigma_{x}^{2}}=\frac{n_{i}}{N}\left(b_{i}^{-1}-b_{i}^{-2}\right) \mathbf{D}^{-1}\left(\boldsymbol{\sigma}_{\mathbf{e}_{i}}^{2}\right) \boldsymbol{\beta}_{\boldsymbol{i}}, i=1, \ldots, p \\
& J_{\boldsymbol{\beta}_{\boldsymbol{i}}, \boldsymbol{\sigma}_{\mathbf{e}_{\mathbf{i}}}^{2^{\prime}}}=-\frac{n_{i}}{N}\left[\left(\sigma_{x}^{2}\right)^{2} b_{i}^{-2} \mathbf{D}^{-1}\left(\boldsymbol{\sigma}_{\mathbf{e}_{i}}^{2}\right) \boldsymbol{\beta}_{\boldsymbol{i}} \boldsymbol{\beta}_{\boldsymbol{i}}{ }^{\prime} \mathbf{D}^{-2}\left(\boldsymbol{\sigma}_{\mathbf{e}_{i}}^{2}\right) \mathbf{D}\left(\boldsymbol{\beta}_{\boldsymbol{i}}\right)-\sigma_{x}^{2} b_{i}^{-1} \mathbf{D}^{-2}\left(\boldsymbol{\sigma}_{\mathbf{e}_{i}}^{2}\right) \mathbf{D}\left(\boldsymbol{\beta}_{\boldsymbol{i}}\right)\right], \\
& i=1, \ldots, p \\
& J_{\boldsymbol{\beta}_{\boldsymbol{i}}, \boldsymbol{\sigma}_{\mathbf{e}_{\mathbf{j}}}^{2^{\prime}}=0, \quad i \neq j, i ; j=1, \ldots, p} \\
& J_{\mu, \mu}=\sum_{i=1}^{p} \frac{n_{i}}{N}\left(1-b_{i}^{-1}\right)\left(\sigma_{x}^{2}\right)^{-1}, J_{\mu, \sigma^{2}}=0, J_{\mu, \sigma_{x}^{2}}=0, \quad J_{\mu, \boldsymbol{\sigma}_{\mathbf{e}_{\mathbf{i}}}^{2^{\prime}}}=\mathbf{0}, i=1, \ldots, p \\
& J_{\sigma^{2}, \sigma^{2}}=\sum_{i=1}^{p} \frac{n_{i}}{2 N} \frac{\left(\sigma^{2} b_{i}-\sigma_{x}^{2}\right)^{2}}{\left(\sigma^{2}\right)^{4} b_{i}^{2}}, \quad J_{\sigma^{2}, \sigma_{x}^{2}}=\sum_{i=1}^{p} \frac{n_{i}}{2 N}\left(\sigma^{2} b_{i}\right)^{-2}
\end{aligned}
$$




$$
\begin{aligned}
& J \sigma^{2}, \boldsymbol{\sigma}_{\mathbf{e}_{\mathbf{i}}}^{2^{\prime}}=\frac{n_{i}}{2 N}\left(\sigma_{x}^{2}\right)^{2} b_{i}^{-2}\left(\sigma^{2}\right)^{-2} \boldsymbol{\beta}_{\boldsymbol{i}}{ }^{\prime} \mathbf{D}\left(\boldsymbol{\beta}_{\boldsymbol{i}}\right) \mathbf{D}^{-2}\left(\boldsymbol{\sigma}_{\mathbf{e}_{i}}^{2}\right), i=1, \ldots, p \\
& J \sigma_{x}^{2}, \sigma_{x}^{2}=\sum_{i=1}^{p} \frac{n_{i}}{2 N}\left(\sigma_{x}^{2}\right)^{-2}\left(b_{i}^{-2}-2 b_{i}^{-1}+1\right), \quad J \sigma_{x}^{2}, \boldsymbol{\sigma}_{\mathbf{e}_{\mathbf{i}}}^{2^{\prime}}=\frac{n_{i}}{2 N} b_{i}^{-2} \boldsymbol{\beta}_{\boldsymbol{i}}{ }^{\prime} \mathbf{D}^{-2}\left(\boldsymbol{\sigma}_{\mathbf{e}_{i}}^{2}\right) \mathbf{D}\left(\boldsymbol{\beta}_{\boldsymbol{i}}\right), i=1, \ldots, p \\
& J \boldsymbol{\sigma}_{\mathbf{e}_{\mathbf{i}}}^{2}, \boldsymbol{\sigma}_{\mathbf{e}_{\mathbf{i}}}^{2^{\prime}}=-\frac{n_{i}}{2 N}\left[-\left(\sigma_{x}^{2}\right)^{2} b i^{-2} \mathbf{D}\left(\boldsymbol{\beta}_{\boldsymbol{i}}\right) \mathbf{D}^{-2}\left(\boldsymbol{\sigma}_{\mathbf{e}_{i}}^{2}\right) \boldsymbol{\beta}_{\boldsymbol{i}} \boldsymbol{\beta}_{\boldsymbol{i}}{ }^{\prime} \mathbf{D}^{-2}\left(\boldsymbol{\sigma}_{\mathbf{e}_{i}}^{2}\right) \mathbf{D}\left(\boldsymbol{\beta}_{\boldsymbol{i}}\right)+\right. \\
& \left.+2 \sigma_{x}^{2} b_{i}^{-1} \mathbf{D}\left(\boldsymbol{\beta}_{\boldsymbol{i}}\right) \mathbf{D}^{-3}\left(\boldsymbol{\sigma}_{\mathbf{e}_{i}}^{2}\right) \mathbf{D}\left(\boldsymbol{\beta}_{\boldsymbol{i}}\right)-\mathbf{D}^{-2}\left(\boldsymbol{\sigma}_{\mathbf{e}_{i}}^{2}\right)\right], i=1, \ldots, p \\
& J \boldsymbol{\sigma}_{\mathbf{e}_{\mathbf{i}}}^{2}, \boldsymbol{\sigma}_{\mathbf{e}_{\mathbf{j}}}^{2^{\prime}}=0, i \neq j, i, j=1, \ldots, p
\end{aligned}
$$

Apresentaremos agora os valores de $J(\widehat{\boldsymbol{\theta}})$. Para facilitar a visualização, seja a partição

$$
J(\widehat{\boldsymbol{\theta}})=\left(\begin{array}{ccc}
J_{11} & J_{12} & J_{13} \\
\left(J_{12}\right)^{\prime} & J_{22} & J_{23} \\
\left(J_{13}\right)^{\prime} & \left(J_{23}\right)^{\prime} & J_{33}
\end{array}\right),
$$

onde:

$$
\begin{aligned}
& J_{11}=\left(E\left[\frac{\partial^{2} L\left(\mathbf{z}_{i}, \theta\right)}{\partial \boldsymbol{\beta} \partial \boldsymbol{\beta}^{\prime}}\right]\right)_{2 p \times 2 p}, \\
& J_{12}=\left(E\left[\frac{\partial^{2} L\left(\mathbf{z}_{i}, \theta\right)}{\partial \boldsymbol{\beta} \partial \mu}\right] \quad E\left[\frac{\partial^{2} L\left(\mathbf{z}_{i}, \theta\right)}{\partial \boldsymbol{\beta} \partial \sigma^{2}}\right] E\left[\frac{\partial^{2} L\left(\mathbf{z}_{i}, \theta\right)}{\partial \boldsymbol{\beta} \partial \sigma_{x}^{2}}\right]\right)_{2 p \times 3}, \\
& J_{13}=\left(E\left[\frac{\partial^{2} L\left(\mathbf{z}_{i}, \theta\right)}{\partial \boldsymbol{\beta} \partial \boldsymbol{\sigma}_{\mathbf{e}}^{2^{\prime}}}\right]\right)_{2 p \times 2 p}, \\
& J_{22}=\left(\begin{array}{lll}
E\left[\frac{\partial^{2} L\left(\mathbf{z}_{i}, \theta\right)}{\partial \mu \partial \mu}\right] & E\left[\frac{\partial^{2} L\left(\mathbf{z}_{i}, \theta\right)}{\partial \mu \partial \sigma^{2}}\right] & E\left[\frac{\partial^{2} L\left(\mathbf{z}_{i}, \theta\right)}{\partial \mu \partial \sigma_{x}^{2}}\right] \\
E\left[\frac{\partial^{2} L\left(\mathbf{z}_{i}, \theta\right)}{\partial \sigma^{2} \partial \mu}\right] & E\left[\frac{\partial^{2} L\left(\mathbf{z}_{i}, \theta\right)}{\partial \sigma^{2} \partial \sigma^{2}}\right] & E\left[\frac{\partial^{2} L\left(\mathbf{z}_{i}, \theta\right)}{\partial \sigma^{2} \partial \sigma_{x}^{2}}\right] \\
E\left[\frac{\partial^{2} L\left(\mathbf{z}_{i}, \theta\right)}{\partial \sigma_{x}^{2} \partial \mu}\right] & E\left[\frac{\partial^{2} L\left(\mathbf{z}_{i}, \theta\right)}{\partial \sigma_{x}^{2} \partial \sigma^{2}}\right] & E\left[\frac{\partial^{2} L\left(\mathbf{z}_{i}, \theta\right)}{\partial \sigma_{x}^{2} \partial \sigma_{x}^{2}}\right]
\end{array}\right)_{3 \times 3}, \\
& J_{23}=\left(\begin{array}{c}
E\left[\frac{\partial^{2} L\left(\mathbf{z}_{i}, \theta\right)}{\partial \mu \partial \boldsymbol{\sigma}_{\mathbf{e}}^{2^{\prime}}}\right] \\
E\left[\frac{\partial^{2} L\left(\mathbf{z}_{i}, \theta\right)}{\partial \sigma^{2} \partial \boldsymbol{\sigma}_{\mathbf{e}}^{2^{\prime}}}\right] \\
E\left[\frac{\partial^{2} L\left(\mathbf{z}_{i}, \theta\right)}{\partial \sigma_{x}^{2} \partial \boldsymbol{\sigma}_{\mathbf{e}}^{2^{\prime}}}\right]
\end{array}\right)_{3 \times 2 p},
\end{aligned}
$$


$J_{33}=\left(E\left[\frac{\partial^{2} L\left(\mathbf{z}_{i}, \theta\right)}{\partial \boldsymbol{\sigma}_{\mathbf{e}}^{2} \partial \boldsymbol{\sigma}_{\mathbf{e}}^{2^{\prime}}}\right]\right)_{2 p \times 2 p}$.

Os elementos de $J(\widehat{\boldsymbol{\theta}})$ são dados por

$J_{11}=\left(\begin{array}{rrrrrr}7.061 & -0.1277 & 0 & 0 & 0 & 0 \\ -0.1277 & 9.3884 & 0 & 0 & 0 & 0 \\ 0 & 0 & 4.7348 & -0.0257 & 0 & 0 \\ 0 & 0 & -0.0257 & 4.7219 & 0 & 0 \\ 0 & 0 & 0 & 0 & 8.6798 & -0.0839 \\ 0 & 0 & 0 & 0 & -0.0839 & 11.5151\end{array}\right)$

$J_{12}=\left(\begin{array}{rrr}0.1638 & -0.6052 & 0.0592 \\ 0.2137 & -0.7895 & 0.0773 \\ 0.0831 & -0.3135 & 0.03 \\ 0.0792 & -0.2989 & 0.0286 \\ 0.1471 & -0.5514 & 0.0531 \\ 0.1587 & -0.5951 & 0.0573\end{array}\right)$,

$J_{13}=\left(\begin{array}{rrrrrr}0.0211 & -0.0005 & 0 & 0 & 0 & 0 \\ -0.0004 & 0.0366 & 0 & 0 & 0 & 0 \\ 0 & 0 & 0.0078 & 0 & 0 & 0 \\ 0 & 0 & 0 & 0.0074 & 0 & 0 \\ 0 & 0 & 0 & 0 & 0.0233 & -0.0002 \\ 0 & 0 & 0 & 0 & -0.0002 & 0.0334\end{array}\right)$,

$J_{22}=\left(\begin{array}{rrr}8.8638 & 0 & 0 \\ 0 & 57.5626 & 37.6046 \\ 0 & 37.6046 & 39.2833\end{array}\right)$,

$J_{23}=\left(\begin{array}{rrrrrr}0 & 0 & 0 & 0 & 0 & 0 \\ 0.6817 & 1.1603 & 0.1915 & 0.174 & 0.5495 & 0.6399 \\ 0.0061 & 0.0104 & 0.0017 & 0.0016 & 0.0049 & 0.0057\end{array}\right)$,

$J_{33}=\left(\begin{array}{rrrrrr}1.7131 & 0.0006 & 0 & 0 & 0 & 0 \\ 0.0006 & 3.0286 & 0 & 0 & 0 & 0 \\ 0 & 0 & 0.8404 & 0 & 0 & 0 \\ 0 & 0 & 0 & 0.8358 & 0 & 0 \\ 0 & 0 & 0 & 0 & 2.5888 & 0.0002 \\ 0 & 0 & 0 & 0 & 0.0002 & 4.5564\end{array}\right)$.

Seja agora $K(\widehat{\boldsymbol{\theta}})=J^{-1}(\widehat{\boldsymbol{\theta}})$. Analogamente consideramos a partição

$$
K(\widehat{\boldsymbol{\theta}})=\left(\begin{array}{ccc}
K_{11} & K_{12} & K_{13} \\
\left(K_{12}\right)^{\prime} & K_{22} & K_{23} \\
\left(K_{13}\right)^{\prime} & \left(K_{23}\right)^{\prime} & K_{33}
\end{array}\right)
$$

Os elementos de $K(\widehat{\boldsymbol{\theta}})=J^{-1}(\widehat{\boldsymbol{\theta}})$ são dados a seguir: 


$$
\begin{aligned}
& K_{11}=\left(\begin{array}{rrrrrr}
0.1422 & 0.0024 & 0.0004 & 0.0004 & 0.0004 & 0.0003 \\
0.0024 & 0.1071 & 0.0004 & 0.0004 & 0.0004 & 0.0003 \\
0.0004 & 0.0004 & 0.2115 & 0.0014 & 0.0003 & 0.0002 \\
0.0004 & 0.0004 & 0.0014 & 0.2121 & 0.0003 & 0.0002 \\
0.0004 & 0.0004 & 0.0003 & 0.0003 & 0.1155 & 0.0011 \\
0.0003 & 0.0003 & 0.0002 & 0.0002 & 0.0011 & 0.087
\end{array}\right), \\
& K_{12}=\left(\begin{array}{ccc}
-0.0027 & 0.0048 & -0.0048 \\
-0.0026 & 0.0047 & -0.0047 \\
-0.0020 & 0.0036 & -0.0036 \\
-0.0019 & 0.0034 & -0.0035 \\
-0.0020 & 0.0035 & -0.0035 \\
-0.0016 & 0.0028 & -0.0028
\end{array}\right) \text {, } \\
& K_{13}=\left(\begin{array}{cccccc}
-0.0036 & -0.0018 & -0.0011 & -0.0010 & -0.0010 & -0.0007 \\
-0.0018 & -0.0031 & -0.0011 & -0.0010 & -0.0010 & -0.0007 \\
-0.0014 & -0.0014 & -0.0028 & -0.0007 & -0.0008 & -0.0005 \\
-0.0014 & -0.0013 & -0.0008 & -0.0026 & -0.0007 & -0.0005 \\
-0.0014 & -0.0013 & -0.0008 & -0.0007 & -0.0018 & -0.0005 \\
-0.0011 & -0.0011 & -0.0006 & -0.0006 & -0.0006 & -0.0010
\end{array}\right) \text {, } \\
& K_{22}=\left(\begin{array}{rrr}
0.1130 & -0.0004 & 0.0004 \\
-0.0004 & 0.0493 & -0.0472 \\
0.0004 & -0.0472 & 0.0706
\end{array}\right) \text {, } \\
& K_{23}=\left(\begin{array}{rrrrrr}
0.0002 & 0.0002 & 0.0001 & 0.0001 & 0.0001 & 0.0001 \\
-0.0195 & -0.0188 & -0.0112 & -0.0102 & -0.0104 & -0.0069 \\
0.0186 & 0.0179 & 0.0106 & 0.0097 & 0.0099 & 0.0066
\end{array}\right) \text {, } \\
& K_{33}=\left(\begin{array}{cccccc}
0.5915 & 0.0073 & 0.0044 & 0.0040 & 0.0041 & 0.0027 \\
0.0073 & 0.3374 & 0.0043 & 0.0039 & 0.0040 & 0.0026 \\
0.0044 & 0.0043 & 1.1925 & 0.0023 & 0.0024 & 0.0016 \\
0.0040 & 0.0039 & 0.0023 & 1.1986 & 0.0022 & 0.0014 \\
0.0041 & 0.0040 & 0.0024 & 0.0022 & 0.3885 & 0.0014 \\
0.0027 & 0.0026 & 0.0016 & 0.0014 & 0.0014 & 0.2204
\end{array}\right) .
\end{aligned}
$$

Utilizando a matriz inversa acima, obtivemos as estimativas para os desvios padrão das estimativas de máxima verossimilhança. A Tabela 4.1 mostra as estimativas para os parâmetros com os respectivos desvios padrão.

Tabela 4.1: Estimativas de máxima verossimilhança dos parâmetros (com desvios padrão)

\begin{tabular}{cccccccccc}
\hline Parâmetro & $\beta_{11}$ & $\beta_{21}$ & $\beta_{12}$ & $\beta_{22}$ & $\beta_{13}$ & $\beta_{23}$ & $\mu$ & $\sigma^{2}$ \\
\hline Estimativa & 0.703 & 0.687 & 0.525 & 0.502 & 0.508 & 0.414 & 2.535 & 0.010 \\
Desvio-padrão & 0.037 & 0.032 & 0.045 & 0.045 & 0.033 & 0.029 & 0.033 & 0.022 \\
\hline Parâmetro & $\sigma_{x}^{2}$ & $\sigma_{e_{11}}^{2}$ & $\sigma_{e_{21}}^{2}$ & $\sigma_{e_{12}}^{2}$ & $\sigma_{e_{22}}^{2}$ & $\sigma_{e_{13}}^{2}$ & $\sigma_{e_{23}}^{2}$ \\
\hline Estimativa & 0.103 & 0.312 & 0.234 & 0.430 & 0.431 & 0.255 & 0.192 \\
Desvio-padrão & 0.026 & 0.075 & 0.057 & 0.107 & 0.107 & 0.061 & 0.046 \\
\hline
\end{tabular}




\section{Capítulo 5}

\section{Testes de hipóteses assintóticos}

Neste capítulo, iremos utilizar os testes de hipóteses assintóticos de Wald, da razão de verossimilhanças e score (como por exemplo em (Sen \& Singer 1993)) para testar as hipóteses de interesse do conjunto de dados de Hadgu \& Koch (1999). Para isto iremos considerar o seguinte teorema:

Teorema 5.1 Seja $(\Omega, \mathcal{F}, P)$ um espaço de probabilidade e $\mathbf{z}_{i}$ um vetor aleatório com função densidade de probabilidade $f_{i}\left(\mathbf{z}_{i}, \boldsymbol{\theta}\right)$, onde $\mathbf{z}_{i}$ assume valores em uma região $R_{i}$ independente de $\boldsymbol{\theta}=\left(\theta_{1}, \ldots, \theta_{k}\right) \in \Theta$ para todo $i=1, \ldots, p$ e $\mathbf{z}_{i}$ independente de $\mathbf{z}_{j}$, para $i \neq j$, como no Teorema 4.1. Novamente, não é necessário que cada $f_{i}$ dependa de todos os parâmetros $\theta_{1}, \ldots, \theta_{k}$. Sejam $\mathbf{z}_{i 1}, \ldots, \mathbf{z}_{i n_{i}} n_{i}$ vetores de observações independentes de $\mathbf{z}_{i}, i=1, \ldots, p$, com função densidade de probabilidade $f_{i_{j}}\left(\mathbf{z}_{i_{j}}, \boldsymbol{\theta}\right)$ para $i=1, \ldots, p$ e $j=1, \ldots, n_{i}$. A função densidade de probabilidade conjunta é dada por

$$
f(\mathbf{z}, \boldsymbol{\theta})=\prod_{i=1}^{p} \prod_{j=1}^{n_{i}} f_{i}\left(\mathbf{z}_{i_{j}}, \boldsymbol{\theta}\right)
$$

Supondo válidas as condições de regularidade descritas no Teorema 4.1, temos que

(I) $\sqrt{N}\left(\widehat{\boldsymbol{\theta}}-\boldsymbol{\theta}_{0}\right) \stackrel{N \rightarrow \infty}{\rightarrow} \mathcal{N}_{k}\left(\mathbf{0}, J^{-1}\left(\boldsymbol{\theta}_{0}\right)\right)$, onde $J(\boldsymbol{\theta})$ é dada em (4.2), e cada elemento de $J\left(\boldsymbol{\theta}_{0}\right)$ é dado por

$$
J\left(\boldsymbol{\theta}_{0}\right)_{r s}=\left.\sum_{i=1}^{p} m_{i} E\left(\frac{\partial \log f_{i}}{\partial \theta_{r}} \frac{\partial \log f_{i}}{\partial \theta_{s}}\right)\right|_{\boldsymbol{\theta}_{=} \boldsymbol{\theta}_{0}}=-\left.\sum_{i=1}^{p} \frac{n_{i}}{N} E\left(\frac{\partial^{2} \log f_{i}}{\partial \theta_{r} \partial \theta_{s}}\right)\right|_{\boldsymbol{\theta}=\boldsymbol{\theta}_{0}},
$$

$\operatorname{com} \widehat{\boldsymbol{\theta}}$ o estimador de máxima verossimilhança de $\boldsymbol{\theta}$ e $\boldsymbol{\theta}_{0}$ o verdadeiro valor de $\boldsymbol{\theta}$.

Seja

$$
H_{0}: \mathbf{h}(\boldsymbol{\theta})=\mathbf{0} \text { versus } H_{1}: \mathbf{h}(\boldsymbol{\theta}) \neq \mathbf{0},
$$

onde $\mathbf{h}(\boldsymbol{\theta})=\left(h_{1}, \ldots, h_{r}\right)^{\prime}: \mathbb{R}^{k} \rightarrow \mathbb{R}^{r} \quad(r \leq k)$ é tal que 
(i) Existem $k-r$ funções adicionais $h^{r+1}(\boldsymbol{\theta}), \ldots, h^{k}(\boldsymbol{\theta})$ tal que as relações inversas $\theta_{1}\left(\boldsymbol{h}^{*}\right), \ldots, \theta_{k}\left(\boldsymbol{h}^{*}\right)$ existem, com $\boldsymbol{h}^{*}=\left(h_{1}(\boldsymbol{\theta}), \ldots, h_{k}(\boldsymbol{\theta})\right)$.

(ii) as derivadas parciais de primeira e segunda ordem de $h_{1}(\boldsymbol{\theta}), \ldots, h_{k}(\boldsymbol{\theta})$ são funções uniformemente contínuas e limitadas de $\boldsymbol{\theta}$.

(iii) o maior limite inferior do valor absoluto do jacobiano $\frac{\partial\left(h_{1}, \ldots, h_{k}\right)^{\prime}}{\partial\left(\theta_{1}, \ldots, \theta_{k}\right)}$ é positivo.

Seja $\mathbf{H}(\boldsymbol{\theta})=\frac{\partial \mathbf{h}(\boldsymbol{\theta})^{\prime}}{\partial \boldsymbol{\theta}}$ uma matriz $(k \times r)$ contínua em $\boldsymbol{\theta}$ com posto $r, \widehat{\boldsymbol{\theta}}$ o estimador de máxima verossimilhança de $\boldsymbol{\theta}$ e $\overline{\boldsymbol{\theta}}$ o estimador de máxima verossimilhança restrito a $H_{0}$, ou seja, tal que $h(\overline{\boldsymbol{\theta}})=0$, e seja

(a) Estatística Wald

$$
Q_{W}=N \mathbf{h}(\widehat{\boldsymbol{\theta}})^{\prime}\left[\mathbf{H}^{\prime}(\widehat{\boldsymbol{\theta}}) J^{-1}(\widehat{\boldsymbol{\theta}}) \mathbf{H}(\widehat{\boldsymbol{\theta}})\right]^{-1} \mathbf{h}(\widehat{\boldsymbol{\theta}}),
$$

(b) Estatística da razão de verossimilhanças

$$
Q_{L}=-2 \log \lambda=2(L(\mathbf{z}, \widehat{\boldsymbol{\theta}})-L(\mathbf{z}, \overline{\boldsymbol{\theta}}))
$$

onde $\lambda=\sup _{\{\boldsymbol{\theta} \in \Theta: \mathbf{h}(\boldsymbol{\theta})=\mathbf{0}\}} L(\mathbf{z}, \boldsymbol{\theta}) / \sup _{\{\boldsymbol{\theta} \in \Theta\}} L(\mathbf{z}, \boldsymbol{\theta})$,

(c) Estatística score

$$
Q_{R}=N^{-1} U^{\prime}(\overline{\boldsymbol{\theta}}) J^{-1}(\overline{\boldsymbol{\theta}}) U(\overline{\boldsymbol{\theta}}),
$$

onde

$$
U(\overline{\boldsymbol{\theta}})=\left.\sum_{i=1}^{p} \sum_{j=1}^{n_{i}} \frac{\partial \log f_{i}\left(\mathbf{z}_{i_{j}}, \boldsymbol{\theta}\right)}{\partial \boldsymbol{\theta} \partial \boldsymbol{\theta}^{\prime}}\right|_{\boldsymbol{\theta}=\overline{\boldsymbol{\theta}}}
$$

(II) Então $Q_{W}, Q_{L}$ e $Q_{R}$ têm distribuição assintótica $\chi_{r}^{2}$ sob $H_{0}$.

Prova: (I) para $Q_{W}, Q_{L}$ e $Q_{R}$ e (II) para $Q_{L}$ estão demonstrados em Bradley \& Gart (1962) (II) $Q_{W}$ : De (I), temos que $\sqrt{N}\left(\widehat{\boldsymbol{\theta}}-\boldsymbol{\theta}_{0}\right) \stackrel{N \rightarrow \infty}{\rightarrow} \mathcal{N}_{k}\left(\mathbf{0}, J^{-1}\left(\boldsymbol{\theta}_{0}\right)\right)$. Assim, utilizando o Método Delta (Lehmann \& Casella 1998, Teorema 8.22 pp 61) temos que:

$$
\sqrt{N}\left(h(\widehat{\boldsymbol{\theta}})-h\left(\boldsymbol{\theta}_{0}\right)\right) \stackrel{N \rightarrow \infty}{\rightarrow} \mathcal{N}_{k}\left(\mathbf{0}, H^{\prime}\left(\boldsymbol{\theta}_{0}\right) J^{-1}\left(\boldsymbol{\theta}_{0}\right) H\left(\boldsymbol{\theta}_{0}\right)\right) \text {, e portanto, sob } H_{0}:
$$

$$
N \mathbf{h}^{\prime}(\widehat{\boldsymbol{\theta}})\left[\mathbf{H}^{\prime}\left(\boldsymbol{\theta}_{0}\right) J^{-1}\left(\boldsymbol{\theta}_{0}\right) \mathbf{H}\left(\boldsymbol{\theta}_{0}\right)\right]^{-1} \mathbf{h}(\widehat{\boldsymbol{\theta}}) \stackrel{N \rightarrow \infty}{\rightarrow} \chi_{r}^{2}
$$


Como $\widehat{\boldsymbol{\theta}}$ é consistente, $\widehat{\boldsymbol{\theta}} \stackrel{P}{\rightarrow} \boldsymbol{\theta}_{0}$. Então

$$
\left[\mathbf{H}^{\prime}(\widehat{\boldsymbol{\theta}}) J^{-1}(\widehat{\boldsymbol{\theta}}) \mathbf{H}(\widehat{\boldsymbol{\theta}})\right]^{-1} \stackrel{P}{\rightarrow}\left[\mathbf{H}^{\prime}\left(\boldsymbol{\theta}_{0}\right) J^{-1}\left(\boldsymbol{\theta}_{0}\right) \mathbf{H}\left(\boldsymbol{\theta}_{0}\right)\right]^{-1}
$$

(Van der Vaart 1998, Teorema 2.3, pp 7) e portanto

$$
\left[\mathbf{H}^{\prime}(\widehat{\boldsymbol{\theta}}) J^{-1}(\widehat{\boldsymbol{\theta}}) \mathbf{H}(\widehat{\boldsymbol{\theta}})\right]^{-1}\left[\mathbf{H}^{\prime}\left(\boldsymbol{\theta}_{0}\right) J^{-1}\left(\boldsymbol{\theta}_{0}\right) \mathbf{H}\left(\boldsymbol{\theta}_{0}\right)\right] \stackrel{P}{\rightarrow} I_{k},
$$

onde $I_{k}$ denota uma matriz identidade de ordem $k$. Assim, utilizando-se o Teorema de Courant (Sen \& Singer (1993), Teorema 1.4.2 pp 28) temos que

$$
\lambda_{k} \leq \frac{\mathbf{h}^{\prime}(\widehat{\boldsymbol{\theta}})\left[\mathbf{H}^{\prime}(\widehat{\boldsymbol{\theta}}) J^{-1}(\widehat{\boldsymbol{\theta}}) \mathbf{H}(\widehat{\boldsymbol{\theta}})\right]^{-1} \mathbf{h}(\widehat{\boldsymbol{\theta}})}{\mathbf{h}^{\prime}(\widehat{\boldsymbol{\theta}})\left[\mathbf{H}^{\prime}\left(\boldsymbol{\theta}_{0}\right) J^{-1}\left(\boldsymbol{\theta}_{0}\right) \mathbf{H}\left(\boldsymbol{\theta}_{0}\right)\right]^{-1} \mathbf{h}(\widehat{\boldsymbol{\theta}})} \leq \lambda_{1},
$$

onde $\lambda_{i}$ é a $i$-ésima raiz característica de $\left[\mathbf{H}^{\prime}(\widehat{\boldsymbol{\theta}}) J^{-1}(\widehat{\boldsymbol{\theta}}) \mathbf{H}(\widehat{\boldsymbol{\theta}})\right]^{-1}\left[\mathbf{H}^{\prime}\left(\boldsymbol{\theta}_{0}\right) J^{-1}\left(\boldsymbol{\theta}_{0}\right) \mathbf{H}\left(\boldsymbol{\theta}_{0}\right)\right]$. Desta forma, temos que $\lambda_{i} \stackrel{P}{\rightarrow} 1$ e $\lambda_{k} \stackrel{P}{\rightarrow} 1$ e portanto

$$
\frac{\mathbf{h}^{\prime}(\widehat{\boldsymbol{\theta}})\left[\mathbf{H}^{\prime}(\widehat{\boldsymbol{\theta}}) J^{-1}(\widehat{\boldsymbol{\theta}}) \mathbf{H}(\widehat{\boldsymbol{\theta}})\right]^{-1} \mathbf{h}(\widehat{\boldsymbol{\theta}})}{\mathbf{h}^{\prime}(\widehat{\boldsymbol{\theta}})\left[\mathbf{H}^{\prime}\left(\boldsymbol{\theta}_{0}\right) J^{-1}\left(\boldsymbol{\theta}_{0}\right) \mathbf{H}\left(\boldsymbol{\theta}_{0}\right)\right]^{-1} \mathbf{h}(\widehat{\boldsymbol{\theta}})} \stackrel{P}{\rightarrow} 1,
$$

e aplicando Slutsky (Van der Vaart 1998, Lema 2.8 pp 11), temos que:

$\mathbf{h}^{\prime}(\widehat{\boldsymbol{\theta}})\left[\mathbf{H}^{\prime}(\widehat{\boldsymbol{\theta}}) J^{-1}(\widehat{\boldsymbol{\theta}}) \mathbf{H}(\widehat{\boldsymbol{\theta}})\right]^{-1} \mathbf{h}(\widehat{\boldsymbol{\theta}})=$

$=\frac{\mathbf{h}^{\prime}(\widehat{\boldsymbol{\theta}})\left[\mathbf{H}^{\prime}(\widehat{\boldsymbol{\theta}}) J^{-1}(\widehat{\boldsymbol{\theta}}) \mathbf{H}(\widehat{\boldsymbol{\theta}})\right]^{-1} \mathbf{h}(\widehat{\boldsymbol{\theta}})}{\mathbf{h}^{\prime}(\widehat{\boldsymbol{\theta}})\left[\mathbf{H}^{\prime}\left(\boldsymbol{\theta}_{0}\right) J^{-1}\left(\boldsymbol{\theta}_{0}\right) \mathbf{H}\left(\boldsymbol{\theta}_{0}\right)\right]^{-1} \mathbf{h}(\widehat{\boldsymbol{\theta}})} \mathbf{h}^{\prime}(\widehat{\boldsymbol{\theta}})\left[\mathbf{H}^{\prime}\left(\boldsymbol{\theta}_{0}\right) J^{-1}\left(\boldsymbol{\theta}_{0}\right) \mathbf{H}\left(\boldsymbol{\theta}_{0}\right)\right]^{-1} \mathbf{h}(\widehat{\boldsymbol{\theta}}) \stackrel{\mathcal{D}}{\rightarrow} \chi_{r}^{2}$

(II) $Q_{R}:$ De Bradley \& Gart (1962), temos que

$$
\begin{gathered}
-\frac{1}{N} \frac{\partial^{2} L(\mathbf{z}, \boldsymbol{\theta})}{\partial \theta_{r} \partial \theta_{s}}=-\frac{1}{N} \sum_{i=1}^{p} \sum_{j=1}^{n_{i}} \frac{\partial^{2} \log f_{i_{j}}}{\partial \theta_{r} \partial \theta_{s}}=-\sum_{i=1}^{p} m_{i} \sum_{j=1}^{n_{i}}\left(\frac{-1}{n_{i}}\right) \frac{\partial^{2} \log f_{i_{j}}}{\partial \theta_{r} \partial \theta_{s}} \rightarrow J_{r s}\left(\boldsymbol{\theta}_{0}\right) \\
N^{-\frac{1}{2}} U(\boldsymbol{\theta}) \stackrel{\mathcal{D}}{\rightarrow} \mathcal{N}(\mathbf{0}, J(\boldsymbol{\theta}))
\end{gathered}
$$

Assim, seguindo os passos de Sen \& Singer (1993) (Teorema 5.6.3 pp 242) e usando (5.1) segue que $Q_{R} \rightarrow \chi_{r}^{2}$.

Utilizando este teorema, testaremos as seguintes hipóteses de interesse do conjunto de dados:

- Se dois líquidos diferentes têm a mesma eficiência nos primeiros três meses de uso, ou seja,

$$
H_{0}: \beta_{1 l}=\beta_{1 m} \text { versus } H_{1}: \beta_{1 l} \neq \beta_{1 m} \text {, para } l, m=1, \ldots, p, l \neq m \text {, }
$$


- Se dois líquidos diferentes têm a mesma eficiência nos seis meses de uso, ou seja,

$$
H_{0}: \beta_{2 l}=\beta_{2 m} \text { versus } H_{1}: \beta_{2 l} \neq \beta_{2 m}, \text { para } l, m=1, \ldots, p, l \neq m
$$

- Se cada líquido continua a diminuir a placa bacteriana após três meses de estudo, ou seja,

$$
H_{0}: \beta_{1 l}=\beta_{2 l} \text { versus } H_{1}: \beta_{1 l} \neq \beta_{2 l}, \text { para } l=1, \ldots, p \text {. }
$$

Para utilizar as estatísticas da razão de verossimilhanças e score, precisaremos das estimativas de máxima verossimilhança sob cada hipótese $H_{0}$. Para calcular tais estimativas, utilizaremos o algoritmo iterativo EM sob $H_{0}$. Neste caso, porém, na Etapa M (maximization) não é possível explicitar os estimadores de todos os parâmetros, como mostraremos na Seção 5.1, o que nos levou a utilizar o método de Newton-Raphson dentro de cada passo M do algoritmo. Este método será descrito na mesma seção.

\subsection{Estimativas de máxima verossimilhança sob $H_{0}$}

Nesta subseção iremos obter as estimativas de máxima verossimilhança sob $H_{0}$, para cada teste de hipótese considerado. Para isto, utilizaremos o Algoritmo EM sob cada hipótese $H_{0}$. Consideremos a hipótese (5.2), em que

$$
H_{0}: \beta_{1 l}=\beta_{1 m}=\beta \text { versus } H_{1}: \beta_{1 l} \neq \beta_{1 m} \text { para } l, m=1, \ldots, p, l \neq m
$$

Na Seção 3.2, havíamos obtido $E(L(\mathbf{w}, \boldsymbol{\theta}) / \mathbf{z})$ (equação $(3.1))$. Seja agora $L_{0}(\mathbf{w}, \boldsymbol{\theta})$ a função de verossimilhança do modelo completo sob a hipótese $H_{0}$. No passo E, as expressões de $\xi_{i_{j}}^{(k+1)}$ e $\left(\xi_{i_{j}}^{2}\right)^{(k+1)}$ permanecem iguais às do algoritmo EM irrestrito para $i \neq l$ e $i \neq m, \operatorname{com} j=1, \ldots, n_{i}$. Para $i=l$ ou $i=m$ temos:

\section{Passo E (expectation)}

$$
\begin{gathered}
\xi_{i_{j}}^{(k+1)}=\frac{\left(\frac{\mu^{(k)}}{\sigma_{x}^{2(k)}}+\frac{x_{i_{j}}}{\sigma^{2(k)}}+\frac{y_{1 i_{j}} \beta^{(k)}}{\sigma_{e_{1 i}}^{2(k)}}+\frac{y_{i_{j}} \beta_{2 i}^{(k)}}{\sigma_{e_{2 i}}^{2(k)}}\right)}{\left(\frac{1}{\sigma^{2(k)}}+\frac{1}{\sigma_{x}^{2(k)}}+\frac{\left(\beta^{(k)}\right)^{2}}{\sigma_{e_{1 i}}^{2(k)}}+\frac{\left(\beta_{2 i}^{(k)}\right)^{2}}{\sigma_{e_{2 i}}^{2(k)}}\right)} \\
\left(\xi_{i_{j}}^{2}\right)^{(k+1)}=E\left[\xi_{i_{j}}^{2} / \mathbf{z}, \boldsymbol{\theta}\right]=\left(\xi_{i_{j}}^{(k+1)}\right)^{2}+\frac{1}{\left(\frac{1}{\sigma^{2(k)}}+\frac{1}{\sigma_{x}^{2(k)}}+\frac{\left(\beta^{(k)}\right)^{2}}{\sigma_{e_{1 i}}^{2(k)}}+\frac{\left(\beta_{2 i}^{(k)}\right)^{2}}{\sigma_{e_{2 i}}^{2(k)}}\right)}
\end{gathered}
$$


No passo M, analogamente, as expressões de $\mu^{(k+2)}, \sigma^{2^{(k+2)}}, \sigma_{x}^{2^{(k+2)}}, \beta_{2 i}^{(k+2)}$ e $\sigma_{e_{2 i}}^{2^{(k+2)}}$ para $i=1, \ldots, p$, permanecem as mesmas. Para $i \neq l$ e $i \neq m$, as expressões de $\beta_{1 i}$ e $\sigma_{e_{1 i}^{2}}^{(k+2)}$ também permanecem iguais. No entanto, não é possível explicitar as expressões de $\beta_{1 l}^{(k+2)}=\beta_{1 m}^{(k+2)}=$ $\beta^{(k+2)}, \sigma_{e_{1 l}^{2}}^{(k+2)}$ e $\sigma_{e_{1 m}^{2}}^{(k+2)}$ já que ao igualar a zero as derivadas parciais de primeira ordem de $E\left(L_{0}(\mathbf{w}, \boldsymbol{\theta}) / \mathbf{z}\right)$ em relação a estes parâmetros, obtemos três equações não-lineares envolvendo as três variáveis:

$$
\begin{gathered}
\sigma_{\mathbf{e}_{1 m}}^{2} \sum_{j=1}^{n_{l}}\left(y_{1 l_{j}} \widehat{\xi}_{l_{j}}-\beta \widehat{\xi}_{l_{j}}^{2}\right)+\sigma_{\mathbf{e}_{1 l}}^{2} \sum_{j=1}^{n_{m}}\left(y_{1 m_{j}} \widehat{\xi}_{m_{j}}-\beta \widehat{\xi}_{m_{j}}^{2}\right)=0 \\
-\sigma_{\mathbf{e}_{1 l}}^{2} n_{l}+\sum_{j=1}^{n_{l}}\left(y_{1 l_{j}}^{2}-2 \beta y_{1 l_{j}} \widehat{\xi}_{l_{j}}+\beta^{2} \widehat{\xi}_{l_{j}}^{2}\right)=0 \\
-\sigma_{\mathbf{e}_{1 m}}^{2} n_{m}+\sum_{j=1}^{n_{m}}\left(y_{1 m_{j}}^{2}-2 \beta y_{1 m_{j}} \widehat{\xi}_{m_{j}}+\beta^{2} \widehat{\xi}_{m_{j}}^{2}\right)=0 .
\end{gathered}
$$

Optamos então pela utilização de um método iterativo para resolver estas equações numericamente (o método de Newton-Raphson).

$\operatorname{Seja} \mathbf{U}\left(\beta, \sigma_{\mathbf{e}_{1 l}}^{2}, \sigma_{\mathbf{e}_{1 m}}^{2}\right)=\left(U_{1}, U_{2}, U_{3}\right)^{\prime}$ onde

$$
\begin{aligned}
& U_{1}\left(\beta, \sigma_{\mathbf{e}_{1 l}}^{2}, \sigma_{\mathbf{e}_{1 m}}^{2}\right)=\sigma_{\mathbf{e}_{1 m}}^{2} \sum_{j=1}^{n_{l}}\left(y_{1 l_{j}} \widehat{\xi}_{l_{j}}-\beta \widehat{\xi}_{l_{j}}^{2}\right)+\sigma_{\mathbf{e}_{1 l}}^{2} \sum_{j=1}^{n_{m}}\left(y_{1 m_{j}} \widehat{\xi}_{m_{j}}-\beta \widehat{\xi}_{m_{j}}^{2}\right), \\
& U_{2}\left(\beta, \sigma_{\mathbf{e}_{1 l}}^{2}, \sigma_{\mathbf{e}_{1 m}}^{2}\right)=-\sigma_{\mathbf{e}_{1 l}}^{2} n_{l}+\sum_{j=1}^{n_{l}}\left(y_{1 l_{j}}^{2}-2 \beta y_{1 l_{j}} \widehat{\xi}_{l_{j}}+\beta^{2} \widehat{\xi}_{l_{j}}^{2}\right), \\
& U_{3}\left(\beta, \sigma_{\mathbf{e}_{1 l}}^{2}, \sigma_{\mathbf{e}_{1 m}}^{2}\right)=-\sigma_{\mathbf{e}_{1 m}}^{2} n_{m}+\sum_{j=1}^{n_{m}}\left(y_{1 m_{j}}^{2}-2 \beta y_{1 m_{j}} \widehat{\xi}_{m_{j}}+\beta^{2} \widehat{\xi}_{m_{j}}^{2}\right) .
\end{aligned}
$$

Assim, teremos a matriz jacobiana

$$
J=\left(\begin{array}{ccc}
\frac{\partial}{\partial \beta} U_{1} & \frac{\partial}{\partial \sigma_{\mathbf{e}_{1 l}}^{2}} U_{1} & \frac{\partial}{\partial \sigma_{\mathbf{e}_{1 m}}^{2}} U_{1} \\
\frac{\partial}{\partial \beta} U_{2} & \frac{\partial}{\partial \sigma_{\mathbf{e}_{1 l}}^{2}} U_{2} & \frac{\partial}{\partial \sigma_{\mathbf{e}_{1 m}}^{2}} U_{2} \\
\frac{\partial}{\partial \beta} U_{3} & \frac{\partial}{\partial \sigma_{\mathbf{e}_{1 l}}^{2}} U_{3} & \frac{\partial}{\partial \sigma_{\mathbf{e}_{1 m}}^{2}} U_{3}
\end{array}\right), \text { onde }
$$




$$
\begin{array}{ll}
\frac{\partial}{\partial \beta} U_{1}=-\sigma_{\mathbf{e}_{1 m}}^{2} \sum_{j=1}^{n_{l}} \widehat{\xi}_{l_{j}}^{2}-\sigma_{\mathbf{e}_{1 l}}^{2} \sum_{j=1}^{n_{m}} \widehat{\xi}_{m_{j}}^{2} ; & \frac{\partial}{\partial \sigma_{\mathbf{e}_{1 l}}^{2}} U_{1}=\sum_{j=1}^{n_{m}}\left(y_{1 m_{j}} \widehat{\xi}_{m_{j}}-\beta \widehat{\xi}_{m_{j}}^{2}\right) ; \\
\frac{\partial}{\partial \sigma_{\mathbf{e}_{1 m}}^{2}} U_{1}=\sum_{j=1}^{n_{l}}\left(y_{1 l_{j}} \widehat{\xi}_{l_{j}}-\beta \widehat{\xi}_{l_{j}}^{2}\right) ; & \frac{\partial}{\partial \beta} U_{2}=\sum_{j=1}^{n_{l}}\left(-2 y_{1 l_{j}} \widehat{\xi}_{l_{j}}+2 \beta \widehat{\xi}_{l_{j}}^{2}\right) ; \\
\frac{\partial}{\partial \sigma_{\mathbf{e}_{1 l}}^{2}} U_{2}=-n_{l} ; & \frac{\partial}{\partial \sigma_{\mathbf{e}_{1 m}}^{2}} U_{2}=\frac{\partial}{\partial \sigma_{\mathbf{e}_{1 l}}^{2}} U_{3}=0 ; \\
\frac{\partial}{\partial \beta} U_{3}=\sum_{j=1}^{n_{m}}\left(-2 y_{1 m_{j}} \widehat{\xi}_{m_{j}}+2 \beta \widehat{\xi}_{m_{j}}^{2}\right) ; & \frac{\partial}{\partial \sigma_{\mathbf{e}_{1 m}}^{2}} U_{3}=-n_{m} .
\end{array}
$$

e então aplicamos o Método de Newton-Raphson, descrito, por exemplo, em Ruggiero \& Lopes (1996), onde

$$
\left(\begin{array}{c}
\beta \\
\sigma_{\mathbf{e}_{1 l}}^{2} \\
\sigma_{\mathbf{e}_{1 m}}^{2}
\end{array}\right)^{(k+1)}=\left(\begin{array}{c}
\beta \\
\sigma_{\mathbf{e}_{1 l}}^{2} \\
\sigma_{\mathbf{e}_{1 m}}^{2}
\end{array}\right)^{(k)}-\left[J^{-1}\left(\begin{array}{c}
\beta \\
\sigma_{\mathbf{e}_{1 l}}^{2} \\
\sigma_{\mathbf{e}_{1 m}}^{2}
\end{array}\right)^{(k)}\right]\left[\mathbf{U}\left(\begin{array}{c}
\beta \\
\sigma_{\mathbf{e}_{1 l}}^{2} \\
\sigma_{\mathbf{e}_{1 m}}^{2}
\end{array}\right)^{(k)}\right]
$$

Desta forma, na $(k+1)$-ésima etapa estimamos $\left(\beta, \sigma_{\mathbf{e}_{1 l}}^{2}, \sigma_{\mathbf{e}_{1 m}}^{2}\right)^{\prime}$ utilizando sua estimativa na ( $k$ )-ésima etapa, dentro do passo M em cada iteração do Algoritmo EM até obter convergência. Considerando os dados de Hadgu \& Koch (1999), não foram necessárias mais que 5 iterações até alcançar a convergência do algoritmo de Newton-Raphson.

Da mesma forma, considerando os testes do tipo

$$
H_{0}: \beta_{2 l}=\beta_{2 m}=\beta \text { versus } H_{1}: \beta_{2 l} \neq \beta_{2 m} \text { para } l, m=1, \ldots, p, l \neq m,
$$

no passo E, as expressões de $\xi_{i_{j}}^{(k+1)}$ e $\left(\xi_{i_{j}}^{2}\right)^{(k+1)}$ permanecem iguais às do algoritmo EM irrestrito para $i \neq l$ e $i \neq m$, com $j=1, \ldots, n_{i}$. Para $i=l$ ou $i=m$ temos:

\section{Passo E (expectation)}

$$
\begin{gathered}
\xi_{i_{j}}^{(k+1)}=\frac{\left(\frac{\mu^{(k)}}{\sigma_{x}^{2(k)}}+\frac{x_{i_{j}}}{\sigma^{2(k)}}+\frac{y_{1 i_{j}} \beta_{1 i}^{(k)}}{\sigma_{e_{1 i}}^{2(k)}}+\frac{y_{i_{j}} \beta^{(k)}}{\sigma_{e_{2 i}}^{2(k)}}\right)}{\left(\frac{1}{\sigma^{2(k)}}+\frac{1}{\sigma_{x}^{2(k)}}+\frac{\left(\beta_{1 i}^{(k)}\right)^{2}}{\sigma_{e_{1 i}}^{2(k)}}+\frac{\left(\beta^{(k)}\right)^{2}}{\sigma_{e_{2 i}}^{2(k)}}\right)} \\
\left(\xi_{i_{j}}^{2}\right)^{(k+1)}=\left(\xi_{i_{j}}^{(k+1)}\right)^{2}+\frac{1}{\left(\frac{1}{\sigma^{2(k)}}+\frac{1}{\sigma_{x}^{2(k)}}+\frac{\left(\beta_{1 i}^{(k)}\right)^{2}}{\sigma_{e_{1 i}}^{2(k)}}+\frac{\left(\beta^{(k)}\right)^{2}}{\sigma_{e_{2 i}}^{2(k)}}\right)}
\end{gathered}
$$

No passo M, as expressões de $\mu^{(k+2)},{\sigma^{2}}^{(k+2)}, \sigma_{x}^{2^{(k+2)}}, \beta_{1 i}^{(k+2)}$ e ${\sigma^{2}}_{e_{1 i}}^{(k+2)}$ para $i=1, \ldots, p$, permanecem as mesmas do algoritmo irrestrito. Para $i \neq l$ e $i \neq m$, as expressões de $\beta_{2 i}$ e $\sigma_{e_{2 i}}^{2(k+2)}$ também permanecem iguais. No entanto, não é possível explicitar as expressões de 
$\beta_{2 l}^{(k+2)}=\beta_{2 m}^{(k+2)}=\beta^{(k+2)}, \sigma_{e_{2 l}}^{2(k+2)}$ e $\sigma_{e_{2 m}^{2}}^{2(k+2)}$, já que ao igualar a zero as derivadas parciais de primeira ordem de $E\left(L_{0}(\mathbf{w}, \boldsymbol{\theta}) / \mathbf{z}\right)$ em relação a estes parâmetros, obtemos três equações nãolineares envolvendo as três variáveis:

$$
\begin{gathered}
\mathbf{U}\left(\beta, \sigma_{\mathbf{e}_{2 l}}^{2}, \sigma_{\mathbf{e}_{2 m}}^{2}\right)=\left(U_{1}, U_{2}, U_{3}\right)^{\prime} \text { onde } \\
U_{1}\left(\beta, \sigma_{\mathbf{e}_{2 l}}^{2}, \sigma_{\mathbf{e}_{2 m}}^{2}\right)=\sigma_{\mathbf{e}_{2 m}}^{2} \sum_{j=1}^{n_{l}}\left(y_{2 l_{j}} \widehat{\xi}_{l_{j}}-\beta \widehat{\xi}_{l_{j}}^{2}\right)+\sigma_{\mathbf{e}_{2 l}}^{2} \sum_{j=1}^{n_{m}}\left(y_{2 m_{j}} \widehat{\xi}_{m_{j}}-\beta \widehat{\xi}_{m_{j}}^{2}\right), \\
U_{2}\left(\beta, \sigma_{\mathbf{e}_{2 l}}^{2}, \sigma_{\mathbf{e}_{2 m}}^{2}\right)=-\sigma_{\mathbf{e}_{2 l}}^{2} n_{l}+\sum_{j=1}^{n_{l}}\left(y_{2 l_{j}}^{2}-2 \beta y_{2 l_{j}} \widehat{\xi}_{l_{j}}+\beta^{2} \widehat{\xi}_{l_{j}}^{2}\right), \\
U_{3}\left(\beta, \sigma_{\mathbf{e}_{2 l}}^{2}, \sigma_{\mathbf{e}_{2 m}}^{2}\right)=-\sigma_{\mathbf{e}_{2 m}}^{2} n_{m}+\sum_{j=1}^{n_{m}}\left(y_{2 m_{j}}^{2}-2 \beta y_{2 m_{j}} \widehat{\xi}_{m_{j}}+\beta^{2} \widehat{\xi}_{m_{j}}^{2}\right) .
\end{gathered}
$$

Assim, teremos a matriz jacobiana

$$
\begin{aligned}
& J=\left(\begin{array}{ccc}
\frac{\partial}{\partial \beta} U_{1} & \frac{\partial}{\partial \sigma_{\mathbf{e}_{2 l}}^{2}} U_{1} & \frac{\partial}{\partial \sigma_{\mathbf{e}_{2 m}}^{2}} U_{1} \\
\frac{\partial}{\partial \beta} U_{2} & \frac{\partial}{\partial \sigma_{\mathbf{e}_{2 l}}^{2}} U_{2} & \frac{\partial}{\partial \sigma_{\mathbf{e}_{2 m}}^{2}} U_{2} \\
\frac{\partial}{\partial \beta} U_{3} & \frac{\partial}{\partial \sigma_{\mathbf{e}_{2 l}}^{2}} U_{3} & \frac{\partial}{\partial \sigma_{\mathbf{e}_{2 m}}^{2}} U_{3}
\end{array}\right), \text { onde } \\
& \frac{\partial}{\partial \beta} U_{1}=-\sigma_{\mathbf{e}_{2 m}}^{2} \sum_{j=1}^{n_{l}} \widehat{\xi}_{l_{j}}^{2}-\sigma_{\mathbf{e}_{2 l}}^{2} \sum_{j=1}^{n_{m}} \widehat{\xi}_{m_{j}}^{2} ; \quad \frac{\partial}{\partial \sigma_{\mathbf{e}_{2 l}}^{2}} U_{1}=\sum_{j=1}^{n_{m}}\left(y_{2 m_{j}} \widehat{\xi}_{m_{j}}-\beta \widehat{\xi}_{m_{j}}^{2}\right) ; \\
& \frac{\partial}{\partial \sigma_{\mathbf{e}_{2 m}}^{2}} U_{1}=\sum_{j=1}^{n_{l}}\left(y_{2 l_{j}} \widehat{\xi}_{l_{j}}-\beta \widehat{\xi}_{l_{j}}^{2}\right) ; \quad \frac{\partial}{\partial \beta} U_{2}=\sum_{j=1}^{n_{l}}\left(-2 y_{2 l_{j}} \widehat{\xi}_{l_{j}}+2 \beta \widehat{\xi}_{l_{j}}^{2}\right) ; \\
& \frac{\partial}{\partial \sigma_{\mathbf{e}_{2 l}}^{2}} U_{2}=-n_{l} ; \quad \frac{\partial}{\partial \sigma_{\mathbf{e}_{2 m}}^{2}} U_{2}=\frac{\partial}{\partial \sigma_{\mathbf{e}_{2 l}}^{2}} U_{3}=0 ; \\
& \frac{\partial}{\partial \beta} U_{3}=\sum_{j=1}^{n_{m}}\left(-2 y_{2 m_{j}} \widehat{\xi}_{m_{j}}+2 \beta \widehat{\xi}_{m_{j}}^{2}\right) ; \quad \frac{\partial}{\partial \sigma_{\mathbf{e}_{2 m}}^{2}} U_{3}=-n_{m} .
\end{aligned}
$$

Analogamente, para os testes do tipo

$$
H_{0}: \beta_{1 l}=\beta_{2 l}=\beta \text { versus } H_{1}: \beta_{1 l} \neq \beta_{2 l} \text { para } l=1, \ldots, p
$$

no passo E, as expressões de $\xi_{i_{j}}^{(k+1)}$ e $\left(\xi_{i_{j}}^{2}\right)^{(k+1)}$ permanecem iguais às do algoritmo EM irrestrito para $i \neq l, \operatorname{com} j=1, \ldots, n_{i}$. Para $i=l$ temos:

\section{Passo E (expectation)}

$$
\xi_{i_{j}}^{(k+1)}=\frac{\left(\frac{\mu^{(k)}}{\sigma_{x}^{2(k)}}+\frac{x_{i_{j}}}{\sigma^{2(k)}}+\frac{y_{1 i_{j}} \beta^{(k)}}{\sigma_{e_{1 i}}^{2(k)}}+\frac{y_{i_{j}} \beta^{(k)}}{\sigma_{e_{2 i}}^{2(k)}}\right)}{\left(\frac{1}{\sigma^{2(k)}}+\frac{1}{\sigma_{x}^{2(k)}}+\frac{\left(\beta^{(k)}\right)^{2}}{\sigma_{e_{1 i}}^{2(k)}}+\frac{\left(\beta^{(k)}\right)^{2}}{\sigma_{e_{2 i}}^{2(k)}}\right)}
$$




$$
\left(\xi_{i_{j}}^{2}\right)^{(k+1)}=\left(\xi_{i_{j}}^{(k+1)}\right)^{2}+\frac{1}{\left(\frac{1}{\sigma^{2(k)}}+\frac{1}{\sigma_{x}^{2(k)}}+\frac{\left(\beta^{(k)}\right)^{2}}{\sigma_{e_{1 i}}^{2(k)}}+\frac{\left(\beta^{(k)}\right)^{2}}{\sigma_{e_{2 i}}^{2(k)}}\right)}
$$

No passo M, as expressões de $\mu^{(k+2)}, \sigma^{2(k+2)}, \sigma_{x}^{2^{(k+2)}}, \beta_{1 i}^{(k+2)}, \beta_{2 i}^{(k+2)}{\sigma^{2}}_{e_{1 i}}^{(k+2)}, \sigma_{e_{2 i}}^{2^{(k+2)}}$ para $i=1, \ldots, p, i \neq l$, permanecem as mesmas do algoritmo irrestrito. No entanto, não é possível explicitar as expressões de $\beta_{1 l}^{(k+2)}=\beta_{2 l}^{(k+2)}=\beta^{(k+2)}, \sigma_{e_{1 l}}^{{ }^{(k+2)}}$ e ${\sigma^{2}}_{e_{2 l}}^{(k+2)}$, já que ao igualar a zero as derivadas parciais de primeira ordem de $E\left(L_{0}(\mathbf{w}, \boldsymbol{\theta}) / \mathbf{z}\right)$ em relação a estes parâmetros, obtemos três equações não-lineares envolvendo as três variáveis:

$\mathbf{U}\left(\beta, \sigma_{\mathbf{e}_{1 l}}^{2}, \sigma_{\mathbf{e}_{2 l}}^{2}\right)=\left(U_{1}, U_{2}, U_{3}\right)^{\prime}$ onde

$$
\begin{aligned}
& U_{1}\left(\beta, \sigma_{\mathbf{e}_{1 l}}^{2}, \sigma_{\mathbf{e}_{2 l}}^{2}\right)=\sum_{j=1}^{n_{l}}\left(\sigma_{\mathbf{e}_{2 l}}^{2} y_{1 l_{j}} \widehat{\xi}_{l_{j}}-\beta \widehat{\xi}_{l_{j}}^{2}\left(\sigma_{\mathbf{e}_{1 l}}^{2}+\sigma_{\mathbf{e}_{2 l}}^{2}\right)+\sigma_{\mathbf{e}_{1 l}}^{2} y_{2 l_{j}} \widehat{\xi}_{l_{j}}\right), \\
& U_{2}\left(\beta, \sigma_{\mathbf{e}_{1 l}}^{2}, \sigma_{\mathbf{e}_{2 l}}^{2}\right)=-\sigma_{\mathbf{e}_{1 l}}^{2} n_{l}+\sum_{j=1}^{n_{l}}\left(y_{1 l_{j}}-2 \beta y_{1 l_{j}} \widehat{\xi}_{l_{j}}+\beta^{2} \widehat{\xi}_{l_{j}}^{2}\right) \\
& U_{3}\left(\beta, \sigma_{\mathbf{e}_{1 l}}^{2}, \sigma_{\mathbf{e}_{2 l}}^{2}\right)=-\sigma_{\mathbf{e}_{2 l}}^{2} n_{l}+\sum_{j=1}^{n_{l}}\left(y_{2 l_{j}}-2 \beta y_{2 l_{j}} \widehat{\xi}_{l_{j}}+\beta^{2} \widehat{\xi}_{l_{j}}^{2}\right) .
\end{aligned}
$$

Assim, teremos a matriz jacobiana

$$
\begin{gathered}
J=\left(\begin{array}{ccc}
\frac{\partial}{\partial \beta} U_{1} & \frac{\partial}{\partial \sigma_{\mathbf{e}_{1 l}}^{2}} U_{1} & \frac{\partial}{\partial \sigma_{\mathbf{e}_{2 l}}^{2}} U_{1} \\
\frac{\partial}{\partial \beta} U_{2} & \frac{\partial}{\partial \sigma_{\mathbf{e}_{1 l}}^{2}} U_{2} & \frac{\partial}{\partial \sigma_{\mathbf{e}_{2 l}}^{2}} U_{2} \\
\frac{\partial}{\partial \beta} U_{3} & \frac{\partial}{\partial \sigma_{\mathbf{e}_{1 l}}^{2}} U_{3} & \frac{\partial}{\partial \sigma_{\mathbf{e}_{2 l}}^{2}} U_{3}
\end{array}\right) \text {, onde } \\
\frac{\partial}{\partial \beta} U_{1}=\sum_{j=1}^{n_{l}}\left(\widehat{\xi}_{l_{j}}^{2}\left(\sigma_{\mathbf{e}_{1 l}}^{2}+\sigma_{\mathbf{e}_{2 l}}^{2}\right)\right) ; \quad \frac{\partial}{\partial \sigma_{\mathbf{e}_{1 l}}^{2}} U_{1}=\beta \sum_{j=1}^{n_{l}}\left(\widehat{\xi}_{l_{j}}^{2}+y_{2 l_{j}} \widehat{\xi}_{l_{j}}\right) ; \\
\frac{\partial}{\partial \sigma_{\mathbf{e}_{2 l}}^{2}} U_{1}=\beta \sum_{j=1}^{n_{l}}\left(\widehat{\xi}_{l_{j}}^{2}+y_{1 l_{j}} \widehat{\xi}_{l_{j}}\right) ; \quad \frac{\partial}{\partial \beta} U_{2}=\sum_{j=1}^{n_{l}}\left(-2 y_{1 l_{j}} \widehat{\xi}_{l_{j}}+2 \beta \widehat{\xi}_{l_{j}}^{2}\right) ; \\
\frac{\partial}{\partial \sigma_{\mathbf{e}_{1 l}}^{2}} U_{2}=-n_{l} ; \quad \frac{\partial}{\partial \sigma_{\mathbf{e}_{2 l}}^{2}} U_{2}=\frac{\partial}{\partial \sigma_{\mathbf{e}_{1 l}}^{2}} U_{3}=0 ; \\
\frac{\partial}{\partial \beta} U_{3}=\sum_{j=1}^{n_{l}}\left(-2 y_{2 l_{j}} \widehat{\xi}_{l_{j}}+2 \beta^{2} \widehat{\xi}_{l_{j}}^{2}\right) ; \quad \frac{\partial}{\partial \sigma_{\mathbf{e}_{2 l}}^{2}} U_{3}=-n_{l} .
\end{gathered}
$$

Finalmente, utilizando o método descrito acima, obtivemos as estimativas para os parâmetros para cada hipótese $H_{0}$, como mostra a Tabela 5.1. 


\begin{tabular}{lllllllll}
\hline Parâmetros & $\beta_{11}$ & $\beta_{21}$ & $\beta_{12}$ & $\beta_{22}$ & $\beta_{13}$ & $\beta_{23}$ & $\mu$ & $\sigma^{2}$ \\
\hline EMV irrestritas & 0.703 & 0.687 & 0.525 & 0.502 & 0.508 & 0.414 & 2.534 & 0.010 \\
EMV sob $H_{0}: \beta_{11}=\beta_{12}$ & 0.634 & 0.686 & 0.634 & 0.503 & 0.508 & 0.414 & 2.534 & 0.010 \\
EMV sob $H_{0}: \beta_{11}=\beta_{13}$ & 0.597 & 0.686 & 0.525 & 0.502 & 0.597 & 0.415 & 2.534 & 0.010 \\
EMV sob $H_{0}: \beta_{12}=\beta_{13}$ & 0.703 & 0.687 & 0.515 & 0.502 & 0.515 & 0.414 & 2.534 & 0.010 \\
EMV sob $H_{0}: \beta_{21}=\beta_{22}$ & 0.702 & 0.630 & 0.526 & 0.630 & 0.508 & 0.414 & 2.534 & 0.010 \\
EMV sob $H_{0}: \beta_{21}=\beta_{23}$ & 0.701 & 0.529 & 0.525 & 0.502 & 0.509 & 0.529 & 2.534 & 0.009 \\
EMV sob $H_{0}: \beta_{22}=\beta_{23}$ & 0.703 & 0.687 & 0.525 & 0.440 & 0.509 & 0.440 & 2.534 & 0.010 \\
EMV sob $H_{0}: \beta_{11}=\beta_{21}$ & 0.694 & 0.694 & 0.525 & 0.502 & 0.508 & 0.414 & 2.534 & 0.010 \\
EMV sob $H_{0}: \beta_{12}=\beta_{22}$ & 0.703 & 0.687 & 0.514 & 0.514 & 0.508 & 0.414 & 2.534 & 0.010 \\
EMV sob $H_{0}: \beta_{13}=\beta_{23}$ & 0.703 & 0.687 & 0.525 & 0.502 & 0.454 & 0.454 & 2.534 & 0.010 \\
\hline
\end{tabular}

Tabela 5.1: Estimativas de máxima verossimilhança irrestritas e restritas às hipóteses $H_{0}$

\begin{tabular}{llllllll}
\hline Parâmetros & $\sigma_{x}^{2}$ & $\sigma_{e_{11}}^{2}$ & $\sigma_{e_{21}}^{2}$ & $\sigma_{e_{12}}^{2}$ & $\sigma_{e_{22}}^{2}$ & $\sigma_{e_{13}}^{2}$ & $\sigma_{e_{23}}^{2}$ \\
\hline EMV irrestritas & 0.103 & 0.312 & 0.234 & 0.430 & 0.431 & 0.255 & 0.192 \\
EMV sob $H_{0}: \beta_{11}=\beta_{12}$ & 0.103 & 0.344 & 0.234 & 0.508 & 0.431 & 0.255 & 0.192 \\
EMV sob $H_{0}: \beta_{11}=\beta_{13}$ & 0.103 & 0.388 & 0.235 & 0.430 & 0.431 & 0.303 & 0.192 \\
EMV sob $H_{0}: \beta_{12}=\beta_{13}$ & 0.103 & 0.312 & 0.234 & 0.431 & 0.431 & 0.255 & 0.192 \\
EMV sob $H_{0}: \beta_{21}=\beta_{22}$ & 0.103 & 0.313 & 0.256 & 0.430 & 0.540 & 0.255 & 0.192 \\
EMV sob $H_{0}: \beta_{21}=\beta_{23}$ & 0.103 & 0.315 & 0.401 & 0.430 & 0.431 & 0.255 & 0.274 \\
EMV sob $H_{0}: \beta_{22}=\beta_{23}$ & 0.103 & 0.312 & 0.234 & 0.430 & 0.458 & 0.255 & 0.197 \\
EMV sob $H_{0}: \beta_{11}=\beta_{21}$ & 0.103 & 0.313 & 0.234 & 0.430 & 0.431 & 0.255 & 0.192 \\
EMV sob $H_{0}: \beta_{12}=\beta_{22}$ & 0.103 & 0.312 & 0.234 & 0.431 & 0.432 & 0.255 & 0.192 \\
EMV sob $H_{0}: \beta_{13}=\beta_{23}$ & 0.103 & 0.312 & 0.234 & 0.430 & 0.431 & 0.274 & 0.202 \\
\hline
\end{tabular}

\subsection{Resultados e conclusões sobre as hipóteses de inter- esse}

Considerando os testes assintóticos de Wald, da razão de verossimilhanças e score descritos no Capítulo 4 e as estimativas de máxima verossimilhança sob $H_{0}$ (Tabela 5.1), obtivemos os resultados dos testes que estão descritos nas Tabelas 5.2 e 5.3.

Tabela 5.2: Valores obtidos pelas estatísticas Wald, razão de verossimilhanças e score e p-valor para testes entre líquidos.

\begin{tabular}{ccccccc}
\hline$H_{0}$ & $\beta_{11}=\beta_{12}$ & $\beta_{11}=\beta_{13}$ & $\beta_{12}=\beta_{13}$ & $\beta_{21}=\beta_{22}$ & $\beta_{21}=\beta_{23}$ & $\beta_{22}=\beta_{23}$ \\
\hline$Q_{W}$ & 9.524 & 15.429 & 0.072 & 11.294 & 40.114 & 2.714 \\
(p-valor) & 0.002 & 0.000 & 0.788 & 0.001 & 0.000 & 0.099 \\
\hline$Q_{L}$ & 9.095 & 13.824 & 0.072 & 10.587 & 31.525 & 2.669 \\
(p-valor) & 0.003 & 0.000 & 0.788 & 0.001 & 0.000 & 0.102 \\
\hline$Q_{S}$ & 11.446 & 16.119 & 3.207 & 13.004 & 30.565 & 5.672 \\
(p-valor) & 0.001 & 0.000 & 0.073 & 0.000 & 0.000 & 0.017 \\
\hline
\end{tabular}


Tabela 5.3: Valores obtidos pelas estatísticas Wald, razão de verossimilhanças e score e p-valor para testes intra-líquidos.

\begin{tabular}{cccc}
\hline$H_{0}$ & $\beta_{11}=\beta_{21}$ & $\beta_{12}=\beta_{22}$ & $\beta_{13}=\beta_{23}$ \\
\hline$Q_{W}$ & 0.113 & 0.124 & 4.687 \\
(p-valor) & 0.737 & 0.724 & 0.030 \\
\hline$Q_{L}$ & 0.115 & 0.128 & 4.335 \\
(p-valor) & 0.735 & 0.721 & 0.037 \\
\hline$Q_{S}$ & 3.163 & 3.220 & 7.152 \\
(p-valor) & 0.075 & 0.073 & 0.007 \\
\hline
\end{tabular}

\section{Conclusões dos testes de hipóteses}

As conclusões para os testes de interesse sobre o conjunto de dados foram:

1. Considerando os níveis de significância $\alpha=1 \%, \alpha=5 \%$ ou $\alpha=10 \%$, os três testes nos levam a concluir que os líquidos de bochecho experimentais A e B são mais eficientes do que o líquido controle tanto nos três quanto nos seis primeiros meses de uso (testes com as hipóteses nulas $\left.H_{0}: \beta_{11}=\beta_{12}, H_{0}: \beta_{11}=\beta_{13}, H_{0}: \beta_{21}=\beta_{22}, H_{0}: \beta_{21}=\beta_{23}\right)$.

2. A comparação dos líquidos experimentais $\mathrm{A}$ e $\mathrm{B}$ após três meses de uso (teste $H_{0}: \beta_{12}=$ $\left.\beta_{13}\right)$ depende do nível de significância considerado. Para $\alpha=1 \%$ e $\alpha=5 \%$ os três testes nos levam a concluir que os líquidos são equivalentes no primeiro período de uso, porém ao nível $\alpha=10 \%$ o teste score dá indícios de que os líquidos A e B não são equivalentes neste período. No entanto, resultados de simulação, mais precisamente a segunda coluna da Tabela E.8, sugerem que a melhor estatística neste caso é a da razão de verossimilhanças, que atinge maior proximidade ao nível de significância mesmo para amostras pequenas. Portanto ao nível de significância de $\alpha=10 \%$ pode-se concluir que os líquidos experimentais A e B são equivalentes após três meses de uso.

3. A comparação dos líquidos experimentais A e B após os seis meses de uso (teste com a hipótese nula $H_{0}: \beta_{22}=\beta_{23}$ ) também depende do nível de significância considerado. Para $\alpha=1 \%$ as três estatísticas de teste dão indícios de que os dois líquidos são equivalentes neste período. Para $\alpha=5 \%$ o teste score nos leva a concluir que os líquidos não são equivalentes, já os testes de Wald e razão de verossimilhanças sugerem que os líquidos são equivalentes após seis meses de uso. Para $\alpha=10 \%$ as estatísticas de Wald e score nos sugerem que os líquidos não são equivalentes após os seis meses de uso, enquanto que o teste da razão de verossimilhanças sugere que os líquidos são equivalentes. Resultados de simulação, mais precisamente a segunda coluna da Tabela E.17 sugerem que a melhor 
estatística neste caso é a da razão de verossimilhanças, portanto aos níveis de significância $\alpha=5 \%$ e $\alpha=10 \%$ pode-se concluir que os líquidos experimentais A e B são equivalentes após seis meses de uso.

4. Ao verificar a eficiência dos líquidos controle e A a longo prazo (testes com a hipótese nula $H_{0}: \beta_{11}=\beta_{21}$ e $H_{0}: \beta_{12}=\beta_{22}$ respectivamente), ao nível de significância $\alpha=1 \%$ e $\alpha=5 \%$, as três estatísticas de teste nos levam a concluir que não há eficiência a longo prazo. Ao considerar $\alpha=10 \%$, o teste score conclui que houve eficiência a longo prazo, porém os testes Wald e razão de verossimilhanças concluem que os líquidos controle e A não continuam a diminuir a placa bacteriana após os três primeiros meses. Os resultados de simulação do tamanho do teste, presentes na segunda coluna das Tabelas E.20 (para testar $H_{0}: \beta_{11}=\beta_{21}$ ) e E.23 (para testar $H_{0}: \beta_{12}=\beta_{22}$ ), sugerem a utilização da estatística da razão de verossimilhanças neste caso, e assim pode-se concluir que os líquidos controle e A não são eficientes a longo prazo, ao nível de significância $\alpha=10 \%$.

5. Ao verificar a eficiência do líquido $\mathrm{B}$ a longo prazo (teste com a hipótese nula $H_{0}: \beta_{13}=$ $\beta_{23}$ ), ao nível de significância $\alpha=1 \%$ o teste score nos leva a concluir que o líquido B é eficiente a longo prazo, e as estatísticas de Wald e razão de verossimilhanças sugerem que o líquido B não continua a diminuir a placa bacteriana após os três primeiros meses de uso. Considerando $\alpha=5 \%$ e $\alpha=10 \%$, as três estatísticas de teste nos levam a concluir que o líquido B continua a diminuir a placa bacteriana. Os resultados de simulação, mais precisamente a segunda coluna da Tabela E.26 sugere a utilização da estatística da razão de verossimilhanças neste caso, assim, para $\alpha=1 \%$ pode-se concluir que o líquido B não continua a diminuir a placa bacteriana a longo prazo, porém ao nível $\alpha=5 \%$ e $\alpha=10 \%$, pode-se concluir que o líquido de bochecho B continua a ter efeito após os primeiros três meses de uso.

No próximo capítulo apresentaremos estudos de simulação para analisar o comportamento dos três testes assintóticos considerando diferentes valores para os parâmetros e para os tamanhos de amostras. 


\section{Capítulo 6}

\section{Estudos de simulação}

Apresentaremos, neste capítulo, estudos de simulação para verificar o comportamento dos testes assintóticos de Wald, razão de verossimilhanças e score quando consideramos diferentes valores para a variância do erro de medição no início do estudo $\left(\sigma^{2}\right)$, para a média do verdadeiro valor dos dados $(\mu)$ e para a sua variância $\left(\sigma_{x}^{2}\right)$. Consideramos para $\mu$ os valores $1,2.5$ e 5 , para $\sigma^{2}$ os valores $0.01,0.05,0.1$ e 0.5 , e para $\sigma_{x}^{2}$ os valores $0.01,0.1$ e 0.5 , e as combinações entre estes valores com a restrição de que $\sigma^{2}$ fosse sempre menor que $\sigma_{x}^{2}$, já que não faria sentido ter uma variância dos erros maior do que a variância do verdadeiro valor dos dados. Para os parâmetros $\beta_{i k} ; i=1,2 ; k=1,2,3$, foram considerados alguns conjuntos de valores, que estão justificados a seguir:

1. $\beta_{11}=0.7, \beta_{12}=0.53, \beta_{13}=0.51, \beta_{21}=0.69, \beta_{22}=0.5, \beta_{23}=0.41$, que são aproximações das estimativas de máxima verossimilhança dos parâmetros para os dados de Hadgu \& Koch (1999),

2. $\beta_{11}=0.6, \beta_{12}=0.52, \beta_{13}=0.51, \beta_{21}=0.54, \beta_{22}=45, \beta_{23}=0.414$, que representariam o caso em que os três líquidos diminuíssem a placa bacteriana nos primeiros três meses de uso dos líquidos e continuassem a diminuir significativamente a placa após três meses de uso.

3. $\beta_{11}=0.7, \beta_{12}=0.57, \beta_{13}=0.63, \beta_{21}=0.65, \beta_{22}=0.52, \beta_{23}=0.56$, que seria equivalente à situação em que um dos líquidos experimentais (líquido A) apresentasse maior eficiência que o outro nos primeiros três meses mas que todos continuassem a diminuir a placa após seis meses de uso,

4. $\beta_{11}=0.73, \beta_{12}=0.57, \beta_{13}=0.56, \beta_{21}=0.71, \beta_{22}=0.55, \beta_{23}=0.54$ que seria equivalente à situação em que os líquidos experimentais apresentassem eficiência nos primeiros três 
meses de uso em relação ao líquido controle porém nenhum dos líquidos continuasse a diminuir a placa bacteriana após três meses de uso,

5. $\beta_{11}=0.77, \beta_{12}=0.74, \beta_{13}=0.75, \beta_{21}=0.74, \beta_{22}=0.72, \beta_{23}=0.73$, que representaria a situação em que nenhum dos líquidos experimentais diminuísse significantemente a placa bacteriana ao longo dos seis meses de uso dos líquidos.

Os diferentes valores considerados para $\beta_{i k} ; i=1,2 ; k=1,2,3$ não afetaram significantemente os resultados obtidos, sendo assim iremos apresentar somente os resultados referentes ao conjunto de valores (1), relativos a aproximações das estimativas de máxima verossimilhança dos parâmetros $\beta_{i k} ; i=1,2 ; k=1,2,3$. Para as variâncias dos erros de medição após o uso dos líquidos foram considerados os valores $\sigma_{e_{11}}^{2}=0.31, \sigma_{e_{21}}^{2}=0.23, \sigma_{e_{12}}^{2}=0.43, \sigma_{e_{22}}^{2}=0.43$, $\sigma_{e_{13}}^{2}=0.25$ e $\sigma_{e_{23}}^{2}=0.19$, que são os valores aproximados das estimativas de máxima verossimilhança destes parâmetros.

Primeiramente fizemos um estudo em relação ao nível de significância do teste, cujos resultados serão apresentados na Seção 6.1. Em seguida, na Seção 6.2 apresentaremos resultados referentes à simulação do poder do teste.

\subsection{Simulação do tamanho dos testes assintóticos}

O estudo de simulação foi desenvolvido utilizando a linguagem Ox (Doornik 2002). Foram simuladas 10000 amostras e obtidas as porcentagens dos valores das estatísticas que foram maiores do que quantis de 1\%, $5 \%$ e $10 \%$ de uma distribuição Chi-quadrado com 1 grau de liberdade. O objetivo destas simulações é avaliar numericamente o comportamento das estatísticas quanto ao tamanho das amostras e quanto aos diferentes valores considerados para os parâmetros. Estes resultados estão apresentados nas Tabelas E.1 a E.27, no Apêndice E.

\subsubsection{Conclusões das simulações do tamanho dos testes assintóticos}

As conclusões do estudo de simulação do tamanho dos testes assintóticos são as seguintes:

1. Para nível de significância $\alpha=0.01$ e amostras pequenas, a estatística de Wald apresentou o pior comportamento entre os três testes, porém quando aumentou-se o tamanho das amostras apresentou boa aproximação ao nível nominal. A estatística score apresentou o melhor comportamento entre as três estatísticas, apresentando boa aproximação ao nível nominal mesmo quando o tamanho das amostras era pequeno. Nos casos em que $\sigma^{2}=$ 
0.01, no entanto, a estatística score não apresentou grande eficiência, sendo melhor neste caso a estatística da razão de verossimilhanças. A estatística da razão de verossimilhanças apresentou bom comportamento em todos os casos, aproximando-se bem do nível nominal quando aumentou-se o tamanho das amostras.

2. Para nível de significância $\alpha=0.05$ e $\alpha=0.1$, a estatística score apresentou o melhor comportamento entre as três estatísticas, exceto nos casos em que $\sigma^{2}=0.01$ e quando $\left(\sigma^{2}, \sigma_{x}^{2}\right)=(0.05,0.5)$. Nestes casos, a estatística score apresentou grandes porcentagens de rejeição e mostrou dificuldade em se aproximar do nível nominal mesmo quando aumentou-se o tamanho das amostras, e a estatística mais eficiente foi a da razão de verossimilhanças.

3. A hipótese testada não apresentou influência tendenciosa no comportamento das estatísticas do teste.

4. Os valores considerados de $\mu$ parecem não afetar o comportamento de nenhuma das três estatísticas de teste.

5. Os valores de $\sigma^{2}$ (variância dos erros de medição no início do estudo) apresentaram influência na estatística score. Fixados $\mu, \sigma_{x}^{2}$ e o tamanho da amostra, o comportamento da estatística apresentou tendência a melhorar quando aumentou-se o valor de $\sigma^{2}$. As outras estatísticas não apresentaram tendência a melhorar ou piorar com a mudança dos valores de $\sigma^{2}$.

6. Os valores de $\sigma_{x}^{2}$ (variância do verdadeiro valor da placa bacteriana no início do estudo) apresentaram influência na estatística score. Fixados $\mu$ e $\sigma^{2}$, o comportamento da estatística apresentou tendência a piorar quando aumentou-se o valor de $\sigma_{x}^{2}$. As outras estatísticas não apresentaram tendência a melhorar ou piorar com a mudança dos valores de $\sigma_{x}^{2}$.

\subsubsection{Gráficos de quantis}

Apresentaremos agora alguns gráficos de quantis ("qq") dos valores obtidos das estatísticas de Wald, da razão de verossimilhançaw e score. Estes gráficos foram construídos a partir dos valores ordenados obtidos nas estatísticas contra os quantis correspondentes da distribuição $\chi_{1}^{2}$. Quanto mais os valores se aproximam desta distribuição, mais próximos estarão os pares de pontos da reta identidade, apresentada em cada gráfico por uma linha reta. 


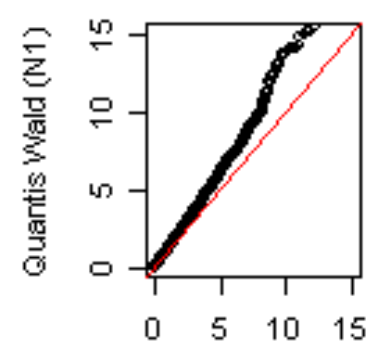

Quantis $Q Q(1)$

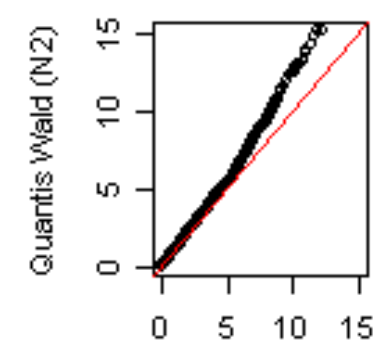

Quantis $Q Q(1)$

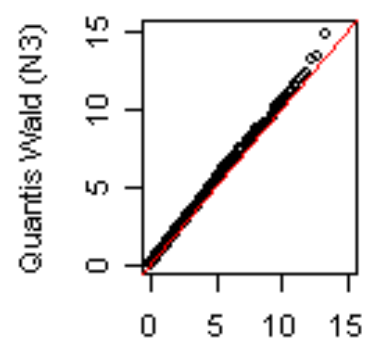

Quantis $Q Q(1)$

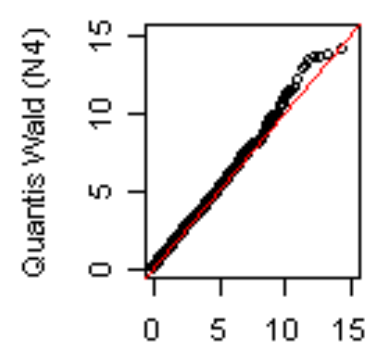

Quantis $Q Q(1)$

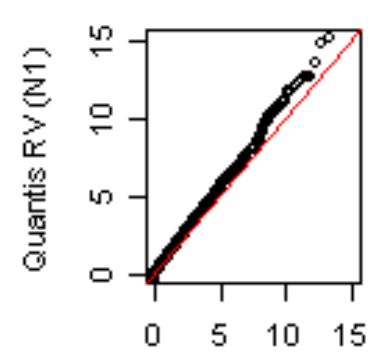

Quantis $Q Q(1)$
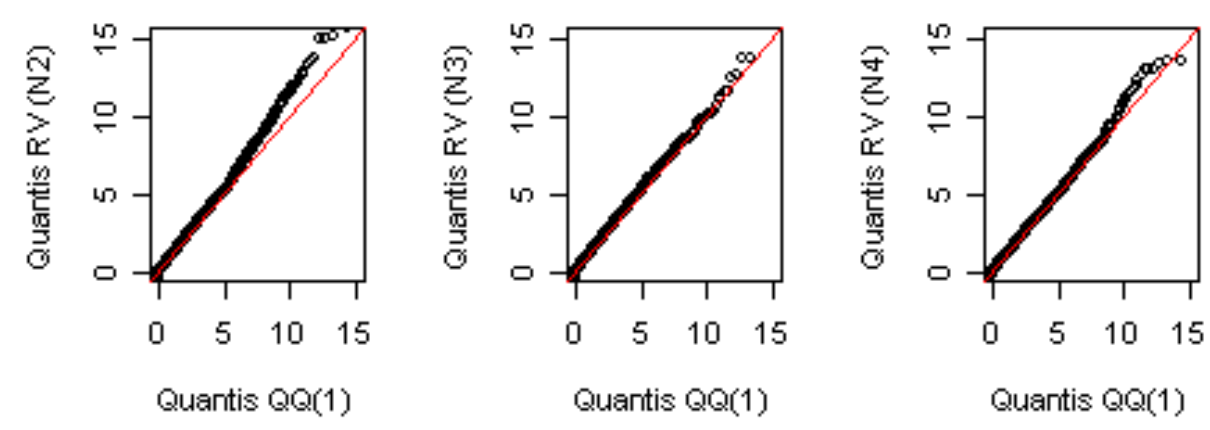

Quantis $Q Q(1)$
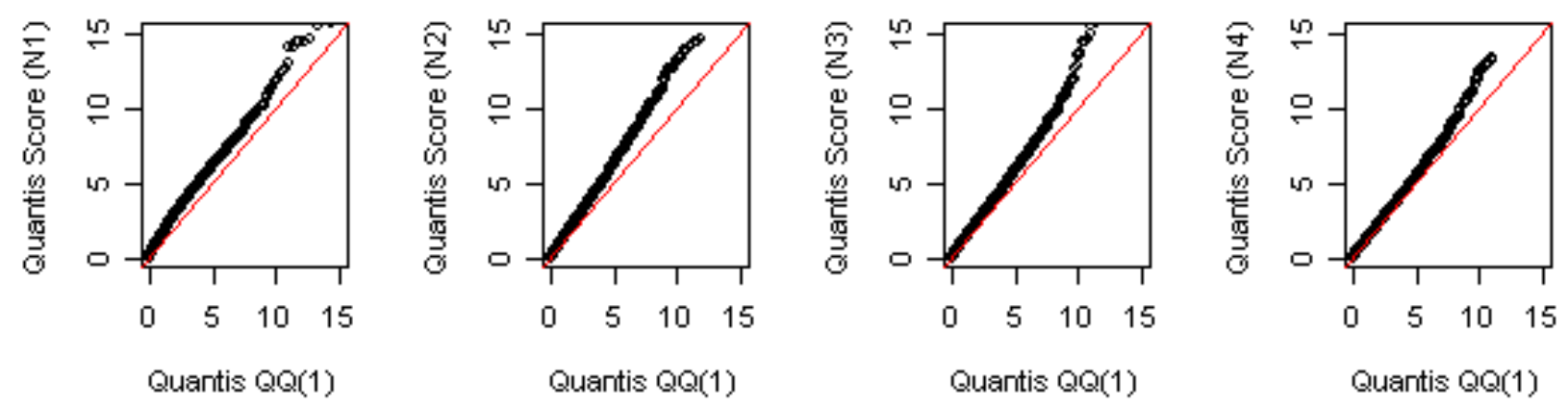

Figura 6.1: Gráficos de quantis para $\sigma^{2}=0.01$ e $\sigma_{x}^{2}=0.01$ para o teste $H_{0}: \beta_{11}=\beta_{13}$ versus $H_{0}: \beta_{11} \neq \beta_{13} \operatorname{com} \mu=2.5$

Para tanto escolhemos $\beta_{11}=0.7, \beta_{12}=0.53, \beta_{13}=0.51, \beta_{21}=0.69, \beta_{22}=0.5, \beta_{23}=0.41$, que são aproximações das estimativas de máxima verossimilhança nos dados de Hadgu \& Koch (1999), $\operatorname{com} \mu=2.5$ e combinações de valores entre a variância do verdadeiro valor dos dados no início do estudo e para a variância dos erros de medição do índice de placa no início do estudo ( $\sigma_{x}^{2}$ e $\sigma^{2}$ respectivamente). Os gráficos estão na seguinte ordem: na primeira linha dispomos os valores obtidos pela estatística de Wald versus quantis de $\chi_{1}^{2}$ conforme aumentamos os tamanhos 


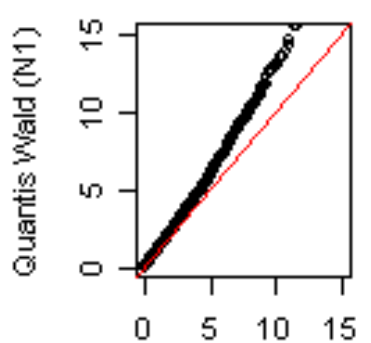

Quantis $Q Q(1)$

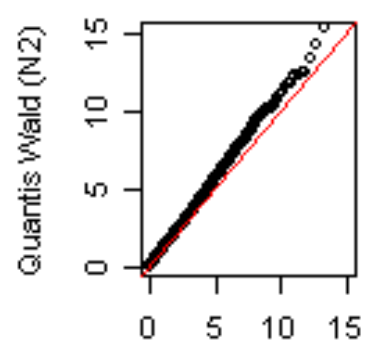

Quantis $Q Q(1)$

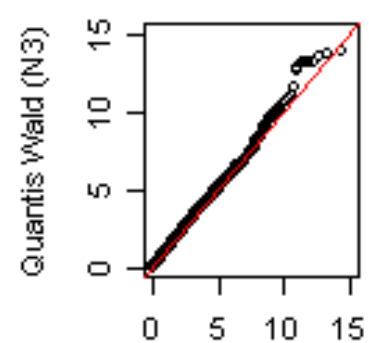

Quantis $Q Q(1)$

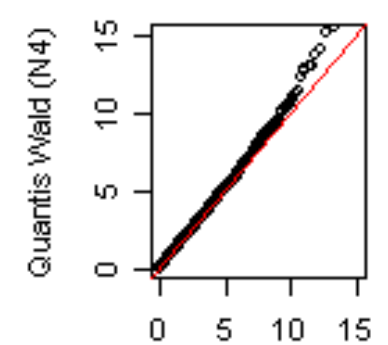

Quantis $Q Q(1)$

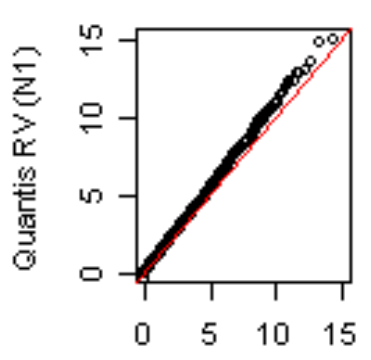

Quantis QQ(1)

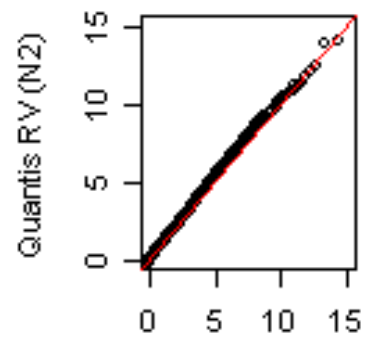

Quantis QQ(1)

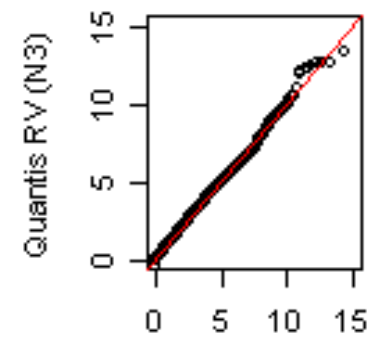

Quantis $Q Q(1)$

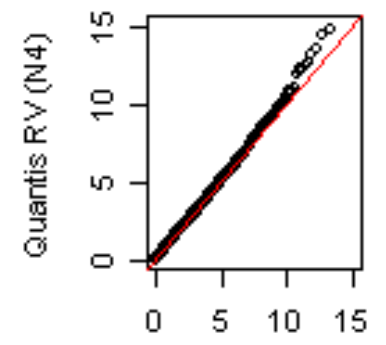

Quantis $Q Q(1)$

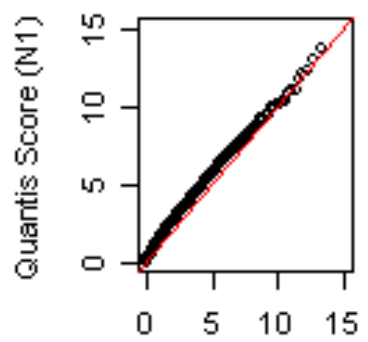

Quantis QQ(1)

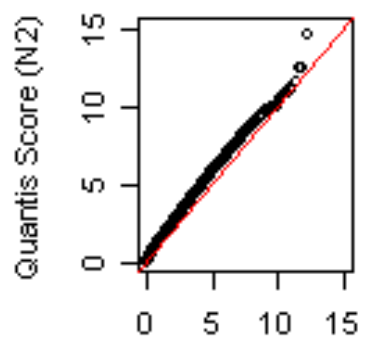

Quantis $Q Q(1)$

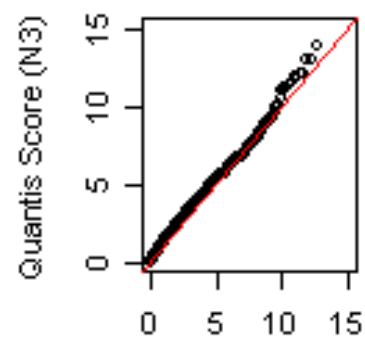

Quantis $\mathrm{QQ}(1)$

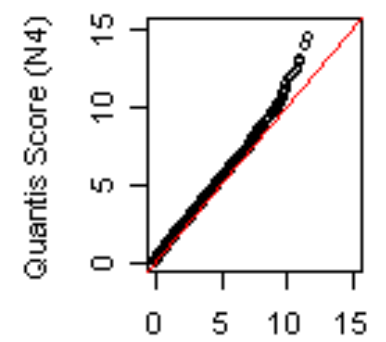

Quantis $Q Q(1)$

Figura 6.2: Gráficos de quantis para $\sigma^{2}=0.01$ e $\sigma_{x}^{2}=0.1$ para o teste $H_{0}: \beta_{11}=\beta_{13}$ versus $H_{0}: \beta_{11} \neq \beta_{13} \operatorname{com} \mu=2.5$

das amostras.

Na segunda linha utilizamos os valores obtidos pela estatística da razão de verossimilhanças e na última linha os valores obtidos pela estatística score, sempre aumentando o tamanho da amostras da esquerda para a direita. Os gráficos das Figuras 6.1 a 6.8 correspondem ao teste de hipóteses $H_{0}: \beta_{11}=\beta_{13}$ versus $H_{0}: \beta_{11} \neq \beta_{13}$, ou seja, que compara o líquido controle com o líquido $\mathrm{B}$ nos primeiros três meses de uso, para alguns valores considerados de $\sigma^{2}$ e $\sigma_{x}^{2}$. A 


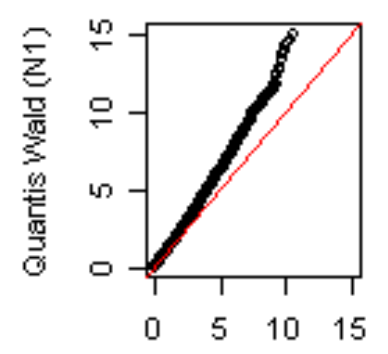

Quantis $Q Q(1)$

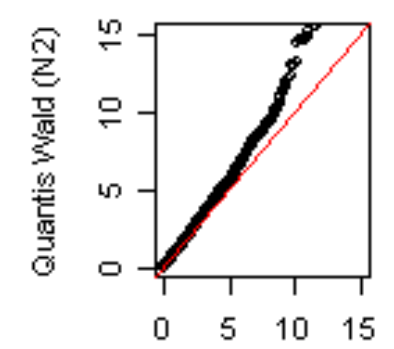

Quantis $Q Q(1)$

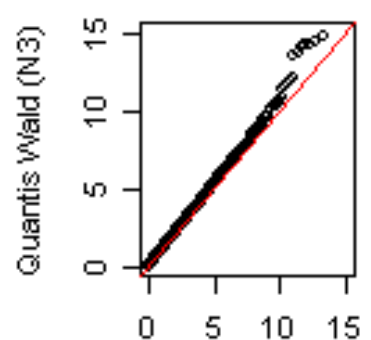

Quantis $Q Q(1)$

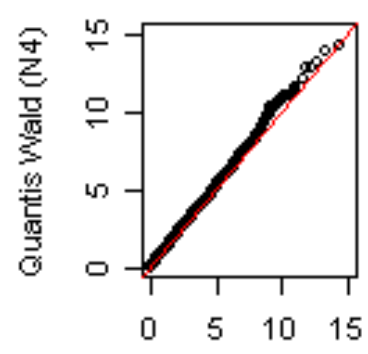

Quantis $Q Q(1)$

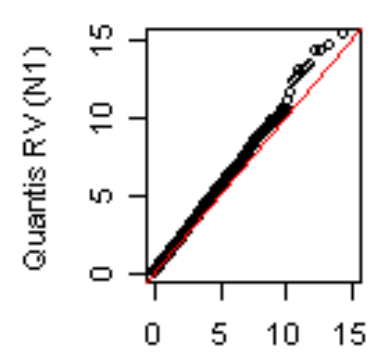

Quantis $Q Q(1)$

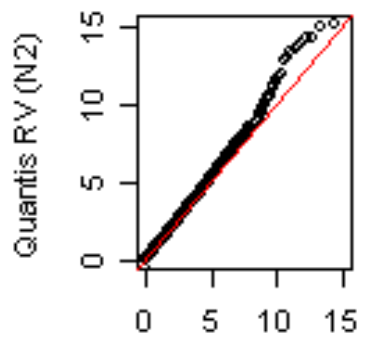

Quantis $Q Q(1)$

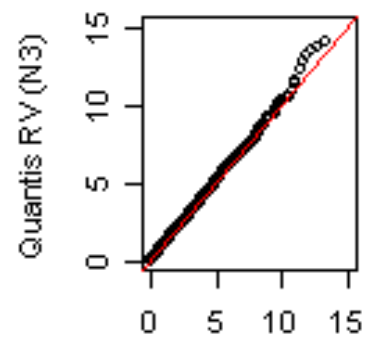

Quantis $Q Q(1)$

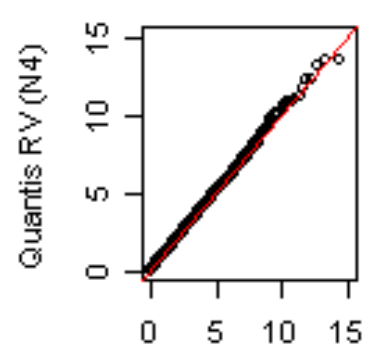

Quantis $Q Q(1)$

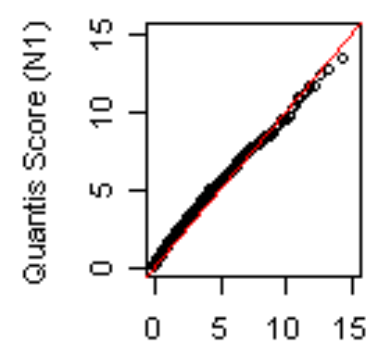

Quantis $Q Q(1)$

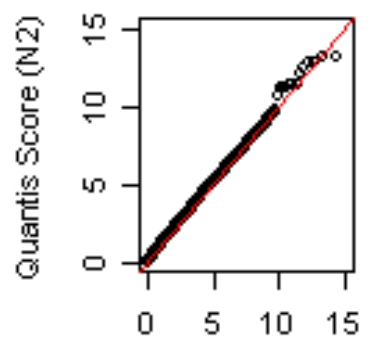

Quantis $Q Q(1)$

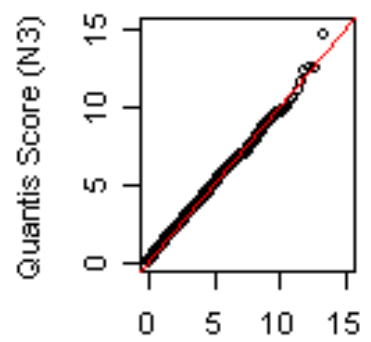

Quantis $Q Q(1)$

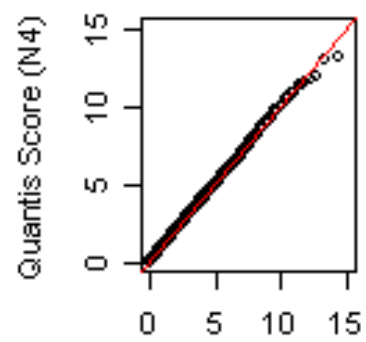

Quantis $Q Q(1)$

Figura 6.3: Gráficos de quantis para $\sigma^{2}=0.05$ e $\sigma_{x}^{2}=0.1$ para o teste $H_{0}: \beta_{11}=\beta_{13}$ versus $H_{0}: \beta_{11} \neq \beta_{13} \operatorname{com} \mu=2.5$

tabela correspondente aos resultados destes gráficos é a Tabela E.5.

\section{Conclusões dos gráficos de quantis}

Em geral, os valores obtidos pelas três estatísticas de teste aproximaram-se dos quantis da distribuição $\chi_{1}^{2}$ quando aumentou-se o tamanho das amostras, com algumas exceções para alguns pontos das caudas. Foram construídos os gráficos para todos os casos apresentados 


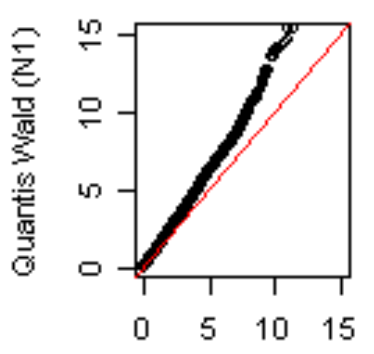

Quantis $Q Q(1)$

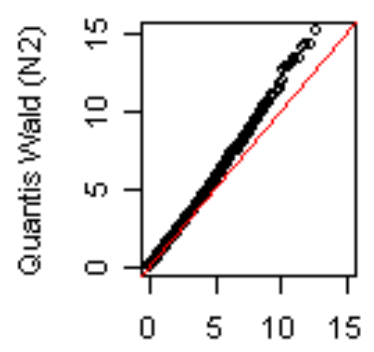

Quantis $Q Q(1)$

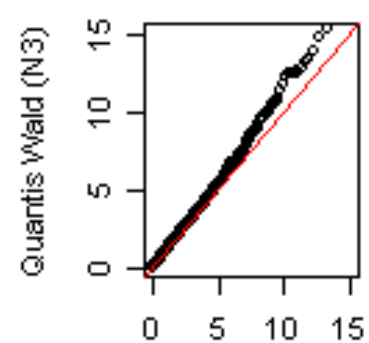

Quantis $Q Q(1)$

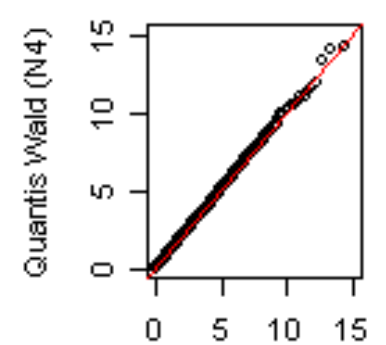

Quantis $Q Q(1)$

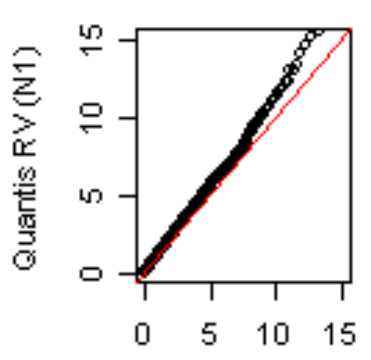

Quantis $Q Q(1)$

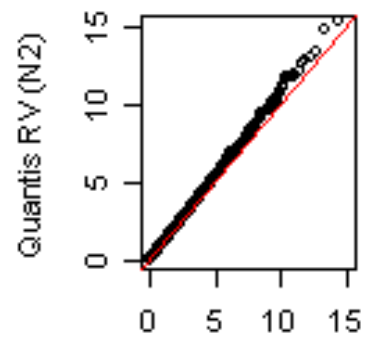

Quantis $Q Q(1)$

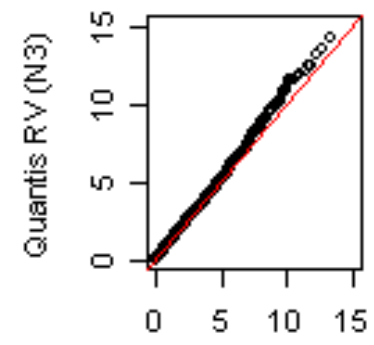

Quantis $Q Q(1)$

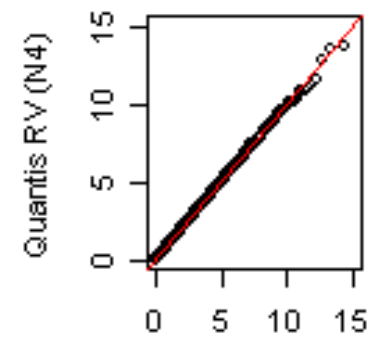

Quantis $Q Q(1)$

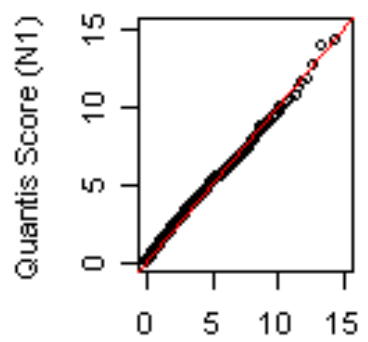

Quantis QQ(1)

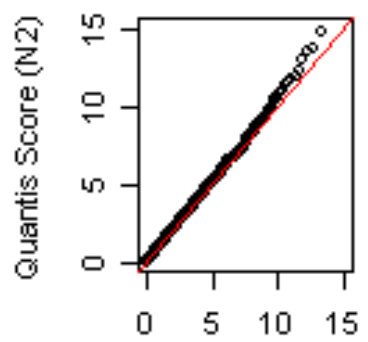

Quantis $Q Q(1)$

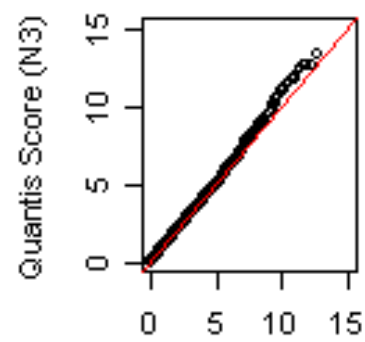

Quantis $\mathrm{QQ}(1)$

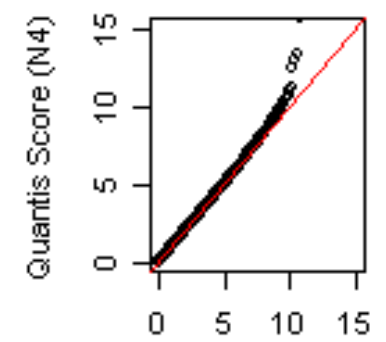

Quantis $Q Q(1)$

Figura 6.4: Gráficos de quantis para $\sigma^{2}=0.1$ e $\sigma_{x}^{2}=0.1$ para o teste $H_{0}: \beta_{11}=\beta_{13}$ versus $H_{0}: \beta_{11} \neq \beta_{13} \operatorname{com} \mu=2.5$

nas tabelas da Seção 6.1 e do Apêndice E e os resultados obtidos foram coerentes com as porcentagens de rejeição obtidas em cada situação. Iremos apresentar aqui somente os gráficos referentes aos casos da Tabela E.5, representados nas Figuras 6.1 a 6.8. As conclusões para estes casos são apresentadas a seguir.

1. Os piores casos para o teste score, quando $\sigma^{2}=0.01, \sigma_{x}^{2}=0.01$ e $\sigma^{2}=0.01, \sigma_{x}^{2}=0.5$, estão representados respectivamente nos gráficos das Figuras 6.1, 6.5. Nestas figuras, podemos 


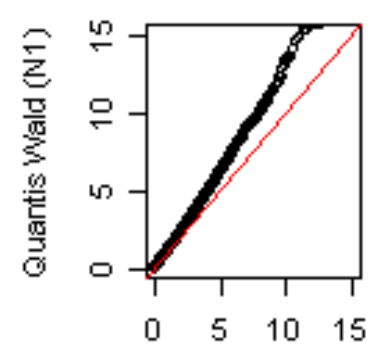

Quantis $Q Q(1)$

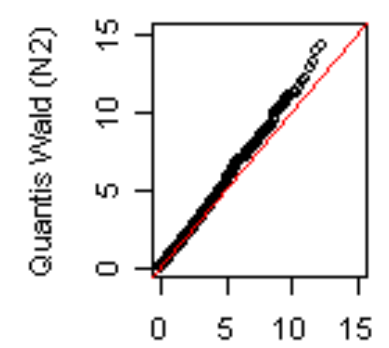

Quantis $Q Q(1)$

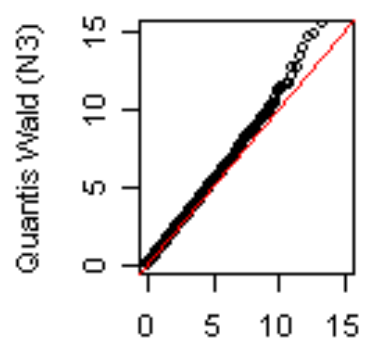

Quantis $Q Q(1)$

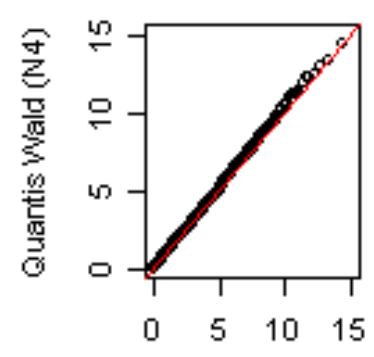

Quantis $Q Q(1)$

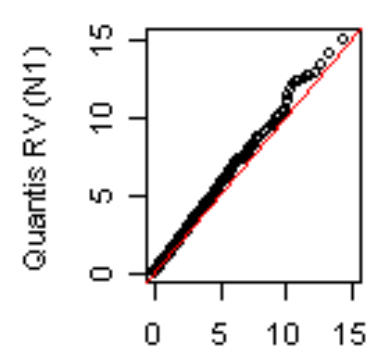

Quantis $Q Q(1)$

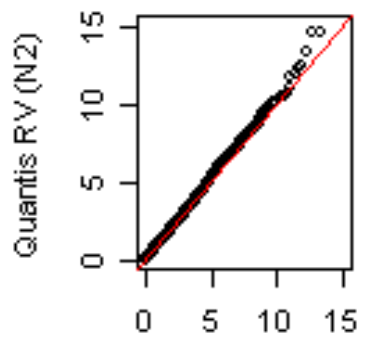

Quantis $Q Q(1)$

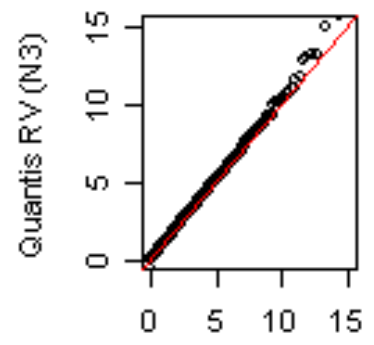

Quantis QQ(1)

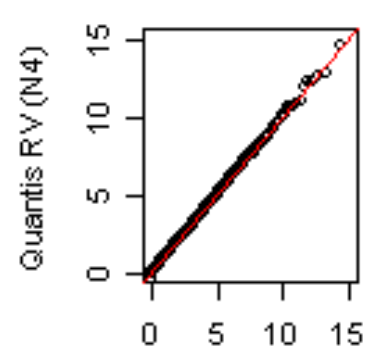

Quantis $Q Q(1)$
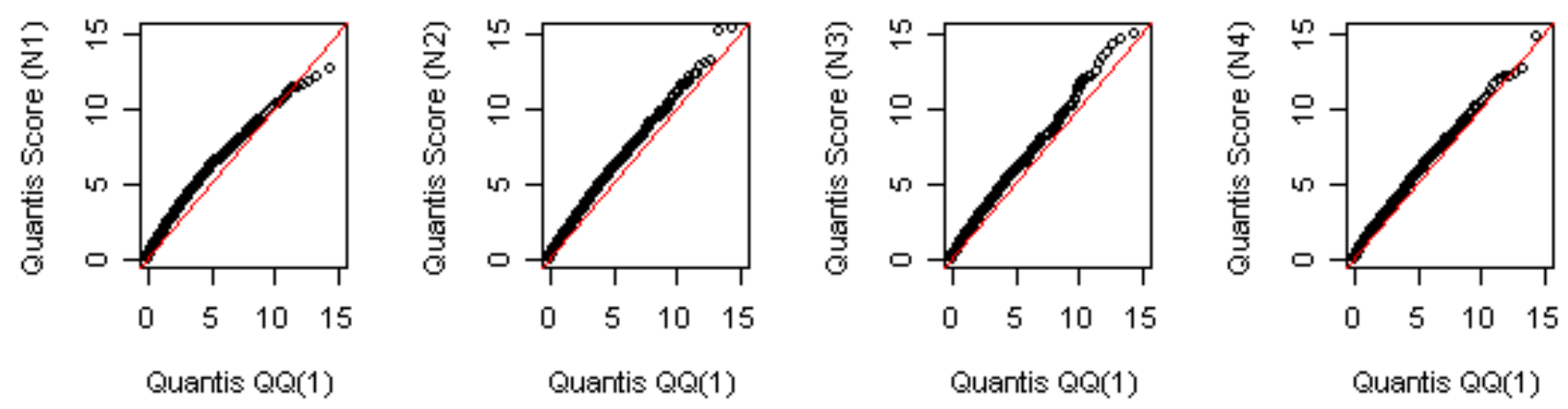

Figura 6.5: Gráficos de quantis para $\sigma^{2}=0.01$ e $\sigma_{x}^{2}=0.5$ para o teste $H_{0}: \beta_{11}=\beta_{13}$ versus $H_{0}: \beta_{11} \neq \beta_{13} \operatorname{com} \mu=2.5$

perceber que há maior distância entre os quantis da estatística score e em relação aos quantis da distribuição $\chi_{1}^{2}$. O caso em que $\sigma^{2}=0.01, \sigma_{x}^{2}=0.1$, em que o teste score também não apresentou desempenho muito bom, está representado no gráfico da Figura 6.2 porém não apresenta tanta distância entre os quantis da estatística score aos quantis da $\chi_{1}^{2}$. Nestes casos, o melhor desempenho foi o da estatística de razão de verossimilhanças, o que se reflete na maior proximidade de seus quantis aos da distribuição $\chi_{1}^{2}$, mesmo para 


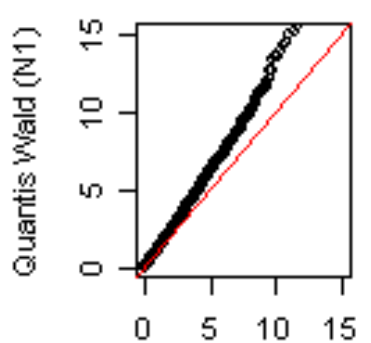

Quantis $Q Q(1)$

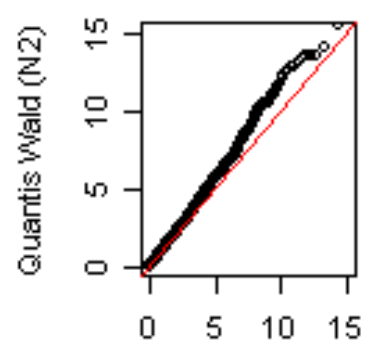

Quantis $Q Q(1)$

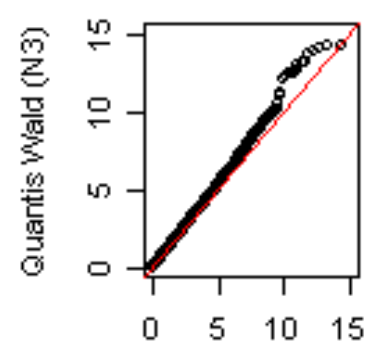

Quantis $Q Q(1)$

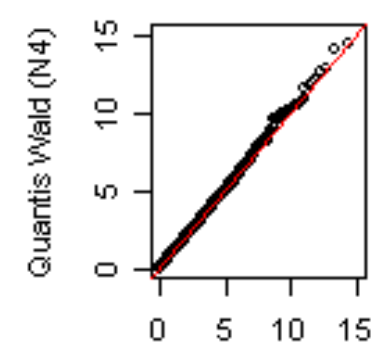

Quantis $Q Q(1)$

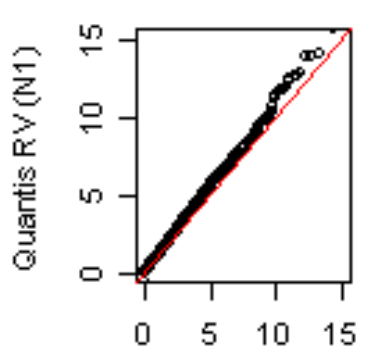

Quantis QQ(1)

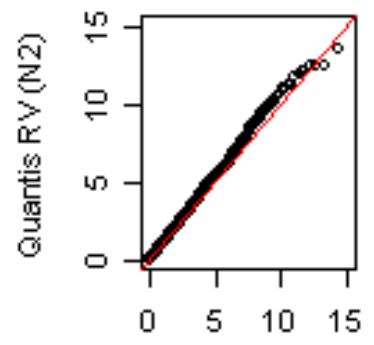

Quantis QQ(1)

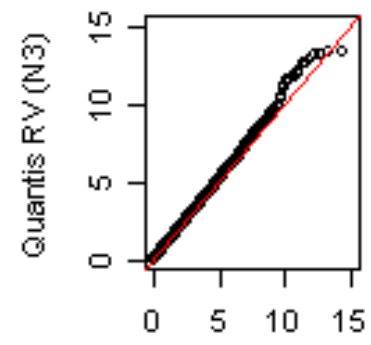

Quantis $Q Q(1)$

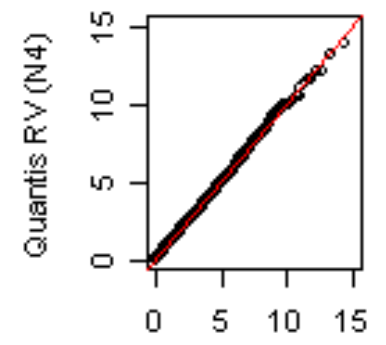

Quantis $Q Q(1)$

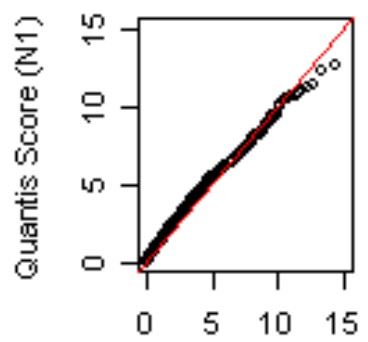

Quantis QQ(1)

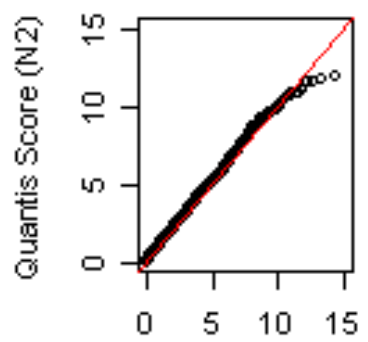

Quantis $Q Q(1)$

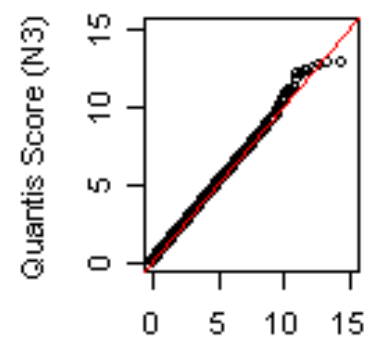

Quantis $\mathrm{QQ}(1)$

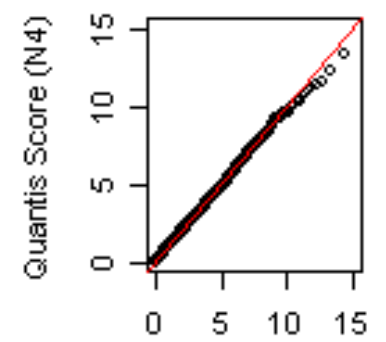

Quantis $Q Q(1)$

Figura 6.6: Gráficos de quantis para $\sigma^{2}=0.05$ e $\sigma_{x}^{2}=0.5$ para o teste $H_{0}: \beta_{11}=\beta_{13}$ versus $H_{0}: \beta_{11} \neq \beta_{13} \operatorname{com} \mu=2.5$

tamanhos pequenos de amostras. Nos demais casos (Figuras 6.3, 6.4, 6.6, 6.7 e 6.8) o teste score apresentou bom desempenho e isto se reflete na boa aproximação dos demais gráficos à reta identidade.

2. O teste de razão de verossimilhanças em geral apresentou bom desempenho mesmo para tamanhos de amostras pequenos, o que está refletido nos gráficos das Figuras 6.1 a 6.8 como a boa aproximação dos quantis desta estatística aos quantis da distribuição $\chi_{1}^{2}$. 


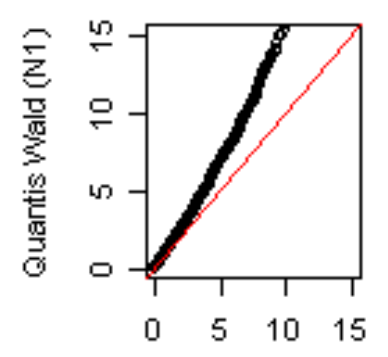

Quantis $Q Q(1)$

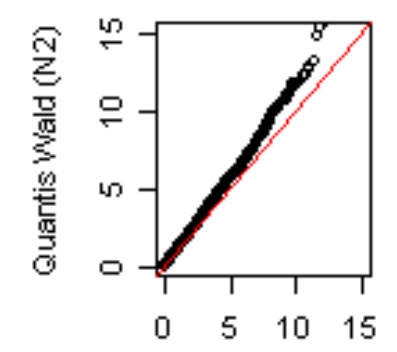

Quantis $Q Q(1)$

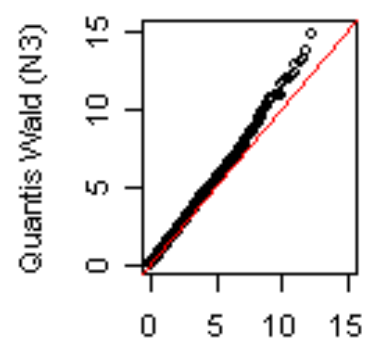

Quantis $Q Q(1)$

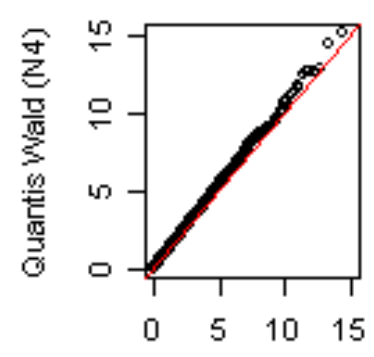

Quantis $Q Q(1)$

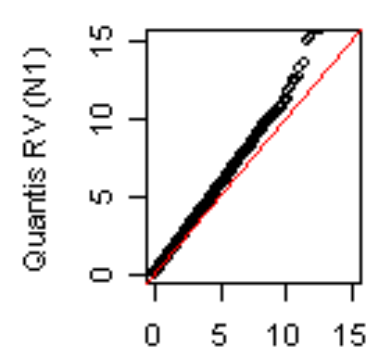

Quantis $Q Q(1)$

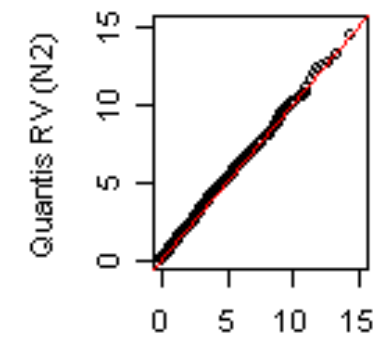

Quantis $Q Q(1)$

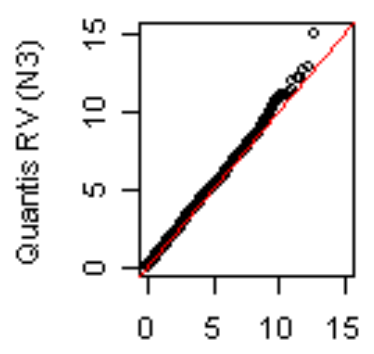

Quantis QQ(1)

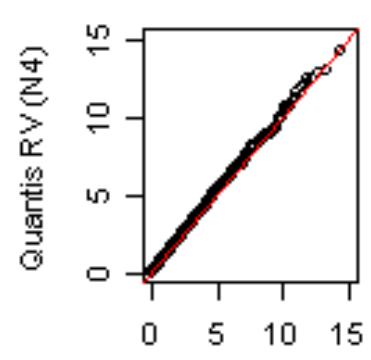

Quantis QQ(1)

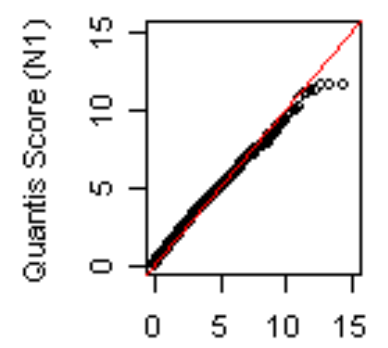

Quantis $Q Q(1)$

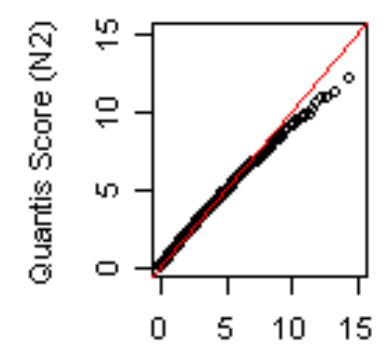

Quantis $Q Q(1)$

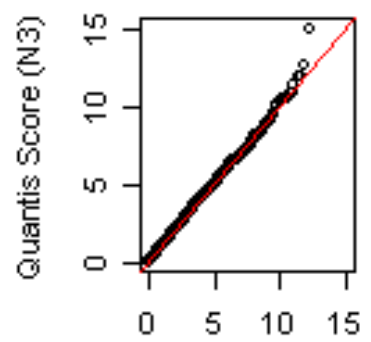

Quantis $Q Q(1)$

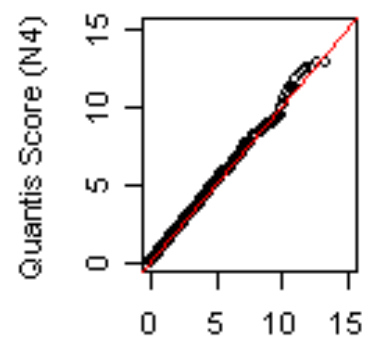

Quantis $Q Q(1)$

Figura 6.7: Gráficos de quantis para $\sigma^{2}=0.1$ e $\sigma_{x}^{2}=0.5$ para o teste $H_{0}: \beta_{11}=\beta_{13}$ versus $H_{0}: \beta_{11} \neq \beta_{13} \operatorname{com} \mu=2.5$

3. O teste de Wald em geral não apresentou bom desempenho para amostras pequenas, o que está refletido na distância entre os quantis desta estatística em relação aos quantis da distribuição $\chi_{1}^{2}$ quando o tamanho das amostras é pequeno, porém ao aumentar o tamanho das amostras ocorre maior aproximação do gráfico de pontos à reta identidade. Este comportamento da estatística Wald apareceu nos gráficos das Figuras 6.1 a 6.8. 


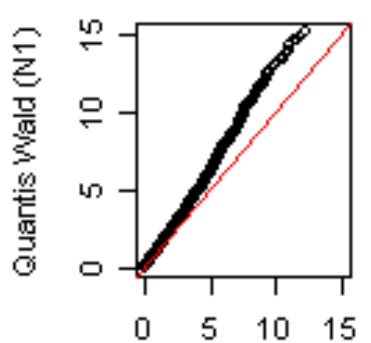

Quantis $Q Q(1)$

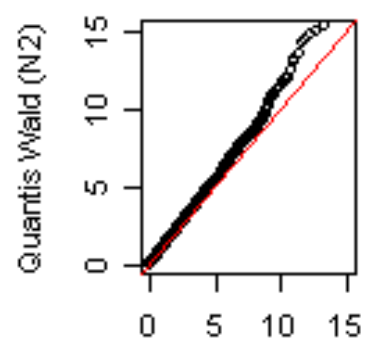

Quantis $Q Q(1)$

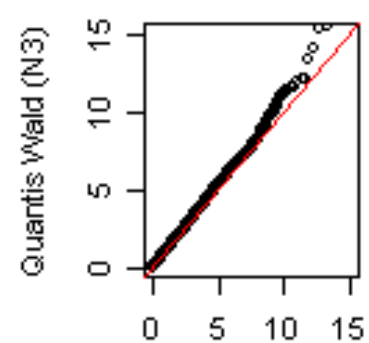

Quantis $Q Q(1)$

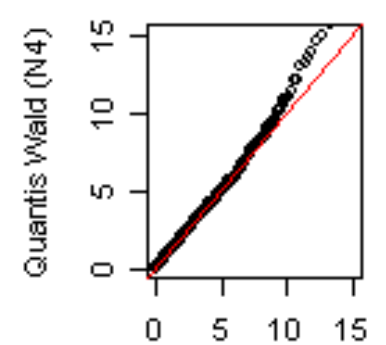

Quantis $Q Q(1)$

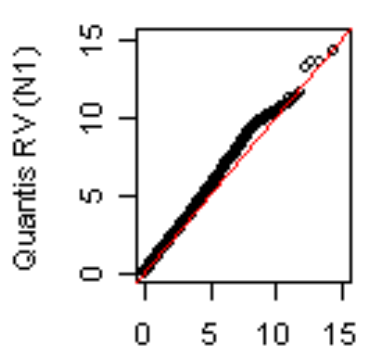

Quantis $Q Q(1)$

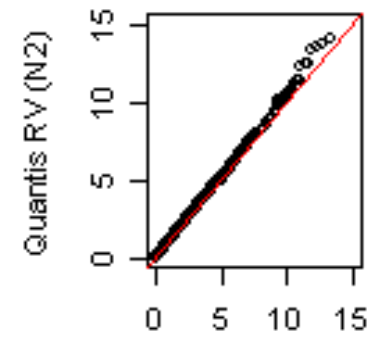

Quantis $Q Q(1)$

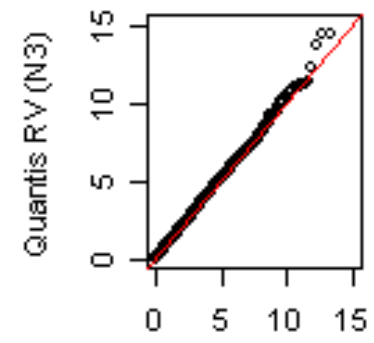

Quantis $Q Q(1)$

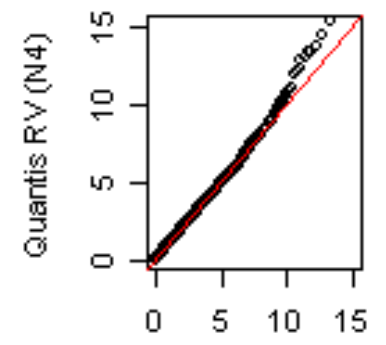

Quantis $Q Q(1)$

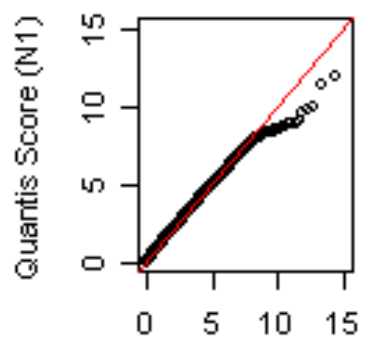

Quantis QQ(1)

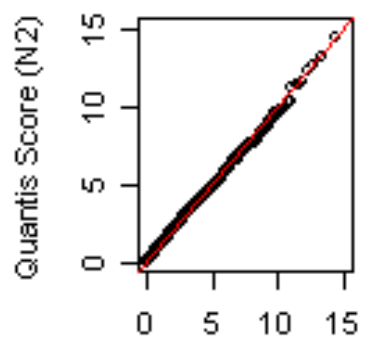

Quantis $Q Q(1)$

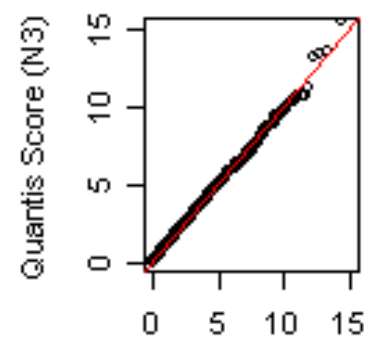

Quantis $\mathrm{QQ}(1)$

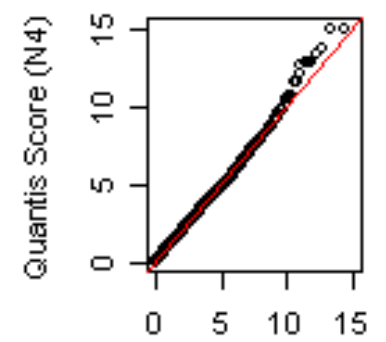

Quantis $Q Q(1)$

Figura 6.8: Gráficos de quantis para $\sigma^{2}=0.5$ e $\sigma_{x}^{2}=0.5$ para o teste $H_{0}: \beta_{11}=\beta_{13}$ versus $H_{0}: \beta_{11} \neq \beta_{13} \operatorname{com} \mu=2.5$

\subsection{Simulação do poder dos testes assintóticos}

Além das simulações do tamanho do teste, realizamos simulações para verificar o comportamento das estatísticas de Wald, score e razão de verossimilhança quanto ao poder do teste. Para avaliar as estatísticas neste sentido, foram geradas 10000 amostras utilizando a linguagem Ox (Doornik 2002) sob as hipóteses alternativas $H_{1}$ tais que $\beta_{i j} \neq \beta_{k l}$, com $i, k=1,2$ e $j, l=1,2,3$. A partir da hipótese $H_{0}$ correspondente, foi considerado um distanciamento gradativo de 0.05, 
valor que foi acrescentado em um dos parâmetros até que se obtivesse um distanciamento de 0.3 entre os valores dos dois parâmetros em questão. Geradas as amostras, foram calculados os valores das três estatísticas e foram obtidas as porcentagens de rejeição comparando-se ao quantil de $95 \%$ de uma $\chi_{1}^{2}$. Os resultados estão apresentados nas Tabelas F.1 a F.27 no Apêndice F.

\subsubsection{Conclusões das simulações do poder dos testes assintóticos}

Fixando-se o tamanho da amostra e distanciando-se da hipótese nula $H_{0}$, o poder das três estatísticas aumentou e se aproximou de 1, como era de se esperar. Esta aproximação também ocorreu ao aumentar o tamanho das amostras, fixando-se o distanciamento de $H_{0}$. As estatísticas que apresentaram melhor comportamento foram score e Wald, sendo que a estatística da razão de verossimilhança apresentou em geral poder menor do que as duas primeiras. As conclusões do estudo de simulação foram as seguintes:

Mais precisamente:

1. As comparações entre os parâmetros (a hipótese $H_{0}$ ) consideradas parecem não influenciar no poder simulado dos testes, fixados os valores para os valores dos parâmetros, o tamanho das amostras e o distanciamento de $H_{0}$.

2. Fixando-se os valores dos parâmetros $\sigma_{x}^{2}, \sigma^{2}$, o tamanho das amostras e o distanciamento da hipótese $H_{0}$ considerado, os valores de $\mu$ considerados parecem influenciar no comportamento das três estatísticas de teste, sendo que conforme aumenta-se o valor de $\mu$, o poder simulado das três estatísticas de teste cresce mais rapidamente.

3. Fixando-se os valores de $\mu$, o tamanho das amostras e o distanciamento da hipótese $H_{0}$ considerado, os valores dos parâmetros $\sigma_{x}^{2}$ e $\sigma^{2}$ não influenciaram tendenciosamente no comportamento das três estatísticas em relação ao poder do teste.

4. Para os casos em que $\sigma^{2}=0.01$, o teste score apresentou-se o melhor dos três testes em relação ao poder, para todos os tamanhos de amostra considerados, quando a hipótese alternativa estava próxima à hipótese nula. Conforme distanciou-se a hipótese alternativa, os três testes tiveram comportamento equivalente. No entanto, nestes casos, em que $\sigma^{2}=0.01$, o teste score já havia apresentado as maiores porcentagens de rejeição nas simulações do tamanho do teste. Comparando-se apenas as estatísticas de Wald e razão de verossimilhanças nestes casos, que tiveram comportamento melhor em relação ao tamanho do teste, a estatística Wald apresentou uma pequena vantagem em relação ao poder, apenas para o menor tamanho de amostra considerado, porém para as amostras de 
tamanho médio o poder do teste da razão de verossimilhança aproximou-se bem do poder do teste Wald, e para amostras de tamanho grande os três testes foram equivalentes, como era de se esperar.

5. Considerando-se $\sigma^{2}=0.05, \sigma^{2}=0.1$ e $\sigma^{2}=0.5$ e amostras de tamanho pequeno e médio, o melhor comportamento foi o da estatística de Wald, seguido pela estatística da razão de verossimilhanças. Nestes casos, as três estatísticas haviam apresentado comportamento semelhante em relação ao tamanho do teste, porém a estatística de Wald já apresentava maiores porcentagens de rejeição para amostras de tamanho pequeno e médio. Conforme distanciou-se a hipótese alternativa da hipótese nula, fixados os tamanhos de amostras, os três testes tiveram comportamento semelhante. Para amostras grandes as três estatísticas tiveram comportamentos equivalentes e alcançaram o valor 1 ao mesmo tempo na grande maioria dos casos, como era de se esperar. 
Análise de um modelo de regressão com erros nas variáveis multivariado com intercepto nulo 


\section{Capítulo 7}

\section{Diagnóstico de influência local}

As técnicas de diagnóstico em modelos de regressão têm como finalidade verificar a robustez de um modelo estatístico quando este sofre algum tipo de perturbação, ou seja, são métodos para estudar as variações em uma análise resultantes de perturbações na formulação do problema. O interesse nesta técnica consiste basicamente em detectar pontos de grande influência no modelo ajustado. Estes pontos têm geralmente comportamento incomum entre os dados, porém podem conter informações importantes e indispensáveis para a análise dos dados em questão, e por isto é interessante dedicar a eles atenção especial e cuidadosa.

Existem várias as técnicas de diagnóstico, como por exemplo as técnicas apresentadas em Belsley et al. (1980), Cook \& Weisberg (1982), e em Cook (1986). Podemos citar, por exemplo, as medidas globais DFBETA, DFFIT e D-Cook. Estas técnicas consistem na exclusão de casos, ou seja, em ajustar novamente o modelo proposto, desconsiderando uma das observações de cada vez, para verificar as mudanças provocadas nos resultados das análises deste modelo.

Em Cook (1986), foi apresentada uma nova metodologia de diagnóstico, com um enfoque local, diferentemente das técnicas de diagnóstico já existentes até então. As técnicas ditas locais procuram avaliar a influência de pequenas perturbações nos dados ou na formulação do problema e desde então, muitos autores têm utilizado esta abordagem, que pode ser aplicada a qualquer modelo desde que se tenha a função de verossimilhança. Na literatura, podem ser encontrados mais de 300 artigos utilizando a técnica de influência local. No entanto, poucos trabalhos podem ser encontrados considerando-se modelos de regressão com erros nas variáveis. Neste trabalho, vamos considerar as técnicas de diagnóstico de influência local propostas por Cook (1986).

Em anos recentes, podemos encontrar aplicações de influência local a vários tipos de modelos estatísticos. Iremos citar alguns.

Em modelos de regressão linear, Paula (1993) utiliza a técnica de influência local com o espaço paramétrico restrito, Lei et al. (2004) calculam a influência local usando técnicas robustas 
de diagnóstico, Poon \& Poon (2001) definem uma nova medida para detectar influência local e Hartless et al. (2003) utiliza técnicas de influência local para detectar a influência de variáveis preditivas. Kim (1996) aplica a técnica de influência local a um modelo normal multivariado. Zou \& Zhang (2005) aplicam o método a um modelo $t$ multivariado com uma estrutura simples de Rao (Rao's simple structure). Lei \& Wang (1996) sugerem uma pequena mudança no enfoque de Cook (1986) para acessar a influência em um modelo multivariado. Jung (2000) adapta as técnicas de influência local para a análise de correlação canônica, considerando uma perturbação baseada na função de distribuição empírica. Tanaka et al. (2002) mostra resultados de invariância de resultados de análise de influência para transformações afins de variáveis no caso de análise de correção canônica. Tanaka (2003) trata influência local como uma técnica equivalente a análise de componentes principais.

Considerando distribuições elípticas, Galea-Rojas et al. (1997) estuda métodos de diagnóstico em modelos elípticos e compara aos métodos de diagnóstico desenvolvidos por Cook (1986) para o modelo linear. Liu (2000) aplica vários esquemas de perturbação no modelo de regressão elíptico e Liu (2002) aplica esquemas de perturbação em modelos de regressão linear multivariados elípticos.

Lesaffre \& Verbeke (1998) aplicam a influência local a modelos mistos para dados longitudinais, e Zhu \& Lee (2003) aplicam esquemas de perturbação a modelos lineares mistos generalizados.

Aplicações a modelos de regressão logística podem ser encontrados em González Sierra \& Suárez Rancel (2000). Farrell \& Cadigan (2000) faz uma comparação entre exclusão de casos e ponderação de casos para modelos de regressão binária.

Aplicações com enfoque bayesiano são apresentadas em Bai \& Fei (2000), Zou \& Zhang (2003). Ouwens et al. (2001) discute a generalização da influência local para respostas normalmente distribuídas para modelos lineares generalizados com efeitos aleatórios.

Lee \& Tang (2004) acessam a influência local em um modelo com variável latente utilizando o algoritmo de Metropolis-Hastings.

Fung \& Tang (1997) propõem que outras funções, como um estimador de um parâmetro ou uma estatística de teste podem ser usadas na análise de influência local.

Tang et al. (2001) utiliza uma medida generalizada de Cook descrita em modelos de dispersão em Poon \& Poon (1999) para acessar influência em modelos não-lineares de dispersão.

Como aplicações em modelos de calibração, temos por exemplo Dávila et al. (2002), que se baseia na parametrização de análise de fatores. Galea-Rojas, Bolfarine \& Castro (2002) aplica esquemas de perturbação em modelos de calibração comparativa, Galea-Rojas et al. (2005) 
aplica a técnica de influência local para modelos estruturais assumindo que as medidas obtidas seguem uma distribuição elíptica multivariada.

Zhu \& Lee (2001) propõem um método para acessar a influência local em modelos com dados incompletos.

Para modelos com erros nas variáveis, considerando técnicas de diagnóstico em geral, podemos encontrar alguns artigos, como Kelly (1984) e Wellman \& Gunst (1991), que trabalham com a função de influência para identificar pontos fora do comum. Carroll \& Spiegelman (1992) sugerem novos métodos gráficos para análise de resíduos em modelos de regressão com erros nas variáveis, Zhao et al. (1994) propõe um estudo de simulação de envelopes para acessar a influência para modelos lineares generalizados com erros nas variáveis. Zhong et al. (2000) propõe um método unificado de influência para modelos com erros nas variáveis baseado na verossimilhança corrigida de Nakamura (1990), Takeuchi (2002) propõe uma maneira de acessar a influência de observações individuais no erro quadrático médio. Também podemos citar os trabalhos de Klepper (1988), Cheng \& Van Ness (1990), Yohai \& Zamar (1990), Fung et al. (2003) entre outros.

Com o enfoque específico de influência local (Cook, 1986), podemos citar Lee \& Zhao (1996) que fazem um estudo de influência local em modelos lineares generalizados com erros nas variáveis, Aoki et al. (n.d.) que apresenta um estudo de diagnóstico de influência local, para o modelo de regressão com erros nas variáveis com o intercepto nulo e com uma possível estrutura de dependência entre as populações, Kim (2000) aplica a influência local a um modelo estrutural, Galea-Rojas, Bolfarine \& Vilca-Labra (2002) que aplica técnicas de influência local a um modelo estrutural com distribuição t-student, Labra et al. (2005) faz um estudo de influência local em um modelo $t$-student com o intercepto nulo e Rasekh (2006) faz um estudo com estimador ridge em um modelo com erros nas variáveis. Lee et al. (2006) aplica a um modelo estrutural não-linear com dados incompletos.

Neste trabalho, faremos um estudo de influência local no modelo com erros nas variáveis multivariado com intercepto nulo ajustado aos dados de Hadgu \& Koch (1999), onde temos duas variáveis resposta e também uma estrutura de dependência entre medições em um determinado indivíduo.

\subsection{Influência local de Cook (1986)}

Nesta seção iremos apresentar a metodologia utilizada no estudo de influência local proposto por Cook (1986). Seja $L(\boldsymbol{\theta})$ a função log da verossimilhança correspondente ao modelo postulado, 
onde $\boldsymbol{\theta}_{p \times 1}$ é um vetor de parâmetros desconhecidos. A perturbação no modelo é introduzida através de um vetor

$$
\boldsymbol{\omega}=\boldsymbol{\omega}_{q \times 1}, \text { onde } \boldsymbol{\omega} \in \Omega \subseteq \mathbb{R}^{q},
$$

$\Omega$ um aberto, e seja $L(\boldsymbol{\theta} / \boldsymbol{\omega})$ a função $\log$-verossimilhança do modelo perturbado. Assumiremos que existe um $\boldsymbol{\omega}_{0} \in \Omega$ tal que $L(\boldsymbol{\theta})=L\left(\boldsymbol{\theta} / \boldsymbol{\omega}_{0}\right), \forall \boldsymbol{\theta}$ e que $L(\boldsymbol{\theta} / \boldsymbol{\omega})$ é duas vezes continuamente diferenciável em $\left(\boldsymbol{\theta}^{\prime}, \boldsymbol{\omega}^{\prime}\right)^{\prime}$. Iremos denotar por $\widehat{\boldsymbol{\theta}}$ e $\widehat{\boldsymbol{\theta}}_{\boldsymbol{\omega}}$ os estimadores de máxima verossimilhança de $\boldsymbol{\theta}$ em $L(\boldsymbol{\theta})$ e $L(\boldsymbol{\theta} / \boldsymbol{\omega})$ respectivamente. Uma medida sugerida por Cook (1986) é definida como:

$$
L D=L D(\boldsymbol{\omega})=2\left[L(\widehat{\boldsymbol{\theta}})-L\left(\widehat{\boldsymbol{\theta}}_{\boldsymbol{\omega}}\right)\right]
$$

e denominada afastamento pela verossimilhança ("likelihood displacement"), de modo que a distância entre $\widehat{\boldsymbol{\theta}}$ e $\widehat{\boldsymbol{\theta}}_{\boldsymbol{\omega}}$ passa a depender da concavidade da log-verossimilhança, ou seja, se $L(\boldsymbol{\theta})$ é suficientemente achatada, $\widehat{\boldsymbol{\theta}}$ e $\widehat{\boldsymbol{\theta}}_{\boldsymbol{\omega}}$ podem ser julgados que estão próximos entre si, enquanto que se $L(\boldsymbol{\theta})$ for suficientemente concentrada em torno de $\widehat{\boldsymbol{\theta}}$ estas estimativas podem estar distantes entre si (Cook, 1987). De acordo com Cook (1986), uma forma de investigar o comportamento local de $L D(\boldsymbol{\omega})$ em torno de $\boldsymbol{\omega}_{0}$ é selecionar uma direção unitária $\mathbf{d}$ que determina o plano normal a $\Omega$ e contém o eixo $L D(\boldsymbol{\omega})$. Para cada direção $\mathbf{d}$, a intersecção deste plano com a superfície pode ser analisada pelo gráfico de $L D\left(\boldsymbol{\omega}_{0}+a \mathbf{d}\right)$ versus $a \in \mathbb{R}$. Como $L D(\boldsymbol{\omega})$ é uma função não negativa com um mínimo global em $\boldsymbol{\omega}_{0}, L D\left(\boldsymbol{\omega}_{0}+a \mathbf{d}\right)$ tem um mínimo local em $a=0$. Cada linha ajustada pode ser caracterizada considerando a curvatura normal $C_{\mathbf{d}}$ em $a=0$, que pode ser interpretada como o círculo de melhor ajuste em $\boldsymbol{\omega}_{0}$. Quanto maior os valores de $C_{\mathbf{d}}$, maior é a sensibilidade à perturbação introduzida na direção d. Cook (1986) sugeriu que a direção $\mathbf{d}_{\max }$, correspondente à maior curvatura $C_{\max }$ contém a informação de diagnóstico mais importante, ou seja, para detectar observações que mais influenciam em $L D(\boldsymbol{\omega})$, basta fazer o gráfico de $\mathbf{d}_{\max }$ pelo índice das observações e verificar quais pontos se destacam dos demais. Ele mostrou que

$$
C_{\mathbf{d}}=2\left|\mathbf{d}^{\prime} \ddot{F} \mathbf{d}\right|
$$

onde $\ddot{F}=\boldsymbol{\Delta}^{\prime}(\ddot{L})^{-1} \boldsymbol{\Delta}$, com $\boldsymbol{\Delta}=\frac{\partial^{2}}{\partial \boldsymbol{\theta} \partial \boldsymbol{\omega}^{\prime}} L(\boldsymbol{\theta} / \boldsymbol{\omega})$ avaliado em $\boldsymbol{\theta}=\widehat{\boldsymbol{\theta}}$ e $\boldsymbol{\omega}=\boldsymbol{\omega}_{0}$ e $-\ddot{L}$ é a matriz de informação observada do modelo postulado, cujos elementos estão descritos no Apêndice D.

A matriz $\Delta$ para as perturbações definidas nas Subseções 7.1.1 a 7.1.5 é dada por

$$
\begin{gathered}
\boldsymbol{\Delta}=\left.\frac{\partial^{2}}{\partial \boldsymbol{\theta} \partial \boldsymbol{\omega}^{\prime}} L(\boldsymbol{\theta} / \boldsymbol{\omega})\right|_{\boldsymbol{\theta}=\widehat{\boldsymbol{\theta}}, \boldsymbol{\omega}=\boldsymbol{\omega}_{0}}=\left.\left(\boldsymbol{\Delta}_{11}, \ldots, \boldsymbol{\Delta}_{p n_{p}}\right)^{\prime}\right|_{\boldsymbol{\theta}=\widehat{\boldsymbol{\theta}}, \boldsymbol{\omega}=\boldsymbol{\omega}_{0}}, \text { onde } \\
\boldsymbol{\Delta}_{\boldsymbol{i}_{j}}=\left(\boldsymbol{\Delta}_{\boldsymbol{\beta}_{1} i j}^{\prime}, \ldots, \boldsymbol{\Delta}_{\boldsymbol{\beta}_{p} i j}^{\prime}, \boldsymbol{\Delta}_{\mu i j}, \boldsymbol{\Delta}_{\sigma_{x}^{2} i j}, \boldsymbol{\Delta}_{\sigma^{2} i j}, \boldsymbol{\Delta}_{\boldsymbol{\sigma}_{\mathbf{e}_{1} i j}^{2}}^{\prime}, \ldots, \boldsymbol{\Delta}_{\boldsymbol{\sigma}_{\mathbf{e}_{p}}^{2} i j}^{\prime}\right)^{\prime}
\end{gathered}
$$


com $i=1, \ldots, p, j=1, \ldots, n_{i}$.

Nas próximas seções, iremos aplicar a técnica de influência local descrita acima para identificar possíveis pontos influentes do conjunto de dados descrito na Seção 2.1 no modelo proposto na Seção 2.2. Para tanto, iremos considerar os esquemas de perturbação de ponderação de casos, perturbação na variável explanatória, nas variáveis resposta, na variância dos erros de medição no início do estudo e nas variâncias dos erros após o uso dos líquidos de bochecho. Para cada esquema de perturbação considerado apresentaremos os elementos que compõem a matriz $\Delta$.

\subsubsection{Ponderação de casos}

O primeiro esquema de perturbação que utilizaremos é o esquema de ponderação de casos. Como definido em Cook (1986), cada caso representa um vetor observado $\mathbf{z}_{i_{j}}$. Para isto, seja o vetor de perturbação $\boldsymbol{\omega}$ de dimensão $N \times 1, \operatorname{com} N=\sum_{i=1}^{p} n_{i}$, tal que $\boldsymbol{\omega}=\left(\boldsymbol{\omega}_{1}, \ldots, \boldsymbol{\omega}_{p}\right)^{\prime}=$ $\left(w_{1_{1}}, \ldots, w_{1_{n_{1}}}, \ldots, w_{p_{1}}, \ldots, w_{p_{n_{p}}}\right)^{\prime}$. A log-verossimilhança do modelo perturbado neste caso é dada por

$$
L(\boldsymbol{\theta} / \boldsymbol{\omega})=-\frac{3}{2} \log (2 \pi) \sum_{i=1}^{p} \sum_{j=1}^{n_{i}} \omega_{i_{j}}-\frac{1}{2} \sum_{i=1}^{p} \log \left|\mathbf{V}_{i}\right| \sum_{j=1}^{n_{i}} \omega_{i_{j}}-\frac{1}{2} \sum_{i=1}^{p} \sum_{j=1}^{n_{i}} \omega_{i_{j}}\left(\mathbf{z}_{i_{j}}-\mathbf{m}_{i}\right)^{\prime} \mathbf{V}_{i}^{-1}\left(\mathbf{z}_{i_{j}}-\mathbf{m}_{i}\right),
$$

com o vetor de não-perturbação dado por $\boldsymbol{\omega}_{0}=\mathbf{1}_{N}=(1, \ldots, 1)^{\prime}$. Definindo

$$
\begin{aligned}
C_{i_{j}}= & \sigma^{-2}\left(x_{i_{j}}-\mu\right)+\left(\left(\mathbf{y}_{i_{j}}-\boldsymbol{\beta}_{i} \mu\right)^{\prime} \mathbf{D}^{-1}\left(\boldsymbol{\sigma}_{\mathbf{e}_{i}}^{2}\right) \boldsymbol{\beta}_{i}\right) \\
G_{i_{j}}= & \sigma^{-4}\left(x_{i_{j}}-\mu\right)^{2}+2 \sigma^{-2}\left(x_{i_{j}}-\mu\right)\left(\mathbf{y}_{i_{j}}-\boldsymbol{\beta}_{i} \mu\right)^{\prime} \mathbf{D}^{-1}\left(\boldsymbol{\sigma}_{\mathbf{e}_{i}}^{2}\right) \boldsymbol{\beta}_{i} \\
& +\left(\mathbf{y}_{i_{j}}-\boldsymbol{\beta}_{i} \mu\right)^{\prime} \mathbf{D}^{-1}\left(\boldsymbol{\sigma}_{\mathbf{e}_{i}}^{2}\right) \boldsymbol{\beta}_{i} \boldsymbol{\beta}_{i}^{\prime} \mathbf{D}^{-1}\left(\boldsymbol{\sigma}_{\mathbf{e}_{i}}^{2}\right)\left(\mathbf{y}_{i_{j}}-\boldsymbol{\beta}_{i} \mu\right),
\end{aligned}
$$

e utilizando $\mathbf{a}_{i}, \mathbf{A}_{i}, \mathbf{B}_{i}$, e $b_{i}$ como definidos em (2.6) e (2.7), as propriedades do Apêndice B.3 e algumas manipulações algébricas, obtivemos os elementos da matriz $\boldsymbol{\Delta}$, para $i=1, \ldots, p, j=$ $1, \ldots, n_{i}$

$$
\boldsymbol{\Delta}_{\boldsymbol{\beta}_{i} i j}^{\prime}=\left\{\mu+\sigma_{x}^{2} b_{i}^{-1} C_{i_{j}}\right\} \mathbf{D}^{-1}\left(\boldsymbol{\sigma}_{\mathbf{e}_{i}}^{2}\right)\left(\mathbf{y}_{i_{j}}-\boldsymbol{\beta}_{i} \mu\right)-\sigma_{x}^{2} b_{i}^{-1}\left\{1+\sigma_{x}^{2} b_{i}^{-1} G_{i_{j}}+\mu C_{i_{j}}\right\} \mathbf{D}^{-1}\left(\boldsymbol{\sigma}_{\mathbf{e}_{i}}^{2}\right) \boldsymbol{\beta}_{i},
$$

$\boldsymbol{\Delta}_{\boldsymbol{\beta}_{k} i j}^{\prime}=\mathbf{0}, \quad k=1, \ldots, p, k \neq i$,

$\Delta_{\mu i j}=b_{i}^{-1} C_{i_{j}}$,

$\boldsymbol{\Delta}_{\sigma^{2} i j}=-\frac{1}{2}\left\{\sigma^{-2}\left[1-\sigma^{-2}\left(\sigma_{x}^{2} b_{i}^{-1}+\left(x_{i_{j}}-\mu\right)^{2}\right)\right]+\sigma_{x}^{2} \sigma^{-4} b_{i}^{-1}\left[2\left(x_{i_{j}}-\mu\right) C_{i_{j}}-\sigma_{x}^{2} b_{i}^{-1} G_{i_{j}}\right]\right\}$,

$\boldsymbol{\Delta}_{\sigma_{x}^{2} i j}=-\frac{1}{2}\left\{\sigma_{x}^{-2}\left(1-b_{i}^{-1}\right)-b_{i}^{-2} G_{i_{j}}\right\}$, 


$$
\begin{aligned}
& \boldsymbol{\Delta}_{\boldsymbol{\sigma}_{\mathbf{e}_{i} i j}^{2}}^{\prime}=\frac{1}{2} \mathbf{D}\left(\mathbf{y}_{i_{j}}-\boldsymbol{\beta}_{i} \mu\right) \mathbf{D}^{-2}\left(\boldsymbol{\sigma}_{\mathbf{e}_{i}}^{2}\right)\left(\mathbf{y}_{i_{j}}-\boldsymbol{\beta}_{i} \mu\right)-\sigma_{x}^{2} b_{i}^{-1} C_{i_{j}} \mathbf{D}\left(\boldsymbol{\beta}_{i}\right) \mathbf{D}^{-2}\left(\boldsymbol{\sigma}_{\mathbf{e}_{i}}^{2}\right)\left(\mathbf{y}_{i_{j}}-\boldsymbol{\beta}_{i} \mu\right) \\
& \quad+\frac{1}{2} \sigma_{x}^{2} b_{i}^{-1}\left\{\sigma_{x}^{2} b_{i}^{-1} G_{i_{j}}+1\right\} \mathbf{D}\left(\boldsymbol{\beta}_{i}\right) \mathbf{D}^{-2}\left(\boldsymbol{\sigma}_{\mathbf{e}_{i}}^{2}\right) \boldsymbol{\beta}_{i}-\frac{1}{2} \mathbf{D}^{-1}\left(\boldsymbol{\sigma}_{\mathbf{e}_{i}}^{2}\right) \mathbf{1}_{2}, \\
& \boldsymbol{\Delta}_{\boldsymbol{\sigma}_{\mathbf{e}_{k}}^{2} i j}^{\prime}=\mathbf{0}, \quad k=1, \ldots, p, k \neq i .
\end{aligned}
$$

\subsubsection{Perturbação na variável explanatória}

Uma forma de perturbar as variáveis explanatórias $x_{i_{j}}$ seria considerando

$$
x_{i_{j}} \rightarrow x_{i_{j}}+S_{x} \omega_{i_{j}}
$$

$i=1, \ldots, p, j=1, \ldots, n_{i}$, onde $S_{x}$ é um fator de escala, por exemplo o desvio padrão amostral de $x$. Neste caso, como os grupos são homogêneos no início do estudo, consideramos um único valor para o desvio padrão amostral.

A log-verossimilhança do modelo perturbado neste caso é dada por

$$
L(\boldsymbol{\theta} / \boldsymbol{\omega})=-\frac{3 N}{2} \log (2 \pi)-\frac{1}{2} \sum_{i=1}^{p} n_{i} \log \left|\mathbf{V}_{i}\right|-\frac{1}{2} \sum_{i=1}^{p} \sum_{j=1}^{n_{i}}\left(\mathbf{z}_{i_{j}}^{\star}-\mathbf{m}_{i}\right)^{\prime} \mathbf{V}_{i}^{-1}\left(\mathbf{z}_{i_{j}}^{\star}-\mathbf{m}_{i}\right),
$$

onde $\mathbf{z}_{i_{j}}^{\star}=\left(x_{i_{j}}+S_{x} \omega_{i_{j}}, y_{1 i_{j}}, y_{2 i_{j}}\right)^{\prime}, i=1, \ldots, p, j=1, \ldots, n_{i}$ e o vetor de não-perturbação é dado por $\boldsymbol{\omega}_{0}=\mathbf{0}_{N}=(0, \ldots, 0)^{\prime}$. Utilizando $\mathbf{a}_{i}, \mathbf{A}_{i}, \mathbf{B}_{i}$, e $b_{i}$ como definidos em (2.6) e (2.7), obtemos, após alguns cálculos utilizando propriedades do Apêndice B.3 os elementos da matriz $\boldsymbol{\Delta}$, com $i=1, \ldots, p, j=1, \ldots, n_{i}$ :

$$
\begin{aligned}
& \boldsymbol{\Delta}_{\boldsymbol{\beta}_{i} i j}^{\prime}=\sigma_{x}^{2} \sigma^{-2} b_{i}^{-1} S_{x}\left\{-2 \sigma_{x}^{2} b_{i}^{-1} C_{i_{j}} \mathbf{D}^{-1}\left(\boldsymbol{\sigma}_{\mathbf{e}_{i}}^{2}\right) \boldsymbol{\beta}_{i}+\mathbf{D}^{-1}\left(\boldsymbol{\sigma}_{\mathbf{e}_{i}}^{2}\right)\left(\mathbf{y}_{i_{j}}-2 \boldsymbol{\beta}_{i} \mu\right)\right\} \\
& \boldsymbol{\Delta}_{\boldsymbol{\beta}_{k} i j}^{\prime}=0, \quad k=1, \ldots, p, k \neq i
\end{aligned}
$$$$
\Delta_{\mu i j}=S_{x} \sigma^{-2} b_{i}^{-1}
$$$$
\boldsymbol{\Delta}_{\sigma^{2} i j}=\sigma^{-4} S_{x}\left(1-\sigma^{-2} \sigma_{x}^{2} b_{i}^{-1}\right)\left[\left(x_{i_{j}}-\mu\right)-\sigma_{x}^{2} C_{i_{j}} b_{i}^{-1}\right],
$$$$
\boldsymbol{\Delta}_{\sigma_{x}^{2} i j}=\sigma^{-2} b_{i}^{-2} S_{x} C_{i_{j}},
$$$$
\boldsymbol{\Delta}_{\boldsymbol{\sigma}_{\mathbf{e}_{i} i j}}^{\prime}=S_{x} \sigma_{x}^{2} b_{i}^{-1} \sigma^{-2}\left\{b_{i}^{-1} \sigma_{x}^{2} C_{i_{j}} \mathbf{D}\left(\boldsymbol{\beta}_{i}\right) \mathbf{D}^{-2}\left(\boldsymbol{\sigma}_{\mathbf{e}_{i}}^{2}\right) \boldsymbol{\beta}_{i}-\mathbf{D}\left(\boldsymbol{\beta}_{i}\right) \mathbf{D}^{-2}\left(\boldsymbol{\sigma}_{\mathbf{e}_{i}}^{2}\right)\left(\mathbf{y}_{i_{j}}-\boldsymbol{\beta}_{i} \mu\right)\right\},
$$$$
\boldsymbol{\Delta}_{\boldsymbol{\sigma}_{\mathbf{e}_{k}}^{2} i j}^{\prime}=\mathbf{0}, \quad k=1, \ldots, p, k \neq i \text {. }
$$

\subsubsection{Perturbação nas variáveis resposta}

Iremos considerar agora um esquema de perturbação nas variáveis resposta, fazendo

$$
\mathbf{y}_{i_{j}} \rightarrow \mathbf{y}_{i_{j}}+\mathbf{S}_{\mathbf{y}_{i}} \omega_{i_{j}}
$$


onde $\mathbf{S}_{\mathbf{y}_{i}}=\left(S_{\mathbf{y}_{1 i}}, S_{\mathbf{y}_{2 i}}\right)^{\prime}$, ou seja,

$$
\left\{\begin{array}{c}
y_{1 i_{j}} \rightarrow y_{1 i_{j}}+S_{\mathbf{y}_{1 i}} \omega_{i_{j}} \\
y_{2 i_{j}} \rightarrow y_{2 i_{j}}+S_{\mathbf{y}_{2 i}} \omega_{i_{j}}
\end{array}\right.
$$

onde $S_{\mathbf{y}_{1 i}}$ e $S_{\mathbf{y}_{2 i}}$ são fatores de escala, por exemplo o desvio padrão amostral de $\mathbf{y}_{1 i}$ e $\mathbf{y}_{2 i}$ respectivamente. A log-verossimilhança do modelo perturbado neste caso é dada por

$$
L(\boldsymbol{\theta} / \boldsymbol{\omega})=-\frac{3 N}{2} \log (2 \pi)-\frac{1}{2} \sum_{i=1}^{p} n_{i} \log \left|\mathbf{V}_{i}\right|-\frac{1}{2} \sum_{i=1}^{p} \sum_{j=1}^{n_{i}}\left(\mathbf{z}_{i_{j}}^{\star \star}-\mathbf{m}_{i}\right)^{\prime} \mathbf{V}_{i}^{-1}\left(\mathbf{z}_{i_{j}}^{\star \star}-\mathbf{m}_{i}\right),
$$

onde $\mathbf{z}_{i_{j}}^{\star \star}=\left(x_{i_{j}}, y_{1 i_{j}}+S_{\mathbf{y}_{1 i}} \omega_{i_{j}}, y_{2 i_{j}}+S_{\mathbf{y}_{2 i}} \omega_{i_{j}}\right)^{\prime}, i=1, \ldots, p, j=1, \ldots, n_{i}$ e o vetor de nãoperturbação é dado por $\boldsymbol{\omega}_{0}=\mathbf{0}_{N}=(0, \ldots, 0)^{\prime}$. Utilizando $\mathbf{a}_{i}, \mathbf{A}_{i}, \mathbf{B}_{i}$, e $b_{i}$ como definidos em (2.6) e (2.7), obtemos, após alguns cálculos utilizando propriedades do Apêndice B.3 os elementos da matriz $\boldsymbol{\Delta}$, com $i=1, \ldots, p, j=1, \ldots, n_{i}$ :

$$
\begin{aligned}
& \boldsymbol{\Delta}_{\boldsymbol{\beta}_{i} i j}^{\prime}=\sigma_{x}^{2} b_{i}^{-1}\left[\boldsymbol{\beta}_{i}^{\prime} \mathbf{D}^{-1}\left(\boldsymbol{\sigma}_{\mathbf{e}_{i}}^{2}\right) \mathbf{S}_{\mathbf{y}_{i}}\right]\left\{\mathbf{D}^{-1}\left(\boldsymbol{\sigma}_{\mathbf{e}_{i}}^{2}\right)\left(\mathbf{y}_{i_{j}}-2 \boldsymbol{\beta}_{i} \mu\right)-2 b_{i}^{-1} \sigma_{x}^{2} C_{i_{j}} \mathbf{D}^{-1}\left(\boldsymbol{\sigma}_{\mathbf{e}_{i}}^{2}\right) \boldsymbol{\beta}_{i}\right\} \\
& \quad+\left(\sigma_{x}^{2} b_{i}^{-1} C_{i_{j}}+\mu\right) \mathbf{D}^{-1}\left(\boldsymbol{\sigma}_{\mathbf{e}_{i}}^{2}\right) \mathbf{S}_{\mathbf{y}_{i}}
\end{aligned}
$$$$
\boldsymbol{\Delta}_{\boldsymbol{\beta}_{k} i j}^{\prime}=0, \quad k=1, \ldots, p, k \neq i
$$$$
\boldsymbol{\Delta}_{\mu i j}=b_{i}^{-1}\left[\boldsymbol{\beta}_{i}^{\prime} \mathbf{D}^{-1}\left(\boldsymbol{\sigma}_{\mathbf{e}_{i}}^{2}\right) \mathbf{S}_{\mathbf{y}_{i}}\right]
$$$$
\boldsymbol{\Delta}_{\sigma^{2} i j}=\sigma_{x}^{2} \sigma^{-4} b_{i}^{-1}\left[\sigma_{x}^{2} b_{i}^{-1} C_{i_{j}}-\left(x_{i_{j}}-\mu\right)\right]\left[\boldsymbol{\beta}_{i}^{\prime} \mathbf{D}^{-1}\left(\boldsymbol{\sigma}_{\mathbf{e}_{i}}^{2}\right) \mathbf{S}_{\mathbf{y}_{i}}\right]
$$$$
\boldsymbol{\Delta}_{\sigma_{x}^{2} i j}=b_{i}^{-2} C_{i_{j}}\left[\boldsymbol{\beta}_{i}^{\prime} \mathbf{D}^{-1}\left(\boldsymbol{\sigma}_{\mathbf{e}_{i}}^{2}\right) \mathbf{S}_{\mathbf{y}_{i}}\right]
$$$$
\boldsymbol{\Delta}_{\boldsymbol{\sigma}_{\mathbf{e}_{i} i j}^{2}}^{\prime}=\mathbf{D}\left(\mathbf{S}_{\mathbf{y}_{i}}\right) \mathbf{D}^{-2}\left(\boldsymbol{\sigma}_{\mathbf{e}_{i}}^{2}\right)\left(\mathbf{y}_{i_{j}}-\boldsymbol{\beta}_{i} \mu\right)-\sigma_{x}^{2} b_{i}^{-1} C_{i_{j}} \mathbf{D}\left(\boldsymbol{\beta}_{i}\right) \mathbf{D}^{-2}\left(\boldsymbol{\sigma}_{\mathbf{e}_{i}}^{2}\right) \mathbf{S}_{\mathbf{y}_{i}}
$$$$
+\sigma_{x}^{2} b_{i}^{-1} \boldsymbol{\beta}_{i}^{\prime} \mathbf{D}^{-1}\left(\boldsymbol{\sigma}_{\mathbf{e}_{i}}^{2}\right) \mathbf{S}_{\mathbf{y}_{i}}\left[\sigma_{x}^{2} b_{i}^{-1} C_{i_{j}} \mathbf{D}\left(\boldsymbol{\beta}_{i}\right) \mathbf{D}^{-2}\left(\boldsymbol{\sigma}_{\mathbf{e}_{i}}^{2}\right) \boldsymbol{\beta}_{i}-\mathbf{D}\left(\boldsymbol{\beta}_{i}\right) \mathbf{D}^{-2}\left(\boldsymbol{\sigma}_{\mathbf{e}_{i}}^{2}\right)\left(\mathbf{y}_{i_{j}}-\boldsymbol{\beta}_{i} \mu\right)\right],
$$

$\boldsymbol{\Delta}_{\boldsymbol{\sigma}_{\mathbf{e}_{k}}^{2} i j}^{\prime}=\mathbf{0}, k=1, \ldots, p, k \neq i$

\subsubsection{Perturbação na variância dos erros de medição no início do estudo}

Neste esquema de perturbação faremos

$$
\sigma^{2} \rightarrow \frac{\sigma^{2}}{\omega_{i_{j}}}
$$

A log-verossimilhança do modelo perturbado neste caso é dada por

$$
L(\boldsymbol{\theta} / \boldsymbol{\omega})=-\frac{3 N}{2} \log (2 \pi)-\frac{1}{2} \sum_{i=1}^{p} \sum_{j=1}^{n_{i}} \log \left|V_{\mathbf{z}_{i_{j}}}^{\star}\right|-\frac{1}{2} \sum_{i=1}^{p} \sum_{j=1}^{n_{i}}\left(\mathbf{z}_{i_{j}}-\mathbf{m}_{i}\right)^{\prime}\left(V_{\mathbf{z}_{i_{j}}}^{\star}\right)^{-1}\left(\mathbf{z}_{i_{j}}-\mathbf{m}_{i}\right), \text { onde }
$$




$$
V_{\mathbf{z}_{i_{j}}}^{\star}=\left(\begin{array}{ccc}
\sigma_{x}^{2}+\frac{\sigma^{2}}{\omega_{i_{j}}} & \beta_{1 i} \sigma_{x}^{2} & \beta_{2 i} \sigma_{x}^{2} \\
\beta_{1 i} \sigma_{x}^{2} & \beta_{1 i}^{2} \sigma_{x}^{2}+\sigma_{e_{1 i}}^{2} & \beta_{1 i} \beta_{2 i} \sigma_{x}^{2} \\
\beta_{2 i} \sigma_{x}^{2} & \beta_{1 i} \beta_{2 i} \sigma_{x}^{2} & \beta_{2 i}^{2} \sigma_{x}^{2}+\sigma_{e_{2 i}}^{2}
\end{array}\right)
$$

e o vetor de não-perturbação é dado por $\boldsymbol{\omega}_{0}=\mathbf{1}_{N}=(1, \ldots, 1)^{\prime}$.

Os elementos da matriz $\boldsymbol{\Delta}$, com $i=1, \ldots, p, j=1, \ldots, n_{i}$.

$$
\begin{aligned}
& \boldsymbol{\Delta}_{\boldsymbol{\beta}_{i} i j}^{\prime}=\left\{\sigma_{x}^{2} \sigma^{-2} b_{i}^{-1}\left[\left(x_{i_{j}}-\mu\right) \mathbf{D}^{-1}\left(\boldsymbol{\sigma}_{\mathbf{e}_{i}}^{2}\right)\left(\mathbf{y}_{i_{j}}-2 \boldsymbol{\beta}_{i} \mu\right)+\sigma_{x}^{2} b_{i}^{-1} \mathbf{D}^{-1}\left(\boldsymbol{\sigma}_{\mathbf{e}_{i}}^{2}\right) \boldsymbol{\beta}_{i}\right]\right. \\
& \left.\quad-\sigma_{x}^{4} b_{i}^{-2} \sigma^{-2} C_{i_{j}} \mathbf{D}^{-1}\left(\boldsymbol{\sigma}_{\mathbf{e}_{i}}^{2}\right)\left(\mathbf{y}_{i_{j}}-2 \boldsymbol{\beta}_{i} \mu\right)-2 \sigma_{x}^{4} \sigma^{-2} b_{i}^{-2}\left[\left(x_{i_{j}}-\mu\right) C_{i_{j}}-\sigma_{x}^{2} b_{i}^{-1} G_{i_{j}}\right] \mathbf{D}^{-1}\left(\boldsymbol{\sigma}_{\mathbf{e}_{i}}^{2}\right) \boldsymbol{\beta}_{i}\right\}
\end{aligned}
$$

$\boldsymbol{\Delta}_{\boldsymbol{\beta}_{k} i j}^{\prime}=0, \quad k=1, \ldots, p, k \neq i$

$\boldsymbol{\Delta}_{\mu i j}=\sigma^{-2} b_{i}^{-1}\left[\left(x_{i_{j}}-\mu\right)-\sigma_{x}^{2} b_{i}^{-1} C_{i_{j}}\right]$

$$
\begin{aligned}
& \Delta_{\sigma^{2} i j}=\frac{1}{2} \sigma^{-4}\left(1-2 \sigma_{x}^{2} b_{i}^{-1} \sigma^{-2}\right)\left(x_{i_{j}}-\mu\right)^{2}+\frac{1}{2} \sigma_{x}^{2} \sigma^{-4} b_{i}^{-1}\left(1-\sigma_{x}^{2} b_{i}^{-1} \sigma^{-2}\right) \\
& -\sigma_{x}^{2} b_{i}^{-1} \sigma^{-4}\left(1-2 \sigma_{x}^{2} \sigma^{-2} b_{i}^{-1}\right)\left(x_{i_{j}}-\mu\right) C_{i_{j}}+\frac{1}{2} \sigma_{x}^{4} b_{i}^{-2} \sigma^{-4}\left(1-2 \sigma_{x}^{2} \sigma^{-2} b_{i}^{-1}\right) G_{i_{j}}
\end{aligned}
$$

$\boldsymbol{\Delta}_{\sigma_{x}^{2} i j}=\sigma^{-2} b_{i}^{-2}\left[\left(-\frac{1}{2}+\left(x_{i_{j}}-\mu\right) C_{i_{j}}\right)-\sigma_{x}^{2} b_{i}^{-1}\left(G_{i_{j}}\right)\right]$

$$
\begin{aligned}
& \boldsymbol{\Delta}_{\boldsymbol{\sigma}_{\mathbf{e}_{i} i j}^{2}}^{\prime}=-\frac{1}{2} \sigma_{x}^{4} b_{i}^{-2} \sigma^{-2} \mathbf{D}\left(\boldsymbol{\beta}_{i}\right) \mathbf{D}^{-2}\left(\boldsymbol{\sigma}_{\mathbf{e}_{i}}^{2}\right) \boldsymbol{\beta}_{i}-\sigma_{x}^{2} b_{i}^{-1} \sigma^{-2}\left(x_{i_{j}}-\mu\right) \mathbf{D}\left(\boldsymbol{\beta}_{i}\right) \mathbf{D}^{-2}\left(\boldsymbol{\sigma}_{\mathbf{e}_{i}}^{2}\right)\left(\mathbf{y}_{i_{j}}-\boldsymbol{\beta}_{i} \mu\right) \\
& \quad+\sigma_{x}^{4} b_{i}^{-2} \sigma^{-2} C_{i_{j}} \mathbf{D}\left(\boldsymbol{\beta}_{i}\right) \mathbf{D}^{-2}\left(\boldsymbol{\sigma}_{\mathbf{e}_{i}}^{2}\right)\left(\mathbf{y}_{i_{j}}-\boldsymbol{\beta}_{i} \mu\right) \\
& \quad+\sigma_{x}^{4} b_{i}^{-2} \sigma^{-2}\left[\left(x_{i_{j}}-\mu\right) C_{i_{j}}-\sigma_{x}^{2} b_{i}^{-1} G_{i_{j}}\right] \mathbf{D}\left(\boldsymbol{\beta}_{i}\right) \mathbf{D}^{-2}\left(\boldsymbol{\sigma}_{\mathbf{e}_{i}}^{2}\right) \boldsymbol{\beta}_{i},
\end{aligned}
$$

$\boldsymbol{\Delta}_{\boldsymbol{\sigma}_{\mathbf{e}_{k}}^{2} i j}^{\prime}=\mathbf{0}, k=1, \ldots, p, k \neq i$

\subsubsection{Perturbação nas variâncias dos erros após o uso dos líquidos}

Neste esquema de perturbação fazemos

$$
\boldsymbol{\sigma}_{\mathbf{e}_{i_{j}}}^{2} \rightarrow \frac{\boldsymbol{\sigma}_{\mathbf{e}_{i}}^{2}}{\omega_{i_{j}}}
$$

A log-verossimilhança do modelo perturbado neste caso é dada por

$$
\begin{gathered}
L(\boldsymbol{\theta} / \boldsymbol{\omega})=-\frac{3 N}{2} \log (2 \pi)-\frac{1}{2} \sum_{i=1}^{p} \sum_{j=1}^{n_{i}} \log \left|V_{\mathbf{z}_{i_{j}}}^{\star \star}\right|-\frac{1}{2} \sum_{i=1}^{p} \sum_{j=1}^{n_{i}}\left(\mathbf{z}_{i_{j}}-\mathbf{m}_{i}\right)^{\prime}\left(V_{\mathbf{z}_{i_{j}}}^{\star \star}\right)^{-1}\left(\mathbf{z}_{i_{j}}-\mathbf{m}_{i}\right), \text { onde } \\
V_{\mathbf{z}_{i_{j}}}^{\star \star}=\left(\begin{array}{ccc}
\sigma_{x}^{2}+\sigma^{2} & \beta_{1 i} \sigma_{x}^{2} & \beta_{2 i} \sigma_{x}^{2} \\
\beta_{1 i} \sigma_{x}^{2} & \beta_{1 i}^{2} \sigma_{x}^{2}+\frac{\sigma_{e_{1 i}}^{2}}{\omega_{i_{j}}} & \beta_{1 i} \beta_{2 i} \sigma_{x}^{2} \\
\beta_{2 i} \sigma_{x}^{2} & \beta_{1 i} \beta_{2 i} \sigma_{x}^{2} & \beta_{2 i}^{2} \sigma_{x}^{2}+\frac{\sigma_{e_{2 i}}^{2}}{\omega_{i_{j}}}
\end{array}\right)
\end{gathered}
$$

e o vetor de não-perturbação é dado por $\boldsymbol{\omega}_{0}=\mathbf{1}_{N}=(1, \ldots, 1)^{\prime}$. Os elementos da matriz $\boldsymbol{\Delta}$, com $i=1, \ldots, p, j=1, \ldots, n_{i}$ : 


$$
\begin{aligned}
& \boldsymbol{\Delta}_{\boldsymbol{\beta}_{i} i j}^{\prime}=-\sigma_{x}^{2} b_{i}^{-2}\left[\left(1+\sigma^{-2} \sigma_{x}^{2}\right)+\sigma_{x}^{2} G_{i_{j}}\left(2\left[b_{i}^{-1}\left(1+\sigma^{-2} \sigma_{x}^{2}\right)\right]-1\right)\right] \mathbf{D}^{-1}\left(\boldsymbol{\sigma}_{\mathbf{e}_{i}}^{2}\right) \boldsymbol{\beta}_{i} \\
& \quad+\mu \mathbf{D}^{-1}\left(\boldsymbol{\sigma}_{\mathbf{e}_{i}}^{2}\right)\left(\mathbf{y}_{i_{j}}-\boldsymbol{\beta}_{i} \mu\right)-2 \sigma_{x}^{4} b_{i}^{-2} C_{i_{j}} \boldsymbol{\beta}_{i}^{\prime} \mathbf{D}^{-1}\left(\boldsymbol{\sigma}_{\mathbf{e}_{i}}^{2}\right)\left(\mathbf{y}_{i_{j}}-\boldsymbol{\beta}_{i} \mu\right) \mathbf{D}^{-1}\left(\boldsymbol{\sigma}_{\mathbf{e}_{i}}^{2}\right) \boldsymbol{\beta}_{i} \\
& \quad+\sigma_{x}^{2} b_{i}^{-1}\left\{C_{i_{j}}\left[b_{i}^{-1}\left(1+\sigma^{-2} \sigma_{x}^{2}\right)\right]+\left[\left(\mathbf{y}_{i_{j}}-\boldsymbol{\beta}_{i} \mu\right)^{\prime} \mathbf{D}^{-1}\left(\boldsymbol{\sigma}_{\mathbf{e}_{i}}^{2}\right) \boldsymbol{\beta}_{i}\right]\right\} \mathbf{D}^{-1}\left(\boldsymbol{\sigma}_{\mathbf{e}_{i}}^{2}\right)\left(\mathbf{y}_{i_{j}}-2 \boldsymbol{\beta}_{i} \mu\right),
\end{aligned}
$$

$\boldsymbol{\Delta}_{\boldsymbol{\beta}_{k} i j}^{\prime}=0, \quad k=1, \ldots, p, k \neq i$

$$
\begin{aligned}
& \left.\left.\boldsymbol{\Delta}_{\mu i j}=b_{i}^{-1}\left[\left(\mathbf{y}_{i_{j}}-\boldsymbol{\beta}_{i} \mu\right)^{\prime} \mathbf{D}^{-1}\left(\boldsymbol{\sigma}_{\mathbf{e}_{i}}^{2}\right) \boldsymbol{\beta}_{i}\right)\right]-\sigma_{x}^{2} b_{i}^{-2} C_{i_{j}}\left[\boldsymbol{\beta}_{i}^{\prime} \mathbf{D}^{-1}\left(\boldsymbol{\sigma}_{\mathbf{e}_{i}}^{2}\right) \boldsymbol{\beta}_{i}\right]\right) \\
& \boldsymbol{\Delta}_{\sigma^{2} i j}=-\frac{1}{2} \sigma_{x}^{4} b_{i}^{-2} \sigma^{-4} \boldsymbol{\beta}_{i}^{\prime} \mathbf{D}^{-1}\left(\boldsymbol{\sigma}_{\mathbf{e}_{i}}^{2}\right) \boldsymbol{\beta}_{i}-\sigma_{x}^{2} b_{i}^{-1} \sigma^{-4}\left(x_{i_{j}}-\mu\right)\left(\mathbf{y}_{i_{j}}-\boldsymbol{\beta}_{i} \mu\right)^{\prime} \mathbf{D}^{-1}\left(\boldsymbol{\sigma}_{\mathbf{e}_{i}}^{2}\right) \boldsymbol{\beta}_{i} \\
& \quad+\sigma_{x}^{4} b_{i}^{-2} \sigma^{-4} C_{i_{j}}\left[\left(x_{i_{j}}-\mu\right) \boldsymbol{\beta}_{i}^{\prime} \mathbf{D}^{-1}\left(\boldsymbol{\sigma}_{\mathbf{e}_{i}}^{2}\right) \boldsymbol{\beta}_{i}+\boldsymbol{\beta}_{i}^{\prime} \mathbf{D}^{-1}\left(\boldsymbol{\sigma}_{\mathbf{e}_{i}}^{2}\right)\left(\mathbf{y}_{i_{j}}-\boldsymbol{\beta}_{i} \mu\right)\right] \\
& \quad-\sigma_{x}^{6} b_{i}^{-3} \sigma^{-4} G_{i_{j}} \boldsymbol{\beta}_{i}^{\prime} \mathbf{D}^{-1}\left(\boldsymbol{\sigma}_{\mathbf{e}_{i}}^{2}\right) \boldsymbol{\beta}_{i}
\end{aligned}
$$$$
\boldsymbol{\Delta}_{\sigma_{x}^{2} i j}=-\frac{1}{2} b_{i}^{-2} \boldsymbol{\beta}_{i}^{\prime} \mathbf{D}^{-1}\left(\boldsymbol{\sigma}_{\mathbf{e}_{i}}^{2}\right) \boldsymbol{\beta}_{i}+b_{i}^{-2}\left(\mathbf{y}_{i_{j}}-\boldsymbol{\beta}_{i} \mu\right)^{\prime} \mathbf{D}^{-1}\left(\boldsymbol{\sigma}_{\mathbf{e}_{i}}^{2}\right) \boldsymbol{\beta}_{i} C_{i_{j}}-\sigma_{x}^{2} b_{i}^{-3} \boldsymbol{\beta}_{i}^{\prime} \mathbf{D}^{-1}\left(\boldsymbol{\sigma}_{\mathbf{e}_{i}}^{2}\right) \boldsymbol{\beta}_{i} G_{i_{j}}
$$$$
\boldsymbol{\Delta}_{\boldsymbol{\sigma}_{\mathbf{e}_{i} i j}^{2}}^{\prime}=\frac{1}{2} \mathbf{D}\left(\mathbf{y}_{i_{j}}-\boldsymbol{\beta}_{i} \mu\right) \mathbf{D}^{-2}\left(\boldsymbol{\sigma}_{\mathbf{e}_{i}}^{2}\right)\left(\mathbf{y}_{i_{j}}-\boldsymbol{\beta}_{i} \mu\right)+\frac{1}{2} \sigma_{x}^{2} b_{i}^{-2}\left(1+\sigma^{-2} \sigma_{x}^{2}\right) \mathbf{D}\left(\boldsymbol{\beta}_{i}\right) \mathbf{D}^{-2}\left(\boldsymbol{\sigma}_{\mathbf{e}_{i}}^{2}\right) \boldsymbol{\beta}_{i}
$$$$
+\sigma_{x}^{4} b_{i}^{-2}\left\{\frac{1}{2} G_{i_{j}}\left[2 b_{i}^{-1}\left(1+\sigma^{-2} \sigma_{x}^{2}\right)-1\right]+\frac{1}{2} \mathbf{D}\left(\mathbf{y}_{i_{j}}-\boldsymbol{\beta}_{i} \mu\right) \mathbf{D}^{-2}\left(\boldsymbol{\sigma}_{\mathbf{e}_{i}}^{2}\right)\left(\mathbf{y}_{i_{j}}-\boldsymbol{\beta}_{i} \mu\right)\right.
$$$$
\left.+C_{i_{j}}\left[\boldsymbol{\beta}_{i}^{\prime} \mathbf{D}^{-1}\left(\boldsymbol{\sigma}_{\mathbf{e}_{i}}^{2}\right)\left(\mathbf{y}_{i_{j}}-\boldsymbol{\beta}_{i} \mu\right)\right]\right\} \mathbf{D}\left(\boldsymbol{\beta}_{i}\right) \mathbf{D}^{-2}\left(\boldsymbol{\sigma}_{\mathbf{e}_{i}}^{2}\right) \boldsymbol{\beta}_{i}
$$$$
-\sigma_{x}^{2} b_{i}^{-1}\left\{b_{i}^{-1}\left(1+\sigma_{x}^{2} \sigma^{-2}\right) C_{i_{j}}+\left[\left(\mathbf{y}_{i_{j}}-\boldsymbol{\beta}_{i} \mu\right)^{\prime} \mathbf{D}^{-1}\left(\boldsymbol{\sigma}_{\mathbf{e}_{i}}^{2}\right) \boldsymbol{\beta}_{i}\right]\right\} \mathbf{D}\left(\mathbf{y}_{i_{j}}-\boldsymbol{\beta}_{i} \mu\right) \mathbf{D}^{-2}\left(\boldsymbol{\sigma}_{\mathbf{e}_{i}}^{2}\right) \boldsymbol{\beta}_{i},
$$

$\boldsymbol{\Delta}_{\boldsymbol{\sigma}_{\mathbf{e}_{k} i j}^{2}}^{\prime}=\mathbf{0}, \quad k=1, \ldots, p, k \neq i$

\subsubsection{Aplicação Numérica}

Com o objetivo de verificar a existência de possíveis pontos influentes nos dados dentários apresentados em Hadgu \& Koch (1999), aplicamos a técnica de diagnóstico de influência local, utilizando os esquemas de perturbação descritos anteriormente nas Seções 7.1.1 a 7.1.5.

\section{Ponderação de Casos}

Uma forma de identificar possíveis observações influentes considerando um determinado esquema de perturbação, segundo Cook (1986), é fazer o gráfico de índices de $d_{\text {max }}$ pelas observações e verificar se existem componentes deste vetor que se destacam entre as demais. O vetor $d_{\max }$ representa a direção referente à maior curvatura de $L D(\boldsymbol{\omega}), C_{\max }$.

Considerando o esquema de perturbação de ponderação de casos para os dados apresentados em Hadgu \& Koch (1999), foi construído o gráfico de índices de $d_{\max }$. No conjunto de dados em questão, as observações estão ordenadas de acordo com os grupos que testaram os líquidos de bochecho: as primeiras 36 observações são de indivíduos que testaram o líquido controle, 
as observações de 37 a 69 representam os indivíduos que testaram o líquido experimental A e as observações de 70 a 105 representam indivíduos que testaram o líquido experimental B. Como mostra a Figura 7.1, a observação 42 (6 $6^{\text {a }}$ observação do grupo que testou o líquido A) está distante das demais. As medições para esta observação no início do estudo, após três e seis meses de uso dos líquidos, respectivamente, são $\mathbf{z}_{2,6}=(3.57,2.8,2.41)^{\prime}$. Esta observação tem a característica de possuir a maior medição no início do estudo, entre todas as observações. Também possui medições relativamente grandes após o uso dos líquidos de bochecho.

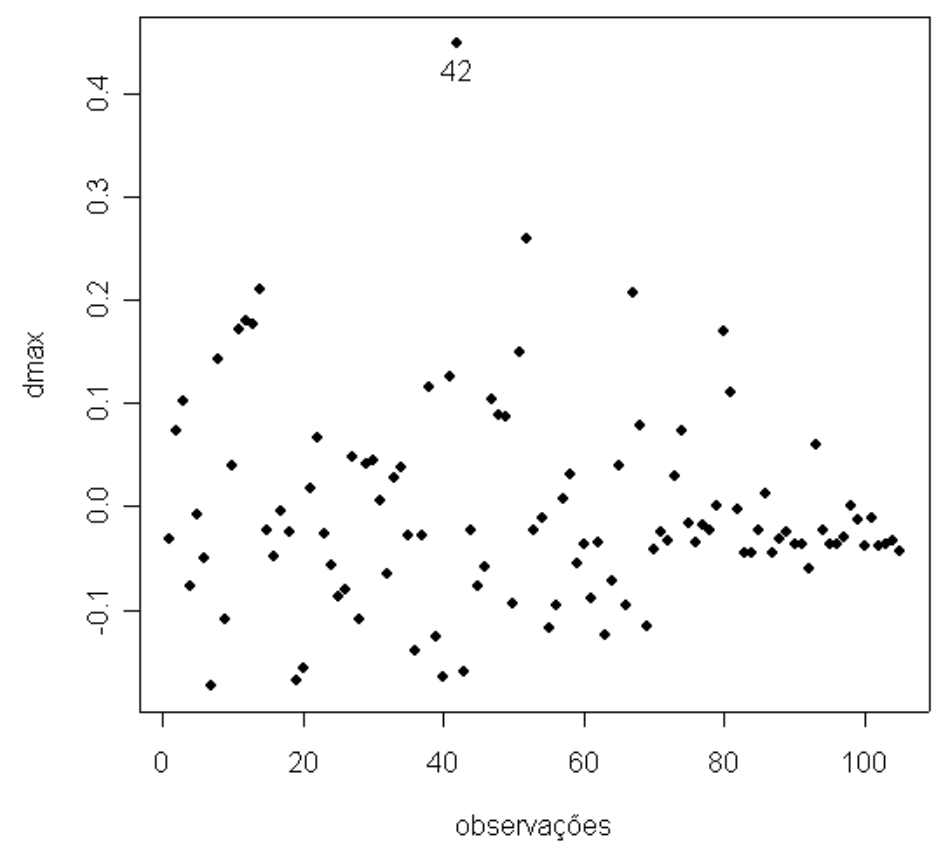

Figura 7.1: Gráfico de $d_{\max }$ para o esquema de perturbação de ponderação de casos

Para acessar a influência individual da j-ésima observação de um conjunto de dados, vamos considerar o vetor $\mathbf{d}_{j}$, que consiste de um vetor de tamanho $N$ com zero em todas as posições exceto na posição $j$, à qual atribui-se o valor -1. Em seguida, podemos construir o gráfico de $L D(\boldsymbol{\omega}(a))$, com $\boldsymbol{\omega}(a)=\boldsymbol{\omega}_{0}+a \mathbf{d}_{j}$, variando-se os valores de $a$, onde $a \in \mathbb{R}$. Como $L D(\boldsymbol{\omega}(a))$ tem um mínimo local em $a=0$, considera-se valores de $a$ em um intervalo em torno do zero, por exemplo entre -1 e 1. A Figura 7.2 mostra que, fazendo o gráfico de $L D(\boldsymbol{\omega}(a))=L D\left(\boldsymbol{\omega}_{0}+a \mathbf{d}_{42}\right)$ o caso 42 parece não exercer muita influência nas variações ocorridas na verossimilhança pois as variações obtidas por $L D\left(\boldsymbol{\omega}_{0}+a \mathbf{d}_{\max }\right)$ são bem maiores do que $L D\left(\boldsymbol{\omega}_{0}+a \mathbf{d}_{42}\right)$, para $-1 \leq a \leq 1$. O valor de $C_{\mathbf{d}_{\max }}$ obtido neste caso foi 2.16, o que segundo Cook (1986) indica que não deve haver grande sensibilidade local neste esquema de perturbação.

Para acessar a sensibilidade das estimativas dos parâmetros em um determinado esquema 


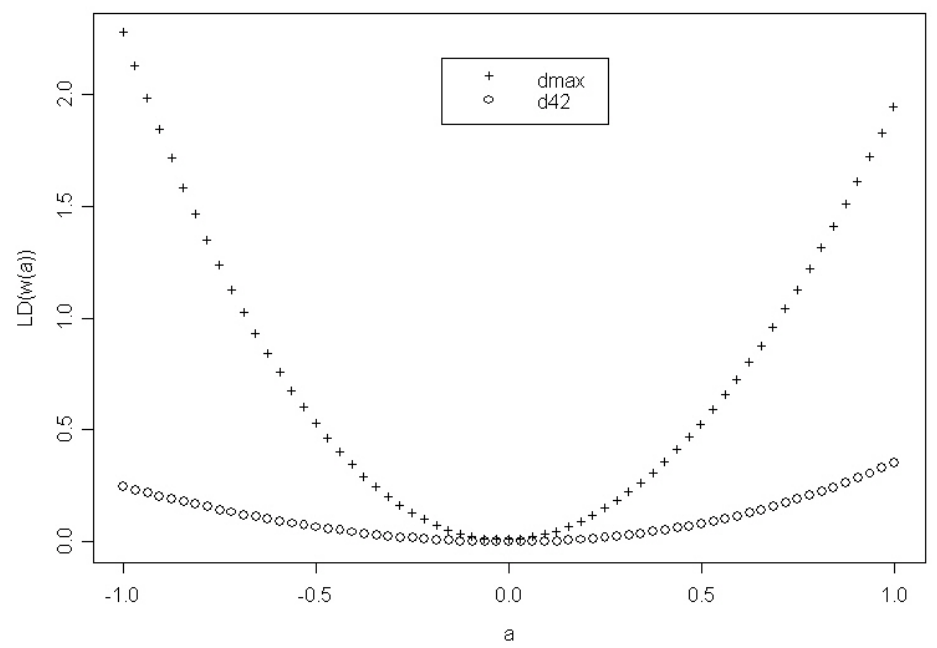

Figura 7.2: Gráfico de $L D(\omega)$ para o esquema de perturbação de ponderação de casos

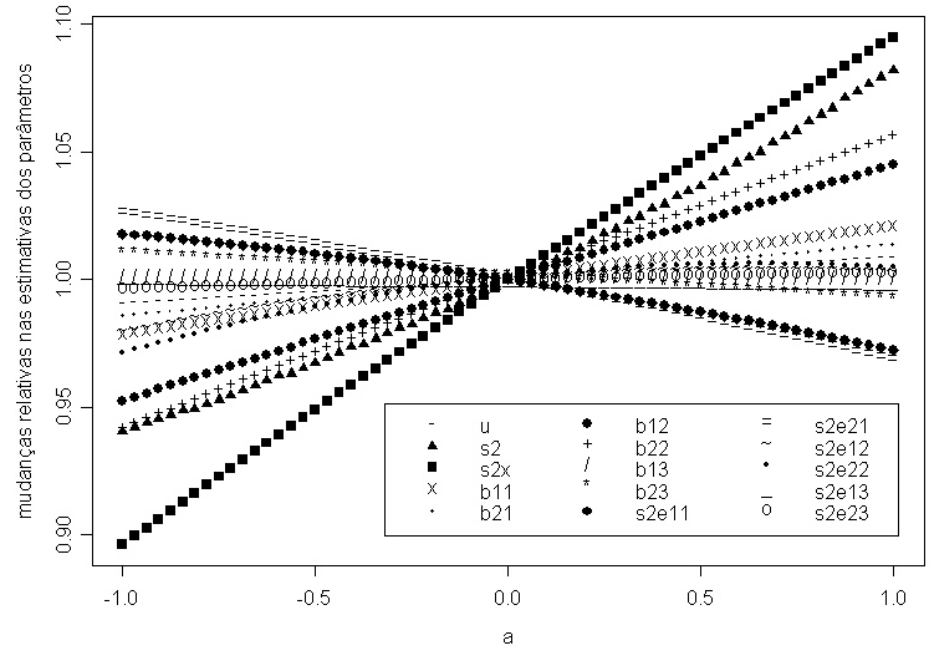

Figura 7.3: Mudanças relativas nas estimativas dos parâmetros no esquema de perturbação de ponderação de casos

de perturbação, podemos obter as mudanças relativas $\frac{\widehat{\theta}_{i}}{{\widehat{\theta_{i}}}_{\boldsymbol{\omega}_{(a)}}}$, onde $\widehat{\theta}_{i}$ é a estimativa de máxima verossimilhança do parâmetro $\theta_{i}$ do modelo postulado e $\widehat{\theta}_{i_{\boldsymbol{\omega}_{(a)}}}$, é a estimativa de máxima verossimilhança do parâmetro $\theta_{i}$ considerando-se o modelo perturbado com $\boldsymbol{\omega}(a)=\boldsymbol{\omega}_{0}+a \mathbf{d}_{\max }$ e $a$ variando de -1 a 1 e $i=1, \ldots, p$. Considerando o esquema de ponderação de casos, o gráfico da Figura 7.3 mostra que os parâmetros que sofrem maior variação quando efetuamos a perturbação na direção máxima são as variâncias $\sigma_{x}^{2}$ e $\sigma^{2}$, respectivamente a variância no ver- 
dadeiro valor das medições dentárias e a variância dos erros de medição. Os outros parâmetros apresentam variações não muito grandes.

\section{Perturbação na variável explanatória}

Ao considerarmos o esquema de perturbação nas variáveis explanatórias e construirmos o gráfico de $d_{\max }$ (Figura 7.4), notamos um comportamento aleatório nos pontos, não dando indícios de nenhum ponto fora do comum. O valor obtido de $C_{\mathbf{d} \max }$ neste caso foi 3.17 .

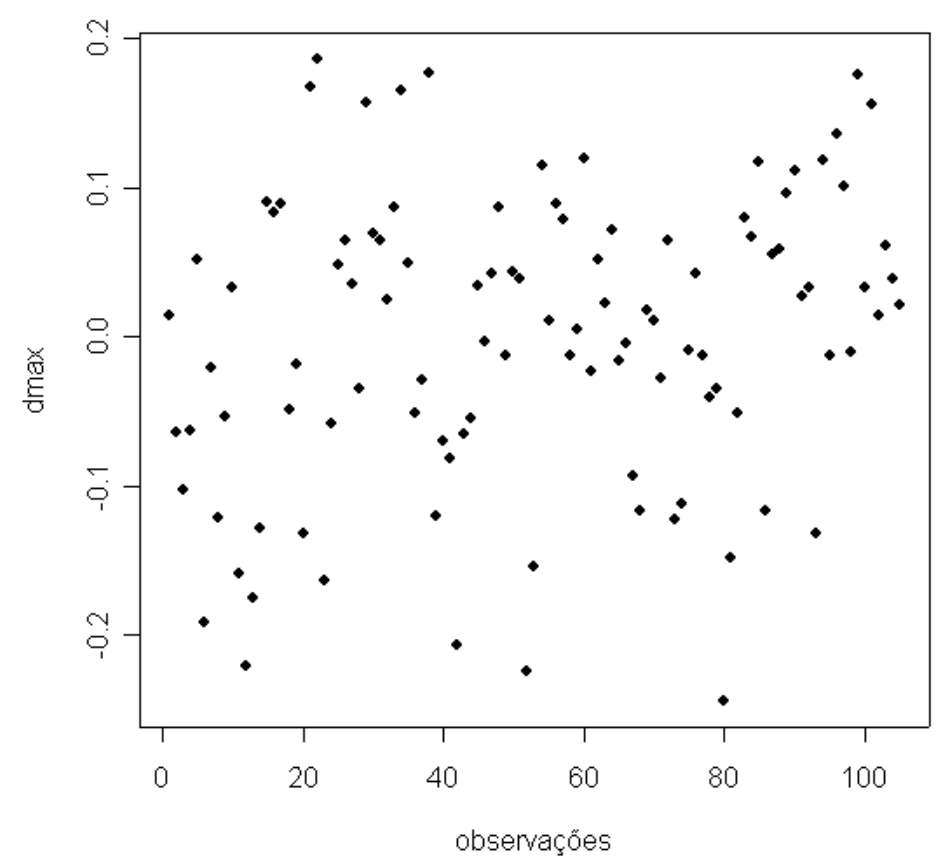

Figura 7.4: Gráfico de $d_{\max }$ para o esquema de perturbação nas variáveis explanatórias

No gráfico da Figura 7.5, vemos que o parâmetro cujas estimativas apresentam maiores mudanças relativas, quando perturbamos as variáveis explanatórias na direção máxima, é a variância dos erros de medição no início do estudo $\sigma^{2}$. Isto poderia indicar influência desta direção nas estimativas deste parâmetro.

\section{Perturbação na variável resposta}

Ao considerarmos o esquema de perturbação nas variáveis resposta, nenhuma observação se apresentou muito distante das demais no gráfico de $d_{\max }$ (Figura 7.6). Porém, notou-se um comportamento diferenciado das observações do grupo que testou o líquido controle (observações de 1 a 36) em relação às observações dos outros grupos. Isto pode significar uma influência 


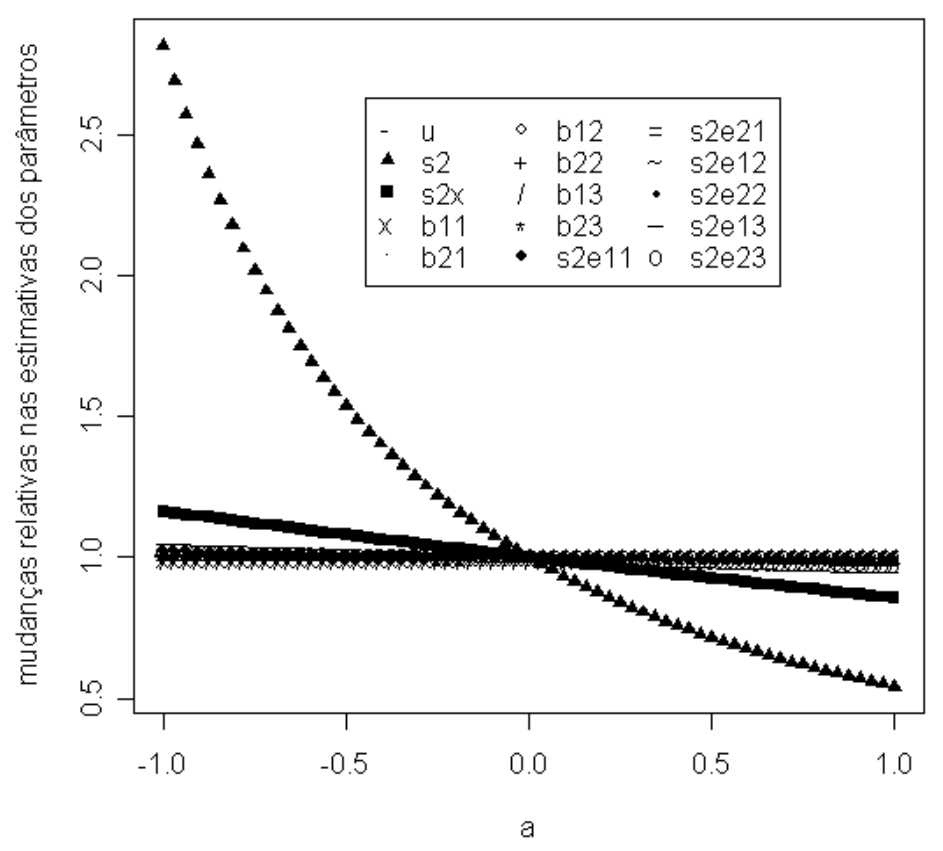

Figura 7.5: Mudanças relativas nas estimativas dos parâmetros no esquema de perturbação nas variáveis explanatórias

conjunta exercida pelas observações deste grupo no esquema de perturbação nas variáveis resposta.

Através do gráfico da Figura 7.7 vemos que $L D\left(\omega_{0}+a \mathbf{d}_{22}\right)$ não está muito próximo de $L D\left(\omega_{0}+a \mathbf{d}_{\max }\right)$, e isto nos dá indícios de que a observação 22 sozinha não influenciaria nas mudanças ocorridas na verossimilhança.

No gráfico da Figura 7.8, vemos que os parâmetros que apresentam maiores mudanças, quando perturbamos as variáveis resposta na direção máxima, são os parâmetros de variância referentes ao grupo controle, $\sigma^{2}{ }_{e_{11}}$ e $\sigma_{e_{21}}^{2}$, respectivamente as variâncias dos erros após três e seis meses de uso dos líquidos, para o grupo que testou o líquido controle (o grupo cujos pontos se destacaram), seguidos do parâmetro $\sigma^{2}$. Na Figura 7.9 estão apresentadas as mudanças apenas nos parâmetros de variância dos erros após o uso dos líquidos. O valor obtido de $C_{\mathbf{d} \text { max }}$ neste caso foi 4.61 .

\section{Pertubação na variância dos erros de medição no início do estudo}

Considerando o esquema de perturbação da variância dos erros de medição no início do estudo, notamos que as observações 42, 52 e 80 se destacaram das demais (Figura 7.10). Estas observações são respectivamente as observações $6^{\mathrm{a}}$ e $16^{\mathrm{a}}$ do grupo que testou o líquido A e a 


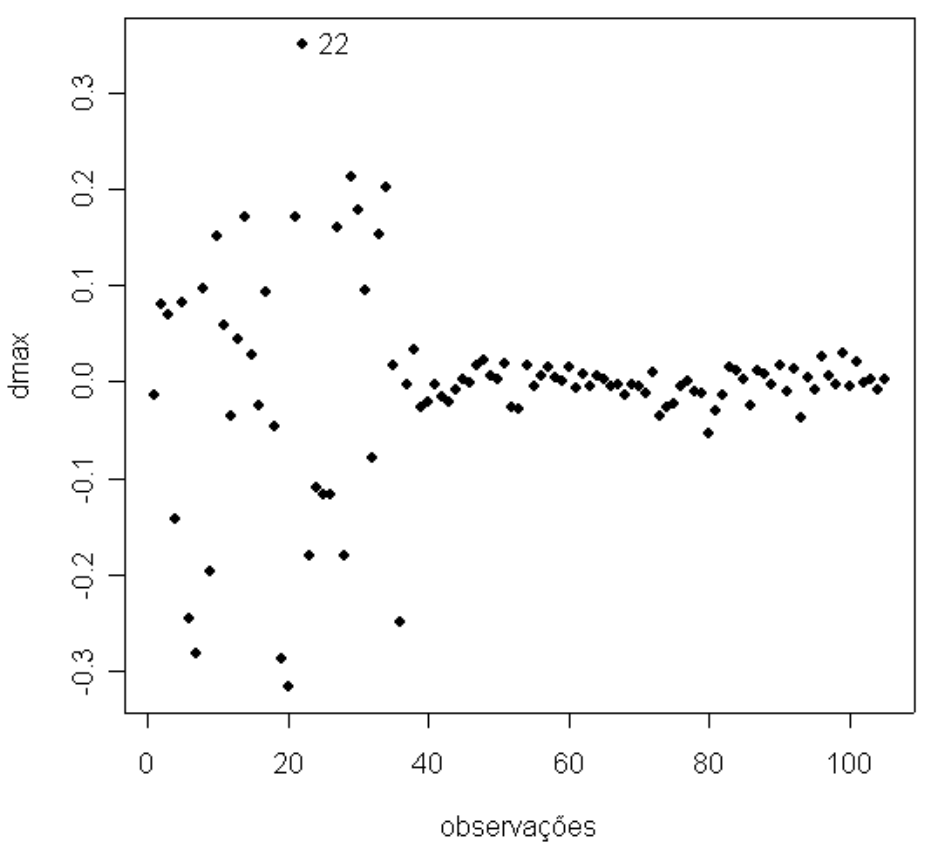

Figura 7.6: Gráfico de $d_{\max }$ para o esquema de perturbação nas variáveis resposta

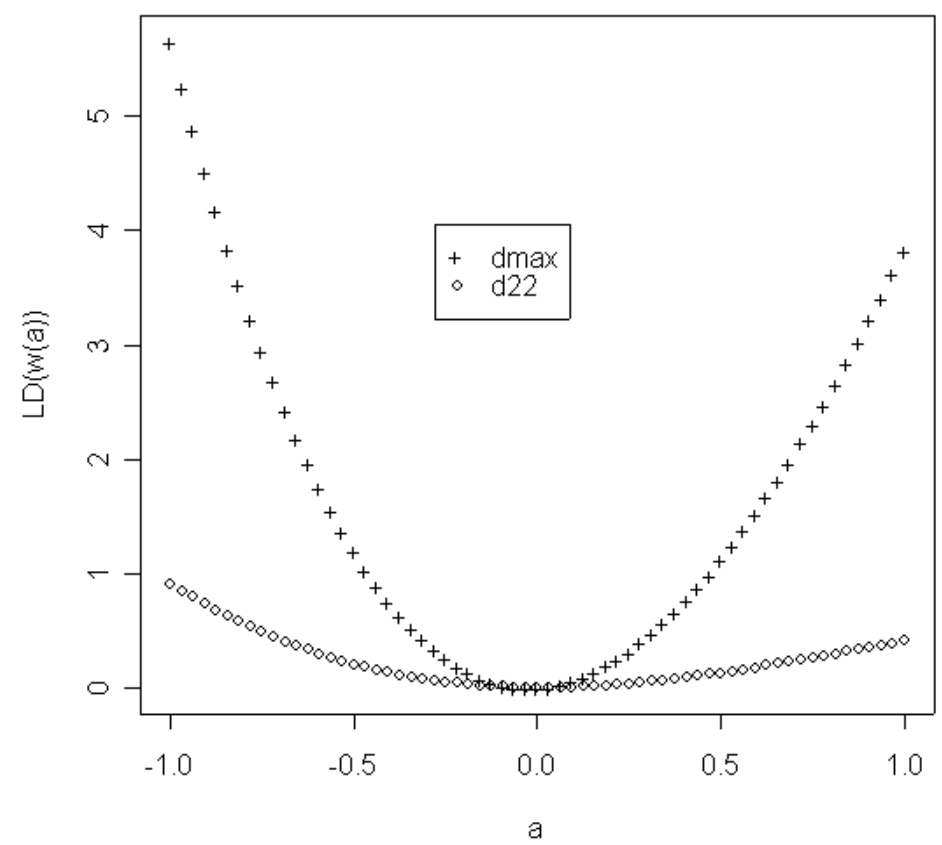

Figura 7.7: Gráfico de $L D(\omega)$ para o esquema de perturbação nas variáveis resposta 


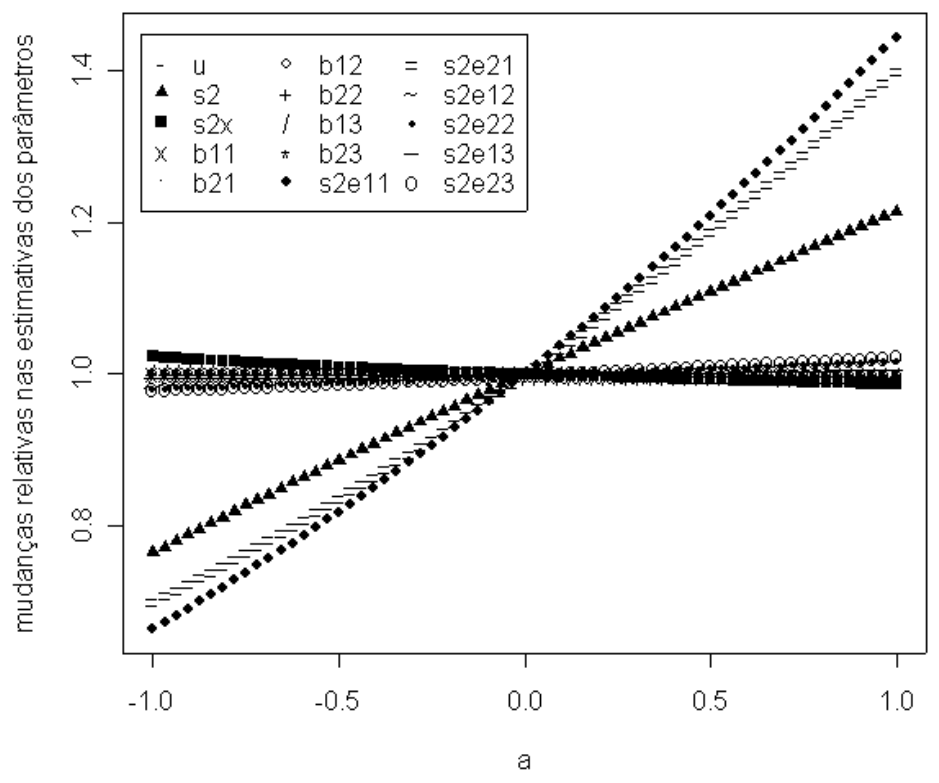

Figura 7.8: Mudanças relativas nas estimativas dos parâmetros no esquema de perturbação nas variáveis resposta

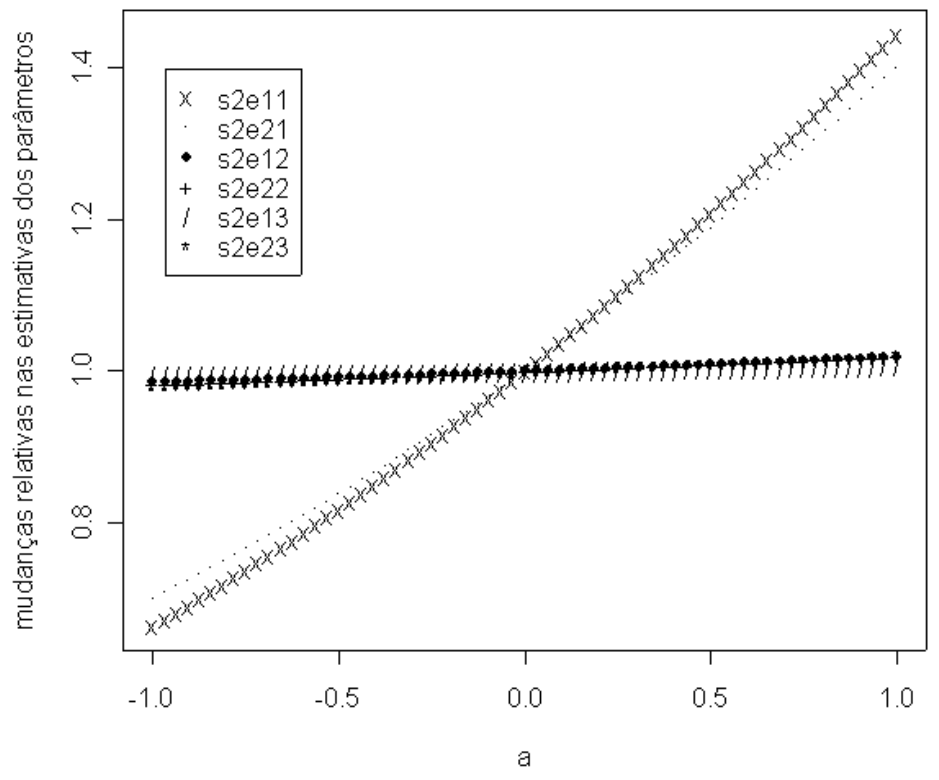

Figura 7.9: Mudanças relativas nas estimativas dos parâmetros de variância dos erros após o uso dos líquidos no esquema de perturbação nas variáveis resposta

$11^{\mathrm{a}}$ observação do grupo que testou o líquido B. A observação 42 já se destacou anteriormente no esquema de perturbação de ponderação de casos (Seção 7.1.6). A observação 52 é dada 
por $\mathbf{z}_{2,16}=(3.4,0.85,2.53)^{\prime}$, que tem uma medição grande inicialmente (a segunda maior entre todas), tem uma medição relativamente pequena após três meses do uso do líquido A e tem uma medição relativamente grande após seis meses do uso do líquido A. Neste caso, pode ter ocorrido erro na obtenção do índice de placa bacteriana após 6 meses do início do estudo. A $80^{\mathrm{a}}$ medição, que representa a $11^{\mathrm{a}}$ observação do grupo que testou o líquido $\mathrm{B}$, é dada por $\mathbf{z}_{2,11}=(3.11,0.93,0.59)^{\prime}$, e caracteriza-se por ter a primeira medição relativamente grande em relação às medições posteriores ao uso do líquido de bochecho. O valor obtido de $C_{\mathbf{d}_{\max }}$ neste caso foi 1.74, o que indicaria que estes pontos não devem influenciar muito as estimativas dos parâmetros.

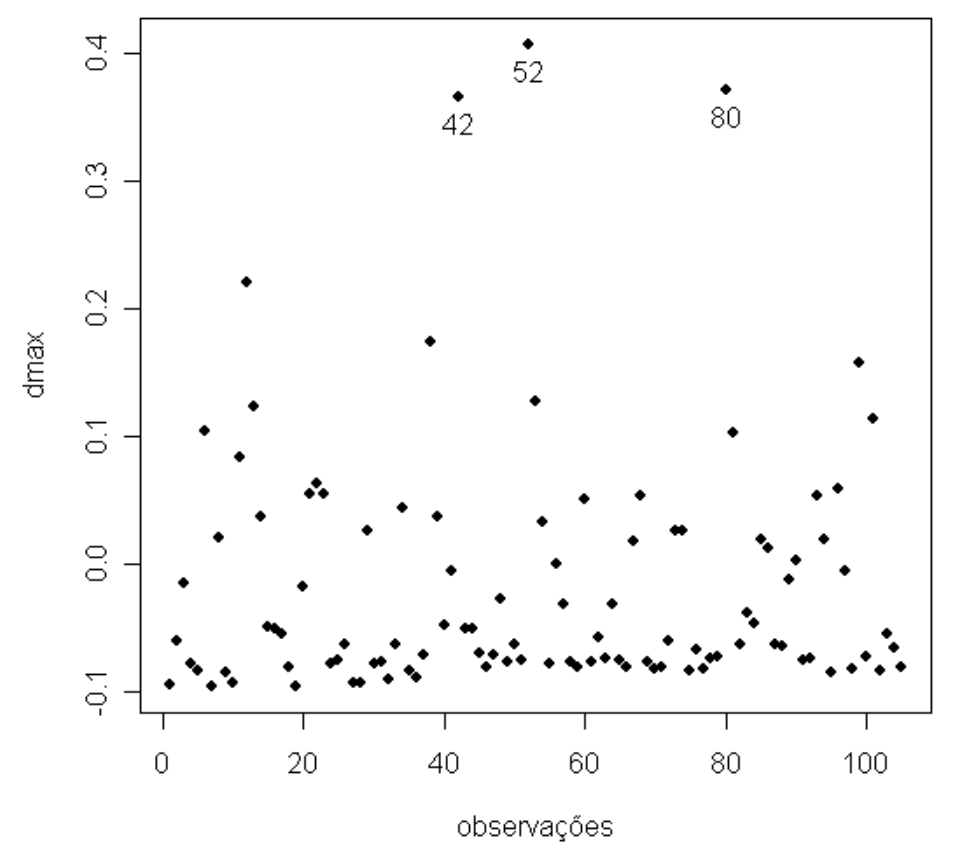

Figura 7.10: Gráfico de $d_{\text {max }}$ para o esquema de perturbação na variância dos erros de medição no início do estudo

Através do gráfico da Figura 7.11 vemos que $L D\left(\omega_{0}+a \mathbf{d}_{42}\right)$ está bem próximo de $L D\left(\omega_{0}+\right.$ $a \mathbf{d}_{\max }$ ), seguida pela observação 52 , e a observação 80 possivelmente não influencia nas mudanças ocorridas.

\section{Pertubação na variância dos erros após o uso dos líquidos}

Considerando o esquema de perturbação na variância dos erros após a utilização dos líquidos de bochecho, as observações 7 e 19 (respectivamente a $7^{\mathrm{a}}$ e $19^{\mathrm{a}}$ observações do grupo que testou o líquido controle) mostraram comportamento incomum entre as demais (7.13). As observações 


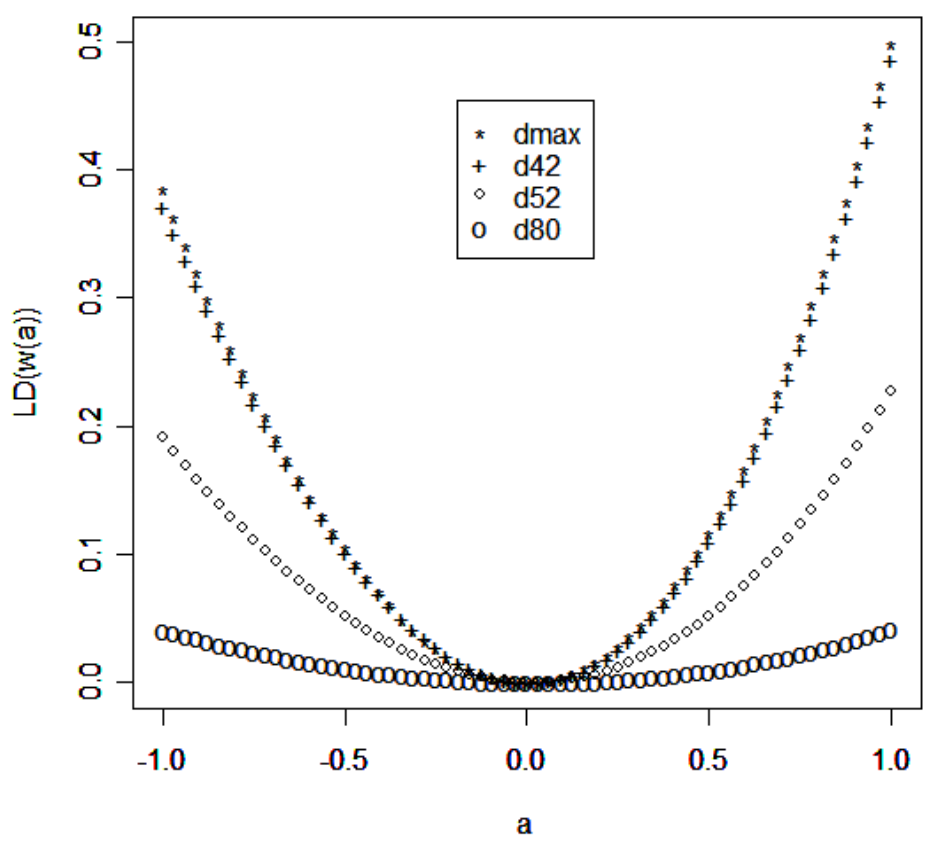

Figura 7.11: Gráfico de $L D(\omega)$ para o esquema de perturbação na variância dos erros de medição no início do estudo

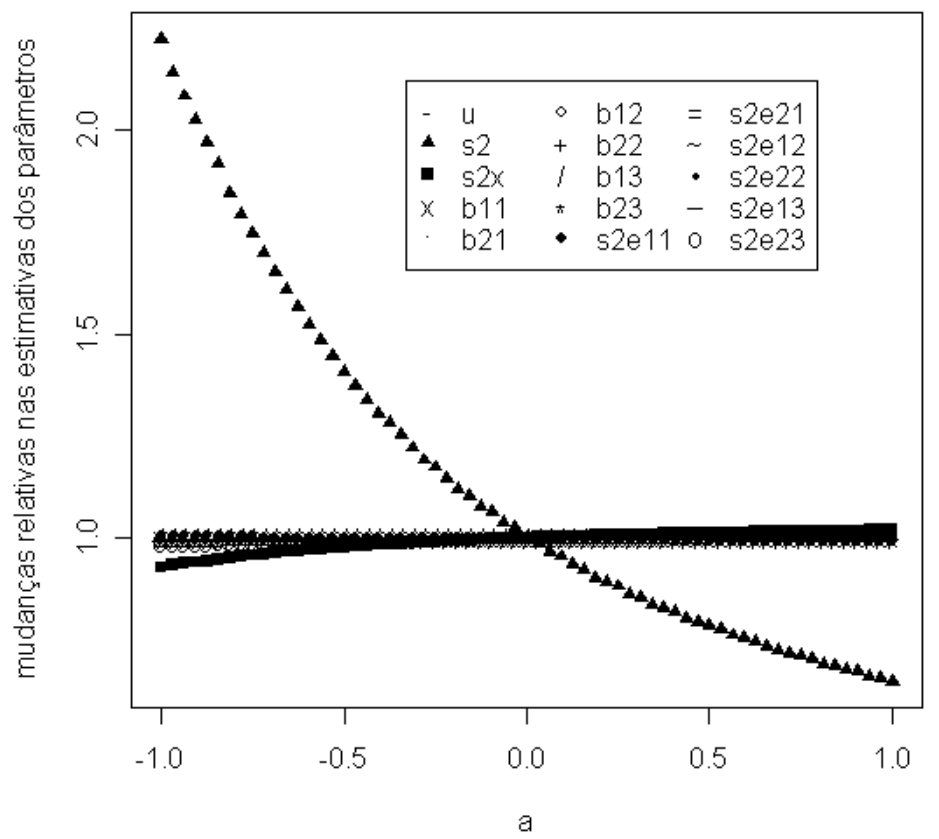

Figura 7.12: Mudanças relativas nas estimativas dos parâmetros no esquema de perturbação na variância dos erros de medição no início do estudo 
são dadas por $\mathbf{z}_{1,7}=(2.08,0.88,0.35)^{\prime}$ e $\mathbf{z}_{1,19}=(2.07,0.7,0.48)^{\prime}$, ou seja, são ambas observações que possuem medições pequenas após o uso do líquido controle em relação à medição inicial (as medidas após seis meses de uso são as duas menores do grupo que testou o líquido controle).

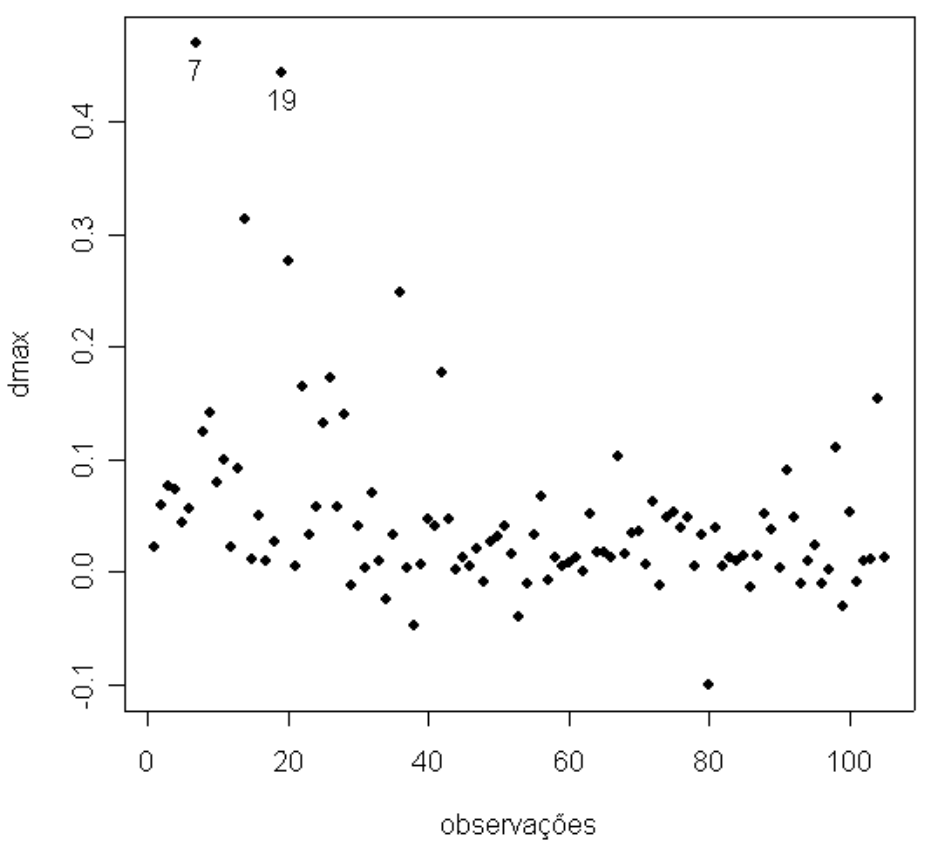

Figura 7.13: Gráfico de $d_{\max }$ para o esquema de perturbação na variância dos erros após o uso dos líquidos

Pelo gráfico da Figura 7.14 vemos que ambas as observações parecem exercer grande influência nas mudanças ocorridas na verossimilhança. O valor de $C_{\mathbf{d} \max }$ neste caso é 3.99.

O gráfico da Figura 7.15 mostra que o parâmetro que obteve a maior variação quando perturbamos o modelo na direção máxima é a variância dos erros após seis meses de uso do líquido, para o grupo que testou o líquido controle. Na Figura 7.15 são apresentadas as mudanças relativas somente nos parâmetros de variância, que são os parâmetros que mais sofreram modificações depois do parâmetro de variância $\sigma^{2}$.

\subsection{Exclusão de casos}

Como uma técnica alternativa à Influência Local de Cook (1986), consideramos também a exclusão de casos, baseando-se nas observações que possivelmente exerciam influência considerandose o enfoque de Cook (1986). A Tabela 7.1 mostra as estimativas de máxima verossimilhança 


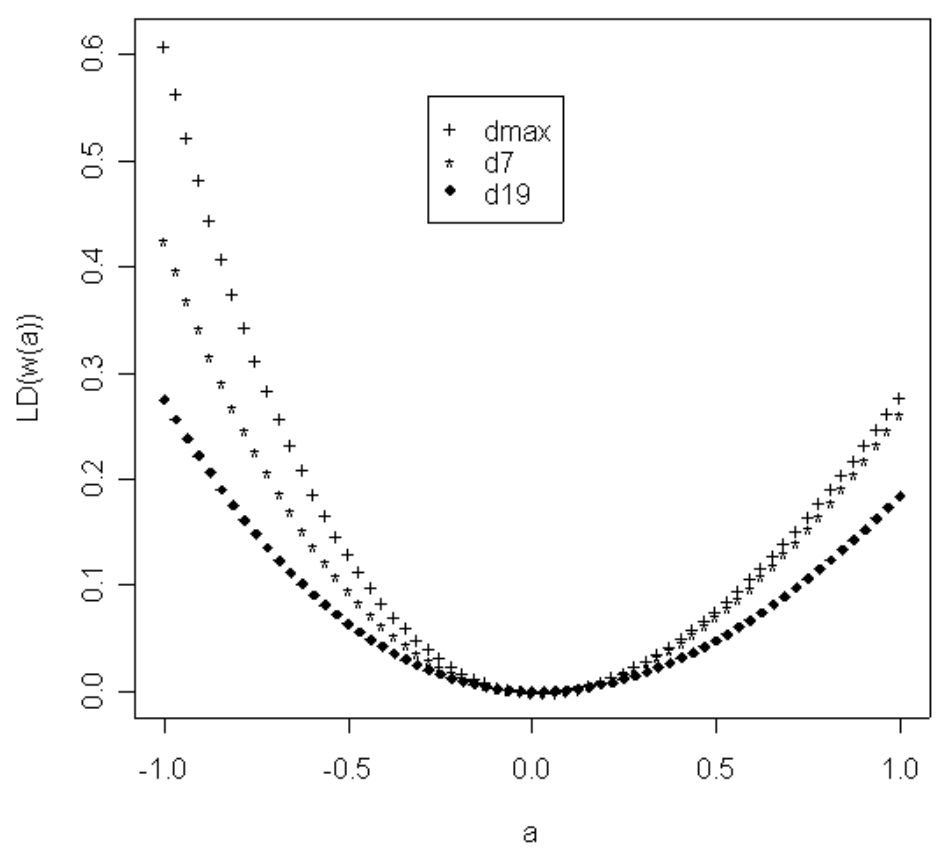

Figura 7.14: Gráfico de $L D(\omega)$ para o esquema de perturbação na variância dos erros após o uso dos líquidos

dos parâmetros resultantes desta técnica. Ao excluir a observação 42, que foi detectada pela ponderação de casos, os parâmetros cujas estimativas mais sofrem influência são o coeficiente angular $\beta_{12}$, que representa a porcentagem de placa bacteriana restante após três meses de uso do líquido experimental A (grupo ao qual pertence a observação 42), a variância do verdadeiro valor das medições dentárias, $\sigma_{x}^{2}$ e a variância dos erros após três meses do uso do líquido A, $\sigma_{e_{12}}^{2}$. Os demais parâmetros sofreram pouca ou nenhuma modificação. Ao excluir a observação 22, que foi detectada no esquema de perturbação nas variáveis resposta, o parâmetro cuja estimativa mais se modificou foi a variância dos erros após o uso do líquido controle (grupo ao qual pertence a observação 22), $\sigma_{e_{21}}^{2}$. A exclusão da observação 52, que foi detectada no esquema de perturbação na variância dos erros de medição no início do estudo, afetou a estimativa da variância dos erros de medição $\sigma^{2}$ e a estimativa do parâmetro $\beta_{12}$, que representa a porcentagem de placa bacteriana restante após três meses de uso do líquido A (grupo ao qual pertence a observação 42). A exclusão da observação 80 teve pouca influência nas estimativas dos parâmetros. Excluindo ao mesmo tempo as observações 42, 52 e 80, que foram detectadas conjuntamente ao perturbar a variância dos erros de medição no início do estudo, obteve-se modificações na estimativa de vários parâmetros, sendo as mudanças relativas mais acentuadas a variância dos erros de medição, $\sigma^{2}$, e a variância do verdadeiro valor das medições, $\sigma_{x}^{2}$, e na 


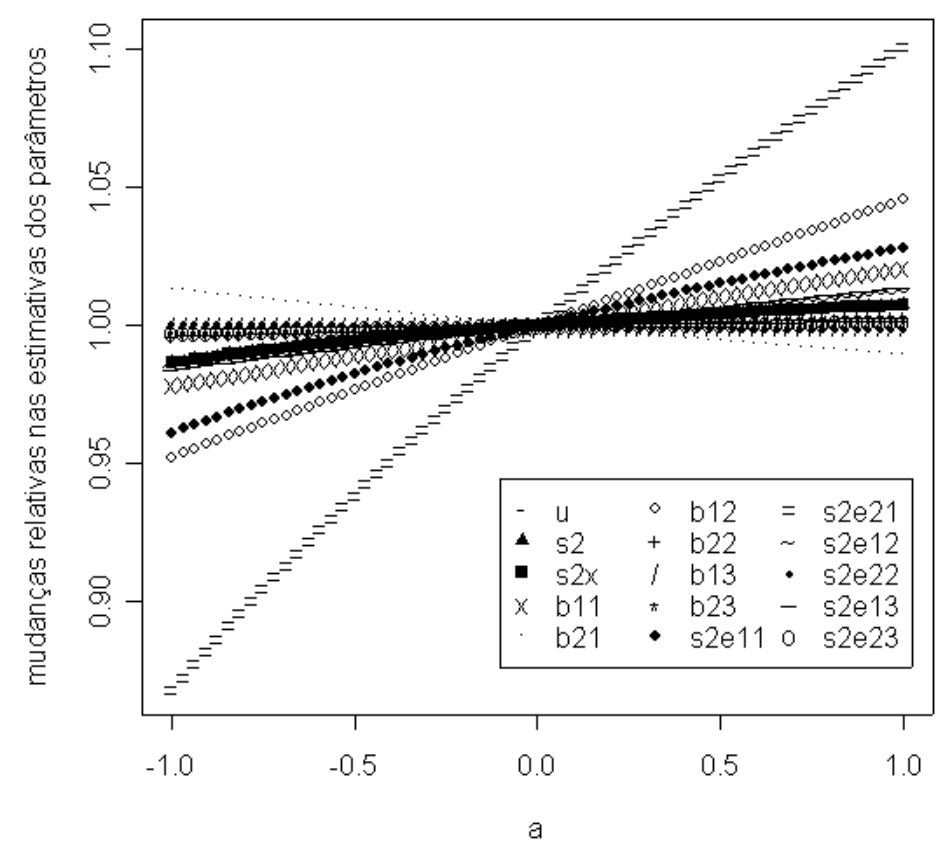

Figura 7.15: Mudanças relativas nas estimativas dos parâmetros no esquema de perturbação na variância dos erros após o uso dos líquidos

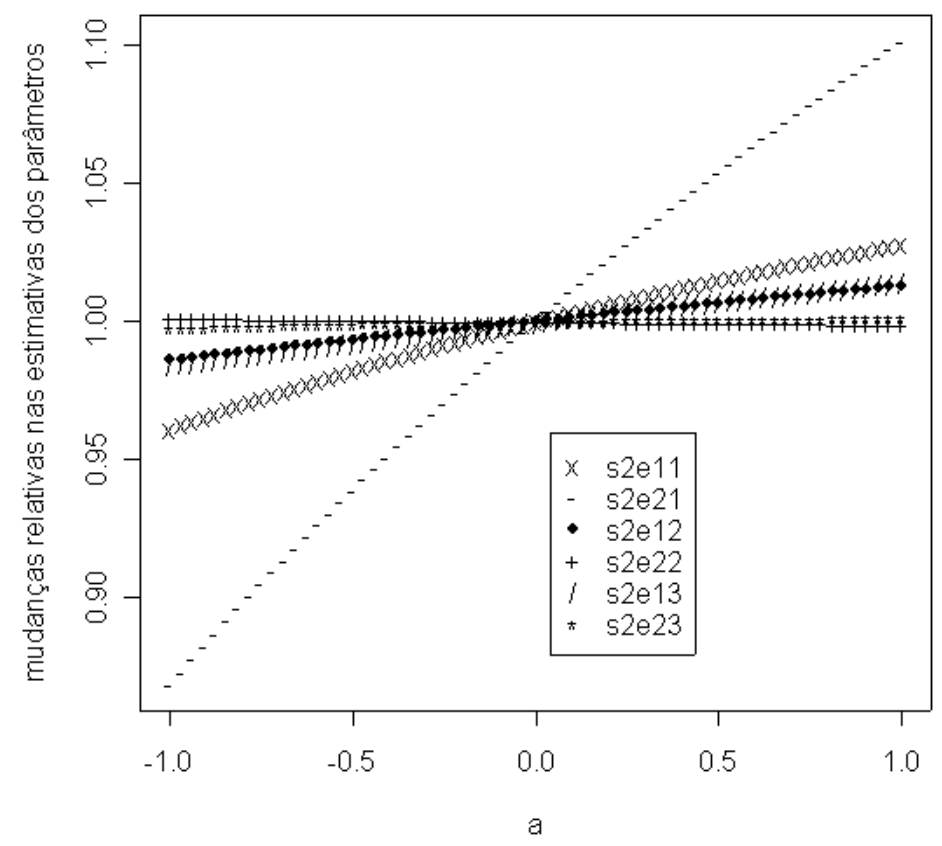

Figura 7.16: Mudanças relativas nas estimativas dos parâmetros de variância dos erros após o uso dos líquidos no esquema de perturbação na variância dos erros após o uso dos líquidos 
Tabela 7.1: Estimativas de máxima verossimilhança considerando a exclusão de casos para as observações detectadas utilizando técnicas de influência local

\begin{tabular}{rrrrrrrrrr}
\hline Parâmetros & $\beta_{11}$ & $\beta_{21}$ & $\beta_{12}$ & $\beta_{22}$ & $\beta_{13}$ & $\beta_{23}$ & $\mu$ & $\sigma^{2}$ \\
\hline conjunto original & 0.703 & 0.687 & 0.525 & 0.502 & 0.508 & 0.414 & 2.534 & 0.010 \\
sem obs 42 (grupo 2) & 0.703 & 0.687 & 0.509 & 0.491 & 0.508 & 0.414 & 2.524 & 0.010 \\
sem obs 22 (grupo 1) & 0.695 & 0.675 & 0.525 & 0.502 & 0.508 & 0.414 & 2.534 & 0.009 \\
sem obs 52 (grupo 2) & 0.703 & 0.687 & 0.540 & 0.489 & 0.508 & 0.414 & 2.526 & 0.009 \\
sem obs 80 (grupo 3) & 0.703 & 0.687 & 0.525 & 0.502 & 0.517 & 0.424 & 2.528 & 0.008 \\
sem obs 42, 52 e 80 (grupos 2 e 3) & 0.703 & 0.687 & 0.523 & 0.476 & 0.518 & 0.424 & 2.510 & 0.007 \\
sem obs 42 e 52 (grupo 2) & 0.703 & 0.688 & 0.523 & 0.476 & 0.509 & 0.414 & 2.515 & 0.009 \\
sem obs 7 (grupo 1) & 0.708 & 0.697 & 0.525 & 0.502 & 0.508 & 0.414 & 2.538 & 0.011 \\
sem obs 19 (grupo 1) & 0.710 & 0.696 & 0.525 & 0.502 & 0.508 & 0.414 & 2.538 & 0.011 \\
sem obs 7 e 19 (grupo 1) & 0.716 & 0.707 & 0.525 & 0.502 & 0.508 & 0.414 & 2.543 & 0.013 \\
\hline Parâmetros & $\sigma_{x}^{2}$ & $\sigma_{e_{11}}^{2}$ & $\sigma_{e_{21}}^{2}$ & $\sigma_{e_{12}}^{2}$ & $\sigma_{e_{22}}^{2}$ & $\sigma_{e_{13}}^{2}$ & $\sigma_{e_{23}}^{2}$ \\
\hline conjunto original & 0.103 & 0.312 & 0.234 & 0.430 & 0.432 & 0.255 & 0.192 \\
sem obs 42 (grupo 2) & 0.094 & 0.313 & 0.234 & 0.413 & 0.431 & 0.255 & 0.192 \\
sem obs 22 (grupo 1) & 0.105 & 0.306 & 0.205 & 0.430 & 0.431 & 0.255 & 0.193 \\
sem obs 52 (grupo 2) & 0.098 & 0.312 & 0.234 & 0.417 & 0.420 & 0.255 & 0.193 \\
sem obs 80 (grupo 3) & 0.102 & 0.312 & 0.234 & 0.430 & 0.431 & 0.251 & 0.185 \\
sem obs 42, 52 e 80 (grupos 2 e 3) & 0.086 & 0.313 & 0.235 & 0.402 & 0.418 & 0.251 & 0.185 \\
sem obs 42 e 52 (grupo 2) & 0.088 & 0.314 & 0.235 & 0.402 & 0.418 & 0.255 & 0.193 \\
sem obs 7 (grupo 1) & 0.101 & 0.310 & 0.205 & 0.430 & 0.432 & 0.255 & 0.192 \\
sem obs 19 (grupo 1) & 0.101 & 0.304 & 0.213 & 0.430 & 0.432 & 0.255 & 0.192 \\
sem obs 7 e 19 (grupo 1) & 0.099 & 0.301 & 0.181 & 0.430 & 0.432 & 0.255 & 0.192 \\
\hline
\end{tabular}

variância dos erros após o uso do líquido A (grupo ao qual pertencem as observações 42 e 52) $\sigma_{e_{12}}^{2}$. Estas mudanças em $\sigma_{x}^{2}$ e $\sigma_{e_{12}}^{2}$ também foram observadas ao excluir somente as observações 42 e 52. A maior modificação nas estimativas ao excluir a observação 7 foi na variância dos erros após seis meses de uso do líquido controle (grupo ao qual pertence a observação 7), $\sigma_{e_{21}}^{2}$. A exclusão da observação 19 não afetou muito nas estimativas, no entanto, ao excluir 7 e 19, afetaram-se as estimativas do coeficiente angular $\beta_{11}$ e $\beta_{21}$, que representam respectivamente a porcentagem de placa restante após três e seis meses de uso do líquido controle (grupo ao qual pertencem as observações 7 e 19). A exclusão das observações 7 e 19 também afetou as estimativa de $\mu, \sigma^{2}$ e das variâncias dos erros após três e seis meses de uso do líquido controle, $\sigma_{e_{11}}^{2}$ e $\sigma_{e_{21}}^{2}$, respectivamente.

\subsection{Novos enfoques para influência local}

Dada a grande importância da técnica de influência local e sua aplicabilidade em diversos modelos estatísticos, alguns autores têm se dedicado à generalização desta técnica. Podemos destacar alguns trabalhos: 
1. Zhu \& Zhang (2004) apresenta um estudo baseado na influência local de Cook (1986) que possibilitaria o teste da discrepância entre o modelo proposto e o verdadeiro modelo que teria gerado os dados, e propõe uma forma de identificação dos pontos onde esta discrepância é mais evidente.

2. Cadigan \& Farrell (2002) argumenta que a influência local está focada no estimador do vetor de parâmetros e muitas vezes o interesse seria em alguma função do vetor de parâmetros. Além disto, as técnicas de influência local não revelariam claramente se os resultados importantes do modelo são sensíveis às suposições do modelo. Neste trabalho é apresentado também uma revisão de medidas alternativas de influência. Além disto, é proposta uma generalização da técnica de influência local de Cook (1986), permitindo calcular o efeito das perturbações usando uma generalização da medida de afastamento pela verossimilhança, dada por

$$
F D=2(F(\widehat{\theta})-F(\widehat{\theta} \boldsymbol{\omega})
$$

onde $\theta$ seria o vetor de parâmetros do modelo postulado, $\widehat{\theta}$ seria a estimativa de $\theta$ sob o modelo não-perturbado e $\widehat{\theta} \boldsymbol{\omega}$ seria a estimativa de $\theta$ sob o modelo perturbado pelo vetor de perturbação $\boldsymbol{\omega}$, e $F$ seria uma função de interesse qualquer, que seja duas vezes diferenciável.

3. Fung \& Kwan (1997) propõem o uso de influência local considerando estimativas de parâmetros ou estatísticas de teste.

Alguns autores sugerem que as medidas de influência local de Cook não seriam invariantes sob reparametrizações de perturbações, e propõem algumas mudanças nestas medidas:

4. Poon \& Poon (1999) afirmam que as medidas seriam invariantes e propõem uma nova medida de curvatura que seria invariante em relação a transformações uniformes de escala.

5. Loynes (2001) usa uma estatística que tem distribuição $F$ e é invariante com relação a reparametrizações de perturbações.

6. Suarez Rancel \& Gonzalez Sierra (2001) citam modificações para a influência local de Cook (1986).

Neste trabalho, iremos aplicar a metodologias desenvolvida por Poon \& Poon (1999). 


\subsubsection{Curvatura normal conformal}

Segundo Poon \& Poon (1999), a curvatura normal utilizada originalmente por Cook (1986) para acessar a influência local apresentaria certa dificuldade de interpretação pois poderia assumir qualquer valor positivo. Além disto, esta medida de influência não seria invariante por reparametrizações de escala. Com o objetivo de contornar então estas dificuldades, Poon \& Poon (1999) utilizam conceitos de geometria diferencial para propor uma nova medida de influência, baseada na curvatura normal, que fosse de fácil aplicação, tivesse boas propriedades e fosse de fácil interpretação.

Considerando LD a medida de afastamento pela verossimilhança como definido em (7.2) e o vetor de perturbação $\boldsymbol{\omega}$ como definido em (7.1), Poon \& Poon (1999) escrevem a curvatura normal utilizando a primeira $(\mathbf{I})$ e segunda $(\Pi)$ formas fundamentais:

$$
C_{\mathbf{l}}=\left.\frac{\mathbf{l}^{\prime} H_{L D} \mathbf{l}}{\mathbf{l}^{\prime}\left(\mathbf{I}_{n}+\nabla_{L D} \nabla_{L D}^{\prime}\right) \mathbf{l}\left(1+\left|\nabla_{L D}\right|^{2}\right)^{\frac{1}{2}}}\right|_{\boldsymbol{\omega}=\boldsymbol{\omega}_{0}}
$$

onde

$$
\begin{gathered}
\mathbf{I}_{i j}=\delta_{i j}+\frac{\partial L D}{\partial \omega_{i}} \frac{\partial L D}{\partial \omega_{j}} \\
\Pi_{i j}=\frac{1}{\left(1+\left|\nabla_{L D}\right|^{2}\right)^{\frac{1}{2}}} \frac{\partial^{2} L D}{\partial \omega_{i} \omega_{j}}
\end{gathered}
$$

com $\delta_{i j}=1$ quando $i=j$ e 0 caso contrário, e $\left|\nabla_{L D}\right|$ representa a norma do vetor gradiente de $L D$. $I_{n}$ representa a matriz identidade de dimensão $n \times n \mathrm{e}$

$$
H_{L D}=\frac{\partial^{2} L D}{\partial \boldsymbol{\omega} \partial \boldsymbol{\omega}^{\prime}}
$$

Uma reta em $\Omega$ passando por $\boldsymbol{\omega}_{0}$ é definida por $\boldsymbol{\omega}_{a}=\boldsymbol{\omega}_{0}+a \mathbf{l}$, com $a \in \mathbb{R}$ e $\mathbf{l}$ são vetores coluna fixos em $\mathbb{R}^{n}$, e $\boldsymbol{\omega}_{0}$ é o vetor de não-perturbação. Se $\boldsymbol{\omega}_{0}$ é um ponto crítico, então $\nabla_{L D}\left(\boldsymbol{\omega}_{0}\right)=0$. Escolhendo uma direção unitária $\mathbf{l}$ tal que $\mathbf{l}^{\prime} \mathbf{l}=1$, então a equação $(7.5)$ se reduz a

$$
C_{\mathbf{l}}=\left.\mathbf{l}^{\prime} H_{L D} \mathbf{l}\right|_{\boldsymbol{\omega}=\boldsymbol{\omega}_{0}}
$$

Sendo $\Delta_{i j}=\frac{\partial^{2}}{\partial \boldsymbol{\theta}_{i} \partial \omega_{j}} L(\boldsymbol{\theta} / \boldsymbol{\omega})$ e $\ddot{L}=\frac{\partial^{2}}{\partial \boldsymbol{\theta}_{i} \partial \boldsymbol{\theta}_{j}} L(\boldsymbol{\theta} / \boldsymbol{\omega})$ avaliado em $\boldsymbol{\theta}=\widehat{\boldsymbol{\theta}}$ e $\boldsymbol{\omega}_{0}$, a equação (7.7) pode ser reescrita como

$$
C_{\mathbf{l}}=-\left.2\left\{\mathbf{l}^{\prime} \Delta^{\prime}(\ddot{L})^{-1} \Delta \mathbf{l}\right\}\right|_{\boldsymbol{\theta}=\widehat{\boldsymbol{\theta}}, \boldsymbol{\omega}=\boldsymbol{\omega}_{0}} .
$$

Poon \& Poon (1999) definem então a curvatura normal conformal no ponto $\boldsymbol{\omega}_{0}$ de um gráfico $\alpha$ na direção de $\mathbf{l}$ como sendo 


$$
B_{\mathbf{l}}=\frac{\Pi(\mathbf{l}, \mathbf{l})}{\left.\mathbf{I}(\mathbf{l}, \mathbf{l})\{\operatorname{tr}(\Pi)\}\right|_{\boldsymbol{\omega}=\boldsymbol{\omega}_{0}}}
$$

onde $\Pi(\mathbf{b}, \mathbf{c})=\mathbf{b}^{\prime} \Pi \mathbf{c}$ e $\mathbf{I}(\mathbf{b}, \mathbf{c})=\mathbf{b}^{\prime} \mathbf{I} \mathbf{c}$

Como a matriz $\Pi$ é simétrica, se os autovalores da segunda forma fundamental são $\lambda_{i}, i=$ $1, \ldots, n$, então $\operatorname{tr}\left(\Pi^{2}\right)=\sum_{i=1}^{n} \lambda_{i}^{2}$. Exceto quando a segunda forma fundamental é identicamente igual a 0, a curvatura normal conformal está bem-definida. Após alguns cálculos, a curvatura normal conformal pode ser escrita como

$$
B_{\mathbf{l}}=-\left.\frac{\mathbf{l}^{\prime} \Delta^{\prime}(\ddot{L})^{-1} \Delta \mathbf{l}}{\sqrt{\operatorname{tr}\left(\Delta^{\prime}(\ddot{L})^{-1} \Delta\right)^{2}}}\right|_{\boldsymbol{\theta}=\widehat{\boldsymbol{\theta}}, \boldsymbol{\omega}=\boldsymbol{\omega}_{0}}
$$

ou seja, é possível calcular facilmente $B_{\mathbf{l}}$ a partir do que foi utilizado para calcular $C_{\mathbf{l}}$.

As medidas $B_{1}$ e $C_{\mathbf{l}}$ diferem apenas por um fator positivo de normalização, e então estas duas medidas de influência são equivalentes. Assim, o autovetor correspondente ao maior autovalor para a curvatura normal irá produzir também a maior curvatura normal conformal. No entanto, $B_{1}$ possui algumas propriedades interessantes, como o fato de que $0 \leq\left|B_{\mathbf{l}}\right| \leq 1$, o que facilita sua interpretação, e além disto $B_{1}$ é invariante sob reparametrizações.

A curvatura do gráfico em um ponto é uniforme em todas as direções se a segunda forma fundamental for diagonalizável a uma constante multiplicando a matriz identidade. Já que $\sum_{i} B_{\mathbf{e}_{i}}^{2}=1$, se as curvaturas normais conformais para todos os autovetores forem idênticas, então todos eles serão iguais a $\frac{1}{\sqrt{n}}$. Poon \& Poon (1999) apresentam então uma forma de decidir se um autovetor é influente ou não, utilizando o valor $\frac{1}{\sqrt{n}}$, com a seguinte definição.

Definição 7.1 Um autovetor e é $q$ influente se $\left|B_{e_{i}}\right| \geq \frac{q}{\sqrt{n}}$.

Ou seja, escolhendo valores de $q$ decidimos se uma direção é influente de acordo com a magnitude do seu autovalor correspondente normalizado.

Poon \& Poon (1999) definem como o $j$-ésimo vetor de perturbação básica do espaço de perturbações, $E_{j}$, um vetor coluna em $\mathbb{R}^{n}$ cuja $j$-ésima entrada é 1 e todas as outras são 0. Assim, podemos escrever cada um dos autovetores de perturbação $\mathbf{e}_{i}$ como sendo $\mathbf{e}_{i}=\sum_{j=1}^{n} a_{i j} E_{j}$, $\operatorname{com} \sum_{j=1}^{n} a_{i j}^{2}=1$. Assim, para cada $i$ fixado, se a contribuição de todos os $a_{i j}$ for uniforme, $a_{i j}=\frac{1}{\sqrt{n}}$. 
Na prática, normalizamos os autovalores de $\Pi$ fazendo $\widehat{\lambda}_{i}=\frac{\lambda_{i}}{\sqrt{\sum_{k=1}^{n} \lambda_{k}^{2}}}$ em seguida podemos ordenar decrescentemente os módulos dos autovalores normalizados, $\mu_{i}=\left|\widehat{\lambda}_{i}\right|$, de forma que

$$
\mu_{\max }=\mu_{1} \geq \ldots \geq \mu_{k} \geq \frac{q}{\sqrt{n}}>\mu_{k+1} \ldots \mu_{n} \geq 0
$$

e denotando $a_{i j}$ o $j$-ésimo elemento do autovetor correspondente a $\mu_{i}$, pode-se obter a contribuição agregada no $j$-ésimo vetor da base a todos os autovetores $q$-influentes fazendo

$$
m(q)_{j}=\sqrt{\sum_{i=1}^{k} \mu_{i} a_{i j}^{2}}
$$

Para a interpretação desta contribuição, pode-se construir um gráfico dos valores obtidos $m(q)_{j}$ pelos índices $j=1, \ldots, n$ e verificar se existem pontos que se distanciam dos demais.

Como $\sum_{j=1}^{n} m(q)_{j}^{2}=\sum_{j=1}^{n}\left(\sum_{i=1}^{k} \mu_{i} a_{i j}^{2}\right)=\sum_{i=1}^{k}\left[\mu_{i} \sum_{j=1}^{n} a_{i j}^{2}\right]=\sum_{i=1}^{k} \mu_{i}$, se a contribuição de todos os vetores de perturbação da base for uniforme então

$$
\bar{m}(q)=\sqrt{\frac{1}{n} \sum_{i=1}^{k} \mu_{i}}
$$

Portanto, podemos nos referir a $\bar{m}(q)$ quando quisermos determinar a significância da contribuição dos vetores individuais da base.

Ao variar os valores de $q$, podemos considerar um valor de $q$ que seja suficientemente grande para agregar somente o maior autovetor $\mathbf{e}_{\max }$. Neste caso, $m(q)_{j}=\sqrt{\mu_{\max }}\left|a_{1 j}\right|$ e este método é equivalente a comparar $a_{1 j} \operatorname{com} \frac{1}{\sqrt{n}}$. Outro extremo é permitir que $q=0$ e então todos os autovalores serão incluídos. A contribuição total é dada então por

$$
m_{j}=m(0)_{j}=\sqrt{\sum_{i=1}^{n} \mu_{i} a_{i j}^{2}}
$$

Se a contribuição de todos os parâmetros da base for uniforme, então cada um é igual a

$$
\bar{m}=\bar{m}(0)=\sqrt{\frac{\sum_{i=1}^{n} \mu_{i}}{n}}=\sqrt{\frac{\sum_{i=1}^{n}\left|\lambda_{i}\right|}{n \sum_{i=1}^{n} \lambda_{i}^{2}}} .
$$


A contribuição total $m_{j}$ e a curvatura normal conformal $B_{E_{j}}$ do vetor de perturbação básico $E_{j}$ estão fortemente relacionados, já que $E_{l}=\sum_{i} a_{i l} \mathbf{e}_{i}$ e $B_{E_{j}}$ pode ser escrito como

$$
B_{E_{j}}=\sum_{i, k} a_{i j} a_{k j} \mathbf{e}_{i}^{\prime} \widehat{\lambda}_{k} \mathbf{e}_{k}=\sum_{i=1}^{n} \widehat{\lambda}_{i} a_{i j}^{2}
$$

Ou seja, se todos os autovalores são não-negativos, $B_{E_{j}}$ é igual ao quadrado da contribuição total do $j$-ésimo vetor da base.

Uma quantidade que será utilizada para decidir se uma direção exerce grande influência ou não será $b$, tal que

$$
b=\frac{\operatorname{tr}(\Pi)}{n} \sqrt{\operatorname{tr} \Pi^{2}}
$$

pois se a contribuição de todos os $B_{E_{j}}$ forem iguais, então cada uma seria igual a $b$.

Portanto, para decidir se um determinado ponto é influente ou não considerando a contribuição agregada dos $k$ maiores autovalores maiores em módulo do que $\frac{q}{\sqrt{n}}$, será utilizada a contribuição média $\bar{m}(q)_{j}$, com $m(q)_{j}$ dado na equação 7.9 , e para decidir se um caso é ou não influente, utiliza-se o valor $b$ dado na equação 7.10. No entanto, Poon \& Poon (1999) propõem $\bar{m}(q)_{j} \sqrt{2}$ como um ponto de corte para a contribuição agregada dos $k$ autovalores e $2 b$ como um ponto de corte da contribuição agregada de todos os autovalores.

\section{Aplicação numérica}

Iremos aplicar a técnica proposta em Poon \& Poon (1999) aos dados apresentados em Hadgu \& Koch (1999), considerando os esquemas de perturbação apresentados na Seção 7.1.

\section{Ponderação de casos}

Primeiramente consideramos o esquema de ponderação de casos apresentado na Seção 7.1.1.

A Figura 7.17 (a) mostra os autovalores em módulo normalizados e os valores de $q$. Para $q=5$ temos apenas um autovalor acima do valor de $\frac{q}{\sqrt{n}}$. No entanto para $q=4$ temos dois autovalores acima do valor de $\frac{q}{\sqrt{n}}$, para $q=3$ temos quatro autovalores acima do valor de $\frac{q}{\sqrt{n}} \mathrm{e}$ assim por diante. A Figura 7.17 (b) mostra a contribuição agregada entre todos os autovetores $(q=0)$. Em Poon \& Poon (1999) foi sugerido o ponto de corte $2 b$, com $b$ dado na equação (7.10). Neste caso $2 b=0.063$. Porém, há muitas observações acima deste valor, e selecionamos apenas as mais distantes para a análise. A Tabela 7.2 mostra que as observações que exercem influência são as observações 42, 52, 14, 7, 19 e 80. Considerando o enfoque de Cook (1986), somente a observação 42 havia sido detectada neste esquema de perturbação. Considerando a contribuição agregada dos autovalores correspondentes aos dois maiores autovalores $(q=4)$, 


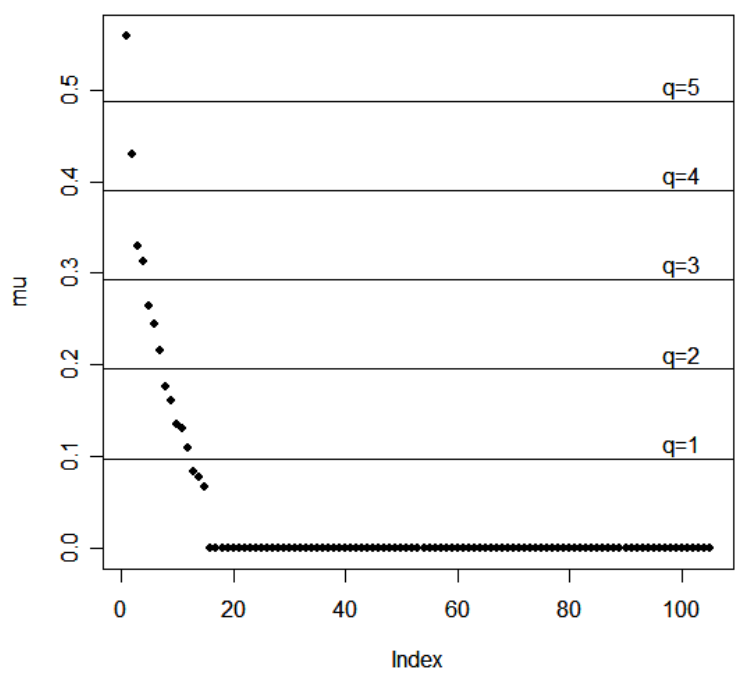

(a)

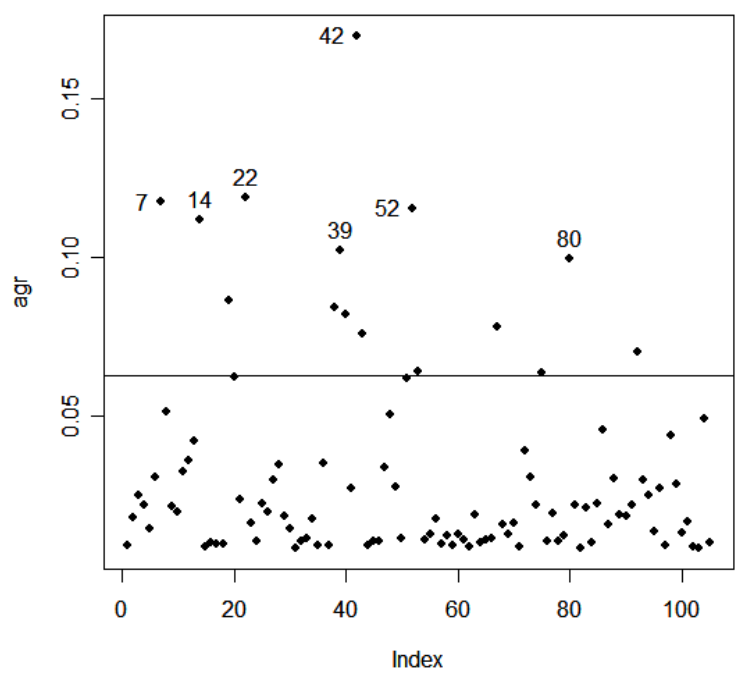

(b)

Figura 7.17: (a) Autovalores normalizados em módulo, $\mu_{i}$, com valores de $q$ e (b) influência devida à contribuição agregada de todos os autovetores para o esquema de ponderação de casos.

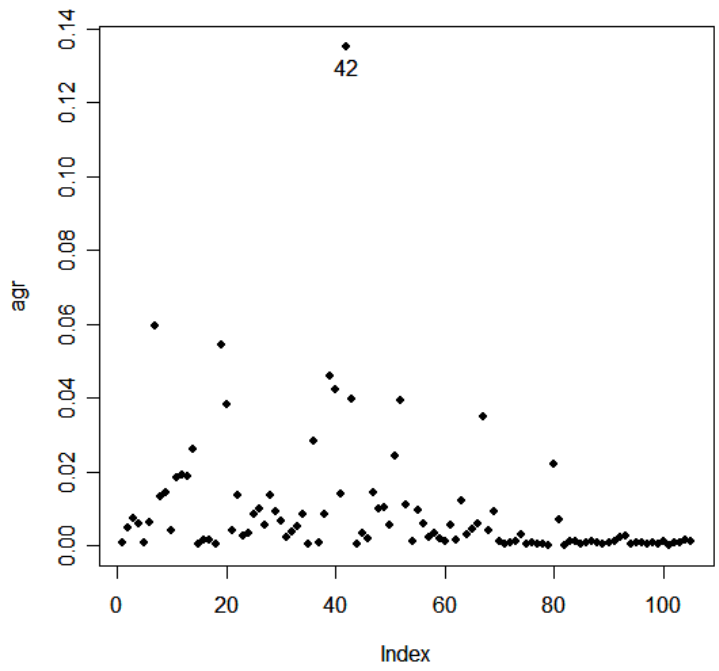

Figura 7.18: Contribuição agregada dos autovetores correspondentes aos dois maiores autovalores $(q=4)$, provenientes da perturbação de ponderação de casos.

vemos claramente que a observação 42 se destaca entre as demais (Figura 7.18). A Figura 7.19 mostra os quatro autovetores correspondentes aos maiores autovalores, com destaque para as observações com maiores entradas no vetor. As observações 42 e 52 têm respectivamente as maiores medições inicial de placa bacteriana. A observação 14 também possui um valor grande para a primeira medição. As observações 7 e 19 possuem as menores medições finais do grupo que testou o líquido 1 (controle) e a observação 80 possui uma grande medição inicial e uma 


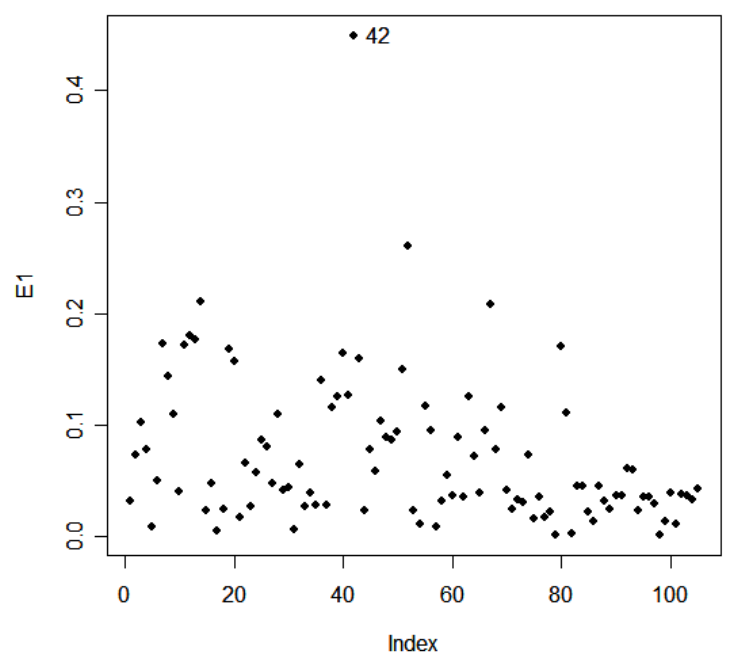

(a)

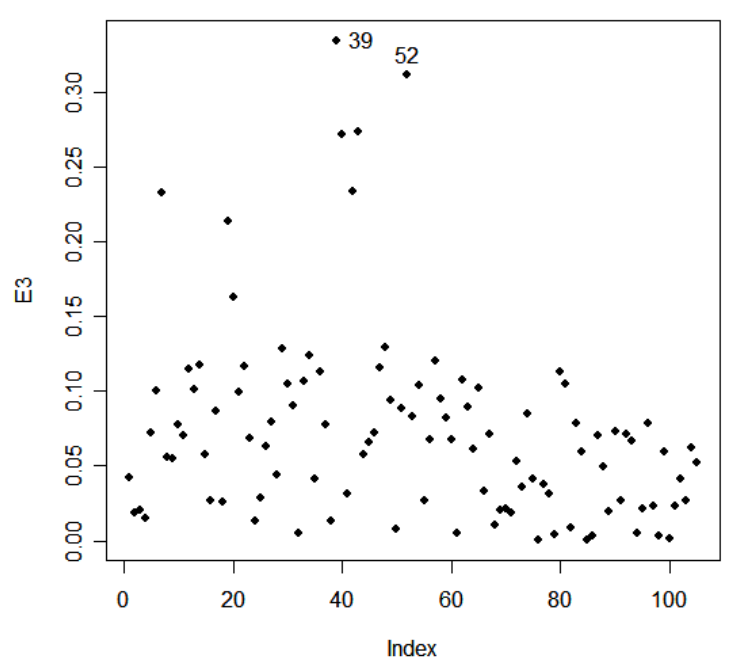

(c)

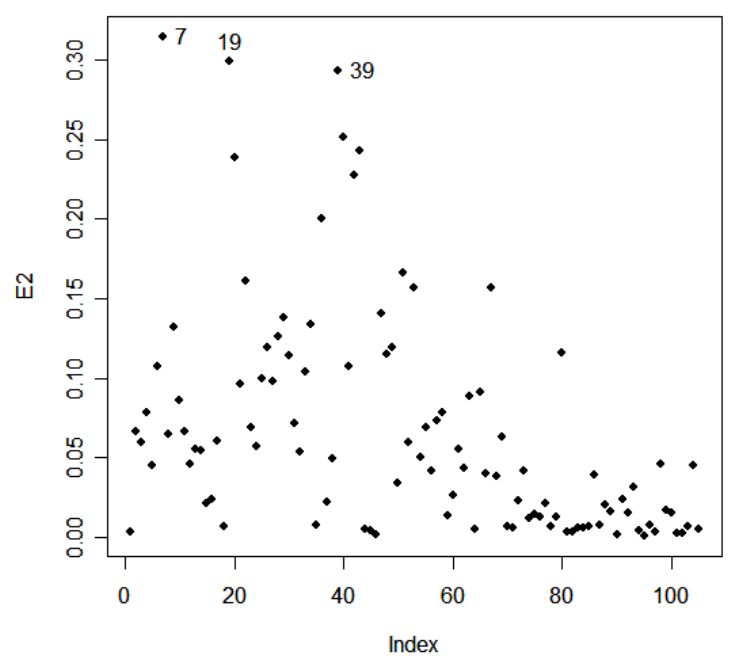

(b)

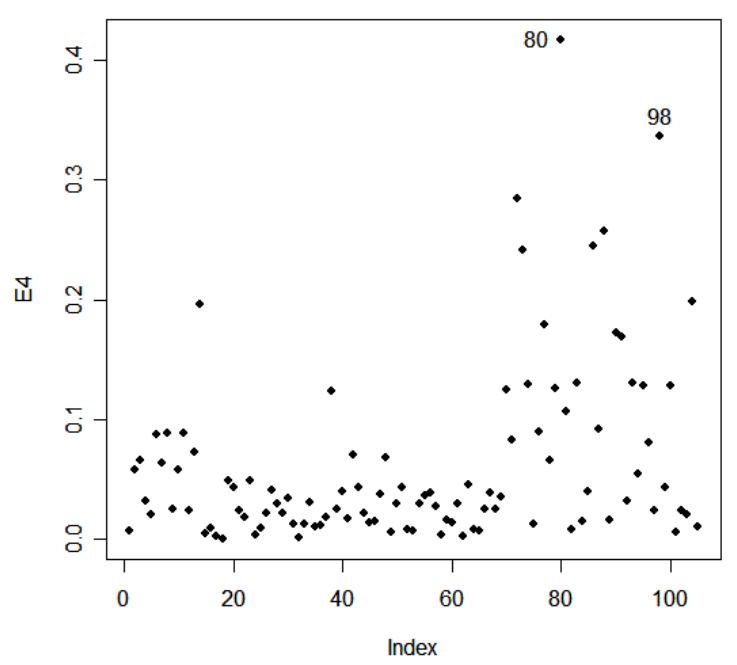

(d)

Figura 7.19: Autovetores e autovalores normalizados, $E_{1}$, com $\mu_{1}=0.559$ (a), $E_{2}$, com $\mu_{2}=$ 0.429 (b), $E_{3}$, com $\mu_{3}=0.329$ (c), $E_{4}$, com $\mu_{4}=0.313$ (d) provenientes da perturbação de ponderação de casos.

pequena medição final. A curvatura $B_{\max }$ obtida neste caso foi 0.56 .

\section{Perturbação nas variáveis explanatórias}

Considerando o esquema de perturbação nas variáveis explanatórias apresentado na Seção 7.1.2 e o enfoque de Cook (1986), havíamos concluído que nenhuma observação era influente, devido ao comportamento aleatório no gráfico de índices do autovetor correspondente ao maior autovalor. No entanto, ao considerarmos o enfoque de Poon \& Poon (1999), é possível destacar o 
Tabela 7.2: Medidas de influência utilizando a curvatura normal conformal para o esquema de ponderação de casos.

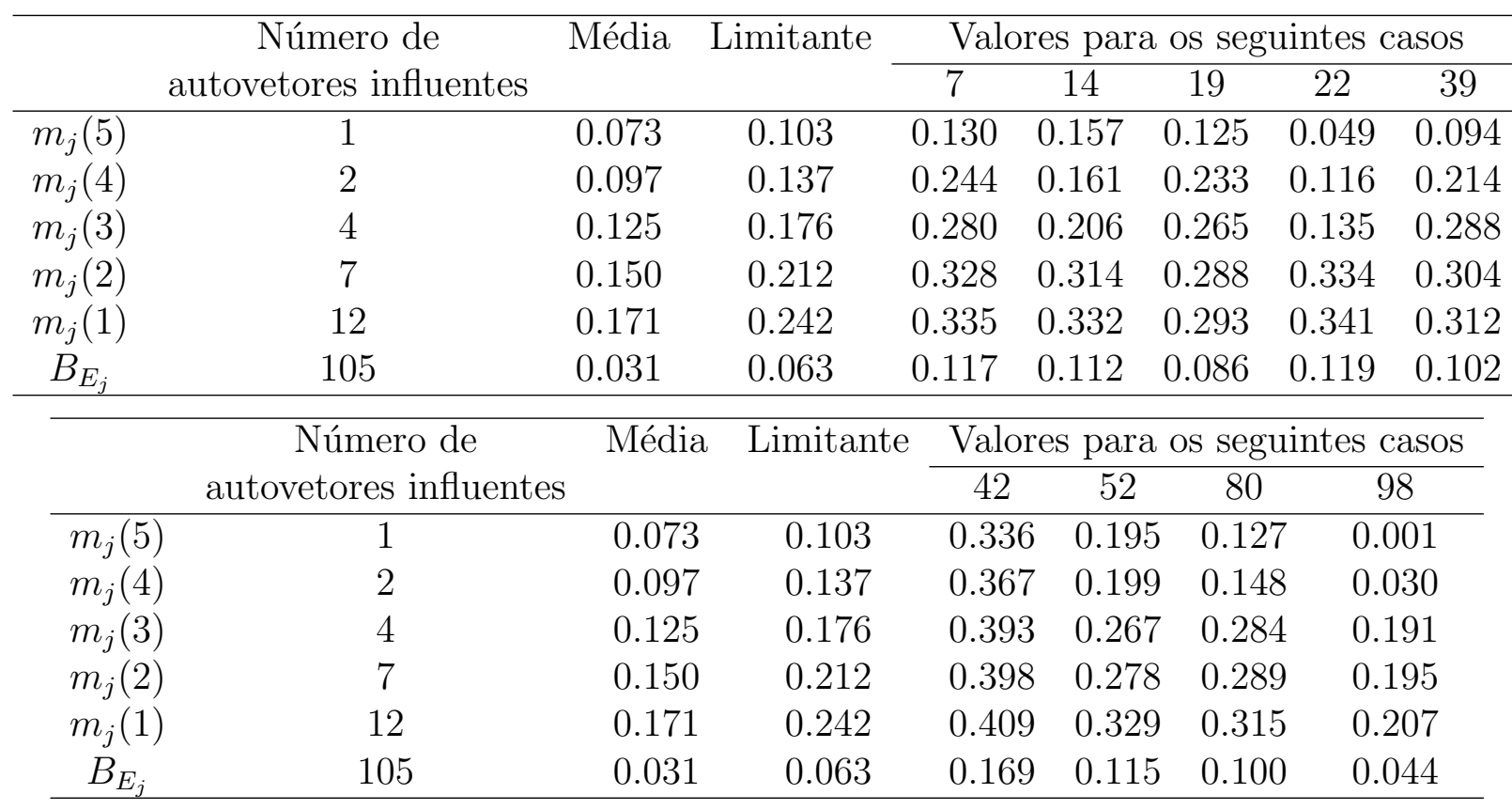

comportamento das observações 22, 42, 52 e 80. A Figura 7.20 mostra, em (a), os autovalores em módulo normalizados e os valores de $q$ e, em (b), a contribuição agregada entre todos os autovetores. A Figura 7.22 mostra que as mesmas observações se destacam ao considerar apenas a contribuição agregada dos autovetores correspondentes aos três maiores autovalores.

Tabela 7.3: Medidas de influência utilizando a curvatura normal conformal para o esquema de perturbação na variável explanatória.

\begin{tabular}{cccccccc}
\hline & Número de & Média & Limitante & \multicolumn{4}{c}{ Valores para os seguintes casos } \\
\cline { 5 - 7 } & autovetores influentes & & & 22 & 42 & 52 & 80 \\
\hline$m_{j}(5)$ & 1 & 0.090 & 0.127 & 0.172 & 0.191 & 0.206 & 0.225 \\
$m_{j}(4)$ & 2 & 0.109 & 0.154 & 0.226 & 0.227 & 0.217 & 0.226 \\
$m_{j}(1)$ & 3 & 0.122 & 0.173 & 0.241 & 0.236 & 0.225 & 0.231 \\
$B_{E_{j}}$ & 105 & 0.018 & 0.036 & 0.062 & 0.060 & 0.055 & 0.061 \\
\hline
\end{tabular}

Através do gráfico da Figura 7.21 vemos que as mudanças ocorridas em $L D\left(\omega_{0}+a \mathbf{d}\right)$ nas direções unitárias relativas a cada observação separadamente não estão muito próximas de $L D\left(\omega_{0}+a \mathbf{d}_{\max }\right)$, o que nos dá indícios de que as observações $22,42,52$ e 80 individualmente não influenciariam nas mudanças ocorridas na verossimilhança. A direção que mais apresentou mudanças foi a direção da observação 42.

De acordo com a Tabela 7.3, as observações 22, 42, 52 e 80 exerceriam influência neste esquema de perturbação. As observações 42, 52 e 80 já se destacaram no esquema de ponderação 


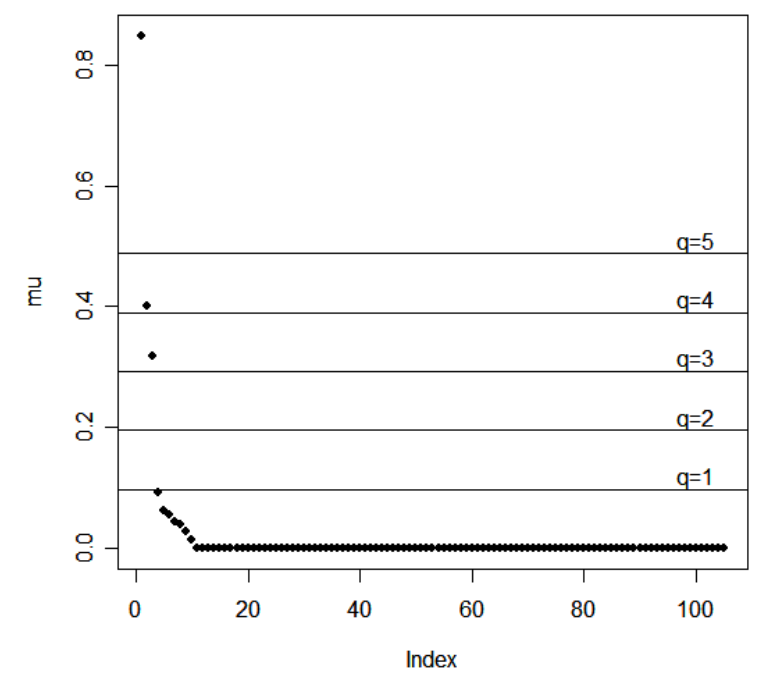

(a)

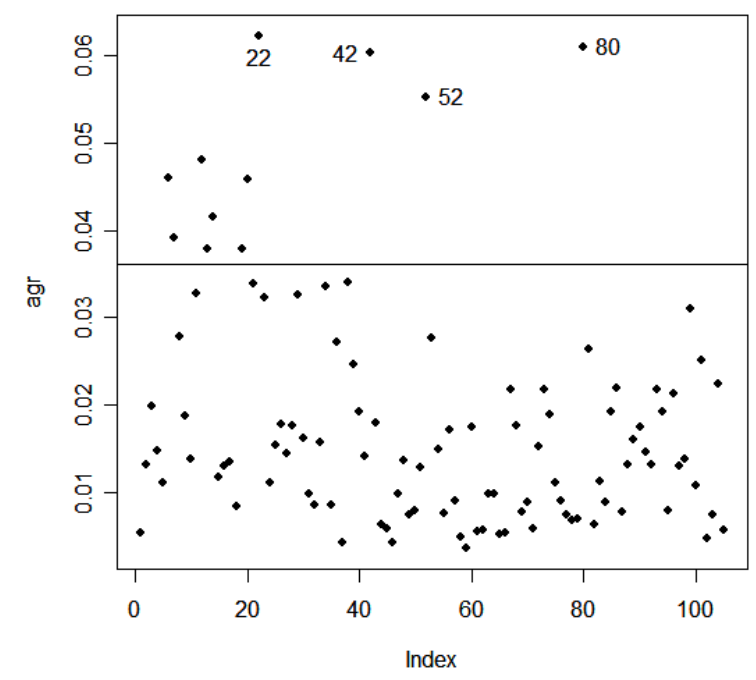

(b)

Figura 7.20: (a) Autovalores normalizados em módulo, $\mu_{i}$, com valores de $q$ e (b) influência devida à contribuição agregada de todos os autovetores para o esquema de perturbação na variável explanatória.

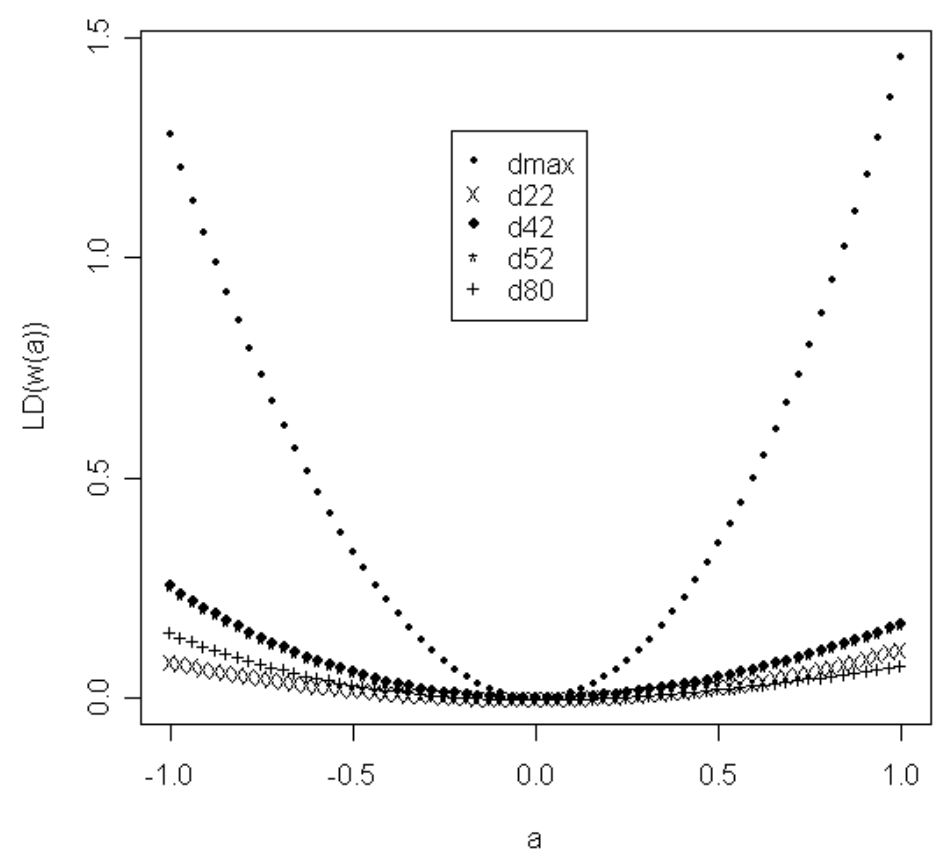

Figura 7.21: Gráfico de $L D(\omega)$ para o esquema de perturbação nas variáveis explanatórias

de casos. A observação 22 não possui nenhuma característica incomum entre as observações, a não ser por ter o comportamento inesperado de ter o índice de placa bacteriana maior depois 


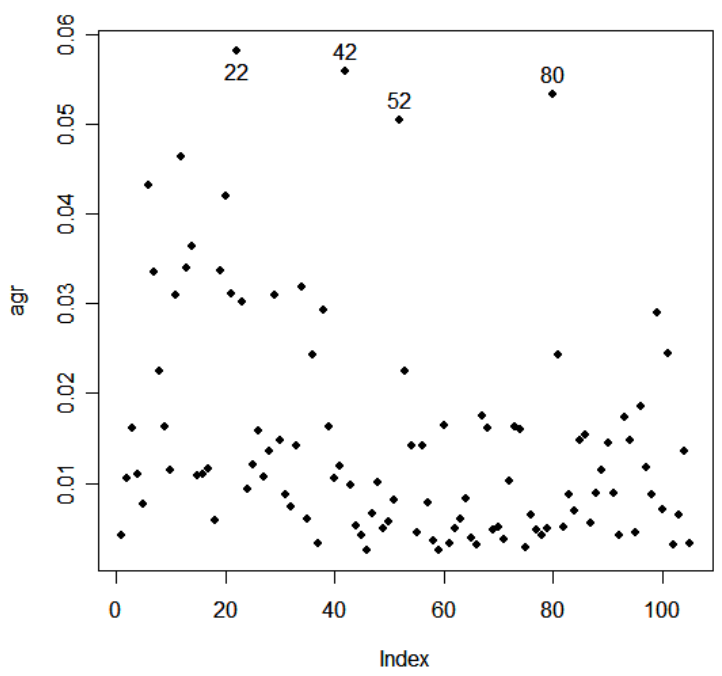

Figura 7.22: Contribuição agregada dos autovetores correspondentes aos três maiores autovalores $(q=1)$, provenientes da perturbação nas variáveis explanatórias.

de utilizar o líquido. Sua medição final é a segunda maior do grupo ao qual pertence. Porém, esta observação faz parte do grupo que testou o líquido controle e portanto este seria um comportamento possível. A Figura 7.23 mostra os gráficos de índices dos três autovetores correspondentes aos maiores autovalores. As observações não possuem grande destaque em relação às demais quando consideramos a contribuição de cada um dos autovetores. A curvatura $B \max$ correspondente à direção de maior influência foi 0.85 neste caso.

\section{Perturbação nas variáveis resposta}

Considerando o esquema de perturbação nas variáveis resposta apresentado na Seção 7.1.3 e o enfoque de Cook (1986), havíamos destacado as observações do grupo controle. Ao considerar o enfoque de Poon \& Poon (1999), é possível destacar o comportamento da observação 22. A Figura 7.24 (a) mostra os autovalores em módulo normalizados e os valores de $q$. Para $q=5$ temos apenas um autovalor acima do valor de $\frac{q}{\sqrt{n}}$. No entanto para $q=4$ temos três autovalores acima de $\frac{q}{\sqrt{n}}$, para $q=3$ temos quatro autovalores acima de $\frac{q}{\sqrt{n}}$ e assim por diante. A Figura 7.24 (b) mostra a contribuição agregada entre todos os autovetores, e a Figura 7.25 mostra a contribuição agregada entre os autovetores correspondentes aos três maiores autovalores. Em ambos os casos a observação 22 se apresentou a maior contribuição entre todas. De acordo com a Tabela 7.4, a observação 22 exerceria influência neste esquema de perturbação.

Uma característica interessante neste esquema de perturbação é que quando fazemos o gráfico separadamente os autovetores correspondentes aos maiores autovalores (Figura 7.26), 


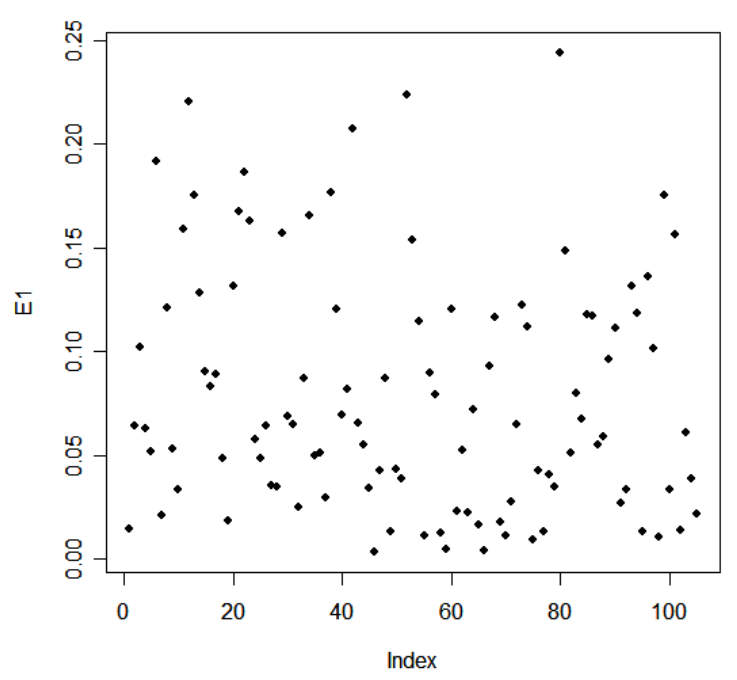

(a)

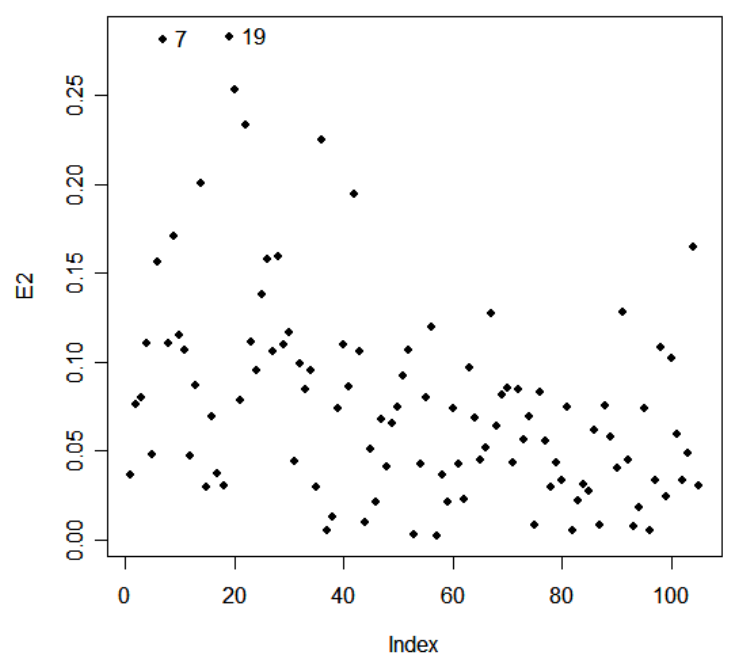

(b)

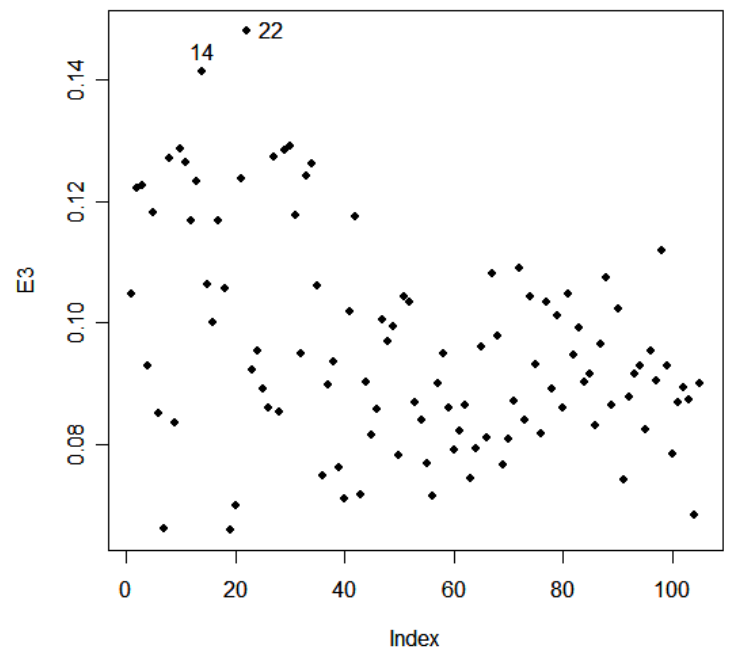

(c)

Figura 7.23: Autovetores e autovalores normalizados, $E_{1}$, com $\mu_{1}=0.845$ (a), $E_{2}$, com $\mu_{2}=$ 0.401 (b), $E_{3}$, com $\mu_{3}=0.317$ (c) provenientes da perturbação na variável explanatória.

as observações aparecem destacadas pelo grupo ao qual pertencem. Por exemplo, o autovetor referente ao maior autovalor possui as maiores entradas para as observações do primeiro grupo, o grupo controle. O segundo autovetor destaca as observações do grupo que testou o líquido B, e o terceiro autovetor destaca as observações do grupo que testou o líquido A. No entanto, ao considerar a contribuição agregada, o comportamento destas observações se apresenta aleatoriamente (Figura 7.24). A curvatura $B \max$ correspondente à direção de maior influência foi 0.51 


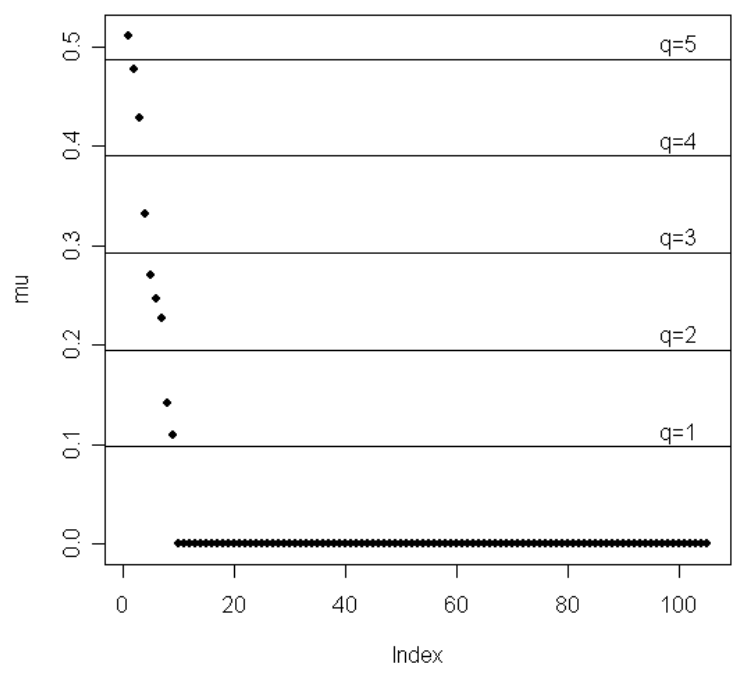

(a)

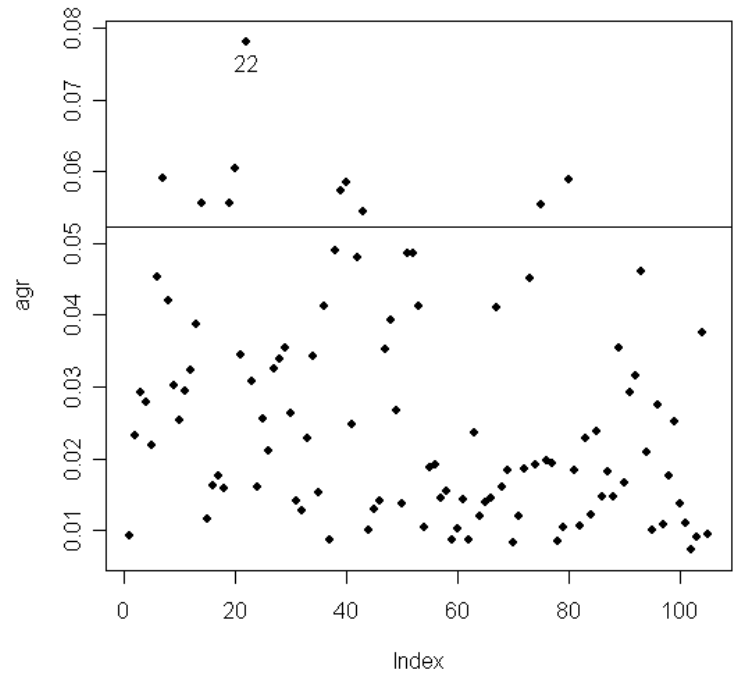

(b)

Figura 7.24: (a) Autovalores normalizados em módulo, $\mu_{i}$, com valores de $q$ e (b) influência devida à contribuição agregada de todos os autovetores para o esquema de perturbação nas variáveis resposta.

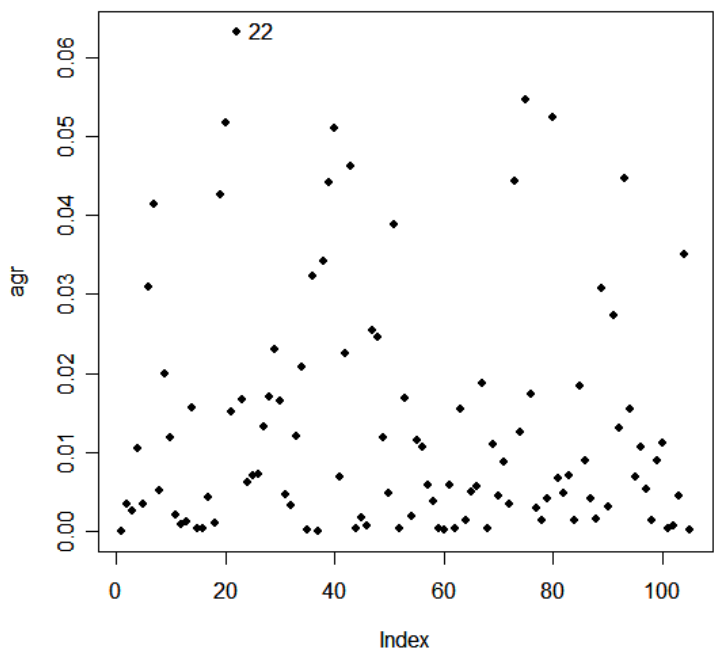

Figura 7.25: Contribuição agregada dos autovetores correspondentes aos três maiores autovalores $(q=4)$, provenientes da perturbação nas variáveis resposta.

neste caso.

\section{Perturbação na variância dos erros de medição no início do estudo}

Considerando o esquema de perturbação na variância dos erros de medição no início do estudo, apresentado na Seção 7.1.4 e o enfoque de Cook (1986), havíamos destacado as observações 42, 


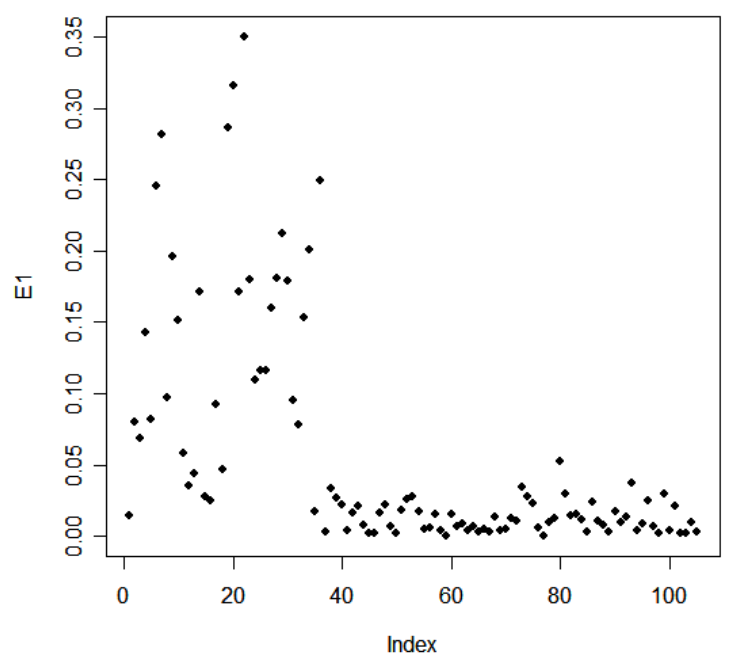

(a)

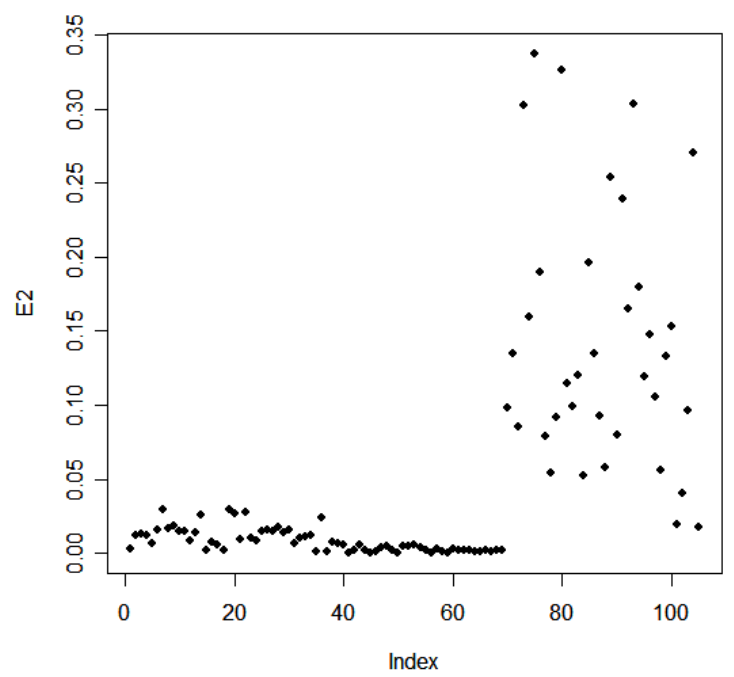

(b)

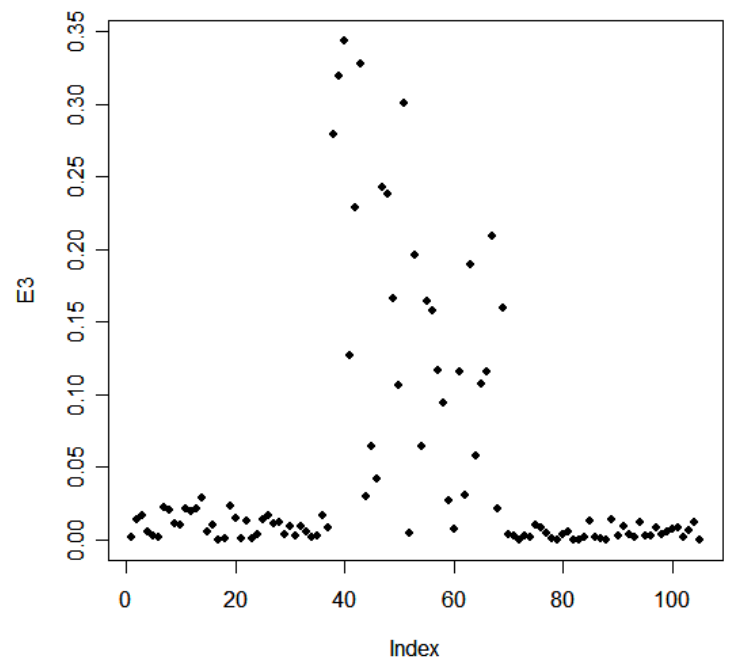

(c)

Figura 7.26: Autovetores e autovalores normalizados, $E_{1}$, com $\mu_{1}=0.511$ (a), $E_{2}$, com $\mu_{2}=$ 0.467 (b), $E_{3}$, com $\mu_{3}=0.361$ (c) provenientes da perturbação na variável resposta.

52 e 80.

Ao considerar o enfoque de Poon \& Poon (1999), as mesmas observações se destacaram, já que o maior autovalor normalizado obtido foi 0.999 e portanto o maior autovetor teria participação muito forte na contribuição agregada. A curvatura $B \max$ correspondente à direção de maior influência foi 0.99 neste caso.

A Figura 7.27 (a) mostra que para $q=1$ já se isola somente o primeiro autovalor. A 
Tabela 7.4: Medidas de influência utilizando a curvatura normal conformal para o esquema de perturbação nas variáveis resposta.

\begin{tabular}{ccccc}
\hline & $\begin{array}{c}\text { Número de } \\
\text { autovetores influentes }\end{array}$ & Média & Limitante & Valores para os seguintes casos \\
\cline { 5 - 5 } & 1 & & 22 \\
\hline$m_{j}(5)$ & 3 & 0.070 & 0.099 & 0.251 \\
$m_{j}(4)$ & 4 & 0.116 & 0.164 & 0.251 \\
$m_{j}(3)$ & 7 & 0.129 & 0.183 & 0.266 \\
$m_{j}(2)$ & 9 & 0.154 & 0.218 & 0.274 \\
$m_{j}(1)$ & 105 & 0.162 & 0.229 & 0.279 \\
$B_{E_{j}}$ & 0.026 & 0.052 & 0.078 \\
\hline
\end{tabular}

Tabela 7.5: Medidas de influência utilizando a curvatura normal conformal para o esquema de perturbação na variância dos erros de medição no início do estudo

\begin{tabular}{ccccccc}
\hline & $\begin{array}{c}\text { Número de } \\
\text { autovetores influentes }\end{array}$ & Média & Limitante & \multicolumn{3}{c}{ Valores para os seguintes casos } \\
\cline { 5 - 7 } & 1 & & & 42 & 52 & 80 \\
\hline$m_{j}(1)$ & 105 & 0.098 & 0.138 & 0.381 & 0.410 & 0.353 \\
$B_{E_{j}}$ & 0.010 & 0.019 & 0.146 & 0.169 & 0.125 \\
\hline
\end{tabular}

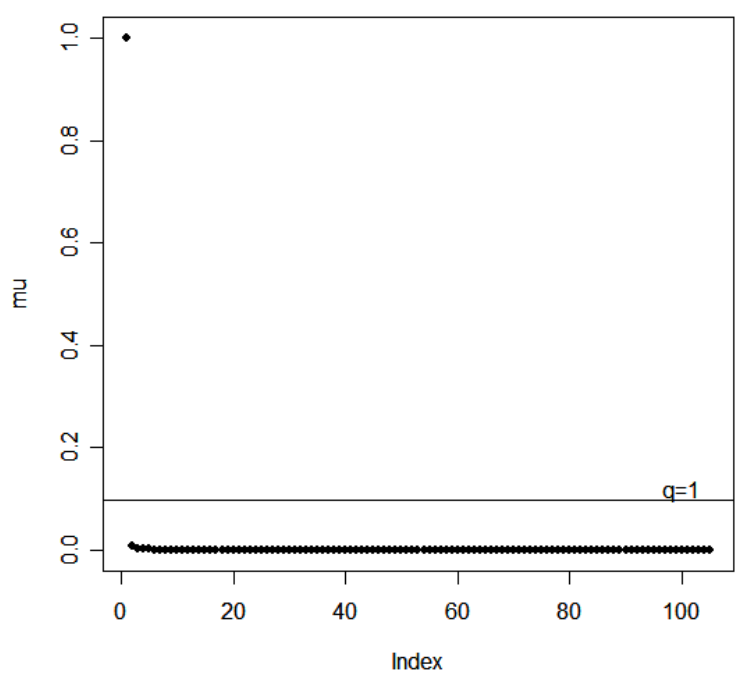

(a)

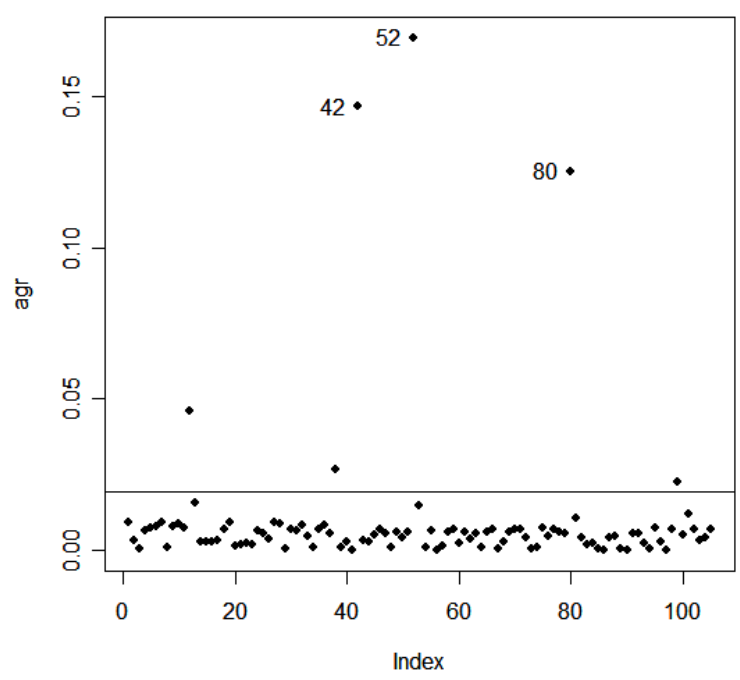

(b)

Figura 7.27: (a) Autovalores normalizados em módulo, $\mu_{i}$, com valores de $q$ e (b) influência devida à contribuição agregada de todos os autovetores para o esquema de perturbação na variância dos erros de medição no início do estudo.

Figura 7.27 (b) mostra a contribuição agregada entre todos os autovetores, e o destaque para as observações 42, 52 e 80 . Ou seja, o resultado foi praticamente o mesmo do enfoque utilizado por Cook (1986). De acordo com a Tabela 7.5, estas três observações seriam influentes para este esquema de perturbação. 


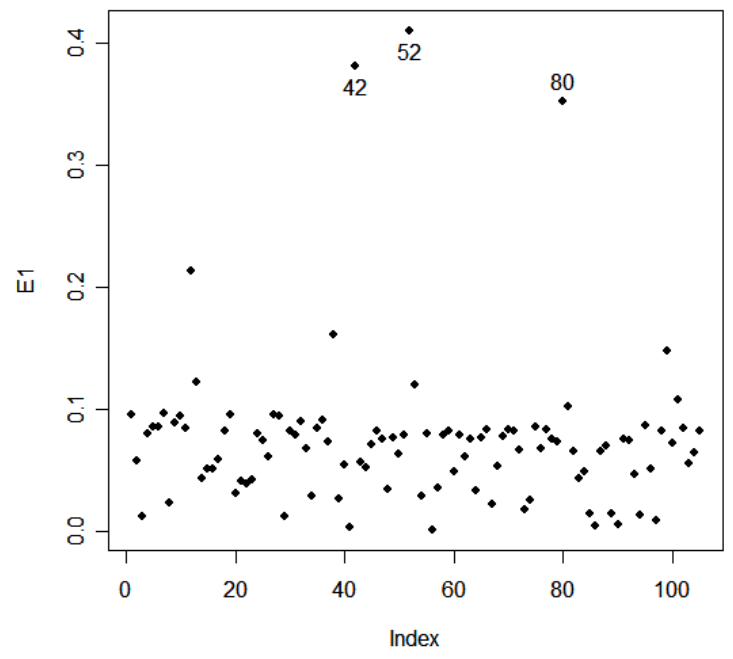

Figura 7.28: Autovetor e autovalor normalizado, $E_{1}$, com $\mu_{1}=0.999$ provenientes da perturbação na variância dos erros de medição no início do estudo.

\section{Perturbação na variância dos erros após o uso dos líquidos}

Tabela 7.6: Medidas de influência utilizando a curvatura normal conformal para o esquema de perturbação na variância dos erros após o uso dos líquidos.

\begin{tabular}{cccccccc}
\hline & Número de & Média & Limitante & \multicolumn{4}{c}{ Valores para os seguintes casos } \\
\cline { 5 - 7 } & autovetores influentes & & & 7 & 14 & 19 & 22 \\
\hline$m_{j}(4)$ & 1 & 0.082 & 0.116 & 0.396 & 0.264 & 0.374 & 0.139 \\
$m_{j}(3)$ & 3 & 0.114 & 0.161 & 0.402 & 0.265 & 0.379 & 0.145 \\
$m_{j}(2)$ & 6 & 0.142 & 0.201 & 0.404 & 0.333 & 0.384 & 0.292 \\
$m_{j}(1)$ & 10 & 0.159 & 0.225 & 0.407 & 0.355 & 0.384 & 0.338 \\
$B_{E_{j}}$ & 105 & 0.027 & 0.054 & 0.176 & 0.128 & 0.149 & 0.115 \\
\hline
\end{tabular}

Considerando o esquema de perturbação na variância dos erros após o uso dos líquidos (Seção 7.1.5) e o enfoque de Cook (1986), havíamos destacado as observações 7 e 19 . Ao considerar o enfoque de Poon \& Poon (1999), destacaram-se as observações 7, 19, 14 e 22. A Figura 7.29 (a) mostra os autovalores em módulo normalizados e os valores de $q$. Para $q=4$ temos apenas um autovalor acima do valor de $\frac{q}{\sqrt{n}}$. No entanto para $q=3$ temos três autovalores acima de $\frac{q}{\sqrt{n}}$, para $q=2$ temos seis autovalores acima de $\frac{q}{\sqrt{n}}$ e assim por diante. A Figura 7.29 (b) mostra a contribuição agregada entre todos os autovetores. Ao considerar apenas os autovetores correspondentes aos três maiores autovalores $(q=3)$, destacam-se apenas as observações 7 e 19, como mostra a Figura 7.30. De acordo com a Tabela 7.6, as observações 7, 14, 19 e 22 exerceriam influência neste esquema de perturbação. A Figura 7.31 mostra a 


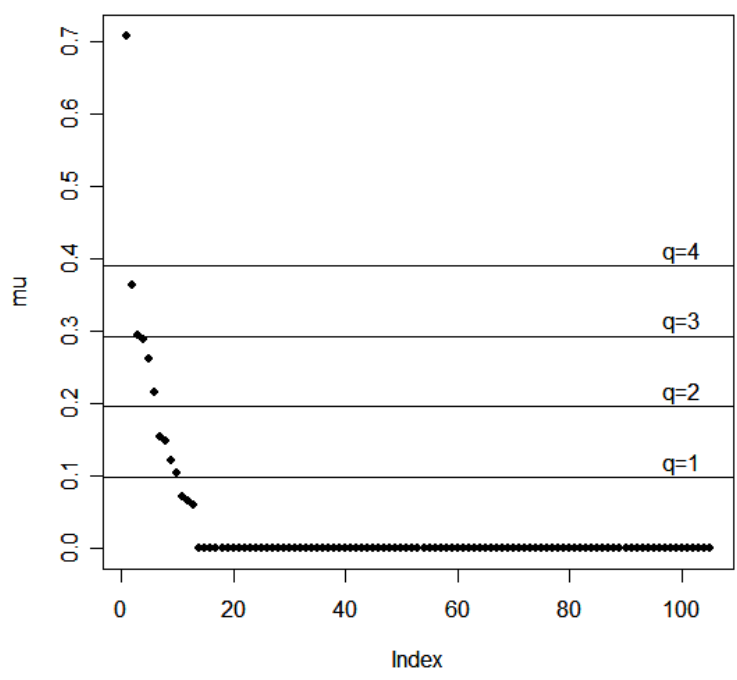

(a)

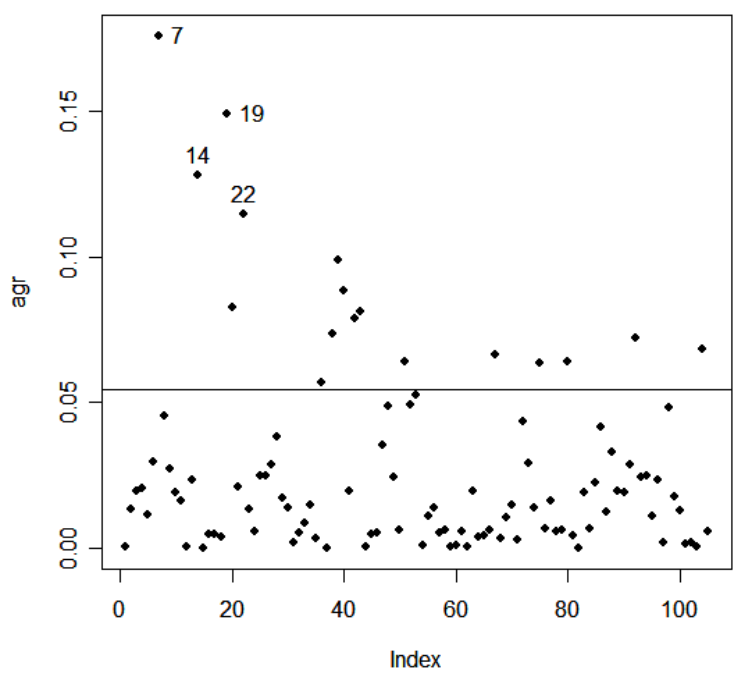

(b)

Figura 7.29: (a) Autovalores normalizados em módulo $\mu_{i}$ com valores de $q$ e (b) influência devida à contribuição agregada de todos os autovetores para o esquema de perturbação na variância dos erros após o uso dos líquidos.

influência dos três autovetores referentes aos maiores autovalores separadamente. A curvatura $B \max$ correspondente à direção de maior influência foi 0.71 neste caso.

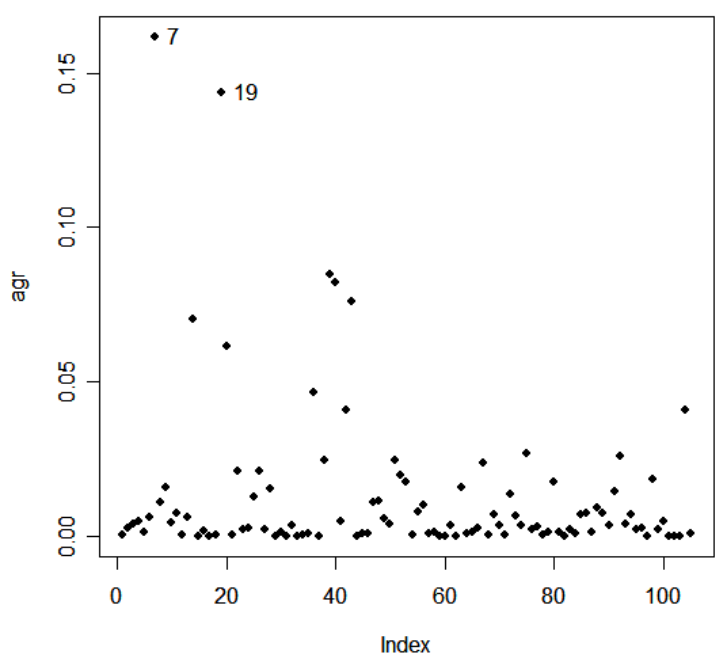

Figura 7.30: Contribuição agregada dos autovetores correspondentes aos três maiores autovalores $(q=3)$, provenientes da perturbação nas variâncias dos erros de medição após o uso dos líquidos. 


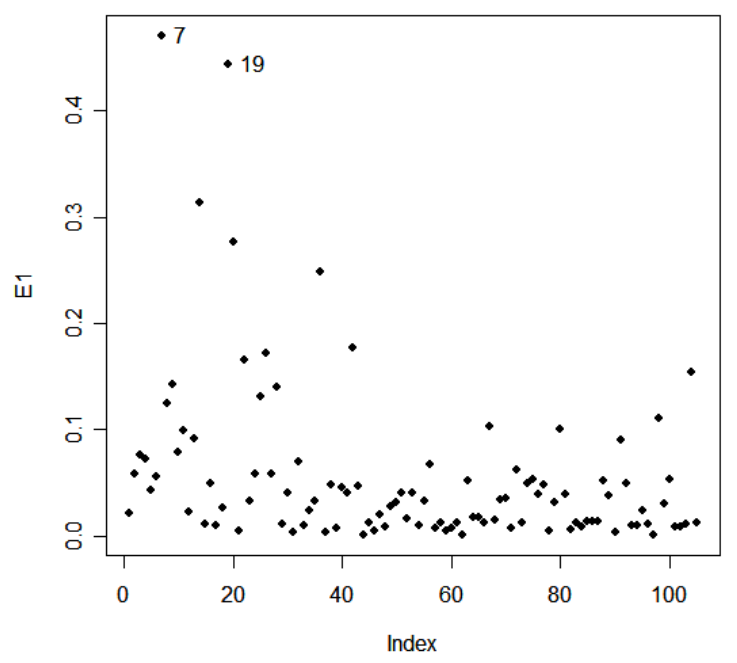

(a)

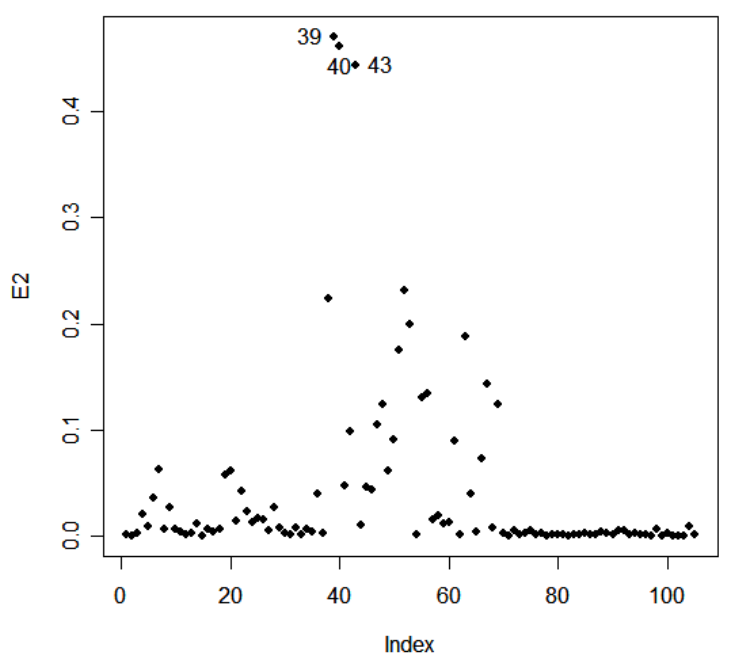

(b)

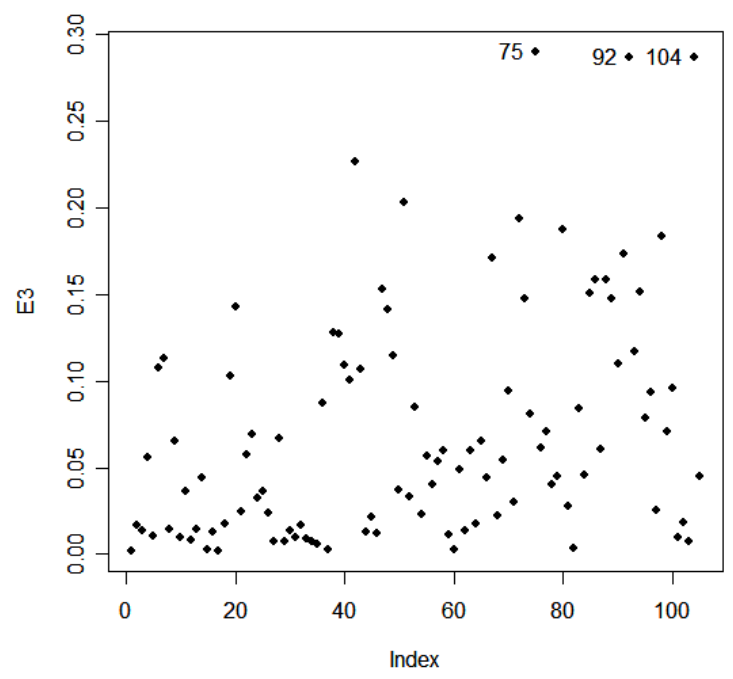

(c)

Figura 7.31: Autovetores e autovalores normalizados, $E_{1}$, com $\mu_{1}=0.707$ (a), $E_{2}$, com $\mu_{2}=$ 0.363 (b), $E_{3}$, com $\mu_{3}=0.295$ (c) provenientes da perturbação na variável resposta.

\subsection{Conclusões dos estudos de diagnóstico de influência}

Considerando a técnica de influência local proposta por Cook (1986) e a curvatura normal conformal proposta por Poon \& Poon (1999), obtivemos as seguintes conclusões: 


\section{Ponderação de casos}

Considerando a técnica de Cook (1986), a observação 42 se destacou entre as demais, como mostra a Figura 7.1, porém parece não exercer muita influência nas variações ocorridas no afastamento pela verossimilhança, como mostra a Figura 7.2. O valor de $C_{\mathbf{d} \text { max }}$ obtido neste caso foi 2.16. A Figura 7.3 mostra que as mudanças nas estimativas dos parâmetros são pequenas ao perturbar o modelo na direção de $d_{\max }$ (o parâmetro cujas estimativas apresentaram maiores mudanças foi $\sigma_{x}^{2}$, e suas mudanças relativas variaram de 0.893 a 1.096). Considerando a exclusão do caso 42, o parâmetro relativamente mais afetado foi $\sigma_{x}^{2}$, que passou de 0.103 a 0.094, como mostra a Tabela 7.1. Considerando o enfoque de Poon \& Poon (1999) a mesma observação se destacou, como mostra a Figura 7.17, porém $B_{\max }$ foi 0.56 neste caso, o que confirma a conclusão de que esta observação não apresentaria muita influência para este esquema de perturbação.

\section{Perturbação nas variáveis explanatórias}

Para este esquema de perturbação, considerando a técnica proposta por Cook (1986), nenhuma observação teve destaque no gráfico de índices de $d_{\max }$, como mostrou a Figura 7.4, e o valor de $C_{\mathbf{d}_{\max }}$ obtido foi de 3.17. Empregando o enfoque de Poon \& Poon (1999), as observações 22, 42, 52 e 80 se destacaram, como mostra a Figura 7.20, e o valor de $B$ max neste caso foi de 0.85, o que representaria influência destas observações neste esquema de perturbação.

\section{Perturbação nas variáveis resposta}

Para o esquema de perturbação nas variáveis resposta, utilizando o enfoque de Cook (1986) houve um destaque do grupo controle em relação às demais observações, como mostrou o gráfico da Figura 7.6, com o maior valor entre os índices da observação 22, que pertence ao grupo que testou o líquido controle. Neste caso, $C_{\mathbf{d} \max }$ obtido foi de 4.61 , o que representaria influência das observações grupo controle neste esquema de perturbação. A Figura 7.8 mostra que os parâmetros que sofreram maiores mudanças relativas foram nas variâncias dos erros de medição do grupo controle após o uso dos líquidos, $\sigma_{e_{11}}^{2}$ e $\sigma_{e_{21}}^{2}$, e suas mudanças relativas foram de 0.663 a 1.445 para $\sigma_{e_{11}}^{2}$ e 0.700 a 1.401 para $\sigma_{e_{21}}^{2}$ ). Considerando a influência global, ou seja, a exclusão do caso 22, o parâmetro relativamente mais afetado foi $\sigma_{e_{21}}^{2}$, que passou de 0.234 para 0.205, como mostra a Tabela 7.1. Utilizando o enfoque de Poon \& Poon (1999), a mesma observação se destacou, como mostra o gráfico da Figura 7.24. O valor obtido de $B \max$ neste caso foi 0.51 . 


\section{Perturbação nas variâncias dos erros de medição no início do estudo}

Para a perturbação na variância dos erros de medição no início do estudo, utilizando o enfoque de Cook (1986), a Figura 7.10 mostra um destaque para as observações 42, 52 e 80, e sugere uma grande influência da observação 42 nas mudanças ocorridas na verossimilhança, de acordo com a Figura 7.2. Neste caso, $C_{\mathbf{d}_{\max }}$ obtido foi de 1.74. A Figura 7.12 mostra que as mudanças relativas nas estimativas do parâmetro $\sigma^{2}$ ao perturbar o modelo na direção de $d_{\text {max }}$ são grandes (as estimativas de $\sigma^{2}$ sofreram mudanças relativas de 2.224 a 0.648 ), e considerando a exclusão dos casos 42, 52 e 80, o parâmetro relativamente mais afetado foi $\sigma^{2}$, cuja estimativa passou de 0.010 para 0.007. Também sofreu "grande" mudança relativa o parâmetro $\sigma_{x}^{2}$, cuja estimativa passou de 0.103 a 0.086, como mostra a Tabela 7.1. Empregando o enfoque de Poon \& Poon (1999), as mesmas observações se destacaram e o valor de $B \max =0.99$ confirma que estas observações exerceriam grande influência neste esquema de perturbação.

\section{Perturbação nas variâncias dos erros após o uso dos líquidos}

Para o esquema de perturbação nas variâncias dos erros após o uso dos líquidos, destacaram-se as observações 7 e 19, como mostra a Figura 7.13 e a Figura 7.14 sugere que estas observações seriam influentes. Neste caso, $C_{\mathbf{d}_{\max }}$ obtido foi de 3.99. As observações 7 e 19 pertencem ao grupo que testou o líquido controle, e a Figura 7.15 mostra que as maiores mudanças relativas ao perturbar o modelo na direção de $d_{\max }$ foram na variância dos erros após seis meses de uso do líquido controle, $\sigma_{e_{21}}^{2} \sigma_{e_{21}}^{2}$, que passou de 0.234 para 0.181 . Utilizando o enfoque de Poon \& Poon (1999), destacaram-se as observações 7, 19, 14 e 22. E o valor obtido de $B \max$ obtido foi de 0.71 , o que indicaria uma influência razoável destas observações neste esquema de perturbação. 


\section{Conclusões}

Apresentamos aqui um modelo de regressão multivariado com erros nas variáveis com intercepto nulo ajustado a dados reais da área de Odontologia. Para estimar os parâmetros do modelo, obtivemos a função de verossimilhança e a função escore, a fim de obter estimativas de máxima verossimilhança para os parâmetros. No entanto, não foi possível obter os estimadores explicitamente, dada a complexidade das expressões envolvidas. Consideramos então o algoritmo iterativo EM para obter estimativas numéricas para os parâmetros. Neste caso, a obtenção das expressões dos passos E e M do algoritmo foram relativamente simples e a convergência não levou mais do que 40 iterações, a partir de estimativas iniciais utilizando o método dos momentos.

Após o ajuste do modelo, desenvolvemos testes de hipóteses assintóticos para testar hipóteses de interesse do problema em questão. Para aplicar os testes da razão de verossimilhanças e score, foi necessário a obtenção de estimativas de máxima verossimilhança restritas a cada hipótese nula de interesse, o que envolveu novas implementações do algoritmo EM restritos a $H_{0}$ e a implementação do algoritmo de Newton-Raphson dentro da etapa de maximização (etapa M). Além disto, para a utilização das estatísticas de Wald e score, foi necessário a obtenção da matriz de informação esperada do modelo em questão. Para tanto, foi necessário utilizar resultados de Bradley \& Gart (1962) para obter a distribuição assintótica dos estimadores de máxima verossimilhança no caso de dados não-identicamente distribuídos. Aplicando os testes aos dados apresentados em Hadgu \& Koch (1999), concluímos que os dois líquidos experimentais realmente eram mais eficientes do que o líquido controle e que apenas o líquido B tem efeito a longo prazo. Estas conclusões coincidem com as conclusões obtidas por Hadgu \& Koch (1999).

Em seguida, para verificar o comportamento das estatísticas de testes assintóticos, realizamos um estudo de simulação envolvendo o tamanho do teste e o poder do teste, variando alguns parâmetros do modelo e considerando alguns tamanhos de amostras. De um modo geral, em relação ao tamanho do teste, a estatística da razão de verossimilhanças apresentou-se a mais eficiente entre as três estatísticas, pois em quase todos os casos apresentou boa aproximação ao nível nominal mesmo para amostras pequenas. Para o poder do teste, para amostras pe- 
quenas, a estatística Wald teve melhor comportamento, nos casos em que o tamanho do teste foi equivalente para as três estatísticas, porém ao aumentar o tamanho das amostras as três estatísticas foram equivalentes, como era de se esperar.

Realizamos também um estudo de diagnóstico de influência para obter possíveis pontos influentes no modelo, primeiramente considerando a abordagem de influência local de Cook (1986) e em seguida utilizando um novo enfoque de diagnóstico baseado na influência local. Consideramos a abordagem de Poon \& Poon (1999), que utiliza a curvatura normal conformal para decidir sobre a significância da curvatura da função de afastamento pela verossimilhança em determinado ponto, utilizando uma medida que fosse invariante sob reparametrizações, com o intuito de complementar os resultados obtidos utilizando a metodologia de Cook (1986). 


\section{Referências Bibliográficas}

Aoki, R., Bolfarine, H. \& Singer, J. M. (2001), 'Null Intercept Measurement Error Regression Models', Test 10, 441-457.

Aoki, R., Bolfarine, H. \& Singer, J. M. (2002), 'Asymptotic Efficiency of Methods of Moments Estimators under Null Intercept Measurement Error Regression Models', Brazilian Journal of Probability and Statistics 16, 157-166.

Aoki, R., Singer, J. M. \& Bolfarine, H. (n.d.), 'Local influence in measurement error regression models for the analysis of pretest/posttest data', To appear in Journal of Applied Statistical Science $\mathbf{1 5 .}$

Bai, P. \& Fei, Y. (2000), 'Bayesian local influence assessments in a growth curve model with general covariance structure', Acta Math. Sci. Ser. B Engl. Ed. 20(4), 563-570.

Belsley, D. A., Kuh, E. \& Welch, R. E. (1980), Regression Diagnostics, Identifying influential data and sources of collinearity, John Wiley.

Bradley, J. J. \& Gart, R. A. (1962), 'The assymptotic properties of ml estimators when sampling from associated populations', Biometrika 49(1 and 2), 205-214.

Cadigan, N. G. \& Farrell, P. J. (2002), 'Generalized local influence with applications to fish stock cohort analysis', Journal of the Royal Statistical Society - Series C 51(4), 469-483.

Carroll, R. J. \& Spiegelman, C. H. (1992), 'Diagnostics for nonlinearity and heteroscedasticity in errors-in-variables regression', Technometrics 34(2), 186-196.

Chan, L. K. \& Mak, T. K. (1979), 'On the maximum likelihood estimation of a linear structural relashionship when the intercept is known', Journal of Multivariate Analysis 9, 304-313.

Cheng, C. L. \& Van Ness, J. W. (1990), 'Bounded influence errors-in-variables regression', Statistical analysis of measurement error models and applications (Arcata, CA, 1989), Contemp. Math. 112, 227-241. 
Cheng, C. L. \& Van Ness, J. W. (1999), Statistical Regression with Measurement Error, 1st edn, Oxford University Press.

Cook, D. (1986), 'Assessment of local influence', Journal of the Royal Statistical Society - Series B 48(2), 133-169.

Cook, R. D. \& Weisberg, S. (1982), Residuals and Influence in Regression, London: Chapman and Hall.

Dempster, A. P., Laird, N. M. \& Rubin, D. B. (1977), 'Maximun likelihood from incomplete data via the em algorithm (with discussion)', Journal of the Royal Statistical Society - Series B 39, 1-38.

Dolby, G. R. (1976), 'The ultra structural relation: A synthesis of the functional and structural relations', Biometrika 63, 39-50.

Doornik, J. A. (2002), Object-Oriented Matrix Programming Using Ox, London: Timberlake Consultants Press and Oxford, http://www.doornik.com.

Dávila, O., Lidia, S. \& Labra, F. E. V. (2002), 'Local influence in the structural comparative calibration model (portuguese)', Rev. Mat. Estatíst. 20, 103-123.

Farrell, P. J. \& Cadigan, N. G. (2000), 'Local influence in binary regression models, and its correspondence with global influence', Comm. Statist. Theory Methods 29, 349-368.

Fuller, A. (1987), Measurement Error Models, 1st edn, Wiley.

Fung, W. K. \& Kwan, C. W. (1997), 'A note on local influence based on normal curvature', Journal of the Royal Statistical Society - Series B 59(4), 839-843.

Fung, W. K. \& Tang, M. K. (1997), 'Assessment of local influence in multivariate regression analysis', Comm. Statist. Theory Methods 26(4), 821-837.

Fung, W. K., Zhong, X. P. \& Wei, B. C. (2003), 'On estimation and influence diagnostics in linear mixed measurement error models', Amer. J. Math. Management Sci. 23(1-2), 37-59.

Galea-Rojas, M., Bolfarine, H. \& Castro, M. (2002), 'Local influence in comparative calibration models', Biometrical Journal 44(1), 59-81.

Galea-Rojas, M., Bolfarine, H. \& Vilca-Labra, F. (2002), 'Influence diagnostics for the structural errors-in-variables model under the student-t distribution', J. Appl. Stat. 29(8), 1191-1204. 
Galea-Rojas, M., Bolfarine, H. \& Vilca-Labra, F. (2005), 'Local influence in comparative calibration models under elliptical t-distributions', Biometrical J 47(5), 691-706.

Galea-Rojas, M., Paula, G. A. \& Bolfarine, H. (1997), 'Local influence in elliptical linear regression models', The Statistician 46(1), 71-79.

González Sierra, M. A. \& Suárez Rancel, M. M. (2000), 'Local influence in logistical regression', Rev. Acad. Canaria Cienc. 12(1-2), 23-31.

Graybill, F. A. (1983), Matrices with Applications in Statistics, 2nd edn, Belmont, Calif.: Wadsworth International Group.

Hadgu, A. \& Koch, G. (1999), 'Application of generalized estimating equations to a dental randomized clinical trial', Journal of Biopharmaceutical Statistics 9(1), 161-178.

Hartless, G., Booth, J. G. \& Littell, R. C. (2003), 'Local influence for predictors in multiple linear regression', Technometrics 45(4), 326-332.

Jung, K. M. (2000), 'Local influence assessment in canonical correlation analysis', J. Appl. Stat. $27(3), 293-301$.

Kelly, G. (1984), 'The influence function in the errors in variables problem', Ann. Statist. 12(1), 87-100.

Kendall, M. G. (1951), 'Structure and functional relationship i’, Biometrika 38, 11-15.

Kendall, M. G. (1952), 'Structure and functional relationship ii', Biometrika 39, 96-108.

Kendall, M. G. \& Stuart, A. (1961), The Advanced Theory of Statistics, London: Griffin.

Kendall, M. G. \& Stuart, A. (1973), The Advanced Theory of Statistics, Vol. v1, v2, 4th edn, London: Griffin.

Kim, M. G. (1996), 'Local influence in multivariate normal data', Journal of Applied Statistics $23(5)$.

Kim, M. G. (2000), 'Outliers and influential observations in the structural errors-in-variables model', Journal of Applied Statistics 27(4), 451-460.

Klepper, S. (1988), 'Regressor diagnostics for the classical errors-in-variables model', Econometrics 37(2), 225-250. 
Labra, F. V., Aoki, R. \& Bolfarine, H. (2005), 'Local influence in null intercept measurement error regression under a student-t model', Journal of Applied Statistics 32(7), 723-740.

Lee, A. H. \& Zhao, Y. (1996), 'Assessing local influence in measurement error models', Biometrical Journal 38(7), 829-841.

Lee, S. Y., Lu, B. \& Song, X. Y. (2006), 'Assessing local influence for nonlinear structural equation models with ignorable missing data', Computational Statistics 85 Data Analysis $50(5), 1356-1377$.

Lee, S. Y. \& Tang, N. S. (2004), 'Local influence analysis of nonlinear structural equation models', Psychometrika 69(4), 573-592.

Lehmann, E. L. \& Casella, G. (1998), Theory of Point Estimation, 2nd edn, Springer.

Lei, S., Li, Y. \& He, L. P. (2004), 'Assessing local influence using robust diagnostic in linear regression', J. Yunnan Univ. Nat. Sci. 26(1), 1-5.

Lei, S. \& Wang, X. (1996), 'Assessment of local influence in multivariate analysis', Acta Math. Scientia 16(3), 257-270.

Lesaffre, E. \& Verbeke, G. (1998), 'Local influence in linear mixed models', Biometrics 54, 570582.

Liu, S. (2000), 'On local influence for elliptical linear models', Statist. Papers 41(2), 211-224.

Liu, S. (2002), 'Local influence in multivariate elliptical linear regression models', Linear Algebra and its Applications 354(1-3), 159-174.

Loynes, R. M. (2001), 'A new measure in local influence', J. Statist. Plann. Inference 92(12), $47-53$.

McLachlan, G. J. \& Krishnan, T. (1997), The EM Algorithm and Extensions, New York: Wiley.

Moran, P. A. P. (1971), 'Estimating structural and functional relationships', Journal of Multivariate Analysis 1, 232-255.

Muirhead, R. J. (1982), Aspects of Multivariate Statistical Theory, New York: Wiley.

Nakamura, T. (1990), 'Corrected score function for errors-in-variables models: Methodology and applications to generalized linear models', Biometrika 77, 127-137. 
Nel, D. G. (1980), 'On matrix differentiation in statistics', South African Statist. J. 14, 137-193.

Ouwens, M. J. N. M., Tan, F. E. S. \& Berger, M. P. F. (2001), 'Local influence to detect influential data structures for generalized linear mixed models', Biometrics 57(4), 1166-1172.

Patefield, W. M. (1985), 'Information from the maximized likelihood function', Biometrics 72(3), 664-668.

Paula, G. A. (1993), 'Assessing local influence in restricted regression models', Computational Statistics \& Data Analysis 16(1), 63-79.

Poon, W. Y. \& Poon, Y. S. (1999), 'Conformal normal curvature and assessment of local influence', Journal of the Royal Statistical Society - Series B 61(1), 51-61.

Poon, W. Y. \& Poon, Y. S. (2001), 'Conditional local influence in case-weights linear regression', Brit J Math Stat Psy 54, 177-191.

Rasekh, A. R. (2006), 'Local influence in measurement error models with ridge estimate', Computational Statistics \& Data Analysis 50(10), 2822-2834.

Ruggiero, M. A. G. \& Lopes, V. L. R. (1996), Cálculo Numérico. Aspectos Teóricos e Computacionais, $2^{\mathrm{a}}$ edn, São Paulo: Makron Books.

Sen, P. K. \& Singer, J. M. (1993), Large Sample Methods in Statistics: An Introduction with Applications, New York: Chapman and Hall.

Singer, J. M. \& Andrade, D. F. (1997), 'Regression models for the analysis of pretest, posttest data', Biometrics 3(3), 729-735.

Sprent, P. (1990), 'Some history of functional and structural relationships', Contemporary Mathematics, American Mathematical Society 112, 3-15.

Suarez Rancel, M. M. \& Gonzalez Sierra, M. A. (2001), 'Regression diagnostic using local influence: a review', Comm. Statist. Theory Methods 30(5), 799-813.

Takeuchi, H. (2002), 'Assessment of influence of individual observations on prediction mean square errors in variable selection problems', J. Japan Statist. Soc. 32(1), 43-55.

Tanaka, Y., Z. F. M. Y. (2003), 'Local influence in principal component analysis: relationship between the local influence and influence function approaches revisited', Computational Statistics \& Data Analysis 44(1-2), 143-160. 
Tanaka, Y., Zhang, F. \& Yang, W. S. (2002), 'On local influence in canonical correlations analysis', Comm. Statist. Theory Methods 31(12), 2325-2347.

Tang, N. S., Wei, B. C. \& Wang, X. R. (2001), 'Local influence in nonlinear reproductive dispersion models', Comm. Statist. Theory Methods 30(3), 435-449.

Van der Vaart, A. W. (1998), Asymptotic Statiscs, Cambridge Series in Statistical and Probabilistic Mathematics, Cambridge University Press.

Wellman, J. M. \& Gunst, R. F. (1991), 'Influence diagnostics for linear measurement error models', Biometrika 78(2), 373-380.

Yohai, V. J. \& Zamar, R. H. (1990), 'Bounded influence estimation in the errors-in-variables model', Statistical analysis of measurement error models and applications (Arcata, CA, 1989) Contemp. Math. 112, 243-248.

Zhao, Y., Lee, A. H. \& Hui, Y. V. (1994), 'Influence diagnostics for generalized linear measurement error models', Biometrics 50, 1117-1128.

Zhong, X. P., Wei, B. C. \& Fung, W. K. (2000), 'Influence analysis for linear measurement error', Models Ann. Inst. Statist. Math. 52(2), 367-379.

Zhu, H. T. \& Lee, S. Y. (2001), 'Local influence for incomplete-data models', Journal of the Royal Statistical Society - Series B 63(1), 111-126.

Zhu, H. T. \& Lee, S. Y. (2003), 'Local influence for generalized linear mixed models', Canad. J. Statist. 31(3), 293-309.

Zhu, H. \& Zhang, H. (2004), 'A diagnostic procedure based on local influence', Biometrika $\mathbf{9 1}(3), 579-589$.

Zou, Q. M. \& Zhang, H. X. (2003), 'Bayesian local influence for a multivariate t-model with rao's simple structure', Math. Appl. (Wuhan) 16(4), 8-17.

Zou, Q. \& Zhang, H. (2005), 'Local influence assessment in a multivariate t-model with rao's simple structure', Acta Math. Sci. Ser. B Engl. Ed. 25(1), 179-192. 


\section{Apêndice A}

\section{Conjunto de Dados}

Conjunto de dados de Hadgu \& Koch (1999):

\begin{tabular}{|c|c|c|c|c|c|c|c|}
\hline OBS & $\operatorname{Sexo}^{a}$ & Idade & Líquido ${ }^{b}$ & Status $\mathrm{f}^{c}$ & $\mathrm{IPD}^{d}$ inicial & IPD 3 meses & IPD 6 meses \\
\hline 1 & 2 & 24 & 1 & 1 & 2.46 & 1.73 & 1.58 \\
\hline 2 & 2 & 29 & 1 & 1 & 2.89 & 2.61 & 1.91 \\
\hline 3 & 1 & 27 & 1 & 0 & 3.00 & 2.75 & 1.89 \\
\hline 4 & 2 & 25 & 1 & 1 & 2.52 & 0.98 & 1.71 \\
\hline 5 & 1 & 27 & 1 & 0 & 2.52 & 1.61 & 2.29 \\
\hline 6 & 2 & 25 & 1 & 0 & 2.78 & 1.25 & 1.34 \\
\hline 7 & 1 & 25 & 1 & 0 & 2.08 & 0.88 & 0.35 \\
\hline 8 & 1 & 29 & 1 & 1 & 3.11 & 3.04 & 1.93 \\
\hline 9 & 1 & 28 & 1 & 0 & 2.38 & 0.90 & 1.28 \\
\hline 10 & 1 & 24 & 1 & 0 & 2.70 & 2.11 & 2.46 \\
\hline 11 & 2 & 38 & 1 & 0 & 3.20 & 2.39 & 2.48 \\
\hline 12 & 2 & 36 & 1 & 0 & 3.25 & 2.23 & 2.23 \\
\hline 13 & 1 & 27 & 1 & 0 & 3.21 & 2.88 & 1.96 \\
\hline 14 & 2 & 23 & 1 & 0 & 3.30 & 2.50 & 3.10 \\
\hline 15 & 2 & 27 & 1 & 0 & 2.27 & 1.61 & 1.61 \\
\hline 16 & 1 & 25 & 1 & 0 & 2.21 & 1.15 & 1.67 \\
\hline 17 & 1 & 25 & 1 & 0 & 2.40 & 1.69 & 2.08 \\
\hline 18 & 1 & 29 & 1 & 0 & 2.64 & 1.45 & 1.96 \\
\hline 19 & 1 & 24 & 1 & 0 & 2.07 & 0.70 & 0.48 \\
\hline 20 & 1 & 28 & 1 & 0 & 2.43 & 0.85 & 0.78 \\
\hline 21 & 1 & 29 & 1 & 0 & 2.27 & 1.71 & 2.27 \\
\hline 22 & 1 & 23 & 1 & 0 & 2.52 & 2.50 & 2.85 \\
\hline 23 & 1 & 28 & 1 & 0 & 2.79 & 1.54 & 1.42 \\
\hline 24 & 1 & 29 & 1 & 0 & 2.54 & 1.58 & 1.35 \\
\hline 25 & 1 & 23 & 1 & 0 & 2.17 & 0.77 & 1.48 \\
\hline 26 & 1 & 26 & 1 & 0 & 2.09 & 1.16 & 0.98 \\
\hline 27 & 1 & 24 & 1 & 0 & 2.68 & 2.74 & 1.88 \\
\hline 28 & 1 & 24 & 1 & 0 & 2.35 & 0.72 & 1.48 \\
\hline 29 & 2 & 29 & 1 & 0 & 2.36 & 2.33 & 2.04 \\
\hline 30 & 2 & 38 & 1 & 0 & 2.61 & 2.43 & 2.16 \\
\hline 31 & 2 & 24 & 1 & 0 & 2.48 & 2.00 & 1.93 \\
\hline 32 & 2 & 29 & 1 & 1 & 2.31 & 1.33 & 1.38 \\
\hline 33 & 1 & 30 & 1 & 0 & 2.50 & 2.32 & 1.96 \\
\hline
\end{tabular}


Conjunto de dados originais (continuação)

\begin{tabular}{|c|c|c|c|c|c|c|c|}
\hline OBS & Sexo & Idade & Líquido & Status f & IPD inicial & IPD 3 meses & IPD 6 meses \\
\hline 34 & 1 & 32 & 1 & 0 & 2.31 & 2.27 & 1.96 \\
\hline 35 & 2 & 32 & 1 & 0 & 2.38 & 1.98 & 1.38 \\
\hline 36 & 1 & 24 & 1 & 0 & 2.27 & 0.75 & 0.96 \\
\hline 37 & 2 & 42 & 2 & 0 & 2.65 & 1.42 & 1.25 \\
\hline 38 & 2 & 48 & 2 & 1 & 2.13 & 2.48 & 1.63 \\
\hline 39 & 2 & 27 & 2 & 0 & 2.70 & 0.00 & 0.59 \\
\hline 40 & 2 & 33 & 2 & 0 & 2.48 & 0.10 & 0.12 \\
\hline 41 & 1 & 25 & 2 & 0 & 2.98 & 1.57 & 2.31 \\
\hline 42 & 2 & 43 & 2 & 0 & 3.57 & 2.80 & 2.41 \\
\hline 43 & 2 & 25 & 2 & 1 & 2.48 & 0.05 & 0.27 \\
\hline 44 & 2 & 23 & 2 & 1 & 2.73 & 1.44 & 1.17 \\
\hline 45 & 2 & 27 & 2 & 0 & 2.35 & 1.35 & 0.65 \\
\hline 46 & 1 & 24 & 2 & 0 & 2.52 & 1.59 & 0.75 \\
\hline 47 & 1 & 25 & 2 & 0 & 2.61 & 1.98 & 2.32 \\
\hline 48 & 2 & 24 & 2 & 0 & 2.43 & 1.63 & 2.43 \\
\hline 49 & 2 & 25 & 2 & 0 & 2.75 & 1.60 & 2.31 \\
\hline 50 & 1 & 25 & 2 & 0 & 2.27 & 0.63 & 0.96 \\
\hline 51 & 2 & 38 & 2 & 0 & 2.68 & 2.24 & 2.52 \\
\hline 52 & 2 & 31 & 2 & 0 & 3.40 & 0.85 & 2.53 \\
\hline 53 & 1 & 25 & 2 & 0 & 2.96 & 1.48 & 0.29 \\
\hline 54 & 2 & 30 & 2 & 0 & 2.16 & 1.46 & 1.20 \\
\hline 55 & 1 & 28 & 2 & 0 & 2.34 & 0.70 & 0.59 \\
\hline 56 & 1 & 25 & 2 & 0 & 2.04 & 0.46 & 0.55 \\
\hline 57 & 2 & 25 & 2 & 0 & 2.35 & 1.78 & 1.43 \\
\hline 58 & 2 & 25 & 2 & 0 & 2.68 & 1.43 & 1.93 \\
\hline 59 & 2 & 29 & 2 & 0 & 2.50 & 1.35 & 1.04 \\
\hline 60 & 2 & 43 & 2 & 0 & 2.07 & 1.29 & 0.80 \\
\hline 61 & 2 & 36 & 2 & 0 & 2.52 & 0.79 & 1.00 \\
\hline 62 & 2 & 26 & 2 & 0 & 2.37 & 1.50 & 1.15 \\
\hline 63 & 1 & 25 & 2 & 0 & 2.27 & 0.39 & 0.64 \\
\hline 64 & 2 & 26 & 2 & 1 & 2.21 & 1.27 & 0.63 \\
\hline 65 & 2 & 23 & 2 & 0 & 2.71 & 1.80 & 1.71 \\
\hline 66 & 2 & 40 & 2 & 1 & 2.45 & 1.10 & 0.65 \\
\hline 67 & 1 & 25 & 2 & 1 & 3.11 & 2.81 & 1.83 \\
\hline 68 & 2 & 22 & 2 & 0 & 3.02 & 1.94 & 1.33 \\
\hline 69 & 1 & 26 & 2 & 0 & 2.32 & 0.73 & 0.57 \\
\hline 70 & 2 & 23 & 3 & 0 & 2.33 & 0.56 & 0.90 \\
\hline 71 & 2 & 38 & 3 & 1 & 2.53 & 1.00 & 0.89 \\
\hline 72 & 1 & 24 & 3 & 0 & 2.61 & 2.11 & 1.61 \\
\hline 73 & 2 & 47 & 3 & 0 & 2.74 & 1.02 & 0.48 \\
\hline 74 & 1 & 23 & 3 & 0 & 3.02 & 2.04 & 1.06 \\
\hline 75 & 2 & 24 & 3 & 0 & 2.56 & 2.04 & 0.29 \\
\hline 76 & 2 & 25 & 3 & 1 & 2.25 & 0.96 & 0.58 \\
\hline
\end{tabular}


Conjunto de dados originais (continuação)

\begin{tabular}{cccccccc}
\hline OBS & Sexo & Idade & Líquido & Status $\mathrm{f}$ & IPD inicial & IPD 3 meses & IPD 6 meses \\
\hline 77 & 1 & 28 & 3 & 0 & 2.74 & 1.54 & 1.65 \\
78 & 1 & 25 & 3 & 0 & 2.60 & 0.88 & 1.17 \\
79 & 1 & 25 & 3 & 0 & 2.76 & 1.82 & 1.14 \\
80 & 1 & 23 & 3 & 0 & 3.11 & 0.93 & 0.59 \\
81 & 1 & 24 & 3 & 0 & 3.13 & 1.85 & 1.25 \\
82 & 2 & 24 & 3 & 0 & 2.71 & 1.36 & 1.09 \\
83 & 2 & 28 & 3 & 0 & 2.42 & 1.36 & 1.62 \\
84 & 1 & 31 & 3 & 0 & 2.32 & 0.92 & 1.36 \\
85 & 2 & 24 & 3 & 0 & 2.19 & 1.85 & 0.54 \\
86 & 1 & 23 & 3 & 0 & 2.72 & 0.50 & 0.98 \\
87 & 1 & 30 & 3 & 1 & 2.45 & 1.20 & 1.55 \\
88 & 2 & 25 & 3 & 1 & 2.60 & 2.06 & 1.52 \\
89 & 1 & 25 & 3 & 0 & 2.17 & 1.60 & 0.35 \\
90 & 2 & 32 & 3 & 0 & 2.38 & 1.78 & 1.48 \\
91 & 2 & 35 & 3 & 0 & 2.18 & 0.55 & 0.39 \\
92 & 2 & 26 & 3 & 0 & 2.38 & 0.33 & 1.73 \\
93 & 1 & 25 & 3 & 0 & 2.88 & 1.53 & 0.55 \\
94 & 2 & 37 & 3 & 1 & 2.21 & 1.90 & 0.60 \\
95 & 2 & 29 & 3 & 0 & 2.42 & 0.67 & 0.88 \\
96 & 2 & 35 & 3 & 0 & 2.21 & 1.17 & 1.60 \\
97 & 2 & 31 & 3 & 0 & 2.22 & 1.46 & 0.83 \\
98 & 1 & 24 & 3 & 0 & 2.86 & 2.20 & 1.64 \\
99 & 2 & 25 & 3 & 0 & 2.06 & 1.13 & 1.48 \\
100 & 1 & 25 & 3 & 0 & 2.23 & 0.61 & 0.68 \\
101 & 1 & 25 & 3 & 0 & 2.02 & 1.00 & 1.11 \\
102 & 2 & 25 & 3 & 0 & 2.45 & 0.98 & 1.14 \\
103 & 1 & 25 & 3 & 0 & 2.29 & 1.13 & 0.89 \\
104 & 2 & 24 & 3 & 0 & 2.06 & 0.27 & 0.23 \\
105 & 1 & 25 & 3 & 0 & 2.44 & 0.88 & 1.31 \\
\hline & & & & & & &
\end{tabular}

a Sexo: 1 Masculino, 2 Feminino

$b$ Líquido: 1 Controle, $2 \mathrm{~A}, 3 \mathrm{~B}$

$c$ Status: 1 Fumante, 2 Não-fumante

$d$ Índice de placa dentária

Obs: Os dados foram reordenados, a partir da forma apresentada em Hadgu \& Koch (1999), de acordo com o líquido de bochecho utilizado, e as observações em que havia dados faltantes foram excluídas para o presente estudo. 


\section{Apêndice B}

\section{Derivadas vetoriais e propriedades}

Neste apêndice, apresentaremos propriedades de derivadas vetoriais que utilizaremos para obter expressões das derivadas primeiras e segundas da função log-verossimilhança. A seguir apresentamos a notação que será utilizada.

\section{B.1 Notação}

1. Escreveremos $\mathbf{x}$ para denotar um vetor de dimensão $n$ :

$$
\mathbf{x}=\left(\begin{array}{c}
x_{1} \\
x_{2} \\
\vdots \\
x_{n}
\end{array}\right)
$$

e $\mathbf{x}^{\prime}$ para o vetor transposto de $\mathbf{x}$ :

$$
\mathbf{x}^{\prime}=\left(\begin{array}{llll}
x_{1} & x_{2} & \ldots & x_{n}
\end{array}\right)
$$

2. Escreveremos A para denotar uma matriz de dimensão $n \times m$ :

$$
\mathbf{A}=\left(\begin{array}{cccc}
a_{11} & a_{12} & \ldots & a_{1 m} \\
a_{21} & a_{22} & \ldots & a_{2 m} \\
\vdots & \vdots & \ddots & \vdots \\
a_{n 1} & a_{n 2} & \ldots & a_{n m}
\end{array}\right)
$$

e $\mathbf{A}^{\prime}$ para a matriz transposta de $\mathbf{A}$ :

$$
\mathbf{A}^{\prime}=\left(\begin{array}{cccc}
a_{11} & a_{21} & \ldots & a_{n 1} \\
a_{12} & a_{22} & \ldots & a_{n 2} \\
\vdots & \vdots & \ddots & \vdots \\
a_{1 m} & a_{2 m} & \ldots & a_{n m}
\end{array}\right)
$$


3. Denotaremos por $\mathbf{D}(\mathbf{x})$ uma matriz diagonal de dimensão $n \times n$ com os elementos do vetor $\mathrm{x}$ em sua diagonal, isto é,

$$
\text { se } \quad \mathbf{x}=\left(\begin{array}{c}
x_{1} \\
x_{2} \\
\vdots \\
x_{n}
\end{array}\right), \quad \text { então } \mathbf{D}(\mathbf{x})=\left(\begin{array}{cccc}
x_{1} & 0 & \ldots & 0 \\
0 & x_{2} & \ddots & \vdots \\
\vdots & \ddots & \ddots & 0 \\
0 & \ldots & 0 & x_{n}
\end{array}\right)
$$

Além disto, escreveremos $\mathbf{D}^{k}(\mathbf{x})$ e $\mathbf{D}^{-k}(\mathbf{x})$ para a seguintes matrizes diagonais

$$
\mathbf{D}^{k}(\mathbf{x})=\left(\begin{array}{cccc}
x_{1}^{k} & 0 & \ldots & 0 \\
0 & x_{2}^{k} & \ddots & \vdots \\
\vdots & \ddots & \ddots & 0 \\
0 & \ldots & 0 & x_{n}^{k}
\end{array}\right) \quad \text { e } \quad \mathbf{D}^{-k}(\mathbf{x})=\left(\begin{array}{cccc}
x_{1}^{-k} & 0 & \ldots & 0 \\
0 & x_{2}^{-k} & \ddots & \vdots \\
\vdots & \ddots & \ddots & 0 \\
0 & \ldots & 0 & x_{n}^{-k}
\end{array}\right)
$$

4. Escreveremos $\mathbf{I}_{n}$ para denotar a matriz identidade de dimensão $n \times n$ e $\mathbf{1}_{n}$ para o vetor de unidades de dimensão $n$, isto é,

$$
\mathbf{I}_{n}=\left(\begin{array}{cccc}
1 & 0 & \ldots & 0 \\
0 & 1 & \ddots & \vdots \\
\vdots & \ddots & \ddots & 0 \\
0 & \ldots & 0 & 1
\end{array}\right) \text { e } \mathbf{1}_{n}=\left(\begin{array}{c}
1 \\
1 \\
\vdots \\
1
\end{array}\right)
$$

5. Utilizaremos como notação a letra minúscula em negrito para um vetor coluna e maiúscula em negrito para uma matriz, por exemplo, $\mathbf{v}_{k}$ é um vetor coluna com $k$ linhas, e $\mathbf{M}_{m \times n}$ é uma matriz com $m$ linhas e $n$ colunas.

\section{B.2 Definições}

Apresentaremos agora as definições de derivadas vetoriais de uma função real e de uma função vetorial, como em Graybill (1983). Utilizando estas definições obteremos na Seção B.3 as propriedades de derivadas que serão utilizadas neste trabalho.

\section{Definição B.1 Derivadas vetoriais de uma função real:}

Seja $\mathbf{x}=\left(x_{1}, x_{2}, \ldots, x_{n}\right)^{\prime}$ e $f$ uma função real de $\mathbf{x}$, isto é,

$$
f(\mathbf{x})=f\left(x_{1}, x_{2}, \ldots, x_{n}\right): \mathbb{R}^{n} \rightarrow \mathbb{R}
$$


1. A derivada de $f$ em relação ao vetor $\mathbf{x}$ é denotada por $\frac{\partial}{\partial \mathbf{x}}(f)$ e é definida por

$$
\frac{\partial}{\partial \mathbf{x}}(f)=\left(\begin{array}{c}
\frac{\partial}{\partial x_{1}}(f) \\
\frac{\partial}{\partial x_{2}}(f) \\
\vdots \\
\frac{\partial}{\partial x_{n}}(f)
\end{array}\right)
$$

2. A derivada de $f$ em relação ao vetor $\mathbf{x}^{\prime}$ é denotada por $\frac{\partial}{\partial \mathbf{x}^{\prime}}(f)$ e é definida por

$$
\frac{\partial}{\partial \mathbf{x}^{\prime}}(f)=\left(\frac{\partial}{\partial x_{1}}(f) \quad \frac{\partial}{\partial x_{2}}(f) \quad \ldots \quad \frac{\partial}{\partial x_{n}}(f)\right)
$$

\section{Definição B.2 Derivadas vetoriais de uma função vetorial:}

Seja $\mathbf{y}: \mathbb{R}^{n} \rightarrow \mathbb{R}^{m}$ uma função vetorial de $\mathbf{x}$, isto é,

$$
\mathbf{y}=\mathbf{y}(\mathbf{x})=\left(\begin{array}{c}
y_{1}(\mathbf{x}) \\
y_{2}(\mathbf{x}) \\
\vdots \\
y_{m}(\mathbf{x})
\end{array}\right)
$$

ou seja, cada elemento de $\mathbf{y}$ é uma função real de $\mathbf{x}$.

1. A derivada de $\mathbf{y}$ em relação ao vetor transposto $\mathbf{x}^{\prime}$ é denotada por $\frac{\partial}{\partial \mathbf{x}^{\prime}}(\mathbf{y})$ e é definida por

$$
\frac{\partial}{\partial \mathbf{x}^{\prime}}(\mathbf{y})=\left(\begin{array}{cccc}
\frac{\partial}{\partial x_{1}}\left(y_{1}(\mathbf{x})\right) & \frac{\partial}{\partial x_{2}}\left(y_{1}(\mathbf{x})\right) & \cdots & \frac{\partial}{\partial x_{n}}\left(y_{1}(\mathbf{x})\right) \\
\frac{\partial}{\partial x_{1}}\left(y_{2}(\mathbf{x})\right) & \frac{\partial}{\partial x_{2}}\left(y_{2}(\mathbf{x})\right) & \cdots & \frac{\partial}{\partial x_{n}}\left(y_{2}(\mathbf{x})\right) \\
\vdots & \vdots & \ddots & \vdots \\
\frac{\partial}{\partial x_{1}}\left(y_{m}(\mathbf{x})\right) & \frac{\partial}{\partial x_{2}}\left(y_{m}(\mathbf{x})\right) & \cdots & \frac{\partial}{\partial x_{n}}\left(y_{m}(\mathbf{x})\right)
\end{array}\right)
$$


2. A derivada de $\mathbf{y}^{\prime}$ em relação ao vetor $\mathbf{x}$ é denotada por $\frac{\partial}{\partial \mathbf{x}}\left(\mathbf{y}^{\prime}\right)$ e é definida por

$$
\frac{\partial}{\partial \mathbf{x}}\left(\mathbf{y}^{\prime}\right)=\left(\begin{array}{cccc}
\frac{\partial}{\partial x_{1}}\left(y_{1}(\mathbf{x})\right) & \frac{\partial}{\partial x_{1}}\left(y_{2}(\mathbf{x})\right) & \cdots & \frac{\partial}{\partial x_{1}}\left(y_{m}(\mathbf{x})\right) \\
\frac{\partial}{\partial x_{2}}\left(y_{1}(\mathbf{x})\right) & \frac{\partial}{\partial x_{2}}\left(y_{2}(\mathbf{x})\right) & \cdots & \frac{\partial}{\partial x_{2}}\left(y_{m}(\mathbf{x})\right) \\
\vdots & \vdots & \ddots & \vdots \\
\frac{\partial}{\partial x_{n}}\left(y_{1}(\mathbf{x})\right) & \frac{\partial}{\partial x_{n}}\left(y_{2}(\mathbf{x})\right) & \cdots & \frac{\partial}{\partial x_{n}}\left(y_{m}(\mathbf{x})\right)
\end{array}\right)
$$

\section{B.3 Propriedades}

Sejam os vetores $\mathbf{x}_{n}, \mathbf{a}_{n}, \mathbf{b}_{n}, \boldsymbol{\phi}_{n}, \mathbf{y}_{p}, \mathbf{z}_{m}$, a matriz quadrada $\mathbf{A}_{n \times n}$, o vetor $\mathbf{1}_{n}$ um vetor coluna de dimensão $n$ com todos os elementos iguais a 1, e a matriz $\mathbf{I}_{n}$ a matriz identidade de dimensão $n$.

Temos as seguintes propriedades:

1. $\frac{\partial}{\partial \mathbf{x}}\left(\mathbf{a}^{\prime} \mathbf{x}\right)=\mathbf{a}$

2. $\frac{\partial}{\partial \mathbf{x}^{\prime}}\left(\mathbf{a}^{\prime} \mathbf{x}\right)=\mathbf{a}^{\prime}$

3. $\frac{\partial}{\partial \mathbf{x}^{\prime}}(\mathbf{A x})=\mathbf{A}$

4. $\frac{\partial}{\partial \mathbf{x}}\left(\mathbf{x}^{\prime} \mathbf{A}\right)=\mathbf{A}$

5. $\frac{\partial}{\partial \mathbf{x}}\left(\mathbf{x}^{\prime} \mathbf{A} \mathbf{x}\right)=\left(\mathbf{A}+\mathbf{A}^{\prime}\right) \mathbf{x}$

6. $\frac{\partial^{2}}{\partial \mathbf{x} \partial \mathbf{x}^{\prime}}\left(\mathbf{x}^{\prime} \mathbf{A} \mathbf{x}\right)=\mathbf{A}+\mathbf{A}^{\prime}$

Em particular, quando A é simétrica, as propriedades (5) e (6) podem ser escritas respectivamente como (7) e (8):

7. $\frac{\partial}{\partial \mathbf{x}}\left(\mathbf{x}^{\prime} \mathbf{A} \mathbf{x}\right)=2 \mathbf{A x} \quad\left(\right.$ se $\left.\mathbf{A}=\mathbf{A}^{\prime}\right)$

8. $\frac{\partial^{2}}{\partial \mathbf{x} \partial \mathbf{x}^{\prime}}\left(\mathbf{x}^{\prime} \mathbf{A} \mathbf{x}\right)=2 \mathbf{A} \quad\left(\right.$ se $\left.\mathbf{A}=\mathbf{A}^{\prime}\right)$

9. $\frac{\partial}{\partial \mathbf{x}^{\prime}}(\mathbf{x})=\mathbf{I}_{n}$ 
10. $\frac{\partial}{\partial \mathbf{x}}\left(\mathbf{x}^{\prime}\right)=\mathbf{I}_{n}$

11. $\frac{\partial}{\partial \mathbf{x}}\left(\mathbf{y}^{\prime}\right)=\left(\frac{\partial}{\partial \mathbf{x}^{\prime}}(\mathbf{y})\right)^{\prime}$

Se $y=y(\mathbf{x}) \in \mathbb{R}$ temos a propriedade (12) como caso particular de (11)

12. $\frac{\partial}{\partial \mathbf{x}}(y)=\left(\frac{\partial}{\partial \mathbf{x}^{\prime}}(y)\right)^{\prime} \quad($ para $y=y(\mathbf{x}) \in \mathbb{R})$

13. Sendo $\mathbf{y}_{p \times 1}=\mathbf{y}(\mathbf{z}(\mathbf{x}))$ e $\mathbf{z}_{p \times 1}=\mathbf{z}(\mathbf{x})$
(a) $\frac{\partial}{\partial \mathbf{x}^{\prime}}(\mathbf{y})=\frac{\partial}{\partial \mathbf{z}^{\prime}}(\mathbf{y}) \frac{\partial}{\partial \mathbf{x}^{\prime}}(\mathbf{z})$
(b) $\frac{\partial}{\partial \mathbf{x}}\left(\mathbf{y}^{\prime}\right)=\frac{\partial}{\partial \mathbf{x}}\left(\mathbf{z}^{\prime}\right) \frac{\partial}{\partial \mathbf{z}}\left(\mathbf{y}^{\prime}\right)$

14. $\frac{\partial}{\partial \phi}(\log |\mathbf{D}(\phi)|)=\mathbf{D}^{-1}(\boldsymbol{\phi}) \mathbf{1}_{n}$

15. $\frac{\partial}{\partial \phi^{\prime}}\left(\mathbf{a}^{\prime} \mathbf{D}^{-1}(\boldsymbol{\phi}) \mathbf{b}\right)=-\mathbf{a}^{\prime} \mathbf{D}^{-2}(\phi) \mathbf{D}(\mathbf{b})=\left(\frac{\partial}{\partial \phi}\left(\mathbf{b}^{\prime} \mathbf{D}^{-1}(\phi) \mathbf{a}\right)\right)^{\prime}$

16. $\frac{\partial}{\partial \phi^{\prime}}\left(\mathbf{D}^{-1}(\boldsymbol{\phi}) \mathbf{a}\right)=-\mathbf{D}^{-2}(\phi) \mathbf{D}(\mathbf{a})=\left(\frac{\partial}{\partial \phi}\left(\mathbf{a}^{\prime} \mathbf{D}^{-1}(\phi)\right)\right)^{\prime}$

17. $\frac{\partial}{\partial \phi^{\prime}}\left(\mathbf{D}^{-2}(\phi) \mathbf{D}(\mathbf{a}) \mathbf{b}\right)=-2 \mathbf{D}^{-3}(\phi) \mathbf{D}(\mathbf{a}) \mathbf{D}(\mathbf{b})=\left(\frac{\partial}{\partial \phi}\left(\mathbf{b}^{\prime} \mathbf{D}(\mathbf{a}) \mathbf{D}^{-2}(\boldsymbol{\phi})\right)\right)^{\prime}$

18. Se $y_{1}=y_{1}(\mathbf{x}) \in \mathbb{R}$ e $y_{2}=y_{2}(\mathbf{x}) \in \mathbb{R}, \mathbf{x}=\left(x_{1}, x_{2}, \ldots, x_{n}\right)^{\prime}$ então
(a) $\frac{\partial}{\partial \mathbf{x}}\left(y_{1} y_{2}\right)=\frac{\partial}{\partial \mathbf{x}}\left(y_{1}\right) y_{2}+y_{1} \frac{\partial}{\partial \mathbf{x}}\left(y_{2}\right)$ e
(b) $\frac{\partial}{\partial \mathbf{x}^{\prime}}\left(y_{1} y_{2}\right)=\frac{\partial}{\partial \mathbf{x}^{\prime}}\left(y_{1}\right) y_{2}+y_{1} \frac{\partial}{\partial \mathbf{x}^{\prime}}\left(y_{2}\right)$

19. Se $y=y(\mathbf{x}) \in \mathbb{R}$ e $\mathbf{z}=\mathbf{z}(\mathbf{x})=\left(z_{1}(\mathbf{x}), z_{2}(\mathbf{x}), \ldots, z_{m}(\mathbf{x})\right)^{\prime}, \mathbf{x}=\left(x_{1}, x_{2}, \ldots, x_{n}\right)^{\prime}$ então
(a) $\frac{\partial}{\partial \mathbf{x}}\left(y \mathbf{z}^{\prime}\right)=\frac{\partial}{\partial \mathbf{x}}(y) \mathbf{z}^{\prime}+y \frac{\partial}{\partial \mathbf{x}}\left(\mathbf{z}^{\prime}\right)$ e
(b) $\frac{\partial}{\partial \mathbf{x}^{\prime}}(y \mathbf{z})=\mathbf{z} \frac{\partial}{\partial \mathbf{x}^{\prime}}(y)+y \frac{\partial}{\partial \mathbf{x}^{\prime}}(\mathbf{z})$

20. Se $\mathbf{y}=\mathbf{y}(\mathbf{x})=\left(y_{1}(\mathbf{x}), y_{2}(\mathbf{x}), \ldots, y_{m}(\mathbf{x})\right)^{\prime}$ e $\mathbf{z}=\mathbf{z}(\mathbf{x})=\left(z_{1}(\mathbf{x}), z_{2}(\mathbf{x}), \ldots, z_{m}(\mathbf{x})\right)^{\prime}, \mathbf{x}=$ $\left(x_{1}, x_{2}, \ldots, x_{n}\right)^{\prime}$, então
(a) $\frac{\partial}{\partial \mathbf{x}}\left(\mathbf{y}^{\prime} \mathbf{z}\right)=\frac{\partial}{\partial \mathbf{x}}\left(\mathbf{y}^{\prime}\right) \mathbf{z}+\frac{\partial}{\partial \mathbf{x}}\left(\mathbf{z}^{\prime}\right) \mathbf{y}$ e
(b) $\frac{\partial}{\partial \mathbf{x}^{\prime}}\left(\mathbf{y}^{\prime} \mathbf{z}\right)=\mathbf{z}^{\prime} \frac{\partial}{\partial \mathbf{x}^{\prime}}(\mathbf{y})+\mathbf{y}^{\prime} \frac{\partial}{\partial \mathbf{x}^{\prime}}(\mathbf{z})$ 


\section{Prova:}

$$
\begin{aligned}
& \text { 1. } \frac{\partial}{\partial \mathbf{x}}\left(\mathbf{a}^{\prime} \mathbf{x}\right)=\frac{\partial}{\partial \mathbf{x}}\left(\left(\begin{array}{llll}
a_{1} & a_{2} & \ldots & a_{n}
\end{array}\right)\left(\begin{array}{c}
x_{1} \\
x_{2} \\
\vdots \\
x_{n}
\end{array}\right)\right)=\frac{\partial}{\partial \mathbf{x}}\left(a_{1} x_{1}+a_{2} x_{2}+\ldots a_{n} x_{n}\right)= \\
& =\frac{\partial}{\partial \mathbf{x}}\left(\sum_{i=1}^{n} a_{i} x_{i}\right)=\left(\begin{array}{c}
\frac{\partial}{\partial x_{1}}\left(\sum_{i=1}^{n} a_{i} x_{i}\right) \\
\frac{\partial}{\partial x_{2}}\left(\sum_{i=1}^{n} a_{i} x_{i}\right) \\
\vdots \\
\frac{\partial}{\partial x_{n}}\left(\sum_{i=1}^{n} a_{i} x_{i}\right)
\end{array}\right)=\left(\begin{array}{c}
a_{1} \\
a_{2} \\
\vdots \\
a_{n}
\end{array}\right)=\mathbf{a} .
\end{aligned}
$$

2. $\frac{\partial}{\partial \mathbf{x}^{\prime}}\left(\mathbf{a}^{\prime} \mathbf{x}\right)=\frac{\partial}{\partial \mathbf{x}^{\prime}}\left(\sum_{i=1}^{n} a_{i} x_{i}\right)=\left(\frac{\partial}{\partial x_{1}}\left(\sum_{i=1}^{n} a_{i} x_{i}\right) \quad \frac{\partial}{\partial x_{2}}\left(\sum_{i=1}^{n} a_{i} x_{i}\right) \quad \ldots \quad \frac{\partial}{\partial x_{n}}\left(\sum_{i=1}^{n} a_{i} x_{i}\right)\right)=$ $=\left(\begin{array}{llll}a_{1} & a_{2} & \ldots & a_{n}\end{array}\right)=\mathbf{a}^{\prime}$.

3. $\frac{\partial}{\partial \mathbf{x}^{\prime}}(\mathbf{A x})=\frac{\partial}{\partial \mathbf{x}^{\prime}}\left(\left(\begin{array}{cccc}a_{11} & a_{12} & \ldots & a_{1 n} \\ a_{21} & a_{22} & \ldots & a_{2 n} \\ \vdots & \vdots & \ddots & \vdots \\ a_{n 1} & a_{n 2} & \ldots & a_{n n}\end{array}\right)\left(\begin{array}{c}x_{1} \\ x_{2} \\ \vdots \\ x_{n}\end{array}\right)\right)=\frac{\partial}{\partial \mathbf{x}^{\prime}}\left(\begin{array}{c}\sum_{j=1}^{n} a_{1 j} x_{j} \\ \sum_{j=1}^{n} a_{2 j} x_{j} \\ \vdots \\ \sum_{j=1}^{n} a_{n j} x_{j}\end{array}\right)=$

$$
\begin{aligned}
& =\left(\begin{array}{cccc}
\frac{\partial}{\partial x_{1}}\left(\sum_{j=1}^{n} a_{1 j} x_{j}\right) & \frac{\partial}{\partial x_{2}}\left(\sum_{j=1}^{n} a_{1 j} x_{j}\right) & \ldots & \frac{\partial}{\partial x_{n}}\left(\sum_{j=1}^{n} a_{1 j} x_{j}\right) \\
\frac{\partial}{\partial x_{1}}\left(\sum_{j=1}^{n} a_{2 j} x_{j}\right) & \frac{\partial}{\partial x_{2}}\left(\sum_{j=1}^{n} a_{2 j} x_{j}\right) & \ldots & \frac{\partial}{\partial x_{n}}\left(\sum_{j=1}^{n} a_{2 j} x_{j}\right) \\
\vdots & \vdots & \ddots & \vdots \\
\frac{\partial}{\partial x_{1}}\left(\sum_{j=1}^{n} a_{n j} x_{j}\right) & \frac{\partial}{\partial x_{2}}\left(\sum_{j=1}^{n} a_{n j} x_{j}\right) & \ldots & \frac{\partial}{\partial x_{n}}\left(\sum_{j=1}^{n} a_{n j} x_{j}\right)
\end{array}\right) \\
& =\left(\begin{array}{cccc}
a_{11} & a_{12} & \ldots & a_{1 n} \\
a_{21} & a_{22} & \ldots & a_{2 n} \\
\vdots & \vdots & \ddots & \vdots \\
a_{n 1} & a_{n 2} & \ldots & a_{n n}
\end{array}\right)=\mathbf{A} .
\end{aligned}
$$

4. $\frac{\partial}{\partial \mathbf{x}}\left(\mathbf{x}^{\prime} \mathbf{A}\right)=\frac{\partial}{\partial \mathbf{x}}\left(\sum_{i=1}^{n} a_{i 1} x_{i} \sum_{i=1}^{n} a_{i 2} x_{i} \quad \ldots \quad \sum_{i=1}^{n} a_{i n} x_{i}\right)=$ 


$$
\begin{aligned}
& =\left(\begin{array}{cccc}
\frac{\partial}{\partial x_{1}}\left(\sum_{i=1}^{n} a_{i 1} x_{i}\right) & \frac{\partial}{\partial x_{1}}\left(\sum_{i=1}^{n} a_{i 2} x_{i}\right) & \ldots & \frac{\partial}{\partial x_{1}}\left(\sum_{i=1}^{n} a_{i n} x_{i}\right) \\
\frac{\partial}{\partial x_{2}}\left(\sum_{i=1}^{n} a_{i 1} x_{i}\right) & \frac{\partial}{\partial x_{2}}\left(\sum_{i=1}^{n} a_{i 2} x_{i}\right) & \ldots & \frac{\partial}{\partial x_{2}}\left(\sum_{i=1}^{n} a_{i n} x_{i}\right) \\
\vdots & & \ddots & \vdots \\
\frac{\partial}{\partial x_{n}}\left(\sum_{i=1}^{n} a_{i 1} x_{i}\right) & \frac{\partial}{\partial x_{n}}\left(\sum_{i=1}^{n} a_{i 2} x_{i}\right) & \ldots & \frac{\partial}{\partial x_{n}}\left(\sum_{i=1}^{n} a_{i n} x_{i}\right)
\end{array}\right)= \\
= & \left(\begin{array}{cccc}
a_{11} & a_{12} & \ldots & a_{1 n} \\
a_{21} & a_{22} & \ldots & a_{2 n} \\
\vdots & \vdots & \ddots & \vdots \\
a_{n 1} & a_{n 2} & \ldots & a_{n n}
\end{array}\right)=\mathbf{A} .
\end{aligned}
$$

5. $\frac{\partial}{\partial \mathbf{x}}\left(\mathbf{x}^{\prime} \mathbf{A} \mathbf{x}\right)=\frac{\partial}{\partial \mathbf{x}}\left(\sum_{j=1}^{n} \sum_{i=1}^{n}\left(x_{i} a_{i j} x_{j}\right)\right)=\left(\begin{array}{c}\sum_{j=1}^{n} a_{1 j} x_{j}+\sum_{i=1}^{n} x_{i} a_{i 1} \\ \sum_{j=1}^{n} a_{2 j} x_{j}+\sum_{i=1}^{n} x_{i} a_{i 2} \\ \vdots \\ \sum_{j=1}^{n} a_{n j} x_{j}+\sum_{i=1}^{n} x_{i} a_{i n}\end{array}\right)=$ $=\left(\begin{array}{c}\sum_{j=1}^{n}\left(a_{1 j} x_{j}\right) \\ \sum_{j=1}^{n}\left(a_{2 j} x_{j}\right) \\ \vdots \\ \sum_{j=1}^{n}\left(a_{n j} x_{j}\right)\end{array}\right)+\left(\begin{array}{c}\sum_{i=1}^{n}\left(x_{i} a_{i 1}\right) \\ \sum_{i=1}^{n}\left(x_{i} a_{i 2}\right) \\ \vdots \\ \sum_{i=1}^{n}\left(x_{i} a_{i n}\right)\end{array}\right)=\mathbf{A} \mathbf{x}+\mathbf{A}^{\prime} \mathbf{x}=\left(\mathbf{A}+\mathbf{A}^{\prime}\right) \mathbf{x}$.

6. $\frac{\partial^{2}}{\partial \mathbf{x} \partial \mathbf{x}^{\prime}}\left(\mathbf{x}^{\prime} \mathbf{A} \mathbf{x}\right)=\frac{\partial^{2}}{\partial \mathbf{x} \partial \mathbf{x}^{\prime}}\left(\sum_{j=1}^{n} \sum_{i=1}^{n}\left(x_{i} a_{i_{j}} x_{j}\right)\right)=\frac{\partial}{\partial \mathbf{x}}\left(\frac{\partial}{\partial \mathbf{x}^{\prime}}\left(\sum_{j=1}^{n} \sum_{i=1}^{n} x_{i} a_{i_{j}} x_{j}\right)\right)=$

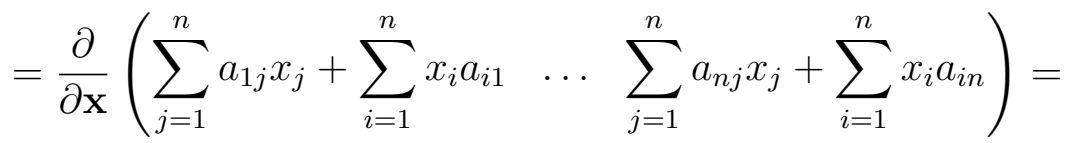
$=\left(\begin{array}{cccc}a_{11}+a_{11} & a_{12}+a_{21} & \ldots & a_{1 n}+a_{n 1} \\ a_{21}+a_{12} & a_{22}+a_{22} & \ldots & a_{2 n}+a_{n 2} \\ \vdots & \vdots & \ddots & \vdots \\ a_{n 1}+a_{1 n} & a_{n 2}+a_{2 n} & \ldots & a_{n n}+a_{n n}\end{array}\right)=\mathbf{A}+\mathbf{A}^{\prime}$.

7. Quando $\mathbf{A}$ é simétrica, $\mathbf{A}=\mathbf{A}^{\prime}$ e portanto $\left(\mathbf{A}+\mathbf{A}^{\prime}\right) \mathbf{x}=2 \mathbf{A x}$. 
8. Análogo a (7) utilizando (6).

9. $\frac{\partial}{\partial \mathbf{x}^{\prime}}(\mathbf{x})=\frac{\partial}{\partial \mathbf{x}^{\prime}}\left(\begin{array}{c}x_{1} \\ x_{2} \\ \vdots \\ x_{n}\end{array}\right)=\left(\begin{array}{cccc}\frac{\partial}{\partial x_{1}}\left(x_{1}\right) & \frac{\partial}{\partial x_{2}}\left(x_{1}\right) & \cdots & \frac{\partial}{\partial x_{n}}\left(x_{1}\right) \\ \frac{\partial}{\partial x_{1}}\left(x_{2}\right) & \frac{\partial}{\partial x_{2}}\left(x_{2}\right) & \ldots & \frac{\partial}{\partial x_{n}}\left(x_{2}\right) \\ \vdots & \vdots & \ddots & \vdots \\ \frac{\partial}{\partial x_{1}}\left(x_{n}\right) & \frac{\partial}{\partial x_{2}}\left(x_{n}\right) & \cdots & \frac{\partial}{\partial x_{n}}\left(x_{n}\right)\end{array}\right)=$

$$
=\left(\begin{array}{cccc}
1 & 0 & \ldots & 0 \\
0 & 1 & \ddots & \vdots \\
\vdots & \ddots & \ddots & 0 \\
0 & \ldots & 0 & 1
\end{array}\right)=\mathbf{I}_{n} .
$$

10. $\frac{\partial}{\partial \mathbf{x}}\left(\mathbf{x}^{\prime}\right)=\left(\begin{array}{cccc}\frac{\partial}{\partial x_{1}}\left(x_{1}\right) & \frac{\partial}{\partial x_{1}}\left(x_{2}\right) & \ldots & \frac{\partial}{\partial x_{1}}\left(x_{n}\right) \\ \frac{\partial}{\partial x_{2}}\left(x_{1}\right) & \frac{\partial}{\partial x_{2}}\left(x_{2}\right) & \ldots & \frac{\partial}{\partial x_{2}}\left(x_{n}\right) \\ \vdots & \vdots & \ddots & \vdots \\ \frac{\partial}{\partial x_{n}}\left(x_{1}\right) & \frac{\partial}{\partial x_{n}}\left(x_{2}\right) & \ldots & \frac{\partial}{\partial x_{n}}\left(x_{n}\right)\end{array}\right)=\left(\begin{array}{cccc}1 & 0 & \ldots & 0 \\ 0 & 1 & \ldots & 0 \\ \vdots & \vdots & \ddots & \vdots \\ 0 & 0 & \ldots & 1\end{array}\right)=\mathbf{I}_{n}$.

11. $\frac{\partial}{\partial \mathbf{x}}\left(\mathbf{y}^{\prime}\right)=\left(\begin{array}{cccc}\frac{\partial}{\partial x_{1}}\left(y_{1}\right) & \frac{\partial}{\partial x_{2}}\left(y_{1}\right) & \cdots & \frac{\partial}{\partial x_{n}}\left(y_{1}\right) \\ \frac{\partial}{\partial x_{1}}\left(y_{2}\right) & \frac{\partial}{\partial x_{2}}\left(y_{2}\right) & \cdots & \frac{\partial}{\partial x_{n}}\left(y_{2}\right) \\ \vdots & \vdots & \ddots & \vdots \\ \frac{\partial}{\partial x_{1}}\left(y_{n}\right) & \frac{\partial}{\partial x_{2}}\left(y_{n}\right) & \cdots & \frac{\partial}{\partial x_{n}}\left(y_{n}\right)\end{array}\right)^{\prime}=\left(\frac{\partial}{\partial \mathbf{x}^{\prime}}(\mathbf{y})\right)^{\prime}$

12. Caso particular de (11) quando $y \in \mathbb{R}$ :

$$
\frac{\partial}{\partial \mathbf{x}}(y)=\left(\begin{array}{c}
\frac{\partial}{\partial x_{1}}(y) \\
\frac{\partial}{\partial x_{2}}(y) \\
\vdots \\
\frac{\partial}{\partial x_{n}}(y)
\end{array}\right)=\left(\frac{\partial}{\partial \mathbf{x}^{\prime}}(y)\right)^{\prime}
$$

13. Omitiremos a prova desta propriedade, que pode ser encontrada em Nel (1980).

14. $\frac{\partial}{\partial \phi}(\log |\mathbf{D}(\phi)|)=\mathbf{D}^{-1}(\phi) \mathbf{1}_{n}$ : 


$$
\begin{aligned}
& \frac{\partial}{\partial \phi}(\log |\mathbf{D}(\phi)|)=\frac{\partial}{\partial \phi} \log \left|\begin{array}{cccc}
\phi_{1} & 0 & \ldots & 0 \\
0 & \phi_{2} & \ddots & \vdots \\
\vdots & \ddots & \ddots & 0 \\
0 & \ldots & 0 & \phi_{n}
\end{array}\right|=\frac{\partial}{\partial \phi} \log \left(\phi_{1} \phi_{2} \ldots \phi_{n}\right)= \\
& =\left(\begin{array}{c}
\frac{\partial}{\partial \phi_{1}}\left(\log \phi_{1}+\log \phi_{2} \ldots+\log \phi_{n}\right) \\
\frac{\partial}{\partial \phi_{2}}\left(\log \phi_{1}+\log \phi_{2} \ldots+\log \phi_{n}\right) \\
\vdots \\
\frac{\partial}{\partial \phi_{n}}\left(\log \phi_{1}+\log \phi_{2} \ldots+\log \phi_{n}\right)
\end{array}\right)=\left(\begin{array}{c}
\frac{1}{\phi_{1}} \\
\frac{1}{\phi_{2}} \\
\vdots \\
\frac{1}{\phi_{n}}
\end{array}\right)= \\
& =\left(\begin{array}{cccc}
\frac{1}{\phi_{1}} & 0 & \ldots & 0 \\
0 & \frac{1}{\phi_{2}} & \ddots & \vdots \\
\vdots & \ddots & \ddots & 0 \\
0 & \ldots & 0 & \frac{1}{\phi_{n}}
\end{array}\right)\left(\begin{array}{c}
1 \\
1 \\
\vdots \\
1
\end{array}\right)=\mathbf{D}^{-1}(\boldsymbol{\phi}) \mathbf{1}_{n}
\end{aligned}
$$

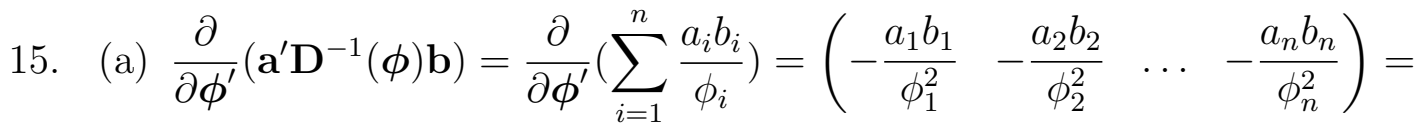

$$
=-\mathbf{a}^{\prime} \mathbf{D}^{-2}(\phi) \mathbf{D}(\mathbf{b})
$$

(b) $\left(\frac{\partial}{\partial \phi}\left(\mathbf{b}^{\prime} \mathbf{D}^{-1}(\phi) \mathbf{a}\right)\right)^{\prime}=-\mathbf{a}^{\prime} \mathbf{D}^{-2}(\phi) \mathbf{D}(\mathbf{b})$ :

Basta utilizar (12) em (15a).

16. (a) $\frac{\partial}{\partial \phi^{\prime}}\left(\mathbf{D}^{-1}(\boldsymbol{\phi}) \mathbf{a}\right)=\frac{\partial}{\partial \phi^{\prime}}\left(\begin{array}{c}\frac{a_{1}}{\phi_{1}} \\ \frac{a_{2}}{\phi_{2}} \\ \vdots \\ \frac{a_{n}}{\phi_{n}}\end{array}\right)=\left(\begin{array}{cccc}-\frac{a_{1}}{\phi_{1}^{2}} & 0 & \cdots & 0 \\ 0 & -\frac{a_{2}}{\phi_{2}^{2}} & \ddots & \vdots \\ & \ddots & \ddots & 0 \\ \vdots & \ldots & 0 & -\frac{a_{n}}{\phi_{n}^{2}}\end{array}\right)=$

$$
=-\left(\begin{array}{cccc}
\phi_{1}^{-2} & 0 & \ldots & 0 \\
0 & \phi_{2}^{-2} & \ddots & \vdots \\
\vdots & \ddots & \ddots & 0 \\
0 & \ldots & 0 & \phi_{n}^{-2}
\end{array}\right)\left(\begin{array}{cccc}
a_{1} & 0 & \ldots & 0 \\
0 & a_{2} & \ddots & \vdots \\
\vdots & \ddots & \ddots & 0 \\
0 & \ldots & 0 & a_{n}
\end{array}\right)=-\mathbf{D}^{-2}(\boldsymbol{\phi}) \mathbf{D}(\mathbf{a}) \text {. }
$$

(b) $\left(\frac{\partial}{\partial \phi}\left(\mathbf{a}^{\prime} \mathbf{D}^{-1}(\phi)\right)\right)^{\prime}=-\mathbf{D}^{-2}(\phi) \mathbf{D}(\mathbf{a})$ 
Basta utilizar (11) em (16a).

17. (a) $\frac{\partial}{\partial \phi^{\prime}}\left(\mathbf{D}^{-2}(\boldsymbol{\phi}) \mathbf{D}(\mathbf{a}) \mathbf{b}\right)=\frac{\partial}{\partial \phi^{\prime}}\left(\begin{array}{c}\frac{a_{1} b_{1}}{\phi_{1}^{2}} \\ \frac{a_{2} b_{2}}{\phi_{2}^{2}} \\ \vdots \\ \frac{a_{n} b_{n}}{\phi_{n}^{2}}\end{array}\right)=\left(\begin{array}{cccc}\frac{-2 a_{1} b_{1}}{\phi_{1}^{3}} & 0 & \cdots & 0 \\ 0 & \frac{-2 a_{2} b_{2}}{\phi_{2}^{3}} & \ddots & \vdots \\ \vdots & \ddots & \ddots & 0 \\ 0 & \ldots & 0 & \frac{-2 a_{n} b_{n}}{\phi_{n}^{3}}\end{array}\right)=$ $=-2 \mathbf{D}^{-3}(\boldsymbol{\phi}) \mathbf{D}(\mathbf{a}) \mathbf{D}(\mathbf{b})$

(b) $\left(\frac{\partial}{\partial \phi}\left(\mathbf{b}^{\prime} \mathbf{D}(\mathbf{a}) \mathbf{D}^{-2}(\boldsymbol{\phi})\right)\right)^{\prime}=-2 \mathbf{D}^{-3}(\boldsymbol{\phi}) \mathbf{D}(\mathbf{a}) \mathbf{D}(\mathbf{b})$ :

Basta utilizar (11) em (17a).

18. Temos $y_{1}=y_{1}(\mathbf{x}) \in \mathbb{R}$ e $y_{2}=y_{2}(\mathbf{x}) \in \mathbb{R}, \mathbf{x}=\left(x_{1}, x_{2}, \ldots, x_{n}\right)^{\prime}$ e queremos mostrar que:

(a) $\frac{\partial}{\partial \mathbf{x}}\left(y_{1} y_{2}\right)=\frac{\partial}{\partial \mathbf{x}}\left(y_{1}\right) y_{2}+y_{1} \frac{\partial}{\partial \mathbf{x}}\left(y_{2}\right)$ :

$\frac{\partial}{\partial \mathbf{x}}\left(y_{1} y_{2}\right)=\frac{\partial}{\partial \mathbf{x}}\left(y_{1}(\mathbf{x}) y_{2}(\mathbf{x})\right)=$

$=\left(\begin{array}{c}\frac{\partial}{\partial x_{1}}\left(y_{1}(\mathbf{x}) y_{2}(\mathbf{x})\right) \\ \frac{\partial}{\partial x_{2}}\left(y_{1}(\mathbf{x}) y_{2}(\mathbf{x})\right) \\ \vdots \\ \frac{\partial}{\partial x_{n}}\left(y_{1}(\mathbf{x}) y_{2}(\mathbf{x})\right)\end{array}\right)=\left(\begin{array}{c}\frac{\partial}{\partial x_{1}}\left(y_{1}(\mathbf{x})\right) y_{2}(\mathbf{x})+y_{1}(\mathbf{x}) \frac{\partial}{\partial x_{1}}\left(y_{2}(\mathbf{x})\right) \\ \frac{\partial}{\partial x_{2}}\left(y_{1}(\mathbf{x})\right) y_{2}(\mathbf{x})+y_{1}(\mathbf{x}) \frac{\partial}{\partial x_{2}}\left(y_{2}(\mathbf{x})\right) \\ \vdots \\ \frac{\partial}{\partial x_{n}}\left(y_{1}(\mathbf{x})\right) y_{2}(\mathbf{x})+y_{1}(\mathbf{x}) \frac{\partial}{\partial x_{n}}\left(y_{2}(\mathbf{x})\right)\end{array}\right)=$

$=y_{2}(\mathbf{x})\left(\begin{array}{c}\frac{\partial}{\partial x_{1}}\left(y_{1}(\mathbf{x})\right) \\ \frac{\partial}{\partial x_{2}}\left(y_{1}(\mathbf{x})\right) \\ \vdots \\ \frac{\partial}{\partial x_{n}}\left(y_{1}(\mathbf{x})\right)\end{array}\right)+y_{1}(\mathbf{x})\left(\begin{array}{c}\frac{\partial}{\partial x_{1}}\left(y_{2}(\mathbf{x})\right) \\ \frac{\partial}{\partial x_{2}}\left(y_{2}(\mathbf{x})\right) \\ \vdots \\ \frac{\partial}{\partial x_{n}}\left(y_{2}(\mathbf{x})\right)\end{array}\right)=\frac{\partial}{\partial \mathbf{x}}\left(y_{1}\right) y_{2}+\frac{\partial}{\partial \mathbf{x}}\left(y_{2}\right) y_{1}$

(b) $\frac{\partial}{\partial \mathbf{x}^{\prime}}\left(y_{1} y_{2}\right)=\frac{\partial}{\partial \mathbf{x}^{\prime}}\left(y_{1}\right) y_{2}+y_{1} \frac{\partial}{\partial \mathbf{x}^{\prime}}\left(y_{2}\right)$ :

$\frac{\partial}{\partial \mathbf{x}^{\prime}}\left(y_{1} y_{2}\right)=\frac{\partial}{\partial \mathbf{x}^{\prime}}\left(y_{1}(\mathbf{x}) y_{2}(\mathbf{x})\right)=$

$=\left(\frac{\partial}{\partial x_{1}}\left(y_{1}(\mathbf{x}) y_{2}(\mathbf{x})\right) \quad \frac{\partial}{\partial x_{2}}\left(y_{1}(\mathbf{x}) y_{2}(\mathbf{x})\right) \quad \ldots \quad \frac{\partial}{\partial x_{n}}\left(y_{1}(\mathbf{x}) y_{2}(\mathbf{x})\right)\right)=$ 


$$
\begin{aligned}
& =y_{2}(\mathbf{x})\left(\frac{\partial}{\partial x_{1}}\left(y_{1}(\mathbf{x})\right) \quad \frac{\partial}{\partial x_{2}}\left(y_{1}(\mathbf{x})\right) \quad \ldots \quad \frac{\partial}{\partial x_{n}}\left(y_{1}(\mathbf{x})\right)\right)+ \\
& +y_{1}(\mathbf{x})\left(\frac{\partial}{\partial x_{1}}\left(y_{2}(\mathbf{x})\right) \quad \frac{\partial}{\partial x_{2}}\left(y_{2}(\mathbf{x})\right) \quad \ldots \quad \frac{\partial}{\partial x_{n}}\left(y_{2}(\mathbf{x})\right)\right)=\frac{\partial}{\partial \mathbf{x}^{\prime}}\left(y_{1}\right) y_{2}+\frac{\partial}{\partial \mathbf{x}^{\prime}}\left(y_{2}\right) y_{1} .
\end{aligned}
$$

19. Temos $y=y(\mathbf{x}) \in \mathbb{R}$ e $\mathbf{z}=\mathbf{z}(\mathbf{x})=\left(z_{1}(\mathbf{x}), z_{2}(\mathbf{x}), \ldots, z_{m}(\mathbf{x})\right)^{\prime}, \mathbf{x}=\left(x_{1}, x_{2}, \ldots, x_{n}\right)^{\prime}$ e queremos mostrar que:

$$
\text { (a) } \begin{aligned}
& \frac{\partial}{\partial \mathbf{x}}\left(y \mathbf{z}^{\prime}\right)=\frac{\partial}{\partial \mathbf{x}}(y) \mathbf{z}^{\prime}+y \frac{\partial}{\partial \mathbf{x}}\left(\mathbf{z}^{\prime}\right): \\
& \frac{\partial}{\partial \mathbf{x}}\left(y \mathbf{z}^{\prime}\right)=\frac{\partial}{\partial \mathbf{x}}\left(y(\mathbf{x})(\mathbf{z}(\mathbf{x}))^{\prime}\right)=\frac{\partial}{\partial \mathbf{x}}\left(\begin{array}{llll}
y(\mathbf{x}) z_{1}(\mathbf{x}) & y(\mathbf{x}) z_{2}(\mathbf{x}) & \ldots & \left.y(\mathbf{x}) z_{m}(\mathbf{x})\right)= \\
= & \left(\begin{array}{cccc}
\frac{\partial}{\partial x_{1}}(y(\mathbf{x})) z_{1}(\mathbf{x}) & \frac{\partial}{\partial x_{1}}(y(\mathbf{x})) z_{2}(\mathbf{x}) & \ldots & \frac{\partial}{\partial x_{1}}(y(\mathbf{x})) z_{m}(\mathbf{x}) \\
\frac{\partial}{\partial x_{2}}(y(\mathbf{x})) z_{1}(\mathbf{x}) & \frac{\partial}{\partial x_{2}}(y(\mathbf{x})) z_{2}(\mathbf{x}) & \ldots & \frac{\partial}{\partial x_{2}}(y(\mathbf{x})) z_{m}(\mathbf{x}) \\
\vdots & \vdots & \ddots & \vdots \\
\frac{\partial}{\partial x_{n}}(y(\mathbf{x})) z_{1}(\mathbf{x}) & \frac{\partial}{\partial x_{n}}(y(\mathbf{x})) z_{2}(\mathbf{x}) & \ldots & \frac{\partial}{\partial x_{n}}(y(\mathbf{x})) z_{m}(\mathbf{x})
\end{array}\right)+ \\
+ & \left(\begin{array}{cccc}
y(\mathbf{x}) \frac{\partial}{\partial x_{1}}\left(z_{1}(\mathbf{x})\right) & y(\mathbf{x}) \frac{\partial}{\partial x_{1}}\left(z_{2}(\mathbf{x})\right) & \ldots & y(\mathbf{x}) \frac{\partial}{\partial x_{1}}\left(z_{m}(\mathbf{x})\right) \\
y(\mathbf{x}) \frac{\partial}{\partial x_{2}}\left(z_{1}(\mathbf{x})\right) & y(\mathbf{x}) \frac{\partial}{\partial x_{2}}\left(z_{2}(\mathbf{x})\right) & \ldots & y(\mathbf{x}) \frac{\partial}{\partial x_{2}}\left(z_{m}(\mathbf{x})\right) \\
\vdots & \vdots & \ddots & \vdots \\
y(\mathbf{x}) \frac{\partial}{\partial x_{n}}\left(z_{1}(\mathbf{x})\right) & y(\mathbf{x}) \frac{\partial}{\partial x_{n}}\left(z_{2}(\mathbf{x})\right) & \ldots & y(\mathbf{x}) \frac{\partial}{\partial x_{n}}\left(z_{m}(\mathbf{x})\right)
\end{array}\right)= \\
= & \frac{\partial}{\partial \mathbf{x}}(y) \mathbf{z}^{\prime}+y \frac{\partial}{\partial \mathbf{x}}\left(\mathbf{z}^{\prime}\right) . &
\end{array}\right)
\end{aligned}
$$

(b) $\frac{\partial}{\partial \mathbf{x}^{\prime}}(y \mathbf{z})=\mathbf{z} \frac{\partial}{\partial \mathbf{x}^{\prime}}(y)+y(\mathbf{x}) \frac{\partial}{\partial \mathbf{x}^{\prime}}(\mathbf{z})$ :

$$
\frac{\partial}{\partial \mathbf{x}^{\prime}}(y \mathbf{z})=\frac{\partial}{\partial \mathbf{x}^{\prime}}(y(\mathbf{x}) \mathbf{z}(\mathbf{x}))=\frac{\partial}{\partial \mathbf{x}^{\prime}}\left(\begin{array}{c}
y(\mathbf{x}) z_{1}(\mathbf{x}) \\
y(\mathbf{x}) z_{2}(\mathbf{x}) \\
\vdots \\
y(\mathbf{x}) z_{m}(\mathbf{x})
\end{array}\right)=
$$




$$
\begin{aligned}
& =\left(\begin{array}{cccc}
\frac{\partial}{\partial x_{1}}(y(x)) z_{1}(\mathbf{x}) & \frac{\partial}{\partial x_{2}}(y(\mathbf{x})) z_{1}(\mathbf{x}) & \ldots & \frac{\partial}{\partial x_{n}}(y(\mathbf{x})) z_{1}(\mathbf{x}) \\
\frac{\partial}{\partial x_{1}}(y(x)) z_{2}(\mathbf{x}) & \frac{\partial}{\partial x_{2}}(y(\mathbf{x})) z_{2}(\mathbf{x}) & \ldots & \frac{\partial}{\partial x_{n}}(y(\mathbf{x})) z_{2}(\mathbf{x}) \\
\vdots & \vdots & \ddots & \vdots \\
\frac{\partial}{\partial x_{1}}(y(x)) z_{m}(\mathbf{x}) & \frac{\partial}{\partial x_{2}}(y(\mathbf{x})) z(\mathbf{x}) & \ldots & \frac{\partial}{\partial x_{n}}(y(\mathbf{x})) z_{m}(\mathbf{x})
\end{array}\right)+ \\
& +\left(\begin{array}{cccc}
y(\mathbf{x}) \frac{\partial}{\partial x_{1}}\left(z_{1}(\mathbf{x})\right) & y(\mathbf{x}) \frac{\partial}{\partial x_{2}}\left(z_{1}(\mathbf{x})\right) & \ldots & y(\mathbf{x}) \frac{\partial}{\partial x_{n}}\left(z_{1}(\mathbf{x})\right) \\
y(\mathbf{x}) \frac{\partial}{\partial x_{1}}\left(z_{2}(\mathbf{x})\right) & y(\mathbf{x}) \frac{\partial}{\partial x_{2}}\left(z_{2}(\mathbf{x})\right) & \ldots & y(\mathbf{x}) \frac{\partial}{\partial x_{n}}\left(z_{2}(\mathbf{x})\right) \\
\vdots & & \ddots & \vdots \\
y(\mathbf{x}) \frac{\partial}{\partial x_{1}}\left(z_{m}(\mathbf{x})\right) & y(\mathbf{x}) \frac{\partial}{\partial x_{2}}\left(z_{m}(\mathbf{x})\right) & \ldots & y(\mathbf{x}) \frac{\partial}{\partial x_{n}}\left(z_{m}(\mathbf{x})\right)
\end{array}\right)= \\
& = \\
& \mathbf{z} \frac{\partial}{\partial \mathbf{x}^{\prime}}(y)+y \frac{\partial}{\partial \mathbf{x}^{\prime}}(\mathbf{z}) .
\end{aligned}
$$

20. Temos $\mathbf{y}=\mathbf{y}(\mathbf{x})=\left(y_{1}(\mathbf{x}), y_{2}(\mathbf{x}), \ldots, y_{m}(\mathbf{x})\right)^{\prime}$ e $\mathbf{z}=\mathbf{z}(\mathbf{x})=\left(z_{1}(\mathbf{x}), z_{2}(\mathbf{x}), \ldots, z_{m}(\mathbf{x})\right)^{\prime}$, $\mathbf{x}=\left(x_{1}, x_{2}, \ldots, x_{n}\right)^{\prime}$, queremos mostrar que

(a) $\frac{\partial}{\partial \mathbf{x}}\left(\mathbf{y}^{\prime} \mathbf{z}\right)=\frac{\partial}{\partial \mathbf{x}}\left(\mathbf{y}^{\prime}\right) \mathbf{z}+\frac{\partial}{\partial \mathbf{x}}\left(\mathbf{z}^{\prime}\right) \mathbf{y}:$

$$
\begin{aligned}
& \frac{\partial}{\partial \mathbf{x}}\left(\mathbf{y}^{\prime} \mathbf{z}\right)=\frac{\partial}{\partial \mathbf{x}}\left(\mathbf{y}^{\prime}(\mathbf{x}) \mathbf{z}(\mathbf{x})\right)=\frac{\partial}{\partial \mathbf{x}}\left(\sum_{i=1}^{m} y_{i}(\mathbf{x}) z_{i}(\mathbf{x})\right)= \\
& =\left(\begin{array}{c}
\left.\sum_{i=1}^{m} \frac{\partial}{\partial x_{1}}\left(y_{i}(\mathbf{x})\right) z_{i}(\mathbf{x})\right) \\
\sum_{i=1}^{m} \frac{\partial}{\partial x_{2}}\left(y_{i}(\mathbf{x})\right) z_{i}(\mathbf{x}) \\
\vdots \\
\sum_{i=1}^{m} \frac{\partial}{\partial x_{n}}\left(y_{i}(\mathbf{x})\right) z_{i}(\mathbf{x})
\end{array}\right)+\left(\begin{array}{c}
\sum_{i=1}^{m} y_{i}(\mathbf{x}) \frac{\partial}{\partial x_{1}}\left(z_{i}(\mathbf{x})\right) \\
\sum_{i=1}^{m} y_{i}(\mathbf{x}) \frac{\partial}{\partial x_{2}}\left(z_{i}(\mathbf{x})\right) \\
\vdots \\
\sum_{i=1}^{m} y_{i}(\mathbf{x}) \frac{\partial}{\partial x_{n}}\left(z_{i}(\mathbf{x})\right)
\end{array}\right)=\frac{\partial}{\partial \mathbf{x}}\left(\mathbf{y}^{\prime}\right) \mathbf{z}+\frac{\partial}{\partial \mathbf{x}}\left(\mathbf{z}^{\prime}\right) \mathbf{y}
\end{aligned}
$$

(b) $\frac{\partial}{\partial \mathbf{x}^{\prime}}\left(\mathbf{y}^{\prime} \mathbf{z}\right)=\mathbf{z}^{\prime} \frac{\partial}{\partial \mathbf{x}^{\prime}}(\mathbf{y})+\mathbf{y}^{\prime} \frac{\partial}{\partial \mathbf{x}^{\prime}}(\mathbf{z})$ :

$$
\begin{aligned}
& \frac{\partial}{\partial \mathbf{x}^{\prime}}\left(\mathbf{y}^{\prime} \mathbf{z}\right)=\frac{\partial}{\partial \mathbf{x}^{\prime}}\left(\mathbf{y}^{\prime}(\mathbf{x}) \mathbf{z}(\mathbf{x})\right)=\frac{\partial}{\partial \mathbf{x}^{\prime}}\left(\sum_{i=1}^{m}\left(y_{i}(\mathbf{x}) z_{i}(\mathbf{x})\right)\right)= \\
& =\left(\sum_{i=1}^{m}\left(\frac{\partial}{\partial x_{1}}\left(y_{i}(\mathbf{x}) z_{i}(\mathbf{x})\right)\right) \quad \ldots \sum_{i=1}^{m}\left(\frac{\partial}{\partial x_{n}}\left(y_{i}(\mathbf{x}) z_{i}(\mathbf{x})\right)\right)\right)= \\
& =\left(\sum_{i=1}^{m}\left(\frac{\partial}{\partial x_{1}}\left(y_{i}(\mathbf{x})\right) z_{i}(\mathbf{x})\right) \quad \ldots \sum_{i=1}^{m}\left(\frac{\partial}{\partial x_{n}}\left(y_{i}(\mathbf{x})\right) z_{i}(\mathbf{x})\right)\right)+
\end{aligned}
$$




$$
\begin{aligned}
& +\left(\sum_{i=1}^{m}\left(y_{i}(\mathbf{x}) \frac{\partial}{\partial x_{1}}\left(z_{i}(\mathbf{x})\right)\right) \ldots \sum_{i=1}^{m}\left(y_{i}(\mathbf{x}) \frac{\partial}{\partial x_{n}}\left(z_{i}(\mathbf{x})\right)\right)\right)= \\
& =\mathbf{z}^{\prime} \frac{\partial}{\partial \mathbf{x}^{\prime}}(\mathbf{y})+\mathbf{y}^{\prime} \frac{\partial}{\partial \mathbf{x}^{\prime}}(\mathbf{z})
\end{aligned}
$$




\section{Apêndice $\mathrm{C}$}

\section{Cálculo da função escore}

Apresentaremos neste apêndice alguns passos do desenvolvimento das derivadas primeiras da log-verossimilhança $L(\mathbf{z}, \boldsymbol{\theta})$ (equação 2.4) em relação aos parâmetros, ou seja, a função escore. As derivadas foram desenvolvidas integralmente, porém apenas indicaremos alguns cálculos. Para tanto, utilizaremos propriedades de derivadas a valores vetoriais (Graybill 1983, Nel 1980) e as propriedades descritas na Seção B.3, e as formas $\mathbf{a}_{i}, \mathbf{A}_{i}, b_{i}$ e $\mathbf{B}_{i}$ definidos em (2.6) e (2.7).

A função log-verossimilhança é dada matricialmente por

$$
L(\mathbf{z}, \boldsymbol{\theta})=-\frac{3 N}{2} \log (2 \pi)-\frac{1}{2} \sum_{i=1}^{p} n_{i} \log \left|\mathbf{V}_{i}\right|-\frac{1}{2} \sum_{i=1}^{p} \sum_{j=1}^{n_{i}}\left(\mathbf{z}_{i_{j}}-\mathbf{m}_{i}\right)^{\prime} \mathbf{V}_{i}^{-1}\left(\mathbf{z}_{i_{j}}-\mathbf{m}_{i}\right) .
$$

Definimos $d_{i_{j}}=\left(\mathbf{z}_{i_{j}}-\mathbf{m}_{i}\right)^{\prime} \mathbf{V}_{i}^{-1}\left(\mathbf{z}_{i_{j}}-\mathbf{m}_{i}\right)$, e apresentaremos alguns cálculos das derivadas primeiras de $\log \left|\mathbf{V}_{i}\right|$ e $d_{i_{j}}$, e em seguida obteremos as expressões das derivadas de $L(\mathbf{z}, \boldsymbol{\theta})$.

Derivadas de $\log \left|\mathbf{V}_{i}\right|$ em relação aos parâmetros

1. $\frac{\partial}{\partial \boldsymbol{\beta}_{i}}\left(\log \left|\mathbf{V}_{i}\right|\right)=\frac{1}{\left|\mathbf{V}_{i}\right|} \frac{\partial}{\partial \boldsymbol{\beta}_{i}}\left(b_{i}\left|\mathbf{A}_{i}\right|\right)=\frac{\left|\mathbf{A}_{i}\right|}{\left|\mathbf{V}_{i}\right|} \frac{\partial}{\partial \boldsymbol{\beta}_{i}}\left(b_{i}\right)=$

$$
=\frac{\left|\mathbf{A}_{i}\right|}{\left|\mathbf{V}_{i}\right|} \frac{\partial}{\partial \boldsymbol{\beta}_{i}}\left(1+\sigma_{x}^{2}\left[\sigma^{-2}+\boldsymbol{\beta}_{i}^{\prime} \mathbf{D}^{-1}\left(\boldsymbol{\sigma}_{e_{i}}^{2}\right) \boldsymbol{\beta}_{i}\right]\right)=2 \sigma_{x}^{2} b_{i}^{-1} \mathbf{D}^{-1}\left(\boldsymbol{\sigma}_{e_{i}}^{2}\right) \boldsymbol{\beta}_{i}
$$

2. $\frac{\partial}{\partial \mu}\left(\log \left|\mathbf{V}_{i}\right|\right)=0$ diretamente.

3. $\frac{\partial}{\partial \sigma_{x}^{2}}\left(\log \left|\mathbf{V}_{i}\right|\right)=\frac{1}{\left|\mathbf{V}_{i}\right|} \frac{\partial}{\partial \sigma_{x}^{2}}\left(b_{i}\left|\mathbf{A}_{i}\right|\right)=\frac{\left|\mathbf{A}_{i}\right|}{\left|\mathbf{V}_{i}\right|} \frac{\partial}{\partial \sigma_{x}^{2}} b_{i}=$

$$
\begin{aligned}
& =\frac{\left|\mathbf{A}_{i}\right|}{\left|\mathbf{V}_{i}\right|} \frac{\partial}{\partial \sigma_{x}^{2}}\left(1+\sigma_{x}^{2}\left[\sigma^{-2}+\boldsymbol{\beta}_{i}^{\prime} \mathbf{D}^{-1}\left(\boldsymbol{\sigma}_{e_{i}}^{2}\right) \boldsymbol{\beta}_{i}\right]\right) \\
& =b_{i}^{-1}\left[\sigma^{-2}+\boldsymbol{\beta}_{i}^{\prime} \mathbf{D}^{-1}\left(\boldsymbol{\sigma}_{e_{i}}^{2}\right) \boldsymbol{\beta}_{i}\right]=b_{i}^{-1} \sigma_{x}^{-2}\left(b_{i}-1\right)=\sigma_{x}^{-2}\left(1-b_{i}^{-1}\right) .
\end{aligned}
$$


4. $\frac{\partial}{\partial \sigma^{2}}\left(\log \left|\mathbf{V}_{i}\right|\right)=\frac{1}{\left|\mathbf{V}_{i}\right|} \frac{\partial}{\partial \sigma^{2}}\left(b_{i}\left|\mathbf{A}_{i}\right|\right)=\frac{1}{\left|\mathbf{V}_{i}\right|}\left\{\frac{\partial}{\partial \sigma^{2}} b_{i}\left|\mathbf{A}_{i}\right|+b_{i} \frac{\partial}{\partial \sigma^{2}}\left(\left|\mathbf{A}_{i}\right|\right)\right\}=$

$=\frac{\partial}{\partial \sigma^{2}}\left\{1+\sigma_{x}^{2}\left[\sigma^{-2}+\boldsymbol{\beta}_{i}^{\prime} \mathbf{D}^{-1}\left(\boldsymbol{\sigma}_{e_{i}}^{2}\right) \boldsymbol{\beta}_{i}\right]\right\}\left|\mathbf{A}_{i}\right|+b_{i} \frac{\partial}{\partial \sigma^{2}}\left\{\sigma^{2}\left|\mathbf{D}\left(\boldsymbol{\sigma}_{e_{i}}^{2}\right)\right|\right\}=$

$=-\sigma_{x}^{2} \sigma^{-4}\left|\mathbf{A}_{i}\right|+b_{i}\left|\mathbf{D}\left(\boldsymbol{\sigma}_{e_{i}}^{2}\right)\right|=\sigma^{-2}\left(1-\sigma_{x}^{2} \sigma^{-2} b_{i}^{-1}\right)$.

5. $\frac{\partial}{\partial \boldsymbol{\sigma}_{e_{i}}^{2}}\left(\log \left|\mathbf{V}_{i}\right|\right)=\frac{1}{\left|\mathbf{V}_{i}\right|} \frac{\partial}{\boldsymbol{\sigma}_{e_{i}}^{2}}\left(b_{i}\left|\mathbf{A}_{i}\right|\right)=$

$=\frac{\partial}{\partial \boldsymbol{\sigma}_{e_{i}}^{2}}\left\{1+\sigma_{x}^{2}\left[\sigma^{-2}+\boldsymbol{\beta}_{i}^{\prime} \mathbf{D}^{-1}\left(\boldsymbol{\sigma}_{e_{i}}^{2}\right) \boldsymbol{\beta}_{i}\right]\right\}\left|\mathbf{A}_{i}\right|+b_{i} \frac{\partial}{\partial \boldsymbol{\sigma}_{e_{i}}^{2}}\left\{\sigma^{2}\left|\mathbf{D}\left(\boldsymbol{\sigma}_{e_{i}}^{2}\right)\right|\right\}=$

$=\frac{\partial}{\partial \boldsymbol{\sigma}_{e_{i}}^{2}}\left(\log \left|\mathbf{V}_{i}\right|\right)=-\sigma_{x}^{2} b_{i}^{-1} \mathbf{D}\left(\boldsymbol{\beta}_{i}\right) \mathbf{D}^{-2}\left(\boldsymbol{\sigma}_{e_{i}}^{2}\right) \boldsymbol{\beta}_{i}+\mathbf{D}^{-1}\left(\boldsymbol{\sigma}_{e_{i}}^{2}\right) \mathbf{1}_{2}$.

\section{Derivadas de $d_{i_{j}}$ em relação aos parâmetros}

Seja

$$
\begin{aligned}
& d_{i_{j}}=\left[\left(\mathbf{z}_{i_{j}}-\mathbf{m}_{i}\right)^{\prime} \mathbf{V}_{i}^{-1}\left(\mathbf{z}_{i_{j}}-\mathbf{m}_{i}\right)\right]= \\
& =\left(\mathbf{z}_{i_{j}}-\mathbf{m}_{i}\right)^{\prime}\left(\mathbf{A}_{i}^{-1}-\sigma_{x}^{2} b_{i}^{-1} \mathbf{B}_{i}\right)\left(\mathbf{z}_{i_{j}}-\mathbf{m}_{i}\right)= \\
& =\left[\left(\mathbf{z}_{i_{j}}-\mathbf{m}_{i}\right)^{\prime} \mathbf{A}_{i}^{-1}\left(\mathbf{z}_{i_{j}}-\mathbf{m}_{i}\right)\right]-\sigma_{x}^{2} b_{i}^{-1}\left[\left(\mathbf{z}_{i_{j}}-\mathbf{m}_{i}\right)^{\prime} \mathbf{B}_{i}\left(\mathbf{z}_{i_{j}}-\mathbf{m}_{i}\right)\right] .
\end{aligned}
$$

Desenvolvendo os produtos ficamos com

$$
\begin{aligned}
& d_{i_{j}}=\sigma^{-2}\left(x_{i_{j}}-\mu\right)^{2}+\left(\mathbf{y}_{i_{j}}-\boldsymbol{\beta}_{i} \mu\right)^{\prime} \mathbf{D}^{-1}\left(\boldsymbol{\sigma}_{e_{i}}^{2}\right)\left(\mathbf{y}_{i_{j}}-\boldsymbol{\beta}_{i} \mu\right) \\
& -\sigma_{x}^{2} b_{i}^{-1}\left\{\sigma^{-4}\left(x_{i_{j}}-\mu\right)^{2}+2 \sigma^{-2}\left(x_{i_{j}}-\mu\right)\left[\left(\mathbf{y}_{i_{j}}-\boldsymbol{\beta}_{i} \mu\right)^{\prime} \mathbf{D}^{-1}\left(\boldsymbol{\sigma}_{e_{i}}^{2}\right) \boldsymbol{\beta}_{i}\right]\right. \\
& \left.+\left[\left(\mathbf{y}_{i_{j}}-\boldsymbol{\beta}_{i} \mu\right)^{\prime} \mathbf{D}^{-1}\left(\boldsymbol{\sigma}_{e_{i}}^{2}\right) \boldsymbol{\beta}_{i} \boldsymbol{\beta}_{i}^{\prime} \mathbf{D}^{-1}\left(\boldsymbol{\sigma}_{e_{i}}^{2}\right)\left(\mathbf{y}_{i_{j}}-\boldsymbol{\beta}_{i} \mu\right)\right]\right\}
\end{aligned}
$$

Assim

$$
\begin{aligned}
& d_{i_{j}}=\left(x_{i_{j}}-\mu\right)^{2}\left(\sigma^{-2}-\sigma_{x}^{2} b_{i}^{-1} \sigma^{-4}\right) \\
& -\sigma^{-2} \sigma_{x}^{2} b_{i}^{-1}\left(x_{i_{j}}-\mu\right)\left[\left(\mathbf{y}_{i_{j}}-\boldsymbol{\beta}_{i} \mu\right)^{\prime} \mathbf{D}^{-1}\left(\boldsymbol{\sigma}_{e_{i}}^{2}\right) \boldsymbol{\beta}_{i}+\boldsymbol{\beta}_{i}^{\prime} \mathbf{D}^{-1}\left(\boldsymbol{\sigma}_{e_{i}}^{2}\right)\left(\mathbf{y}_{i_{j}}-\boldsymbol{\beta}_{i} \mu\right)\right] \\
& +\left(\mathbf{y}_{i_{j}}-\boldsymbol{\beta}_{i} \mu\right)^{\prime} \mathbf{D}^{-1}\left(\boldsymbol{\sigma}_{e_{i}}^{2}\right)\left(\mathbf{y}_{i_{j}}-\boldsymbol{\beta}_{i} \mu\right) \\
& -\sigma_{x}^{2} b_{i}^{-1}\left[\left(\mathbf{y}_{i_{j}}-\boldsymbol{\beta}_{i} \mu\right)^{\prime} \mathbf{D}^{-1}\left(\boldsymbol{\sigma}_{e_{i}}^{2}\right) \boldsymbol{\beta}_{i} \boldsymbol{\beta}_{i}^{\prime} \mathbf{D}^{-1}\left(\boldsymbol{\sigma}_{e_{i}}^{2}\right)\left(\mathbf{y}_{i_{j}}-\boldsymbol{\beta}_{i} \mu\right)\right]
\end{aligned}
$$

Para os cálculos, derivamos a expressão (C.1) em relação a cada um dos parâmetros.

1. $\frac{\partial}{\partial \boldsymbol{\beta}_{i}}\left(d_{i_{j}}\right)=\frac{\partial}{\partial \boldsymbol{\beta}_{i}}\left(\left(x_{i_{j}}-\mu\right)^{2}\left(\sigma^{-2}-\sigma_{x}^{2} b_{i}^{-1} \sigma^{-4}\right)\right)$ 


$$
\begin{aligned}
& -\frac{\partial}{\partial \boldsymbol{\beta}_{i}}\left(\sigma^{-2} \sigma_{x}^{2} b_{i}^{-1}\left(x_{i_{j}}-\mu\right)\left[\left(\mathbf{y}_{i_{j}}-\boldsymbol{\beta}_{i} \mu\right)^{\prime} \mathbf{D}^{-1}\left(\boldsymbol{\sigma}_{e_{i}}^{2}\right) \boldsymbol{\beta}_{i}+\boldsymbol{\beta}_{i}^{\prime} \mathbf{D}^{-1}\left(\boldsymbol{\sigma}_{e_{i}}^{2}\right)\left(\mathbf{y}_{i_{j}}-\boldsymbol{\beta}_{i} \mu\right)\right]\right) \\
& +\frac{\partial}{\partial \boldsymbol{\beta}_{i}}\left(\left(\mathbf{y}_{i_{j}}-\boldsymbol{\beta}_{i} \mu\right)^{\prime} \mathbf{D}^{-1}\left(\boldsymbol{\sigma}_{e_{i}}^{2}\right)\left(\mathbf{y}_{i_{j}}-\boldsymbol{\beta}_{i} \mu\right)\right) \\
& -\frac{\partial}{\partial \boldsymbol{\beta}_{i}}\left(\sigma_{x}^{2} b_{i}^{-1}\left[\left(\mathbf{y}_{i_{j}}-\boldsymbol{\beta}_{i} \mu\right)^{\prime} \mathbf{D}^{-1}\left(\boldsymbol{\sigma}_{e_{i}}^{2}\right) \boldsymbol{\beta}_{i} \boldsymbol{\beta}_{i}^{\prime} \mathbf{D}^{-1}\left(\boldsymbol{\sigma}_{e_{i}}^{2}\right)\left(\mathbf{y}_{i_{j}}-\boldsymbol{\beta}_{i} \mu\right)\right]\right)= \\
& =2 \sigma_{x}^{4} b_{i}^{-2}\left[\left(\mathbf{z}_{i_{j}}-\mathbf{m}_{i}\right)^{\prime} \mathbf{B}_{i}\left(\mathbf{z}_{i_{j}}-\mathbf{m}_{i}\right)\right] \mathbf{D}^{-1}\left(\boldsymbol{\sigma}_{e_{i}}^{2}\right) \boldsymbol{\beta}_{i} \\
& -2 \sigma_{x}^{2} b_{i}^{-1}\left[\mathbf{a}_{i}^{\prime} \mathbf{A}_{i}^{-1}\left(\mathbf{z}_{i_{j}}-\mathbf{m}_{i}\right)\right] \mathbf{D}^{-1}\left(\boldsymbol{\sigma}_{e_{i}}^{2}\right)\left(\mathbf{y}_{i_{j}}-2 \boldsymbol{\beta}_{i} \mu\right)-2 \mu \mathbf{D}^{-1}\left(\boldsymbol{\sigma}_{e_{i}}^{2}\right)\left(\mathbf{y}_{i_{j}}-\boldsymbol{\beta}_{i} \mu\right) .
\end{aligned}
$$

2. $\frac{\partial}{\partial \mu}\left(d_{i_{j}}\right)=-2 b_{i}^{-1}\left[\mathbf{a}_{i}^{\prime} \mathbf{A}_{i}^{-1}\left(\mathbf{z}_{i_{j}}-\mathbf{m}_{i}\right)\right]$.

3. $\frac{\partial}{\partial \sigma_{x}^{2}}\left(d_{i_{j}}\right)=-b_{i}^{-2}\left[\left(\mathbf{z}_{i_{j}}-\mathbf{m}_{i}\right)^{\prime} \mathbf{B}_{i}\left(\mathbf{z}_{i_{j}}-\mathbf{m}_{i}\right)\right]$

4. $\frac{\partial}{\partial \sigma^{2}}\left(d_{i_{j}}\right)==\left(1-\sigma_{x}^{2} b_{i}^{-1} \sigma^{-2}\right)\left[\sigma_{x}^{2} b_{i}^{-1} \sigma^{-2}\left[\left(\mathbf{z}_{i_{j}}-\mathbf{m}_{i}\right)^{\prime} \mathbf{B}_{i}\left(\mathbf{z}_{i_{j}}-\mathbf{m}_{i}\right)\right]\right.$

$\left.-\sigma^{-4}\left(x_{i_{j}}-\mu\right)^{2}\right]-\sigma_{x}^{2} \sigma^{-2} b_{i}^{-1}\left[\left(\mathbf{y}_{i_{j}}-\boldsymbol{\beta}_{i} \mu\right)^{\prime} \mathbf{D}^{-1}\left(\boldsymbol{\sigma}_{e_{i}}^{2}\right) \boldsymbol{\beta}_{i} \boldsymbol{\beta}_{i}^{\prime} \mathbf{D}^{-1}\left(\boldsymbol{\sigma}_{e_{i}}^{2}\right)\left(\mathbf{y}_{i_{j}}-\boldsymbol{\beta}_{i} \mu\right)\right]$.

5. $\frac{\partial}{\partial \boldsymbol{\sigma}_{e_{i}}^{2}}\left(d_{i_{j}}\right)==-\sigma_{x}^{4} b_{i}^{-2}\left[\left(\mathbf{z}_{i_{j}}-\mathbf{m}_{i}\right)^{\prime} \mathbf{B}_{i}\left(\mathbf{z}_{i_{j}}-\mathbf{m}_{i}\right)\right] \mathbf{D}\left(\boldsymbol{\beta}_{i}\right) \mathbf{D}^{-2}\left(\boldsymbol{\sigma}_{e_{i}}^{2}\right) \boldsymbol{\beta}_{i}$

$+2 \sigma_{x}^{2} b_{i}^{-1}\left[\mathbf{a}_{i}^{\prime} \mathbf{A}_{i}^{-1}\left(\mathbf{z}_{i_{j}}-\mathbf{m}_{i}\right)\right] \mathbf{D}\left(\mathbf{y}_{i_{j}}-\boldsymbol{\beta}_{i} \mu\right) \mathbf{D}^{-2}\left(\boldsymbol{\sigma}_{e_{i}}^{2}\right) \boldsymbol{\beta}_{i}$

$$
-\mathbf{D}\left(\mathbf{y}_{i_{j}}-\boldsymbol{\beta}_{i} \mu\right) \mathbf{D}^{-2}\left(\boldsymbol{\sigma}_{e_{i}}^{2}\right)\left(\mathbf{y}_{i_{j}}-\boldsymbol{\beta}_{i} \mu\right)
$$

Considerando novamente a equação (2.4), escreveremos agora as expressões das derivadas primeiras de $L(\mathbf{z}, \boldsymbol{\theta})$ em relação aos parâmetros.

\section{Derivadas primeiras da log-verossimilhança}

Temos que a função $L(\mathbf{z}, \boldsymbol{\theta})$ é dada por

$$
L(\mathbf{z}, \boldsymbol{\theta})=\log \left(f_{\mathbf{z}}\right)=-\frac{3 N}{2} \log (2 \pi)-\frac{1}{2} \sum_{i=1}^{p} n_{i} \log \left|\mathbf{V}_{i}\right|-\frac{1}{2} \sum_{i=1}^{p} \sum_{j=1}^{n_{i}}\left(\mathbf{z}_{i_{j}}-\mathbf{m}_{i}\right)^{\prime} \mathbf{V}_{i}^{-1}\left(\mathbf{z}_{i_{j}}-\mathbf{m}_{i}\right),
$$

onde $\boldsymbol{\theta}_{(4 p+3) \times 1}=\left(\boldsymbol{\beta}_{1}^{\prime}, \ldots, \boldsymbol{\beta}_{p}^{\prime}, \mu, \sigma_{x}^{2}, \sigma^{2}, \boldsymbol{\sigma}_{e_{1}^{\prime}}^{2}, \ldots, \boldsymbol{\sigma}_{e_{p}^{\prime}}^{2}\right)$. Para obter o estimador de máxima verossimilhança iremos derivar $L(\mathbf{z}, \boldsymbol{\theta})$ em relação a cada parâmetro $\theta_{i}$. Ou seja,

$$
\begin{aligned}
\frac{\partial}{\partial \theta_{i}} L(\mathbf{z}, \boldsymbol{\theta}) & =\frac{\partial}{\partial \theta_{i}}\left(-\frac{3 N}{2} \log (2 \pi)-\frac{1}{2} \sum_{i=1}^{p} n_{i} \log \left|\mathbf{V}_{i}\right|-\frac{1}{2} \sum_{i=1}^{p} \sum_{j=1}^{n_{i}}\left(\mathbf{z}_{i_{j}}-\mathbf{m}_{i}\right)^{\prime} \mathbf{V}_{i}^{-1}\left(\mathbf{z}_{i_{j}}-\mathbf{m}_{i}\right)\right)= \\
= & -\frac{\partial}{\partial \theta_{i}}\left(\frac{1}{2} \sum_{i=1}^{p} n_{i} \log \left|\mathbf{V}_{i}\right|\right)-\frac{\partial}{\partial \theta_{i}}\left(\frac{1}{2} \sum_{i=1}^{p} \sum_{j=1}^{n_{i}}\left(\mathbf{z}_{i_{j}}-\mathbf{m}_{i}\right)^{\prime} \mathbf{V}_{i}^{-1}\left(\mathbf{z}_{i_{j}}-\mathbf{m}_{i}\right)\right)=
\end{aligned}
$$


Portanto

$$
\frac{\partial}{\partial \theta_{i}} L(\mathbf{z}, \boldsymbol{\theta})=-\frac{1}{2} \sum_{i=1}^{p} \sum_{j=1}^{n_{i}}\left(\frac{\partial}{\partial \theta_{i}} \log \left|\mathbf{V}_{i}\right|+\frac{\partial}{\partial \theta_{i}} d_{i_{j}}\right) .
$$

Partindo então das derivadas de $\log \left|\mathbf{V}_{i}\right|$ e de $d_{i_{j}}$ obtidas anteriormente, obtemos as expressões apresentadas na Seção 2.3. 


\section{Apêndice D}

\section{Matriz de informação observada}

Neste Apêndice apresentaremos os elementos da matriz de informação observada para os dados de Hadgu \& Koch (1999). Sabemos que a matriz de informação observada é dada por

$$
I_{o}(\theta)=-\frac{\partial^{2} L(\mathbf{z}, \boldsymbol{\theta})}{\partial \boldsymbol{\theta} \partial \boldsymbol{\theta}^{\prime}}
$$

$\operatorname{com} L(\mathbf{z}, \boldsymbol{\theta})$ como em $(2.4)$ e $\boldsymbol{\theta}_{(4 p+3) \times 1}=\left(\boldsymbol{\beta}_{1}^{\prime}, \ldots, \boldsymbol{\beta}_{p}^{\prime}, \mu, \sigma^{2}, \sigma_{x}^{2}, \boldsymbol{\sigma}_{\mathbf{e}_{1}}^{2^{\prime}}, \ldots, \boldsymbol{\sigma}_{\mathbf{e}_{p}}^{2^{\prime}}\right)^{\prime}$, com $p$ o número de líquidos de bochecho. Definindo $\boldsymbol{\beta}^{\prime}=\left(\boldsymbol{\beta}_{1}^{\prime}, \ldots, \boldsymbol{\beta}_{p}^{\prime}\right)$ e $\boldsymbol{\sigma}_{\mathbf{e}}^{2^{\prime}}=\left(\boldsymbol{\sigma}_{\mathbf{e}_{1}}^{2^{\prime}}, \ldots, \boldsymbol{\sigma}_{\mathbf{e}_{p}}^{2^{\prime}}\right), \operatorname{com} \boldsymbol{\beta}_{i}=\left(\beta_{1 i}, \beta_{2 i}\right)^{\prime}$ e $\boldsymbol{\sigma}_{\mathbf{e}_{i}}^{2}=\left(\sigma_{\mathbf{e}_{1 i}}^{2}, \sigma_{\mathbf{e}_{2 i}}^{2}\right)^{\prime}, i=1, \ldots, p$, temos que

$$
I_{o}(\theta)=-\left(\begin{array}{ccccc}
\frac{\partial^{2} L(\mathbf{z}, \boldsymbol{\theta})}{\partial \boldsymbol{\beta} \partial \boldsymbol{\beta}^{\prime}} & \frac{\partial^{2} L(\mathbf{z}, \boldsymbol{\theta})}{\partial \boldsymbol{\beta} \partial \mu} & \frac{\partial^{2} L(\mathbf{z}, \boldsymbol{\theta})}{\partial \boldsymbol{\beta} \partial \sigma^{2}} & \frac{\partial^{2} L(\mathbf{z}, \boldsymbol{\theta})}{\partial \boldsymbol{\beta} \partial \sigma_{x}^{2}} & \frac{\partial^{2} L(\mathbf{z}, \boldsymbol{\theta})}{\partial \boldsymbol{\beta} \partial \boldsymbol{\sigma}_{\mathbf{e}}^{2^{\prime}}} \\
\frac{\partial^{2} L(\mathbf{z}, \boldsymbol{\theta})}{\partial \mu \partial \boldsymbol{\beta}^{\prime}} & \frac{\partial^{2} L(\mathbf{z}, \boldsymbol{\theta})}{\partial \mu \partial \mu} & \frac{\partial^{2} L(\mathbf{z}, \boldsymbol{\theta})}{\partial \mu \partial \sigma^{2}} & \frac{\partial^{2} L(\mathbf{z}, \boldsymbol{\theta})}{\partial \mu \partial \sigma_{x}^{2}} & \frac{\partial^{2} L(\mathbf{z}, \boldsymbol{\theta})}{\partial \mu \partial \boldsymbol{\sigma}_{\mathbf{e}}^{2^{\prime}}} \\
\frac{\partial^{2} L(\mathbf{z}, \boldsymbol{\theta})}{\partial \sigma^{2} \partial \boldsymbol{\beta}^{\prime}} & \frac{\partial^{2} L(\mathbf{z}, \boldsymbol{\theta})}{\partial \sigma^{2} \partial \mu} & \frac{\partial^{2} L(\mathbf{z}, \boldsymbol{\theta})}{\partial \sigma^{2} \partial \sigma^{2}} & \frac{\partial^{2} L(\mathbf{z}, \boldsymbol{\theta})}{\partial \sigma^{2} \partial \sigma_{x}^{2}} & \frac{\partial^{2} L(\mathbf{z}, \boldsymbol{\theta})}{\partial \sigma^{2} \partial \boldsymbol{\sigma}_{\mathbf{e}}^{2^{\prime}}} \\
\frac{\partial^{2} L(\mathbf{z}, \boldsymbol{\theta})}{\partial \sigma_{x}^{2} \partial \boldsymbol{\beta}^{\prime}} & \frac{\partial^{2} L(\mathbf{z}, \boldsymbol{\theta})}{\partial \sigma_{x}^{2} \partial \mu} & \frac{\partial^{2} L(\mathbf{z}, \boldsymbol{\theta})}{\partial \sigma_{x}^{2} \partial \sigma^{2}} & \frac{\partial^{2} L(\mathbf{z}, \boldsymbol{\theta})}{\partial \sigma_{x}^{2} \partial \sigma_{x}^{2}} & \frac{\partial^{2} L(\mathbf{z}, \boldsymbol{\theta})}{\partial \sigma_{x}^{2} \partial \boldsymbol{\sigma}_{\mathbf{e}}^{2^{\prime}}} \\
\frac{\partial^{2} L(\mathbf{z}, \boldsymbol{\theta})}{\partial \boldsymbol{\sigma}_{\mathbf{e}}^{2} \partial \boldsymbol{\beta}^{\prime}} & \frac{\partial^{2} L(\mathbf{z}, \boldsymbol{\theta})}{\partial \boldsymbol{\sigma}_{\mathbf{e}}^{2} \partial \mu} & \frac{\partial^{2} L(\mathbf{z}, \boldsymbol{\theta})}{\partial \boldsymbol{\sigma}_{\mathbf{e}}^{2} \partial \sigma^{2}} & \frac{\partial^{2} L(\mathbf{z}, \boldsymbol{\theta})}{\partial \boldsymbol{\sigma}_{\mathbf{e}}^{2} \partial \sigma_{x}^{2}} & \frac{\partial^{2} L(\mathbf{z}, \boldsymbol{\theta})}{\partial \boldsymbol{\sigma}_{\mathbf{e}}^{2} \partial \boldsymbol{\sigma}_{\mathbf{e}}^{2^{\prime}}}
\end{array}\right),
$$

Seja $\mathbf{a}_{i}, \mathbf{A}_{i}, \mathbf{B}_{i}$, e $b_{i}$ como definidos em (2.6) e (2.7). 
A partir das derivadas primeiras de $L(\mathbf{z}, \boldsymbol{\theta})$, apresentadas no Apêndice C e utilizando as propriedades de derivadas vetoriais apresentadas no Apêndice B.3, calculamos as expressões das derivadas segundas de $L(\mathbf{z}, \boldsymbol{\theta})$.

\section{Derivadas segundas}

$$
\begin{aligned}
& \frac{\partial^{2} L(\mathbf{z}, \boldsymbol{\theta})}{\partial \boldsymbol{\beta}_{i} \partial \boldsymbol{\beta}_{i}^{\prime}}=\left\{\sigma_{x}^{2} b_{i}^{-1} \mathbf{D}^{-1}\left(\boldsymbol{\sigma}_{\mathbf{e}_{i}}^{2}\right)\left(\mathbf{y}_{i_{j}}-2 \boldsymbol{\beta}_{i} \mu\right)\left(\mathbf{y}_{i_{j}}-2 \boldsymbol{\beta}_{i} \mu\right)^{\prime} \mathbf{D}^{-1}\left(\boldsymbol{\sigma}_{\mathbf{e}_{i}}^{2}\right)\right. \\
& -4 \sigma_{x}^{4} b_{i}^{-2}\left[\mathbf{a}_{i}^{\prime} \mathbf{A}_{i}^{-1}\left(\mathbf{z}_{i_{j}}-\mathbf{m}_{i}\right)\right] \mathbf{D}^{-1}\left(\boldsymbol{\sigma}_{\mathbf{e}_{i}}^{2}\right) \boldsymbol{\beta}_{i}\left(\mathbf{y}_{i_{j}}-2 \boldsymbol{\beta}_{i} \mu\right)^{\prime} \mathbf{D}^{-1}\left(\boldsymbol{\sigma}_{\mathbf{e}_{i}}^{2}\right) \\
& +2 \sigma_{x}^{4} b_{i}^{-2}\left(1+2 \sigma_{x}^{2} b_{i}^{-1}\left[\left(\mathbf{z}_{i_{j}}-\mathbf{m}_{i}\right)^{\prime} \mathbf{B}_{i}\left(\mathbf{z}_{i_{j}}-\mathbf{m}_{i}\right)\right]\right) \mathbf{D}^{-1}\left(\boldsymbol{\sigma}_{\mathbf{e}_{i}}^{2}\right) \boldsymbol{\beta}_{i} \boldsymbol{\beta}_{i}^{\prime} \mathbf{D}^{-1}\left(\boldsymbol{\sigma}_{\mathbf{e}_{i}}^{2}\right) \\
& -\left\{u^{2}+\sigma_{x}^{2} b_{i}^{-1}\left[1+2 u\left[\mathbf{a}_{i}^{\prime} \mathbf{A}_{i}^{-1}\left(\mathbf{z}_{i_{j}}-\mathbf{m}_{i}\right)\right]+\right.\right. \\
& \left.\left.\left.\sigma_{x}^{2} b_{i}^{-1}\left[\left(\mathbf{z}_{i_{j}}-\mathbf{m}_{i}\right)^{\prime} \mathbf{B}_{i}\left(\mathbf{z}_{i_{j}}-\mathbf{m}_{i}\right)\right]\right]\right\} \mathbf{D}^{-1}\left(\boldsymbol{\sigma}_{\mathbf{e}_{i}}^{2}\right)\right\}, i=1, \ldots, p \\
& \frac{\partial^{2} L(\mathbf{z}, \boldsymbol{\theta})}{\partial \boldsymbol{\beta}_{i} \partial \boldsymbol{\beta}_{j}^{\prime}}=0, \text { para } i \neq j, i, j=1, \ldots, p \\
& \frac{\partial^{2} L(\mathbf{z}, \boldsymbol{\theta})}{\partial \boldsymbol{\beta}_{i} \partial \mu}=-2 b_{i}^{-2}\left[\mathbf{a}_{i}^{\prime} \mathbf{A}_{i}^{-1}\left(\mathbf{z}_{i_{j}}-\mathbf{m}_{i}\right)\right] \sigma_{x}^{2} \mathbf{D}^{-1}\left(\boldsymbol{\sigma}_{\mathbf{e}_{i}}^{2}\right) \boldsymbol{\beta}_{i}+b_{i}^{-1} \mathbf{D}^{-1}\left(\boldsymbol{\sigma}_{\mathbf{e}_{i}}^{2}\right)\left(\mathbf{y}_{i_{j}}-2 \mu \boldsymbol{\beta}_{i}\right), \\
& i=1, \ldots, p \\
& \frac{\partial^{2} L(\mathbf{z}, \boldsymbol{\theta})}{\partial \boldsymbol{\beta}_{i} \partial \sigma^{2}}=-\sigma_{x}^{4} \sigma^{-4} b_{i}^{-2}\left\{1-2\left(x_{i_{j}}-\mu\right)\left[\mathbf{a}_{i}^{\prime} \mathbf{A}_{i}^{-1}\left(\mathbf{z}_{i_{j}}-\mathbf{m}_{i}\right)\right]\right. \\
& \left.+2 \sigma_{x}^{2} b_{i}^{-1}\left[\left(\mathbf{z}_{i_{j}}-\mathbf{m}_{i}\right)^{\prime} \mathbf{B}_{i}\left(\mathbf{z}_{i_{j}}-\mathbf{m}_{i}\right)\right]\right\} \mathbf{D}^{-1}\left(\boldsymbol{\sigma}_{\mathbf{e}_{i}}^{2}\right) \boldsymbol{\beta}_{i} \\
& -\sigma_{x}^{2} \sigma^{-4} b_{i}^{-1}\left\{\left(x_{i_{j}}-\mu\right)-\sigma_{x}^{2} b_{i}^{-1}\left[\mathbf{a}_{i}^{\prime} \mathbf{A}_{i}^{-1}\left(\mathbf{z}_{i_{j}}-\mathbf{m}_{i}\right)\right]\right\} \mathbf{D}^{-1}\left(\boldsymbol{\sigma}_{\mathbf{e}_{i}}^{2}\right)\left(\mathbf{y}_{i_{j}}-2 u \boldsymbol{\beta}_{i}\right), \\
& i=1, \ldots, p \\
& \frac{\partial^{2} L(\mathbf{z}, \boldsymbol{\theta})}{\partial \boldsymbol{\beta}_{i} \partial \sigma_{x}^{2}}=-b_{i}^{-2} \mathbf{D}^{-1}\left(\boldsymbol{\sigma}_{\mathbf{e}_{i}}^{2}\right) \boldsymbol{\beta}_{i}-2 b_{i}^{-3} \sigma_{x}^{2}\left[\left(\mathbf{z}_{i_{j}}-\mathbf{m}_{i}\right)^{\prime} \mathbf{B}_{i}\left(\mathbf{z}_{i_{j}}-\mathbf{m}_{i}\right)\right] \mathbf{D}^{-1}\left(\boldsymbol{\sigma}_{\mathbf{e}_{i}}^{2}\right) \boldsymbol{\beta}_{i} \\
& +b_{i}^{-2}\left[\mathbf{a}_{i}^{\prime} \mathbf{A}_{i}^{-1}\left(\mathbf{z}_{i_{j}}-\mathbf{m}_{i}\right)\right] \mathbf{D}^{-1}\left(\boldsymbol{\sigma}_{\mathbf{e}_{i}}^{2}\right)\left(\mathbf{y}_{i_{j}}-2 \mu \boldsymbol{\beta}_{i}\right), i=1, \ldots, p
\end{aligned}
$$




$$
\begin{aligned}
& \frac{\partial^{2} L(\mathbf{z}, \boldsymbol{\theta})}{\partial \boldsymbol{\beta}_{i} \partial \boldsymbol{\sigma}_{\mathbf{e}_{i}}^{2^{\prime}}}=\sigma_{x}^{2} b_{i}^{-1}\left\{-\mathbf{D}^{-1}\left(\boldsymbol{\sigma}_{\mathbf{e}_{i}}^{2}\right)\left(\mathbf{y}_{i_{j}}-2 \boldsymbol{\beta}_{i} \mu\right)\left(\mathbf{y}_{i_{j}}-\boldsymbol{\beta}_{i} \mu\right)^{\prime} \mathbf{D}^{-2}\left(\boldsymbol{\sigma}_{\mathbf{e}_{i}}^{2}\right) \mathbf{D}\left(\boldsymbol{\beta}_{i}\right)\right. \\
& \left.+\mathbf{D}^{-2}\left(\boldsymbol{\sigma}_{\mathbf{e}_{i}}^{2}\right) \mathbf{D}\left(\boldsymbol{\beta}_{i}\right)-\sigma_{x}^{2} b_{i}^{-1} \mathbf{D}^{-1}\left(\boldsymbol{\sigma}_{\mathbf{e}_{i}}^{2}\right) \boldsymbol{\beta}_{i} \boldsymbol{\beta}_{i}^{\prime} \mathbf{D}^{-2}\left(\boldsymbol{\sigma}_{\mathbf{e}_{i}}^{2}\right) \mathbf{D}\left(\boldsymbol{\beta}_{i}\right)\right\} \\
& -\mu \mathbf{D}^{-2}\left(\boldsymbol{\sigma}_{\mathbf{e}_{i}}^{2}\right) \mathbf{D}\left(\mathbf{y}_{i_{j}}-\boldsymbol{\beta}_{i} \mu\right)-\sigma_{x}^{4} b_{i}^{-2}\left[\left(\mathbf{z}_{i_{j}}-\mathbf{m}_{i}\right)^{\prime} \mathbf{B}_{i}\left(\mathbf{z}_{i_{j}}-\mathbf{m}_{i}\right)\right] \\
& \left\{2 \sigma_{x}^{2} b_{i}^{-1} \mathbf{D}^{-1}\left(\boldsymbol{\sigma}_{\mathbf{e}_{i}}^{2}\right) \boldsymbol{\beta}_{i} \boldsymbol{\beta}_{i}^{\prime} \mathbf{D}^{-2}\left(\boldsymbol{\sigma}_{\mathbf{e}_{i}}^{2}\right) \mathbf{D}\left(\boldsymbol{\beta}_{i}\right)-\mathbf{D}^{-2}\left(\boldsymbol{\sigma}_{\mathbf{e}_{i}}^{2}\right) \mathbf{D}\left(\boldsymbol{\beta}_{i}\right)\right\} \\
& -\sigma_{x}^{2} b_{i}^{-1}\left[\mathbf{a}_{i}^{\prime} \mathbf{A}_{i}^{-1}\left(\mathbf{z}_{i_{j}}-\mathbf{m}_{i}\right)\right]\left\{\mathbf{D}^{-2}\left(\boldsymbol{\sigma}_{\mathbf{e}_{i}}^{2}\right) \mathbf{D}\left(\mathbf{y}_{i_{j}}-2 \boldsymbol{\beta}_{i} \mu\right)\right. \\
& \left.-\sigma_{x}^{2} b_{i}^{-1} \mathbf{D}^{-1}\left(\boldsymbol{\sigma}_{\mathbf{e}_{i}}^{2}\right) \boldsymbol{\beta}_{i}\left(3 \mathbf{y}_{i_{j}}-4 \boldsymbol{\beta}_{i} \mu\right)^{\prime} \mathbf{D}^{-2}\left(\boldsymbol{\sigma}_{\mathbf{e}_{i}}^{2}\right) \mathbf{D}\left(\boldsymbol{\beta}_{i}\right)\right\}, i=1, \ldots, p \\
& \frac{\partial^{2} L(\mathbf{z}, \boldsymbol{\theta})}{\partial \boldsymbol{\beta}_{i} \partial \boldsymbol{\sigma}_{\mathbf{e}_{j}}^{2}}=0, i \neq j, i, j=1, \ldots, p \\
& \frac{\partial^{2} L(\mathbf{z}, \boldsymbol{\theta})}{\partial \mu \partial \mu}=-\sum_{i=1}^{p} \sum_{j=1}^{n_{i}}\left\{\left(1-b_{i}^{-1}\right) \sigma_{x}^{-2}\right\} \\
& \frac{\partial^{2} L(\mathbf{z}, \boldsymbol{\theta})}{\partial \mu \partial \sigma^{2}}=-\sum_{i=1}^{p} \sum_{j=1}^{n_{i}} b_{i}^{-1} \sigma^{-4}\left\{\left(x_{i_{j}}-\mu\right)-\sigma_{x}^{2} b_{i}^{-1}\left[\mathbf{a}_{i}^{\prime} \mathbf{A}_{i}^{-1}\left(\mathbf{z}_{i_{j}}-\mathbf{m}_{i}\right)\right]\right\} \\
& \frac{\partial^{2} L(\mathbf{z}, \boldsymbol{\theta})}{\partial \mu \partial \sigma_{x}^{2}}=-\sum_{i=1}^{p} \sum_{j=1}^{n_{i}}\left(b_{i}^{-1}-b_{i}^{-2}\right) \sigma_{x}^{-2}\left[\mathbf{a}_{i}^{\prime} \mathbf{A}_{i}^{-1}\left(\mathbf{z}_{i_{j}}-\mathbf{m}_{i}\right)\right] \\
& \frac{\partial^{2} L(\mathbf{z}, \boldsymbol{\theta})}{\partial \mu \partial \boldsymbol{\sigma}_{\mathbf{e}_{i}}^{2{ }^{\prime}}}=b_{i}^{-2} \sigma_{x}^{2}\left[\mathbf{a}_{i}^{\prime} \mathbf{A}_{i}^{-1}\left(\mathbf{z}_{i_{j}}-\mathbf{m}_{i}\right)\right] \boldsymbol{\beta}_{i}^{\prime} \mathbf{D}^{-2}\left(\boldsymbol{\sigma}_{\mathbf{e}_{i}}^{2}\right) \mathbf{D}\left(\boldsymbol{\beta}_{i}\right) \\
& -b_{i}^{-1}\left(\mathbf{y}_{i_{j}}-\boldsymbol{\beta}_{i} \mu\right)^{\prime} \mathbf{D}^{-2}\left(\boldsymbol{\sigma}_{\mathbf{e}_{i}}^{2}\right) \mathbf{D}\left(\boldsymbol{\beta}_{i}\right), i=1, \ldots, p \\
& \frac{\partial^{2} L(\mathbf{z}, \boldsymbol{\theta})}{\partial \sigma^{2} \partial \sigma^{2}}=-\frac{1}{2} \sum_{i=1}^{p} \sum_{j=1}^{n_{i}}\left\{-\sigma^{-4}+\left(2-\sigma_{x}^{2} \sigma^{-2} b_{i}^{-1}\right) \sigma^{-6} \sigma_{x}^{2} b_{i}^{-1}\right. \\
& -4 \sigma_{x}^{2} \sigma^{-6} b_{i}^{-1}\left(1-\sigma_{x}^{2} \sigma^{-2} b_{i}^{-1}\right)\left(x_{i_{j}}-\mu\right)\left[\mathbf{a}_{i}^{\prime} \mathbf{A}_{i}^{-1}\left(\mathbf{z}_{i_{j}}-\mathbf{m}_{i}\right)\right] \\
& +2 \sigma_{x}^{4} \sigma^{-6} b_{i}^{-2}\left(1-\sigma_{x}^{2} \sigma^{-2} b_{i}^{-1}\right)\left[\left(\mathbf{z}_{i_{j}}-\mathbf{m}_{i}\right)^{\prime} \mathbf{B}_{i}\left(\mathbf{z}_{i_{j}}-\mathbf{m}_{i}\right)\right] \\
& \left.+2 \sigma^{-6}\left(1-\sigma_{x}^{2} \sigma^{-2} b_{i}^{-1}\right)\left(x_{i_{j}}-\mu\right)^{2}\right\}
\end{aligned}
$$




$$
\begin{aligned}
& \frac{\partial^{2} L(\mathbf{z}, \boldsymbol{\theta})}{\partial \sigma^{2} \partial \sigma_{x}^{2}}=-\frac{1}{2} \sum_{i=1}^{p} \sum_{j=1}^{n_{i}}\left\{-\sigma^{-4} b_{i}^{-2}-2 b_{i}^{-3} \sigma_{x}^{2} \sigma^{-4}\left[\left(\mathbf{z}_{i_{j}}-\mathbf{m}_{i}\right)^{\prime} \mathbf{B}_{i}\left(\mathbf{z}_{i_{j}}-\mathbf{m}_{i}\right)\right]\right. \\
& \left.+2 b_{i}^{-2} \sigma^{-4}\left(x_{i_{j}}-\mu\right)\left[\mathbf{a}_{i}^{\prime} \mathbf{A}_{i}^{-1}\left(\mathbf{z}_{i_{j}}-\mathbf{m}_{i}\right)\right]\right\} \\
& \frac{\partial^{2} L(\mathbf{z}, \boldsymbol{\theta})}{\partial \sigma^{2} \partial \boldsymbol{\sigma}_{\mathbf{e}_{i}}^{2{ }^{\prime}}}=-\frac{1}{2}\left\{b _ { i } ^ { - 2 } \sigma ^ { - 4 } \left\{\sigma _ { x } ^ { 4 } \left[2\left(x_{i_{j}}-\mu\right)\left[\mathbf{a}_{i}^{\prime} \mathbf{A}_{i}^{-1}\left(\mathbf{z}_{i_{j}}-\mathbf{m}_{i}\right)\right]\right.\right.\right. \\
& \left.\left.-2 \sigma_{x}^{2}\left[\left(\mathbf{z}_{i_{j}}-\mathbf{m}_{i}\right)^{\prime} \mathbf{B}_{i}\left(\mathbf{z}_{i_{j}}-\mathbf{m}_{i}\right)\right] b_{i}^{-1}-1\right] \mathbf{D}\left(\boldsymbol{\beta}_{i}\right) \mathbf{D}^{-2}\left(\boldsymbol{\sigma}_{\mathbf{e}_{i}}^{2}\right) \boldsymbol{\beta}_{i}\right\} \\
& -b_{i}^{-1} \sigma^{-4}\left[2 \sigma_{x}^{2}\left[x_{i_{j}}-\mu-\sigma_{x}^{2} b_{i}^{-1}\left[\mathbf{a}_{i}^{\prime} \mathbf{A}_{i}^{-1}\left(\mathbf{z}_{i_{j}}-\mathbf{m}_{i}\right)\right]\right]\right. \\
& \left.\left.\mathbf{D}\left(\boldsymbol{\beta}_{i}\right) \mathbf{D}^{-2}\left(\boldsymbol{\sigma}_{\mathbf{e}_{i}}^{2}\right)\left(\mathbf{y}_{i_{j}}-\boldsymbol{\beta}_{i} \mu\right)\right]\right\}, i=1, \ldots, p \\
& \frac{\partial^{2} L(\mathbf{z}, \boldsymbol{\theta})}{\partial \sigma_{x}^{2} \partial \sigma_{x}^{2}}=-\frac{1}{2} \sum_{i=1}^{p} \sum_{j=1}^{n_{i}}\left\{-\sigma_{x}^{-4}\left[1-b_{i}^{-1}\left(2-b_{i}^{-1}\right)\right]\right. \\
& \left.+2 \sigma_{x}^{-2} b_{i}^{-2}\left(1-b_{i}^{-1}\right)\left[\left(\mathbf{z}_{i_{j}}-\mathbf{m}_{i}\right)^{\prime} \mathbf{B}_{i}\left(\mathbf{z}_{i_{j}}-\mathbf{m}_{i}\right)\right]\right\} \\
& \frac{\partial^{2} L(\mathbf{z}, \boldsymbol{\theta})}{\partial \sigma_{x}^{2} \partial \boldsymbol{\sigma}_{\mathbf{e}_{i}}^{2{ }^{\prime}}}=\frac{1}{2}\left\{b_{i}^{-2}\left(1+2 b_{i}^{-1} \sigma_{x}^{2}\left[\left(\mathbf{z}_{i_{j}}-\mathbf{m}_{i}\right)^{\prime} \mathbf{B}_{i}\left(\mathbf{z}_{i_{j}}-\mathbf{m}_{i}\right)\right]\right) \mathbf{D}\left(\boldsymbol{\beta}_{i}\right) \mathbf{D}^{-2}\left(\boldsymbol{\sigma}_{\mathbf{e}_{i}}^{2}\right) \boldsymbol{\beta}_{i}\right. \\
& \left.-2 b_{i}^{-2}\left[\mathbf{a}_{i}^{\prime} \mathbf{A}_{i}^{-1}\left(\mathbf{z}_{i_{j}}-\mathbf{m}_{i}\right)\right] \mathbf{D}\left(\boldsymbol{\beta}_{i}\right) \mathbf{D}^{-2}\left(\boldsymbol{\sigma}_{\mathbf{e}_{i}}^{2}\right)\left(\mathbf{y}_{i_{j}}-\boldsymbol{\beta}_{i} \mu\right)\right\}, i=1, \ldots, p \\
& \frac{\partial^{2} L(\mathbf{z}, \boldsymbol{\theta})}{\partial \boldsymbol{\sigma}_{\mathbf{e}_{i}}^{2} \partial \boldsymbol{\sigma}_{\mathbf{e}_{i}}^{2{ }^{\prime}}}=\frac{1}{2}\left\{-2 \mathbf{D}\left(\mathbf{y}_{i_{j}}-\boldsymbol{\beta}_{i} \mu\right) \mathbf{D}^{-3}\left(\boldsymbol{\sigma}_{\mathbf{e}_{i}}^{2}\right) \mathbf{D}\left(\mathbf{y}_{i_{j}}-\boldsymbol{\beta}_{i} \mu\right)\right. \\
& +2 \sigma_{x}^{2} b_{i}^{-1} \mathbf{D}\left(\boldsymbol{\beta}_{i}\right) \mathbf{D}^{-2}\left(\boldsymbol{\sigma}_{\mathbf{e}_{i}}^{2}\right)\left(\mathbf{y}_{i_{j}}-\boldsymbol{\beta}_{i} \mu\right)\left(\mathbf{y}_{i_{j}}-\boldsymbol{\beta}_{i} \mu\right)^{\prime} \mathbf{D}^{-2}\left(\boldsymbol{\sigma}_{\mathbf{e}_{i}}^{2}\right) \mathbf{D}\left(\boldsymbol{\beta}_{i}\right) \\
& -\sigma_{x}^{2} b_{i}^{-1}\left[2 \mathbf{D}\left(\boldsymbol{\beta}_{i}\right) \mathbf{D}^{-3}\left(\boldsymbol{\sigma}_{\mathbf{e}_{i}}^{2}\right) \mathbf{D}\left(\boldsymbol{\beta}_{i}\right)-\sigma_{x}^{2} b_{i}^{-1} \mathbf{D}\left(\boldsymbol{\beta}_{i}\right) \mathbf{D}^{-2}\left(\boldsymbol{\sigma}_{\mathbf{e}_{i}}^{2}\right) \boldsymbol{\beta}_{i} \boldsymbol{\beta}_{i}^{\prime} \mathbf{D}^{-2}\left(\boldsymbol{\sigma}_{\mathbf{e}_{i}}^{2}\right) \mathbf{D}\left(\boldsymbol{\beta}_{i}\right)\right] \\
& -2 \sigma_{x}^{4} b_{i}^{-2}\left[\left(\mathbf{z}_{i_{j}}-\mathbf{m}_{i}\right)^{\prime} \mathbf{B}_{i}\left(\mathbf{z}_{i_{j}}-\mathbf{m}_{i}\right)\right]\left[\mathbf{D}\left(\boldsymbol{\beta}_{i}\right) \mathbf{D}^{-3}\left(\boldsymbol{\sigma}_{\mathbf{e}_{i}}^{2}\right) \mathbf{D}\left(\boldsymbol{\beta}_{i}\right)\right. \\
& \left.-\sigma_{x}^{2} b_{i}^{-1} \mathbf{D}\left(\boldsymbol{\beta}_{i}\right) \mathbf{D}^{-2}\left(\boldsymbol{\sigma}_{\mathbf{e}_{i}}^{2}\right) \boldsymbol{\beta}_{i} \boldsymbol{\beta}_{i}^{\prime} \mathbf{D}^{-2}\left(\boldsymbol{\sigma}_{\mathbf{e}_{i}}^{2}\right) \mathbf{D}\left(\boldsymbol{\beta}_{i}\right)\right] \\
& +4 \sigma_{x}^{2} b_{i}^{-1}\left[\mathbf{a}_{i}^{\prime} \mathbf{A}_{i}^{-1}\left(\mathbf{z}_{i_{j}}-\mathbf{m}_{i}\right)\right]\left[\mathbf{D}\left(\boldsymbol{\beta}_{i}\right) \mathbf{D}^{-3}\left(\boldsymbol{\sigma}_{\mathbf{e}_{i}}^{2}\right) \mathbf{D}\left(\mathbf{y}_{i_{j}}-\boldsymbol{\beta}_{i} \mu\right)\right. \\
& \left.\left.-\sigma_{x}^{2} b_{i}^{-1} \mathbf{D}\left(\boldsymbol{\beta}_{i}\right) \mathbf{D}^{-2}\left(\boldsymbol{\sigma}_{\mathbf{e}_{i}}^{2}\right) \boldsymbol{\beta}_{i}\left(\mathbf{y}_{i_{j}}-\boldsymbol{\beta}_{i} \mu\right)^{\prime} \mathbf{D}^{-2}\left(\boldsymbol{\sigma}_{\mathbf{e}_{i}}^{2}\right) \mathbf{D}\left(\boldsymbol{\beta}_{i}\right)\right]+\mathbf{D}^{-2}\left(\boldsymbol{\sigma}_{\mathbf{e}_{i}}^{2}\right)\right\}, \\
& i=1, \ldots, p \\
& \frac{\partial^{2} L(\mathbf{z}, \boldsymbol{\theta})}{\partial \boldsymbol{\sigma}_{\mathbf{e}_{i}}^{2} \partial \boldsymbol{\sigma}_{\mathbf{e}_{j}}^{2{ }^{\prime}}}=0, i \neq j, i, j=1, \ldots, p
\end{aligned}
$$




\section{Apêndice E}

\section{Resultados das simulações do tamanho do teste}

Apresentamos aqui os resultados das simulações do tamanho dos testes assintóticos para as hipóteses apresentadas no Capítulo 5. Entre elas, estão os testes por exemplo do tipo $H_{0}$ : $\beta_{11}=\beta_{12}$ e $H_{0}: \beta_{12}=\beta_{13}$, que comparam respectivamente o desempenho do líquido controle com o líquido experimental a e o desempenho dos líquidos experimentais a e b após três meses de uso, $H_{0}: \beta_{21}=\beta_{22}$ e $H_{0}: \beta_{22}=\beta_{23}$, que comparam respectivamente o desempenho do líquido controle com o líquido a e o desempenho dos líquidos experimentais a e b após seis meses de uso, $H_{0}: \beta_{11}=\beta_{21}$, que compara o desempenho do líquido controle após três e seis meses de uso e $H_{0}: \beta_{12}=\beta_{22}$, que compara o desempenho do líquido a após três e seis meses de uso. Os resultados são apresentados nas Tabelas a E.1 a E.24. Para tanto, foi considerado o procedimento descrito na Seção 6.1, e as conclusões foram análogas às apresentadas na mesma seção. 
Tabela E.1: Tamanhos empíricos dos testes Wald, razão de verossimilhança e score para o teste $H_{0}: \beta_{11}=\beta_{12}$, com $\mu=1$.

\begin{tabular}{|c|c|c|c|c|c|c|c|c|c|c|c|}
\hline \multirow[b]{2}{*}{ Nível } & \multirow[b]{2}{*}{ Teste } & \multirow[b]{2}{*}{$\left(n_{1}, n_{2}, n_{3}\right)$} & \multirow{2}{*}{$\begin{array}{l}\sigma_{x}^{2} \\
\sigma^{2} \\
\end{array}$} & \multirow{2}{*}{$\begin{array}{l}0.01 \\
0.01\end{array}$} & \multicolumn{3}{|c|}{0.1} & \multicolumn{4}{|c|}{0.5} \\
\hline & & & & & 0.01 & 0.05 & 0.1 & 0.01 & 0.05 & 0.1 & 0.5 \\
\hline \multirow[t]{12}{*}{0.01} & W & $(17,14,20)$ & & 0.020 & 0.021 & 0.023 & 0.021 & 0.021 & 0.025 & 0.024 & 0.027 \\
\hline & W & $(35,28,36)$ & & 0.015 & 0.015 & 0.015 & 0.014 & 0.015 & 0.018 & 0.014 & 0.015 \\
\hline & W & $(50,46,55)$ & & 0.013 & 0.011 & 0.013 & 0.013 & 0.013 & 0.014 & 0.014 & 0.014 \\
\hline & W & $(101,95,105)$ & & 0.011 & 0.010 & 0.012 & 0.012 & 0.011 & 0.011 & 0.012 & 0.011 \\
\hline & $\overline{\mathrm{RV}}$ & $(17,14,20)$ & & 0.015 & 0.014 & 0.015 & 0.013 & 0.012 & 0.014 & 0.014 & 0.017 \\
\hline & RV & $(35,28,36)$ & & 0.013 & 0.012 & 0.012 & 0.012 & 0.012 & 0.012 & 0.011 & 0.012 \\
\hline & RV & $(50,46,55)$ & & 0.012 & 0.010 & 0.012 & 0.012 & 0.011 & 0.012 & 0.011 & 0.012 \\
\hline & $\mathrm{RV}$ & $(101,95,105)$ & & 0.011 & 0.009 & 0.012 & 0.011 & 0.010 & 0.010 & 0.011 & 0.010 \\
\hline & $\mathrm{S}$ & $(17,14,20)$ & & 0.018 & 0.015 & 0.009 & 0.007 & 0.018 & 0.012 & 0.009 & 0.007 \\
\hline & $\mathrm{S}$ & $(35,28,36)$ & & 0.018 & 0.014 & 0.009 & 0.008 & 0.017 & 0.010 & 0.008 & 0.009 \\
\hline & $\mathrm{S}$ & $(50,46,55)$ & & 0.017 & 0.011 & 0.010 & 0.010 & 0.016 & 0.010 & 0.010 & 0.010 \\
\hline & $\mathrm{S}$ & $(101,95,105)$ & & 0.016 & 0.011 & 0.010 & 0.010 & 0.016 & 0.009 & 0.010 & 0.009 \\
\hline \multirow[t]{12}{*}{0.05} & W & $(17,14,20)$ & & 0.069 & 0.076 & 0.073 & 0.072 & 0.078 & 0.078 & 0.077 & 0.080 \\
\hline & $\mathrm{W}$ & $(35,28,36)$ & & 0.063 & 0.065 & 0.059 & 0.059 & 0.060 & 0.067 & 0.063 & 0.063 \\
\hline & W & $(50,46,55)$ & & 0.054 & 0.054 & 0.056 & 0.058 & 0.053 & 0.060 & 0.060 & 0.060 \\
\hline & W & $(101,95,105)$ & & 0.053 & 0.052 & 0.051 & 0.056 & 0.052 & 0.053 & 0.054 & 0.053 \\
\hline & $\overline{\mathrm{RV}}$ & $(17,14,20)$ & & 0.060 & 0.063 & 0.061 & 0.060 & 0.060 & 0.059 & 0.059 & 0.066 \\
\hline & RV & $(35,28,36)$ & & 0.060 & 0.059 & 0.054 & 0.054 & 0.053 & 0.058 & 0.056 & 0.056 \\
\hline & RV & $(50,46,55)$ & & 0.052 & 0.050 & 0.053 & 0.054 & 0.049 & 0.053 & 0.055 & 0.056 \\
\hline & $\mathrm{RV}$ & $(101,95,105)$ & & 0.052 & 0.050 & 0.049 & 0.053 & 0.051 & 0.051 & 0.052 & 0.050 \\
\hline & $\mathrm{S}$ & $(17,14,20)$ & & 0.089 & 0.078 & 0.053 & 0.048 & 0.096 & 0.064 & 0.052 & 0.051 \\
\hline & $\mathrm{S}$ & $(35,28,36)$ & & 0.079 & 0.071 & 0.050 & 0.048 & 0.088 & 0.057 & 0.050 & 0.049 \\
\hline & $\mathrm{S}$ & $(50,46,55)$ & & 0.068 & 0.062 & 0.050 & 0.052 & 0.088 & 0.052 & 0.050 & 0.051 \\
\hline & $\mathrm{S}$ & $(101,95,105)$ & & 0.063 & 0.058 & 0.049 & 0.052 & 0.080 & 0.049 & 0.050 & 0.049 \\
\hline \multirow[t]{12}{*}{0.1} & $\mathrm{~W}$ & $(17,14,20)$ & & 0.127 & 0.130 & 0.131 & 0.131 & 0.132 & 0.131 & 0.135 & 0.137 \\
\hline & W & $(35,28,36)$ & & 0.120 & 0.117 & 0.111 & 0.115 & 0.112 & 0.121 & 0.121 & 0.118 \\
\hline & W & $(50,46,55)$ & & 0.109 & 0.105 & 0.110 & 0.114 & 0.106 & 0.115 & 0.118 & 0.111 \\
\hline & $\mathrm{W}$ & $(101,95,105)$ & & 0.105 & 0.103 & 0.104 & 0.105 & 0.105 & 0.104 & 0.105 & 0.104 \\
\hline & RV & $(17,14,20)$ & & 0.116 & 0.117 & 0.118 & 0.116 & 0.113 & 0.115 & 0.116 & 0.119 \\
\hline & $\mathrm{RV}$ & $(35,28,36)$ & & 0.116 & 0.110 & 0.104 & 0.109 & 0.105 & 0.111 & 0.112 & 0.109 \\
\hline & $\mathrm{RV}$ & $(50,46,55)$ & & 0.106 & 0.102 & 0.103 & 0.108 & 0.102 & 0.108 & 0.111 & 0.107 \\
\hline & RV & $(101,95,105)$ & & 0.104 & 0.101 & 0.102 & 0.104 & 0.101 & 0.103 & 0.102 & 0.101 \\
\hline & $\mathrm{S}$ & $(17,14,20)$ & & 0.168 & 0.152 & 0.111 & 0.104 & 0.182 & 0.129 & 0.107 & 0.103 \\
\hline & $\mathrm{S}$ & $(35,28,36)$ & & 0.152 & 0.138 & 0.099 & 0.102 & 0.178 & 0.118 & 0.105 & 0.099 \\
\hline & $\mathrm{S}$ & $(50,46,55)$ & & 0.129 & 0.127 & 0.101 & 0.102 & 0.167 & 0.109 & 0.105 & 0.101 \\
\hline & $\mathrm{S}$ & $(101,95,105)$ & & 0.116 & 0.116 & 0.101 & 0.101 & 0.147 & 0.102 & 0.099 & 0.099 \\
\hline
\end{tabular}


Tabela E.2: Tamanhos empíricos dos testes Wald, razão de verossimilhança e score para o teste $H_{0}: \beta_{11}=\beta_{12}, \operatorname{com} \mu=2.5$.

\begin{tabular}{|c|c|c|c|c|c|c|c|c|c|c|c|}
\hline \multirow[b]{2}{*}{ Nível } & \multirow[b]{2}{*}{ Teste } & \multirow[b]{2}{*}{$\left(n_{1}, n_{2}, n_{3}\right)$} & \multirow{2}{*}{$\begin{array}{l}\sigma_{x}^{2} \\
\sigma^{2}\end{array}$} & \multirow{2}{*}{$\begin{array}{l}0.01 \\
0.01\end{array}$} & \multicolumn{3}{|c|}{0.1} & \multicolumn{4}{|c|}{0.5} \\
\hline & & & & & 0.01 & 0.05 & 0.1 & 0.01 & 0.05 & 0.1 & 0.5 \\
\hline \multirow[t]{12}{*}{0.01} & W & $(17,14,20)$ & & 0.020 & 0.020 & 0.022 & 0.020 & 0.019 & 0.022 & 0.023 & 0.023 \\
\hline & W & $(35,28,36)$ & & 0.014 & 0.016 & 0.015 & 0.016 & 0.014 & 0.015 & 0.015 & 0.014 \\
\hline & W & $(50,46,55)$ & & 0.013 & 0.013 & 0.013 & 0.013 & 0.011 & 0.012 & 0.014 & 0.014 \\
\hline & $\mathrm{W}$ & $(101,95,105)$ & & 0.011 & 0.010 & 0.010 & 0.011 & 0.010 & 0.009 & 0.013 & 0.010 \\
\hline & $\overline{\mathrm{RV}}$ & $(17,14,20)$ & & 0.013 & 0.013 & 0.015 & 0.013 & 0.012 & 0.014 & 0.015 & 0.015 \\
\hline & RV & $(35,28,36)$ & & 0.011 & 0.013 & 0.012 & 0.012 & 0.010 & 0.012 & 0.012 & 0.011 \\
\hline & RV & $(50,46,55)$ & & 0.011 & 0.012 & 0.011 & 0.012 & 0.010 & 0.010 & 0.012 & 0.011 \\
\hline & RV & $(101,95,105)$ & & 0.010 & 0.010 & 0.009 & 0.011 & 0.009 & 0.009 & 0.012 & 0.010 \\
\hline & $\mathrm{S}$ & $(17,14,20)$ & & 0.020 & 0.013 & 0.009 & 0.007 & 0.017 & 0.011 & 0.011 & 0.006 \\
\hline & $\mathrm{S}$ & $(35,2$ & & 0.016 & 0.013 & 0.009 & 0.011 & 0.016 & 0.009 & 0.009 & 0.009 \\
\hline & $\mathrm{S}$ & $(50,46,55)$ & & 0.015 & 0.013 & 0.010 & 0.011 & 0.016 & 0.009 & 0.011 & 0.011 \\
\hline & $\mathrm{S}$ & $(101,95,105)$ & & 0.014 & 0.011 & 0.009 & 0.011 & 0.015 & 0.009 & 0.011 & 0.009 \\
\hline \multirow[t]{12}{*}{0.05} & $\mathrm{~W}$ & $(17,14,20)$ & & 0.068 & 0.073 & 0.075 & 0.068 & 0.071 & 0.075 & 0.075 & 0.079 \\
\hline & W & $(35$, & & 0.057 & 0.062 & 0.059 & 0.059 & 0.061 & 0.061 & 0.060 & 0.062 \\
\hline & W & $(50,46,55)$ & & 0.057 & 0.057 & 0.058 & 0.051 & 0.053 & 0.056 & 0.058 & 0.058 \\
\hline & W & $(101,95,105)$ & & 0.055 & 0.055 & 0.052 & 0.051 & 0.052 & 0.051 & 0.057 & 0.054 \\
\hline & RV & $(17,14,20)$ & & 0.059 & 0.062 & 0.064 & 0.058 & 0.060 & 0.061 & 0.062 & 0.065 \\
\hline & RV & $(35$, & & 0.053 & 0.057 & 0.055 & 0.054 & 0.056 & 0.055 & 0.055 & 0.056 \\
\hline & RV & $(50,46,55)$ & & 0.053 & 0.053 & 0.055 & 0.049 & 0.050 & 0.054 & 0.054 & 0.054 \\
\hline & RV & $(101,95,105)$ & & 0.053 & 0.053 & 0.051 & 0.051 & 0.050 & 0.050 & 0.054 & 0.053 \\
\hline & $\mathrm{S}$ & $(17,14,20)$ & & 0.095 & 0.080 & 0.058 & 0.048 & 0.084 & 0.065 & 0.054 & 0.052 \\
\hline & $\mathrm{S}$ & $(35,28,36)$ & & 0.069 & 0.070 & 0.052 & 0.051 & 0.083 & 0.056 & 0.051 & 0.051 \\
\hline & $\mathrm{S}$ & $(50$, & & 0.068 & 0.065 & 0.051 & 0.049 & 0.074 & 0.053 & 0.050 & 0.051 \\
\hline & $\mathrm{S}$ & $(101,95,105)$ & & 0.063 & 0.060 & 0.050 & 0.051 & 0.073 & 0.048 & 0.050 & 0.051 \\
\hline \multirow[t]{12}{*}{0.1} & $\mathrm{~W}$ & $(17,14,20)$ & & 0.123 & 0.128 & 0.131 & 0.125 & 0.124 & 0.132 & 0.131 & 0.138 \\
\hline & W & $(35,28,36)$ & & 0.112 & 0.116 & 0.116 & 0.112 & 0.113 & 0.113 & 0.120 & 0.114 \\
\hline & W & $(50,46,55)$ & & 0.109 & 0.106 & 0.113 & 0.102 & 0.107 & 0.106 & 0.111 & 0.105 \\
\hline & $\mathrm{W}$ & $(101,95,105)$ & & 0.104 & 0.104 & 0.102 & 0.102 & 0.103 & 0.099 & 0.106 & 0.105 \\
\hline & RV & $(17,14,20)$ & & 0.114 & 0.117 & 0.118 & 0.114 & 0.114 & 0.119 & 0.118 & 0.122 \\
\hline & RV & $(35,28,36)$ & & 0.106 & 0.111 & 0.109 & 0.106 & 0.107 & 0.107 & 0.109 & 0.109 \\
\hline & RV & $(50,46,55)$ & & 0.105 & 0.103 & 0.109 & 0.100 & 0.103 & 0.102 & 0.109 & 0.102 \\
\hline & RV & $(101,95,105)$ & & 0.103 & 0.103 & 0.099 & 0.100 & 0.102 & 0.098 & 0.104 & 0.101 \\
\hline & $\mathrm{S}$ & $(17,14,20)$ & & 0.177 & 0.148 & 0.113 & 0.102 & 0.159 & 0.133 & 0.109 & 0.108 \\
\hline & $\mathrm{S}$ & $(35,28,36)$ & & 0.138 & 0.137 & 0.105 & 0.102 & 0.155 & 0.110 & 0.108 & 0.102 \\
\hline & $\mathrm{S}$ & $(50,46,55)$ & & 0.131 & 0.126 & 0.105 & 0.098 & 0.149 & 0.103 & 0.104 & 0.098 \\
\hline & $\mathrm{S}$ & $(101,95,105)$ & & 0.118 & 0.117 & 0.098 & 0.102 & 0.146 & 0.097 & 0.102 & 0.102 \\
\hline
\end{tabular}


Tabela E.3: Tamanhos empíricos dos testes Wald, razão de verossimilhança e score para o teste $H_{0}: \beta_{11}=\beta_{12}$, com $\mu=5$.

\begin{tabular}{|c|c|c|c|c|c|c|c|c|c|c|c|}
\hline \multirow[b]{2}{*}{ Nível } & \multirow[b]{2}{*}{ Teste } & \multirow[b]{2}{*}{$\left(n_{1}, n_{2}, n_{3}\right)$} & \multirow{2}{*}{$\begin{array}{l}\sigma_{x}^{2} \\
\sigma^{2} \\
\end{array}$} & \multirow{2}{*}{$\begin{array}{l}0.01 \\
0.01\end{array}$} & \multicolumn{3}{|c|}{0.1} & \multicolumn{4}{|c|}{0.5} \\
\hline & & & & & 0.01 & 0.05 & 0.1 & 0.01 & 0.05 & 0.1 & 0.5 \\
\hline \multirow[t]{12}{*}{0.01} & W & $(17,14,20)$ & & 0.018 & 0.018 & 0.022 & 0.019 & 0.021 & 0.018 & 0.020 & 0.022 \\
\hline & W & $(35,28,36)$ & & 0.016 & 0.013 & 0.013 & 0.013 & 0.016 & 0.013 & 0.016 & 0.015 \\
\hline & W & $(50,46,55)$ & & 0.014 & 0.011 & 0.013 & 0.012 & 0.012 & 0.013 & 0.012 & 0.015 \\
\hline & W & $(101,95,105)$ & & 0.011 & 0.010 & 0.011 & 0.011 & 0.011 & 0.010 & 0.012 & 0.011 \\
\hline & $\overline{\mathrm{RV}}$ & $(17,14,20)$ & & 0.016 & 0.013 & 0.015 & 0.013 & 0.013 & 0.012 & 0.014 & 0.014 \\
\hline & RV & $(35,28,36)$ & & 0.015 & 0.010 & 0.013 & 0.012 & 0.012 & 0.009 & 0.013 & 0.012 \\
\hline & RV & $(50,46,55)$ & & 0.012 & 0.010 & 0.012 & 0.011 & 0.011 & 0.011 & 0.010 & 0.012 \\
\hline & $\mathrm{RV}$ & $(101,95,105)$ & & 0.010 & 0.009 & 0.011 & 0.011 & 0.011 & 0.009 & 0.010 & 0.011 \\
\hline & $\mathrm{S}$ & $(17,14,20)$ & & 0.019 & 0.013 & 0.008 & 0.007 & 0.018 & 0.008 & 0.009 & 0.007 \\
\hline & $\mathrm{S}$ & $(35,28,36)$ & & 0.018 & 0.013 & 0.008 & 0.009 & 0.017 & 0.009 & 0.009 & 0.008 \\
\hline & $\mathrm{S}$ & $(50,46,55)$ & & 0.016 & 0.013 & 0.011 & 0.011 & 0.016 & 0.010 & 0.009 & 0.011 \\
\hline & $\mathrm{S}$ & $(101,95,105)$ & & 0.015 & 0.012 & 0.010 & 0.011 & 0.015 & 0.009 & 0.011 & 0.010 \\
\hline \multirow[t]{12}{*}{0.05} & $\mathrm{~W}$ & $(17,14,20)$ & & 0.063 & 0.065 & 0.074 & 0.072 & 0.069 & 0.066 & 0.070 & 0.077 \\
\hline & W & $(35,28,36)$ & & 0.058 & 0.061 & 0.060 & 0.055 & 0.059 & 0.058 & 0.062 & 0.061 \\
\hline & W & $(50,46,55)$ & & 0.054 & 0.051 & 0.056 & 0.053 & 0.053 & 0.054 & 0.060 & 0.060 \\
\hline & W & $(101,95,105)$ & & 0.051 & 0.051 & 0.053 & 0.049 & 0.049 & 0.049 & 0.054 & 0.051 \\
\hline & $\overline{\mathrm{RV}}$ & $(17,14,20)$ & & 0.055 & 0.056 & 0.062 & 0.061 & 0.057 & 0.057 & 0.059 & 0.064 \\
\hline & RV & $(35,28,36)$ & & 0.054 & 0.056 & 0.054 & 0.058 & 0.056 & 0.053 & 0.058 & 0.055 \\
\hline & RV & $(50,46,55)$ & & 0.051 & 0.049 & 0.053 & 0.057 & 0.050 & 0.052 & 0.056 & 0.054 \\
\hline & $\mathrm{RV}$ & $(101,95,105)$ & & 0.051 & 0.051 & 0.051 & 0.048 & 0.049 & 0.048 & 0.053 & 0.049 \\
\hline & $\mathrm{S}$ & $(17,14,20)$ & & 0.089 & 0.073 & 0.055 & 0.049 & 0.093 & 0.058 & 0.055 & 0.052 \\
\hline & $\mathrm{S}$ & $(35,28,36)$ & & 0.077 & 0.073 & 0.049 & 0.048 & 0.090 & 0.053 & 0.054 & 0.048 \\
\hline & $\mathrm{S}$ & $(50,46,55)$ & & 0.065 & 0.064 & 0.050 & 0.049 & 0.088 & 0.052 & 0.054 & 0.052 \\
\hline & $\mathrm{S}$ & $(101,95,105)$ & & 0.061 & 0.061 & 0.050 & 0.049 & 0.073 & 0.049 & 0.052 & 0.048 \\
\hline \multirow[t]{12}{*}{0.1} & $\mathrm{~W}$ & $(17,14,20)$ & & 0.120 & 0.118 & 0.127 & 0.129 & 0.124 & 0.120 & 0.129 & 0.137 \\
\hline & W & $(35,28,36)$ & & 0.116 & 0.113 & 0.116 & 0.110 & 0.111 & 0.112 & 0.116 & 0.114 \\
\hline & W & $(50,46,55)$ & & 0.102 & 0.101 & 0.107 & 0.110 & 0.108 & 0.107 & 0.113 & 0.109 \\
\hline & $\mathrm{W}$ & $(101,95,105)$ & & 0.102 & 0.101 & 0.107 & 0.099 & 0.101 & 0.100 & 0.102 & 0.100 \\
\hline & $\mathrm{RV}$ & $(17,14,20)$ & & 0.112 & 0.108 & 0.117 & 0.118 & 0.112 & 0.109 & 0.118 & 0.121 \\
\hline & $\mathrm{RV}$ & $(35,28,36)$ & & 0.111 & 0.107 & 0.110 & 0.115 & 0.106 & 0.107 & 0.111 & 0.108 \\
\hline & $\mathrm{RV}$ & $(50,46,55)$ & & 0.099 & 0.097 & 0.104 & 0.111 & 0.105 & 0.105 & 0.109 & 0.106 \\
\hline & RV & $(101,95,105)$ & & 0.101 & 0.101 & 0.103 & 0.098 & 0.099 & 0.098 & 0.100 & 0.098 \\
\hline & $\mathrm{S}$ & $(17,14,20)$ & & 0.175 & 0.150 & 0.112 & 0.108 & 0.180 & 0.122 & 0.110 & 0.109 \\
\hline & $\mathrm{S}$ & $(35,28,36)$ & & 0.141 & 0.144 & 0.105 & 0.099 & 0.170 & 0.111 & 0.105 & 0.103 \\
\hline & $\mathrm{S}$ & $(50,46,55)$ & & 0.122 & 0.127 & 0.101 & 0.101 & 0.167 & 0.105 & 0.105 & 0.103 \\
\hline & $\mathrm{S}$ & $(101,95,105)$ & & 0.117 & 0.119 & 0.100 & 0.099 & 0.142 & 0.097 & 0.098 & 0.099 \\
\hline
\end{tabular}


Tabela E.4: Tamanhos empíricos dos testes Wald, razão de verossimilhança e score para o teste $H_{0}: \beta_{11}=\beta_{13}$, com $\mu=1$.

\begin{tabular}{|c|c|c|c|c|c|c|c|c|c|c|c|}
\hline \multirow[b]{2}{*}{ Nível } & \multirow[b]{2}{*}{ Teste } & \multirow[b]{2}{*}{$\left(n_{1}, n_{2}, n_{3}\right)$} & \multirow{2}{*}{$\begin{array}{l}\sigma_{x}^{2} \\
\sigma^{2}\end{array}$} & \multirow{2}{*}{$\begin{array}{l}0.01 \\
0.01\end{array}$} & \multicolumn{3}{|c|}{0.1} & \multicolumn{4}{|c|}{0.5} \\
\hline & & & & & 0.01 & 0.05 & 0.1 & 0.01 & 0.05 & 0.1 & 0.5 \\
\hline \multirow[t]{12}{*}{0.01} & $\mathrm{~W}$ & $(17,14,20)$ & & 0.020 & 0.019 & 0.018 & 0.021 & 0.023 & 0.018 & 0.018 & 0.018 \\
\hline & $\mathrm{W}$ & $(35,28,36)$ & & 0.014 & 0.015 & 0.014 & 0.016 & 0.015 & 0.017 & 0.012 & 0.017 \\
\hline & W & $(50,46,55)$ & & 0.013 & 0.012 & 0.014 & 0.014 & 0.013 & 0.015 & 0.012 & 0.011 \\
\hline & $\mathrm{W}$ & $(101,95,105)$ & & 0.011 & 0.012 & 0.012 & 0.009 & 0.011 & 0.011 & 0.011 & 0.010 \\
\hline & $\mathrm{RV}$ & $(17,14,20)$ & & 0.015 & 0.013 & 0.013 & 0.014 & 0.015 & 0.013 & 0.013 & 0.013 \\
\hline & RV & $(35,28,36)$ & & 0.012 & 0.011 & 0.011 & 0.013 & 0.010 & 0.013 & 0.010 & 0.013 \\
\hline & $\mathrm{RV}$ & $(50,46,55)$ & & 0.011 & 0.010 & 0.011 & 0.012 & 0.010 & 0.012 & 0.010 & 0.009 \\
\hline & $\mathrm{RV}$ & $(101,95,105)$ & & 0.011 & 0.010 & 0.011 & 0.009 & 0.010 & 0.011 & 0.010 & 0.009 \\
\hline & S & $(17,14,20)$ & & 0.015 & 0.016 & 0.009 & 0.007 & 0.016 & 0.013 & 0.008 & 0.008 \\
\hline & S & $(35,28,36)$ & & 0.013 & 0.015 & 0.009 & 0.010 & 0.015 & 0.013 & 0.008 & 0.012 \\
\hline & $\mathrm{S}$ & $(50,46,55)$ & & 0.012 & 0.013 & 0.011 & 0.010 & 0.015 & 0.011 & 0.009 & 0.008 \\
\hline & $\mathrm{S}$ & $(101,95,105)$ & & 0.010 & 0.013 & 0.010 & 0.010 & 0.015 & 0.011 & 0.009 & 0.009 \\
\hline \multirow[t]{12}{*}{0.05} & $\mathrm{~W}$ & $(17,14,20)$ & & 0.066 & 0.069 & 0.072 & 0.077 & 0.073 & 0.074 & 0.071 & 0.071 \\
\hline & $\mathrm{W}$ & $(35,28,36)$ & & 0.062 & 0.060 & 0.059 & 0.063 & 0.063 & 0.067 & 0.058 & 0.064 \\
\hline & $\mathrm{W}$ & $(50,46,55)$ & & 0.058 & 0.057 & 0.058 & 0.057 & 0.059 & 0.058 & 0.058 & 0.050 \\
\hline & W & $(101,95,105)$ & & 0.052 & 0.054 & 0.054 & 0.051 & 0.051 & 0.053 & 0.052 & 0.050 \\
\hline & RV & $(17,14,20)$ & & 0.059 & 0.059 & 0.060 & 0.066 & 0.059 & 0.061 & 0.057 & 0.061 \\
\hline & $\mathrm{RV}$ & $(35,28,36)$ & & 0.058 & 0.055 & 0.053 & 0.058 & 0.055 & 0.061 & 0.052 & 0.059 \\
\hline & $\mathrm{RV}$ & $(50,46,55)$ & & 0.055 & 0.054 & 0.053 & 0.054 & 0.054 & 0.054 & 0.052 & 0.047 \\
\hline & RV & $(101,95,105)$ & & 0.051 & 0.052 & 0.053 & 0.050 & 0.049 & 0.051 & 0.051 & 0.051 \\
\hline & $\mathrm{S}$ & $(17,14,20)$ & & 0.075 & 0.081 & 0.052 & 0.056 & 0.084 & 0.065 & 0.053 & 0.053 \\
\hline & S & $(35,28,36)$ & & 0.068 & 0.078 & 0.052 & 0.053 & 0.084 & 0.065 & 0.047 & 0.053 \\
\hline & S & $(50,46,55)$ & & 0.061 & 0.073 & 0.052 & 0.051 & 0.084 & 0.059 & 0.052 & 0.047 \\
\hline & $\mathrm{S}$ & $(101,95,105)$ & & 0.054 & 0.070 & 0.051 & 0.049 & 0.082 & 0.056 & 0.050 & 0.049 \\
\hline \multirow[t]{12}{*}{0.1} & $\mathrm{~W}$ & $(17,14,20)$ & & 0.121 & 0.125 & 0.129 & 0.135 & 0.130 & 0.130 & 0.127 & 0.129 \\
\hline & $\mathrm{W}$ & $(35,28,36)$ & & 0.117 & 0.118 & 0.113 & 0.118 & 0.114 & 0.118 & 0.116 & 0.121 \\
\hline & $\mathrm{W}$ & $(50,46,55)$ & & 0.108 & 0.108 & 0.111 & 0.111 & 0.112 & 0.108 & 0.115 & 0.107 \\
\hline & $\mathrm{W}$ & $(101,95,105)$ & & 0.101 & 0.104 & 0.104 & 0.106 & 0.100 & 0.106 & 0.102 & 0.106 \\
\hline & RV & $(17,14,20)$ & & 0.115 & 0.114 & 0.119 & 0.123 & 0.116 & 0.114 & 0.112 & 0.115 \\
\hline & $\mathrm{RV}$ & $(35,28,36)$ & & 0.114 & 0.111 & 0.107 & 0.111 & 0.108 & 0.111 & 0.107 & 0.115 \\
\hline & $\mathrm{RV}$ & $(50,46,55)$ & & 0.105 & 0.103 & 0.107 & 0.107 & 0.107 & 0.104 & 0.106 & 0.102 \\
\hline & $\mathrm{RV}$ & $(101,95,105)$ & & 0.100 & 0.102 & 0.102 & 0.103 & 0.098 & 0.103 & 0.101 & 0.102 \\
\hline & $\mathrm{S}$ & $(17,14,20)$ & & 0.152 & 0.158 & 0.115 & 0.114 & 0.167 & 0.131 & 0.108 & 0.107 \\
\hline & $\mathrm{S}$ & $(35,28,36)$ & & 0.137 & 0.154 & 0.104 & 0.105 & 0.162 & 0.128 & 0.106 & 0.106 \\
\hline & $\mathrm{S}$ & $(50,46,55)$ & & 0.122 & 0.138 & 0.104 & 0.103 & 0.160 & 0.118 & 0.105 & 0.097 \\
\hline & S & $(101,95,105)$ & & 0.110 & 0.133 & 0.100 & 0.100 & 0.154 & 0.112 & 0.098 & 0.102 \\
\hline
\end{tabular}


Tabela E.5: Tamanhos empíricos dos testes Wald, razão de verossimilhança e score para o teste $H_{0}: \beta_{11}=\beta_{13}$, com $\mu=2.5$.

\begin{tabular}{|c|c|c|c|c|c|c|c|c|c|c|c|}
\hline \multirow[b]{2}{*}{ Nível } & \multirow[b]{2}{*}{ Teste } & \multirow[b]{2}{*}{$\left(n_{1}, n_{2}, n_{3}\right)$} & \multirow{2}{*}{$\begin{array}{l}\sigma_{x}^{2} \\
\sigma^{2}\end{array}$} & \multirow{2}{*}{$\begin{array}{l}0.01 \\
0.01\end{array}$} & \multicolumn{3}{|c|}{0.1} & \multicolumn{4}{|c|}{0.5} \\
\hline & & & & & 0.01 & 0.05 & 0.1 & 0.01 & 0.05 & 0.1 & 0.5 \\
\hline \multirow[t]{12}{*}{0.01} & W & $(17,14,20)$ & & 0.017 & 0.018 & 0.020 & 0.018 & 0.018 & 0.018 & 0.018 & 0.017 \\
\hline & W & $(35,28,36)$ & & 0.015 & 0.016 & 0.015 & 0.016 & 0.013 & 0.012 & 0.011 & 0.015 \\
\hline & W & $(50,46,55)$ & & 0.012 & 0.014 & 0.012 & 0.013 & 0.012 & 0.012 & 0.011 & 0.015 \\
\hline & W & $(101,95,105)$ & & 0.012 & 0.012 & 0.012 & 0.011 & 0.011 & 0.009 & 0.011 & 0.011 \\
\hline & RV & $(17,14,20)$ & & 0.013 & 0.013 & 0.014 & 0.013 & 0.013 & 0.012 & 0.013 & 0.013 \\
\hline & RV & $(35,28,36)$ & & 0.013 & 0.013 & 0.012 & 0.013 & 0.013 & 0.012 & 0.009 & 0.012 \\
\hline & RV & $(50,46,55)$ & & 0.010 & 0.012 & 0.011 & 0.011 & 0.012 & 0.012 & 0.011 & 0.012 \\
\hline & RV & $(101,95,105)$ & & 0.010 & 0.011 & 0.011 & 0.011 & 0.011 & 0.008 & 0.011 & 0.010 \\
\hline & $\mathrm{S}$ & $(17,14,20)$ & & 0.014 & 0.016 & 0.009 & 0.009 & 0.017 & 0.011 & 0.009 & 0.008 \\
\hline & $\mathrm{S}$ & $(35,28,36)$ & & 0.013 & 0.016 & 0.011 & 0.011 & 0.015 & 0.009 & 0.009 & 0.011 \\
\hline & $\mathrm{S}$ & $(50,46,55)$ & & 0.012 & 0.015 & 0.009 & 0.009 & 0.014 & 0.011 & 0.011 & 0.011 \\
\hline & $\mathrm{S}$ & $(101,95,105)$ & & 0.012 & 0.014 & 0.011 & 0.009 & 0.014 & 0.008 & 0.011 & 0.009 \\
\hline \multirow[t]{12}{*}{0.05} & W & $(17,14,20)$ & & 0.065 & 0.065 & 0.069 & 0.067 & 0.069 & 0.070 & 0.072 & 0.065 \\
\hline & W & $(35,28,36)$ & & 0.058 & 0.064 & 0.061 & 0.060 & 0.056 & 0.059 & 0.059 & 0.058 \\
\hline & W & $(50,46,55)$ & & 0.053 & 0.059 & 0.054 & 0.057 & 0.055 & 0.055 & 0.055 & 0.057 \\
\hline & $\mathrm{W}$ & $(101,95,105)$ & & 0.053 & 0.049 & 0.054 & 0.052 & 0.052 & 0.051 & 0.051 & 0.052 \\
\hline & $\mathrm{RV}$ & $(17,14,20)$ & & 0.057 & 0.059 & 0.060 & 0.057 & 0.058 & 0.060 & 0.062 & 0.057 \\
\hline & RV & $(35,28,36)$ & & 0.054 & 0.059 & 0.056 & 0.056 & 0.052 & 0.054 & 0.055 & 0.054 \\
\hline & RV & $(50,46,55)$ & & 0.051 & 0.056 & 0.053 & 0.053 & 0.051 & 0.052 & 0.051 & 0.053 \\
\hline & RV & $(101,95,105)$ & & 0.051 & 0.049 & 0.053 & 0.050 & 0.050 & 0.051 & 0.049 & 0.051 \\
\hline & $\mathrm{S}$ & $(17,14,20)$ & & 0.074 & 0.081 & 0.056 & 0.051 & 0.085 & 0.065 & 0.054 & 0.048 \\
\hline & $\mathrm{S}$ & $(35,28,36)$ & & 0.067 & 0.081 & 0.053 & 0.051 & 0.079 & 0.056 & 0.052 & 0.052 \\
\hline & $\mathrm{S}$ & $(50,46,55)$ & & 0.057 & 0.073 & 0.049 & 0.051 & 0.077 & 0.052 & 0.048 & 0.052 \\
\hline & $\mathrm{S}$ & $(101,95,105)$ & & 0.055 & 0.065 & 0.051 & 0.049 & 0.072 & 0.050 & 0.048 & 0.049 \\
\hline \multirow[t]{12}{*}{0.1} & $\mathrm{~W}$ & $(17,14,20)$ & & 0.121 & 0.121 & 0.126 & 0.123 & 0.122 & 0.129 & 0.128 & 0.126 \\
\hline & W & $(35,28,36)$ & & 0.106 & 0.118 & 0.113 & 0.112 & 0.105 & 0.113 & 0.111 & 0.110 \\
\hline & W & $(50,46,55)$ & & 0.106 & 0.114 & 0.105 & 0.109 & 0.105 & 0.104 & 0.106 & 0.110 \\
\hline & $\mathrm{W}$ & $(101,95,105)$ & & 0.105 & 0.101 & 0.103 & 0.103 & 0.102 & 0.103 & 0.099 & 0.102 \\
\hline & RV & $(17,14,20)$ & & 0.112 & 0.113 & 0.117 & 0.112 & 0.111 & 0.119 & 0.117 & 0.116 \\
\hline & RV & $(35,28,36)$ & & 0.103 & 0.113 & 0.108 & 0.108 & 0.103 & 0.107 & 0.105 & 0.109 \\
\hline & RV & $(50,46,55)$ & & 0.103 & 0.111 & 0.103 & 0.105 & 0.101 & 0.100 & 0.103 & 0.109 \\
\hline & RV & $(101,95,105)$ & & 0.102 & 0.100 & 0.101 & 0.102 & 0.101 & 0.100 & 0.097 & 0.101 \\
\hline & $\mathrm{S}$ & $(17,14,20)$ & & 0.152 & 0.157 & 0.112 & 0.104 & 0.165 & 0.132 & 0.115 & 0.107 \\
\hline & $\mathrm{S}$ & $(35,28,36)$ & & 0.126 & 0.153 & 0.106 & 0.104 & 0.152 & 0.112 & 0.102 & 0.102 \\
\hline & $\mathrm{S}$ & $(50,46,55)$ & & 0.120 & 0.145 & 0.101 & 0.102 & 0.152 & 0.103 & 0.100 & 0.102 \\
\hline & $\mathrm{S}$ & $(101,95,105)$ & & 0.113 & 0.133 & 0.100 & 0.101 & 0.143 & 0.102 & 0.100 & 0.100 \\
\hline
\end{tabular}


Tabela E.6: Tamanhos empíricos dos testes Wald, razão de verossimilhança e score para o teste $H_{0}: \beta_{11}=\beta_{13}$, com $\mu=5$.

\begin{tabular}{|c|c|c|c|c|c|c|c|c|c|c|}
\hline \multirow[b]{2}{*}{ Nível } & \multirow[b]{2}{*}{ Teste } & \multirow[b]{2}{*}{$\left(n_{1}, n_{2}, n_{3}\right)$} & \multirow{2}{*}{$\begin{array}{l}0.01 \\
0.01\end{array}$} & \multicolumn{3}{|c|}{0.1} & \multicolumn{4}{|c|}{0.5} \\
\hline & & & & 0.01 & 0.05 & 0.1 & 0.01 & 0.05 & 0.1 & 0.5 \\
\hline \multirow[t]{12}{*}{0.01} & $\mathrm{~W}$ & $(17,14,20)$ & 0.018 & 0.017 & 0.018 & 0.020 & 0.020 & 0.017 & 0.018 & 0.018 \\
\hline & $\mathrm{W}$ & $(35,28,36)$ & 0.015 & 0.015 & 0.012 & 0.015 & 0.015 & 0.014 & 0.015 & 0.014 \\
\hline & $\mathrm{W}$ & $(50,46,55)$ & 0.012 & 0.014 & 0.012 & 0.013 & 0.015 & 0.012 & 0.011 & 0.014 \\
\hline & $\mathrm{W}$ & $(101,95,105)$ & 0.011 & 0.010 & 0.012 & 0.009 & 0.011 & 0.010 & 0.011 & 0.012 \\
\hline & $\mathrm{RV}$ & $(17,14,20)$ & 0.013 & 0.012 & 0.013 & 0.015 & 0.014 & 0.012 & 0.015 & 0.013 \\
\hline & RV & $(35,28,36)$ & 0.013 & 0.012 & 0.013 & 0.012 & 0.013 & 0.012 & 0.014 & 0.013 \\
\hline & $\mathrm{RV}$ & $(50,46,55)$ & 0.010 & 0.012 & 0.012 & 0.011 & 0.012 & 0.011 & 0.012 & 0.012 \\
\hline & $\mathrm{RV}$ & $(101,95,105)$ & 0.010 & 0.009 & 0.012 & 0.009 & 0.011 & 0.009 & 0.011 & 0.011 \\
\hline & $\mathrm{S}$ & $(17,14,20)$ & 0.015 & 0.015 & 0.009 & 0.011 & 0.019 & 0.010 & 0.011 & 0.009 \\
\hline & S & $(35,28,36)$ & 0.013 & 0.013 & 0.009 & 0.011 & 0.018 & 0.010 & 0.011 & 0.010 \\
\hline & $\mathrm{S}$ & $(50,46,55)$ & 0.011 & 0.013 & 0.011 & 0.009 & 0.017 & 0.010 & 0.009 & 0.010 \\
\hline & $\mathrm{S}$ & $(101,95,105)$ & 0.011 & 0.012 & 0.011 & 0.009 & 0.017 & 0.010 & 0.010 & 0.010 \\
\hline \multirow[t]{12}{*}{0.05} & $\mathrm{~W}$ & $(17,14,20)$ & 0.069 & 0.065 & 0.068 & 0.071 & 0.068 & 0.068 & 0.070 & 0.065 \\
\hline & $\mathrm{W}$ & $(35,28,36)$ & 0.061 & 0.059 & 0.059 & 0.056 & 0.064 & 0.059 & 0.063 & 0.063 \\
\hline & $\mathrm{W}$ & $(50,46,55)$ & 0.054 & 0.059 & 0.058 & 0.055 & 0.058 & 0.059 & 0.055 & 0.054 \\
\hline & W & $(101,95,105)$ & 0.054 & 0.049 & 0.055 & 0.049 & 0.051 & 0.052 & 0.053 & 0.052 \\
\hline & RV & $(17,14,20)$ & 0.061 & 0.058 & 0.059 & 0.059 & 0.060 & 0.057 & 0.060 & 0.059 \\
\hline & $\mathrm{RV}$ & $(35,28,36)$ & 0.056 & 0.054 & 0.055 & 0.055 & 0.060 & 0.054 & 0.058 & 0.059 \\
\hline & $\mathrm{RV}$ & $(50,46,55)$ & 0.051 & 0.053 & 0.055 & 0.054 & 0.055 & 0.054 & 0.052 & 0.052 \\
\hline & RV & $(101,95,105)$ & 0.051 & 0.047 & 0.054 & 0.048 & 0.050 & 0.050 & 0.052 & 0.051 \\
\hline & $\mathrm{S}$ & $(17,14,20)$ & 0.080 & 0.080 & 0.052 & 0.052 & 0.092 & 0.060 & 0.055 & 0.049 \\
\hline & S & $(35,28,36)$ & 0.065 & 0.074 & 0.052 & 0.048 & 0.090 & 0.056 & 0.054 & 0.051 \\
\hline & S & $(50,46,55)$ & 0.057 & 0.074 & 0.052 & 0.052 & 0.085 & 0.055 & 0.049 & 0.049 \\
\hline & $\mathrm{S}$ & $(101,95,105)$ & 0.057 & 0.063 & 0.052 & 0.048 & 0.075 & 0.050 & 0.051 & 0.050 \\
\hline \multirow[t]{12}{*}{0.1} & $\mathrm{~W}$ & $(17,14,20)$ & 0.121 & 0.123 & 0.122 & 0.128 & 0.121 & 0.118 & 0.127 & 0.121 \\
\hline & $\mathrm{W}$ & $(35,28,36)$ & 0.110 & 0.110 & 0.112 & 0.110 & 0.118 & 0.108 & 0.113 & 0.114 \\
\hline & $\mathrm{W}$ & $(50,46,55)$ & 0.106 & 0.109 & 0.111 & 0.108 & 0.109 & 0.108 & 0.104 & 0.107 \\
\hline & $\mathrm{W}$ & $(101,95,105)$ & 0.105 & 0.100 & 0.106 & 0.103 & 0.104 & 0.104 & 0.101 & 0.107 \\
\hline & $\mathrm{RV}$ & $(17,14,20)$ & 0.112 & 0.114 & 0.114 & 0.119 & 0.112 & 0.111 & 0.118 & 0.111 \\
\hline & $\mathrm{RV}$ & $(35,28,36)$ & 0.107 & 0.107 & 0.109 & 0.106 & 0.111 & 0.106 & 0.110 & 0.110 \\
\hline & $\mathrm{RV}$ & $(50,46,55)$ & 0.104 & 0.107 & 0.107 & 0.105 & 0.105 & 0.105 & 0.101 & 0.103 \\
\hline & RV & $(101,95,105)$ & 0.103 & 0.099 & 0.104 & 0.101 & 0.103 & 0.102 & 0.099 & 0.103 \\
\hline & $\mathrm{S}$ & $(17,14,20)$ & 0.153 & 0.156 & 0.109 & 0.110 & 0.171 & 0.122 & 0.113 & 0.108 \\
\hline & $\mathrm{S}$ & $(35,28,36)$ & 0.133 & 0.147 & 0.105 & 0.098 & 0.169 & 0.112 & 0.107 & 0.105 \\
\hline & $\mathrm{S}$ & $(50,46,55)$ & 0.120 & 0.145 & 0.105 & 0.102 & 0.159 & 0.108 & 0.098 & 0.100 \\
\hline & S & $(101,95,105)$ & 0.114 & 0.129 & 0.103 & 0.099 & 0.149 & 0.103 & 0.098 & 0.100 \\
\hline
\end{tabular}


Tabela E.7: Tamanhos empíricos dos testes Wald, razão de verossimilhança e score para o teste $H_{0}: \beta_{12}=\beta_{13}$, com $\mu=1$.

\begin{tabular}{|c|c|c|c|c|c|c|c|c|c|c|c|}
\hline \multirow[b]{2}{*}{ Nível } & \multirow[b]{2}{*}{ Teste } & \multirow[b]{2}{*}{$\left(n_{1}, n_{2}, n_{3}\right)$} & \multirow{2}{*}{$\begin{array}{l}\sigma_{x}^{2} \\
\sigma^{2}\end{array}$} & \multirow{2}{*}{$\begin{array}{l}0.01 \\
0.01\end{array}$} & \multicolumn{3}{|c|}{0.1} & \multicolumn{4}{|c|}{0.5} \\
\hline & & & & & 0.01 & 0.05 & 0.1 & 0.01 & 0.05 & 0.1 & 0.5 \\
\hline \multirow[t]{12}{*}{0.01} & W & $(17,14,20)$ & & 0.020 & 0.020 & 0.022 & 0.021 & 0.021 & 0.022 & 0.028 & 0.028 \\
\hline & W & $(35,28,36)$ & & 0.017 & 0.016 & 0.016 & 0.015 & 0.015 & 0.016 & 0.014 & 0.017 \\
\hline & W & $(50,46,55)$ & & 0.011 & 0.014 & 0.016 & 0.014 & 0.013 & 0.012 & 0.013 & 0.014 \\
\hline & $\mathrm{W}$ & $(101,95,105)$ & & 0.009 & 0.012 & 0.009 & 0.011 & 0.012 & 0.011 & 0.012 & 0.012 \\
\hline & $\overline{\mathrm{RV}}$ & $(17,14,20)$ & & 0.014 & 0.014 & 0.014 & 0.013 & 0.012 & 0.013 & 0.016 & 0.017 \\
\hline & RV & $(35,28,36)$ & & 0.014 & 0.012 & 0.012 & 0.012 & 0.011 & 0.011 & 0.012 & 0.012 \\
\hline & RV & $(50,46,55)$ & & 0.009 & 0.012 & 0.012 & 0.012 & 0.011 & 0.010 & 0.011 & 0.012 \\
\hline & RV & $(101,95,105)$ & & 0.009 & 0.011 & 0.008 & 0.010 & 0.011 & 0.010 & 0.011 & 0.012 \\
\hline & $\mathrm{S}$ & $(17,14,20)$ & & 0.019 & 0.014 & 0.008 & 0.007 & 0.019 & 0.011 & 0.009 & 0.009 \\
\hline & $\mathrm{S}$ & $(35,28,36)$ & & 0.019 & 0.014 & 0.012 & 0.010 & 0.019 & 0.009 & 0.008 & 0.009 \\
\hline & $\mathrm{S}$ & $(50,46,55)$ & & 0.015 & 0.013 & 0.012 & 0.010 & 0.018 & 0.009 & 0.010 & 0.011 \\
\hline & $\mathrm{S}$ & $(101,95,105)$ & & 0.013 & 0.013 & 0.008 & 0.010 & 0.017 & 0.011 & 0.010 & 0.010 \\
\hline \multirow[t]{12}{*}{0.05} & $\mathrm{~W}$ & $(17,14,20)$ & & 0.069 & 0.072 & 0.072 & 0.066 & 0.071 & 0.072 & 0.082 & 0.084 \\
\hline & W & $(35,28,36)$ & & 0.065 & 0.061 & 0.066 & 0.064 & 0.057 & 0.062 & 0.066 & 0.068 \\
\hline & W & $(50,46,55)$ & & 0.056 & 0.060 & 0.058 & 0.061 & 0.056 & 0.055 & 0.060 & 0.055 \\
\hline & W & $(101,95,105)$ & & 0.051 & 0.054 & 0.053 & 0.054 & 0.053 & 0.052 & 0.057 & 0.055 \\
\hline & $\overline{R V}$ & $(17,14,20)$ & & 0.060 & 0.060 & 0.061 & 0.058 & 0.055 & 0.057 & 0.066 & 0.068 \\
\hline & $\mathrm{RV}$ & $(35,28,36)$ & & 0.059 & 0.054 & 0.060 & 0.058 & 0.054 & 0.056 & 0.057 & 0.060 \\
\hline & RV & $(50,46,55)$ & & 0.055 & 0.053 & 0.054 & 0.057 & 0.054 & 0.051 & 0.055 & 0.051 \\
\hline & RV & $(101,95,105)$ & & 0.050 & 0.052 & 0.052 & 0.053 & 0.051 & 0.051 & 0.054 & 0.051 \\
\hline & $\mathrm{S}$ & $(17,14,20)$ & & 0.086 & 0.072 & 0.055 & 0.054 & 0.088 & 0.058 & 0.054 & 0.055 \\
\hline & $\mathrm{S}$ & $(35,28,36)$ & & 0.077 & 0.069 & 0.055 & 0.053 & 0.088 & 0.057 & 0.052 & 0.053 \\
\hline & $\mathrm{S}$ & $(50,46,55)$ & & 0.067 & 0.068 & 0.051 & 0.053 & 0.085 & 0.049 & 0.050 & 0.048 \\
\hline & $\mathrm{S}$ & $(101,95,105)$ & & 0.060 & 0.061 & 0.051 & 0.052 & 0.076 & 0.049 & 0.050 & 0.052 \\
\hline \multirow[t]{12}{*}{0.1} & $\mathrm{~W}$ & $(17,14,20)$ & & 0.125 & 0.128 & 0.129 & 0.125 & 0.127 & 0.128 & 0.135 & 0.144 \\
\hline & W & $(35,28,36)$ & & 0.117 & 0.112 & 0.119 & 0.117 & 0.107 & 0.112 & 0.116 & 0.120 \\
\hline & W & $(50,46,55)$ & & 0.108 & 0.112 & 0.109 & 0.112 & 0.108 & 0.108 & 0.110 & 0.110 \\
\hline & $\mathrm{W}$ & $(101,95,105)$ & & 0.106 & 0.107 & 0.109 & 0.102 & 0.101 & 0.103 & 0.109 & 0.105 \\
\hline & RV & $(17,14,20)$ & & 0.113 & 0.111 & 0.114 & 0.109 & 0.107 & 0.110 & 0.119 & 0.124 \\
\hline & RV & $(35,28,36)$ & & 0.112 & 0.106 & 0.113 & 0.108 & 0.105 & 0.104 & 0.107 & 0.114 \\
\hline & $\mathrm{RV}$ & $(50,46,55)$ & & 0.104 & 0.105 & 0.105 & 0.107 & 0.103 & 0.104 & 0.106 & 0.106 \\
\hline & $\mathrm{RV}$ & $(101,95,105)$ & & 0.103 & 0.105 & 0.105 & 0.100 & 0.100 & 0.101 & 0.106 & 0.102 \\
\hline & $\mathrm{S}$ & $(17,14,20)$ & & 0.165 & 0.147 & 0.110 & 0.097 & 0.171 & 0.123 & 0.111 & 0.103 \\
\hline & $\mathrm{S}$ & $(35,28,36)$ & & 0.141 & 0.135 & 0.108 & 0.104 & 0.168 & 0.112 & 0.106 & 0.103 \\
\hline & $\mathrm{S}$ & $(50,46,55)$ & & 0.125 & 0.125 & 0.107 & 0.103 & 0.167 & 0.103 & 0.102 & 0.101 \\
\hline & $\mathrm{S}$ & $(101,95,105)$ & & 0.118 & 0.122 & 0.106 & 0.099 & 0.147 & 0.099 & 0.102 & 0.100 \\
\hline
\end{tabular}


Tabela E.8: Tamanhos empíricos dos testes Wald, razão de verossimilhança e score para o teste $H_{0}: \beta_{12}=\beta_{13}, \operatorname{com} \mu=2.5$.

\begin{tabular}{|c|c|c|c|c|c|c|c|c|c|c|}
\hline \multirow[b]{2}{*}{ Nível } & \multirow[b]{2}{*}{ Teste } & \multirow[b]{2}{*}{$\left(n_{1}, n_{2}, n_{3}\right)$} & \multirow{2}{*}{$\begin{array}{l}0.01 \\
0.01\end{array}$} & \multicolumn{3}{|c|}{0.1} & \multicolumn{4}{|c|}{0.5} \\
\hline & & & & 0.01 & 0.05 & 0.1 & 0.01 & 0.05 & 0.1 & 0.5 \\
\hline \multirow[t]{12}{*}{0.01} & W & $(17,14,20)$ & 0.021 & 0.019 & 0.018 & 0.021 & 0.020 & 0.022 & 0.023 & 0.025 \\
\hline & W & $(35,28,36)$ & 0.017 & 0.015 & 0.015 & 0.018 & 0.014 & 0.014 & 0.015 & 0.014 \\
\hline & W & $(50,46,55)$ & 0.011 & 0.013 & 0.014 & 0.013 & 0.014 & 0.014 & 0.012 & 0.013 \\
\hline & $\mathrm{W}$ & $(101,95,105)$ & 0.011 & 0.012 & 0.010 & 0.011 & 0.010 & 0.010 & 0.010 & 0.011 \\
\hline & RV & $(17,14,20)$ & 0.015 & 0.013 & 0.008 & 0.013 & 0.013 & 0.013 & 0.015 & 0.017 \\
\hline & RV & $(35,28,36)$ & 0.014 & 0.012 & 0.012 & 0.012 & 0.011 & 0.011 & 0.012 & 0.013 \\
\hline & RV & $(50,46,55)$ & 0.010 & 0.012 & 0.011 & 0.011 & 0.011 & 0.011 & 0.010 & 0.012 \\
\hline & RV & $(101,95,105)$ & 0.010 & 0.011 & 0.010 & 0.011 & 0.010 & 0.009 & 0.009 & 0.010 \\
\hline & $\mathrm{S}$ & $(17,14,20)$ & 0.019 & 0.013 & 0.008 & 0.009 & 0.019 & 0.011 & 0.009 & 0.007 \\
\hline & $\mathrm{S}$ & $(35,28,36)$ & 0.018 & 0.013 & 0.011 & 0.011 & 0.018 & 0.009 & 0.008 & 0.008 \\
\hline & $\mathrm{S}$ & $(50,46,55)$ & 0.015 & 0.013 & 0.011 & 0.010 & 0.018 & 0.011 & 0.009 & 0.010 \\
\hline & S & $(101,95,105)$ & 0.014 & 0.012 & 0.009 & 0.010 & 0.017 & 0.008 & 0.009 & 0.009 \\
\hline \multirow[t]{12}{*}{0.05} & W & $(17,14,20)$ & 0.071 & 0.071 & 0.070 & 0.071 & 0.066 & 0.075 & 0.075 & 0.079 \\
\hline & $\mathrm{W}$ & $(35,28,36)$ & 0.064 & 0.061 & 0.065 & 0.064 & 0.060 & 0.062 & 0.061 & 0.060 \\
\hline & W & $(50,46,55)$ & 0.052 & 0.056 & 0.057 & 0.059 & 0.055 & 0.059 & 0.056 & 0.056 \\
\hline & $\mathrm{W}$ & $(101,95,105)$ & 0.052 & 0.055 & 0.051 & 0.055 & 0.051 & 0.051 & 0.054 & 0.052 \\
\hline & RV & $(17,14,20)$ & 0.063 & 0.060 & 0.058 & 0.060 & 0.055 & 0.062 & 0.062 & 0.065 \\
\hline & RV & $(35,28,36)$ & 0.060 & 0.057 & 0.055 & 0.060 & 0.053 & 0.056 & 0.054 & 0.054 \\
\hline & RV & $(50,46,55)$ & 0.050 & 0.054 & 0.054 & 0.056 & 0.053 & 0.055 & 0.053 & 0.053 \\
\hline & RV & $(101,95,105)$ & 0.050 & 0.054 & 0.048 & 0.054 & 0.050 & 0.049 & 0.052 & 0.051 \\
\hline & S & $(17,14,20)$ & 0.090 & 0.073 & 0.051 & 0.055 & 0.087 & 0.061 & 0.054 & 0.051 \\
\hline & $\mathrm{S}$ & $(35,28,36)$ & 0.078 & 0.071 & 0.053 & 0.054 & 0.086 & 0.055 & 0.049 & 0.049 \\
\hline & $\mathrm{S}$ & $(50,46,55)$ & 0.064 & 0.063 & 0.053 & 0.054 & 0.085 & 0.054 & 0.049 & 0.051 \\
\hline & $\mathrm{S}$ & $(101,95,105)$ & 0.064 & 0.061 & 0.047 & 0.053 & 0.076 & 0.048 & 0.051 & 0.049 \\
\hline \multirow[t]{12}{*}{0.1} & $\mathrm{~W}$ & $(17,14,20)$ & 0.126 & 0.127 & 0.119 & 0.130 & 0.117 & 0.130 & 0.127 & 0.138 \\
\hline & W & $(35,28,36)$ & 0.118 & 0.111 & 0.118 & 0.117 & 0.113 & 0.116 & 0.112 & 0.113 \\
\hline & $\mathrm{W}$ & $(50,46,55)$ & 0.102 & 0.107 & 0.109 & 0.109 & 0.109 & 0.108 & 0.104 & 0.105 \\
\hline & $\mathrm{W}$ & $(101,95,105)$ & 0.102 & 0.107 & 0.103 & 0.106 & 0.102 & 0.103 & 0.103 & 0.101 \\
\hline & RV & $(17,14,20)$ & 0.117 & 0.116 & 0.115 & 0.117 & 0.107 & 0.117 & 0.115 & 0.122 \\
\hline & RV & $(35,28,36)$ & 0.112 & 0.105 & 0.113 & 0.111 & 0.107 & 0.110 & 0.106 & 0.106 \\
\hline & RV & $(50,46,55)$ & 0.100 & 0.104 & 0.106 & 0.106 & 0.105 & 0.105 & 0.101 & 0.101 \\
\hline & RV & $(101,95,105)$ & 0.100 & 0.103 & 0.101 & 0.105 & 0.100 & 0.101 & 0.101 & 0.099 \\
\hline & $\mathrm{S}$ & $(17,14,20)$ & 0.168 & 0.149 & 0.108 & 0.105 & 0.169 & 0.125 & 0.105 & 0.108 \\
\hline & $\mathrm{S}$ & $(35,28,36)$ & 0.142 & 0.136 & 0.107 & 0.105 & 0.168 & 0.112 & 0.101 & 0.099 \\
\hline & $\mathrm{S}$ & $(50,46,55)$ & 0.122 & 0.125 & 0.103 & 0.102 & 0.168 & 0.107 & 0.099 & 0.099 \\
\hline & $\mathrm{S}$ & $(101,95,105)$ & 0.117 & 0.119 & 0.099 & 0.102 & 0.146 & 0.100 & 0.101 & 0.097 \\
\hline
\end{tabular}


Tabela E.9: Tamanhos empíricos dos testes Wald, razão de verossimilhança e score para o teste $H_{0}: \beta_{12}=\beta_{13}$, com $\mu=5$.

\begin{tabular}{|c|c|c|c|c|c|c|c|c|c|c|c|}
\hline \multirow[b]{2}{*}{ Nível } & \multirow[b]{2}{*}{ Teste } & \multirow[b]{2}{*}{$\left(n_{1}, n_{2}, n_{3}\right)$} & \multirow{2}{*}{$\begin{array}{l}\sigma_{x}^{2} \\
\sigma^{2}\end{array}$} & \multirow{2}{*}{$\begin{array}{l}0.01 \\
0.01\end{array}$} & \multicolumn{3}{|c|}{0.1} & \multicolumn{4}{|c|}{0.5} \\
\hline & & & & & 0.01 & 0.05 & 0.1 & 0.01 & 0.05 & 0.1 & 0.5 \\
\hline \multirow[t]{12}{*}{0.01} & W & $(17,14,20)$ & & 0.019 & 0.018 & 0.022 & 0.021 & 0.019 & 0.021 & 0.023 & 0.025 \\
\hline & W & $(35,28,36)$ & & 0.014 & 0.014 & 0.015 & 0.014 & 0.015 & 0.014 & 0.013 & 0.014 \\
\hline & W & $(50,46,55)$ & & 0.012 & 0.011 & 0.013 & 0.012 & 0.012 & 0.012 & 0.013 & 0.013 \\
\hline & $\mathrm{W}$ & $(101,95,105)$ & & 0.010 & 0.009 & 0.011 & 0.012 & 0.011 & 0.012 & 0.010 & 0.010 \\
\hline & $\overline{\mathrm{RV}}$ & $(17,14,20)$ & & 0.012 & 0.011 & 0.013 & 0.013 & 0.012 & 0.016 & 0.015 & 0.017 \\
\hline & RV & $(35,28,36)$ & & 0.011 & 0.011 & 0.012 & 0.011 & 0.012 & 0.012 & 0.011 & 0.011 \\
\hline & RV & $(50,46,55)$ & & 0.010 & 0.010 & 0.012 & 0.011 & 0.010 & 0.010 & 0.011 & 0.011 \\
\hline & RV & $(101,95,105)$ & & 0.009 & 0.009 & 0.011 & 0.011 & 0.010 & 0.010 & 0.010 & 0.009 \\
\hline & $\mathrm{S}$ & $(17,14,20)$ & & 0.021 & 0.013 & 0.007 & 0.006 & 0.018 & 0.012 & 0.008 & 0.009 \\
\hline & $\mathrm{S}$ & $(35,28,36)$ & & 0.020 & 0.013 & 0.009 & 0.008 & 0.017 & 0.009 & 0.008 & 0.008 \\
\hline & $\mathrm{S}$ & $(50,46,55)$ & & 0.015 & 0.012 & 0.011 & 0.010 & 0.016 & 0.010 & 0.009 & 0.009 \\
\hline & $\mathrm{S}$ & $(101,95,105)$ & & 0.015 & 0.010 & 0.011 & 0.010 & 0.016 & 0.010 & 0.009 & 0.009 \\
\hline \multirow[t]{12}{*}{0.05} & $\mathrm{~W}$ & $(17,14,20)$ & & 0.070 & 0.069 & 0.074 & 0.075 & 0.068 & 0.067 & 0.073 & 0.077 \\
\hline & W & $(35,28,36)$ & & 0.063 & 0.058 & 0.061 & 0.061 & 0.057 & 0.055 & 0.060 & 0.057 \\
\hline & W & $(50,46,55)$ & & 0.055 & 0.056 & 0.054 & 0.058 & 0.056 & 0.055 & 0.057 & 0.057 \\
\hline & W & $(101,95,105)$ & & 0.048 & 0.048 & 0.053 & 0.052 & 0.048 & 0.055 & 0.050 & 0.052 \\
\hline & $\overline{R V}$ & $(17,14,20)$ & & 0.060 & 0.058 & 0.065 & 0.062 & 0.058 & 0.057 & 0.061 & 0.065 \\
\hline & $\mathrm{RV}$ & $(35,28,36)$ & & 0.059 & 0.054 & 0.055 & 0.056 & 0.052 & 0.051 & 0.055 & 0.051 \\
\hline & RV & $(50,46,55)$ & & 0.052 & 0.053 & 0.052 & 0.054 & 0.052 & 0.051 & 0.055 & 0.051 \\
\hline & RV & $(101,95,105)$ & & 0.048 & 0.048 & 0.051 & 0.050 & 0.047 & 0.051 & 0.049 & 0.051 \\
\hline & $\mathrm{S}$ & $(17,14,20)$ & & 0.093 & 0.076 & 0.057 & 0.052 & 0.091 & 0.059 & 0.051 & 0.051 \\
\hline & $\mathrm{S}$ & $(35,28,36)$ & & 0.079 & 0.070 & 0.051 & 0.052 & 0.090 & 0.051 & 0.051 & 0.049 \\
\hline & $\mathrm{S}$ & $(50,46,55)$ & & 0.066 & 0.067 & 0.050 & 0.052 & 0.090 & 0.050 & 0.051 & 0.051 \\
\hline & $\mathrm{S}$ & $(101,95,105)$ & & 0.060 & 0.056 & 0.050 & 0.050 & 0.072 & 0.050 & 0.048 & 0.049 \\
\hline \multirow[t]{12}{*}{0.1} & $\mathrm{~W}$ & $(17,14,20)$ & & 0.122 & 0.126 & 0.126 & 0.128 & 0.117 & 0.120 & 0.129 & 0.132 \\
\hline & W & $(35,28,36)$ & & 0.114 & 0.109 & 0.119 & 0.111 & 0.111 & 0.107 & 0.111 & 0.109 \\
\hline & W & $(50,46,55)$ & & 0.106 & 0.107 & 0.107 & 0.111 & 0.106 & 0.106 & 0.111 & 0.108 \\
\hline & $\mathrm{W}$ & $(101,95,105)$ & & 0.099 & 0.099 & 0.107 & 0.101 & 0.103 & 0.105 & 0.099 & 0.102 \\
\hline & RV & $(17,14,20)$ & & 0.113 & 0.113 & 0.116 & 0.117 & 0.107 & 0.110 & 0.117 & 0.119 \\
\hline & RV & $(35,28,36)$ & & 0.109 & 0.104 & 0.113 & 0.106 & 0.105 & 0.102 & 0.108 & 0.103 \\
\hline & $\mathrm{RV}$ & $(50,46,55)$ & & 0.102 & 0.104 & 0.104 & 0.105 & 0.104 & 0.102 & 0.108 & 0.102 \\
\hline & $\mathrm{RV}$ & $(101,95,105)$ & & 0.098 & 0.097 & 0.104 & 0.100 & 0.101 & 0.102 & 0.096 & 0.100 \\
\hline & $\mathrm{S}$ & $(17,14,20)$ & & 0.174 & 0.154 & 0.109 & 0.106 & 0.171 & 0.120 & 0.111 & 0.106 \\
\hline & $\mathrm{S}$ & $(35,28,36)$ & & 0.140 & 0.136 & 0.108 & 0.102 & 0.170 & 0.105 & 0.106 & 0.097 \\
\hline & $\mathrm{S}$ & $(50,46,55)$ & & 0.122 & 0.132 & 0.102 & 0.102 & 0.169 & 0.103 & 0.105 & 0.102 \\
\hline & $\mathrm{S}$ & $(101,95,105)$ & & 0.114 & 0.115 & 0.102 & 0.099 & 0.145 & 0.102 & 0.095 & 0.099 \\
\hline
\end{tabular}


Tabela E.10: Tamanhos empíricos dos testes Wald, razão de verossimilhança e score para o teste $H_{0}: \beta_{21}=\beta_{22}$, com $\mu=1$.

\begin{tabular}{|c|c|c|c|c|c|c|c|c|c|c|c|}
\hline \multirow[b]{2}{*}{ Nível } & \multirow[b]{2}{*}{ Teste } & \multirow[b]{2}{*}{$\left(n_{1}, n_{2}, n_{3}\right)$} & \multirow{2}{*}{$\begin{array}{l}\sigma_{x}^{2} \\
\sigma^{2}\end{array}$} & \multirow{2}{*}{$\begin{array}{l}0.01 \\
0.01\end{array}$} & \multicolumn{3}{|c|}{0.1} & \multicolumn{4}{|c|}{0.5} \\
\hline & & & & & 0.01 & 0.05 & 0.1 & 0.01 & 0.05 & 0.1 & 0.5 \\
\hline \multirow[t]{12}{*}{0.01} & W & $(17,14,20)$ & & 0.020 & 0.019 & 0.021 & 0.021 & 0.022 & 0.025 & 0.024 & 0.027 \\
\hline & W & $(35,28,36)$ & & 0.015 & 0.014 & 0.015 & 0.015 & 0.016 & 0.016 & 0.016 & 0.016 \\
\hline & W & $(50,46,55)$ & & 0.012 & 0.014 & 0.013 & 0.013 & 0.015 & 0.013 & 0.012 & 0.013 \\
\hline & $\mathrm{W}$ & $(101,95,105)$ & & 0.011 & 0.011 & 0.011 & 0.011 & 0.008 & 0.012 & 0.010 & 0.011 \\
\hline & RV & $(17,14,20)$ & & 0.014 & 0.011 & 0.014 & 0.013 & 0.013 & 0.016 & 0.014 & 0.015 \\
\hline & $\mathrm{RV}$ & $(35,28,36)$ & & 0.012 & 0.011 & 0.011 & 0.012 & 0.012 & 0.012 & 0.012 & 0.013 \\
\hline & $\mathrm{RV}$ & $(50,46,55)$ & & 0.011 & 0.010 & 0.011 & 0.011 & 0.012 & 0.011 & 0.010 & 0.011 \\
\hline & $\mathrm{RV}$ & $(101,95,105)$ & & 0.010 & 0.010 & 0.010 & 0.010 & 0.007 & 0.011 & 0.010 & 0.011 \\
\hline & $\mathrm{S}$ & $(17,14,20)$ & & 0.022 & 0.014 & 0.009 & 0.006 & 0.019 & 0.012 & 0.009 & 0.007 \\
\hline & S & $(35,28,36)$ & & 0.020 & 0.014 & 0.008 & 0.009 & 0.018 & 0.011 & 0.010 & 0.009 \\
\hline & $\mathrm{S}$ & $(50,46,55)$ & & 0.020 & 0.013 & 0.010 & 0.011 & 0.017 & 0.010 & 0.009 & 0.010 \\
\hline & S & $(101,95,105)$ & & 0.015 & 0.013 & 0.010 & 0.010 & 0.013 & 0.009 & 0.009 & 0.009 \\
\hline \multirow[t]{12}{*}{0.05} & $\mathrm{~W}$ & $(17,14,20)$ & & 0.069 & 0.071 & 0.076 & 0.069 & 0.073 & 0.080 & 0.072 & 0.086 \\
\hline & W & $(35,28,36)$ & & 0.057 & 0.057 & 0.059 & 0.060 & 0.061 & 0.062 & 0.063 & 0.065 \\
\hline & W & $(50,46,55)$ & & 0.054 & 0.056 & 0.055 & 0.058 & 0.057 & 0.061 & 0.053 & 0.063 \\
\hline & W & $(101,95,105)$ & & 0.054 & 0.048 & 0.052 & 0.052 & 0.047 & 0.053 & 0.052 & 0.054 \\
\hline & $\mathrm{RV}$ & $(17,14,20)$ & & 0.062 & 0.060 & 0.064 & 0.056 & 0.059 & 0.062 & 0.056 & 0.067 \\
\hline & $\mathrm{RV}$ & $(35$ & & 0.052 & 0.052 & 0.054 & 0.054 & 0.054 & 0.054 & 0.055 & 0.058 \\
\hline & $\mathrm{RV}$ & $(50,46,55)$ & & 0.052 & 0.052 & 0.053 & 0.054 & 0.052 & 0.053 & 0.049 & 0.057 \\
\hline & $\mathrm{RV}$ & $(101,95,105)$ & & 0.051 & 0.048 & 0.051 & 0.051 & 0.048 & 0.053 & 0.049 & 0.052 \\
\hline & $\mathrm{S}$ & $(17,14,20)$ & & 0.107 & 0.084 & 0.057 & 0.045 & 0.098 & 0.066 & 0.052 & 0.048 \\
\hline & S & $(35,28,36)$ & & 0.081 & 0.072 & 0.051 & 0.048 & 0.094 & 0.057 & 0.051 & 0.051 \\
\hline & S & $(50,46,5$ & & 0.072 & 0.070 & 0.051 & 0.052 & 0.090 & 0.054 & 0.049 & 0.051 \\
\hline & $\mathrm{S}$ & $(101,95,105)$ & & 0.067 & 0.060 & 0.051 & 0.050 & 0.077 & 0.050 & 0.049 & 0.050 \\
\hline \multirow[t]{12}{*}{0.1} & W & $(17,14,20)$ & & 0.125 & 0.130 & 0.128 & 0.124 & 0.130 & 0.137 & 0.131 & 0.145 \\
\hline & W & $(35,28,36)$ & & 0.112 & 0.103 & 0.115 & 0.113 & 0.116 & 0.114 & 0.118 & 0.116 \\
\hline & W & $(50,46,55)$ & & 0.110 & 0.102 & 0.110 & 0.110 & 0.108 & 0.113 & 0.106 & 0.115 \\
\hline & W & $(101,95,105)$ & & 0.109 & 0.098 & 0.106 & 0.107 & 0.099 & 0.102 & 0.105 & 0.106 \\
\hline & RV & $(17,14,20)$ & & 0.116 & 0.116 & 0.114 & 0.111 & 0.112 & 0.116 & 0.110 & 0.124 \\
\hline & $\mathrm{RV}$ & $(35,28,36)$ & & 0.108 & 0.098 & 0.108 & 0.106 & 0.107 & 0.104 & 0.108 & 0.110 \\
\hline & $\mathrm{RV}$ & $(50,46,55)$ & & 0.107 & 0.102 & 0.105 & 0.106 & 0.103 & 0.103 & 0.100 & 0.110 \\
\hline & $\mathrm{RV}$ & $(101,95,105)$ & & 0.107 & 0.096 & 0.103 & 0.105 & 0.098 & 0.101 & 0.100 & 0.103 \\
\hline & $\mathrm{S}$ & $(17,14,20)$ & & 0.197 & 0.161 & 0.112 & 0.101 & 0.182 & 0.133 & 0.105 & 0.105 \\
\hline & $\mathrm{S}$ & $(35,28,36)$ & & 0.152 & 0.137 & 0.102 & 0.099 & 0.181 & 0.111 & 0.101 & 0.101 \\
\hline & S & $(50,46,55)$ & & 0.136 & 0.136 & 0.102 & 0.101 & 0.175 & 0.111 & 0.099 & 0.101 \\
\hline & $\mathrm{S}$ & $(101,95,105)$ & & 0.124 & 0.117 & 0.101 & 0.101 & 0.145 & 0.099 & 0.100 & 0.101 \\
\hline
\end{tabular}


Tabela E.11: Tamanhos empíricos dos testes Wald, razão de verossimilhança e score para o teste $H_{0}: \beta_{21}=\beta_{22}$, com $\mu=2.5$.

\begin{tabular}{|c|c|c|c|c|c|c|c|c|c|c|c|}
\hline \multirow[b]{2}{*}{ Nível } & \multirow[b]{2}{*}{ Teste } & \multirow[b]{2}{*}{$\left(n_{1}, n_{2}, n_{3}\right)$} & \multirow{2}{*}{$\begin{array}{l}\sigma_{x}^{2} \\
\sigma^{2}\end{array}$} & \multirow{2}{*}{$\begin{array}{l}0.01 \\
0.01\end{array}$} & \multicolumn{3}{|c|}{0.1} & \multicolumn{4}{|c|}{0.5} \\
\hline & & & & & 0.01 & 0.05 & 0.1 & 0.01 & 0.05 & 0.1 & 0.5 \\
\hline \multirow[t]{12}{*}{0.01} & W & $(17,14,20)$ & & 0.020 & 0.022 & 0.020 & 0.022 & 0.023 & 0.019 & 0.021 & 0.023 \\
\hline & W & $(35,28,36)$ & & 0.015 & 0.014 & 0.019 & 0.013 & 0.017 & 0.015 & 0.012 & 0.014 \\
\hline & W & $(50,46,55)$ & & 0.013 & 0.013 & 0.012 & 0.013 & 0.013 & 0.011 & 0.012 & 0.013 \\
\hline & $\mathrm{W}$ & $(101,95,105)$ & & 0.013 & 0.011 & 0.010 & 0.012 & 0.013 & 0.011 & 0.012 & 0.012 \\
\hline & $\overline{\mathrm{RV}}$ & $(17,14,20)$ & & 0.013 & 0.014 & 0.016 & 0.015 & 0.015 & 0.013 & 0.014 & 0.014 \\
\hline & RV & $(35,28,36)$ & & 0.013 & 0.012 & 0.016 & 0.011 & 0.013 & 0.011 & 0.011 & 0.011 \\
\hline & RV & $(50,46,55)$ & & 0.012 & 0.011 & 0.011 & 0.011 & 0.011 & 0.009 & 0.011 & 0.011 \\
\hline & RV & $(101,95,105)$ & & 0.012 & 0.011 & 0.010 & 0.011 & 0.011 & 0.010 & 0.011 & 0.011 \\
\hline & $\mathrm{S}$ & $(17,14,20)$ & & 0.021 & 0.015 & 0.007 & 0.009 & 0.022 & 0.009 & 0.008 & 0.007 \\
\hline & $\mathrm{S}$ & $(35,28,36)$ & & 0.021 & 0.014 & 0.012 & 0.009 & 0.018 & 0.010 & 0.008 & 0.008 \\
\hline & $\mathrm{S}$ & $(50,46,55)$ & & 0.018 & 0.014 & 0.010 & 0.011 & 0.018 & 0.009 & 0.009 & 0.009 \\
\hline & $\mathrm{S}$ & $(101,95,105)$ & & 0.017 & 0.013 & 0.009 & 0.011 & 0.018 & 0.010 & 0.011 & 0.011 \\
\hline \multirow[t]{12}{*}{0.05} & $\mathrm{~W}$ & $(17,14,20)$ & & 0.069 & 0.075 & 0.076 & 0.076 & 0.073 & 0.071 & 0.076 & 0.077 \\
\hline & W & $(35,28,36)$ & & 0.058 & 0.060 & 0.067 & 0.064 & 0.058 & 0.058 & 0.062 & 0.058 \\
\hline & W & $(50,46,55)$ & & 0.056 & 0.056 & 0.054 & 0.056 & 0.054 & 0.056 & 0.057 & 0.056 \\
\hline & W & $(101,95,105)$ & & 0.055 & 0.054 & 0.050 & 0.054 & 0.054 & 0.056 & 0.052 & 0.054 \\
\hline & $\overline{R V}$ & $(17,14,20)$ & & 0.060 & 0.063 & 0.064 & 0.063 & 0.060 & 0.057 & 0.062 & 0.062 \\
\hline & $\mathrm{RV}$ & $(35,28,36)$ & & 0.055 & 0.055 & 0.062 & 0.058 & 0.052 & 0.053 & 0.054 & 0.054 \\
\hline & RV & $(50,46,55)$ & & 0.053 & 0.053 & 0.051 & 0.053 & 0.050 & 0.051 & 0.054 & 0.053 \\
\hline & RV & $(101,95,105)$ & & 0.052 & 0.052 & 0.049 & 0.053 & 0.050 & 0.051 & 0.051 & 0.053 \\
\hline & $\mathrm{S}$ & $(17,14,20)$ & & 0.099 & 0.079 & 0.056 & 0.053 & 0.094 & 0.062 & 0.053 & 0.048 \\
\hline & $\mathrm{S}$ & $(35,28,36)$ & & 0.082 & 0.071 & 0.055 & 0.052 & 0.089 & 0.054 & 0.049 & 0.048 \\
\hline & $\mathrm{S}$ & $(50,46,55)$ & & 0.072 & 0.066 & 0.048 & 0.052 & 0.088 & 0.051 & 0.051 & 0.051 \\
\hline & $\mathrm{S}$ & $(101,95,105)$ & & 0.063 & 0.065 & 0.048 & 0.052 & 0.088 & 0.051 & 0.050 & 0.051 \\
\hline \multirow[t]{12}{*}{0.1} & $\mathrm{~W}$ & $(17,14,20)$ & & 0.122 & 0.130 & 0.132 & 0.126 & 0.126 & 0.122 & 0.132 & 0.135 \\
\hline & W & $(35,28,36)$ & & 0.109 & 0.110 & 0.117 & 0.117 & 0.109 & 0.109 & 0.117 & 0.115 \\
\hline & W & $(50,46,55)$ & & 0.107 & 0.108 & 0.103 & 0.105 & 0.100 & 0.106 & 0.109 & 0.108 \\
\hline & $\mathrm{W}$ & $(101,95,105)$ & & 0.107 & 0.101 & 0.100 & 0.104 & 0.100 & 0.105 & 0.104 & 0.102 \\
\hline & RV & $(17,14,20)$ & & 0.111 & 0.116 & 0.119 & 0.116 & 0.114 & 0.111 & 0.120 & 0.120 \\
\hline & RV & $(35,28,36)$ & & 0.106 & 0.106 & 0.111 & 0.110 & 0.103 & 0.104 & 0.111 & 0.107 \\
\hline & $\mathrm{RV}$ & $(50,46,55)$ & & 0.104 & 0.104 & 0.101 & 0.102 & 0.097 & 0.102 & 0.105 & 0.106 \\
\hline & $\mathrm{RV}$ & $(101,95,105)$ & & 0.104 & 0.099 & 0.098 & 0.102 & 0.097 & 0.102 & 0.101 & 0.100 \\
\hline & $\mathrm{S}$ & $(17,14,20)$ & & 0.181 & 0.154 & 0.116 & 0.106 & 0.178 & 0.126 & 0.115 & 0.105 \\
\hline & $\mathrm{S}$ & $(35,28,36)$ & & 0.147 & 0.139 & 0.106 & 0.106 & 0.175 & 0.111 & 0.107 & 0.099 \\
\hline & $\mathrm{S}$ & $(50,46,55)$ & & 0.133 & 0.129 & 0.099 & 0.103 & 0.164 & 0.103 & 0.101 & 0.101 \\
\hline & $\mathrm{S}$ & $(101,95,105)$ & & 0.120 & 0.123 & 0.097 & 0.102 & 0.164 & 0.102 & 0.099 & 0.098 \\
\hline
\end{tabular}


Tabela E.12: Tamanhos empíricos dos testes Wald, razão de verossimilhança e score para o teste $H_{0}: \beta_{21}=\beta_{22}$, com $\mu=5$.

\begin{tabular}{|c|c|c|c|c|c|c|c|c|c|c|}
\hline \multirow[b]{2}{*}{ Nível } & \multirow[b]{2}{*}{ Teste } & \multirow[b]{2}{*}{$\left(n_{1}, n_{2}, n_{3}\right)$} & \multirow{2}{*}{$\begin{array}{l}0.01 \\
0.01\end{array}$} & \multicolumn{3}{|c|}{0.1} & \multicolumn{4}{|c|}{0.5} \\
\hline & & & & 0.01 & 0.05 & 0.1 & 0.01 & 0.05 & 0.1 & 0.5 \\
\hline \multirow[t]{12}{*}{0.01} & W & $(17,14,20)$ & 0.021 & 0.018 & 0.011 & 0.022 & 0.021 & 0.020 & 0.021 & 0.024 \\
\hline & $\mathrm{W}$ & $(35,28,36)$ & 0.015 & 0.013 & 0.011 & 0.015 & 0.015 & 0.015 & 0.017 & 0.017 \\
\hline & $\mathrm{W}$ & $(50,46,55)$ & 0.013 & 0.012 & 0.011 & 0.012 & 0.015 & 0.014 & 0.012 & 0.014 \\
\hline & $\mathrm{W}$ & $(101,95,105)$ & 0.012 & 0.012 & 0.011 & 0.011 & 0.012 & 0.011 & 0.011 & 0.011 \\
\hline & $\overline{R V}$ & $(17,14,20)$ & 0.015 & 0.013 & 0.011 & 0.015 & 0.013 & 0.013 & 0.014 & 0.015 \\
\hline & RV & $(35,28,36)$ & 0.012 & 0.011 & 0.011 & 0.012 & 0.012 & 0.012 & 0.013 & 0.014 \\
\hline & $\mathrm{RV}$ & $(50,46,55)$ & 0.012 & 0.010 & 0.011 & 0.010 & 0.012 & 0.012 & 0.011 & 0.013 \\
\hline & $\mathrm{RV}$ & $(101,95,105)$ & 0.011 & 0.010 & 0.011 & 0.010 & 0.011 & 0.011 & 0.011 & 0.010 \\
\hline & $\mathrm{S}$ & $(17,14,20)$ & 0.024 & 0.015 & 0.010 & 0.008 & 0.018 & 0.009 & 0.009 & 0.008 \\
\hline & $\mathrm{S}$ & $(35,28,36)$ & 0.018 & 0.015 & 0.010 & 0.010 & 0.018 & 0.011 & 0.010 & 0.011 \\
\hline & S & $(50,46,55)$ & 0.017 & 0.015 & 0.010 & 0.010 & 0.018 & 0.010 & 0.010 & 0.011 \\
\hline & S & $(101,95,105)$ & 0.016 & 0.014 & 0.010 & 0.010 & 0.016 & 0.010 & 0.010 & 0.008 \\
\hline \multirow[t]{12}{*}{0.05} & $\mathrm{~W}$ & $(17,14,20)$ & 0.071 & 0.067 & 0.053 & 0.079 & 0.071 & 0.072 & 0.074 & 0.078 \\
\hline & $\mathrm{W}$ & $(35,28,36)$ & 0.061 & 0.058 & 0.053 & 0.062 & 0.058 & 0.061 & 0.062 & 0.062 \\
\hline & $\mathrm{W}$ & $(50,46,55)$ & 0.056 & 0.057 & 0.053 & 0.054 & 0.056 & 0.058 & 0.054 & 0.057 \\
\hline & $\mathrm{W}$ & $(101,95,105)$ & 0.056 & 0.053 & 0.053 & 0.053 & 0.053 & 0.054 & 0.052 & 0.050 \\
\hline & $\mathrm{RV}$ & $(17,14,20)$ & 0.062 & 0.055 & 0.051 & 0.068 & 0.060 & 0.062 & 0.063 & 0.065 \\
\hline & $\mathrm{RV}$ & $(35,28,36)$ & 0.057 & 0.053 & 0.051 & 0.057 & 0.053 & 0.056 & 0.057 & 0.058 \\
\hline & RV & $(50,46,55)$ & 0.053 & 0.053 & 0.051 & 0.052 & 0.052 & 0.054 & 0.051 & 0.054 \\
\hline & RV & $(101,95,105)$ & 0.052 & 0.051 & 0.051 & 0.052 & 0.052 & 0.051 & 0.051 & 0.050 \\
\hline & $\mathrm{S}$ & $(17,14,20)$ & 0.101 & 0.079 & 0.050 & 0.057 & 0.097 & 0.064 & 0.055 & 0.051 \\
\hline & S & $(35,28,36)$ & 0.083 & 0.073 & 0.050 & 0.052 & 0.092 & 0.058 & 0.052 & 0.051 \\
\hline & $\mathrm{S}$ & $(50,46,55)$ & 0.072 & 0.070 & 0.050 & 0.050 & 0.092 & 0.054 & 0.048 & 0.051 \\
\hline & $\mathrm{S}$ & $(101,95,105)$ & 0.064 & 0.063 & 0.050 & 0.050 & 0.081 & 0.050 & 0.050 & 0.049 \\
\hline \multirow[t]{12}{*}{0.1} & $\mathrm{~W}$ & $(17,14,20)$ & 0.126 & 0.121 & 0.107 & 0.130 & 0.120 & 0.123 & 0.129 & 0.134 \\
\hline & $\mathrm{W}$ & $(35,28,36)$ & 0.114 & 0.113 & 0.107 & 0.114 & 0.107 & 0.114 & 0.114 & 0.113 \\
\hline & $\mathrm{W}$ & $(50,46,55)$ & 0.108 & 0.107 & 0.107 & 0.110 & 0.105 & 0.108 & 0.104 & 0.108 \\
\hline & $\mathrm{W}$ & $(101,95,105)$ & 0.103 & 0.102 & 0.107 & 0.106 & 0.100 & 0.105 & 0.103 & 0.103 \\
\hline & RV & $(17,14,20)$ & 0.119 & 0.111 & 0.105 & 0.121 & 0.110 & 0.112 & 0.117 & 0.119 \\
\hline & RV & $(35,28,36)$ & 0.110 & 0.107 & 0.105 & 0.110 & 0.102 & 0.108 & 0.109 & 0.107 \\
\hline & $\mathrm{RV}$ & $(50,46,55)$ & 0.105 & 0.103 & 0.105 & 0.107 & 0.101 & 0.105 & 0.101 & 0.104 \\
\hline & $\mathrm{RV}$ & $(101,95,105)$ & 0.101 & 0.100 & 0.105 & 0.105 & 0.099 & 0.103 & 0.101 & 0.102 \\
\hline & $\mathrm{S}$ & $(17,14,20)$ & 0.188 & 0.155 & 0.104 & 0.113 & 0.182 & 0.130 & 0.111 & 0.106 \\
\hline & $\mathrm{S}$ & $(35,28,36)$ & 0.152 & 0.147 & 0.104 & 0.106 & 0.172 & 0.116 & 0.106 & 0.101 \\
\hline & $\mathrm{S}$ & $(50,46,55)$ & 0.133 & 0.139 & 0.104 & 0.104 & 0.171 & 0.107 & 0.099 & 0.101 \\
\hline & S & $(101,95,105)$ & 0.115 & 0.125 & 0.104 & 0.103 & 0.149 & 0.102 & 0.100 & 0.100 \\
\hline
\end{tabular}


Tabela E.13: Tamanhos empíricos dos testes Wald, razão de verossimilhança e score para o teste $H_{0}: \beta_{21}=\beta_{23}$, com $\mu=1$.

\begin{tabular}{|c|c|c|c|c|c|c|c|c|c|c|c|}
\hline \multirow[b]{2}{*}{ Nível } & \multirow[b]{2}{*}{ Teste } & \multirow[b]{2}{*}{$\left(n_{1}, n_{2}, n_{3}\right)$} & \multirow{2}{*}{$\begin{array}{l}\sigma_{x}^{2} \\
\sigma^{2}\end{array}$} & \multirow{2}{*}{$\begin{array}{l}0.01 \\
0.01\end{array}$} & \multicolumn{3}{|c|}{0.1} & \multicolumn{4}{|c|}{0.5} \\
\hline & & & & & 0.01 & 0.05 & 0.1 & 0.01 & 0.05 & 0.1 & 0.5 \\
\hline \multirow[t]{12}{*}{0.01} & W & $(17,14,20)$ & & 0.019 & 0.021 & 0.018 & 0.017 & 0.022 & 0.021 & 0.019 & 0.019 \\
\hline & W & $(35,28,36)$ & & 0.015 & 0.014 & 0.013 & 0.014 & 0.018 & 0.014 & 0.014 & 0.015 \\
\hline & W & $(50,46,55)$ & & 0.013 & 0.013 & 0.012 & 0.014 & 0.013 & 0.013 & 0.013 & 0.011 \\
\hline & W & $(101,95,105)$ & & 0.013 & 0.012 & 0.012 & 0.011 & 0.013 & 0.010 & 0.011 & 0.011 \\
\hline & RV & $(17,14,20)$ & & 0.017 & 0.016 & 0.014 & 0.015 & 0.016 & 0.015 & 0.014 & 0.015 \\
\hline & RV & $(35,28,36)$ & & 0.016 & 0.015 & 0.014 & 0.015 & 0.016 & 0.014 & 0.013 & 0.015 \\
\hline & RV & $(50,46,55)$ & & 0.012 & 0.013 & 0.012 & 0.015 & 0.012 & 0.012 & 0.013 & 0.011 \\
\hline & RV & $(101,95,105)$ & & 0.012 & 0.011 & 0.011 & 0.011 & 0.012 & 0.010 & 0.011 & 0.011 \\
\hline & $\mathrm{S}$ & $(17,14,20)$ & & 0.013 & 0.015 & 0.007 & 0.005 & 0.020 & 0.009 & 0.008 & 0.005 \\
\hline & $\mathrm{S}$ & $(35,28,36)$ & & 0.013 & 0.015 & 0.007 & 0.006 & 0.019 & 0.011 & 0.009 & 0.013 \\
\hline & $\mathrm{S}$ & $(50,46,55)$ & & 0.009 & 0.015 & 0.009 & 0.010 & 0.018 & 0.009 & 0.009 & 0.007 \\
\hline & $\mathrm{S}$ & $(101,95,105)$ & & 0.011 & 0.014 & 0.010 & 0.010 & 0.016 & 0.009 & 0.009 & 0.008 \\
\hline \multirow[t]{12}{*}{0.05} & W & $(17,14,20)$ & & 0.069 & 0.072 & 0.073 & 0.065 & 0.074 & 0.076 & 0.070 & 0.070 \\
\hline & W & $(35,28,36)$ & & 0.062 & 0.062 & 0.058 & 0.061 & 0.062 & 0.064 & 0.064 & 0.059 \\
\hline & W & $(50,46,55)$ & & 0.054 & 0.059 & 0.054 & 0.059 & 0.058 & 0.057 & 0.059 & 0.055 \\
\hline & $\mathrm{W}$ & $(101,95,105)$ & & 0.052 & 0.055 & 0.053 & 0.054 & 0.056 & 0.053 & 0.051 & 0.051 \\
\hline & $\mathrm{RV}$ & $(17,14,20)$ & & 0.065 & 0.070 & 0.069 & 0.069 & 0.073 & 0.069 & 0.070 & 0.079 \\
\hline & RV & $(35,28,36)$ & & 0.065 & 0.070 & 0.069 & 0.068 & 0.072 & 0.068 & 0.063 & 0.078 \\
\hline & RV & $(50,46,55)$ & & 0.057 & 0.062 & 0.055 & 0.062 & 0.059 & 0.057 & 0.061 & 0.061 \\
\hline & RV & $(101,95,105)$ & & 0.053 & 0.057 & 0.055 & 0.056 & 0.056 & 0.054 & 0.052 & 0.053 \\
\hline & $\mathrm{S}$ & $(17,14,20)$ & & 0.081 & 0.084 & 0.053 & 0.043 & 0.095 & 0.065 & 0.046 & 0.045 \\
\hline & $\mathrm{S}$ & $(35,28,36)$ & & 0.074 & 0.084 & 0.053 & 0.053 & 0.093 & 0.065 & 0.055 & 0.055 \\
\hline & $\mathrm{S}$ & $(50,46,55)$ & & 0.061 & 0.076 & 0.047 & 0.052 & 0.091 & 0.057 & 0.051 & 0.051 \\
\hline & $\mathrm{S}$ & $(101,95,105)$ & & 0.055 & 0.075 & 0.049 & 0.051 & 0.091 & 0.055 & 0.049 & 0.049 \\
\hline \multirow[t]{12}{*}{0.1} & $\mathrm{~W}$ & $(17,14,20)$ & & 0.127 & 0.126 & 0.135 & 0.120 & 0.132 & 0.134 & 0.127 & 0.128 \\
\hline & W & $(35,28,36)$ & & 0.113 & 0.114 & 0.112 & 0.108 & 0.114 & 0.122 & 0.117 & 0.116 \\
\hline & W & $(50,46,55)$ & & 0.108 & 0.108 & 0.105 & 0.107 & 0.113 & 0.107 & 0.115 & 0.107 \\
\hline & $\mathrm{W}$ & $(101,95,105)$ & & 0.101 & 0.108 & 0.099 & 0.106 & 0.107 & 0.102 & 0.099 & 0.105 \\
\hline & RV & $(17,14,20)$ & & 0.127 & 0.125 & 0.134 & 0.119 & 0.128 & 0.139 & 0.124 & 0.131 \\
\hline & RV & $(35,28,36)$ & & 0.120 & 0.124 & 0.132 & 0.119 & 0.127 & 0.137 & 0.123 & 0.130 \\
\hline & RV & $(50,46,55)$ & & 0.117 & 0.116 & 0.114 & 0.119 & 0.121 & 0.116 & 0.119 & 0.120 \\
\hline & RV & $(101,95,105)$ & & 0.106 & 0.113 & 0.103 & 0.110 & 0.110 & 0.105 & 0.102 & 0.112 \\
\hline & $\mathrm{S}$ & $(17,14,20)$ & & 0.156 & 0.159 & 0.116 & 0.090 & 0.187 & 0.149 & 0.104 & 0.093 \\
\hline & $\mathrm{S}$ & $(35,28,36)$ & & 0.156 & 0.158 & 0.115 & 0.110 & 0.187 & 0.149 & 0.104 & 0.107 \\
\hline & $\mathrm{S}$ & $(50,46,55)$ & & 0.130 & 0.148 & 0.100 & 0.106 & 0.177 & 0.123 & 0.103 & 0.106 \\
\hline & $\mathrm{S}$ & $(101,95,105)$ & & 0.114 & 0.147 & 0.100 & 0.103 & 0.173 & 0.115 & 0.097 & 0.105 \\
\hline
\end{tabular}


Tabela E.14: Tamanhos empíricos dos testes Wald, razão de verossimilhança e score para o teste $H_{0}: \beta_{21}=\beta_{23}$, com $\mu=2.5$.

\begin{tabular}{|c|c|c|c|c|c|c|c|c|c|c|}
\hline \multirow[b]{2}{*}{ Nível } & \multirow[b]{2}{*}{ Teste } & \multirow[b]{2}{*}{$\left(n_{1}, n_{2}, n_{3}\right)$} & \multirow{2}{*}{$\begin{array}{l}0.01 \\
0.01\end{array}$} & \multicolumn{3}{|c|}{0.1} & \multicolumn{4}{|c|}{0.5} \\
\hline & & & & 0.01 & 0.05 & 0.1 & 0.01 & 0.05 & 0.1 & 0.5 \\
\hline \multirow{12}{*}{0.01} & W & $(17,14,20)$ & 0.019 & 0.018 & 0.018 & 0.019 & 0.020 & 0.020 & 0.020 & 0.018 \\
\hline & W & $(35,28,36)$ & 0.015 & 0.014 & 0.014 & 0.011 & 0.014 & 0.014 & 0.014 & 0.016 \\
\hline & $\mathrm{W}$ & $(50,46,55)$ & 0.015 & 0.011 & 0.014 & 0.011 & 0.012 & 0.011 & 0.014 & 0.015 \\
\hline & $\mathrm{W}$ & $(101,95,105)$ & 0.012 & 0.011 & 0.013 & 0.011 & 0.012 & 0.009 & 0.011 & 0.012 \\
\hline & RV & $(17,14,20)$ & 0.016 & 0.016 & 0.016 & 0.015 & 0.016 & 0.015 & 0.015 & 0.015 \\
\hline & $\mathrm{RV}$ & $(35,28,36)$ & 0.016 & 0.015 & 0.015 & 0.012 & 0.014 & 0.015 & 0.015 & 0.015 \\
\hline & $\mathrm{RV}$ & $(50,46,55)$ & 0.014 & 0.011 & 0.014 & 0.012 & 0.012 & 0.011 & 0.014 & 0.015 \\
\hline & $\mathrm{RV}$ & $(101,95,105)$ & 0.012 & 0.011 & 0.013 & 0.010 & 0.012 & 0.010 & 0.011 & 0.012 \\
\hline & $\mathrm{S}$ & $(17,14,20)$ & 0.012 & 0.016 & 0.007 & 0.006 & 0.016 & 0.011 & 0.008 & 0.007 \\
\hline & $\mathrm{S}$ & $(35,28,36)$ & 0.012 & 0.016 & 0.007 & 0.006 & 0.015 & 0.009 & 0.008 & 0.007 \\
\hline & $\mathrm{S}$ & $(50,46,55)$ & 0.011 & 0.013 & 0.009 & 0.009 & 0.015 & 0.009 & 0.010 & 0.010 \\
\hline & $\mathrm{S}$ & $(101,95,105)$ & 0.011 & 0.013 & 0.011 & 0.009 & 0.014 & 0.009 & 0.010 & 0.010 \\
\hline \multirow[t]{12}{*}{0.05} & $\mathrm{~W}$ & $(17,14,20)$ & 0.068 & 0.071 & 0.071 & 0.069 & 0.070 & 0.073 & 0.071 & 0.069 \\
\hline & $\mathrm{W}$ & $(35,28,36)$ & 0.062 & 0.059 & 0.058 & 0.058 & 0.057 & 0.058 & 0.061 & 0.061 \\
\hline & W & $(50,46,55)$ & 0.060 & 0.052 & 0.055 & 0.057 & 0.057 & 0.048 & 0.056 & 0.056 \\
\hline & W & $(101,95,105)$ & 0.054 & 0.049 & 0.051 & 0.055 & 0.057 & 0.048 & 0.048 & 0.055 \\
\hline & RV & $(17,14,20)$ & 0.075 & 0.070 & 0.067 & 0.072 & 0.069 & 0.073 & 0.075 & 0.083 \\
\hline & $\mathrm{RV}$ & $(35,28,36)$ & 0.065 & 0.070 & 0.067 & 0.071 & 0.068 & 0.072 & 0.073 & 0.081 \\
\hline & $\mathrm{RV}$ & $(50,46,55)$ & 0.064 & 0.056 & 0.058 & 0.060 & 0.061 & 0.051 & 0.059 & 0.062 \\
\hline & RV & $(101,95,105)$ & 0.056 & 0.051 & 0.053 & 0.056 & 0.057 & 0.049 & 0.050 & 0.058 \\
\hline & $\mathrm{S}$ & $(17,14,20)$ & 0.076 & 0.083 & 0.049 & 0.047 & 0.083 & 0.063 & 0.051 & 0.047 \\
\hline & $\mathrm{S}$ & $(35,28,36)$ & 0.076 & 0.082 & 0.051 & 0.052 & 0.083 & 0.060 & 0.051 & 0.053 \\
\hline & $\mathrm{S}$ & $(50,46,55)$ & 0.067 & 0.070 & 0.051 & 0.052 & 0.082 & 0.046 & 0.051 & 0.052 \\
\hline & $\mathrm{S}$ & $(101,95,105)$ & 0.059 & 0.069 & 0.049 & 0.051 & 0.080 & 0.047 & 0.049 & 0.052 \\
\hline \multirow[t]{12}{*}{0.1} & W & $(17,14,20)$ & 0.122 & 0.129 & 0.124 & 0.127 & 0.122 & 0.130 & 0.131 & 0.124 \\
\hline & W & $(35,28,36)$ & 0.113 & 0.111 & 0.109 & 0.111 & 0.113 & 0.110 & 0.111 & 0.116 \\
\hline & $\mathrm{W}$ & $(50,46,55)$ & 0.111 & 0.103 & 0.103 & 0.111 & 0.112 & 0.098 & 0.107 & 0.105 \\
\hline & W & $(101,95,105)$ & 0.104 & 0.103 & 0.103 & 0.108 & 0.106 & 0.099 & 0.100 & 0.104 \\
\hline & RV & $(17,14,20)$ & 0.123 & 0.138 & 0.125 & 0.128 & 0.120 & 0.137 & 0.131 & 0.127 \\
\hline & $\mathrm{RV}$ & $(35,28,36)$ & 0.122 & 0.137 & 0.124 & 0.126 & 0.118 & 0.136 & 0.130 & 0.125 \\
\hline & $\mathrm{RV}$ & $(50,46,55)$ & 0.120 & 0.113 & 0.111 & 0.122 & 0.117 & 0.105 & 0.118 & 0.121 \\
\hline & $\mathrm{RV}$ & $(101,95,105)$ & 0.108 & 0.107 & 0.109 & 0.113 & 0.111 & 0.101 & 0.106 & 0.114 \\
\hline & $\mathrm{S}$ & $(17,14,20)$ & 0.158 & 0.172 & 0.114 & 0.113 & 0.165 & 0.131 & 0.117 & 0.103 \\
\hline & $\mathrm{S}$ & $(35,28,36)$ & 0.155 & 0.171 & 0.114 & 0.110 & 0.165 & 0.129 & 0.115 & 0.102 \\
\hline & $\mathrm{S}$ & $(50,46,55)$ & 0.135 & 0.146 & 0.102 & 0.108 & 0.160 & 0.103 & 0.104 & 0.102 \\
\hline & $\mathrm{S}$ & $(101,95,105)$ & 0.119 & 0.138 & 0.101 & 0.106 & 0.154 & 0.099 & 0.099 & 0.102 \\
\hline
\end{tabular}


Tabela E.15: Tamanhos empíricos dos testes Wald, razão de verossimilhança e score para o teste $H_{0}: \beta_{21}=\beta_{23}$, com $\mu=5$.

\begin{tabular}{|c|c|c|c|c|c|c|c|c|c|c|c|}
\hline \multirow[b]{2}{*}{ Nível } & \multirow[b]{2}{*}{ Teste } & \multirow[b]{2}{*}{$\left(n_{1}, n_{2}, n_{3}\right)$} & \multirow{2}{*}{$\begin{array}{l}\sigma_{x}^{2} \\
\sigma^{2}\end{array}$} & \multirow{2}{*}{$\begin{array}{l}0.01 \\
0.01\end{array}$} & \multicolumn{3}{|c|}{0.1} & \multicolumn{4}{|c|}{0.5} \\
\hline & & & & & 0.01 & 0.05 & 0.1 & 0.01 & 0.05 & 0.1 & 0.5 \\
\hline \multirow[t]{12}{*}{0.01} & W & $(17,14,20)$ & & 0.019 & 0.019 & 0.019 & 0.019 & 0.018 & 0.021 & 0.018 & 0.021 \\
\hline & W & $(35,28,36)$ & & 0.015 & 0.012 & 0.014 & 0.013 & 0.014 & 0.013 & 0.013 & 0.016 \\
\hline & W & $(50,46,55)$ & & 0.011 & 0.012 & 0.013 & 0.013 & 0.013 & 0.012 & 0.012 & 0.012 \\
\hline & W & $(101,95,105)$ & & 0.011 & 0.011 & 0.013 & 0.012 & 0.012 & 0.011 & 0.012 & 0.012 \\
\hline & RV & $(17,14,20)$ & & 0.014 & 0.013 & 0.015 & 0.013 & 0.014 & 0.014 & 0.012 & 0.015 \\
\hline & RV & $(35,28,36)$ & & 0.013 & 0.008 & 0.013 & 0.012 & 0.012 & 0.010 & 0.011 & 0.013 \\
\hline & RV & $(50,46,55)$ & & 0.010 & 0.012 & 0.011 & 0.012 & 0.011 & 0.010 & 0.010 & 0.010 \\
\hline & RV & $(101,95,105)$ & & 0.010 & 0.012 & 0.011 & 0.011 & 0.011 & 0.010 & 0.010 & 0.010 \\
\hline & $\mathrm{S}$ & $(17,14,20)$ & & 0.021 & 0.014 & 0.011 & 0.008 & 0.018 & 0.010 & 0.008 & 0.011 \\
\hline & $\mathrm{S}$ & $(35,28,36)$ & & 0.021 & 0.013 & 0.011 & 0.009 & 0.017 & 0.010 & 0.010 & 0.011 \\
\hline & $\mathrm{S}$ & $(50,46,55)$ & & 0.018 & 0.013 & 0.010 & 0.011 & 0.016 & 0.010 & 0.010 & 0.009 \\
\hline & $\mathrm{S}$ & $(101,95,105)$ & & 0.016 & 0.013 & 0.010 & 0.011 & 0.016 & 0.010 & 0.010 & 0.010 \\
\hline \multirow[t]{12}{*}{0.05} & W & $(17,14,20)$ & & 0.068 & 0.069 & 0.071 & 0.069 & 0.069 & 0.072 & 0.067 & 0.075 \\
\hline & W & $(35,28,36)$ & & 0.057 & 0.053 & 0.059 & 0.060 & 0.059 & 0.057 & 0.057 & 0.058 \\
\hline & W & $(50,46,55)$ & & 0.053 & 0.052 & 0.057 & 0.059 & 0.058 & 0.056 & 0.055 & 0.054 \\
\hline & W & $(101,95,105)$ & & 0.052 & 0.051 & 0.056 & 0.056 & 0.053 & 0.053 & 0.051 & 0.054 \\
\hline & $\overline{\mathrm{RV}}$ & $(17,14,20)$ & & 0.061 & 0.061 & 0.060 & 0.061 & 0.062 & 0.064 & 0.059 & 0.064 \\
\hline & RV & $(35,28,36)$ & & 0.053 & 0.059 & 0.056 & 0.055 & 0.055 & 0.054 & 0.052 & 0.053 \\
\hline & RV & $(50,46,55)$ & & 0.051 & 0.058 & 0.055 & 0.055 & 0.054 & 0.052 & 0.052 & 0.052 \\
\hline & RV & $(101,95,105)$ & & 0.051 & 0.057 & 0.054 & 0.055 & 0.053 & 0.051 & 0.050 & 0.052 \\
\hline & $\mathrm{S}$ & $(17,14,20)$ & & 0.095 & 0.077 & 0.056 & 0.054 & 0.091 & 0.068 & 0.053 & 0.053 \\
\hline & $\mathrm{S}$ & $(35,28,36)$ & & 0.083 & 0.067 & 0.053 & 0.053 & 0.085 & 0.058 & 0.049 & 0.048 \\
\hline & $\mathrm{S}$ & $(50,46,55)$ & & 0.070 & 0.066 & 0.053 & 0.053 & 0.082 & 0.052 & 0.050 & 0.049 \\
\hline & $\mathrm{S}$ & $(101,95,105)$ & & 0.065 & 0.066 & 0.053 & 0.052 & 0.080 & 0.050 & 0.049 & 0.051 \\
\hline \multirow[t]{12}{*}{0.1} & W & $(17,14,20)$ & & 0.123 & 0.121 & 0.125 & 0.124 & 0.127 & 0.123 & 0.125 & 0.130 \\
\hline & $\mathrm{W}$ & $(35,28,36)$ & & 0.110 & 0.106 & 0.112 & 0.115 & 0.111 & 0.112 & 0.113 & 0.112 \\
\hline & W & $(50,46,55)$ & & 0.108 & 0.105 & 0.108 & 0.104 & 0.107 & 0.107 & 0.106 & 0.108 \\
\hline & W & $(101,95,105)$ & & 0.101 & 0.102 & 0.107 & 0.104 & 0.106 & 0.107 & 0.100 & 0.108 \\
\hline & RV & $(17,14,20)$ & & 0.116 & 0.115 & 0.115 & 0.115 & 0.120 & 0.116 & 0.114 & 0.119 \\
\hline & RV & $(35,28,36)$ & & 0.107 & 0.114 & 0.106 & 0.110 & 0.107 & 0.106 & 0.107 & 0.106 \\
\hline & RV & $(50,46,55)$ & & 0.105 & 0.114 & 0.105 & 0.101 & 0.104 & 0.104 & 0.102 & 0.106 \\
\hline & RV & $(101,95,105)$ & & 0.100 & 0.113 & 0.105 & 0.101 & 0.103 & 0.103 & 0.100 & 0.105 \\
\hline & $\mathrm{S}$ & $(17,14,20)$ & & 0.183 & 0.151 & 0.113 & 0.108 & 0.171 & 0.130 & 0.109 & 0.108 \\
\hline & $\mathrm{S}$ & $(35,28,36)$ & & 0.147 & 0.143 & 0.106 & 0.107 & 0.156 & 0.115 & 0.104 & 0.105 \\
\hline & $\mathrm{S}$ & $(50,46,55)$ & & 0.134 & 0.143 & 0.104 & 0.099 & 0.152 & 0.108 & 0.099 & 0.103 \\
\hline & $\mathrm{S}$ & $(101,95,105)$ & & 0.117 & 0.132 & 0.103 & 0.101 & 0.150 & 0.106 & 0.099 & 0.103 \\
\hline
\end{tabular}


Tabela E.16: Tamanhos empíricos dos testes Wald, razão de verossimilhança e score para o teste $H_{0}: \beta_{22}=\beta_{23}$, com $\mu=1$.

\begin{tabular}{|c|c|c|c|c|c|c|c|c|c|c|c|}
\hline \multirow[b]{2}{*}{ Nível } & \multirow[b]{2}{*}{ Teste } & \multirow[b]{2}{*}{$\left(n_{1}, n_{2}, n_{3}\right)$} & \multirow{2}{*}{$\begin{array}{l}\sigma_{x}^{2} \\
\sigma^{2}\end{array}$} & \multirow{2}{*}{$\begin{array}{l}0.01 \\
0.01\end{array}$} & \multicolumn{3}{|c|}{0.1} & \multicolumn{4}{|c|}{0.5} \\
\hline & & & & & 0.01 & 0.05 & 0.1 & 0.01 & 0.05 & 0.1 & 0.5 \\
\hline \multirow[t]{12}{*}{0.01} & W & $(17,14,20)$ & & 0.020 & 0.021 & 0.026 & 0.020 & 0.022 & 0.025 & 0.023 & 0.026 \\
\hline & W & $(35,28,36)$ & & 0.015 & 0.017 & 0.016 & 0.015 & 0.017 & 0.015 & 0.014 & 0.018 \\
\hline & W & $(50,46,55)$ & & 0.013 & 0.011 & 0.011 & 0.012 & 0.013 & 0.013 & 0.013 & 0.012 \\
\hline & $\mathrm{W}$ & $(101,95,105)$ & & 0.011 & 0.011 & 0.011 & 0.012 & 0.010 & 0.013 & 0.012 & 0.011 \\
\hline & RV & $(17,14,20)$ & & 0.015 & 0.012 & 0.017 & 0.013 & 0.014 & 0.015 & 0.013 & 0.015 \\
\hline & RV & $(35,28,36)$ & & 0.012 & 0.012 & 0.011 & 0.010 & 0.011 & 0.011 & 0.012 & 0.015 \\
\hline & RV & $(50,46,55)$ & & 0.011 & 0.010 & 0.009 & 0.010 & 0.011 & 0.011 & 0.011 & 0.010 \\
\hline & RV & $(101,95,105)$ & & 0.011 & 0.009 & 0.010 & 0.010 & 0.009 & 0.011 & 0.010 & 0.010 \\
\hline & $\mathrm{S}$ & $(17,14,20)$ & & 0.022 & 0.018 & 0.009 & 0.006 & 0.019 & 0.012 & 0.008 & 0.006 \\
\hline & $\mathrm{S}$ & $(35,28,36)$ & & 0.021 & 0.017 & 0.008 & 0.007 & 0.019 & 0.010 & 0.007 & 0.011 \\
\hline & $\mathrm{S}$ & $(50,46,55)$ & & 0.017 & 0.014 & 0.008 & 0.009 & 0.017 & 0.010 & 0.009 & 0.009 \\
\hline & $\mathrm{S}$ & $(101,95,105)$ & & 0.015 & 0.012 & 0.009 & 0.010 & 0.015 & 0.010 & 0.009 & 0.010 \\
\hline \multirow[t]{12}{*}{0.05} & $\mathrm{~W}$ & $(17,14,20)$ & & 0.072 & 0.070 & 0.078 & 0.075 & 0.074 & 0.076 & 0.077 & 0.088 \\
\hline & $\mathrm{W}$ & $(35,28,36)$ & & 0.060 & 0.063 & 0.066 & 0.061 & 0.064 & 0.063 & 0.062 & 0.067 \\
\hline & W & $(50,46,55)$ & & 0.053 & 0.057 & 0.051 & 0.056 & 0.055 & 0.059 & 0.059 & 0.055 \\
\hline & $\mathrm{W}$ & $(101,95,105)$ & & 0.051 & 0.054 & 0.051 & 0.052 & 0.053 & 0.055 & 0.055 & 0.055 \\
\hline & $\overline{\mathrm{RV}}$ & $(17,14,20)$ & & 0.062 & 0.059 & 0.064 & 0.061 & 0.062 & 0.059 & 0.060 & 0.069 \\
\hline & RV & $(35,28,36)$ & & 0.055 & 0.057 & 0.060 & 0.055 & 0.055 & 0.054 & 0.054 & 0.059 \\
\hline & RV & $(50,46,55)$ & & 0.051 & 0.054 & 0.047 & 0.052 & 0.050 & 0.053 & 0.053 & 0.050 \\
\hline & RV & $(101,95,105)$ & & 0.050 & 0.052 & 0.051 & 0.052 & 0.050 & 0.052 & 0.053 & 0.050 \\
\hline & $\mathrm{S}$ & $(17,14,20)$ & & 0.096 & 0.081 & 0.056 & 0.047 & 0.099 & 0.064 & 0.053 & 0.048 \\
\hline & $\mathrm{S}$ & $(35,28,36)$ & & 0.079 & 0.075 & 0.054 & 0.051 & 0.090 & 0.053 & 0.049 & 0.051 \\
\hline & $\mathrm{S}$ & $(50,46,55)$ & & 0.064 & 0.068 & 0.046 & 0.049 & 0.086 & 0.053 & 0.049 & 0.049 \\
\hline & $\mathrm{S}$ & $(101,95,105)$ & & 0.060 & 0.062 & 0.050 & 0.050 & 0.079 & 0.051 & 0.051 & 0.051 \\
\hline \multirow[t]{12}{*}{0.1} & $\mathrm{~W}$ & $(17,14,20)$ & & 0.123 & 0.123 & 0.137 & 0.131 & 0.129 & 0.130 & 0.135 & 0.145 \\
\hline & W & $(35,28,36)$ & & 0.114 & 0.112 & 0.118 & 0.113 & 0.116 & 0.115 & 0.114 & 0.121 \\
\hline & $\mathrm{W}$ & $(50,46,55)$ & & 0.103 & 0.109 & 0.103 & 0.109 & 0.105 & 0.112 & 0.111 & 0.109 \\
\hline & $\mathrm{W}$ & $(101,95,105)$ & & 0.102 & 0.103 & 0.101 & 0.109 & 0.102 & 0.105 & 0.106 & 0.108 \\
\hline & RV & $(17,14,20)$ & & 0.114 & 0.110 & 0.120 & 0.116 & 0.119 & 0.114 & 0.117 & 0.125 \\
\hline & RV & $(35,28,36)$ & & 0.106 & 0.106 & 0.112 & 0.105 & 0.107 & 0.107 & 0.106 & 0.112 \\
\hline & RV & $(50,46,55)$ & & 0.102 & 0.105 & 0.099 & 0.104 & 0.100 & 0.105 & 0.104 & 0.106 \\
\hline & RV & $(101,95,105)$ & & 0.100 & 0.101 & 0.100 & 0.103 & 0.100 & 0.102 & 0.104 & 0.106 \\
\hline & $\mathrm{S}$ & $(17,14,20)$ & & 0.177 & 0.153 & 0.114 & 0.105 & 0.180 & 0.130 & 0.109 & 0.104 \\
\hline & $\mathrm{S}$ & $(35,28,36)$ & & 0.144 & 0.146 & 0.109 & 0.102 & 0.171 & 0.112 & 0.098 & 0.103 \\
\hline & $\mathrm{S}$ & $(50,46,55)$ & & 0.122 & 0.137 & 0.096 & 0.102 & 0.164 & 0.105 & 0.099 & 0.102 \\
\hline & $\mathrm{S}$ & $(101,95,105)$ & & 0.112 & 0.122 & 0.097 & 0.102 & 0.143 & 0.100 & 0.101 & 0.102 \\
\hline
\end{tabular}


Tabela E.17: Tamanhos empíricos dos testes Wald, razão de verossimilhança e score para o teste $H_{0}: \beta_{22}=\beta_{23}$, com $\mu=2.5$.

\begin{tabular}{|c|c|c|c|c|c|c|c|c|c|c|c|}
\hline \multirow[b]{2}{*}{ Nível } & \multirow[b]{2}{*}{ Teste } & \multirow[b]{2}{*}{$\left(n_{1}, n_{2}, n_{3}\right)$} & \multirow{2}{*}{$\begin{array}{l}\sigma_{x}^{2} \\
\sigma^{2}\end{array}$} & \multirow{2}{*}{$\begin{array}{l}0.01 \\
0.01\end{array}$} & \multicolumn{3}{|c|}{0.1} & \multicolumn{4}{|c|}{0.5} \\
\hline & & & & & 0.01 & 0.05 & 0.1 & 0.01 & 0.05 & 0.1 & 0.5 \\
\hline \multirow[t]{12}{*}{0.01} & W & $(17,14,20)$ & & 0.019 & 0.018 & 0.022 & 0.020 & 0.012 & 0.023 & 0.023 & 0.023 \\
\hline & W & $(35,28,36)$ & & 0.016 & 0.014 & 0.014 & 0.015 & 0.012 & 0.016 & 0.019 & 0.017 \\
\hline & W & $(50,46,55)$ & & 0.013 & 0.014 & 0.014 & 0.013 & 0.011 & 0.014 & 0.013 & 0.012 \\
\hline & W & $(101,95,105)$ & & 0.010 & 0.011 & 0.011 & 0.011 & 0.011 & 0.011 & 0.011 & 0.012 \\
\hline & RV & $(17,14,20)$ & & 0.013 & 0.011 & 0.014 & 0.012 & 0.011 & 0.013 & 0.016 & 0.015 \\
\hline & RV & $(35,28,36)$ & & 0.013 & 0.011 & 0.012 & 0.012 & 0.011 & 0.012 & 0.015 & 0.014 \\
\hline & RV & $(50,46,55)$ & & 0.011 & 0.011 & 0.012 & 0.012 & 0.009 & 0.011 & 0.010 & 0.010 \\
\hline & RV & $(101,95,105)$ & & 0.010 & 0.010 & 0.011 & 0.010 & 0.011 & 0.011 & 0.010 & 0.010 \\
\hline & $\mathrm{S}$ & $(17,14,20)$ & & 0.020 & 0.015 & 0.007 & 0.007 & 0.016 & 0.010 & 0.009 & 0.006 \\
\hline & $\mathrm{S}$ & $(35,28,36)$ & & 0.018 & 0.014 & 0.009 & 0.009 & 0.016 & 0.010 & 0.011 & 0.009 \\
\hline & $\mathrm{S}$ & $(50,46,55)$ & & 0.018 & 0.014 & 0.010 & 0.011 & 0.015 & 0.009 & 0.008 & 0.009 \\
\hline & $\mathrm{S}$ & $(101,95,105)$ & & 0.014 & 0.012 & 0.010 & 0.010 & 0.014 & 0.010 & 0.009 & 0.010 \\
\hline \multirow[t]{12}{*}{0.05} & W & $(17,14,20)$ & & 0.069 & 0.071 & 0.071 & 0.071 & 0.049 & 0.073 & 0.078 & 0.078 \\
\hline & W & $(35,28,36)$ & & 0.064 & 0.061 & 0.059 & 0.060 & 0.049 & 0.060 & 0.066 & 0.066 \\
\hline & W & $(50,46,55)$ & & 0.057 & 0.056 & 0.057 & 0.059 & 0.051 & 0.056 & 0.056 & 0.054 \\
\hline & $\mathrm{W}$ & $(101,95,105)$ & & 0.051 & 0.047 & 0.055 & 0.052 & 0.051 & 0.049 & 0.054 & 0.054 \\
\hline & $\mathrm{RV}$ & $(17,14,20)$ & & 0.059 & 0.059 & 0.059 & 0.059 & 0.048 & 0.061 & 0.064 & 0.064 \\
\hline & RV & $(35,28,36)$ & & 0.058 & 0.056 & 0.057 & 0.054 & 0.048 & 0.056 & 0.060 & 0.059 \\
\hline & RV & $(50,46,55)$ & & 0.054 & 0.053 & 0.056 & 0.053 & 0.052 & 0.052 & 0.053 & 0.051 \\
\hline & RV & $(101,95,105)$ & & 0.049 & 0.047 & 0.053 & 0.051 & 0.052 & 0.048 & 0.052 & 0.051 \\
\hline & $\mathrm{S}$ & $(17,14,20)$ & & 0.091 & 0.076 & 0.052 & 0.048 & 0.076 & 0.061 & 0.055 & 0.049 \\
\hline & $\mathrm{S}$ & $(35,28,36)$ & & 0.079 & 0.075 & 0.048 & 0.050 & 0.076 & 0.056 & 0.055 & 0.051 \\
\hline & $\mathrm{S}$ & $(50,46,55)$ & & 0.070 & 0.068 & 0.052 & 0.050 & 0.075 & 0.052 & 0.049 & 0.048 \\
\hline & $\mathrm{S}$ & $(101,95,105)$ & & 0.060 & 0.057 & 0.052 & 0.050 & 0.073 & 0.048 & 0.051 & 0.051 \\
\hline \multirow[t]{12}{*}{0.1} & $\mathrm{~W}$ & $(17,14,20)$ & & 0.124 & 0.129 & 0.129 & 0.128 & 0.098 & 0.128 & 0.135 & 0.134 \\
\hline & W & $(35,28,36)$ & & 0.119 & 0.112 & 0.113 & 0.113 & 0.098 & 0.112 & 0.120 & 0.122 \\
\hline & W & $(50,46,55)$ & & 0.107 & 0.106 & 0.110 & 0.112 & 0.104 & 0.107 & 0.109 & 0.105 \\
\hline & $\mathrm{W}$ & $(101,95,105)$ & & 0.100 & 0.096 & 0.105 & 0.102 & 0.103 & 0.100 & 0.105 & 0.105 \\
\hline & RV & $(17,14,20)$ & & 0.115 & 0.117 & 0.117 & 0.117 & 0.097 & 0.116 & 0.122 & 0.118 \\
\hline & RV & $(35,28,36)$ & & 0.114 & 0.107 & 0.107 & 0.107 & 0.097 & 0.106 & 0.115 & 0.115 \\
\hline & RV & $(50,46,55)$ & & 0.104 & 0.102 & 0.106 & 0.106 & 0.103 & 0.102 & 0.105 & 0.101 \\
\hline & RV & $(101,95,105)$ & & 0.098 & 0.098 & 0.103 & 0.101 & 0.103 & 0.098 & 0.103 & 0.101 \\
\hline & $\mathrm{S}$ & $(17,14,20)$ & & 0.174 & 0.156 & 0.111 & 0.105 & 0.143 & 0.126 & 0.114 & 0.103 \\
\hline & $\mathrm{S}$ & $(35,28,36)$ & & 0.150 & 0.142 & 0.103 & 0.103 & 0.143 & 0.110 & 0.109 & 0.103 \\
\hline & $\mathrm{S}$ & $(50,46,55)$ & & 0.128 & 0.131 & 0.103 & 0.103 & 0.141 & 0.104 & 0.101 & 0.097 \\
\hline & $\mathrm{S}$ & $(101,95,105)$ & & 0.114 & 0.114 & 0.102 & 0.100 & 0.139 & 0.097 & 0.101 & 0.102 \\
\hline
\end{tabular}


Tabela E.18: Tamanhos empíricos dos testes Wald, razão de verossimilhança e score para o teste $H_{0}: \beta_{22}=\beta_{23}$, com $\mu=5$.

\begin{tabular}{|c|c|c|c|c|c|c|c|c|c|c|}
\hline \multirow[b]{2}{*}{ Nível } & \multirow[b]{2}{*}{ Teste } & \multirow[b]{2}{*}{$\left(n_{1}, n_{2}, n_{3}\right)$} & \multirow{2}{*}{$\begin{array}{l}0.01 \\
0.01\end{array}$} & \multicolumn{3}{|c|}{0.1} & \multicolumn{4}{|c|}{0.5} \\
\hline & & & & 0.01 & 0.05 & 0.1 & 0.01 & 0.05 & 0.1 & 0.5 \\
\hline \multirow[t]{12}{*}{0.01} & W & $(17,14,20)$ & 0.018 & 0.020 & 0.021 & 0.022 & 0.020 & 0.022 & 0.021 & 0.024 \\
\hline & W & $(35,28,36)$ & 0.014 & 0.013 & 0.016 & 0.015 & 0.015 & 0.014 & 0.015 & 0.015 \\
\hline & W & $(50,46,55)$ & 0.014 & 0.013 & 0.011 & 0.013 & 0.012 & 0.014 & 0.012 & 0.011 \\
\hline & $\mathrm{W}$ & $(101,95,105)$ & 0.012 & 0.010 & 0.011 & 0.013 & 0.011 & 0.011 & 0.011 & 0.011 \\
\hline & RV & $(17,14,20)$ & 0.012 & 0.013 & 0.014 & 0.013 & 0.013 & 0.015 & 0.013 & 0.016 \\
\hline & RV & $(35,28,36)$ & 0.011 & 0.010 & 0.013 & 0.012 & 0.012 & 0.012 & 0.012 & 0.011 \\
\hline & RV & $(50,46,55)$ & 0.011 & 0.010 & 0.009 & 0.011 & 0.010 & 0.012 & 0.010 & 0.010 \\
\hline & RV & $(101,95,105)$ & 0.011 & 0.009 & 0.011 & 0.011 & 0.010 & 0.010 & 0.010 & 0.010 \\
\hline & $\mathrm{S}$ & $(17,14,20)$ & 0.020 & 0.013 & 0.008 & 0.007 & 0.018 & 0.011 & 0.008 & 0.007 \\
\hline & $\mathrm{S}$ & $(35,28,36)$ & 0.020 & 0.012 & 0.010 & 0.009 & 0.018 & 0.010 & 0.008 & 0.009 \\
\hline & $\mathrm{S}$ & $(50,46,55)$ & 0.019 & 0.011 & 0.009 & 0.010 & 0.018 & 0.010 & 0.009 & 0.009 \\
\hline & $\mathrm{S}$ & $(101,95,105)$ & 0.015 & 0.011 & 0.010 & 0.010 & 0.017 & 0.010 & 0.009 & 0.010 \\
\hline \multirow[t]{12}{*}{0.05} & $\mathrm{~W}$ & $(17,14,20)$ & 0.069 & 0.071 & 0.071 & 0.073 & 0.070 & 0.074 & 0.072 & 0.078 \\
\hline & $\mathrm{W}$ & $(35,28,36)$ & 0.059 & 0.057 & 0.064 & 0.059 & 0.062 & 0.062 & 0.061 & 0.061 \\
\hline & W & $(50,46,55)$ & 0.057 & 0.056 & 0.052 & 0.057 & 0.053 & 0.057 & 0.056 & 0.055 \\
\hline & $\mathrm{W}$ & $(101,95,105)$ & 0.053 & 0.051 & 0.052 & 0.056 & 0.050 & 0.052 & 0.052 & 0.053 \\
\hline & RV & $(17,14,20)$ & 0.059 & 0.059 & 0.059 & 0.061 & 0.058 & 0.062 & 0.059 & 0.065 \\
\hline & RV & $(35$ & 0.055 & 0.051 & 0.057 & 0.054 & 0.056 & 0.056 & 0.056 & 0.054 \\
\hline & RV & $(50,46,55)$ & 0.054 & 0.051 & 0.049 & 0.053 & 0.050 & 0.054 & 0.052 & 0.052 \\
\hline & RV & $(101,95,105)$ & 0.052 & 0.049 & 0.051 & 0.053 & 0.048 & 0.051 & 0.050 & 0.052 \\
\hline & $\mathrm{S}$ & $(17,14,20)$ & 0.098 & 0.073 & 0.052 & 0.051 & 0.089 & 0.063 & 0.051 & 0.051 \\
\hline & $\mathrm{S}$ & $(35,28,36)$ & 0.077 & 0.071 & 0.052 & 0.049 & 0.089 & 0.055 & 0.051 & 0.051 \\
\hline & $\mathrm{S}$ & $(50,46$ & 0.070 & 0.070 & 0.049 & 0.051 & 0.083 & 0.054 & 0.049 & 0.050 \\
\hline & S & $(101,95,105)$ & 0.064 & 0.058 & 0.050 & 0.051 & 0.077 & 0.049 & 0.049 & 0.050 \\
\hline \multirow[t]{12}{*}{0.1} & W & $(17,14,20)$ & 0.125 & 0.124 & 0.128 & 0.131 & 0.119 & 0.129 & 0.129 & 0.133 \\
\hline & W & $(35,28,36)$ & 0.115 & 0.108 & 0.116 & 0.110 & 0.114 & 0.116 & 0.117 & 0.113 \\
\hline & W & $(50,46,55)$ & 0.107 & 0.106 & 0.105 & 0.110 & 0.106 & 0.111 & 0.107 & 0.102 \\
\hline & W & $(101,95,105)$ & 0.103 & 0.102 & 0.105 & 0.105 & 0.104 & 0.101 & 0.105 & 0.101 \\
\hline & $\overline{R V}$ & $(17,14,20)$ & 0.115 & 0.113 & 0.116 & 0.119 & 0.110 & 0.117 & 0.116 & 0.119 \\
\hline & RV & $(35,28,36)$ & 0.110 & 0.103 & 0.111 & 0.105 & 0.108 & 0.110 & 0.111 & 0.107 \\
\hline & RV & $(50,46,55)$ & 0.103 & 0.102 & 0.102 & 0.104 & 0.103 & 0.107 & 0.104 & 0.099 \\
\hline & RV & $(101,95,105)$ & 0.102 & 0.100 & 0.102 & 0.103 & 0.103 & 0.100 & 0.102 & 0.101 \\
\hline & $\mathrm{S}$ & $(17,14,20)$ & 0.181 & 0.150 & 0.111 & 0.108 & 0.171 & 0.129 & 0.108 & 0.105 \\
\hline & $\mathrm{S}$ & $(35,28,36)$ & 0.142 & 0.137 & 0.105 & 0.106 & 0.170 & 0.117 & 0.106 & 0.105 \\
\hline & $\mathrm{S}$ & $(50,46,55)$ & 0.129 & 0.135 & 0.099 & 0.105 & 0.163 & 0.108 & 0.099 & 0.096 \\
\hline & $\mathrm{S}$ & $(101,95,105)$ & 0.118 & 0.119 & 0.101 & 0.102 & 0.151 & 0.099 & 0.100 & 0.102 \\
\hline
\end{tabular}


Tabela E.19: Tamanhos empíricos dos testes Wald, razão de verossimilhança e score para o teste $H_{0}: \beta_{11}=\beta_{21}$, com $\mu=1$.

\begin{tabular}{|c|c|c|c|c|c|c|c|c|c|c|}
\hline \multirow[b]{2}{*}{ Nível } & \multirow[b]{2}{*}{ Teste } & \multirow[b]{2}{*}{$\left(n_{1}, n_{2}, n_{3}\right)$} & \multirow{2}{*}{$\begin{array}{l}0.01 \\
0.01\end{array}$} & \multicolumn{3}{|c|}{0.1} & \multicolumn{4}{|c|}{0.5} \\
\hline & & & & 0.01 & 0.05 & 0.1 & 0.01 & 0.05 & 0.1 & 0.5 \\
\hline \multirow[t]{12}{*}{0.01} & W & $(17,14,20)$ & 0.018 & 0.018 & 0.018 & 0.018 & 0.024 & 0.024 & 0.022 & 0.026 \\
\hline & W & $(35,28,36)$ & 0.013 & 0.014 & 0.013 & 0.016 & 0.015 & 0.014 & 0.015 & 0.015 \\
\hline & W & $(50,46,55)$ & 0.011 & 0.012 & 0.012 & 0.013 & 0.011 & 0.014 & 0.015 & 0.014 \\
\hline & $\mathrm{W}$ & $(101,95,105)$ & 0.009 & 0.010 & 0.012 & 0.011 & 0.011 & 0.013 & 0.011 & 0.013 \\
\hline & RV & $(17,14,20)$ & 0.014 & 0.011 & 0.012 & 0.013 & 0.013 & 0.014 & 0.011 & 0.016 \\
\hline & $\mathrm{RV}$ & $(35,28,36)$ & 0.012 & 0.011 & 0.011 & 0.013 & 0.012 & 0.011 & 0.010 & 0.012 \\
\hline & $\mathrm{RV}$ & $(50,46,55)$ & 0.010 & 0.010 & 0.011 & 0.011 & 0.008 & 0.011 & 0.010 & 0.011 \\
\hline & $\mathrm{RV}$ & $(101,95,105)$ & 0.009 & 0.009 & 0.011 & 0.010 & 0.011 & 0.011 & 0.010 & 0.011 \\
\hline & $\mathrm{S}$ & $(17,14,20)$ & 0.025 & 0.017 & 0.009 & 0.007 & 0.022 & 0.013 & 0.009 & 0.008 \\
\hline & $\mathrm{S}$ & $(35,28,36)$ & 0.021 & 0.017 & 0.011 & 0.010 & 0.021 & 0.010 & 0.009 & 0.008 \\
\hline & $\mathrm{S}$ & $(50,46,55)$ & 0.017 & 0.014 & 0.011 & 0.010 & 0.019 & 0.010 & 0.010 & 0.009 \\
\hline & $\mathrm{S}$ & $(101,95,105)$ & 0.013 & 0.011 & 0.011 & 0.010 & 0.018 & 0.010 & 0.009 & 0.009 \\
\hline \multirow[t]{12}{*}{0.05} & W & $(17,14,20)$ & 0.066 & 0.066 & 0.068 & 0.067 & 0.075 & 0.076 & 0.075 & 0.082 \\
\hline & $\mathrm{W}$ & $(35,28,36)$ & 0.058 & 0.055 & 0.060 & 0.063 & 0.060 & 0.059 & 0.061 & 0.064 \\
\hline & W & $(50,46,55)$ & 0.055 & 0.055 & 0.057 & 0.057 & 0.057 & 0.058 & 0.059 & 0.056 \\
\hline & $\mathrm{W}$ & $(101,95,105)$ & 0.055 & 0.049 & 0.054 & 0.054 & 0.055 & 0.057 & 0.055 & 0.056 \\
\hline & RV & $(17,14,20)$ & 0.060 & 0.057 & 0.056 & 0.057 & 0.058 & 0.061 & 0.059 & 0.067 \\
\hline & $\mathrm{RV}$ & $(35,28,36)$ & 0.055 & 0.052 & 0.055 & 0.056 & 0.053 & 0.055 & 0.055 & 0.055 \\
\hline & $\mathrm{RV}$ & $(50,46,55)$ & 0.053 & 0.052 & 0.053 & 0.054 & 0.053 & 0.052 & 0.055 & 0.052 \\
\hline & $\mathrm{RV}$ & $(101,95,105)$ & 0.053 & 0.048 & 0.052 & 0.053 & 0.052 & 0.052 & 0.051 & 0.052 \\
\hline & $\mathrm{S}$ & $(17,14,20)$ & 0.099 & 0.086 & 0.051 & 0.046 & 0.106 & 0.070 & 0.053 & 0.051 \\
\hline & $\mathrm{S}$ & $(35,28,36)$ & 0.080 & 0.072 & 0.051 & 0.053 & 0.101 & 0.053 & 0.053 & 0.049 \\
\hline & $\mathrm{S}$ & $(50,46,55)$ & 0.070 & 0.068 & 0.051 & 0.051 & 0.092 & 0.051 & 0.053 & 0.049 \\
\hline & $\mathrm{S}$ & $(101,95,105)$ & 0.063 & 0.059 & 0.051 & 0.051 & 0.081 & 0.051 & 0.049 & 0.051 \\
\hline \multirow[t]{12}{*}{0.1} & W & $(17,14,20)$ & 0.118 & 0.121 & 0.124 & 0.121 & 0.128 & 0.133 & 0.129 & 0.140 \\
\hline & W & $(35,28,36)$ & 0.109 & 0.106 & 0.112 & 0.118 & 0.116 & 0.111 & 0.114 & 0.118 \\
\hline & W & $(50,46,55)$ & 0.102 & 0.105 & 0.111 & 0.105 & 0.108 & 0.108 & 0.113 & 0.108 \\
\hline & $\mathrm{W}$ & $(101,95,105)$ & 0.102 & 0.099 & 0.105 & 0.104 & 0.105 & 0.107 & 0.103 & 0.108 \\
\hline & RV & $(17,14,20)$ & 0.112 & 0.109 & 0.112 & 0.110 & 0.116 & 0.119 & 0.110 & 0.122 \\
\hline & $\mathrm{RV}$ & $(35,28,36)$ & 0.105 & 0.098 & 0.108 & 0.110 & 0.108 & 0.102 & 0.108 & 0.107 \\
\hline & RV & $(50,46,55)$ & 0.100 & 0.101 & 0.108 & 0.101 & 0.105 & 0.102 & 0.108 & 0.104 \\
\hline & $\mathrm{RV}$ & $(101,95,105)$ & 0.100 & 0.099 & 0.105 & 0.101 & 0.101 & 0.101 & 0.100 & 0.103 \\
\hline & $\mathrm{S}$ & $(17,14,20)$ & 0.180 & 0.161 & 0.109 & 0.108 & 0.198 & 0.139 & 0.108 & 0.104 \\
\hline & $\mathrm{S}$ & $(35,28,36)$ & 0.148 & 0.140 & 0.105 & 0.108 & 0.181 & 0.108 & 0.099 & 0.097 \\
\hline & $\mathrm{S}$ & $(50,46,55)$ & 0.130 & 0.135 & 0.104 & 0.099 & 0.172 & 0.104 & 0.101 & 0.098 \\
\hline & $\mathrm{S}$ & $(101,95,105)$ & 0.120 & 0.118 & 0.103 & 0.101 & 0.145 & 0.103 & 0.099 & 0.102 \\
\hline
\end{tabular}


Tabela E.20: Tamanhos empíricos dos testes Wald, razão de verossimilhança e score para o teste $H_{0}: \beta_{11}=\beta_{21}$, com $\mu=2.5$.

\begin{tabular}{|c|c|c|c|c|c|c|c|c|c|c|}
\hline \multirow[b]{2}{*}{ Nível } & \multirow[b]{2}{*}{ Teste } & \multirow[b]{2}{*}{$\left(n_{1}, n_{2}, n_{3}\right)$} & \multirow{2}{*}{$\begin{array}{l}0.01 \\
0.01\end{array}$} & \multicolumn{3}{|c|}{0.1} & \multicolumn{4}{|c|}{0.5} \\
\hline & & & & 0.01 & 0.05 & 0.1 & 0.01 & 0.05 & 0.1 & 0.5 \\
\hline \multirow[t]{12}{*}{0.01} & W & $(17,14,20)$ & 0.019 & 0.018 & 0.019 & 0.021 & 0.021 & 0.024 & 0.023 & 0.029 \\
\hline & W & $(35,28,36)$ & 0.013 & 0.013 & 0.014 & 0.012 & 0.013 & 0.016 & 0.014 & 0.016 \\
\hline & $\mathrm{W}$ & $(50,46,55)$ & 0.011 & 0.011 & 0.012 & 0.011 & 0.013 & 0.016 & 0.013 & 0.014 \\
\hline & $\mathrm{W}$ & $(101,95,105)$ & 0.011 & 0.011 & 0.011 & 0.010 & 0.012 & 0.016 & 0.011 & 0.011 \\
\hline & RV & $(17,14,20)$ & 0.015 & 0.013 & 0.013 & 0.014 & 0.013 & 0.015 & 0.014 & 0.018 \\
\hline & RV & $(35,28,36)$ & 0.011 & 0.010 & 0.012 & 0.010 & 0.011 & 0.013 & 0.010 & 0.012 \\
\hline & RV & $(50,46,55)$ & 0.010 & 0.009 & 0.010 & 0.009 & 0.011 & 0.013 & 0.010 & 0.011 \\
\hline & RV & $(101,95,105)$ & 0.010 & 0.010 & 0.010 & 0.009 & 0.011 & 0.013 & 0.010 & 0.010 \\
\hline & $\mathrm{S}$ & $(17,14,20)$ & 0.022 & 0.015 & 0.011 & 0.008 & 0.021 & 0.013 & 0.008 & 0.007 \\
\hline & $\mathrm{S}$ & $(35,28,36)$ & 0.020 & 0.013 & 0.009 & 0.008 & 0.020 & 0.012 & 0.008 & 0.009 \\
\hline & $\mathrm{S}$ & $(50,46,55)$ & 0.017 & 0.012 & 0.009 & 0.009 & 0.020 & 0.012 & 0.009 & 0.009 \\
\hline & $\mathrm{S}$ & $(101,95,105)$ & 0.016 & 0.012 & 0.010 & 0.010 & 0.016 & 0.012 & 0.009 & 0.009 \\
\hline \multirow[t]{12}{*}{0.05} & $\mathrm{~W}$ & $(17,14,20)$ & 0.068 & 0.062 & 0.066 & 0.072 & 0.071 & 0.074 & 0.073 & 0.082 \\
\hline & $\mathrm{W}$ & $(35,28,36)$ & 0.059 & 0.056 & 0.061 & 0.058 & 0.059 & 0.060 & 0.058 & 0.061 \\
\hline & W & $(50,46,55)$ & 0.053 & 0.052 & 0.057 & 0.051 & 0.056 & 0.060 & 0.052 & 0.054 \\
\hline & $\mathrm{W}$ & $(101,95,105)$ & 0.049 & 0.052 & 0.052 & 0.051 & 0.049 & 0.060 & 0.050 & 0.053 \\
\hline & $\overline{\mathrm{RV}}$ & $(17,14,20)$ & 0.062 & 0.054 & 0.057 & 0.063 & 0.061 & 0.061 & 0.061 & 0.067 \\
\hline & RV & $(35,28,36)$ & 0.056 & 0.053 & 0.057 & 0.054 & 0.053 & 0.054 & 0.052 & 0.055 \\
\hline & RV & $(50,46,55)$ & 0.051 & 0.050 & 0.054 & 0.049 & 0.051 & 0.054 & 0.048 & 0.051 \\
\hline & RV & $(101,95,105)$ & 0.049 & 0.050 & 0.051 & 0.050 & 0.049 & 0.053 & 0.048 & 0.051 \\
\hline & $\mathrm{S}$ & $(17,14,20)$ & 0.096 & 0.079 & 0.053 & 0.056 & 0.102 & 0.067 & 0.052 & 0.051 \\
\hline & $\mathrm{S}$ & $(35,28,36)$ & 0.080 & 0.073 & 0.053 & 0.049 & 0.093 & 0.058 & 0.047 & 0.049 \\
\hline & $\mathrm{S}$ & $(50,46,55)$ & 0.068 & 0.067 & 0.053 & 0.049 & 0.092 & 0.058 & 0.047 & 0.048 \\
\hline & $\mathrm{S}$ & $(101,95,105)$ & 0.059 & 0.065 & 0.050 & 0.050 & 0.076 & 0.058 & 0.049 & 0.049 \\
\hline \multirow[t]{12}{*}{0.1} & $\mathrm{~W}$ & $(17,14,20)$ & 0.116 & 0.119 & 0.123 & 0.124 & 0.127 & 0.128 & 0.127 & 0.136 \\
\hline & W & $(35,28,36)$ & 0.111 & 0.108 & 0.115 & 0.108 & 0.110 & 0.110 & 0.109 & 0.116 \\
\hline & $\mathrm{W}$ & $(50,46,55)$ & 0.105 & 0.101 & 0.108 & 0.105 & 0.110 & 0.110 & 0.101 & 0.102 \\
\hline & $\mathrm{W}$ & $(101,95,105)$ & 0.101 & 0.101 & 0.099 & 0.104 & 0.098 & 0.110 & 0.099 & 0.101 \\
\hline & RV & $(17,14,20)$ & 0.112 & 0.111 & 0.114 & 0.116 & 0.116 & 0.117 & 0.115 & 0.120 \\
\hline & RV & $(35,28,36)$ & 0.107 & 0.105 & 0.112 & 0.104 & 0.105 & 0.105 & 0.103 & 0.108 \\
\hline & RV & $(50,46,55)$ & 0.103 & 0.099 & 0.104 & 0.103 & 0.105 & 0.105 & 0.099 & 0.099 \\
\hline & RV & $(101,95,105)$ & 0.099 & 0.101 & 0.098 & 0.103 & 0.097 & 0.105 & 0.099 & 0.099 \\
\hline & $\mathrm{S}$ & $(17,14,20)$ & 0.176 & 0.158 & 0.111 & 0.110 & 0.191 & 0.136 & 0.112 & 0.103 \\
\hline & $\mathrm{S}$ & $(35,28,36)$ & 0.146 & 0.141 & 0.109 & 0.109 & 0.180 & 0.112 & 0.109 & 0.102 \\
\hline & $\mathrm{S}$ & $(50,46,55)$ & 0.128 & 0.131 & 0.103 & 0.100 & 0.177 & 0.112 & 0.096 & 0.098 \\
\hline & $\mathrm{S}$ & $(101,95,105)$ & 0.114 & 0.124 & 0.097 & 0.100 & 0.143 & 0.112 & 0.097 & 0.099 \\
\hline
\end{tabular}


Tabela E.21: Tamanhos empíricos dos testes Wald, razão de verossimilhança e score para o teste $H_{0}: \beta_{11}=\beta_{21}$, com $\mu=5$.

\begin{tabular}{|c|c|c|c|c|c|c|c|c|c|c|}
\hline \multirow[b]{2}{*}{ Nível } & \multirow[b]{2}{*}{ Teste } & \multirow[b]{2}{*}{$\left(n_{1}, n_{2}, n_{3}\right)$} & \multirow{2}{*}{$\begin{array}{l}0.01 \\
0.01\end{array}$} & \multicolumn{3}{|c|}{0.1} & \multicolumn{4}{|c|}{0.5} \\
\hline & & & & 0.01 & 0.05 & 0.1 & 0.01 & 0.05 & 0.1 & 0.5 \\
\hline \multirow[t]{12}{*}{0.01} & W & $(17,14,20)$ & 0.018 & 0.017 & 0.017 & 0.020 & 0.017 & 0.018 & 0.021 & 0.025 \\
\hline & W & $(35,28,36)$ & 0.014 & 0.013 & 0.012 & 0.014 & 0.014 & 0.014 & 0.013 & 0.015 \\
\hline & W & $(50,46,55)$ & 0.014 & 0.013 & 0.012 & 0.013 & 0.012 & 0.014 & 0.012 & 0.013 \\
\hline & W & $(101,95,105)$ & 0.010 & 0.011 & 0.012 & 0.011 & 0.012 & 0.013 & 0.010 & 0.013 \\
\hline & $\overline{\mathrm{RV}}$ & $(17,14,20)$ & 0.013 & 0.012 & 0.012 & 0.011 & 0.012 & 0.012 & 0.013 & 0.014 \\
\hline & RV & $(35,28,36)$ & 0.012 & 0.011 & 0.011 & 0.011 & 0.012 & 0.012 & 0.010 & 0.011 \\
\hline & RV & $(50,46,55)$ & 0.012 & 0.011 & 0.011 & 0.011 & 0.011 & 0.012 & 0.010 & 0.011 \\
\hline & RV & $(101,95,105)$ & 0.010 & 0.010 & 0.011 & 0.010 & 0.011 & 0.011 & 0.010 & 0.011 \\
\hline & $\mathrm{S}$ & $(17,14,20)$ & 0.021 & 0.015 & 0.008 & 0.007 & 0.019 & 0.012 & 0.008 & 0.006 \\
\hline & $\mathrm{S}$ & $(35,28,36)$ & 0.020 & 0.015 & 0.008 & 0.011 & 0.019 & 0.011 & 0.008 & 0.007 \\
\hline & $\mathrm{S}$ & $(50,46,55)$ & 0.020 & 0.014 & 0.011 & 0.011 & 0.019 & 0.011 & 0.009 & 0.009 \\
\hline & $\mathrm{S}$ & $(101,95,105)$ & 0.013 & 0.012 & 0.011 & 0.010 & 0.018 & 0.011 & 0.009 & 0.011 \\
\hline \multirow[t]{12}{*}{0.05} & W & $(17$, & 0.065 & 0.067 & 0.066 & 0.072 & 0.067 & 0.065 & 0.074 & 0.078 \\
\hline & W & $(35,28,36)$ & 0.060 & 0.058 & 0.056 & 0.054 & 0.060 & 0.061 & 0.056 & 0.062 \\
\hline & W & $(50,46,55)$ & 0.057 & 0.054 & 0.054 & 0.054 & 0.053 & 0.056 & 0.055 & 0.060 \\
\hline & W & $(101,95,105)$ & 0.054 & 0.054 & 0.053 & 0.054 & 0.052 & 0.055 & 0.054 & 0.054 \\
\hline & $\overline{\mathrm{RV}}$ & $(17,14,20)$ & 0.059 & 0.060 & 0.057 & 0.062 & 0.056 & 0.057 & 0.063 & 0.063 \\
\hline & RV & $(35,28,36)$ & 0.055 & 0.054 & 0.052 & 0.051 & 0.055 & 0.056 & 0.053 & 0.056 \\
\hline & RV & $(50,46,55)$ & 0.053 & 0.052 & 0.052 & 0.051 & 0.051 & 0.053 & 0.052 & 0.056 \\
\hline & RV & $(101,95,105)$ & 0.052 & 0.051 & 0.052 & 0.051 & 0.051 & 0.053 & 0.052 & 0.052 \\
\hline & $\mathrm{S}$ & $(17,14,20)$ & 0.096 & 0.086 & 0.052 & 0.053 & 0.099 & 0.065 & 0.055 & 0.047 \\
\hline & $\mathrm{S}$ & $(35,28,36)$ & 0.080 & 0.076 & 0.049 & 0.047 & 0.097 & 0.057 & 0.048 & 0.049 \\
\hline & $\mathrm{S}$ & $(50,46,55)$ & 0.071 & 0.068 & 0.051 & 0.050 & 0.088 & 0.051 & 0.051 & 0.051 \\
\hline & $\mathrm{S}$ & $(101,95,105)$ & 0.061 & 0.061 & 0.051 & 0.050 & 0.084 & 0.051 & 0.050 & 0.051 \\
\hline \multirow[t]{12}{*}{0.1} & W & $(17,14,20)$ & 0.119 & 0.123 & 0.116 & 0.127 & 0.124 & 0.121 & 0.129 & 0.131 \\
\hline & W & $(35,28,36)$ & 0.108 & 0.108 & 0.109 & 0.104 & 0.110 & 0.114 & 0.107 & 0.120 \\
\hline & W & $(50,46,55)$ & 0.108 & 0.106 & 0.107 & 0.103 & 0.103 & 0.110 & 0.106 & 0.114 \\
\hline & W & $(101,95,105)$ & 0.101 & 0.101 & 0.107 & 0.103 & 0.101 & 0.107 & 0.100 & 0.108 \\
\hline & RV & $(17,14,20)$ & 0.113 & 0.115 & 0.108 & 0.118 & 0.113 & 0.109 & 0.118 & 0.118 \\
\hline & RV & $(35,28,36)$ & 0.104 & 0.105 & 0.105 & 0.107 & 0.105 & 0.109 & 0.105 & 0.113 \\
\hline & RV & $(50,46,55)$ & 0.103 & 0.104 & 0.104 & 0.100 & 0.101 & 0.105 & 0.105 & 0.111 \\
\hline & RV & $(101,95,105)$ & 0.100 & 0.100 & 0.103 & 0.100 & 0.101 & 0.105 & 0.099 & 0.105 \\
\hline & $\mathrm{S}$ & $(17,14,20)$ & 0.178 & 0.161 & 0.108 & 0.109 & 0.190 & 0.126 & 0.114 & 0.107 \\
\hline & $\mathrm{S}$ & $(35,28,36)$ & 0.144 & 0.144 & 0.103 & 0.097 & 0.178 & 0.116 & 0.100 & 0.106 \\
\hline & $\mathrm{S}$ & $(50,46,55)$ & 0.126 & 0.135 & 0.103 & 0.103 & 0.170 & 0.106 & 0.100 & 0.105 \\
\hline & $\mathrm{S}$ & $(101,95,105)$ & 0.113 & 0.121 & 0.102 & 0.101 & 0.153 & 0.105 & 0.099 & 0.104 \\
\hline
\end{tabular}


Tabela E.22: Tamanhos empíricos dos testes Wald, razão de verossimilhança e score para o teste $H_{0}: \beta_{12}=\beta_{22}$, com $\mu=1$.

\begin{tabular}{|c|c|c|c|c|c|c|c|c|c|c|}
\hline \multirow[b]{2}{*}{ Nível } & \multirow[b]{2}{*}{ Teste } & \multirow[b]{2}{*}{$\left(n_{1}, n_{2}, n_{3}\right)$} & \multirow{2}{*}{$\begin{array}{l}0.01 \\
0.01\end{array}$} & \multicolumn{3}{|c|}{0.1} & \multicolumn{4}{|c|}{0.5} \\
\hline & & & & 0.01 & 0.05 & 0.1 & 0.01 & 0.05 & 0.1 & 0.5 \\
\hline \multirow[t]{12}{*}{0.01} & $\mathrm{~W}$ & $(17,14,20)$ & 0.019 & 0.021 & 0.023 & 0.021 & 0.026 & 0.027 & 0.023 & 0.030 \\
\hline & $\mathrm{W}$ & $(35,28,36)$ & 0.015 & 0.014 & 0.017 & 0.015 & 0.016 & 0.016 & 0.016 & 0.017 \\
\hline & $\mathrm{W}$ & $(50,46,55)$ & 0.012 & 0.013 & 0.013 & 0.010 & 0.014 & 0.015 & 0.016 & 0.015 \\
\hline & $\mathrm{W}$ & $(101,95,105)$ & 0.012 & 0.010 & 0.012 & 0.010 & 0.012 & 0.011 & 0.012 & 0.012 \\
\hline & RV & $(17,14,20)$ & 0.013 & 0.014 & 0.015 & 0.014 & 0.016 & 0.015 & 0.014 & 0.017 \\
\hline & $\mathrm{RV}$ & $(35,28,36)$ & 0.013 & 0.011 & 0.013 & 0.012 & 0.013 & 0.011 & 0.013 & 0.013 \\
\hline & RV & $(50,46,55)$ & 0.011 & 0.011 & 0.011 & 0.009 & 0.011 & 0.011 & 0.013 & 0.012 \\
\hline & $\mathrm{RV}$ & $(101,95,105)$ & 0.011 & 0.009 & 0.011 & 0.010 & 0.011 & 0.009 & 0.012 & 0.011 \\
\hline & $\mathrm{S}$ & $(17,14,20)$ & 0.022 & 0.015 & 0.009 & 0.008 & 0.026 & 0.015 & 0.013 & 0.007 \\
\hline & $\mathrm{S}$ & $(35,28,36)$ & 0.019 & 0.015 & 0.012 & 0.010 & 0.024 & 0.011 & 0.012 & 0.009 \\
\hline & $\mathrm{S}$ & $(50,46,55)$ & 0.016 & 0.015 & 0.011 & 0.010 & 0.020 & 0.011 & 0.012 & 0.011 \\
\hline & S & $(101,95,105)$ & 0.016 & 0.012 & 0.011 & 0.010 & 0.020 & 0.009 & 0.011 & 0.010 \\
\hline \multirow[t]{12}{*}{0.05} & $\mathrm{~W}$ & $(17,14,20)$ & 0.067 & 0.074 & 0.078 & 0.075 & 0.083 & 0.079 & 0.081 & 0.088 \\
\hline & W & $(35,28,36)$ & 0.059 & 0.058 & 0.064 & 0.064 & 0.064 & 0.065 & 0.065 & 0.063 \\
\hline & W & $(50,46,55)$ & 0.056 & 0.055 & 0.056 & 0.055 & 0.058 & 0.057 & 0.059 & 0.059 \\
\hline & $\mathrm{W}$ & $(101,95,105)$ & 0.055 & 0.050 & 0.056 & 0.052 & 0.056 & 0.056 & 0.054 & 0.054 \\
\hline & RV & $(17,14,20)$ & 0.059 & 0.061 & 0.066 & 0.061 & 0.066 & 0.063 & 0.062 & 0.070 \\
\hline & $\mathrm{RV}$ & $(35$ & 0.056 & 0.053 & 0.059 & 0.058 & 0.056 & 0.056 & 0.057 & 0.056 \\
\hline & RV & $(50,46,55)$ & 0.053 & 0.051 & 0.053 & 0.049 & 0.055 & 0.054 & 0.054 & 0.056 \\
\hline & $\mathrm{RV}$ & $(101,95,105)$ & 0.053 & 0.049 & 0.052 & 0.051 & 0.054 & 0.054 & 0.053 & 0.053 \\
\hline & $\mathrm{S}$ & $(17,14,20)$ & 0.097 & 0.086 & 0.060 & 0.055 & 0.109 & 0.074 & 0.057 & 0.052 \\
\hline & $\mathrm{S}$ & $(35,28,36)$ & 0.080 & 0.075 & 0.056 & 0.053 & 0.099 & 0.057 & 0.053 & 0.048 \\
\hline & $\mathrm{S}$ & $(50,46$ & 0.071 & 0.067 & 0.051 & 0.051 & 0.094 & 0.054 & 0.051 & 0.052 \\
\hline & $\mathrm{S}$ & $(101,95,105)$ & 0.064 & 0.060 & 0.051 & 0.050 & 0.083 & 0.051 & 0.051 & 0.051 \\
\hline \multirow[t]{12}{*}{0.1} & $\mathrm{~W}$ & $(17,14,20)$ & 0.118 & 0.128 & 0.135 & 0.131 & 0.136 & 0.136 & 0.135 & 0.150 \\
\hline & W & $(35,28,36)$ & 0.115 & 0.109 & 0.119 & 0.117 & 0.113 & 0.114 & 0.113 & 0.117 \\
\hline & W & $(50,46,55)$ & 0.108 & 0.104 & 0.104 & 0.106 & 0.111 & 0.109 & 0.112 & 0.112 \\
\hline & $\mathrm{W}$ & $(101,95,105)$ & 0.106 & 0.103 & 0.104 & 0.103 & 0.106 & 0.102 & 0.105 & 0.105 \\
\hline & RV & $(17,14,20)$ & 0.112 & 0.116 & 0.123 & 0.121 & 0.118 & 0.119 & 0.116 & 0.129 \\
\hline & RV & $(35,28,36)$ & 0.112 & 0.103 & 0.113 & 0.112 & 0.108 & 0.105 & 0.105 & 0.110 \\
\hline & RV & $(50,46,55)$ & 0.105 & 0.100 & 0.101 & 0.103 & 0.107 & 0.104 & 0.104 & 0.106 \\
\hline & $\mathrm{RV}$ & $(101,95,105)$ & 0.105 & 0.100 & 0.101 & 0.102 & 0.105 & 0.101 & 0.101 & 0.104 \\
\hline & $\mathrm{S}$ & $(17,14,20)$ & 0.179 & 0.164 & 0.118 & 0.110 & 0.196 & 0.140 & 0.112 & 0.109 \\
\hline & $\mathrm{S}$ & $(35,28,36)$ & 0.151 & 0.144 & 0.111 & 0.108 & 0.179 & 0.116 & 0.103 & 0.104 \\
\hline & $\mathrm{S}$ & $(50,46,55)$ & 0.130 & 0.133 & 0.101 & 0.099 & 0.176 & 0.108 & 0.102 & 0.103 \\
\hline & S & $(101,95,105)$ & 0.120 & 0.121 & 0.101 & 0.101 & 0.150 & 0.100 & 0.100 & 0.102 \\
\hline
\end{tabular}


Tabela E.23: Tamanhos empíricos dos testes Wald, razão de verossimilhança e score para o teste $H_{0}: \beta_{12}=\beta_{22}$, com $\mu=2.5$.

\begin{tabular}{|c|c|c|c|c|c|c|c|c|c|c|c|}
\hline \multirow[b]{2}{*}{ Nível } & \multirow[b]{2}{*}{ Teste } & \multirow[b]{2}{*}{$\left(n_{1}, n_{2}, n_{3}\right)$} & \multirow{2}{*}{$\begin{array}{l}\sigma_{x}^{2} \\
\sigma^{2}\end{array}$} & \multirow{2}{*}{$\begin{array}{l}0.01 \\
0.01\end{array}$} & \multicolumn{3}{|c|}{0.1} & \multicolumn{4}{|c|}{0.5} \\
\hline & & & & & 0.01 & 0.05 & 0.1 & 0.01 & 0.05 & 0.1 & 0.5 \\
\hline \multirow[t]{12}{*}{0.01} & W & $(17,14,20)$ & & 0.019 & 0.019 & 0.024 & 0.019 & 0.025 & 0.021 & 0.021 & 0.024 \\
\hline & W & $(35,28,36)$ & & 0.014 & 0.014 & 0.015 & 0.014 & 0.015 & 0.016 & 0.014 & 0.016 \\
\hline & W & $(50,46,55)$ & & 0.013 & 0.014 & 0.012 & 0.012 & 0.012 & 0.013 & 0.013 & 0.011 \\
\hline & W & $(101,95,105)$ & & 0.011 & 0.011 & 0.012 & 0.009 & 0.010 & 0.013 & 0.012 & 0.010 \\
\hline & $\overline{\mathrm{RV}}$ & $(17,14,20)$ & & 0.013 & 0.012 & 0.016 & 0.013 & 0.015 & 0.013 & 0.013 & 0.013 \\
\hline & RV & $(35,28,36)$ & & 0.011 & 0.012 & 0.013 & 0.012 & 0.010 & 0.013 & 0.011 & 0.011 \\
\hline & RV & $(50,46,55)$ & & 0.011 & 0.012 & 0.011 & 0.011 & 0.010 & 0.010 & 0.011 & 0.009 \\
\hline & RV & $(101,95,105)$ & & 0.011 & 0.011 & 0.011 & 0.009 & 0.009 & 0.010 & 0.011 & 0.009 \\
\hline & $\mathrm{S}$ & $(17,14,20)$ & & 0.018 & 0.014 & 0.011 & 0.007 & 0.021 & 0.012 & 0.008 & 0.005 \\
\hline & $\mathrm{S}$ & $(35,28,36)$ & & 0.018 & 0.013 & 0.011 & 0.010 & 0.019 & 0.011 & 0.008 & 0.008 \\
\hline & $\mathrm{S}$ & $(50,46,55)$ & & 0.017 & 0.013 & 0.010 & 0.010 & 0.019 & 0.009 & 0.010 & 0.008 \\
\hline & $\mathrm{S}$ & $(101,95,105)$ & & 0.015 & 0.012 & 0.010 & 0.009 & 0.015 & 0.010 & 0.010 & 0.009 \\
\hline \multirow[t]{12}{*}{0.05} & $\mathrm{~W}$ & $(17,14,20)$ & & 0.067 & 0.071 & 0.078 & 0.073 & 0.076 & 0.070 & 0.072 & 0.075 \\
\hline & W & $(35,28,36)$ & & 0.058 & 0.058 & 0.060 & 0.059 & 0.057 & 0.061 & 0.063 & 0.062 \\
\hline & W & $(50,46,55)$ & & 0.056 & 0.058 & 0.056 & 0.058 & 0.052 & 0.057 & 0.054 & 0.057 \\
\hline & W & $(101,95,105)$ & & 0.053 & 0.050 & 0.054 & 0.048 & 0.052 & 0.052 & 0.052 & 0.056 \\
\hline & $\overline{R V}$ & $(17,14,20)$ & & 0.059 & 0.060 & 0.067 & 0.061 & 0.064 & 0.058 & 0.060 & 0.060 \\
\hline & RV & $(35,28,36)$ & & 0.054 & 0.052 & 0.056 & 0.056 & 0.052 & 0.055 & 0.055 & 0.057 \\
\hline & RV & $(50,46,55)$ & & 0.053 & 0.052 & 0.053 & 0.055 & 0.049 & 0.053 & 0.049 & 0.055 \\
\hline & RV & $(101,95,105)$ & & 0.052 & 0.050 & 0.053 & 0.046 & 0.050 & 0.051 & 0.051 & 0.053 \\
\hline & $\mathrm{S}$ & $(17,14,20)$ & & 0.092 & 0.083 & 0.061 & 0.051 & 0.104 & 0.067 & 0.053 & 0.045 \\
\hline & $\mathrm{S}$ & $(35,28,36)$ & & 0.080 & 0.072 & 0.053 & 0.051 & 0.095 & 0.056 & 0.053 & 0.053 \\
\hline & $\mathrm{S}$ & $(50,46,55)$ & & 0.072 & 0.072 & 0.051 & 0.051 & 0.084 & 0.054 & 0.047 & 0.052 \\
\hline & $\mathrm{S}$ & $(101,95,105)$ & & 0.063 & 0.059 & 0.051 & 0.049 & 0.078 & 0.050 & 0.052 & 0.052 \\
\hline \multirow[t]{12}{*}{0.1} & $\mathrm{~W}$ & $(17,14,20)$ & & 0.121 & 0.124 & 0.132 & 0.126 & 0.133 & 0.126 & 0.127 & 0.134 \\
\hline & W & $(35,28,36)$ & & 0.112 & 0.106 & 0.109 & 0.112 & 0.110 & 0.114 & 0.119 & 0.115 \\
\hline & W & $(50,46,55)$ & & 0.110 & 0.106 & 0.107 & 0.107 & 0.102 & 0.106 & 0.105 & 0.108 \\
\hline & W & $(101,95,105)$ & & 0.103 & 0.102 & 0.104 & 0.097 & 0.099 & 0.104 & 0.103 & 0.107 \\
\hline & RV & $(17,14,20)$ & & 0.114 & 0.114 & 0.123 & 0.115 & 0.122 & 0.112 & 0.117 & 0.118 \\
\hline & RV & $(35,28,36)$ & & 0.109 & 0.102 & 0.104 & 0.106 & 0.104 & 0.107 & 0.113 & 0.108 \\
\hline & RV & $(50,46,55)$ & & 0.108 & 0.102 & 0.103 & 0.104 & 0.100 & 0.103 & 0.101 & 0.105 \\
\hline & RV & $(101,95,105)$ & & 0.103 & 0.099 & 0.102 & 0.097 & 0.099 & 0.103 & 0.101 & 0.105 \\
\hline & $\mathrm{S}$ & $(17,14,20)$ & & 0.173 & 0.163 & 0.121 & 0.106 & 0.191 & 0.130 & 0.111 & 0.102 \\
\hline & $\mathrm{S}$ & $(35,28,36)$ & & 0.150 & 0.136 & 0.102 & 0.103 & 0.177 & 0.112 & 0.108 & 0.101 \\
\hline & $\mathrm{S}$ & $(50,46,55)$ & & 0.136 & 0.136 & 0.101 & 0.102 & 0.167 & 0.107 & 0.105 & 0.100 \\
\hline & $\mathrm{S}$ & $(101,95,105)$ & & 0.118 & 0.121 & 0.101 & 0.098 & 0.150 & 0.102 & 0.102 & 0.100 \\
\hline
\end{tabular}


Tabela E.24: Tamanhos empíricos dos testes Wald, razão de verossimilhança e score para o teste $H_{0}: \beta_{12}=\beta_{22}$, com $\mu=5$.

\begin{tabular}{|c|c|c|c|c|c|c|c|c|c|c|}
\hline \multirow[b]{2}{*}{ Nível } & \multirow[b]{2}{*}{ Teste } & \multirow[b]{2}{*}{$\left(n_{1}, n_{2}, n_{3}\right)$} & \multirow{2}{*}{$\begin{array}{l}0.01 \\
0.01\end{array}$} & \multicolumn{3}{|c|}{0.1} & \multicolumn{4}{|c|}{0.5} \\
\hline & & & & 0.01 & 0.05 & 0.1 & 0.01 & 0.05 & 0.1 & 0.5 \\
\hline \multirow[t]{12}{*}{0.01} & $\mathrm{~W}$ & $(17,14,20)$ & 0.019 & 0.019 & 0.022 & 0.021 & 0.020 & 0.019 & 0.022 & 0.026 \\
\hline & $\mathrm{W}$ & $(35,28,36)$ & 0.013 & 0.015 & 0.014 & 0.014 & 0.015 & 0.016 & 0.016 & 0.017 \\
\hline & $\mathrm{W}$ & $(50,46,55)$ & 0.012 & 0.013 & 0.012 & 0.012 & 0.011 & 0.014 & 0.013 & 0.015 \\
\hline & $\mathrm{W}$ & $(101,95,105)$ & 0.010 & 0.012 & 0.012 & 0.011 & 0.010 & 0.011 & 0.012 & 0.012 \\
\hline & RV & $(17,14,20)$ & 0.013 & 0.013 & 0.015 & 0.014 & 0.013 & 0.012 & 0.016 & 0.014 \\
\hline & $\mathrm{RV}$ & $(35,28,36)$ & 0.011 & 0.013 & 0.011 & 0.011 & 0.012 & 0.012 & 0.013 & 0.012 \\
\hline & RV & $(50,46,55)$ & 0.011 & 0.012 & 0.011 & 0.010 & 0.010 & 0.012 & 0.012 & 0.012 \\
\hline & $\mathrm{RV}$ & $(101,95,105)$ & 0.010 & 0.011 & 0.011 & 0.010 & 0.010 & 0.010 & 0.011 & 0.011 \\
\hline & $\mathrm{S}$ & $(17,14,20)$ & 0.023 & 0.015 & 0.009 & 0.007 & 0.021 & 0.009 & 0.009 & 0.005 \\
\hline & $\mathrm{S}$ & $(35,28,36)$ & 0.019 & 0.015 & 0.009 & 0.008 & 0.019 & 0.011 & 0.009 & 0.009 \\
\hline & $\mathrm{S}$ & $(50,46,55)$ & 0.019 & 0.015 & 0.010 & 0.010 & 0.018 & 0.011 & 0.009 & 0.011 \\
\hline & S & $(101,95,105)$ & 0.015 & 0.014 & 0.010 & 0.010 & 0.015 & 0.009 & 0.010 & 0.010 \\
\hline \multirow[t]{12}{*}{0.05} & $\mathrm{~W}$ & $(17,14,20)$ & 0.070 & 0.071 & 0.072 & 0.071 & 0.072 & 0.071 & 0.073 & 0.078 \\
\hline & W & $(35,28,36)$ & 0.057 & 0.061 & 0.061 & 0.058 & 0.061 & 0.059 & 0.061 & 0.064 \\
\hline & W & $(50,46,55)$ & 0.054 & 0.056 & 0.057 & 0.056 & 0.051 & 0.054 & 0.059 & 0.054 \\
\hline & $\mathrm{W}$ & $(101,95,105)$ & 0.053 & 0.051 & 0.054 & 0.053 & 0.051 & 0.053 & 0.053 & 0.053 \\
\hline & $\mathrm{RV}$ & $(17,14,20)$ & 0.062 & 0.061 & 0.062 & 0.061 & 0.063 & 0.060 & 0.061 & 0.063 \\
\hline & $\mathrm{RV}$ & $(35$ & 0.052 & 0.056 & 0.058 & 0.054 & 0.057 & 0.054 & 0.055 & 0.057 \\
\hline & RV & $(50,46,55)$ & 0.052 & 0.054 & 0.054 & 0.053 & 0.049 & 0.051 & 0.055 & 0.051 \\
\hline & $\mathrm{RV}$ & $(101,95,105)$ & 0.052 & 0.050 & 0.052 & 0.052 & 0.051 & 0.051 & 0.052 & 0.051 \\
\hline & $\mathrm{S}$ & $(17,14,20)$ & 0.098 & 0.083 & 0.056 & 0.054 & 0.101 & 0.060 & 0.054 & 0.047 \\
\hline & $\mathrm{S}$ & $(35,28,36)$ & 0.077 & 0.073 & 0.055 & 0.053 & 0.098 & 0.056 & 0.052 & 0.051 \\
\hline & $\mathrm{S}$ & $(50,46$ & 0.069 & 0.071 & 0.051 & 0.051 & 0.088 & 0.050 & 0.051 & 0.049 \\
\hline & $\mathrm{S}$ & $(101,95,105)$ & 0.062 & 0.059 & 0.051 & 0.051 & 0.080 & 0.050 & 0.051 & 0.050 \\
\hline \multirow[t]{12}{*}{0.1} & $\mathrm{~W}$ & $(17,14,20)$ & 0.125 & 0.131 & 0.127 & 0.129 & 0.124 & 0.125 & 0.128 & 0.137 \\
\hline & W & $(35,28,36)$ & 0.109 & 0.113 & 0.114 & 0.110 & 0.117 & 0.113 & 0.114 & 0.117 \\
\hline & W & $(50,46,55)$ & 0.102 & 0.109 & 0.111 & 0.110 & 0.107 & 0.107 & 0.110 & 0.106 \\
\hline & $\mathrm{W}$ & $(101,95,105)$ & 0.102 & 0.101 & 0.100 & 0.103 & 0.106 & 0.104 & 0.102 & 0.098 \\
\hline & RV & $(17,14,20)$ & 0.116 & 0.121 & 0.118 & 0.118 & 0.114 & 0.114 & 0.117 & 0.121 \\
\hline & RV & $(35,28,36)$ & 0.106 & 0.109 & 0.108 & 0.105 & 0.112 & 0.108 & 0.109 & 0.112 \\
\hline & RV & $(50,46,55)$ & 0.099 & 0.106 & 0.107 & 0.104 & 0.104 & 0.104 & 0.106 & 0.103 \\
\hline & $\mathrm{RV}$ & $(101,95,105)$ & 0.101 & 0.099 & 0.099 & 0.101 & 0.104 & 0.102 & 0.100 & 0.097 \\
\hline & $\mathrm{S}$ & $(17,14,20)$ & 0.178 & 0.164 & 0.118 & 0.109 & 0.184 & 0.129 & 0.110 & 0.106 \\
\hline & $\mathrm{S}$ & $(35,28,36)$ & 0.144 & 0.141 & 0.105 & 0.102 & 0.184 & 0.113 & 0.104 & 0.106 \\
\hline & $\mathrm{S}$ & $(50,46,55)$ & 0.125 & 0.138 & 0.104 & 0.101 & 0.169 & 0.107 & 0.104 & 0.100 \\
\hline & S & $(101,95,105)$ & 0.120 & 0.120 & 0.099 & 0.101 & 0.151 & 0.101 & 0.099 & 0.099 \\
\hline
\end{tabular}


Tabela E.25: Tamanhos empíricos dos testes Wald, razão de verossimilhança e score para o teste $H_{0}: \beta_{13}=\beta_{23}$, com $\mu=1$.

\begin{tabular}{|c|c|c|c|c|c|c|c|c|c|c|c|}
\hline \multirow[b]{2}{*}{ Nível } & \multirow[b]{2}{*}{ Teste } & \multirow[b]{2}{*}{$\left(n_{1}, n_{2}, n_{3}\right)$} & \multirow{2}{*}{$\begin{array}{l}\sigma_{x}^{2} \\
\sigma^{2}\end{array}$} & \multirow{2}{*}{$\begin{array}{l}0.01 \\
0.01\end{array}$} & \multicolumn{3}{|c|}{0.1} & \multicolumn{4}{|c|}{0.5} \\
\hline & & & & & 0.01 & 0.05 & 0.1 & 0.01 & 0.05 & 0.1 & 0.5 \\
\hline \multirow[t]{12}{*}{0.01} & W & $(17,14,20)$ & & 0.017 & 0.016 & 0.016 & 0.016 & 0.017 & 0.019 & 0.021 & 0.020 \\
\hline & W & $(35,28,36)$ & & 0.013 & 0.015 & 0.015 & 0.016 & 0.016 & 0.015 & 0.017 & 0.016 \\
\hline & W & $(50,46,55)$ & & 0.012 & 0.012 & 0.014 & 0.014 & 0.014 & 0.013 & 0.013 & 0.013 \\
\hline & W & $(101,95,105)$ & & 0.012 & 0.012 & 0.011 & 0.011 & 0.014 & 0.011 & 0.012 & 0.012 \\
\hline & RV & $(17,14,20)$ & & 0.013 & 0.012 & 0.012 & 0.011 & 0.012 & 0.012 & 0.015 & 0.014 \\
\hline & RV & $(35,28,36)$ & & 0.011 & 0.012 & 0.011 & 0.011 & 0.011 & 0.011 & 0.013 & 0.014 \\
\hline & RV & $(50,46,55)$ & & 0.011 & 0.011 & 0.011 & 0.011 & 0.010 & 0.011 & 0.011 & 0.012 \\
\hline & RV & $(101,95,105)$ & & 0.011 & 0.011 & 0.011 & 0.010 & 0.010 & 0.011 & 0.011 & 0.011 \\
\hline & $\mathrm{S}$ & $(17,14,20)$ & & 0.024 & 0.016 & 0.009 & 0.007 & 0.022 & 0.013 & 0.011 & 0.007 \\
\hline & $\mathrm{S}$ & $(35,28,36)$ & & 0.020 & 0.016 & 0.009 & 0.009 & 0.021 & 0.012 & 0.011 & 0.010 \\
\hline & $\mathrm{S}$ & $(50,46,55)$ & & 0.017 & 0.015 & 0.011 & 0.011 & 0.020 & 0.010 & 0.010 & 0.010 \\
\hline & $\mathrm{S}$ & $(101,95,105)$ & & 0.015 & 0.014 & 0.010 & 0.010 & 0.020 & 0.010 & 0.010 & 0.010 \\
\hline \multirow[t]{12}{*}{0.05} & W & $(17,14,20)$ & & 0.061 & 0.066 & 0.067 & 0.063 & 0.068 & 0.068 & 0.070 & 0.073 \\
\hline & W & $(35,28,36)$ & & 0.058 & 0.056 & 0.058 & 0.061 & 0.058 & 0.061 & 0.063 & 0.067 \\
\hline & W & $(50,46,55)$ & & 0.049 & 0.055 & 0.058 & 0.055 & 0.057 & 0.056 & 0.054 & 0.056 \\
\hline & $\mathrm{W}$ & $(101,95,105)$ & & 0.050 & 0.054 & 0.056 & 0.054 & 0.057 & 0.055 & 0.053 & 0.056 \\
\hline & $\mathrm{RV}$ & $(17,14,20)$ & & 0.057 & 0.060 & 0.058 & 0.056 & 0.056 & 0.058 & 0.061 & 0.060 \\
\hline & RV & $(35,28,36)$ & & 0.056 & 0.054 & 0.056 & 0.055 & 0.055 & 0.054 & 0.057 & 0.060 \\
\hline & RV & $(50,46,55)$ & & 0.048 & 0.050 & 0.055 & 0.052 & 0.054 & 0.052 & 0.050 & 0.053 \\
\hline & RV & $(101,95,105)$ & & 0.049 & 0.050 & 0.054 & 0.052 & 0.054 & 0.052 & 0.050 & 0.053 \\
\hline & $\mathrm{S}$ & $(17,14,20)$ & & 0.097 & 0.086 & 0.057 & 0.049 & 0.103 & 0.069 & 0.059 & 0.047 \\
\hline & $\mathrm{S}$ & $(35,28,36)$ & & 0.083 & 0.076 & 0.056 & 0.051 & 0.097 & 0.055 & 0.053 & 0.053 \\
\hline & $\mathrm{S}$ & $(50,46,55)$ & & 0.065 & 0.067 & 0.055 & 0.050 & 0.091 & 0.053 & 0.047 & 0.050 \\
\hline & $\mathrm{S}$ & $(101,95,105)$ & & 0.057 & 0.066 & 0.054 & 0.050 & 0.091 & 0.052 & 0.048 & 0.050 \\
\hline \multirow[t]{12}{*}{0.1} & $\mathrm{~W}$ & $(17,14,20)$ & & 0.108 & 0.119 & 0.120 & 0.119 & 0.117 & 0.122 & 0.125 & 0.127 \\
\hline & W & $(35,28,36)$ & & 0.107 & 0.108 & 0.108 & 0.111 & 0.115 & 0.114 & 0.116 & 0.119 \\
\hline & W & $(50,46,55)$ & & 0.101 & 0.104 & 0.108 & 0.105 & 0.108 & 0.109 & 0.102 & 0.108 \\
\hline & $\mathrm{W}$ & $(101,95,105)$ & & 0.101 & 0.103 & 0.102 & 0.104 & 0.108 & 0.107 & 0.102 & 0.107 \\
\hline & RV & $(17,14,20)$ & & 0.109 & 0.112 & 0.112 & 0.111 & 0.107 & 0.110 & 0.111 & 0.114 \\
\hline & RV & $(35,28,36)$ & & 0.108 & 0.105 & 0.105 & 0.108 & 0.106 & 0.106 & 0.110 & 0.112 \\
\hline & RV & $(50,46,55)$ & & 0.099 & 0.101 & 0.104 & 0.104 & 0.103 & 0.106 & 0.099 & 0.106 \\
\hline & RV & $(101,95,105)$ & & 0.101 & 0.101 & 0.102 & 0.104 & 0.103 & 0.105 & 0.101 & 0.106 \\
\hline & $\mathrm{S}$ & $(17,14,20)$ & & 0.180 & 0.160 & 0.114 & 0.104 & 0.195 & 0.137 & 0.113 & 0.096 \\
\hline & $\mathrm{S}$ & $(35,28,36)$ & & 0.146 & 0.144 & 0.104 & 0.104 & 0.179 & 0.116 & 0.107 & 0.104 \\
\hline & $\mathrm{S}$ & $(50,46,55)$ & & 0.126 & 0.138 & 0.102 & 0.101 & 0.170 & 0.109 & 0.095 & 0.102 \\
\hline & $\mathrm{S}$ & $(101,95,105)$ & & 0.115 & 0.128 & 0.101 & 0.101 & 0.170 & 0.103 & 0.101 & 0.102 \\
\hline
\end{tabular}


Tabela E.26: Tamanhos empíricos dos testes Wald, razão de verossimilhança e score para o teste $H_{0}: \beta_{13}=\beta_{23}$, com $\mu=2.5$.

\begin{tabular}{|c|c|c|c|c|c|c|c|c|c|c|}
\hline \multirow[b]{2}{*}{ Nível } & \multirow[b]{2}{*}{ Teste } & \multirow[b]{2}{*}{$\left(n_{1}, n_{2}, n_{3}\right)$} & \multirow{2}{*}{$\begin{array}{l}0.01 \\
0.01\end{array}$} & \multicolumn{3}{|c|}{0.1} & \multicolumn{4}{|c|}{0.5} \\
\hline & & & & 0.01 & 0.05 & 0.1 & 0.01 & 0.05 & 0.1 & 0.5 \\
\hline \multirow[t]{12}{*}{0.01} & W & $(17,14,20)$ & 0.019 & 0.017 & 0.019 & 0.018 & 0.016 & 0.017 & 0.017 & 0.019 \\
\hline & W & $(35,28,36)$ & 0.015 & 0.013 & 0.015 & 0.015 & 0.013 & 0.016 & 0.015 & 0.015 \\
\hline & $\mathrm{W}$ & $(50,46,55)$ & 0.013 & 0.013 & 0.014 & 0.013 & 0.012 & 0.012 & 0.012 & 0.012 \\
\hline & $\mathrm{W}$ & $(101,95,105)$ & 0.011 & 0.011 & 0.011 & 0.010 & 0.012 & 0.012 & 0.010 & 0.012 \\
\hline & RV & $(17,14,20)$ & 0.015 & 0.011 & 0.015 & 0.012 & 0.011 & 0.012 & 0.012 & 0.014 \\
\hline & $\mathrm{RV}$ & $(35,28,36)$ & 0.013 & 0.011 & 0.013 & 0.012 & 0.011 & 0.012 & 0.012 & 0.013 \\
\hline & $\mathrm{RV}$ & $(50,46,55)$ & 0.012 & 0.011 & 0.012 & 0.011 & 0.011 & 0.011 & 0.010 & 0.010 \\
\hline & $\mathrm{RV}$ & $(101,95,105)$ & 0.010 & 0.011 & 0.011 & 0.010 & 0.011 & 0.011 & 0.010 & 0.010 \\
\hline & $\mathrm{S}$ & $(17,14,20)$ & 0.016 & 0.015 & 0.010 & 0.009 & 0.018 & 0.012 & 0.009 & 0.009 \\
\hline & $\mathrm{S}$ & $(35,28,36)$ & 0.015 & 0.014 & 0.010 & 0.010 & 0.018 & 0.012 & 0.009 & 0.009 \\
\hline & $\mathrm{S}$ & $(50,46,55)$ & 0.013 & 0.014 & 0.010 & 0.010 & 0.017 & 0.010 & 0.009 & 0.009 \\
\hline & $\mathrm{S}$ & $(101,95,105)$ & 0.010 & 0.014 & 0.010 & 0.010 & 0.016 & 0.010 & 0.010 & 0.010 \\
\hline \multirow[t]{12}{*}{0.05} & $\mathrm{~W}$ & $(17,14,20)$ & 0.065 & 0.065 & 0.068 & 0.067 & 0.066 & 0.066 & 0.065 & 0.068 \\
\hline & $\mathrm{W}$ & $(35,28,36)$ & 0.060 & 0.061 & 0.061 & 0.059 & 0.058 & 0.063 & 0.061 & 0.061 \\
\hline & W & $(50,46,55)$ & 0.057 & 0.053 & 0.058 & 0.059 & 0.058 & 0.055 & 0.059 & 0.056 \\
\hline & W & $(101,95,105)$ & 0.054 & 0.051 & 0.051 & 0.053 & 0.057 & 0.055 & 0.052 & 0.051 \\
\hline & RV & $(17,14,20)$ & 0.059 & 0.059 & 0.061 & 0.057 & 0.057 & 0.059 & 0.057 & 0.059 \\
\hline & $\mathrm{RV}$ & $(35,28,36)$ & 0.058 & 0.057 & 0.057 & 0.057 & 0.054 & 0.059 & 0.056 & 0.055 \\
\hline & $\mathrm{RV}$ & $(50,46,55)$ & 0.055 & 0.050 & 0.055 & 0.057 & 0.054 & 0.051 & 0.055 & 0.053 \\
\hline & RV & $(101,95,105)$ & 0.053 & 0.050 & 0.050 & 0.053 & 0.052 & 0.051 & 0.050 & 0.048 \\
\hline & $\mathrm{S}$ & $(17,14,20)$ & 0.080 & 0.083 & 0.059 & 0.052 & 0.090 & 0.065 & 0.053 & 0.051 \\
\hline & $\mathrm{S}$ & $(35,28,36)$ & 0.070 & 0.080 & 0.053 & 0.051 & 0.086 & 0.063 & 0.053 & 0.051 \\
\hline & $\mathrm{S}$ & $(50,46,55)$ & 0.064 & 0.072 & 0.053 & 0.051 & 0.084 & 0.053 & 0.052 & 0.051 \\
\hline & $\mathrm{S}$ & $(101,95,105)$ & 0.058 & 0.070 & 0.049 & 0.051 & 0.083 & 0.053 & 0.050 & 0.049 \\
\hline \multirow[t]{12}{*}{0.1} & W & $(17,14,20)$ & 0.117 & 0.122 & 0.123 & 0.118 & 0.122 & 0.120 & 0.117 & 0.124 \\
\hline & W & $(35,28,36)$ & 0.116 & 0.115 & 0.110 & 0.110 & 0.110 & 0.112 & 0.114 & 0.114 \\
\hline & $\mathrm{W}$ & $(50,46,55)$ & 0.108 & 0.101 & 0.108 & 0.108 & 0.110 & 0.104 & 0.108 & 0.106 \\
\hline & W & $(101,95,105)$ & 0.103 & 0.100 & 0.103 & 0.101 & 0.109 & 0.104 & 0.102 & 0.104 \\
\hline & RV & $(17,14,20)$ & 0.113 & 0.113 & 0.115 & 0.112 & 0.112 & 0.111 & 0.108 & 0.112 \\
\hline & $\mathrm{RV}$ & $(35,28,36)$ & 0.112 & 0.109 & 0.107 & 0.105 & 0.105 & 0.108 & 0.108 & 0.108 \\
\hline & $\mathrm{RV}$ & $(50,46,55)$ & 0.105 & 0.099 & 0.105 & 0.105 & 0.104 & 0.101 & 0.106 & 0.102 \\
\hline & $\mathrm{RV}$ & $(101,95,105)$ & 0.102 & 0.099 & 0.101 & 0.100 & 0.102 & 0.101 & 0.100 & 0.102 \\
\hline & $\mathrm{S}$ & $(17,14,20)$ & 0.159 & 0.161 & 0.117 & 0.108 & 0.172 & 0.131 & 0.110 & 0.105 \\
\hline & $\mathrm{S}$ & $(35,28,36)$ & 0.139 & 0.151 & 0.106 & 0.101 & 0.164 & 0.119 & 0.108 & 0.102 \\
\hline & $\mathrm{S}$ & $(50,46,55)$ & 0.127 & 0.142 & 0.103 & 0.101 & 0.161 & 0.107 & 0.106 & 0.102 \\
\hline & $\mathrm{S}$ & $(101,95,105)$ & 0.113 & 0.131 & 0.100 & 0.099 & 0.156 & 0.105 & 0.099 & 0.101 \\
\hline
\end{tabular}


Tabela E.27: Tamanhos empíricos dos testes Wald, razão de verossimilhança e score para o teste $H_{0}: \beta_{13}=\beta_{23}$, com $\mu=5$.

\begin{tabular}{|c|c|c|c|c|c|c|c|c|c|c|c|}
\hline \multirow[b]{2}{*}{ Nível } & \multirow[b]{2}{*}{ Teste } & \multirow[b]{2}{*}{$\left(n_{1}, n_{2}, n_{3}\right)$} & \multirow{2}{*}{$\begin{array}{l}\sigma_{x}^{2} \\
\sigma^{2}\end{array}$} & \multirow{2}{*}{$\begin{array}{l}0.01 \\
0.01\end{array}$} & \multicolumn{3}{|c|}{0.1} & \multicolumn{4}{|c|}{0.5} \\
\hline & & & & & 0.01 & 0.05 & 0.1 & 0.01 & 0.05 & 0.1 & 0.5 \\
\hline \multirow[t]{12}{*}{0.01} & W & $(17,14,20)$ & & 0.017 & 0.016 & 0.018 & 0.018 & 0.018 & 0.018 & 0.019 & 0.018 \\
\hline & W & $(35,28,36)$ & & 0.014 & 0.014 & 0.015 & 0.015 & 0.014 & 0.014 & 0.013 & 0.015 \\
\hline & W & $(50,46,55)$ & & 0.012 & 0.010 & 0.013 & 0.014 & 0.012 & 0.012 & 0.011 & 0.013 \\
\hline & W & $(101,95,105)$ & & 0.011 & 0.010 & 0.013 & 0.009 & 0.011 & 0.009 & 0.011 & 0.013 \\
\hline & RV & $(17,14,20)$ & & 0.013 & 0.013 & 0.013 & 0.013 & 0.012 & 0.013 & 0.013 & 0.012 \\
\hline & RV & $(35,28,36)$ & & 0.012 & 0.012 & 0.012 & 0.013 & 0.012 & 0.011 & 0.010 & 0.011 \\
\hline & RV & $(50,46,55)$ & & 0.010 & 0.009 & 0.011 & 0.013 & 0.011 & 0.011 & 0.010 & 0.010 \\
\hline & RV & $(101,95,105)$ & & 0.010 & 0.009 & 0.011 & 0.008 & 0.010 & 0.009 & 0.010 & 0.010 \\
\hline & $\mathrm{S}$ & $(17,14,20)$ & & 0.015 & 0.016 & 0.010 & 0.009 & 0.019 & 0.011 & 0.009 & 0.007 \\
\hline & $\mathrm{S}$ & $(35,28,36)$ & & 0.012 & 0.014 & 0.010 & 0.010 & 0.019 & 0.010 & 0.009 & 0.009 \\
\hline & $\mathrm{S}$ & $(50,46,55)$ & & 0.011 & 0.013 & 0.010 & 0.010 & 0.017 & 0.010 & 0.009 & 0.010 \\
\hline & $\mathrm{S}$ & $(101,95,105)$ & & 0.011 & 0.013 & 0.010 & 0.010 & 0.016 & 0.010 & 0.009 & 0.010 \\
\hline \multirow[t]{12}{*}{0.05} & W & $(17,14,20)$ & & 0.067 & 0.063 & 0.067 & 0.064 & 0.069 & 0.066 & 0.066 & 0.064 \\
\hline & W & $(35,28,36)$ & & 0.057 & 0.060 & 0.059 & 0.061 & 0.062 & 0.060 & 0.057 & 0.059 \\
\hline & W & $(50,46,55)$ & & 0.055 & 0.054 & 0.052 & 0.057 & 0.053 & 0.056 & 0.056 & 0.053 \\
\hline & $\mathrm{W}$ & $(101,95,105)$ & & 0.052 & 0.050 & 0.051 & 0.051 & 0.052 & 0.051 & 0.056 & 0.053 \\
\hline & $\mathrm{RV}$ & $(17,14,20)$ & & 0.060 & 0.055 & 0.061 & 0.058 & 0.062 & 0.059 & 0.059 & 0.055 \\
\hline & RV & $(35,28,36)$ & & 0.055 & 0.054 & 0.056 & 0.057 & 0.057 & 0.054 & 0.052 & 0.054 \\
\hline & RV & $(50,46,55)$ & & 0.054 & 0.052 & 0.051 & 0.055 & 0.051 & 0.053 & 0.052 & 0.049 \\
\hline & RV & $(101,95,105)$ & & 0.051 & 0.049 & 0.049 & 0.049 & 0.050 & 0.050 & 0.054 & 0.051 \\
\hline & $\mathrm{S}$ & $(17,14,20)$ & & 0.082 & 0.080 & 0.059 & 0.052 & 0.092 & 0.068 & 0.054 & 0.046 \\
\hline & $\mathrm{S}$ & $(35,28,36)$ & & 0.068 & 0.078 & 0.053 & 0.052 & 0.091 & 0.059 & 0.049 & 0.049 \\
\hline & $\mathrm{S}$ & $(50,46,55)$ & & 0.063 & 0.075 & 0.048 & 0.052 & 0.084 & 0.055 & 0.051 & 0.049 \\
\hline & $\mathrm{S}$ & $(101,95,105)$ & & 0.055 & 0.069 & 0.049 & 0.048 & 0.081 & 0.051 & 0.051 & 0.050 \\
\hline \multirow[t]{12}{*}{0.1} & $\mathrm{~W}$ & $(17,14,20)$ & & 0.123 & 0.116 & 0.123 & 0.120 & 0.124 & 0.124 & 0.121 & 0.116 \\
\hline & W & $(35,28,36)$ & & 0.105 & 0.109 & 0.113 & 0.111 & 0.117 & 0.111 & 0.109 & 0.110 \\
\hline & W & $(50,46,55)$ & & 0.104 & 0.104 & 0.103 & 0.108 & 0.106 & 0.104 & 0.109 & 0.105 \\
\hline & $\mathrm{W}$ & $(101,95,105)$ & & 0.104 & 0.100 & 0.100 & 0.097 & 0.106 & 0.103 & 0.105 & 0.101 \\
\hline & RV & $(17,14,20)$ & & 0.117 & 0.110 & 0.117 & 0.113 & 0.115 & 0.115 & 0.113 & 0.107 \\
\hline & RV & $(35,28,36)$ & & 0.108 & 0.105 & 0.109 & 0.107 & 0.113 & 0.106 & 0.103 & 0.105 \\
\hline & RV & $(50,46,55)$ & & 0.106 & 0.101 & 0.101 & 0.106 & 0.102 & 0.105 & 0.103 & 0.102 \\
\hline & RV & $(101,95,105)$ & & 0.103 & 0.099 & 0.099 & 0.095 & 0.102 & 0.105 & 0.103 & 0.099 \\
\hline & $\mathrm{S}$ & $(17,14,20)$ & & 0.166 & 0.159 & 0.120 & 0.109 & 0.177 & 0.136 & 0.114 & 0.098 \\
\hline & $\mathrm{S}$ & $(35,28,36)$ & & 0.135 & 0.148 & 0.108 & 0.104 & 0.168 & 0.115 & 0.105 & 0.101 \\
\hline & $\mathrm{S}$ & $(50,46,55)$ & & 0.126 & 0.144 & 0.099 & 0.104 & 0.158 & 0.108 & 0.103 & 0.099 \\
\hline & $\mathrm{S}$ & $(101,95,105)$ & & 0.113 & 0.133 & 0.099 & 0.097 & 0.155 & 0.106 & 0.103 & 0.099 \\
\hline
\end{tabular}




\section{Apêndice F}

\section{Resultados das simulações do poder do teste}

Apresentamos aqui os resultados das simulações do poder dos testes assintóticos para as hipóteses $H_{0}: \beta_{11}=\beta_{12}$ e $H_{0}: \beta_{12}=\beta_{13}$, que comparam respectivamente o desempenho do líquido controle com o líquido experimental A e o desempenho dos líquidos experimentais A e B após três meses de uso, $H_{0}: \beta_{21}=\beta_{22}$ e $H_{0}: \beta_{22}=\beta_{23}$, que comparam respectivamente o desempenho do líquido controle com o líquido A e o desempenho dos líquidos experimentais A e B após seis meses de uso, $H_{0}: \beta_{11}=\beta_{21} \mathrm{e}$ $H_{0}: \beta_{12}=\beta_{22}$, que comparam respectivamente o desempenho do líquido controle após três e seis meses de uso e o desempenho do líquido A após três e seis meses de uso. Os resultados estão apresentados da seguinte maneira: em cada tabela foi considerado um teste de hipóteses do tipo $H_{0}: \beta_{i j}=\beta_{k l}$ contra $\beta_{i j} \neq \beta_{k l}$, com $i, k=1,2$ e $j, l=1,2,3$ de acordo com as hipóteses de interesse discutidas no Capítulo 5, para um dado valor de $\mu$ e para as combinações de valores considerados entre $\sigma_{x}^{2}$ e $\sigma^{2}$, descritos na Seção 6.1. $N_{i}$ representa o tamanho $i$ de amostras consideradas para os três grupos, ou seja, $N_{1}$ representa os tamanhos $\left(n_{1}, n_{2}, n_{3}\right)=(17,14,20), N_{2}$ representa $\left(n_{1}, n_{2}, n_{3}\right)=(35,28,36), N_{3}$ representa $\left(n_{1}, n_{2}, n_{3}\right)=(50,46,55), N_{4}$ representa $\left(n_{1}, n_{2}, n_{3}\right)=(101,95,105)$, onde $n_{i}$ é o tamanho da amostra do $i$-ésimo grupo. Verticalmente dentro de cada subtabela para o tamanho de amostra $N_{i}$ apresenta-se as porcentagens de rejeição conforme distancia-se da hipótese $H_{0}$. A coluna correspondente a $\mathrm{W}$ representa as porcentagens obtidas pela estatística Wald, RV representa as porcentagens obtidas pela estatística da razão de verossimilhanças e $\mathrm{S}$ representa as porcentagens obtidas pela estatística score. Selecionamos os valores $\beta_{11}=0.7, \beta_{12}=0.53, \beta_{13}=0.51, \beta_{21}=0.69, \beta_{22}=0.5, \beta_{23}=0.414$, $\mu=2.5$ e variamos os valores de $\sigma^{2}$ e $\sigma_{x}^{2}$. Apresentaremos aqui, nas Tabelas F.4 a F.27, alguns dos resultados dos estudos de simulação relativos ao poder dos testes, relativos às hipóteses $H_{0}: \beta_{11}=\beta_{13}$, $H_{0}: \beta_{21}=\beta_{23}$ e $H_{0}: \beta_{13}=\beta_{23}$, como foi apresentado para as simulações do tamanho dos testes. Os resultados são apresentados nas Tabelas a F.1 a F.24. Para tanto, foi considerado o procedimento descrito na Seção 6.2, e as conclusões foram análogas às apresentadas na mesma seção. 
Tabela F.1: Poder simulado dos testes para a hipótese $H_{0}: \beta_{11}=\beta_{12}$, com $\mu=1$.

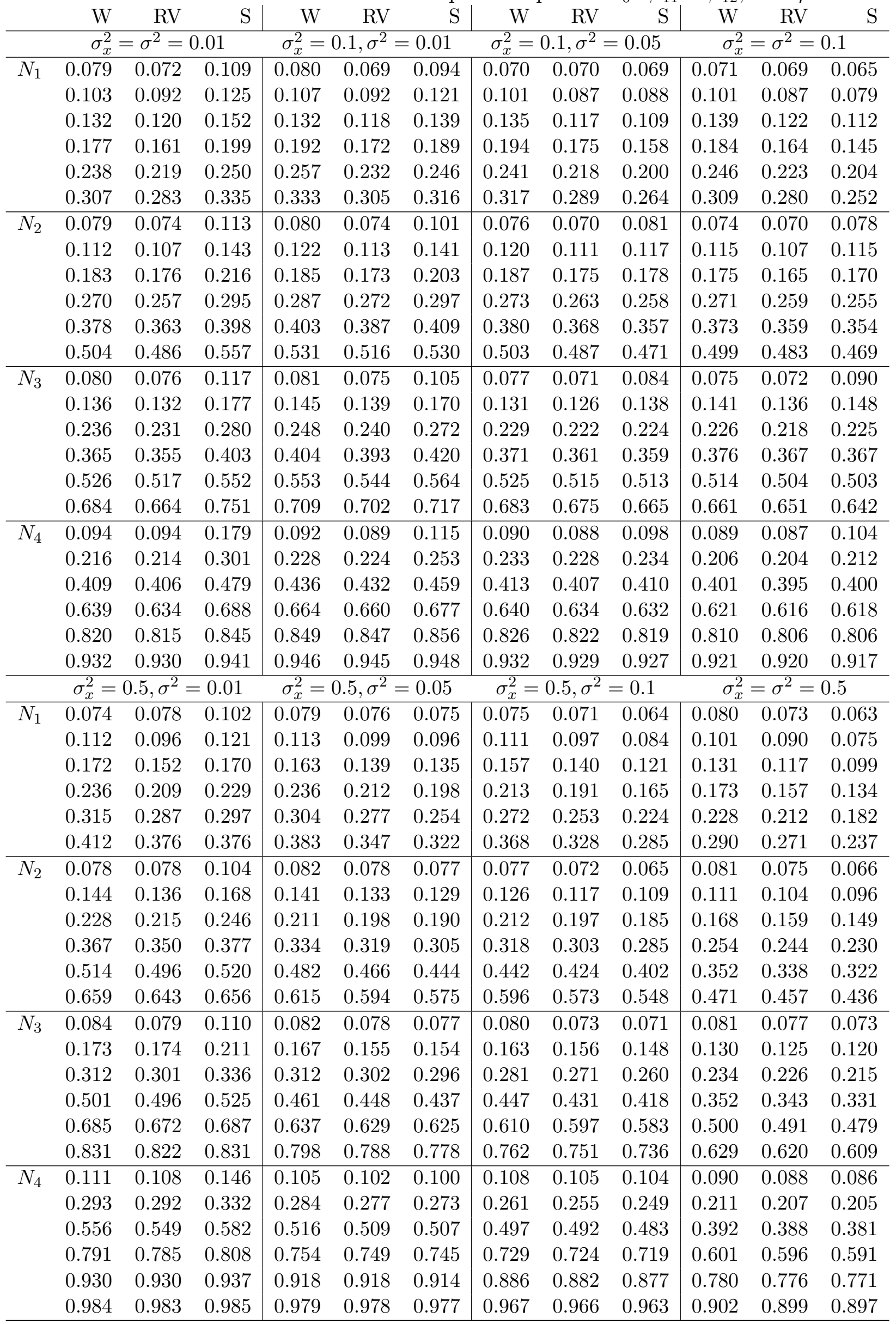


Tabela F.2: Poder simulado dos testes para a hipótese $H_{0}: \beta_{11}=\beta_{12}$, com $\mu=2.5$.

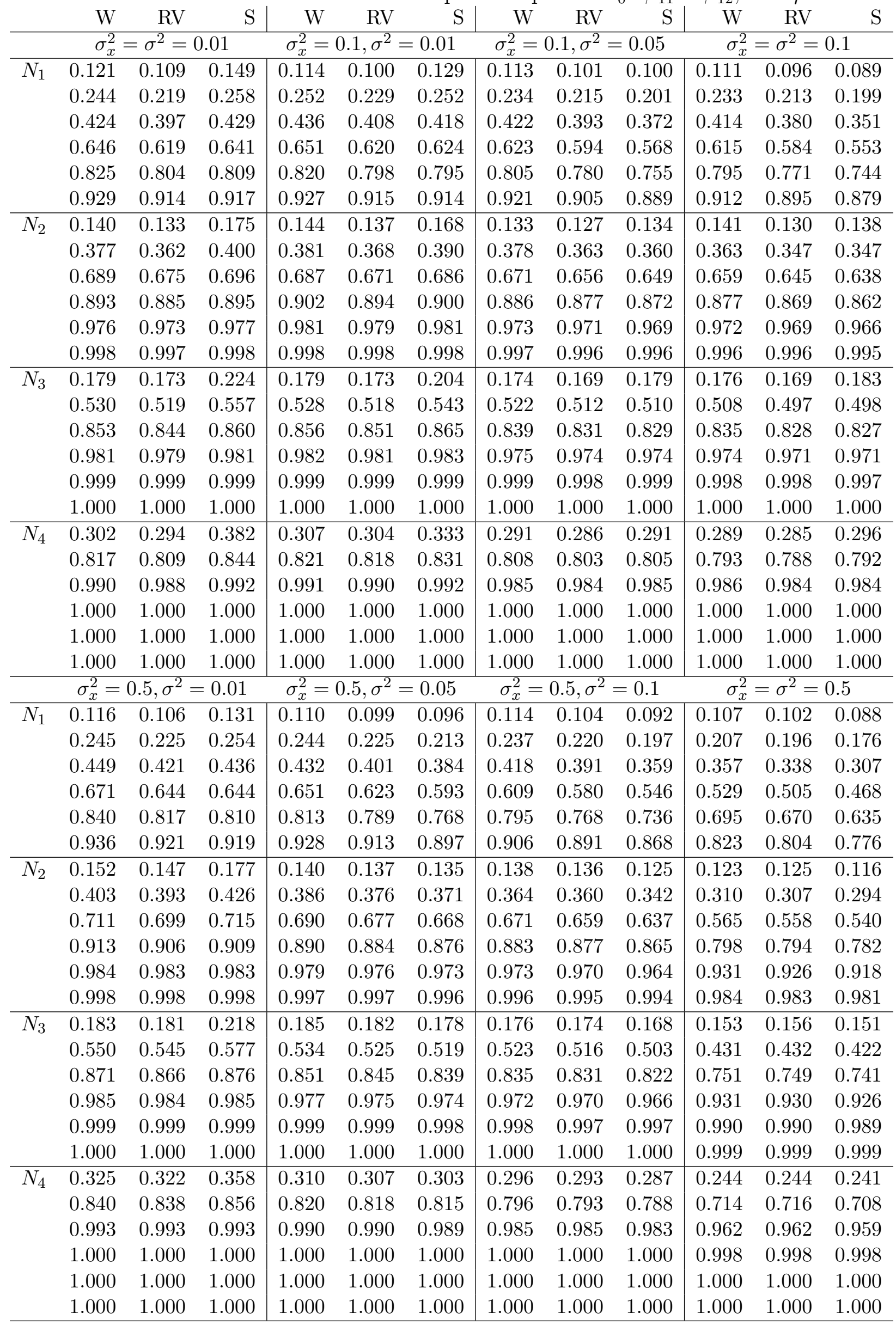


Tabela F.3: Poder simulado dos testes para a hipótese $H_{0}: \beta_{11}=\beta_{12}$, com $\mu=5$.

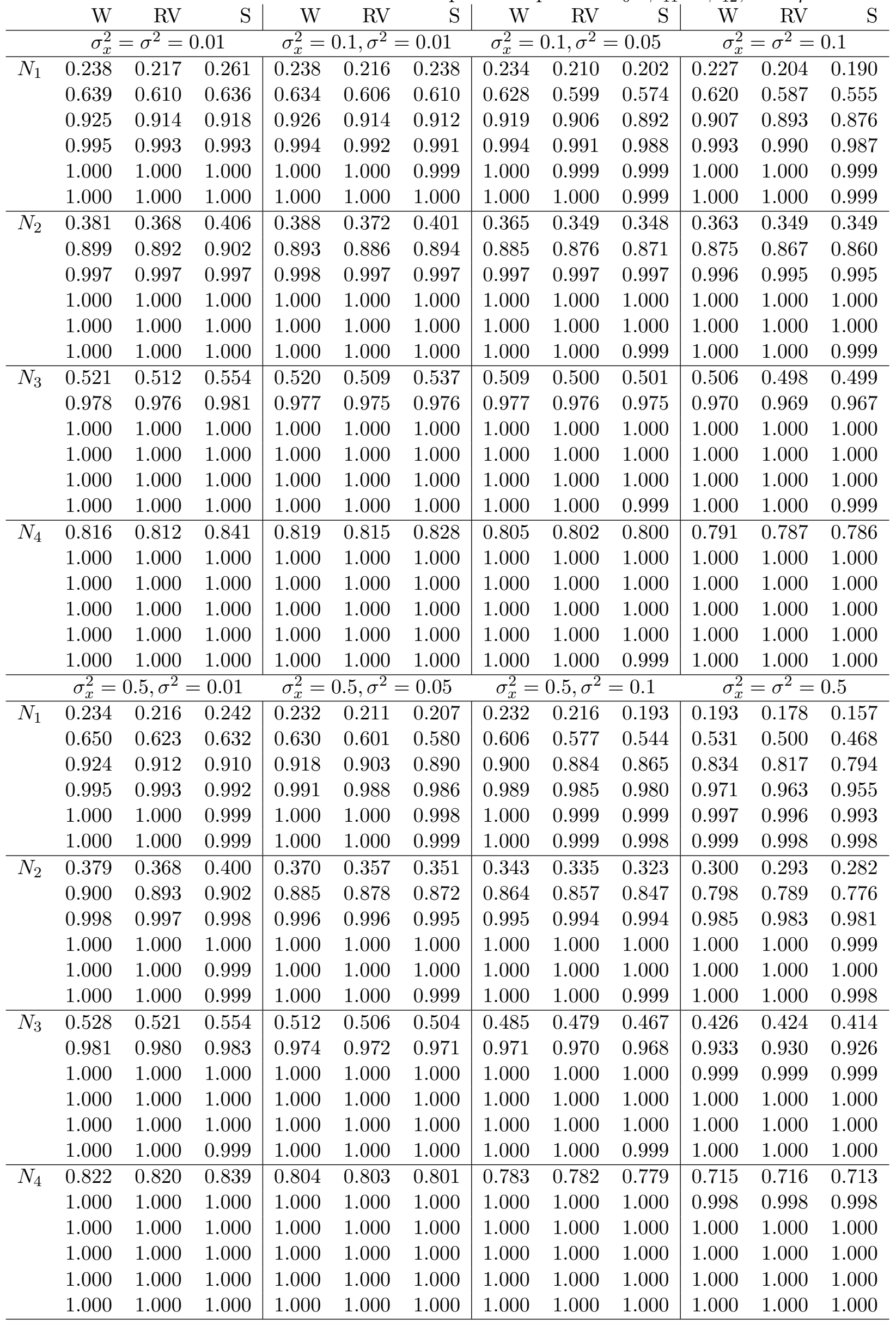


Tabela F.4: Poder simulado dos testes para a hipótese $H_{0}: \beta_{11}=\beta_{13}$, com $\mu=1$.

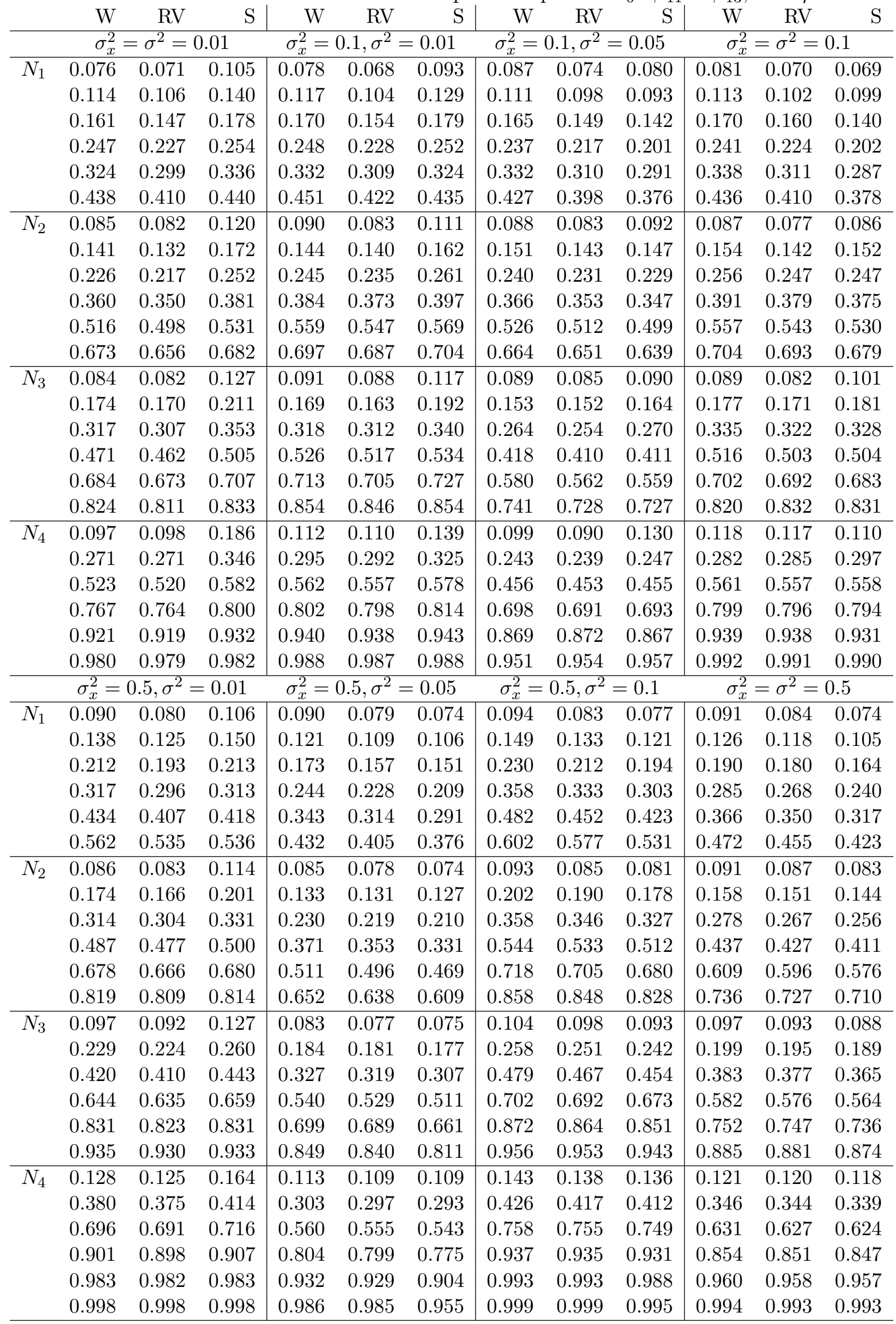


Tabela F.5: Poder simulado dos testes para a hipótese $H_{0}: \beta_{11}=\beta_{13}$, com $\mu=2.5$.

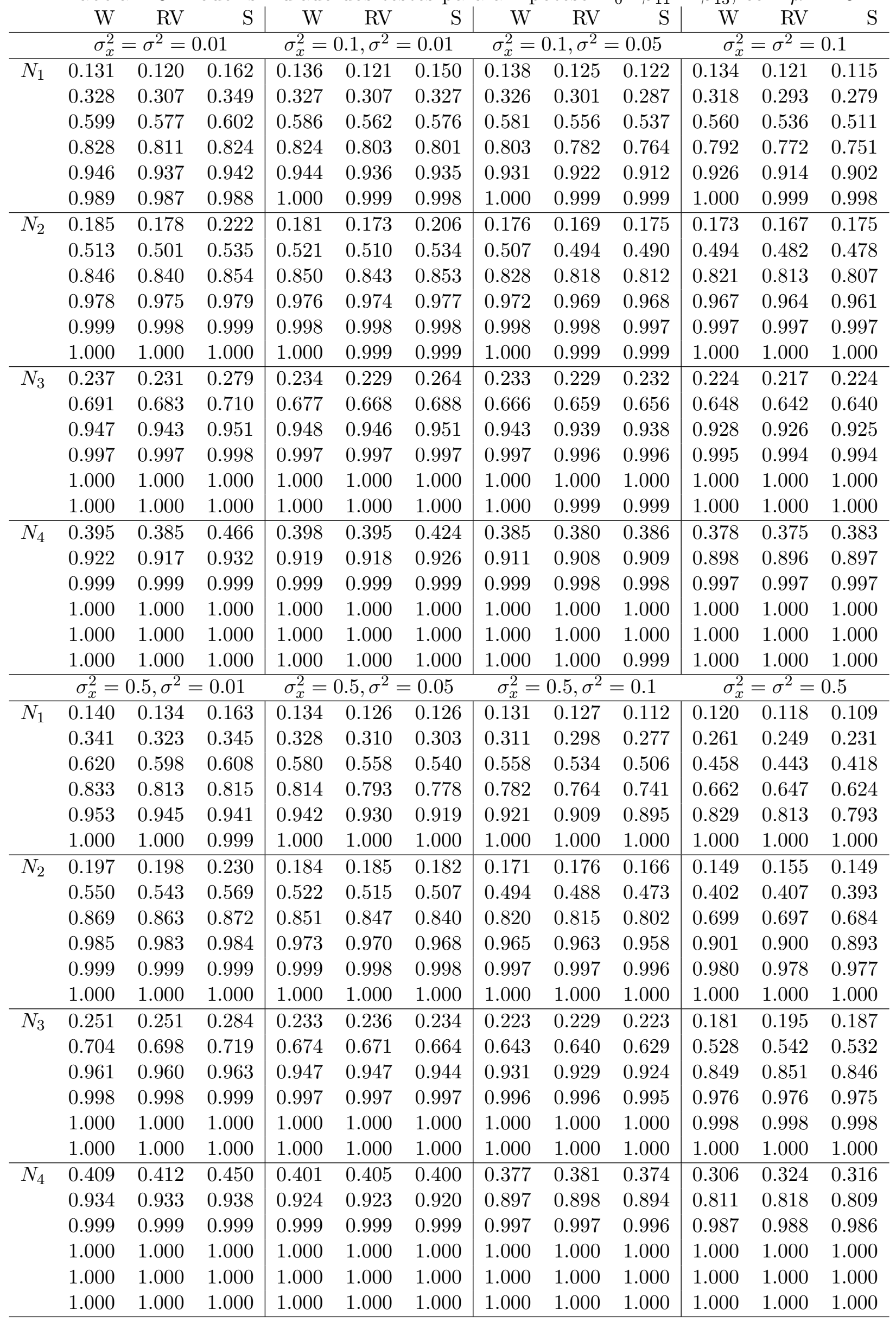


Tabela F.6: Poder simulado dos testes para a hipótese $H_{0}: \beta_{11}=\beta_{13}$, com $\mu=5$.

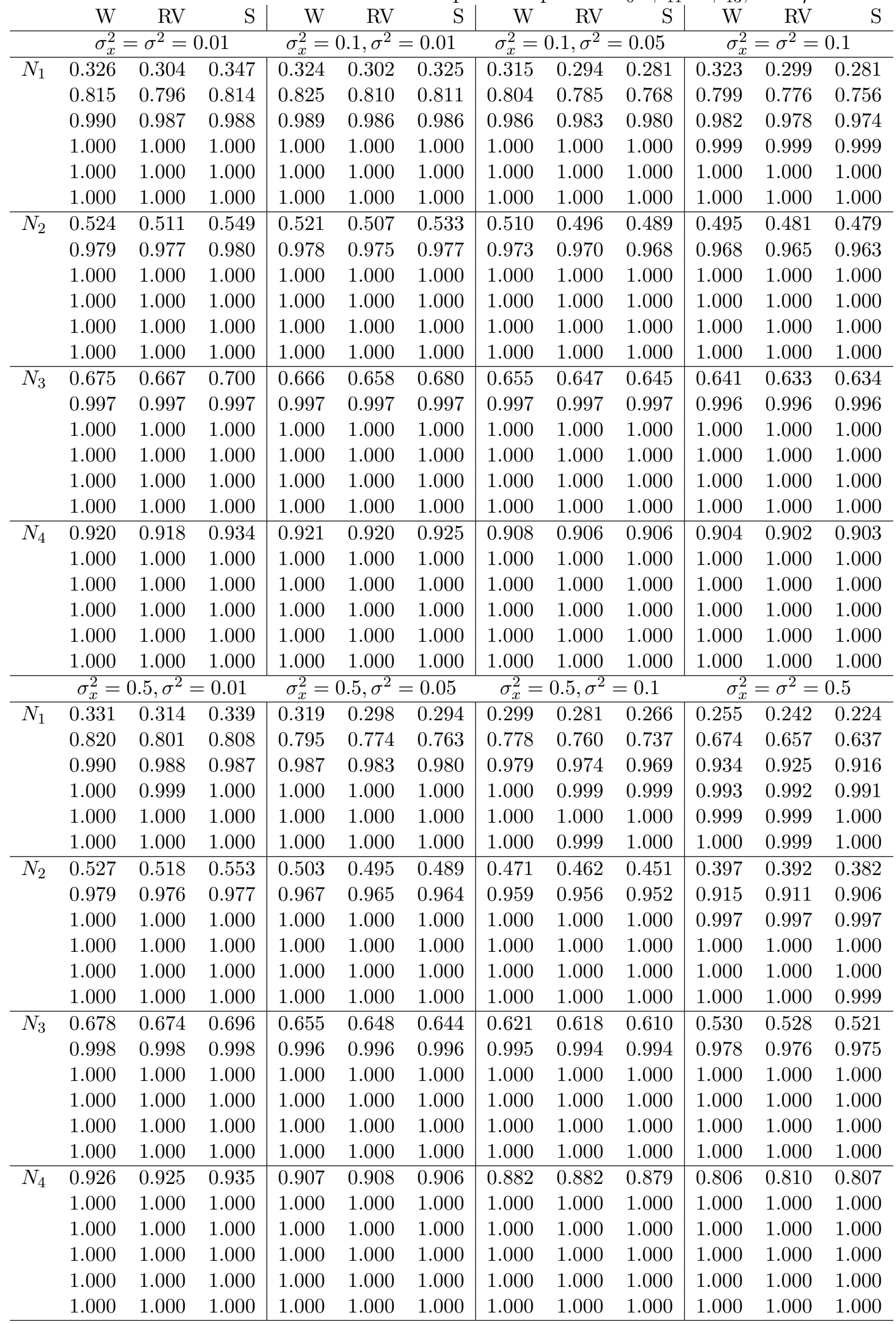


180 Análise de um modelo de regressão com erros nas variáveis multivariado com intercepto nulo

Tabela F.7: Poder simulado dos testes para a hipótese $H_{0}: \beta_{12}=\beta_{13}$, com $\mu=1$.

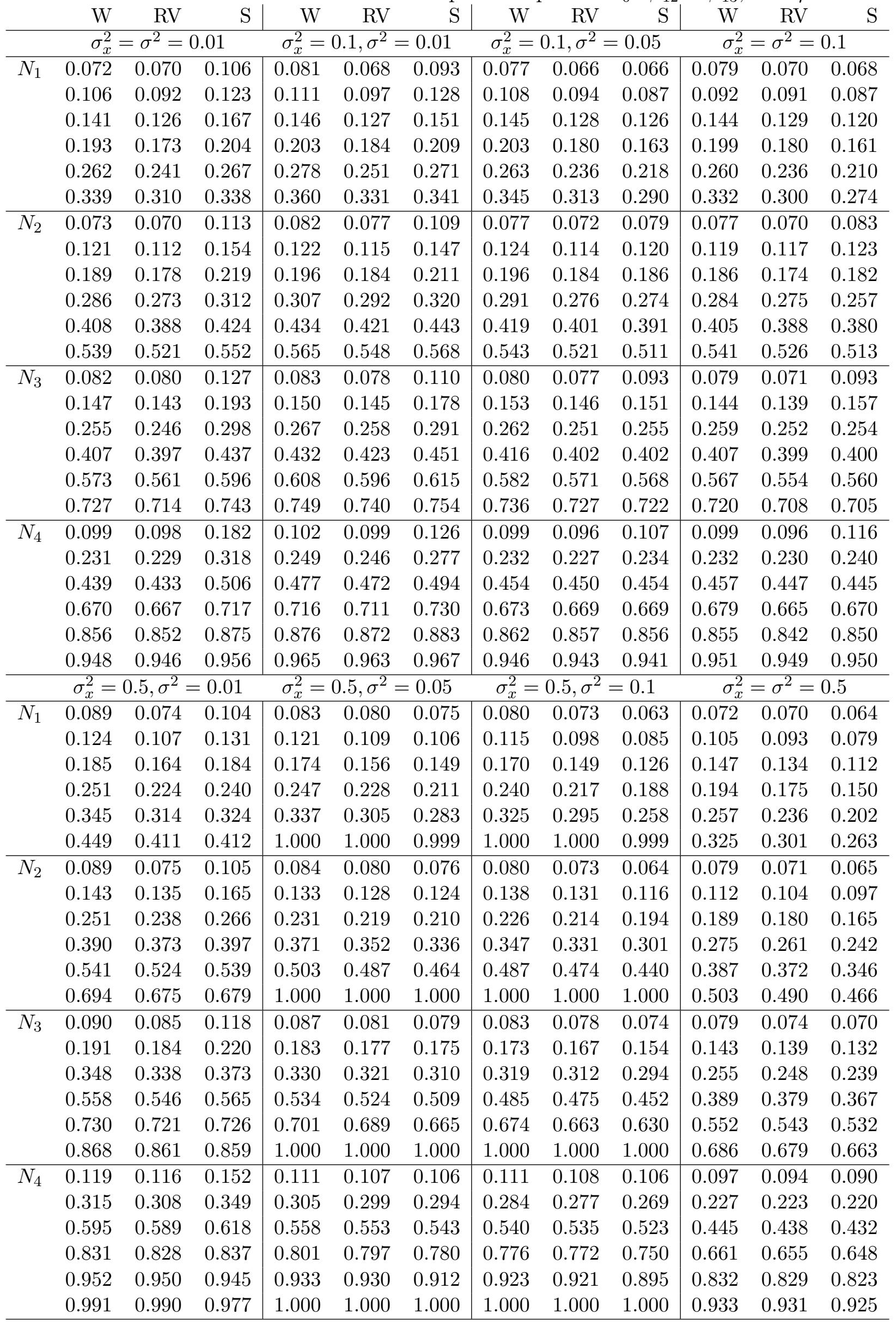


Tabela F.8: Poder simulado dos testes para a hipótese $H_{0}: \beta_{12}=\beta_{13}$, com $\mu=2.5$.

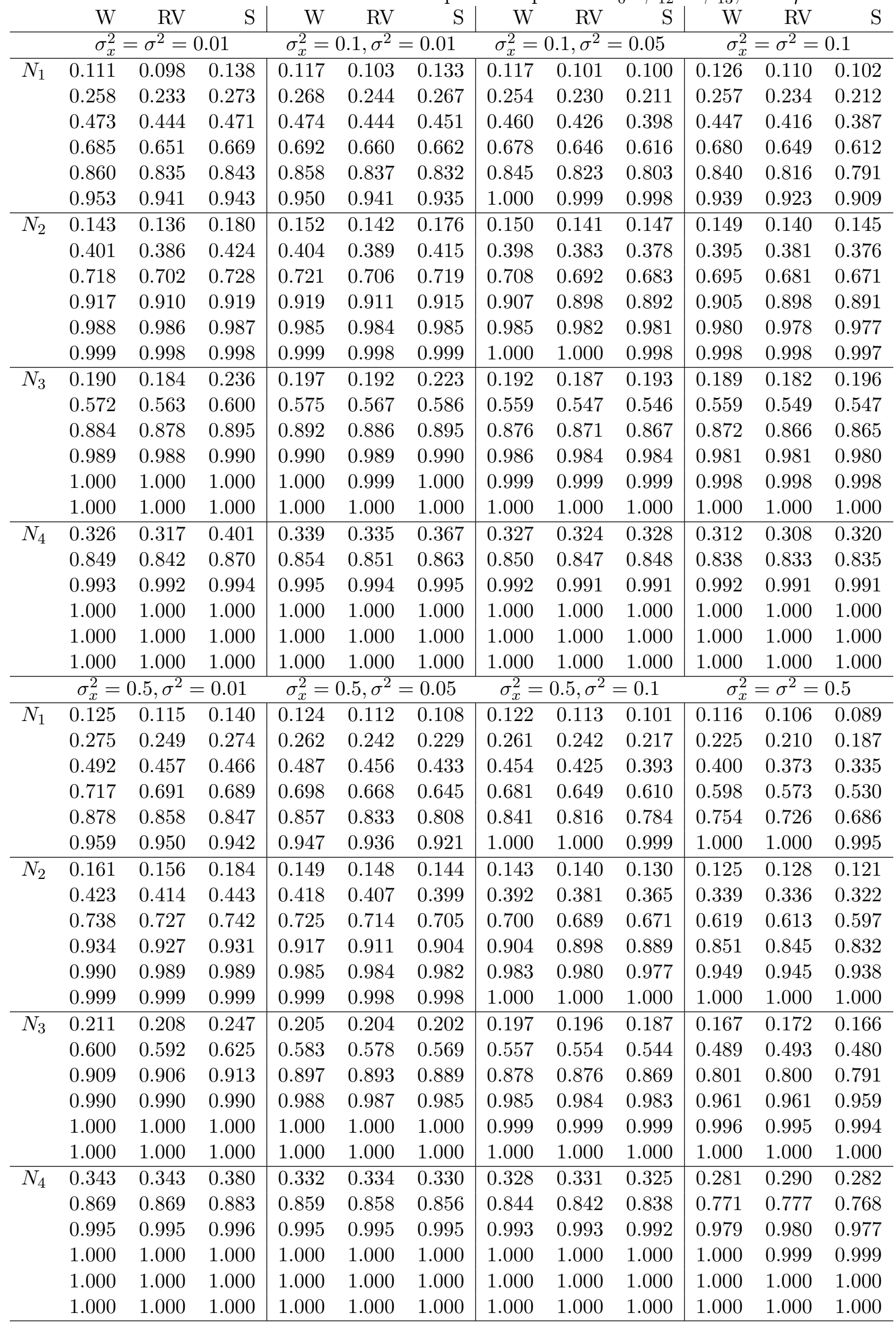


Tabela F.9: Poder simulado dos testes para a hipótese $H_{0}: \beta_{12}=\beta_{13}$, com $\mu=5$.

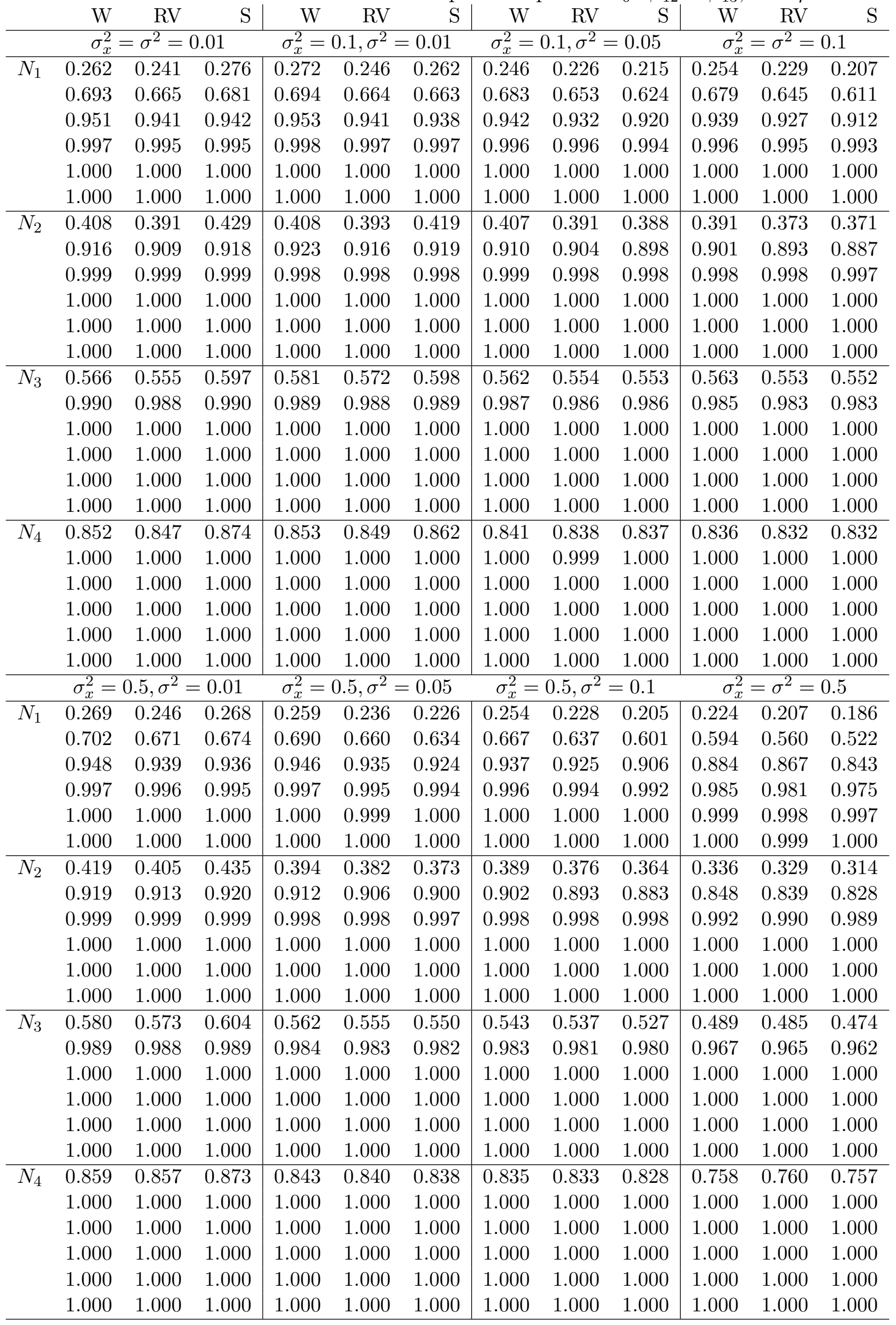


Tabela F.10: Poder simulado dos testes para a hipótese $H_{0}: \beta_{21}=\beta_{22}$, com $\mu=1$.

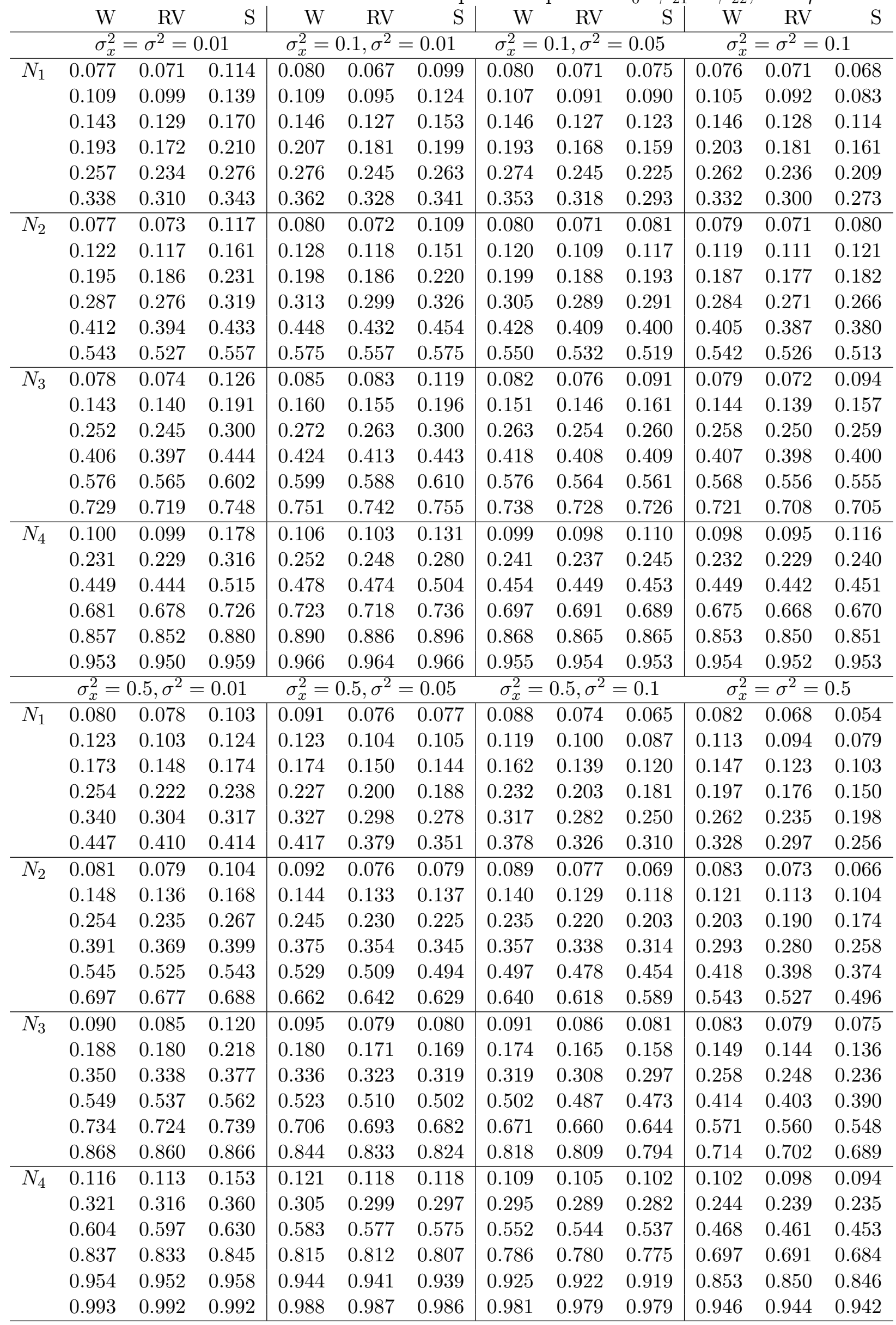


Tabela F.11: Poder simulado dos testes para a hipótese $H_{0}: \beta_{21}=\beta_{22}$, com $\mu=2.5$.

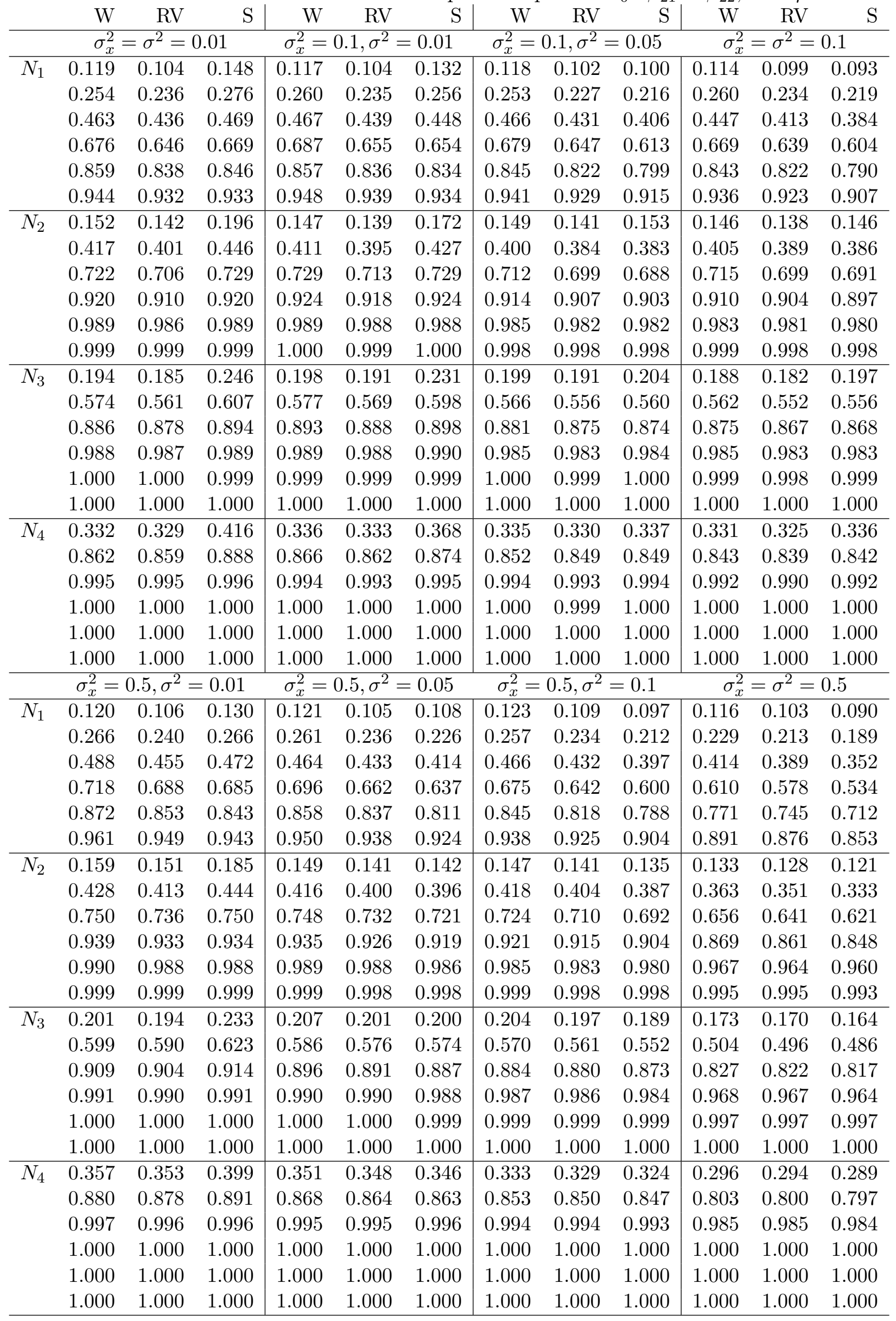


Tabela F.12: Poder simulado dos testes para a hipótese $H_{0}: \beta_{21}=\beta_{22}$, com $\mu=5$.

\begin{tabular}{|c|c|c|c|c|c|c|c|c|c|c|c|c|}
\hline & W & RV & $\mathrm{S}$ & W & RV & S & W & RV & S & W & RV & $\mathrm{S}$ \\
\hline & \multicolumn{3}{|c|}{$\sigma_{x}^{2}=\sigma^{2}=0.01$} & \multicolumn{3}{|c|}{$\sigma_{x}^{2}=0.1, \sigma^{2}=0.01$} & \multicolumn{3}{|c|}{$\sigma_{x}^{2}=0.1, \sigma^{2}=0.05$} & \multicolumn{3}{|c|}{$\sigma_{x}^{2}=\sigma^{2}=0.1$} \\
\hline \multirow[t]{6}{*}{$N_{1}$} & 0.255 & 0.231 & 0.278 & 0.259 & 0.234 & 0.256 & 0.257 & 0.233 & 0.221 & 0.260 & 0.235 & 0.216 \\
\hline & 0.689 & 0.658 & 0.681 & 0.677 & 0.650 & 0.656 & 0.676 & 0.646 & 0.619 & 0.673 & 0.637 & 0.606 \\
\hline & 0.945 & 0.934 & 0.937 & 0.948 & 0.937 & 0.935 & 0.941 & 0.930 & 0.919 & 0.944 & 0.931 & 0.918 \\
\hline & 0.996 & 0.995 & 0.994 & 0.996 & 0.996 & 0.995 & 0.996 & 0.995 & 0.994 & 0.996 & 0.995 & 0.992 \\
\hline & 1.000 & 1.000 & 1.000 & 1.000 & 1.000 & 1.000 & 1.000 & 1.000 & 0.999 & 1.000 & 1.000 & 0.999 \\
\hline & 1.000 & 1.000 & 1.000 & 1.000 & 1.000 & 0.998 & 1.000 & 1.000 & 0.999 & 1.000 & 1.000 & 0.998 \\
\hline \multirow[t]{6}{*}{$N_{2}$} & 0.401 & 0.386 & 0.433 & 0.418 & 0.402 & 0.437 & 0.402 & 0.385 & 0.384 & 0.400 & 0.387 & 0.383 \\
\hline & 0.925 & 0.918 & 0.927 & 0.920 & 0.914 & 0.919 & 0.913 & 0.907 & 0.902 & 0.909 & 0.902 & 0.897 \\
\hline & 0.999 & 0.999 & 0.999 & 0.998 & 0.998 & 0.998 & 0.999 & 0.999 & 0.998 & 0.999 & 0.999 & 0.998 \\
\hline & 1.000 & 1.000 & 1.000 & 1.000 & 1.000 & 1.000 & 1.000 & 1.000 & 1.000 & 1.000 & 1.000 & 1.000 \\
\hline & 1.000 & 1.000 & 1.000 & 1.000 & 1.000 & 1.000 & 1.000 & 1.000 & 1.000 & 1.000 & 1.000 & 1.000 \\
\hline & 1.000 & 1.000 & 1.000 & 1.000 & 1.000 & 1.000 & 1.000 & 1.000 & 0.999 & 1.000 & 1.000 & 0.998 \\
\hline \multirow[t]{6}{*}{$N_{3}$} & 0.562 & 0.553 & 0.601 & 0.573 & 0.564 & 0.590 & 0.558 & 0.545 & 0.548 & 0.557 & 0.547 & 0.551 \\
\hline & 0.989 & 0.988 & 0.990 & 0.988 & 0.987 & 0.988 & 0.987 & 0.986 & 0.986 & 0.985 & 0.984 & 0.984 \\
\hline & 1.000 & 1.000 & 1.000 & 1.000 & 1.000 & 1.000 & 1.000 & 1.000 & 1.000 & 1.000 & 1.000 & 1.000 \\
\hline & 1.000 & 1.000 & 1.000 & 1.000 & 1.000 & 1.000 & 1.000 & 1.000 & 1.000 & 1.000 & 1.000 & 1.000 \\
\hline & 1.000 & 1.000 & 1.000 & 1.000 & 1.000 & 1.000 & 1.000 & 1.000 & 1.000 & 1.000 & 1.000 & 1.000 \\
\hline & 1.000 & 1.000 & 1.000 & 1.000 & 1.000 & 1.000 & 1.000 & 1.000 & 1.000 & 1.000 & 1.000 & 0.998 \\
\hline \multirow[t]{7}{*}{$N_{4}$} & 0.860 & 0.855 & 0.879 & 0.863 & 0.860 & 0.871 & 0.850 & 0.847 & 0.847 & 0.846 & 0.842 & 0.842 \\
\hline & 1.000 & 1.000 & 1.000 & 1.000 & 1.000 & 1.000 & 1.000 & 1.000 & 1.000 & 1.000 & 1.000 & 1.000 \\
\hline & 1.000 & 1.000 & 1.000 & 1.000 & 1.000 & 1.000 & 1.000 & 1.000 & 1.000 & 1.000 & 1.000 & 1.000 \\
\hline & 1.000 & 1.000 & 1.000 & 1.000 & 1.000 & 1.000 & 1.000 & 1.000 & 1.000 & 1.000 & 1.000 & 1.000 \\
\hline & 1.000 & 1.000 & 1.000 & 1.000 & 1.000 & 1.000 & 1.000 & 1.000 & 1.000 & 1.000 & 1.000 & 1.000 \\
\hline & 1.000 & 1.000 & 1.000 & 1.000 & 1.000 & 1.000 & 1.000 & 1.000 & 1.000 & 1.000 & 1.000 & 0.998 \\
\hline & \multicolumn{3}{|c|}{$\sigma_{x}^{2}=0.5, \sigma^{2}=0.01$} & \multicolumn{3}{|c|}{$\sigma_{x}^{2}=0.5, \sigma^{2}=0.05$} & \multicolumn{3}{|c|}{$\sigma_{x}^{2}=0.5, \sigma^{2}=0.1$} & \multicolumn{3}{|c|}{$\sigma_{x}^{2}=\sigma^{2}=0.5$} \\
\hline \multirow[t]{6}{*}{$N_{1}$} & 0.269 & 0.246 & 0.271 & 0.255 & 0.232 & 0.225 & 0.249 & 0.224 & 0.206 & 0.230 & 0.209 & 0.185 \\
\hline & 0.696 & 0.664 & 0.669 & 0.684 & 0.655 & 0.631 & 0.668 & 0.633 & 0.600 & 0.614 & 0.581 & 0.543 \\
\hline & 0.948 & 0.939 & 0.935 & 0.943 & 0.931 & 0.921 & 0.937 & 0.924 & 0.907 & 0.904 & 0.886 & 0.865 \\
\hline & 0.997 & 0.997 & 0.996 & 0.997 & 0.995 & 0.993 & 0.995 & 0.994 & 0.991 & 0.985 & 0.981 & 0.977 \\
\hline & 1.000 & 1.000 & 0.999 & 1.000 & 0.999 & 0.998 & 1.000 & 1.000 & 0.998 & 0.999 & 0.998 & 0.997 \\
\hline & 1.000 & 0.999 & 0.996 & 1.000 & 1.000 & 0.997 & 1.000 & 0.999 & 0.998 & 1.000 & 0.999 & 0.997 \\
\hline \multirow[t]{6}{*}{$N_{2}$} & 0.413 & 0.400 & 0.434 & 0.400 & 0.387 & 0.384 & 0.384 & 0.371 & 0.357 & 0.355 & 0.342 & 0.326 \\
\hline & 0.927 & 0.922 & 0.927 & 0.917 & 0.910 & 0.906 & 0.908 & 0.900 & 0.892 & 0.870 & 0.860 & 0.849 \\
\hline & 0.999 & 0.999 & 0.999 & 0.999 & 0.999 & 0.999 & 0.998 & 0.998 & 0.997 & 0.995 & 0.995 & 0.994 \\
\hline & 1.000 & 1.000 & 1.000 & 1.000 & 1.000 & 1.000 & 1.000 & 1.000 & 1.000 & 1.000 & 1.000 & 1.000 \\
\hline & 1.000 & 1.000 & 0.999 & 1.000 & 1.000 & 1.000 & 1.000 & 1.000 & 0.998 & 1.000 & 1.000 & 1.000 \\
\hline & 1.000 & 1.000 & 0.999 & 1.000 & 1.000 & 0.997 & 1.000 & 1.000 & 0.998 & 1.000 & 1.000 & 0.997 \\
\hline \multirow[t]{6}{*}{$N_{3}$} & 0.577 & 0.569 & 0.602 & 0.565 & 0.559 & 0.559 & 0.551 & 0.544 & 0.535 & 0.502 & 0.497 & 0.488 \\
\hline & 0.989 & 0.989 & 0.989 & 0.987 & 0.986 & 0.986 & 0.985 & 0.984 & 0.982 & 0.972 & 0.970 & 0.969 \\
\hline & 1.000 & 1.000 & 1.000 & 1.000 & 1.000 & 1.000 & 1.000 & 1.000 & 1.000 & 1.000 & 1.000 & 1.000 \\
\hline & 1.000 & 1.000 & 1.000 & 1.000 & 1.000 & 1.000 & 1.000 & 1.000 & 1.000 & 1.000 & 1.000 & 1.000 \\
\hline & 1.000 & 1.000 & 0.999 & 1.000 & 1.000 & 1.000 & 1.000 & 1.000 & 0.999 & 1.000 & 1.000 & 1.000 \\
\hline & 1.000 & 1.000 & 0.999 & 1.000 & 1.000 & 1.000 & 1.000 & 1.000 & 0.999 & 1.000 & 1.000 & 0.997 \\
\hline \multirow[t]{6}{*}{$\overline{N_{4}}$} & 0.864 & 0.862 & 0.876 & 0.862 & 0.860 & 0.858 & 0.844 & 0.841 & 0.838 & 0.788 & 0.787 & 0.784 \\
\hline & 1.000 & 1.000 & 1.000 & 1.000 & 1.000 & 1.000 & 1.000 & 1.000 & 1.000 & 1.000 & 1.000 & 1.000 \\
\hline & 1.000 & 1.000 & 1.000 & 1.000 & 1.000 & 1.000 & 1.000 & 1.000 & 1.000 & 1.000 & 1.000 & 1.000 \\
\hline & 1.000 & 1.000 & 1.000 & 1.000 & 1.000 & 1.000 & 1.000 & 1.000 & 1.000 & 1.000 & 1.000 & 1.000 \\
\hline & 1.000 & 1.000 & 1.000 & 1.000 & 1.000 & 1.000 & 1.000 & 1.000 & 1.000 & 1.000 & 1.000 & 1.000 \\
\hline & 1.000 & 1.000 & 1.000 & 1.000 & 1.000 & 1.000 & 1.000 & 1.000 & 1.000 & 1.000 & 1.000 & 1.000 \\
\hline
\end{tabular}


186 Análise de um modelo de regressão com erros nas variáveis multivariado com intercepto nulo

Tabela F.13: Poder simulado dos testes para a hipótese $H_{0}: \beta_{21}=\beta_{23}$, com $\mu=1$.

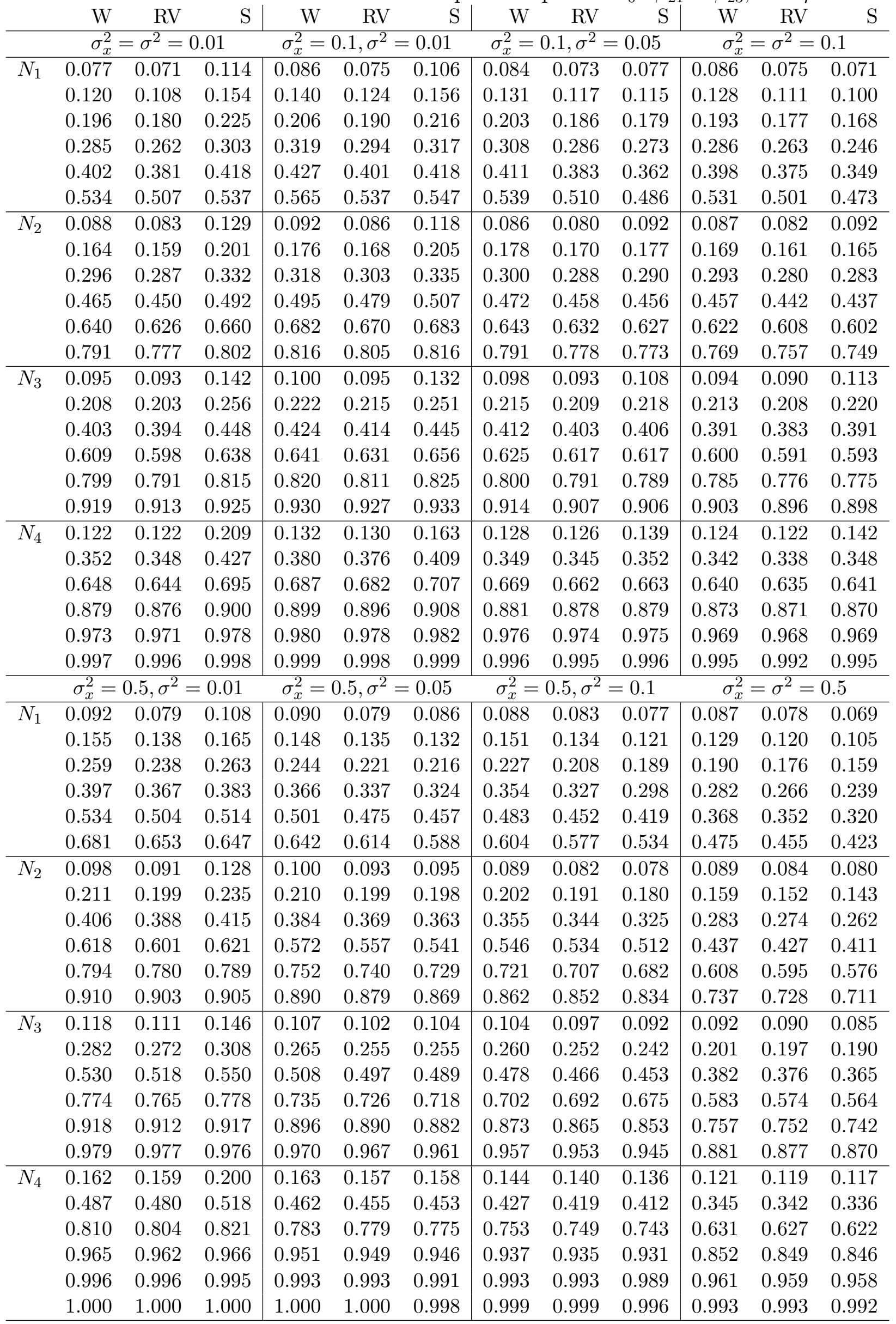


Tabela F.14: Poder simulado dos testes para a hipótese $H_{0}: \beta_{21}=\beta_{23}$, com $\mu=2.5$.

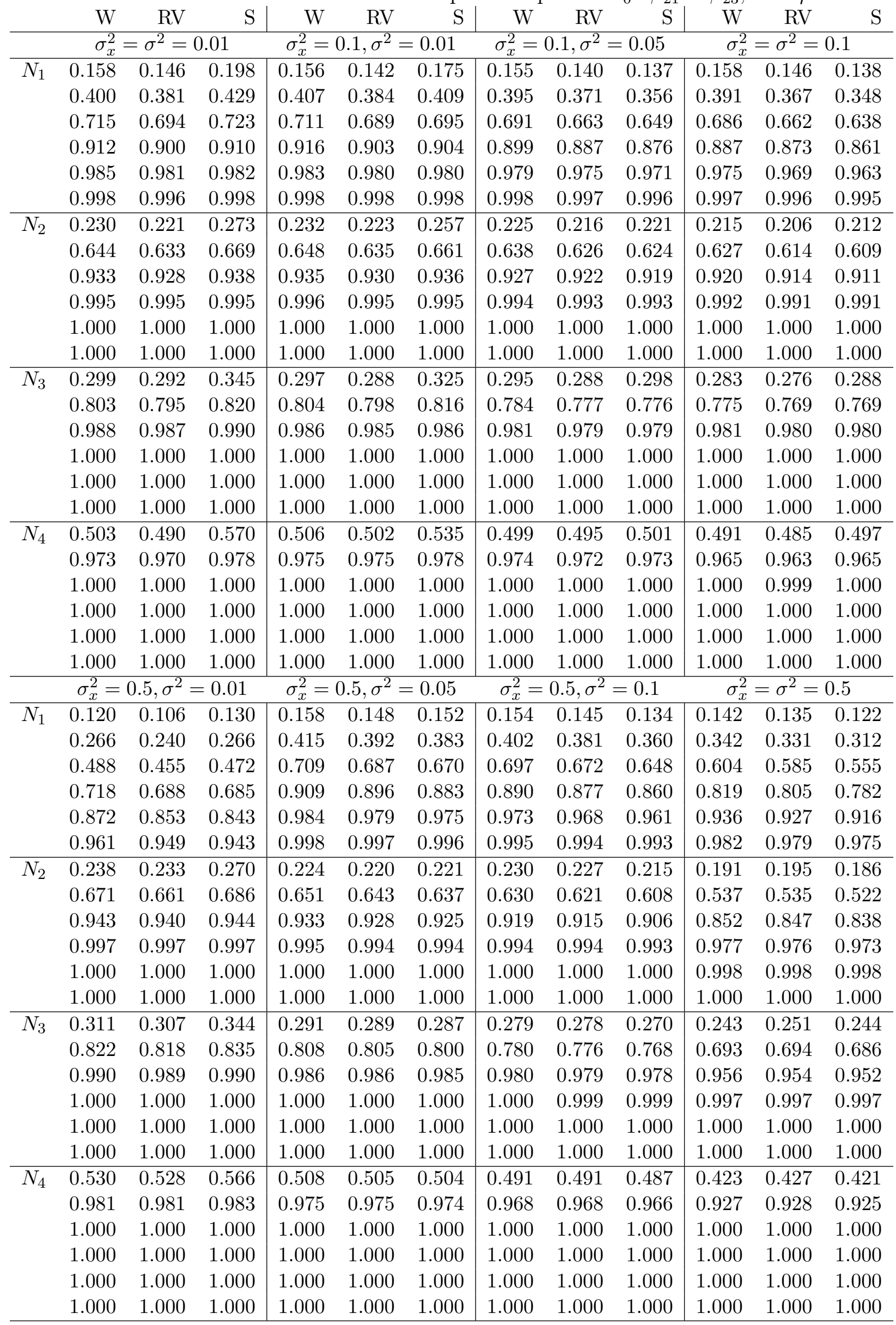


188 Análise de um modelo de regressão com erros nas variáveis multivariado com intercepto nulo

Tabela F.15: Poder simulado dos testes para a hipótese $H_{0}: \beta_{21}=\beta_{23}$, com $\mu=5$.

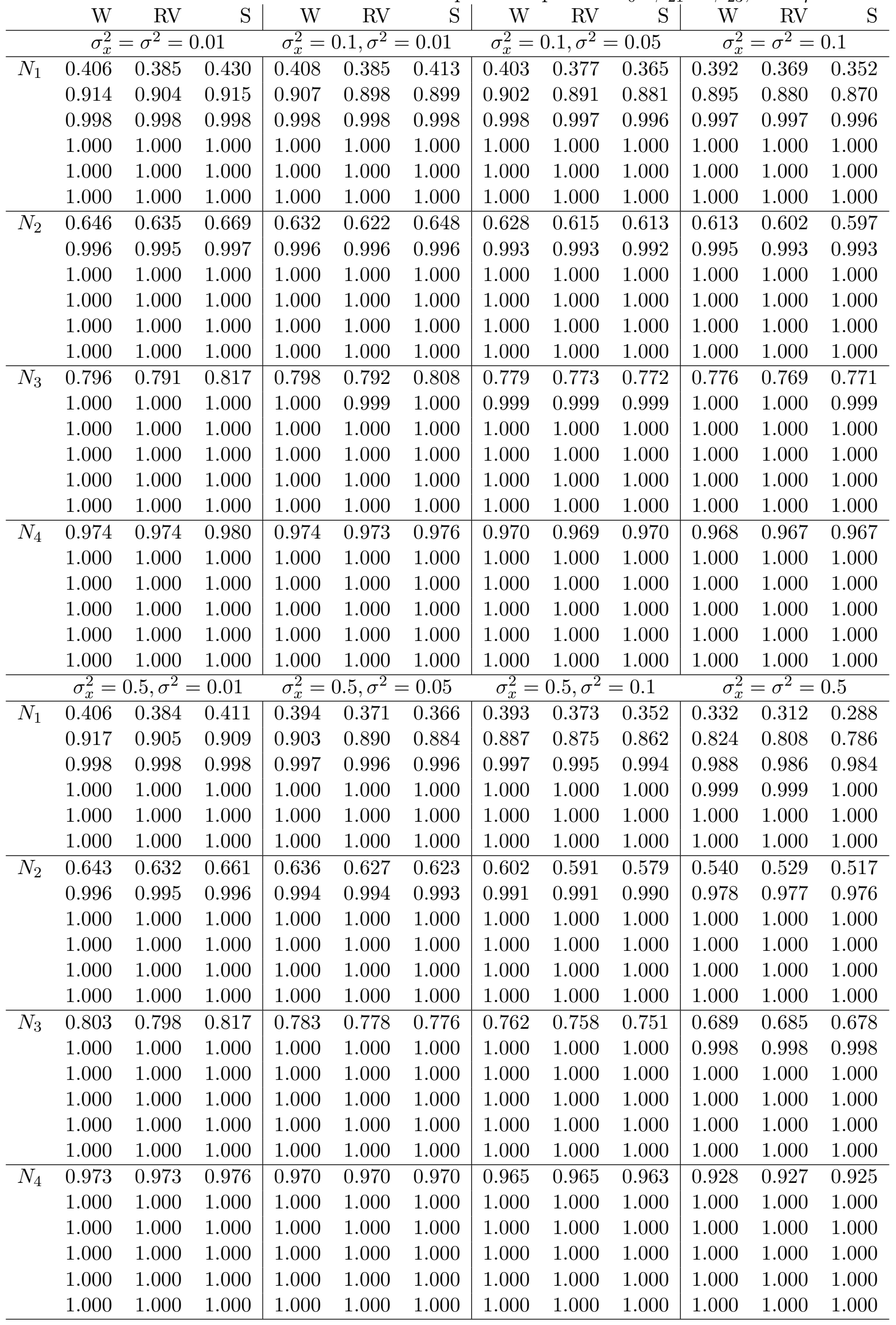


Tabela F.16: Poder simulado dos testes para a hipótese $H_{0}: \beta_{22}=\beta_{23}$, com $\mu=1$.

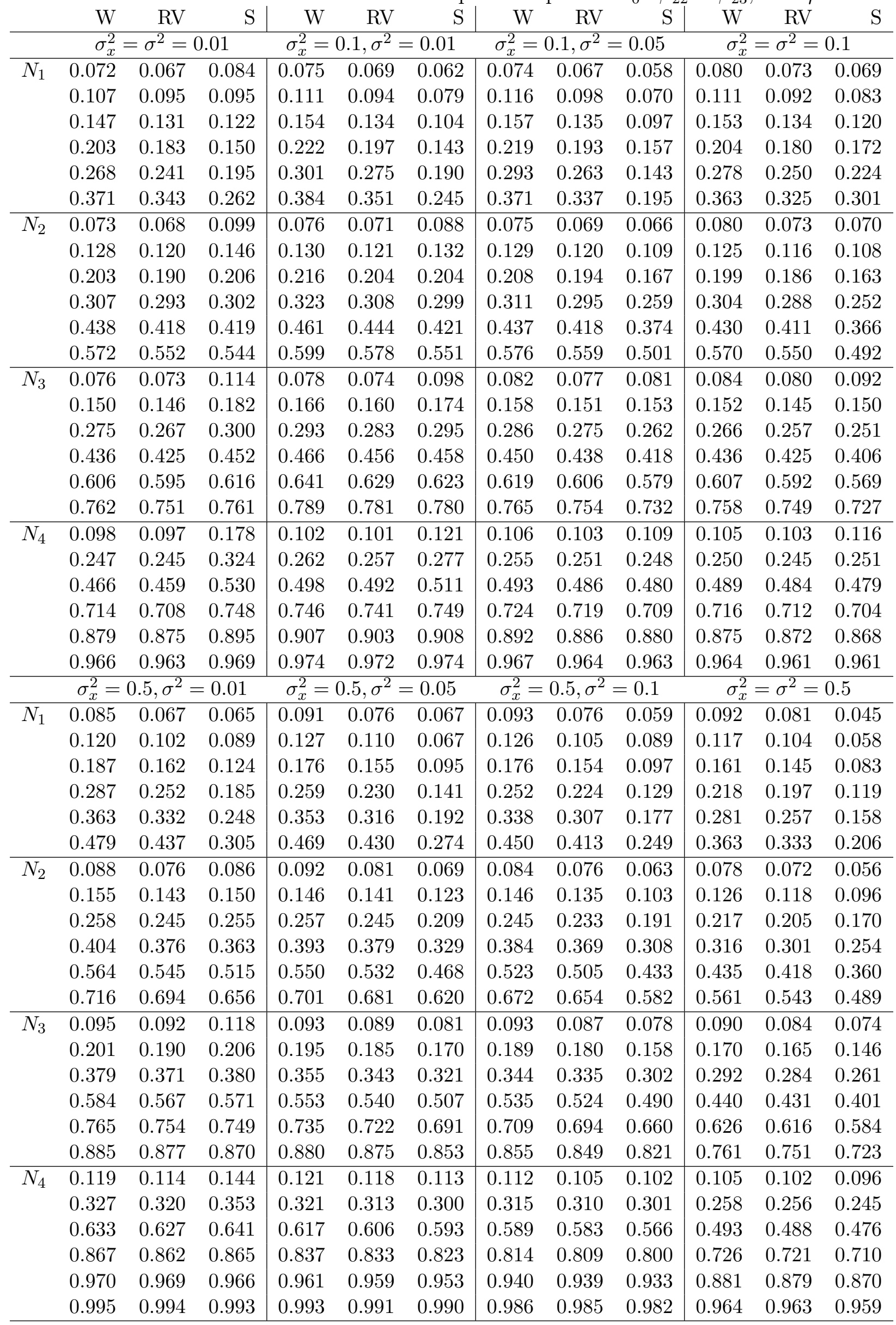


Tabela F.17: Poder simulado dos testes para a hipótese $H_{0}: \beta_{22}=\beta_{23}$, com $\mu=2.5$.

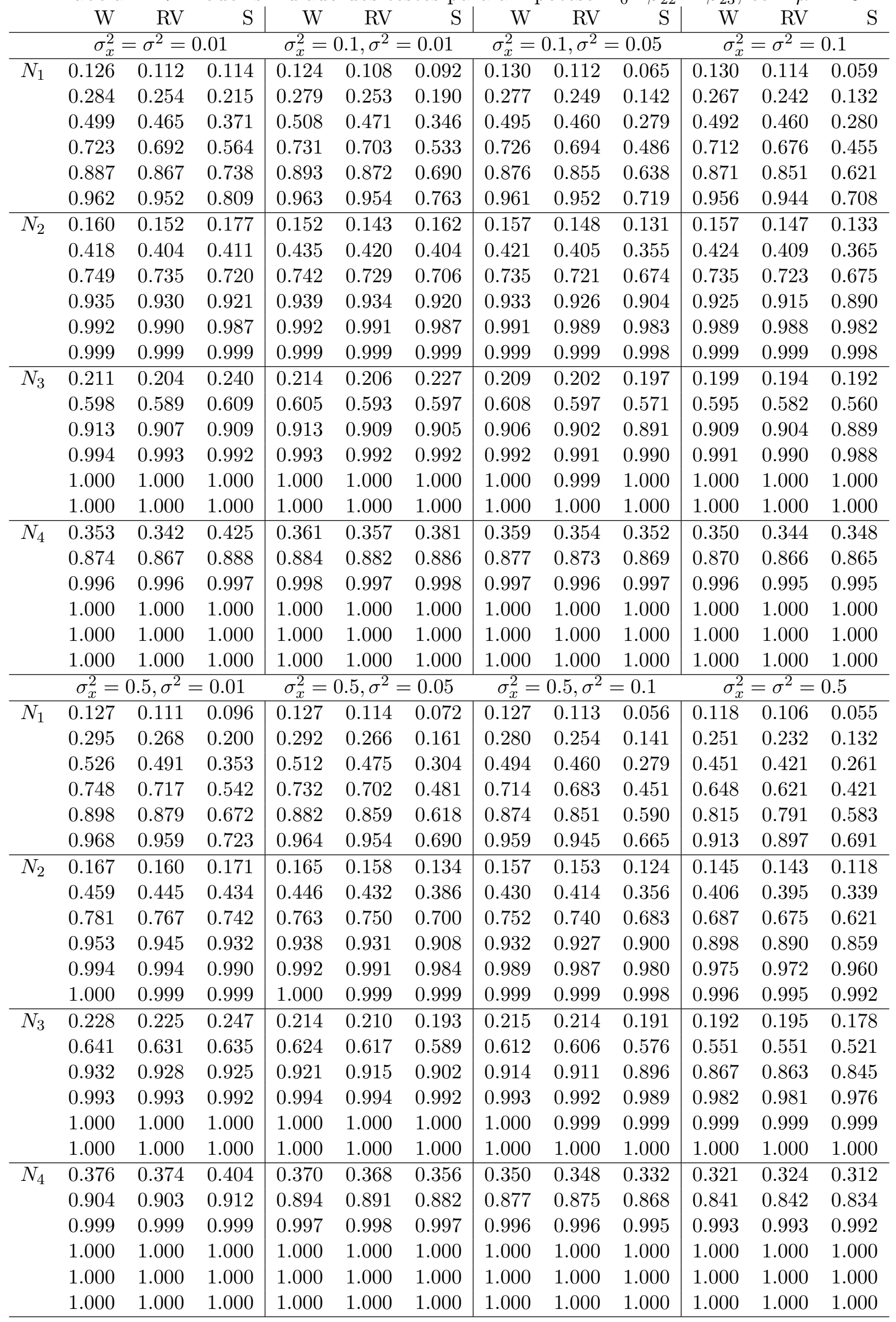


Tabela F.18: Poder simulado dos testes para a hipótese $H_{0}: \beta_{22}=\beta_{23}$, com $\mu=5$.

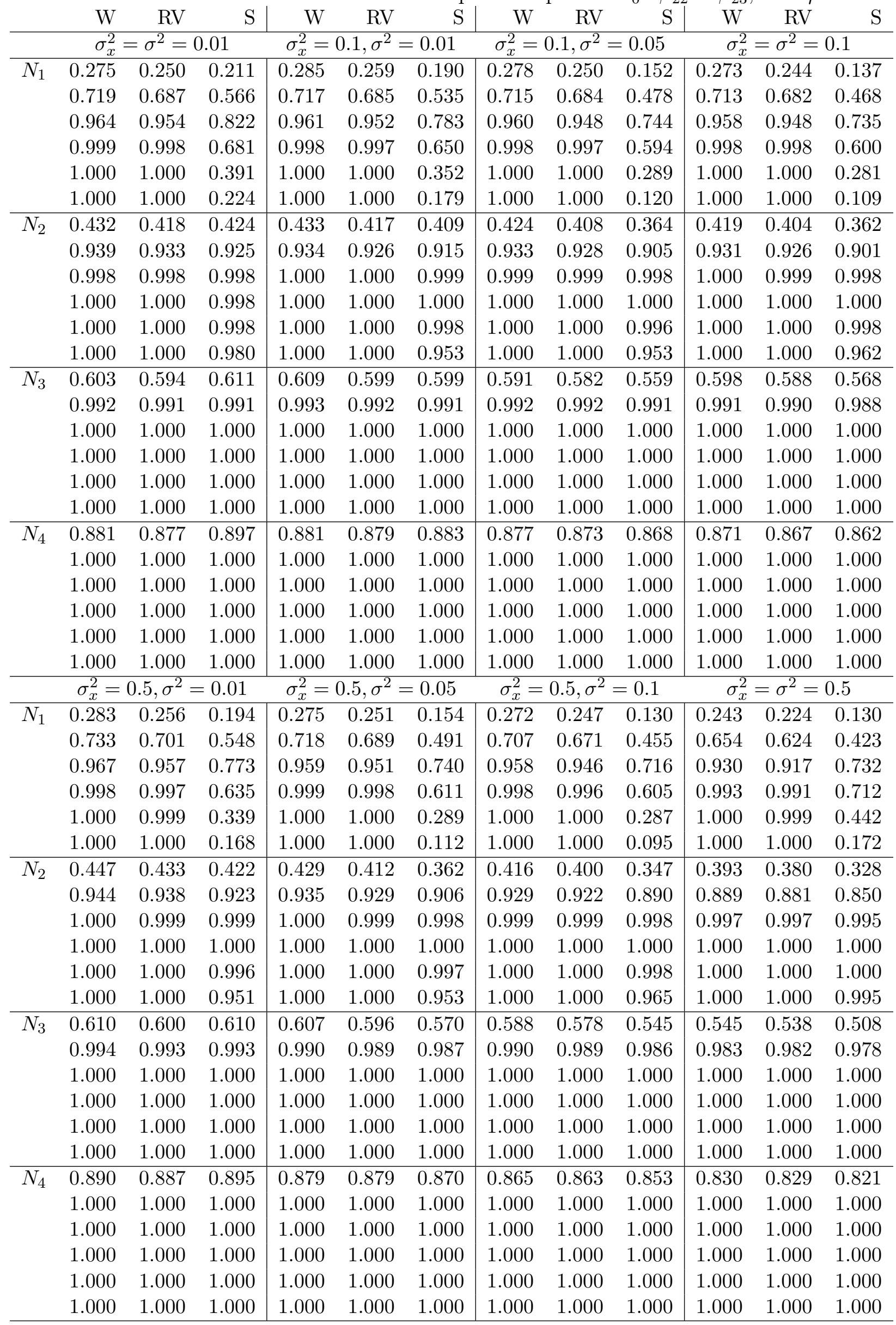


Tabela F.19: Poder simulado dos testes para a hipótese $H_{0}: \beta_{11}=\beta_{21}$, com $\mu=1$.

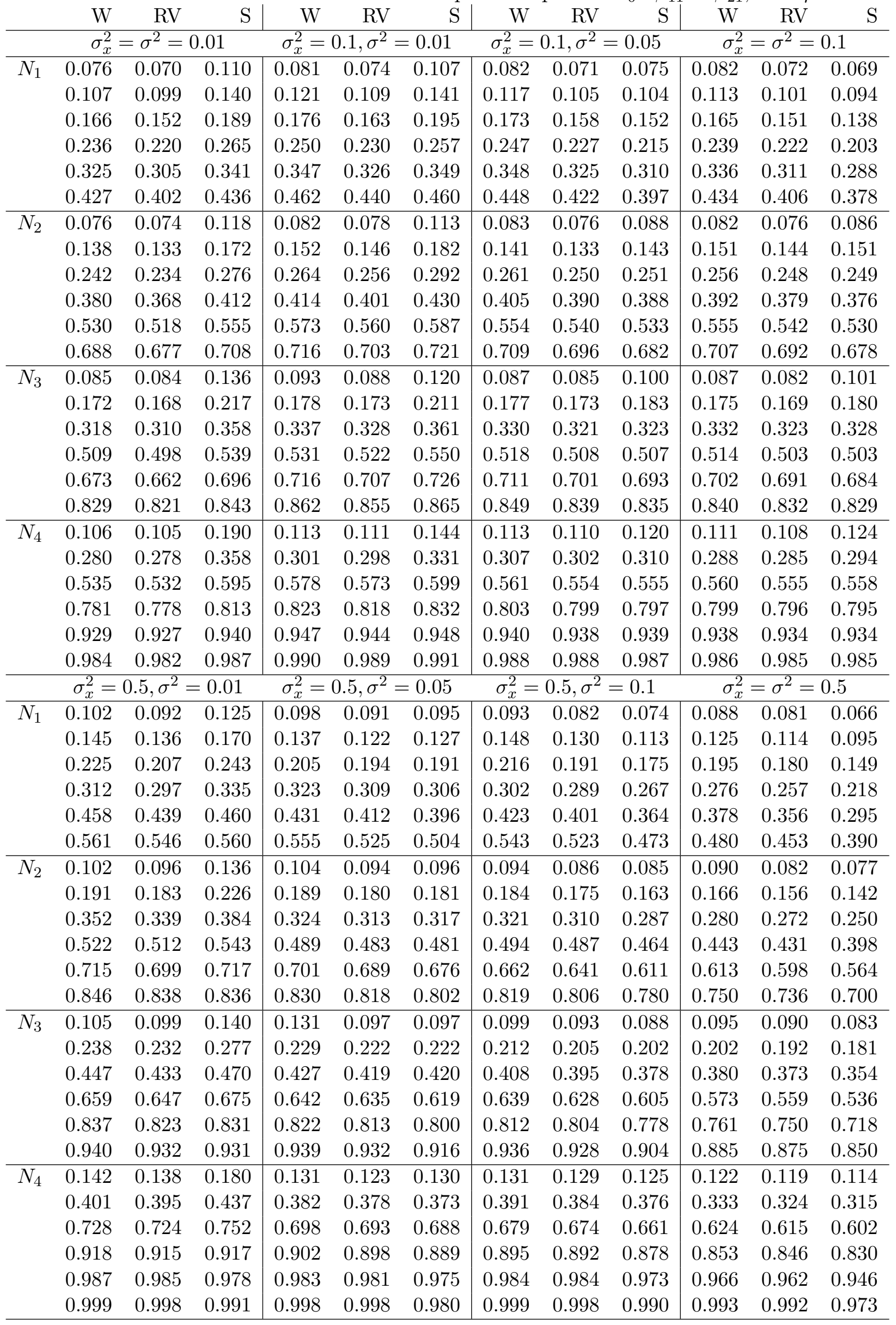


Tabela F.20: Poder simulado dos testes para a hipótese $H_{0}: \beta_{11}=\beta_{21}$, com $\mu=2.5$.

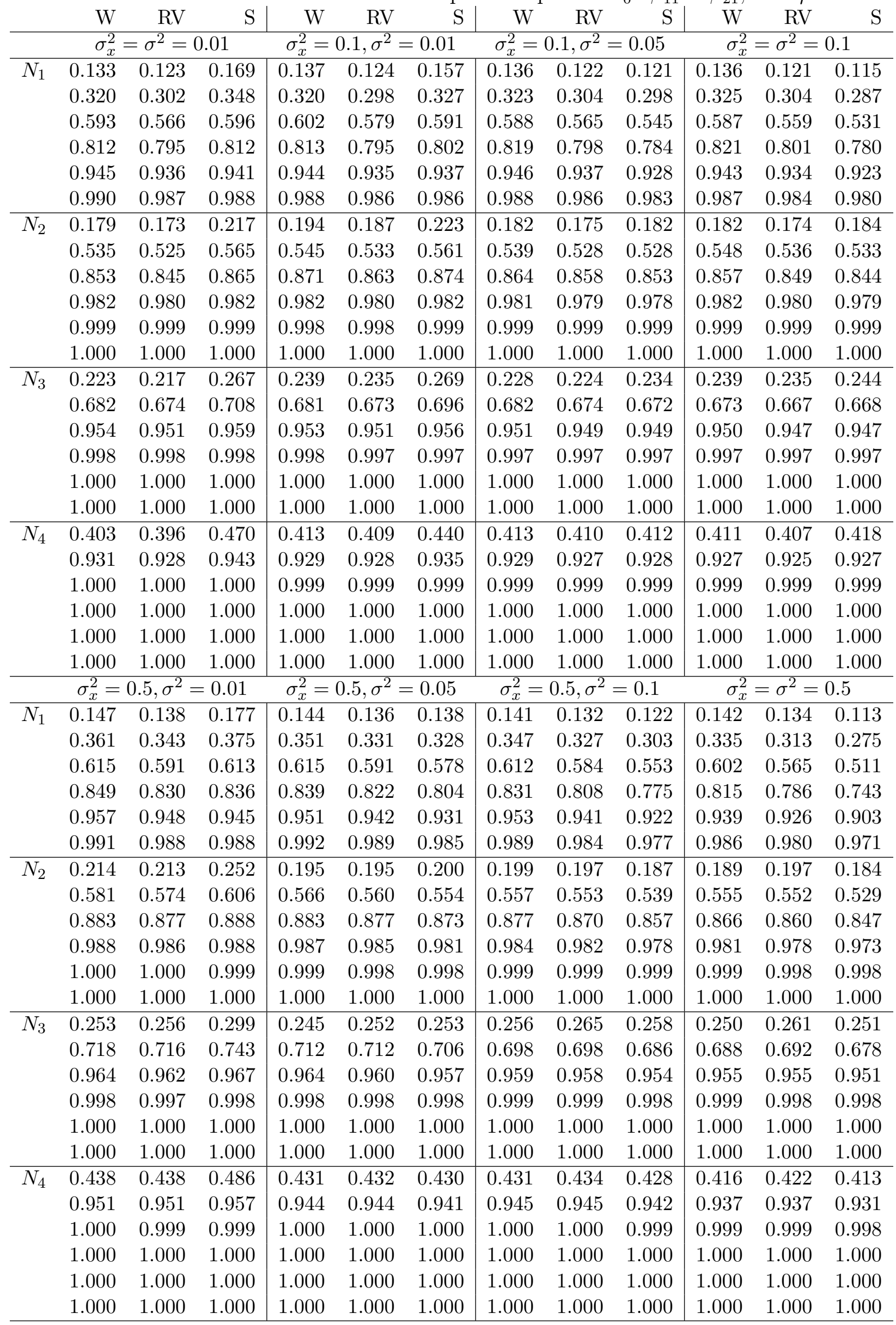


Tabela F.21: Poder simulado dos testes para a hipótese $H_{0}: \beta_{11}=\beta_{21}$, com $\mu=5$.

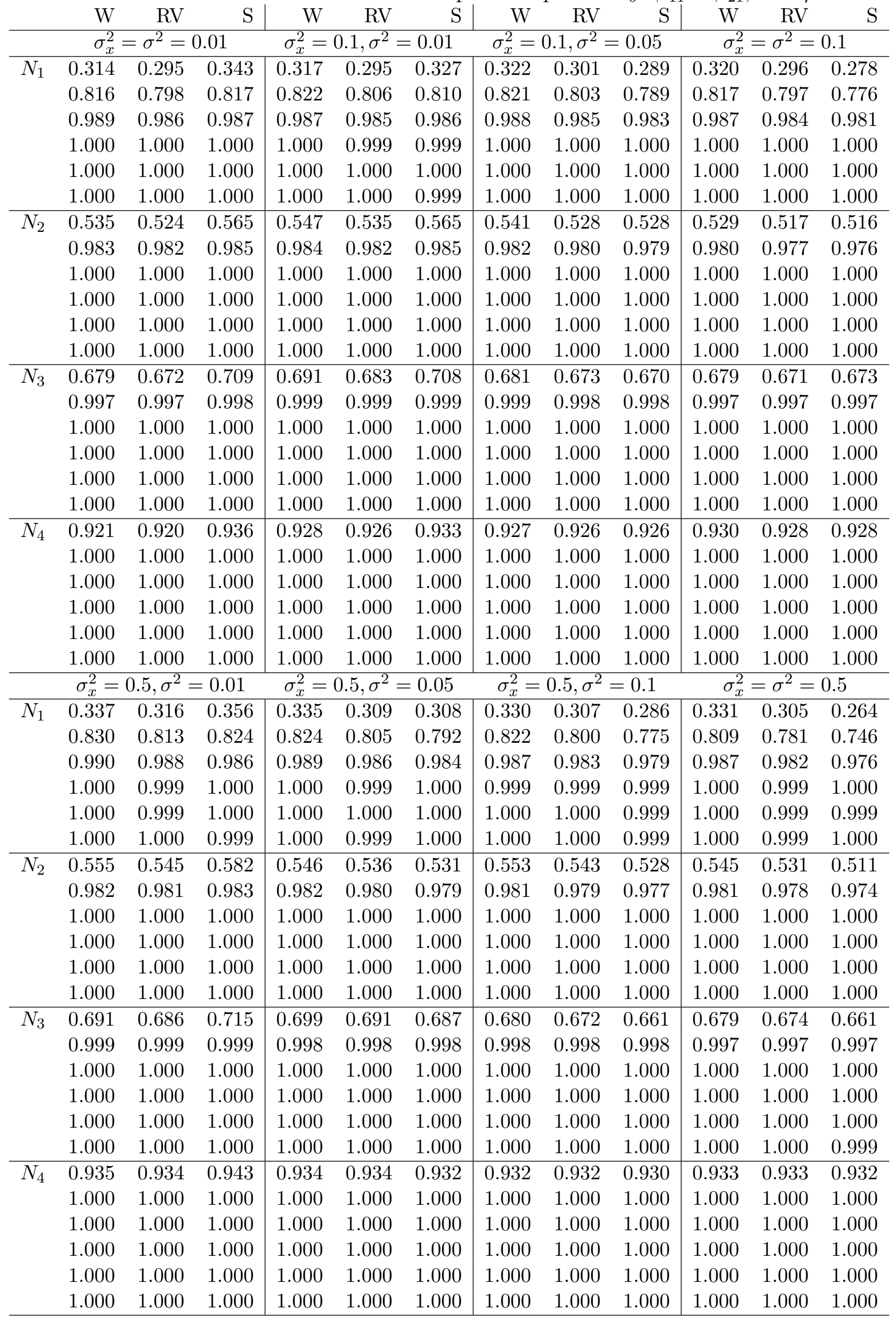


Tabela F.22: Poder simulado dos testes para a hipótese $H_{0}: \beta_{12}=\beta_{22}$, com $\mu=1$.

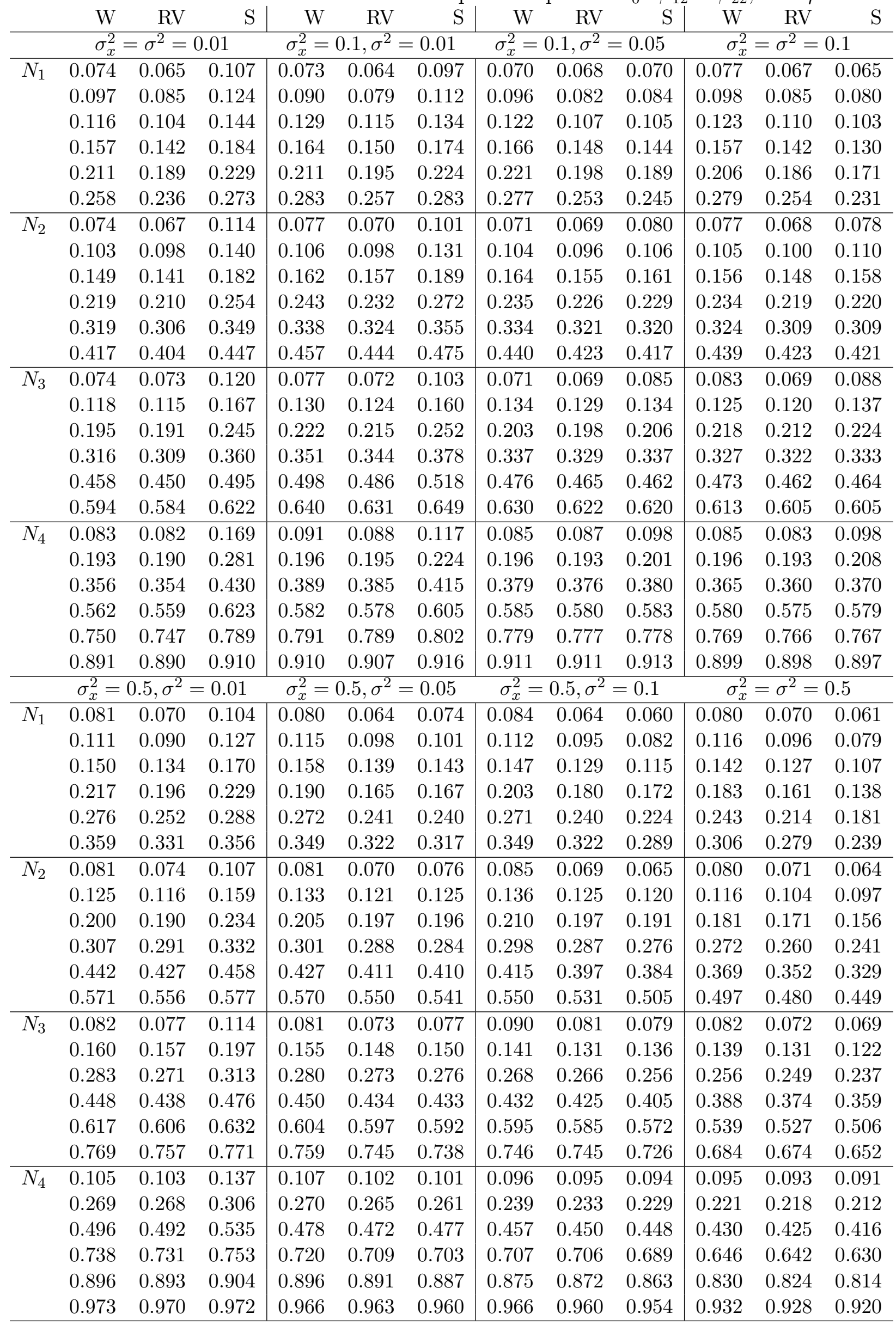


Tabela F.23: Poder simulado dos testes para a hipótese $H_{0}: \beta_{12}=\beta_{22}$, com $\mu=2.5$.

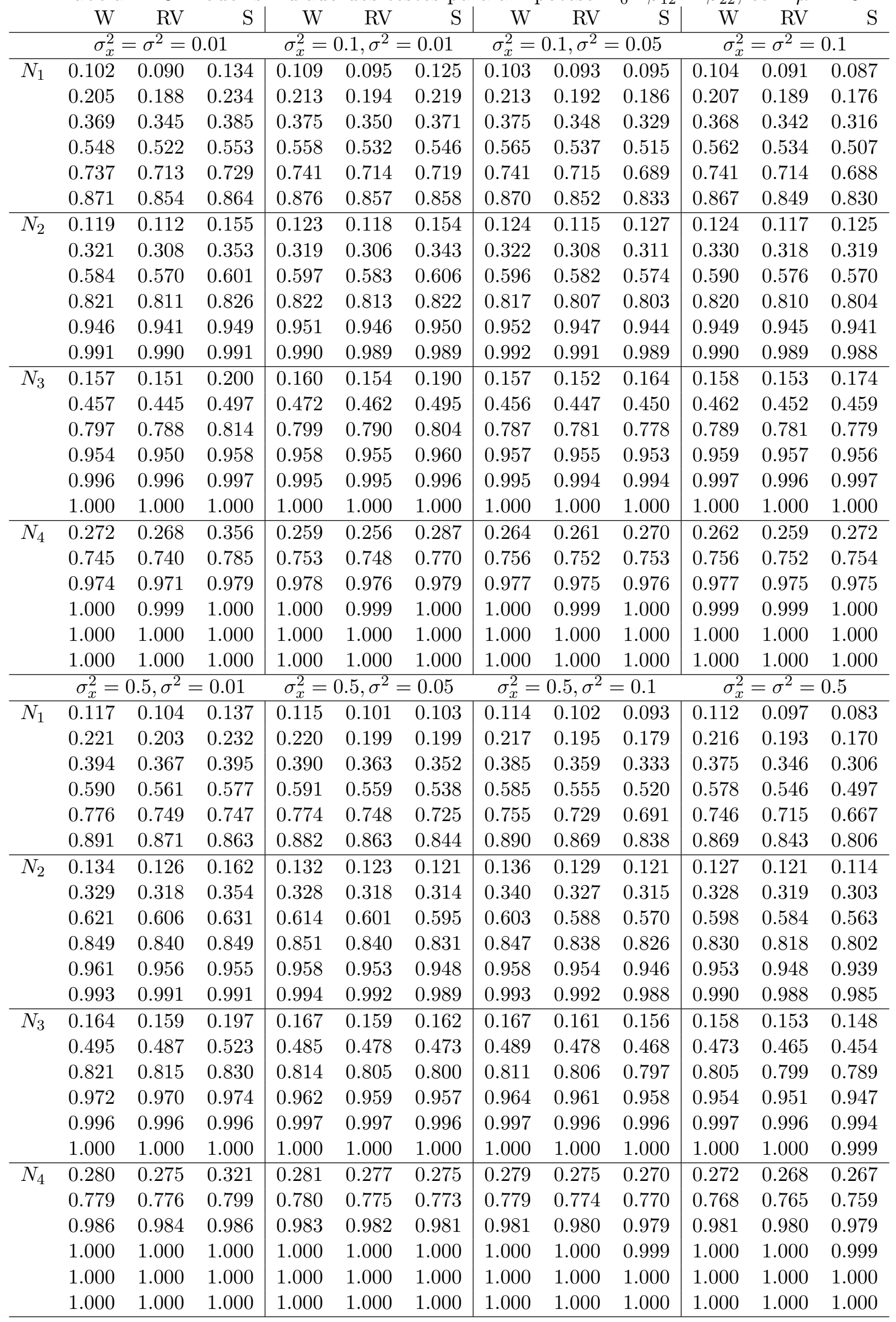


Tabela F.24: Poder simulado dos testes para a hipótese $H_{0}: \beta_{12}=\beta_{22}$, com $\mu=5$.

\begin{tabular}{|c|c|c|c|c|c|c|c|c|c|c|c|c|}
\hline & W & RV & $\mathrm{S}$ & W & RV & S & W & RV & S & W & RV & $\mathrm{S}$ \\
\hline & \multicolumn{3}{|c|}{$\sigma_{x}^{2}=\sigma^{2}=0.01$} & \multicolumn{3}{|c|}{$\sigma_{x}^{2}=0.1, \sigma^{2}=0.01$} & \multicolumn{3}{|c|}{$\sigma_{x}^{2}=0.1, \sigma^{2}=0.05$} & \multicolumn{3}{|c|}{$\sigma_{x}^{2}=\sigma^{2}=0.1$} \\
\hline \multirow[t]{6}{*}{$N_{1}$} & 0.210 & 0.190 & 0.235 & 0.202 & 0.181 & 0.212 & 0.205 & 0.185 & 0.181 & 0.198 & 0.178 & 0.167 \\
\hline & 0.564 & 0.537 & 0.571 & 0.549 & 0.524 & 0.542 & 0.561 & 0.534 & 0.517 & 0.555 & 0.527 & 0.500 \\
\hline & 0.864 & 0.847 & 0.860 & 0.864 & 0.851 & 0.853 & 0.866 & 0.851 & 0.835 & 0.865 & 0.847 & 0.828 \\
\hline & 0.982 & 0.978 & 0.979 & 0.981 & 0.978 & 0.978 & 0.982 & 0.979 & 0.974 & 0.981 & 0.977 & 0.971 \\
\hline & 0.999 & 0.998 & 0.999 & 0.999 & 0.999 & 0.998 & 1.000 & 0.999 & 0.999 & 1.000 & 0.999 & 0.998 \\
\hline & 1.000 & 1.000 & 1.000 & 1.000 & 1.000 & 1.000 & 1.000 & 1.000 & 1.000 & 1.000 & 1.000 & 1.000 \\
\hline \multirow[t]{6}{*}{$N_{2}$} & 0.316 & 0.304 & 0.353 & 0.324 & 0.312 & 0.350 & 0.323 & 0.310 & 0.311 & 0.315 & 0.301 & 0.302 \\
\hline & 0.820 & 0.810 & 0.830 & 0.828 & 0.816 & 0.827 & 0.825 & 0.815 & 0.809 & 0.819 & 0.809 & 0.805 \\
\hline & 0.991 & 0.989 & 0.991 & 0.991 & 0.990 & 0.991 & 0.990 & 0.989 & 0.988 & 0.991 & 0.989 & 0.990 \\
\hline & 1.000 & 1.000 & 1.000 & 1.000 & 1.000 & 1.000 & 1.000 & 1.000 & 1.000 & 1.000 & 1.000 & 1.000 \\
\hline & 1.000 & 1.000 & 1.000 & 1.000 & 1.000 & 1.000 & 1.000 & 1.000 & 1.000 & 1.000 & 1.000 & 1.000 \\
\hline & 1.000 & 1.000 & 1.000 & 1.000 & 1.000 & 1.000 & 1.000 & 1.000 & 1.000 & 1.000 & 1.000 & 1.000 \\
\hline \multirow[t]{6}{*}{$N_{3}$} & 0.459 & 0.451 & 0.502 & 0.461 & 0.452 & 0.485 & 0.460 & 0.452 & 0.457 & 0.450 & 0.443 & 0.450 \\
\hline & 0.956 & 0.953 & 0.960 & 0.958 & 0.957 & 0.960 & 0.958 & 0.956 & 0.955 & 0.961 & 0.959 & 0.958 \\
\hline & 0.999 & 0.999 & 0.999 & 1.000 & 1.000 & 1.000 & 1.000 & 1.000 & 1.000 & 1.000 & 1.000 & 1.000 \\
\hline & 1.000 & 1.000 & 1.000 & 1.000 & 1.000 & 1.000 & 1.000 & 1.000 & 1.000 & 1.000 & 1.000 & 1.000 \\
\hline & 1.000 & 1.000 & 1.000 & 1.000 & 1.000 & 1.000 & 1.000 & 1.000 & 1.000 & 1.000 & 1.000 & 1.000 \\
\hline & 1.000 & 1.000 & 1.000 & 1.000 & 1.000 & 1.000 & 1.000 & 1.000 & 1.000 & 1.000 & 1.000 & 1.000 \\
\hline \multirow[t]{7}{*}{$N_{4}$} & 0.752 & 0.747 & 0.789 & 0.750 & 0.747 & 0.766 & 0.760 & 0.756 & 0.757 & 0.751 & 0.747 & 0.749 \\
\hline & 1.000 & 1.000 & 1.000 & 1.000 & 1.000 & 1.000 & 0.999 & 0.999 & 0.999 & 0.999 & 0.999 & 0.999 \\
\hline & 1.000 & 1.000 & 1.000 & 1.000 & 1.000 & 1.000 & 1.000 & 1.000 & 1.000 & 1.000 & 1.000 & 1.000 \\
\hline & 1.000 & 1.000 & 1.000 & 1.000 & 1.000 & 1.000 & 1.000 & 1.000 & 1.000 & 1.000 & 1.000 & 1.000 \\
\hline & 1.000 & 1.000 & 1.000 & 1.000 & 1.000 & 1.000 & 1.000 & 1.000 & 1.000 & 1.000 & 1.000 & 1.000 \\
\hline & 1.000 & 1.000 & 1.000 & 1.000 & 1.000 & 1.000 & 1.000 & 1.000 & 1.000 & 1.000 & 1.000 & 1.000 \\
\hline & \multicolumn{3}{|c|}{$\sigma_{x}^{2}=0.5, \sigma^{2}=0.01$} & \multicolumn{3}{|c|}{$\sigma_{x}^{2}=0.5, \sigma^{2}=0.05$} & \multicolumn{3}{|c|}{$\sigma_{x}^{2}=0.5, \sigma^{2}=0.1$} & \multicolumn{3}{|c|}{$\sigma_{x}^{2}=\sigma^{2}=0.5$} \\
\hline \multirow[t]{6}{*}{$N_{1}$} & 0.212 & 0.190 & 0.222 & 0.204 & 0.186 & 0.185 & 0.214 & 0.192 & 0.177 & 0.207 & 0.186 & 0.164 \\
\hline & 0.577 & 0.547 & 0.569 & 0.560 & 0.531 & 0.516 & 0.566 & 0.535 & 0.504 & 0.555 & 0.518 & 0.478 \\
\hline & 0.877 & 0.859 & 0.862 & 0.877 & 0.859 & 0.844 & 0.868 & 0.849 & 0.825 & 0.871 & 0.852 & 0.822 \\
\hline & 0.982 & 0.977 & 0.975 & 0.984 & 0.980 & 0.975 & 0.985 & 0.981 & 0.974 & 0.982 & 0.976 & 0.968 \\
\hline & 0.999 & 0.998 & 0.998 & 0.999 & 0.998 & 0.998 & 0.999 & 0.998 & 0.997 & 0.999 & 0.998 & 0.997 \\
\hline & 1.000 & 0.999 & 0.999 & 1.000 & 1.000 & 0.999 & 1.000 & 1.000 & 0.999 & 1.000 & 0.999 & 0.998 \\
\hline \multirow[t]{6}{*}{$N_{2}$} & 0.323 & 0.311 & 0.351 & 0.322 & 0.312 & 0.311 & 0.323 & 0.313 & 0.301 & 0.320 & 0.308 & 0.292 \\
\hline & 0.832 & 0.823 & 0.840 & 0.830 & 0.819 & 0.814 & 0.825 & 0.813 & 0.801 & 0.826 & 0.814 & 0.801 \\
\hline & 0.990 & 0.989 & 0.990 & 0.992 & 0.990 & 0.990 & 0.991 & 0.990 & 0.989 & 0.990 & 0.988 & 0.986 \\
\hline & 1.000 & 1.000 & 1.000 & 1.000 & 1.000 & 1.000 & 1.000 & 1.000 & 1.000 & 1.000 & 1.000 & 1.000 \\
\hline & 1.000 & 1.000 & 1.000 & 1.000 & 1.000 & 1.000 & 1.000 & 1.000 & 1.000 & 1.000 & 1.000 & 1.000 \\
\hline & 1.000 & 1.000 & 1.000 & 1.000 & 1.000 & 1.000 & 1.000 & 1.000 & 1.000 & 1.000 & 1.000 & 1.000 \\
\hline \multirow[t]{6}{*}{$N_{3}$} & 0.464 & 0.459 & 0.494 & 0.467 & 0.460 & 0.458 & 0.462 & 0.454 & 0.446 & 0.455 & 0.450 & 0.438 \\
\hline & 0.957 & 0.956 & 0.961 & 0.960 & 0.957 & 0.956 & 0.959 & 0.956 & 0.953 & 0.959 & 0.957 & 0.954 \\
\hline & 1.000 & 1.000 & 1.000 & 1.000 & 1.000 & 1.000 & 1.000 & 1.000 & 1.000 & 1.000 & 0.999 & 0.999 \\
\hline & 1.000 & 1.000 & 1.000 & 1.000 & 1.000 & 1.000 & 1.000 & 1.000 & 1.000 & 1.000 & 1.000 & 1.000 \\
\hline & 1.000 & 1.000 & 1.000 & 1.000 & 1.000 & 1.000 & 1.000 & 1.000 & 1.000 & 1.000 & 1.000 & 1.000 \\
\hline & 1.000 & 1.000 & 1.000 & 1.000 & 1.000 & 1.000 & 1.000 & 1.000 & 1.000 & 1.000 & 1.000 & 0.999 \\
\hline \multirow[t]{6}{*}{$\overline{N_{4}}$} & 0.763 & 0.759 & 0.784 & 0.756 & 0.753 & 0.753 & 0.763 & 0.761 & 0.756 & 0.748 & 0.747 & 0.744 \\
\hline & 1.000 & 1.000 & 1.000 & 0.999 & 0.999 & 0.999 & 1.000 & 1.000 & 1.000 & 0.999 & 0.999 & 0.999 \\
\hline & 1.000 & 1.000 & 1.000 & 1.000 & 1.000 & 1.000 & 1.000 & 1.000 & 1.000 & 1.000 & 1.000 & 1.000 \\
\hline & 1.000 & 1.000 & 1.000 & 1.000 & 1.000 & 1.000 & 1.000 & 1.000 & 1.000 & 1.000 & 1.000 & 1.000 \\
\hline & 1.000 & 1.000 & 1.000 & 1.000 & 1.000 & 1.000 & 1.000 & 1.000 & 1.000 & 1.000 & 1.000 & 1.000 \\
\hline & 1.000 & 1.000 & 1.000 & 1.000 & 1.000 & 1.000 & 1.000 & 1.000 & 1.000 & 1.000 & 1.000 & 1.000 \\
\hline
\end{tabular}


Tabela F.25: Poder simulado dos testes para a hipótese $H_{0}: \beta_{13}=\beta_{23}$, com $\mu=1$.

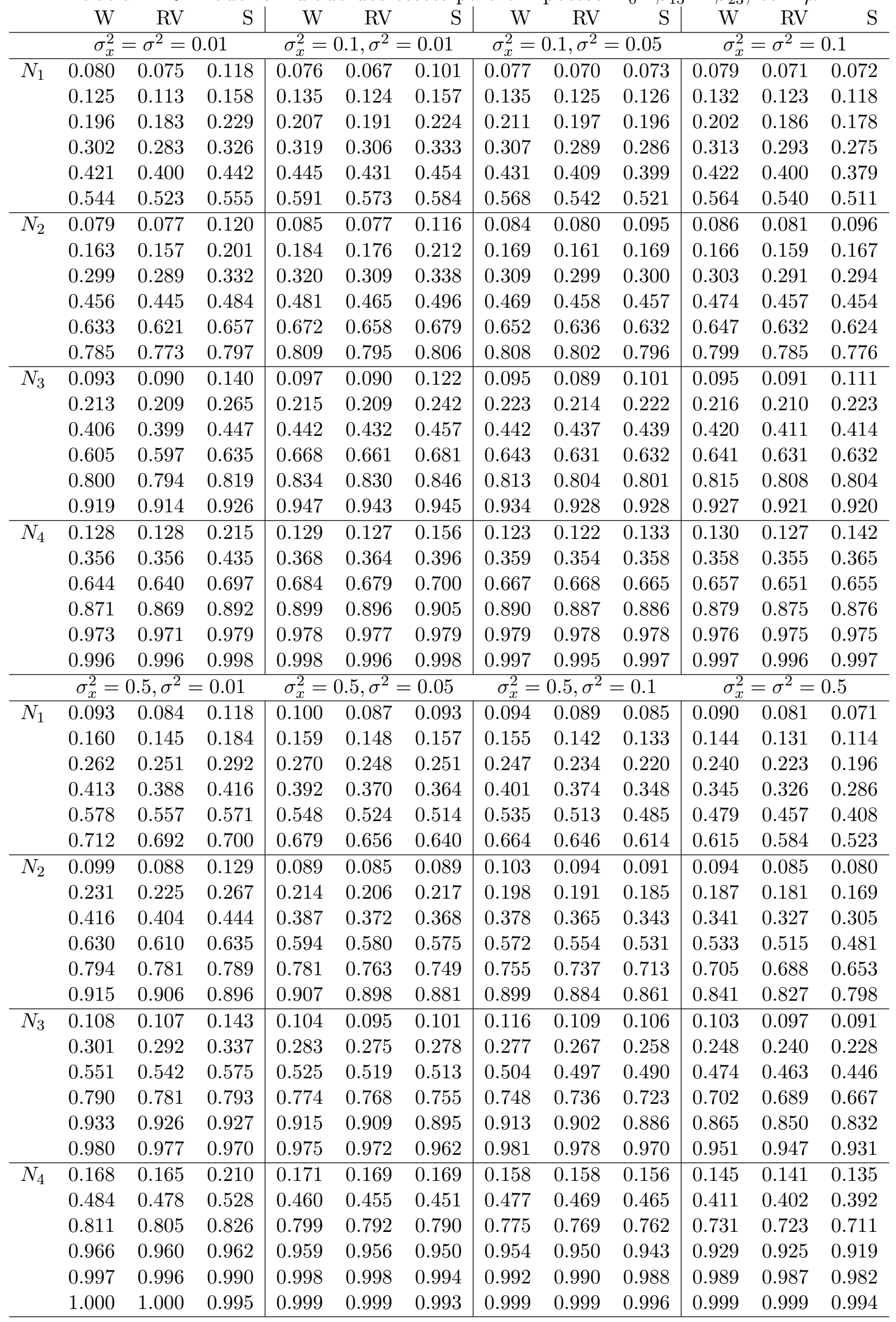


Tabela F.26: Poder simulado dos testes para a hipótese $H_{0}: \beta_{13}=\beta_{23}$, com $\mu=2.5$.

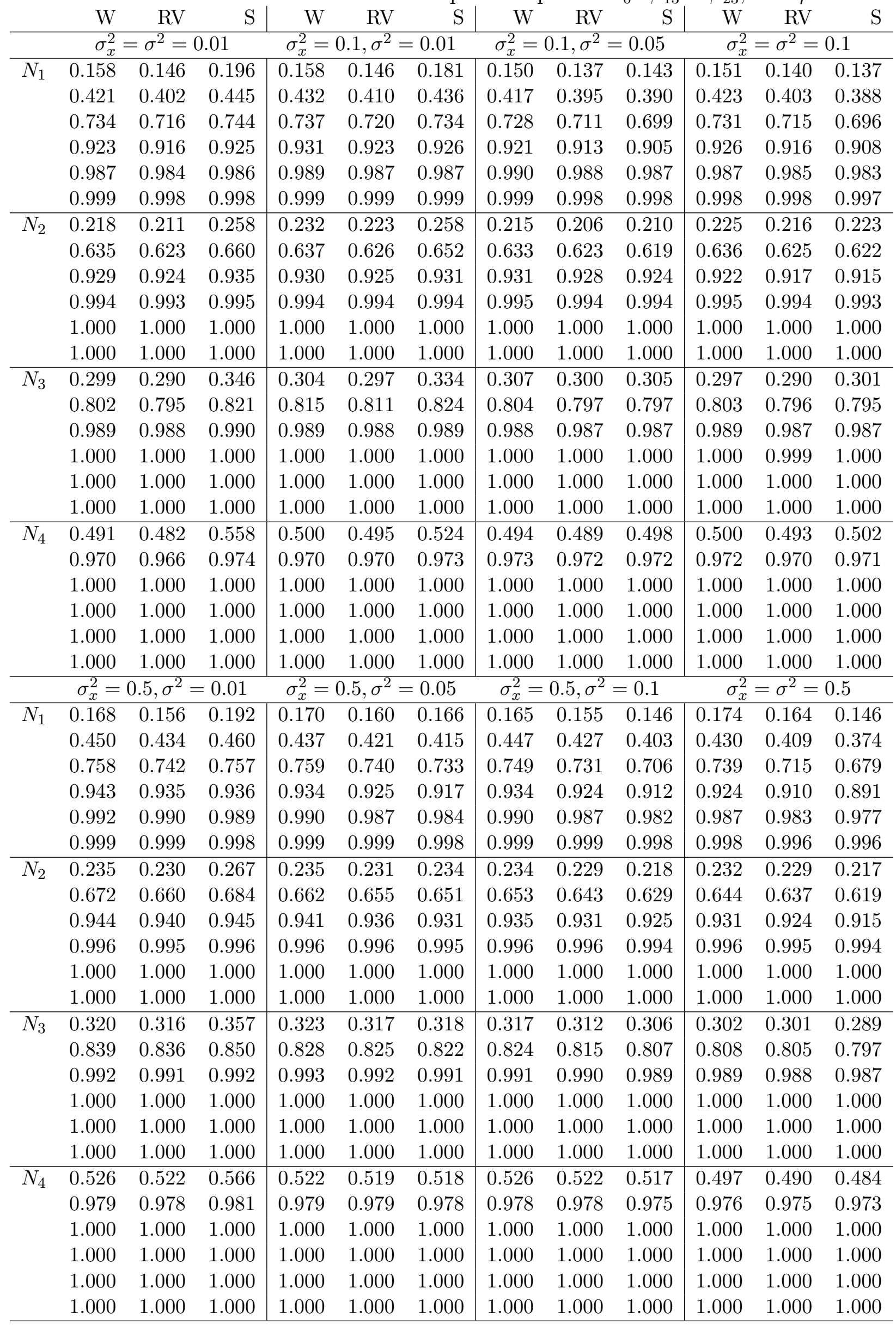


Tabela F.27: Poder simulado dos testes para a hipótese $H_{0}: \beta_{13}=\beta_{23}$, com $\mu=5$.

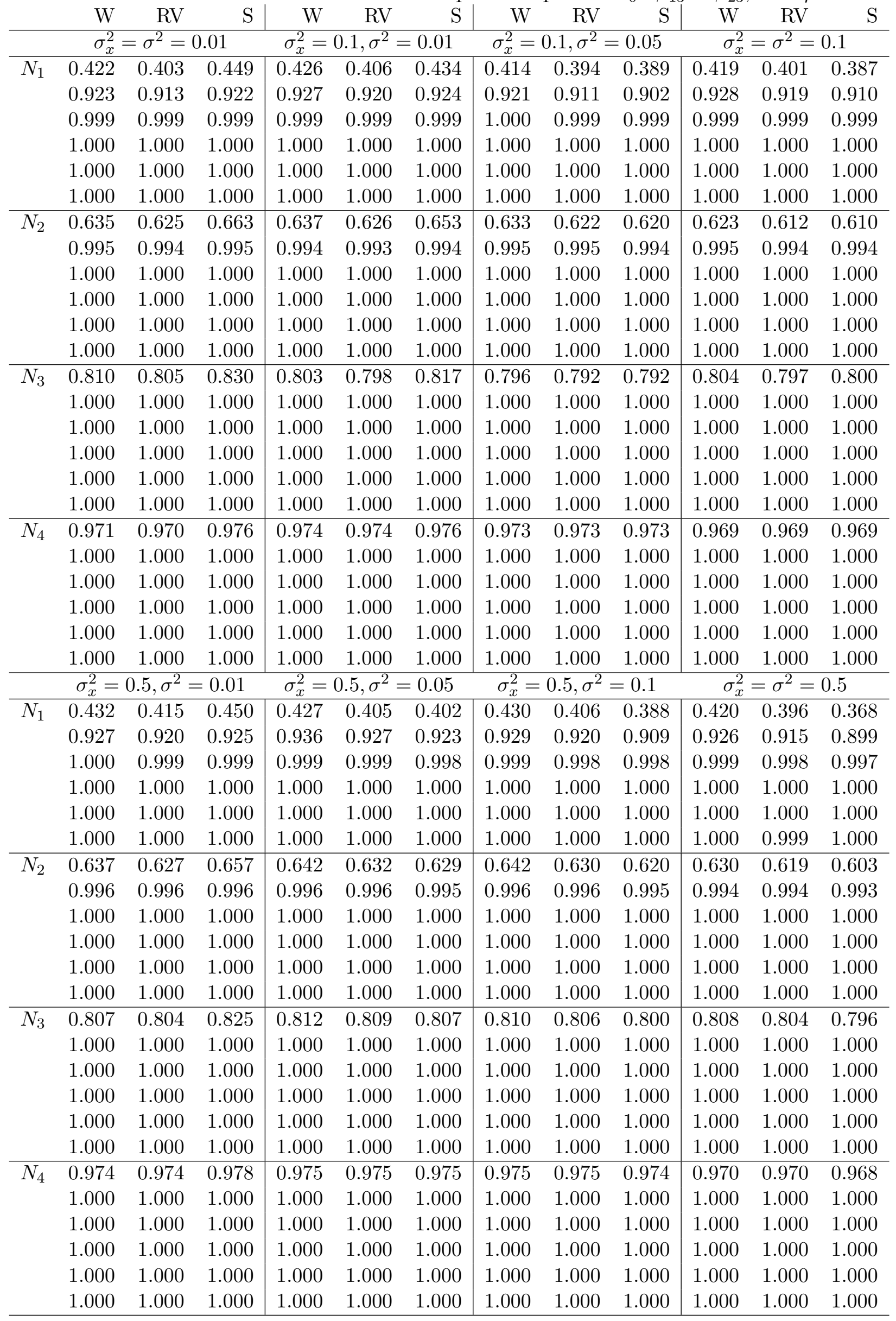

